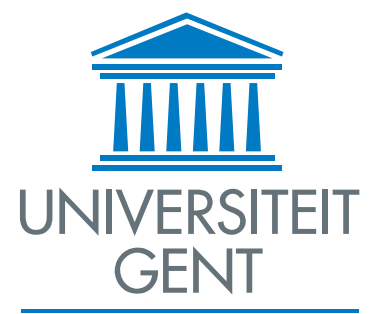

Faculteit Wetenschappen

Vakgroep Subatomaire en Stralingsfysica

Academiejaar 2005-2006

\title{
Transverse Spin Structure of the Proton Studied in Semi-inclusive DIS
}

\author{
Transversale Spinstructuur van het Proton bestudeerd in Semi-inclusieve \\ Diep-inelastische Verstrooiing
}

Promotor: Prof. Dr. D. Ryckbosch

Proefschrift ingediend tot het verkrijgen van de graad van

Doctor in de Wetenschappen, groep Natuurkunde

door

Ulrike Elschenbroich 



\section{Contents}

1 Introduction 1

2 The Inner Structure of the Nucleon $\quad 5$

2.1 Inclusive Deep-Inelastic Scattering . . . . . . . . . . . . . . . . . . 5

2.1.1 The Unpolarised Cross Section . . . . . . . . . . . . . . . . . . . . . . . . . 9

2.1.2 The Polarised Cross Section . . . . . . . . . . . . . . . . . . . . . . . . . . . 9

2.1.3 Forward Virtual Compton Scattering . . . . . . . . . . . . . . . . . . . . 11

2.2 The Quark Parton Model . . . . . . . . . . . . . . . . . . . . . . . . . . . . . . . . 12

2.3 Quantum Chromodynamics ... . . . . . . . . . . . . . . . . . . . . . . . . . . . . 14

2.4 Inclusive DIS in the QCD Improved QPM . . . . . . . . . . . . . . . . . . . . . 16

2.4 .1 Transversity . . . . . . . . . . . . . . . . . . . . . . . . 17

2.4.2 Transverse-Momentum Dependent Distribution Functions . . . . . . . 18

2.4.3 Gauge Link and T-Odd Distribution Functions . . . . . . . . . . . . . . . 20

2.4.4 Subleading-Twist Distribution Functions . . . . . . . . . . . . . . . . . . 21

2.5 Semi-Inclusive Deep-Inelastic Scattering . . . . . . . . . . . . . . . . . . . 22

2.5.1 Fragmentation Functions . . . . . . . . . . . . . . . . . . . . . . . . 22

2.5.2 Cross Sections and Azimuthal Asymmetry Moments . . . . . . . . . . . 24

2.5 .3 The Purity Formalism . . . . . . . . . . . . . . . . . . . . . . . . . . . . . . 29

2.6 Universality . . . . . . . . . . . . . . . . . . . . . . . . . . . . . . 30

3 The HERMES Experiment 33

3.1 The Hera Positron Beam . . . . . . . . . . . . . . . . . . . . . . . . . . . . . . . . . . . . 33

3.2 The Target . . . . . . . . . . . . . . . . . . . . . . . . . . . . . . . . . . . . . . . . 34

3.2.1 The Atomic Beam Source . . . . . . . . . . . . . . . . . . . . . . . . . . . 35

3.2 .2 The Storage Cell . . . . . . . . . . . . . . . . . . . . . . . . . . . . . 36

3.2 .3 The Target Gas Analyser . . . . . . . . . . . . . . . . . . . . . . . . . . . . . 37

3.2 .4 The Breit-Rabi Polarimeter . . . . . . . . . . . . . . . . . . . . . . . 37

3.2 .5 The Target Magnet . . . . . . . . . . . . . . . . . . . . . . . . . . . . . . . . . . . . . . . 39

3.2 .6 The Unpolarised Gas Feed System . . . . . . . . . . . . . . . . . . . . . . . . . 39

3.3 The Spectrometer . . . . . . . . . . . . . . . . . . . . . . . . . . . . . . . . . . . . 40

3.3.1 The Tracking System . . . . . . . . . . . . . . . . . . . . . . . . . . . . . . 40

3.3.2 Particle Identification Detectors . . . . . . . . . . . . . . . . . . . 43

3.3.3 The Luminosity Monitor . . . . . . . . . . . . . . . . . . . . . . . . . . . . . 47

3.3 .4 The Trigger System . . . . . . . . . . . . . . . . . . . . . . . . . . . . . . . . 48

3.4 Data Acquisition and Processing . . . . . . . . . . . . . . . . . . . . . . . . . . . 48 
4 Azimuthal Asymmetry Moments $\quad 51$

4.1 Data Quality . . . . . . . . . . . . . . . . . . . . . . . . . . . . . . . . . . . . . . . . 51

4.2 Event Selection . . . . . . . . . . . . . . . . . . . . . . . . . . . . . 52

4.2 .1 Geometry Cuts . . . . . . . . . . . . . . . . . . . . . . . . . . 53

4.2 .2 Particle ldentification . . . . . . . . . . . . . . . . . 53

4.2 .3 Kinematic Cuts . . . . . . . . . . . . . . . . . . . . . . . . . . . . . 58

4.3 The Extraction of Asymmetry Moments . . . . . . . . . . . . . . . . . . . . . 61

4.4 Systematic Studies . . . . . . . . . . . . . . . . . . . . . . . . . . . . . . . . 65

4.4.1 Event Number Distributions in the Azimuthal Bins . . . . . . . . . . . . 65

4.4.2 Compatibility of Data Taking Periods . . . . . . . . . . . . . . . . 66

4.4 .3 Luminosity Normalisation . . . . . . . . . . . . . . . . . . . . . . . . . 67

4.4.4 Additional Sine Moments and Unpolarised Cosine Moments . . . . . . 69

4.4 .5 Beam Polarisation . . . . . . . . . . . . . . . . . . . . . . . 72

4.4.6 Hadron Classification with the $\mathrm{RICH} \ldots \ldots \ldots$

4.4.7 Target Magnet Field Correction . . . . . . . . . . . . . . . . . . . . . . . 74

4.4 .8 Misalignment . . . . . . . . . . . . . . . . . . . . . . . . . . . . . . . . . . . . 75

4.4 .9 Fake Asymmetry Amplitudes . . . . . . . . . . . . . . . . . . . . . . . 77

4.5 The Correction for Neutral Pion Combinatorial Background . . . . . . . . . . 78

5 Monte Carlo Studies $\quad \mathbf{8 1}$

5.1 The Unpolarised Monte Carlo Generators . . . . . . . . . . . . . . . . . . . . . 81

5.1.1 Asymmetry Amplitude Scan . . . . . . . . . . . . . . . . . . . . . . 85

5.1 .2 Binning Effect . . . . . . . . . . . . . . . . . . . . . . . . 86

5.1 .3 Spectrometer Acceptance Effects . . . . . . . . . . . . . . . . . . . . . 90

5.1.4 Target Magnet Field Correction . . . . . . . . . . . . . . . . . . . . . . . 90 90

5.1 .5 Radiative Effects . . . . . . . . . . . . . . . . . . . . . . . . . . . . . . . . . . 92

5.1 .6 Detector Misalignment . . . . . . . . . . . . . . . . . . . . . . . . . . . . . . . . 92

$5.1 .7 \quad P_{h \perp}$-Weighted Asymmetry Amplitudes . . . . . . . . . . . . . . . . . . 94

5.1 .8 Estimated Systematic Uncertainty . . . . . . . . . . . . . . . . . . . . . . . . . . 94

5.2 The Monte Carlo Generator gmc_trans . . . . . . . . . . . . . . . . . . . . . . 96

5.2.1 Unweighted Asymmetry Amplitudes . . . . . . . . . . . . . . . . . . . . . . 99

5.2.2 $P_{h \perp}$-Weighted Asymmetry Amplitudes . . . . . . . . . . . . . . . . 107

5.3 Concluding Remarks . . . . . . . . . . . . . . . . . . . . . . . . . . 107

6 Contribution from Exclusively Produced Vector Mesons 109

6.1 The Vector Meson Dominance Model . . . . . . . . . . . . . . . . . . . . . . . . 109

6.2 Exclusively Produced Vector Mesons as Background Events . . . . . . . . . . 111

6.2 .1 The Vector Meson Fraction . . . . . . . . . . . . . . . . . . . . . . 111

6.2 .2 The Transfer Coefficients . . . . . . . . . . . . . . . . . . . . . . . . . . . . . 113

6.2.3 Asymmetry Amplitudes Acquired during the Decay Process . . . . . . 117

6.2.4 Asymmetry Amplitudes of Exclusively Produced $\rho^{0} \ldots \ldots$. . . . . . . . . 121

6.2 .5 Interpretive Uncertainties . . . . . . . . . . . . . . . . . . 126

6.3 Vector Meson Suppression . . . . . . . . . . . . . . . . . . . . . . . . . . . . . . 127

6.3.1 Suppression of Exclusively Produced $\rho^{0}$ Mesons . . . . . . . . . . . . . . . 128

6.3.2 Suppression of Exclusively Produced $\omega$ Mesons . . . . . . . . . . . . . . . 133

6.4 Amplitudes of the Pion-Yield-Difference Asymmetry . . . . . . . . . . . . . . . 135 
6.5 Correction for the Vector Meson Contribution . . . . . . . . . . . . . . . . . 136

7 Separation of the Photon-Axis Asymmetry Moments 139

7.1 Spin Components in the Two Reference Systems . . . . . . . . . . . . . . . . . . 139

7.2 The Reviewed Analysis of the Longitudinal Data . . . . . . . . . . . . . . . . . 140

7.3 The Subleading-Twist Cross Section Component . . . . . . . . . . . . . . . . . 142

7.3.1 Exclusively Produced Vector Meson Contribution . . . . . . . . . . . . . 145

7.4 Contribution to the Lepton-Axis Collins and Sivers Amplitudes . . . . . . . . . 146

8 Interpretation of the Asymmetry Moments $\quad 149$

8.1 The Makins Relation . . . . . . . . . . . . . . . . . . . . . . . . . . . . . . . . . . . 149

8.2 Virtual-Photon Asymmetry Moments . . . . . . . . . . . . . . . . . . . . . . . . 154

8.3 The Collins Moments . . . . . . . . . . . . . . . . . . . . . . . . . . . . . . 155

8.4 The Sivers Moments . . . . . . . . . . . . . . . . . . . . . . . . . . . . . . . . . 162

8.5 Extraction of the Quark Distribution Functions . . . . . . . . . . . . . . . 168

8.5.1 Sivers Distribution Function . . . . . . . . . . . . . . . . . . . 168

8.5 .2 Transversity . . . . . . . . . . . . . . . . . . . . . . . . . 170

8.6 Other Options to Measure the Transversity and Sivers Functions . . . . . . . . 171

8.6.1 Possibilities at Hermes . . . . . . . . . . . . . . . . . . . . . . . . . . . 171

8.6.2 Other Semi-Inclusive DIS Experiments . . . . . . . . . . . . . . . . . . 175

8.6.3 Hadron-Hadron Scattering Experiments . . . . . . . . . . . . . . . . 176

$9 \begin{array}{ll}9 \text { Summary and Conclusion } & 177\end{array}$

$\begin{array}{ll}\text { A Conventions } & 181\end{array}$

A. 1 Light-cone Vectors . . . . . . . . . . . . . . . . . . . . . . . . . . . 181

A.2 Dirac Matrices . . . . . . . . . . . . . . . . . . . . . . . . . . . . . . . . . . . . 182

B The Luminosity Constants for the Year $2002 \quad 183$

C Angular Decay Distribution for Exclusive $\rho^{0}$ Production $\quad 185$

C. 1 The Decay Angles . . . . . . . . . . . . . . . . . . . . . . . . . . . 185

C.2 The Terms of the Angular Decay Distribution . . . . . . . . . . . . . . . . . . 186

$\begin{array}{ll}\text { D Asymmetry Estimate Using Positivity Limits } & 189\end{array}$

$\begin{array}{llr}\text { E } & \text { Additional Figures } & 191\end{array}$

$\begin{array}{lll}\text { F } & \text { Additional Tables } & 195\end{array}$

$\begin{array}{lr}\text { Samenvatting } & 219\end{array}$

$\begin{array}{ll}\text { Acknowledgements } & 223\end{array}$ 

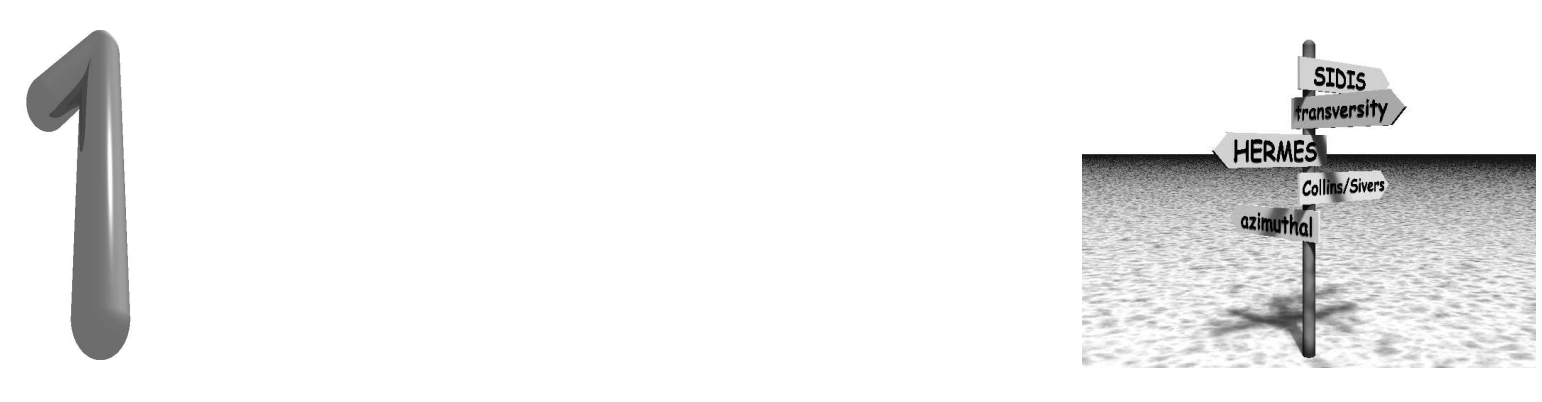

\section{Introduction}

In the investigation of the structure of the nucleon, the question about the origin of the nucleon spin is still not completely solved. The contribution of the spin of the partons, the constituents of the nucleon, is given by the helicity distribution. This distribution is the difference of the numbers of partons with their spins aligned and antialigned with the nucleon spin in a frame in which the nucleon has a very large momentum (infinite momentum frame) and the direction of its spin is longitudinal to its momentum. In the naivve quark parton model the partons are identified with the quarks and their spins add up to yield the total nucleon spin. However, measurements at the CERN and SLAC laboratories yielded helicity distributions of all quarks of less than 30\% (see [Ant00] and references therein). In quantum chromodynamics the interaction of quarks is described by the exchange of gluons, the gauge bosons of the strong interaction. These gluons dress the quarks in the nucleon with a sea of virtual quark-antiquark pairs and gluons. Besides the spin of the gluons, also the orbital angular momenta of quarks and gluons can contribute to the nucleon spin in addition to the spin of the quarks. To date, indirect and direct measurements of the gluon helicity distribution gave only vague information about its size and a direct measurement of the orbital angular momenta is not possible so far.

Not only the helicity distribution but also a transverse spin of the constituents can reveal information about the structure of the nucleon. In contrast to the helicity distribution, the transversity distribution is defined for a nucleon spin transverse to its direction of motion. As in the case of a longitudinal nucleon spin, the transversity distribution is the difference of the numbers of partons with their spins aligned and antialigned to the nucleon spin. The helicity and transversity distributions seem to be similar but their interpretation is valid only in the infinite momentum frame in which the direction of the nucleon momentum breaks rotational symmetry. In addition, helicity conservation forbids contributions from the gluon to the transversity distribution in the nucleon.

Besides the helicity and the transversity distribution functions, six other quark distribution functions exist for each quark flavour in leading twist. However, only the unpolarised distribution function - the quark number density in the nucleon-and the helicity and transversity distributions do not vanish when they are integrated over the intrinsic transverse quark momenta. These three distributions are the fundamental degrees of freedom of the quarks inside the nucleon and are of equal relevance. The transversity distribution function was first mentioned already in 1979 [Ral79] but remains unmeasured 
to date. The reason is its chiral-odd nature which requires the combination with another chiral-odd object in helicity conserving processes. One possibility to access transversity is the semi-inclusive deep-inelastic scattering process in which the distribution functions are combined with fragmentation functions. The combination of the transversity function and the chiral-odd Collins fragmentation function causes a left-right asymmetry in the cross section for a transversely polarised nucleon. The interest in this asymmetry increased when a first measurement with the SMC experiment in 1999 [Bra99] indicated a positive asymmetry for positive hadrons produced in deep-inelastic scattering off a transversely polarised hydrogen target. Semi-inclusive deep-inelastic scattering allows furthermore the measurement of another distribution function, the Sivers function, which describes the correlation of quark transverse momenta with the transverse spin of the nucleon. The investigation of the Sivers function is motivated by the requirement of nonzero orbital angular momenta of the quarks for the existence of this distribution function. Like the combination of transversity and Collins functions, the Sivers function appears together with the unpolarised fragmentation function in the cross section for a transversely polarised nucleon and produces a different left-right asymmetry.

The deep-inelastic scattering experiment HERMES (HERa MEasurement of Spin) uses the lepton beam provided by the HERA (Hadron-Elektron-RingAnlage) facility at the DESY (Deutsches Elektronen-SYnchrotron) laboratory in Hamburg, Germany. In the years 1996 to 2000, the lepton beam was scattered off fixed hydrogen and deuterium targets which were longitudinally polarised. Published azimuthal asymmetry moments in pion production [AirO0, AirO1, AirO3] did not allow the disentanglement of the cross-section terms containing the Sivers function, the Collins function, and a twist-three component from these data. Since 2002, the operation of a target which is polarised transversely to the direction of the lepton beam allows the individual measurement of Collins and Sivers asymmetry moments and furthermore the separation of the twist-three component from the measurement on a longitudinally polarised hydrogen target. The extraction of the Collins and Sivers moments and the separation of the twist-three component are presented in this thesis.

The theory of the inner structure of the nucleon is reviewed in Chapter 2 with emphasis on the concept of the factorisation of the deep-inelastic scattering process into distribution and fragmentation functions. Since the field of distribution and fragmentation functions dependent on quark transverse momenta is quite young, many notations for the various functions exist. Details about the chosen notation and further conventions are given in the subsequent chapters and in Appendix A. In Chapter 3 the HERMES experiment is introduced with its main components: the polarised hydrogen gas target, the tracking and particle identification detectors of the spectrometer, and the data acquisition and processing. The formalism of the extraction of Collins and Sivers moments from the experimental data and systematic studies of the obtained moments are presented in the subsequent chapter. In Chapter 5 the systematic uncertainties are investigated using a newly developed Monte Carlo generator which simulates azimuthal asymmetries arising from intrinsic quark momenta. Monte Carlo simulations are also used for the estimation of the contribution by decay hadrons from exclusively produced vector mesons in Chapter 6. In the same chapter, measurements of asymmetry amplitudes for exclusive $\rho^{0}$ vector mesons are presented as well as an asymmetry in the difference between positive and negative pion yields where the vector meson contribution cancel. The mea- 
sured Collins and Sivers moments can be used to separate the twist-three cross-section component from the extracted amplitudes of the measurements on the longitudinally polarised hydrogen target, which is described in Chapter 7. The discussion and interpretation of the measured Collins and Sivers moments together with the comparison to model predictions can be found in Chapter 8 before the summary and conclusion of this work. 

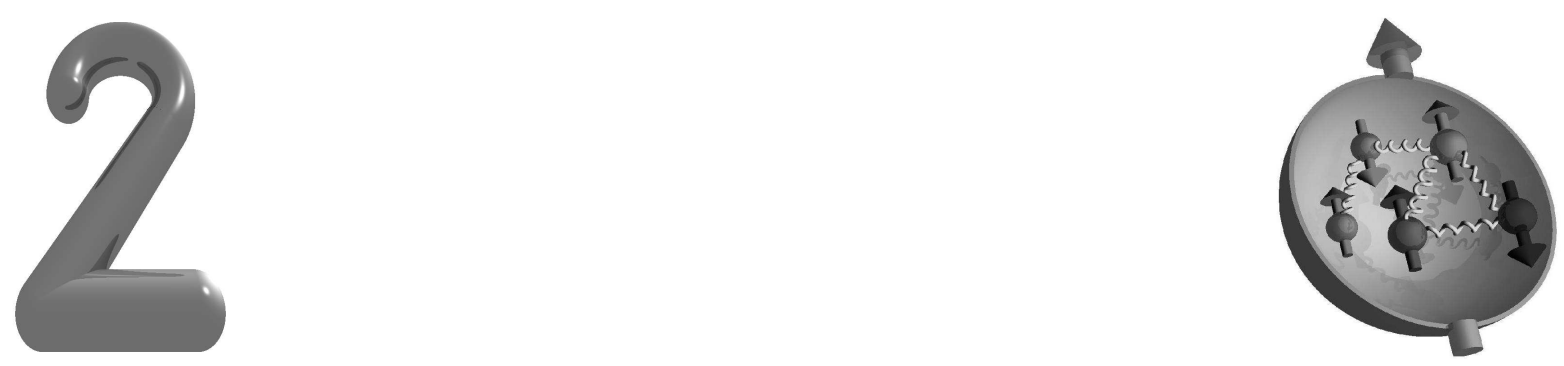

\section{The Inner Structure of the Nucleon}

A very successful tool to gain information about the inner structure of the nucleon is the deep-inelastic scattering process which is described in this chapter. Inclusive deepinelastic scattering was the first process in which point-like partons were identified inside the nucleon. Quark distribution and fragmentation functions, which appear in the cross section for semi-inclusive deep-inelastic scattering, are introduced and their properties described. The topics of this thesis are the transversity and Sivers distribution functions and the Collins fragmentation function which are addressed in Sections 2.4. 1, 2.4.3, and 2.5. 1. Furthermore, the concept of cross-section asymmetries-the measured quantities in deep-inelastic scattering experiments for the extraction of quark distribution functionsis presented.

The definition of twist as proposed in [Jaf96] is used, i.e., a leading-twist or respectively twist-two term is identified with a term of leading order in a $1 / Q$ expansion. The notation of Mulders and Tangermann [Mul96] is applied to the distribution and fragmentation functions except for the three leading-twist distribution functions that do not vanish when integrated over transverse quark momenta. For these three functions, $q\left(x,\left|\vec{p}_{T}\right|^{2}\right)$ refers to the unpolarised distribution $f_{1}^{q}\left(x,\left|\vec{p}_{T}\right|^{2}\right), \Delta q\left(x,\left|\vec{p}_{T}\right|^{2}\right)$ to the helicity distribution $g_{1 L}^{q}\left(x,\left|\vec{p}_{T}\right|^{2}\right)$, and $\delta q\left(x,\left|\vec{p}_{T}\right|^{2}\right)$ to the transversity distribution $h_{1}^{q}\left(x,\left|\vec{p}_{T}\right|^{2}\right)$, where $\vec{p}_{T}$ is the intrinsic quark transverse momentum. The definitions of the azimuthal angles and the asymmetries follow the Trento conventions [Bac04a]. Throughout this work, four-vectors are written as bold symbols, e.g., q, space vectors with three or two components are indicated by arrows, e.g., $\vec{p}=\left(\vec{p}_{T}, p_{z}\right)$, where transverse vectors (with two components) have an additional subscript $T$. Further notations and conventions can be found in Appendix $A$.

\subsection{Inclusive Deep-Inelastic Scattering}

In the deep-inelastic scattering (DIS) process a lepton scatters off a nucleon via the exchange of a virtual boson. For charged leptons and moderate squared momentum transfers to the target $Q^{2}$, the exchange of a single virtual photon, as shown in Figure 2. 1. 1, is the dominant process during which the nucleon breaks up and forms a final hadronic state $X$. The maximum possible momentum transfer is determined by the centre-of-mass energy $\sqrt{s}$ which is only about 7.2 GeV at the HERMES experiment. In inclusive DIS only 


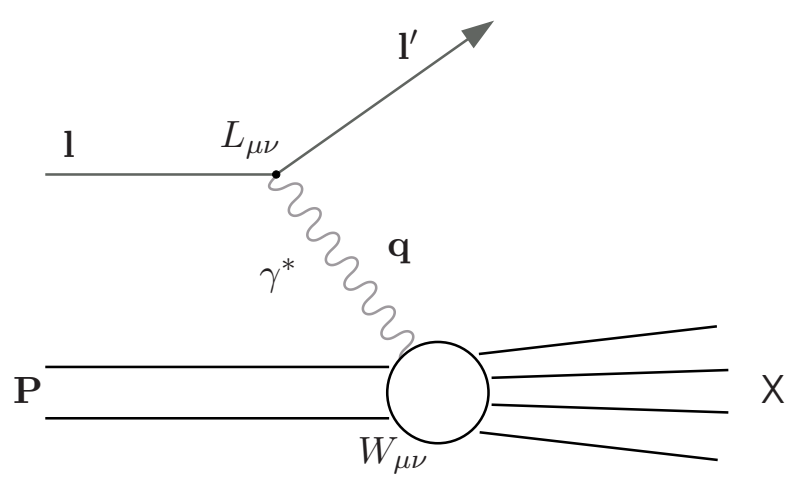

Figure 2.1.1: DIS with one-photon exchange in the laboratory system.

the scattered lepton is detected whereas additional hadrons of the final state $X$ are detected in semi-inclusive DIS processes. In contrast to inclusive and semi-inclusive DIS the hadronic state $X$ is fully reconstructed in exclusive DIS. In the laboratory frame the fourmomentum of the incoming (outgoing) lepton is $\mathbf{l}=(E, \vec{l})\left(\mathbf{l}^{\prime}=\left(E^{\prime}, \overrightarrow{l^{\prime}}\right)\right)$. For a fixed target experiment like HERMES, only the first component of the four-momentum of the nucleoni $\mathbf{P}=(M, \overrightarrow{0})$ differs from zero, where $M$ is the rest mass of the nucleon. The relevant kinematic variables for the inclusive DIS process are summarised in Table 2.1.1.

\begin{tabular}{|c|c|}
\hline$\theta$ & $\begin{array}{l}\text { polar scattering angle } \\
\text { in the laboratory frame }\end{array}$ \\
\hline $\mathbf{q}=\mathbf{l}-\mathbf{l}^{\prime}$ & four-momentum transfer to the target \\
\hline$s=(\mathbf{P}+\mathbf{l})^{2} \stackrel{\text { lab }}{\approx} M^{2}+2 M E$ & squared centre-of-mass energy \\
\hline$\nu=\frac{\mathbf{P} \cdot \mathbf{q}}{M} \stackrel{\text { lab }}{=} E-E^{\prime}$ & energy transfer to the target \\
\hline$y=\frac{\mathbf{P} \cdot \mathbf{q}}{\mathbf{P} \cdot \mathbf{l}} \stackrel{\text { lab }}{=} \frac{\nu}{E}$ & fractional energy transfer to the target \\
\hline$Q^{2}=-\mathbf{q}^{2} \stackrel{\operatorname{lab}}{\approx} 4 E E^{\prime} \sin ^{2} \frac{\theta}{2}$ & squared invariant mass of the virtual photon \\
\hline$W^{2}=(\mathbf{P}+\mathbf{q})^{2} \stackrel{\text { lab }}{=} M^{2}+2 M \nu-Q^{2}$ & squared mass of the final state \\
\hline$x=\frac{Q^{2}}{2 \mathbf{P} \cdot \mathbf{q}} \stackrel{\operatorname{lab}}{=} \frac{Q^{2}}{2 M \nu}$ & Bjorken scaling variable \\
\hline
\end{tabular}

Table 2.1.1: Kinematic variables used in the description of DIS. The (near-) equalities marked 'lab' hold in the laboratory frame for a fixed target, neglecting the lepton rest mass.

The squared momentum transfer to the target $Q^{2}$ is a measure of the spatial resolution in the scattering process. In DIS processes, $Q^{2}$ is large enough to resolve the constituents of the nucleon. The dimensionless Bjorken scaling variable $x$ describes the inelasticity

'Throughout this work natural units are used, i.e., $\hbar=c=1$. 
of the process. Mass is conserved in elastic scattering processes, i.e., $W^{2}=M^{2}$ which implies $x=1$. The squared mass of the final state becomes larger than the nucleon mass in inelastic processes, and consequently $0<x<1$. As additional degree of freedom, the mass difference between final hadronic state and target nucleon - the excitation energy - requires two independent variables for the description of the scattering process, e.g., $Q^{2}$ and $x$.

The differential cross section for a scattered lepton which is detected within a solid angle $\mathrm{d} \Omega$ and in an energy range $\left[E^{\prime}, E^{\prime}+\mathrm{d} E^{\prime}\right]$ can be written as (see e.g. [Ans95]):

$$
\frac{\mathrm{d}^{2} \sigma}{\mathrm{d} \Omega \mathrm{d} E^{\prime}}=\frac{\alpha^{2}}{2 M Q^{4}} \frac{E^{\prime}}{E} L_{\mu \nu} W^{\mu \nu}=\frac{\alpha^{2}}{2 M Q^{4}} \frac{E^{\prime}}{E}\left[L_{\mu \nu}^{(\mathrm{S})} W^{\mu \nu(\mathrm{S})}-L_{\mu \nu}^{(\mathrm{A})} W^{\mu \nu(\mathrm{A})}\right],
$$

where $\alpha$ is the electromagnetic coupling constant. $L_{\mu \nu}$ and $W_{\mu \nu}$ are the leptonic and hadronic tensors, describing the interaction at the leptonic and hadronic vertices of the DIS process (cf. Figure 2.1.1). They can be split up in two parts which are symmetric and antisymmetric under parity transformation, respectively:

$$
L_{\mu \nu}=L_{\mu \nu}^{(\mathrm{S})}+i L_{\mu \nu}^{(\mathrm{A})}, \quad W_{\mu \nu}=W_{\mu \nu}^{(\mathrm{S})}+i W_{\mu \nu}^{(\mathrm{A})},
$$

where only the antisymmetric parts are spin-dependent. In the cross section of Eq. (2. 1.1), antisymmetric combinations of $L_{\mu \nu}$ and $W_{\mu \nu}$ do not appear due to the parity conservation of the electromagnetic interaction.

Since the lepton is a point-like spin- $\frac{1}{2}$ particle, the leptonic tensor can be calculated in the Dirac formalism of QED. The small electromagnetic coupling constant $\alpha \approx 1 / 137$ and the fact that photons do not couple to themselves allow the application of perturbative QED. Using Dirac matrices (cf. Appendix A) and the Dirac spinor $u(1, \mathbf{s})$ for spin- $\frac{1}{2}$ particles with four-momentum 1 and spin four-vector s, the leptonic tensor reads:

$$
L_{\mu \nu}\left(\mathbf{l}, \mathbf{s} ; \mathbf{l}^{\prime}, \mathbf{s}^{\prime}\right)=\left[\bar{u}^{\prime}\left(\mathbf{l}^{\prime}, \mathbf{s}^{\prime}\right) \gamma_{\mu} u(\mathbf{l}, \mathbf{s})\right]^{*}\left[\bar{u}^{\prime}\left(\mathbf{l}^{\prime}, \mathbf{s}^{\prime}\right) \gamma_{\nu} u(\mathbf{l}, \mathbf{s})\right] .
$$

The polarisation of the scattered lepton is not measured in the experiment, so the leptonic tensor can be summed over the spin four-vector $\mathrm{s}^{\prime}$ of the scattered lepton. This results in the following symmetric and antisymmetric parts of the tensor:

$$
\begin{aligned}
L_{\mu \nu}^{(\mathrm{S})}\left(\mathbf{l} ; \mathbf{l}^{\prime}\right) & =2\left[l_{\mu} l_{\nu}^{\prime}+l_{\mu}^{\prime} l_{\nu}-g_{\mu \nu}\left(\mathbf{l} \cdot \mathbf{l}^{\prime}-m_{l}^{2}\right)\right], \\
L_{\mu \nu}^{(\mathrm{A})}\left(\mathbf{l}, \mathbf{s} ; \mathbf{l}^{\prime}\right) & =2 m_{l} \epsilon_{\mu \nu \lambda \kappa} s^{\lambda}\left(l^{\kappa}-l^{\prime \kappa}\right) .
\end{aligned}
$$

Here, $g_{\mu \nu}$ denotes the metric tensor, $\epsilon_{\mu \nu \lambda \kappa}$ the Levi-Civita tensor normalised so that $\epsilon^{0123}=$ 1 , and $m_{l}$ the lepton mass.

The complex inner structure of the nucleon prevents the exact calculation of the hadronic tensor. Only a parametrisation in terms of structure functions is possible. A general decomposition of $W_{\mu \nu}$ in symmetric and antisymmetric parts:

$$
\begin{aligned}
W_{\mu \nu}^{(\mathrm{S})}(\mathbf{q} ; \mathbf{P})= & 2\left(-g_{\mu \nu}+\frac{q_{\mu} q_{\nu}}{\mathbf{q}^{2}}\right) F_{1}\left(x, Q^{2}\right)+ \\
& \frac{2}{\mathbf{P} \cdot \mathbf{q}}\left(P_{\mu}-\frac{\mathbf{P} \cdot \mathbf{q}}{\mathbf{q}^{2}} q_{\mu}\right)\left(P_{\nu}-\frac{\mathbf{P} \cdot \mathbf{q}}{\mathbf{q}^{2}} q_{\nu}\right) F_{2}\left(x, Q^{2}\right), \\
W_{\mu \nu}^{(\mathrm{A})}(\mathbf{q} ; \mathbf{P}, \mathbf{S})= & \epsilon_{\mu \nu \lambda \kappa} \frac{2 M q^{\lambda}}{\mathbf{P} \cdot \mathbf{q}}\left[S^{\kappa} g_{1}\left(x, Q^{2}\right)+\left(S^{\kappa}-\frac{\mathbf{S} \cdot \mathbf{q}}{\mathbf{P} \cdot \mathbf{q}} P^{\kappa}\right) g_{2}\left(x, Q^{2}\right)\right],
\end{aligned}
$$


fulfils additional symmetry requirements as Lorentz covariance, gauge invariance, and parity conservation of the electromagnetic interaction. The Lorentz-invariant structure functions $F_{1}, F_{2}, g_{1}, g_{2}$ depend on the two independent variables $x$ and $Q^{2}$ in DIS. The two structure functions $F_{1}$ and $F_{2}$ appear in the symmetric part of the hadronic tensor while the antisymmetric part contains the structure function pair $g_{1}, g_{2}$.

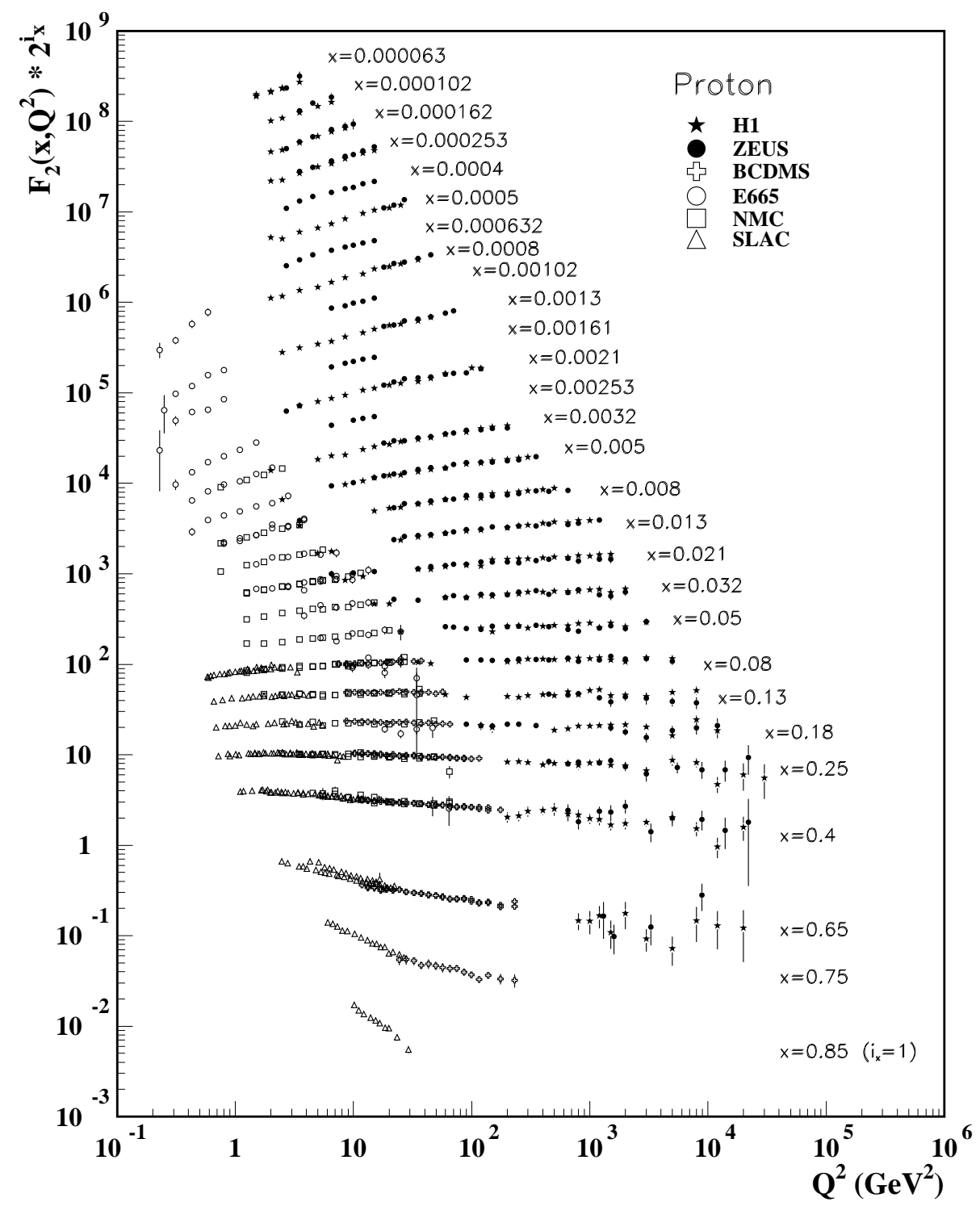

Figure 2.1.2: The proton structure function $F_{2}$ measured in electromagnetic scattering of positrons on protons (ZEUS [Che01], H1 [AdlO1, Adl03]) and for electrons (SLAC [Whi92]) and muons (BCDMS [Ben89], E665 [Ada96], NMc [Arn97a]) on a fixed target. Statistical and systematic uncertainties added in quadrature are shown. The data are plotted as a function of $Q^{2}$ in bins of fixed $x$. Some points have been slightly offset in $Q^{2}$ for clarity. For the purpose of plotting, $F_{2}$ has been multiplied by $2^{i_{x}}$, where $i_{x}$ is the number of the $x$ bin, ranging from $i_{x}=1(x=0.85)$ to $i_{x}=28(x=0.000063)$. This plot is taken from [Eid04]. 


\subsubsection{The Unpolarised Cross Section}

Averaging over all spin states in the initial state of the scattering process, only the spinindependent symmetric parts of the leptonic and hadronic tensors do not vanish and the unpolarised cross section [Ans95],

$$
\frac{\mathrm{d}^{2} \sigma_{\text {unpol }}}{\mathrm{d} x \mathrm{~d} y}=\frac{4 \pi \alpha^{2}}{s x^{2} y^{2}}\left\{x y^{2} F_{1}\left(x, Q^{2}\right)+\left(1-y-\frac{\gamma^{2} y^{2}}{4}\right) F_{2}\left(x, Q^{2}\right)\right\}
$$

with $\gamma=(2 M x) / Q$ is obtained. The structure functions $F_{1}\left(x, Q^{2}\right)$ and $F_{2}\left(x, Q^{2}\right)$ reflect the unpolarised inner structure of the nucleon. They describe the deviation from the cross section for the scattering of a relativistic spin- $\frac{1}{2}$ particle off a point-like central potential. Thus, they correspond to the electric and magnetic form factors in elastic electronnucleon scattering, which describe the Fourier transform of the electric charge distribution and the magnetic moment of the nucleon, respectively. A selection of world data for the structure function $F_{2}$ as a function of $Q^{2}$ for different $x$ can be found in Figure 2. 1.2.

\subsubsection{The Polarised Cross Section}

In contrast to the symmetric part, the antisymmetric part of the cross section depends on the lepton and the nucleon spins. For a lepton with its spin and momentum aligned, i.e., longitudinally aligned, the cross section depends on the azimuthal and polar angles, $\phi_{S}^{l}$ and $\theta_{S}^{l}$, of the target spin $\vec{S}$ with respect to the lepton beam (cf. Figure 2.1.3). In the difference between two opposite target spin states $\pm S$, the unpolarised cross section

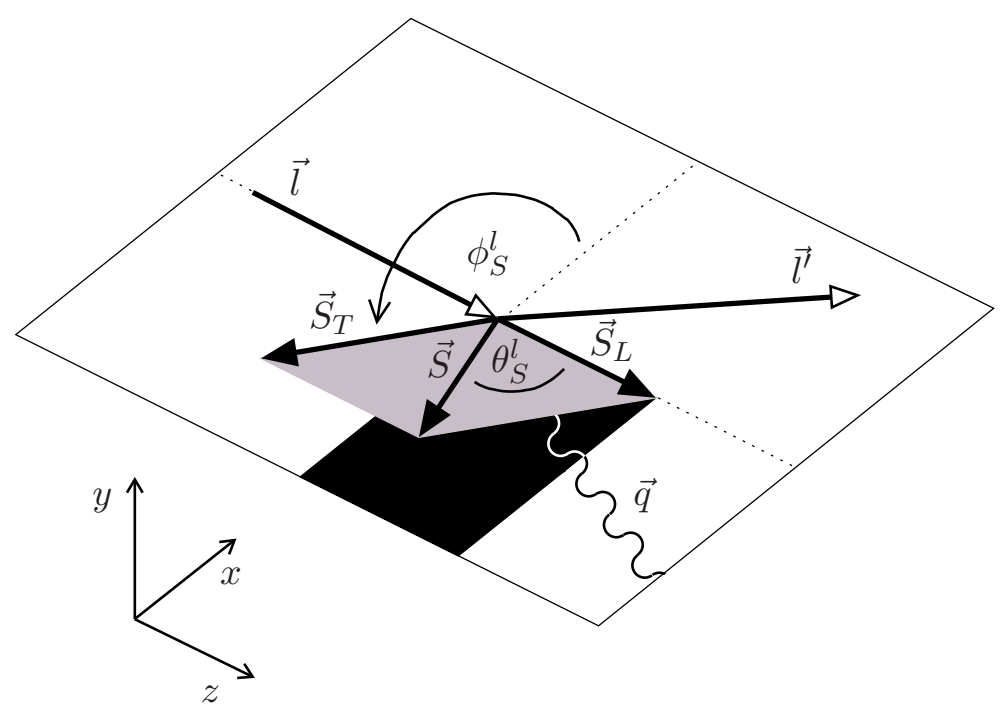

Figure 2.1.3: Definition of azimuthal and polar angles of the target spin $\vec{S}$. 


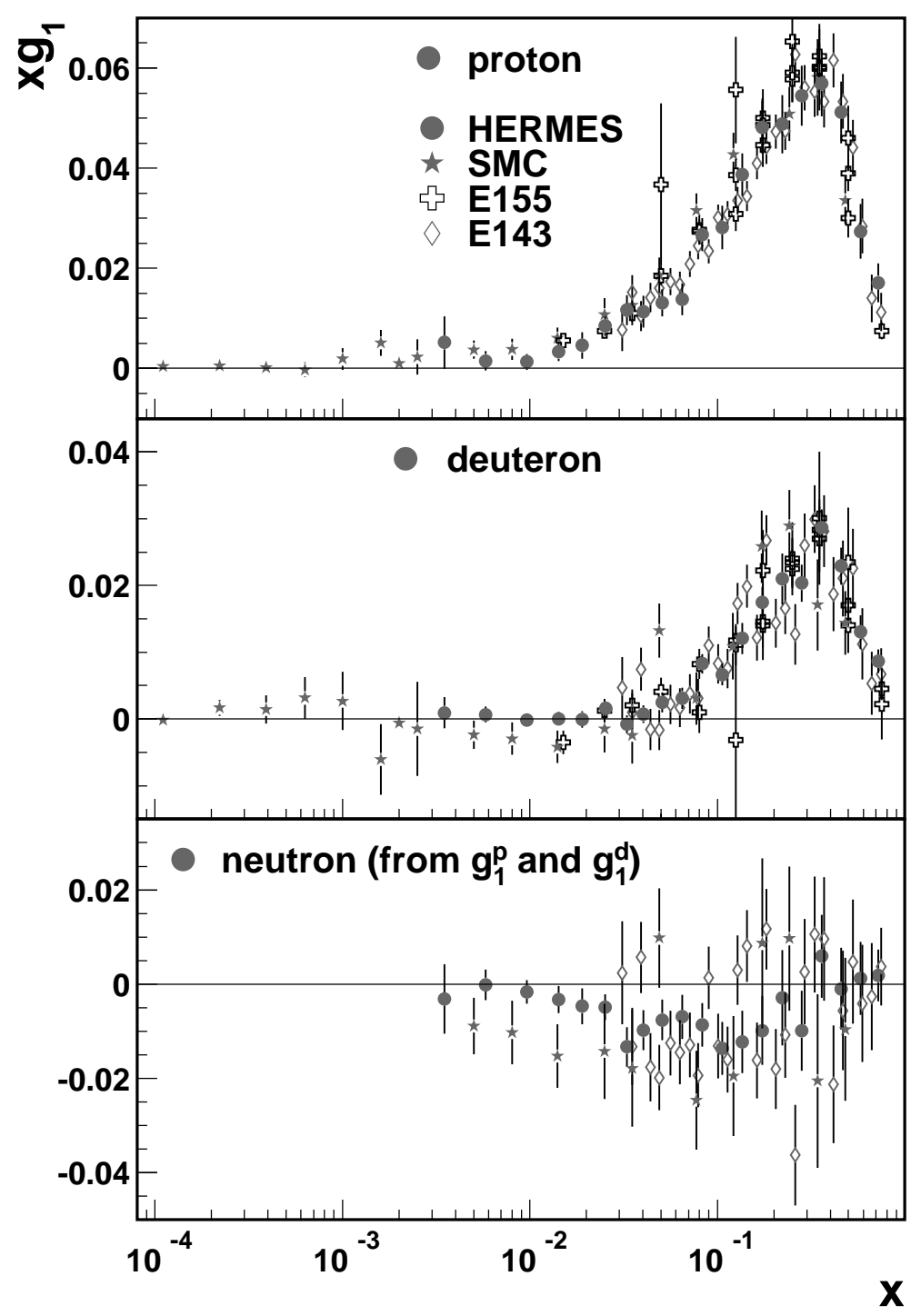

Figure 2.1.4: The spin-dependent structure function $x g_{1}$ of the proton, deuteron, and neutron measured as function of $x$ in DIS of polarised leptons (HERMES: [Reg05], SMc: [Ade98, Ade99], E155: [Ant99, Ant00], E143: [Abe98]). Statistical and systematic uncertainties added in quadrature are shown.

cancels and one obtains the polarised cross section [Ans95]:

$$
\begin{aligned}
\frac{\mathrm{d}^{3} \sigma_{\mathrm{pol}}}{\mathrm{d} x \mathrm{~d} y \mathrm{~d} \phi_{S}^{l}}= & \frac{\mathrm{d}^{3} \sigma(-S)}{\mathrm{d} x \mathrm{~d} y \mathrm{~d} \phi_{S}^{l}}-\frac{\mathrm{d}^{3} \sigma(+S)}{\mathrm{d} x \mathrm{~d} y \mathrm{~d} \phi_{S}^{l}} \\
= & \frac{4 \alpha^{2}}{s x y}\left\{\left[\left(2-y-\frac{\gamma^{2} y^{2}}{2}\right) g_{1}\left(x, Q^{2}\right)-\gamma^{2} y g_{2}\left(x, Q^{2}\right)\right] \cos \theta_{S}^{l}+\right. \\
& \left.\gamma \sqrt{1-y-\frac{\gamma^{2} y^{2}}{4}}\left[\gamma g_{1}\left(x, Q^{2}\right)+2 g_{2}\left(x, Q^{2}\right)\right] \sin \theta_{S}^{l} \cos \phi_{S}^{l}\right\}
\end{aligned}
$$


If the target nucleons are also longitudinally polarised, $\theta_{S}^{l}=0$ and the cross section reduces to:

$$
\frac{\mathrm{d}^{3} \sigma^{\rightleftarrows}}{\mathrm{d} x \mathrm{~d} y \mathrm{~d} \phi_{S}^{l}}-\frac{\mathrm{d}^{3} \sigma^{\rightrightarrows}}{\mathrm{d} x \mathrm{~d} y \mathrm{~d} \phi_{S}^{l}}=\frac{4 \alpha^{2}}{s x y}\left\{\left[\left(2-y-\frac{\gamma^{2} y^{2}}{2}\right) g_{1}\left(x, Q^{2}\right)-\gamma^{2} y g_{2}\left(x, Q^{2}\right)\right]\right\} .
$$

Here, $\rightarrow$ indicates the spin orientation of the incoming lepton and $\Leftarrow, \Rightarrow$ the different spin states of the target nucleon. The cross section is dominated by the structure function $g_{1}\left(x, Q^{2}\right)$ because $g_{2}\left(x, Q^{2}\right)$ is suppressed by $\gamma^{2} \sim 1 / Q^{2}$ and $g_{2}\left(x, Q^{2}\right)$ is small by itself and even vanishes in the simple quark parton model. The contribution of $g_{2}\left(x, Q^{2}\right)$ vanishes completely in case of a target polarised longitudinally with respect to the virtual photon. Experimentally, however, a polarisation with respect to the virtual photon is not achievable. Figure 2.1.4 shows measurements on longitudinally polarised proton and deuteron targets of different experiments. The structure function $g_{2}\left(x, Q^{2}\right)$ is usually taken into account by a parametrisation of world data. The precision of the parametrisation has only a marginal effect on the $g_{1}\left(x, Q^{2}\right)$ measurement and is part of the systematic uncertainty. The neutron structure function is extracted from the difference of the results for the deuteron and the proton. It can be measured also directly with a polarised ${ }^{3} \mathrm{He}$ target, which is an effective polarised neutron target due to the almost complete cancellation of the proton spins (see e.g. [Ack97]).

In the special case of a target polarised transverse to the incoming lepton beam, $\theta_{S}^{l}=\pi / 2$ and the polarised cross section remains $\phi_{S}^{l}$ dependent:

$$
\frac{\mathrm{d}^{3} \sigma^{\rightarrow \Downarrow}}{\mathrm{d} x \mathrm{~d} y \mathrm{~d} \phi_{S}^{l}}-\frac{\mathrm{d}^{3} \sigma^{\rightarrow \Uparrow}}{\mathrm{d} x \mathrm{~d} y \mathrm{~d} \phi_{S}^{l}}=\frac{4 \alpha^{2}}{s x y} \gamma \sqrt{1-y-\frac{\gamma^{2} y^{2}}{4}}\left\{\gamma g_{1}\left(x, Q^{2}\right)+2 g_{2}\left(x, Q^{2}\right)\right\} \cos \phi_{S}^{l} .
$$

Here, neither $g_{1}\left(x, Q^{2}\right)$ nor $g_{2}\left(x, Q^{2}\right)$ dominates the cross section. The combination of measurements on longitudinally and transversely polarised targets allows the extraction of $g_{2}\left(x, Q^{2}\right)$. First measurements of $g_{2}\left(x, Q^{2}\right)$ exist [Ant03].

\subsubsection{Forward Virtual Compton Scattering}

The optical theorem relates the hadronic tensor to the imaginary part of the forward virtual Compton scattering amplitude $T_{\mu \nu}$ :

$$
W_{\mu \nu}=\frac{1}{2 \pi} \operatorname{Im} T_{\mu \nu}
$$

In the basis of helicity eigenstates (helicity basis), only four independent photon-hadron amplitudes $T\left(h, H ; h^{\prime}, H^{\prime}\right)$ exist because of helicity conservation:

$$
T\left(1, \frac{1}{2} ; 1, \frac{1}{2}\right), \quad T\left(1,-\frac{1}{2} ; 1,-\frac{1}{2}\right), \quad T\left(0, \frac{1}{2} ; 0, \frac{1}{2}\right), \quad T\left(0, \frac{1}{2} ; 1,-\frac{1}{2}\right) .
$$

They depend on the helicities of the initial (final) nucleon $H\left(H^{\prime}\right)$ and photon $h\left(h^{\prime}\right)$ (cf. Figure 2.1.5). Combinations of the four amplitudes are proportional to the unpolarised and 


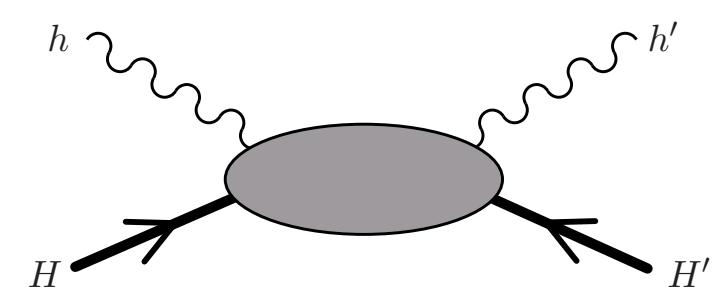

Figure 2.1.5: The forward virtual Compton scattering amplitude.

polarised structure functions:

$$
\begin{aligned}
F_{1} & \sim \frac{1}{2} \operatorname{Im}\left[T\left(1, \frac{1}{2} ; 1, \frac{1}{2}\right)+T\left(1,-\frac{1}{2} ; 1,-\frac{1}{2}\right)\right] \\
\left(1+\gamma^{2}\right) \frac{F_{2}}{2 x}-F_{1} & \sim \operatorname{Im} T\left(0, \frac{1}{2} ; 0, \frac{1}{2}\right) \\
g_{1}-\gamma^{2} g_{2} & \sim \frac{1}{2} \operatorname{Im}\left[T\left(1, \frac{1}{2} ; 1, \frac{1}{2}\right)-T\left(1,-\frac{1}{2} ; 1,-\frac{1}{2}\right)\right] \\
\gamma\left(g_{1}+g_{2}\right) & \sim \operatorname{Im} T\left(0, \frac{1}{2} ; 1,-\frac{1}{2}\right) .
\end{aligned}
$$

The hadronic tensor yields the virtual photo-absorption cross sections $\sigma_{h h^{\prime}}^{H H^{\prime}}$ :

$$
\begin{aligned}
\sigma_{++}^{++} & \sim \operatorname{Im} T\left(1, \frac{1}{2} ; 1, \frac{1}{2}\right), & & \sigma_{++}^{--} \sim \operatorname{Im} T\left(1,-\frac{1}{2} ; 1,-\frac{1}{2}\right), \\
\sigma_{00}^{++} & \sim \operatorname{Im} T\left(0, \frac{1}{2} ; 0, \frac{1}{2}\right), & & \sigma_{0+}^{+-} \sim \operatorname{Im} T\left(0, \frac{1}{2} ; 1,-\frac{1}{2}\right) .
\end{aligned}
$$

The ratio of the photo-absorption cross sections of longitudinal and transverse virtual photons,

$$
R\left(x, Q^{2}\right)=\frac{\sigma_{\mathrm{L}}\left(x, Q^{2}\right)}{\sigma_{\mathrm{T}}\left(x, Q^{2}\right)}=\frac{\sigma_{00}^{++}}{\frac{1}{2}\left(\sigma_{++}^{++}+\sigma_{++}^{--}\right)},
$$

relates the structure functions $F_{1}\left(x, Q^{2}\right)$ and $F_{2}\left(x, Q^{2}\right)$ to each other by:

$$
R\left(x, Q^{2}\right)=\frac{\left(1+\gamma^{2}\right) F_{2}\left(x, Q^{2}\right)-2 x F_{1}\left(x, Q^{2}\right)}{2 x F_{1}\left(x, Q^{2}\right)} .
$$

In the Bjorken limit where $Q^{2} \rightarrow \infty$ and $\nu \rightarrow \infty$ such that $x$ remains constant, the kinematic factor $\gamma$ can be neglected and the photo-absorption cross section $\sigma_{\mathrm{L}}$ for longitudinal photons with helicity 0 vanishes as a consequence of the requirement of helicity conservation at the virtual photon-parton scattering vertex. Thus, $R \rightarrow 0$ and Eq. (2.1.19) yields the Callan-Gross relation [Cal69],

$$
F_{2}(x)=2 x F_{1}(x),
$$

for scattering off point-like constituents of the nucleon with spin $\frac{1}{2}$.

\subsection{The Quark Parton Model}

In the 1960s, experiments in which high-energetic electrons were scattered off protons [Blo69, Bre69] yielded results which could not be interpreted with a diffusely distributed 


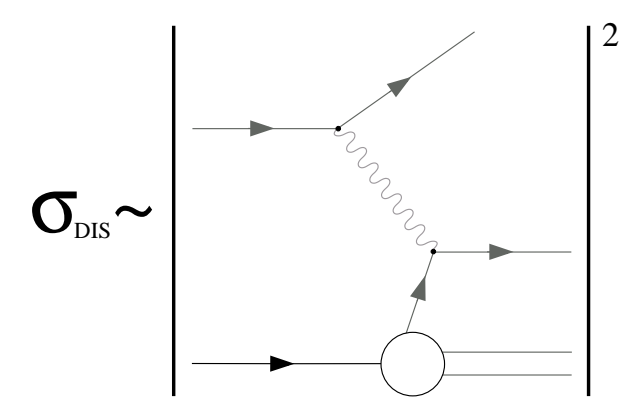

Figure 2.2.1: DIS in the parton model. The target is a cloud of partons and the lepton scatters elastically from one of the partons by exchanging a virtual photon.

proton charge. Instead, the measurements indicated discrete scattering centres. These point-like components of the protons were called partons. They could be identified with the quarks which were proposed by Gell-Mann [Gel64] and Zweig [Zwe64] as fundamental entities of the proton, neutron, and other hadrons to explain results from particle spectroscopy.

In the Quark Parton Model (QPM), the essential assumption is that for large energy and momentum transfer to the nucleon, the mediator interacts incoherently with free partons in the nucleon. In a frame where the nucleon is moving with large (infinite) momentum, the nucleon and quark masses can be neglected. If the target moves in the $z$ direction in this so-called infinite momentum frame, the three-momentum of a quark in the nucleon can be written as follows:

$$
\vec{p}=\left(\vec{p}_{T}, x P\right),
$$

where $P$ is the absolute nucleon momentum and $\vec{p}_{T}$ are the components of the quark momentum transverse to the $z$ direction. Also the transverse motion of the quark during the interaction time is small if $P \rightarrow \infty$. Hence, the Bjorken scaling variable $x$ can be interpreted as the momentum fraction of the nucleon carried by the struck quark.

In the QPM, the total cross section for lepton scattering from the partons as a whole, i.e., the nucleon (cf. Figure 2.2.1), is the sum of the individual contributions:

$$
\frac{\mathrm{d}^{2} \sigma}{\mathrm{d} Q^{2} \mathrm{~d} \nu}=\sum_{q, \bar{q}} \int \mathrm{d} x q(x) \frac{\mathrm{d}^{2} \sigma^{l q}}{\mathrm{~d} Q^{2} \mathrm{~d} \nu},
$$

where $\mathrm{d}^{2} \sigma^{l q} / \mathrm{d} Q^{2} \mathrm{~d} \nu$ is the cross section for the elastic lepton-quark scattering and the sum is performed over all quark and antiquark flavours in the nucleon, $q$ and $\bar{q}$. The unpolarised quark distribution function $q(x)$ multiplied by the differential momentum fraction $\mathrm{d} x$ gives the probability to find a quark of flavour $q$ carrying a fraction of the target momentum in the range $[x, x+\mathrm{d} x]$. Momentum conservation in the elastic scattering process yields that the momentum fraction $x$ is almost equal to the Bjorken scaling variable defined in Table 2.1.1, as already mentioned above. The comparison of the QPM cross section with the cross section in Eq. (2.1.8) obtained with the parametrisation of the hadronic tensor, yields relations between the unpolarised structure functions and the unpolarised 
distribution function (DF) [Clo79]:

$$
\begin{aligned}
& F_{1}\left(x, Q^{2}\right)=\frac{1}{2} \sum_{q, \bar{q}} e_{q}^{2} q(x), \\
& F_{2}\left(x, Q^{2}\right)=\sum_{q, \bar{q}} x e_{q}^{2} q(x),
\end{aligned}
$$

where $e_{q}$ is the electric quark charge in units of the elementary charge $e$. Since the right hand sides do not depend on $Q^{2}$, the QPM predicts the so-called Bjorken scaling [Bjo69], i.e., that for fixed $x$ the structure functions will be independent of $Q^{2}$.

In the helicity base, the DF $q(x)$ can be split up in functions for quarks with parallel $(+)$ and antiparallel $(-)$ orientation of the spin with respect to the nucleon spin: $q(x)=$ $q^{+}(x)+q^{-}(x)$. The polarised or helicity DF is then defined as $\Delta q(x)=q^{+}(x)-q^{-}(x)$ and can be related to the polarised structure function $g_{1}(x)$ :

$$
g_{1}(x)=\frac{1}{2} \sum_{q, \bar{q}} e_{q}^{2} \Delta q(x)=\frac{1}{2} \sum_{q, \bar{q}} e_{q}^{2}\left[q^{+}(x)-q^{-}(x)\right] .
$$

The second polarised structure function vanishes in the QPM: $g_{2}(x)=0$.

\subsection{Quantum Chromodynamics}

As visible in Figure 2.1.2, the Bjorken scaling is approximately fulfilled only in the small kinematic range $0.18 \lesssim x \lesssim 0.25$. The violation of scaling can be explained if quarks interact with each other in the nucleon. In the late 1970s, a field theory of Quantum Chromodynamics (QCD) was developed whose basic fields are quarks interacting via electrically neutral vector gluons. $Q C D$ is the non-Abelian gauge theory of the strong interaction and part of the Standard Model. Quarks couple to the strong interaction through three different colours. In contrast to Quantum Electrodynamics (QED) where the photons have no electrical charge and cannot couple to each other, the field quanta of the strong interaction, i.e., the gluons, do carry colour charge. This causes a scale or energy dependence of the strong coupling 'constant' $\alpha_{s}$ :

$$
\alpha_{s}\left(Q^{2}\right)=\frac{12 \pi}{\left(33-2 n_{f}\right) \ln \left(Q^{2} / \Lambda_{\mathrm{QCD}}^{2}\right)} .
$$

The QCD scale parameter $\Lambda_{\mathrm{QCD}}$ depends on the number of quark flavours $n_{f}$ and the renormalisation scheme. It is of the order of a couple of hundred MeV. Eq. (2.3.1) is only valid for $Q^{2} \gg \Lambda_{\mathrm{QCD}}^{2}$ and shows that the coupling decreases with increasing $Q^{2}$ and reaches zero for $Q^{2} \rightarrow \infty$. This feature, which only appears in non-Abelian gauge theories, is called asymptotic freedom. It explains the success of the description of experimental results with the QPM in which the quarks are treated as free partons. For $\alpha_{s} \ll$ 1 perturbative QCD is applicable and only lower order diagrams have to be taken into account. But the coupling constant is not necessarily smaller than 1 for small $Q^{2}$ so that perturbation theory fails. In the so-called confinement region $\left(Q^{2} \lesssim 1 \mathrm{GeV}^{2}\right)$, where $\alpha_{s}$ is of the order of 1 or larger, phenomenological models or QCD calculations on the lattice have to be used to describe the experimental data. In fact, also the QED coupling constant $\alpha$ depends slightly on $Q^{2}$ because of the shielding effect of vacuum fluctuations into 
lepton-antilepton pairs. However, the $Q^{2}$ dependence of $\alpha$ has the opposite tendency than for $\alpha_{s}$ and is much weaker.

Due to the fact that quarks and gluons interact, gluons dress the quarks in the nucleon with a cloud of gluons and virtual quark-antiquark pairs, so-called sea quarks. With increasing $Q^{2}$ the wavelength of the virtual photon decreases and the resolution of the external probing current increases. A quark which is not resolved at low $Q^{2}$ can therefore be resolved at larger $Q^{2}$ as a quark with lower momentum plus gluon (see Fig. 2.3.1(a)). Hence, there is a depletion of high momentum partons and an increase in the low momentum parton distribution as $Q^{2}$ increases. This behaviour can be seen in Figure 2.1.2 where the structure function $F_{2}$, i.e., the sum of the distribution functions of the different quark and antiquark flavours weighted by $x$, is plotted dependent on $Q^{2}$ for different values of $x$. In addition, there is an enhancement of partons at small $x$ due to resolved gluons. At low $Q^{2}$ a photon does not interact with the electrically neutral gluon. With sufficient large $Q^{2}$ the gluon can be resolved in a quark-antiquark pair and the photon can interact with one of them (see Fig. 2.3.1(b)).

In leading order perturbative QCD, the structure functions have the same form as in the QPM (Eq. (2.2.3) - (2.2.5)):

$$
\begin{aligned}
& F_{1}\left(x, Q^{2}\right)=\frac{1}{2} \sum_{q, \bar{q}} e_{q}^{2} q\left(x, Q^{2}\right), \\
& g_{1}\left(x, Q^{2}\right)=\frac{1}{2} \sum_{q, \bar{q}} e_{q}^{2} \Delta q\left(x, Q^{2}\right),
\end{aligned}
$$

but the DFs $q\left(x, Q^{2}\right)$ and $\Delta q\left(x, Q^{2}\right)$ are now $Q^{2}$ dependent. Their logarithmic $Q^{2}$ behaviour is described by the Dokshitzer-Gribov-Lipatov-Altarelli-Parisi (DGLAP) equations [Dok77, Gri72, Lip75, Alt77]. The photo-absorption cross-section ratio $R$ vanishes in leading order perturbative QCD. Hence, the Callan-Gross relation (2.1.21) holds also in the so-called QCD improved QPM. In contrast to the naïve QPM, in the QCD improved parton model the structure function $g_{2}\left(x, Q^{2}\right)$ does not vanish but arises from quark-gluon interactions.

(a)

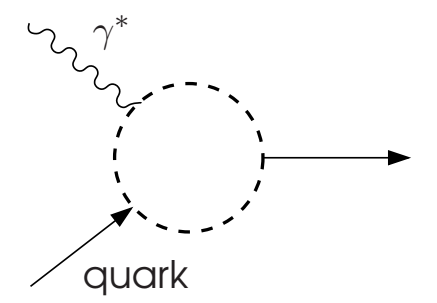

(b)

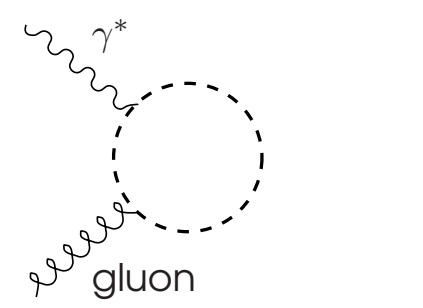

increasing $Q^{2}$
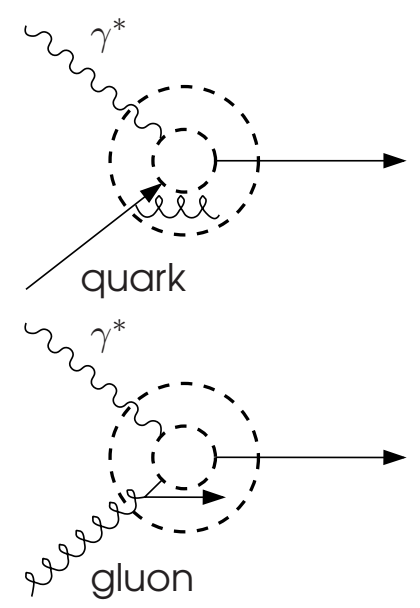

Figure 2.3.1: Improving resolution for increasing $Q^{2}$. 


\subsection{Inclusive DIS in the QCD Improved QPM}

In the QCD improved parton model, the scattering process can be described as elastic quark-lepton scattering inside the nucleon. In the interaction with a virtual photon with sufficiently large $Q^{2}$, quarks behave like quasi-free particles.

The handbag diagram representing the hadronic tensor $W^{\mu \nu}$ is shown in Figure 2.4.1. The virtual photon is absorbed by a quark with initial four momentum $\mathbf{p}$. Thus, the four momentum of the outgoing quark is $\mathbf{k}=\mathbf{p}+\mathbf{q}$. Neglecting quark masses, the hadronic tensor can be written as [BarO3]:

$$
W^{\mu \nu}=\sum_{q, \bar{q}} e_{q}^{2} \int \mathrm{d}^{4} \mathbf{p} \delta\left((\mathbf{p}+\mathbf{q})^{2}\right) \operatorname{Tr}\left[\Phi \gamma^{\mu}(\not \mathbf{p}+\mathbf{q}) \gamma^{\nu}\right]
$$

performing the sum over the different quark and antiquark flavours, $q$ and $\bar{q}$. The Dirac matrices $\gamma^{\mu}$ are defined in Appendix A. The quark-quark correlation matrix $\Phi_{i j}$ describes the confinement of the quarks inside the nucleon and depends on the four momentum of the quark (p) and the proton (P) and the spin of the proton (S):

$$
\Phi_{i j}(\mathbf{p}, \mathbf{P}, \mathbf{S})=\frac{1}{(2 \pi)^{4}} \int \mathrm{d}^{4} \boldsymbol{\xi} \mathrm{e}^{i \mathbf{p} \cdot \boldsymbol{\xi}}\left\langle\mathbf{P S}\left|\bar{\psi}_{j}(\mathbf{0}) \psi_{i}(\boldsymbol{\xi})\right| \mathbf{P S}\right\rangle .
$$

Here, $\psi$ is the quark spinor with $i, j$ being Dirac indices. The summation over colour is not indicated for clarity. The integration is performed over all possible separations $\xi$ of the $i$ th component of the quark spinor.

The correlation function can be decomposed in a basis of Dirac matrices,

$$
\mathbb{1}, \gamma^{\mu}, \gamma^{\mu} \gamma_{5}, i \gamma_{5}, i \sigma^{\mu \nu} \gamma_{5}
$$

where $\sigma^{\mu \nu}$ is defined as $\frac{i}{2}\left[\gamma^{\mu}, \gamma^{\nu}\right]$ :

$$
\Phi(\mathbf{p}, \mathbf{P}, \mathbf{S})=\frac{1}{2}\left\{\mathcal{S} \mathbb{1}+\mathcal{V}_{\mu} \gamma^{\mu}+\mathcal{A}_{\mu} \gamma_{5} \gamma^{\mu}+i \mathcal{P}_{5} \gamma_{5}+i \mathcal{T}_{\mu \nu} \sigma^{\mu \nu} \gamma_{5}\right\}
$$

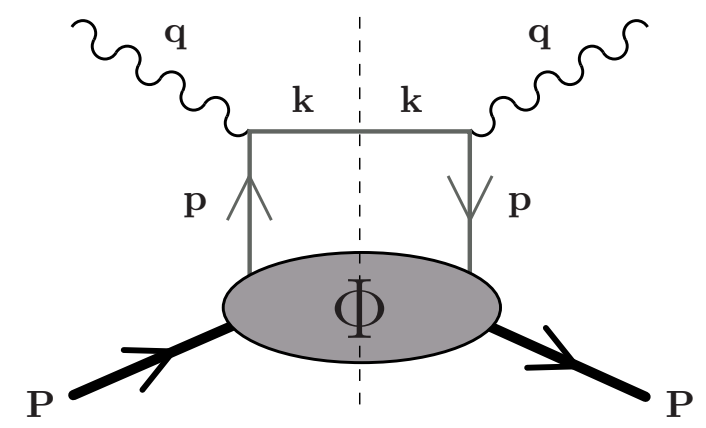

Figure 2.4.1: Handbag diagram for inclusive DIS. It is equivalent to the lower part of Figure 2.2.1. 
The scalar $\mathcal{S}$, vector $\mathcal{V}_{\mu}$, axial-vector $\mathcal{A}_{\mu}$, tensor $\mathcal{T}_{\mu \nu}$, and pseudo-scalar $\mathcal{P}_{5}$ parameters depend on the vectors $\mathbf{p}, \mathbf{P}$, and $\mathbf{S}$. They can be ordered according to powers of $1 / P^{+}$ where the leading term is $\left(1 / P^{+}\right)^{-1}=P^{+}$and the next-to-leading term is $\left(1 / P^{+}\right)^{0}=1$. The different powers correspond to the twist expansion according to [Jaf96], where the leading term is twist-two.

If transverse momenta are neglected, in leading twist ( = twist-two), only the vector, axial-vector, and tensor components are non-zero. They can be expressed by three real amplitudes $A_{i}\left(\mathbf{p}^{2}, \mathbf{p} \cdot \mathbf{P}\right)$ satisfying hermiticity and parity invariance:

$$
\Phi(\mathbf{p}, \mathbf{P}, \mathbf{S})=\frac{1}{2}\left\{A_{1} \not \mathbf{P}+A_{2} \lambda \gamma_{5} \not \mathbf{P}+A_{3} \not \mathbf{P} \gamma_{5} \mathbf{S}_{\perp}\right\},
$$

where the nucleon spin is $\mathbf{S} \approx \lambda \frac{\mathbf{P}}{M}+\mathbf{S}_{\perp}$ with the nucleon helicity $\lambda$. Integrating the amplitudes $A_{i}$ over $\mathbf{p}$ with the constraint $x=p^{+} / P^{+}$, the three leading twist DFs are obtained:

$$
\begin{aligned}
q(x) & =\int \frac{\mathrm{d}^{4} \mathbf{p}}{(2 \pi)^{4}} A_{1}\left(\mathbf{p}^{2}, \mathbf{p} \cdot \mathbf{P}\right) \delta\left(x-\frac{p^{+}}{P^{+}}\right), \\
\Delta q(x) & =\int \frac{\mathrm{d}^{4} \mathbf{p}}{(2 \pi)^{4}} A_{2}\left(\mathbf{p}^{2}, \mathbf{p} \cdot \mathbf{P}\right) \delta\left(x-\frac{p^{+}}{P^{+}}\right), \\
\delta q(x) & =\int \frac{\mathrm{d}^{4} \mathbf{p}}{(2 \pi)^{4}} A_{3}\left(\mathbf{p}^{2}, \mathbf{p} \cdot \mathbf{P}\right) \delta\left(x-\frac{p^{+}}{P^{+}}\right) .
\end{aligned}
$$

Here and in the following, the implicit $Q^{2}$ dependence of the DFs is indicated only when relevant. The integrated quark-quark correlation function over the quark momentum $\mathbf{p}$ in leading twist then reads:

$$
\Phi(x)=\frac{1}{2}\left\{q(x) \not \mathbf{P}+\lambda \Delta q(x) \gamma_{5} \not \mathbf{P}+\delta q(x) \not \gamma_{5} \boldsymbol{\$}_{\perp}\right\} .
$$

\subsubsection{Transversity}

Two of the three leading twist DFs in Eq. (2.4.6) - (2.4.8) were already introduced in Section 2.2. The third DF $\delta q(x)$, called transversity, cannot be diagonalised in the helicity basis and hence has no probabilistic interpretation, unlike $q(x)$ and $\Delta q(x)$. In the basis of transverse spin eigenstates, $|\perp\rangle$ and $|T\rangle$, which are defined as linear combinations of helicity eigenstates, $|+\rangle$ and $|-\rangle$,

$$
|\perp\rangle=\frac{1}{2}(|+\rangle+i|-\rangle), \quad|\top\rangle=\frac{1}{2}(|+\rangle-i|-\rangle),
$$

$\delta q(x)$ can be interpreted as the probability to find a quark with its spin aligned along the transverse spin of the nucleon minus the probability to find it oppositely aligned:

$$
\delta q(x)=q^{\Uparrow \uparrow}(x)-q^{\Uparrow \downarrow}(x) .
$$

The quark-hadron scattering amplitude which transversity is related to includes helicity flips of the quark and the hadron. In the infinite momentum frame, masses can be neglected and helicity is equal to chirality. Thus, transversity is a chiral-odd function. In Figure 2.4.2, the handbag diagrams of inclusive DIS with the different possible configurations of the nucleon and quark helicities are shown. The sum (difference) of configurations (a) 
and (b) is proportional to the unpolarised (polarised) DF $q(x)(\Delta q(x))$. The third configuration (c) includes helicity flips of quark and nucleon and is proportional to the transversity DF $\delta q(x)$. Helicity conservation in hard scattering processes forbids its existence and prevents therefore the measurement of transversity in inclusive DIS. Also no direct relation between $\delta q(x)$ and the second polarised structure function $g_{2}(x)$ exists, which can be accessed in inclusive DIS on a transversely polarised target.

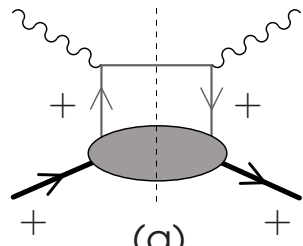

(a)

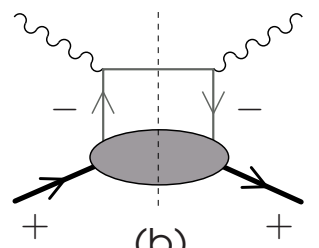

(b)

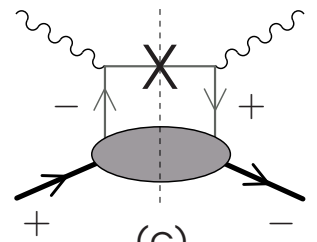

(c)

Figure 2.4.2: The three independent configurations of quark and nucleon helicities in the handbag diagram.

The basis of transverse spin eigenstates can be transferred to the helicity basis by rotation. Thus, only in relativistic regimes where Lorentz boost and rotation do not commute, differences of $\delta q(x)$ and $\Delta q(x)$ are expected. In contrast to the helicity distribution, no transversity distribution of gluons exists in nucleons. Such a distribution would imply a helicity flip of gluon and nucleon which is impossible because of the integer gluon helicity of \pm 1 and the conservation of helicity. This results in a different $Q^{2}$ evolution for transversity and helicity DFs. Even if $\delta q(x)=\Delta q(x)$ for a certain $Q_{0}^{2}$, the functions may differ at another scale $Q_{1}^{2}$.

The probabilistic interpretations of the DFs allow the relation $q(x)=q^{+}(x)+q^{-}(x)=$ $q^{\Uparrow \uparrow}(x)+q^{\Uparrow \downarrow}(x)$ from which the following trivial bounds on the three leading twist DFs are obtained:

$$
|\Delta q(x)| \leq q(x), \quad|\delta q(x)| \leq q(x) .
$$

The derivation of the Soffer inequality [Sof95] is more complicated:

$$
2|\delta q(x)| \leq q(x)+\Delta q(x),
$$

because the three quantities are not diagonal in the same basis. All three inequalities are preserved by QCD evolution.

\subsubsection{Transverse-Momentum Dependent Distribution Functions}

So far the quark transverse momentum $\vec{p}_{T}$ has been neglected because it is small compared to the longitudinal component. Nevertheless, it remains unchanged by the quarkparton interaction and influences the final momenta of the produced hadrons. For the analysis of semi-inclusive DIS on a transversely polarised target (see Section 2.5), it will be therefore necessary to consider also quark transverse-momentum dependent DFs. Taking into account non-zero $\vec{p}_{T}$, in leading twist additional amplitudes $A_{i}$ appear in the vector $\mathcal{V}_{\mu}$, axial-vector $\mathcal{A}_{\mu}$, and tensor $\mathcal{T}_{\mu \nu}$ component of the correlation function. All amplitudes are related to eight different $\vec{p}_{T}$-dependent DFs. They are listed in Figure 2.4.3 
together with illustrations of their probabilistic interpretations. Nucleon and quark are represented by light and dark grey circles, respectively. Their spin orientations with respect to the incident virtual photon which is thought of entering Figure 2.4.3 from the left side, are indicated by arrows.

\begin{tabular}{|c|c|c|c|}
\hline \multicolumn{2}{|c|}{ T-even } & \multicolumn{2}{|c|}{ T-odd } \\
\hline chiral-even & chiral-odd & chiral-even & chiral-odd \\
\hline$q$ & & & $\frac{1}{1}(\hat{0})-$ \\
\hline & & & \\
\hline & & & \\
\hline & & 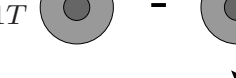 & \\
\hline & - & & \\
\hline
\end{tabular}

Figure 2.4.3: Leading twist transverse-momentum dependent quark distribution functions. All DFs depend on $x$ and $p_{T}^{2}=\left|\vec{p}_{T}\right|^{2}$. For clarity the index $q$ of the quark flavour is omitted unless it contributes to the name of the DF.

The functions are grouped according to their behaviour under chirality and timereversal operations. Integrating the DFs over the transverse momentum of the quark, most of the functions vanish and the three DFs $(2.4 .6)-(2.4 .7)$ are retrieved:

$$
\begin{aligned}
q(x) & =\int \mathrm{d}^{2} \vec{p}_{T} q\left(x, p_{T}^{2}\right) \\
\Delta q(x) & =\int \mathrm{d}^{2} \vec{p}_{T} \Delta q\left(x, p_{T}^{2}\right) \\
\delta q(x) & =\int \mathrm{d}^{2} \vec{p}_{T}\left\{h_{1 T}^{q}\left(x, p_{T}^{2}\right)+\frac{p_{T}^{2}}{2 M} h_{1 T}^{\perp q}\left(x, p_{T}^{2}\right)\right\} \equiv \int \mathrm{d}^{2} p_{T}^{2} \delta q\left(x, p_{T}^{2}\right) .
\end{aligned}
$$

It is convenient to express integrated cross sections in terms of the following moments of any $\operatorname{DF} d^{q}\left(x, p_{T}^{2}\right)$ :

$$
\begin{aligned}
d^{(1 / 2) q}(x) & \equiv \int \mathrm{d}^{2} \vec{p}_{T} d^{(1 / 2) q}\left(x, p_{T}^{2}\right) \equiv \int \mathrm{d}^{2} \vec{p}_{T} \frac{\left|\vec{p}_{T}\right|}{2 M} d^{q}\left(x, p_{T}^{2}\right), \\
d^{(n) q}(x) & \equiv \int \mathrm{d}^{2} \vec{p}_{T} d^{(n) q}\left(x, p_{T}^{2}\right) \equiv \int \mathrm{d}^{2} \vec{p}_{T}\left(\frac{p_{T}^{2}}{2 M^{2}}\right)^{n} d^{q}\left(x, p_{T}^{2}\right),
\end{aligned}
$$

for integer values of $n$. 


\subsubsection{Gauge Link and T-Odd Distribution Functions}

In $Q C D$, a path-dependent link operator $\mathcal{L}$ must be inserted between the quark fields to obtain a gauge invariant correlation function $\Phi$ [Boe00, Ell83]:

$$
\Phi_{i j}(\mathbf{p}, \mathbf{P}, \mathbf{S})=\frac{1}{(2 \pi)^{4}} \int \mathrm{d}^{4} \boldsymbol{\xi} \mathrm{e}^{i \mathbf{p} \cdot \boldsymbol{\xi}}\left\langle\mathbf{P S}\left|\bar{\psi}_{j}(\mathbf{0}) \mathcal{L}(\mathbf{0}, \boldsymbol{\xi}) \psi_{i}(\boldsymbol{\xi})\right| \mathbf{P S}\right\rangle .
$$

The gauge link $\mathcal{L}$, which is also called Wilson line,

$$
\mathcal{L}(\mathbf{0}, \boldsymbol{\xi})=\mathcal{P} \exp \left(-i \sqrt{4 \pi \alpha_{s}} \int_{0}^{\xi} \mathrm{d} s^{\mu} A_{\mu}(s)\right),
$$

is a bilocal operator connecting the quark fields $\psi$ in two different points in space and time, $\mathbf{0}$ and $\boldsymbol{\xi}$. Here, $\mathcal{P}$ indicates the path-ordering of the integral over the gauge field $A_{\mu}$. The Wilson line corresponds to the summation of all diagrams with soft gluon exchange. As example, the handbag diagram for one-gluon exchange is shown in Figure 2.4.4. In the light-cone gauge $\left(A_{+}=0\right)$ the gauge link is equal to unity for $\xi_{T}=0$ and was therefore neglected so far.

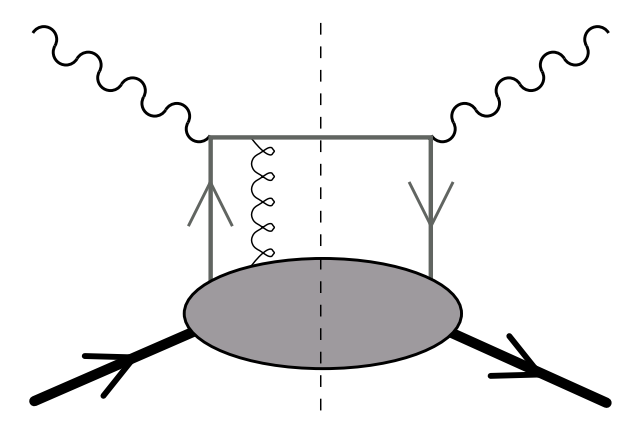

Figure 2.4.4: The handbag diagram with one additional gluon exchanged between quark line and the nucleon remnant.

The Wilson line plays an important role for the existence of time-reversal odd (T-odd) DFs. Invariance under the time-reversal operation which transforms initial states into final states, implies the following constraint on the correlation function $\Phi$ [BarO3] :

$$
\Phi^{*}(\mathbf{p}, \mathbf{P}, \mathbf{S})=\gamma_{5} C \Phi(\tilde{\mathbf{p}}, \tilde{\mathbf{P}}, \tilde{\mathbf{S}}) C^{\dagger} \gamma_{5},
$$

where $C=i \gamma^{2} \gamma^{0}$ and the tilde four-vectors are defined as $\tilde{p}^{\mu}=\left(p^{0},-\vec{p}\right)$. T-odd terms in $\Phi$ will change sign on the left-hand side and are therefore forbidden. With the insertion of $\mathcal{L}$ in $\Phi$, time-reversal invariance does not constrain the T-odd DFs- $f_{1 T}^{\perp q}\left(x, p_{T}^{2}\right)$ and $h_{1}^{\perp q}\left(x, p_{T}^{2}\right)$ in leading twist-to be zero but gives a relation between processes that probe Wilson lines pointing in opposite directions [Col02].

Figure 2.4.4 can also be interpreted in terms of final-state interactions via soft gluons [Bro02]. The colliding particles interact strongly with non-trivial relative phases and 
the transformation of interacting final states into initial states is not as simple as for noninteracting final states. Therefore, time-reversal invariance cannot be implemented by nailvely imposing the condition (2.4.21). T-odd, rather than violating time-reversal invariance, means that the condition (2.4.21) is not satisfied and is often referred to as naive T-odd.

The naïve T-odd DF $f_{1 T}^{\perp q}\left(x, p_{T}^{2}\right)$ was first proposed by Sivers [Siv90] to explain single-spin asymmetries observed in pion production in proton-proton scattering. It is hence referred to as the Sivers function. This DF describes the correlation between the intrinsic transverse momentum of the quarks and the transverse polarisation of the nucleon (cf. Figure 2.4.3). An interesting prerequisite for a non-zero Sivers function is the existence of orbital angular momentum of the quarks, contributing to the spin of the nucleon [Bro02, BurO4].

Also for the T-odd DFs, bounds can be found to ensure positivity of any matrix element of the spin matrix [Bac00]:

$$
\begin{aligned}
& \left|f_{1 T}^{\perp(1) q}\left(x, p_{T}^{2}\right)\right| \leq q^{(1 / 2)}\left(x, p_{T}^{2}\right), \\
& \left|h_{1}^{\perp(1) q}\left(x, p_{T}^{2}\right)\right| \leq q^{(1 / 2)}\left(x, p_{T}^{2}\right) .
\end{aligned}
$$

\subsubsection{Subleading-Twist Distribution Functions}

Taking into account also terms of order $\left(1 / P^{+}\right)^{0}$ in the correlation function, more DFs enter $\Phi(\mathbf{p}, \mathbf{P}, \mathbf{S})$. Six of these twist-three DFs survive the integration over the transverse quark momentum. Three of the functions are T-even functions, $e^{q}(x), h_{L}^{q}(x), g_{T}^{q}(x)$, and three of them are T-odd functions, $h^{q}(x), e_{L}^{q}(x), f_{T}^{q}(x)$, where $L$ and $T$ indicate a longitudinally and transversely polarised nucleon, respectively. Different kinematic and dynamic effects contribute to higher twist: quark masses, intrinsic transverse motion, and gluon interaction. Due to the quark-quark-gluon correlations all subleading-twist DFs have no simple partonic meaning. In general, twist-three DFs can be decomposed into a quark mass term, a term which is related to a twist-two DF, and an interaction dependent term. The latter arises from non-handbag diagrams like the one illustrated in Figure 2.4.4, where the gluon is now a hard gluon. Hence, the interaction dependent term may differ for different processes. The non-handbag diagrams for hard gluons require the introduction of quark-quark-gluon correlation functions.

One important twist-three function is $g_{T}^{q}(x)$ which is related to the second polarised structure function $g_{2}(x)$ by:

$$
g_{1}(x)+g_{2}(x)=\frac{1}{2} \sum_{q, \bar{q}} e_{q}^{2} g_{T}^{q}(x)
$$

The decomposition of $g_{T}^{q}(x)$ contains, among other terms, the transversity DF suppressed by the quark mass $m_{q}[$ Mul96]:

$$
g_{T}^{q}(x)=\frac{m_{q}}{M x} \delta q(x)+\frac{1}{x} g_{1 T}^{(1) q}(x)+\tilde{g}_{T}^{q}(x) .
$$

The interaction dependent term is indicated by a tilde. Thus, only an indirect relation between the polarised structure function $g_{2}(x)$ and transversity $\delta q(x)$ exists. 


\subsection{Semi-Inclusive Deep-Inelastic Scattering}

\subsubsection{Fragmentation Functions}

As explained in the previous section, transversity cannot be measured in inclusive DIS. In order to access the chiral-odd transversity function, it has to be combined with another chiral-odd object. One possibility is the semi-inclusive DIS process where fragmentation functions (FF) enter the cross section in conjunction with the DFs. In a semi-inclusive DIS measurement, at least one produced hadron $h$ with four-momentum $\mathbf{P}_{h}$ is detected in addition to the scattered lepton. The detected hadron has an energy fraction

$$
z=\frac{\mathbf{P} \cdot \mathbf{P}_{h}}{\mathbf{P} \cdot \mathbf{q}} \stackrel{\text { lab }}{=} \frac{E_{h}}{\nu}
$$

and a spin four-vector $\mathbf{S}_{h}$, which goes undetected in most experiments.

Figure 2.5. 1 shows the extended handbag diagram for semi-inclusive DIS, representing the hadronic tensor [Bac02b]:

$$
W^{\mu \nu}=\sum_{q, \bar{q}} e_{q}^{2} \int \mathrm{d}^{4} \mathbf{p} \mathrm{d}^{4} \mathbf{k} \delta(\mathbf{p}+\mathbf{q}-\mathbf{k}) \operatorname{Tr}\left[\Phi(\mathbf{p}, \mathbf{P}, \mathbf{S}) \gamma^{\mu} \Xi\left(\mathbf{k}, \mathbf{P}_{h}, \mathbf{S}_{h}\right) \gamma^{\nu}\right] .
$$

Here, $\Phi$ is the correlation function as defined in Eq. (2.4.2) and $\Xi$ is a new quark-quarkcorrelation function [BarO3],

$$
\Xi_{i j}\left(\mathbf{k}, \mathbf{P}_{h}, \mathbf{S}_{h}\right)=\frac{1}{(2 \pi)^{4}} \int \mathrm{d}^{4} \boldsymbol{\xi} \mathrm{e}^{i \mathbf{k} \cdot \boldsymbol{\xi}}\left\langle\mathbf{0}\left|\psi_{i}(\boldsymbol{\xi})\right| \mathbf{P}_{h}, \mathbf{S}_{h}\right\rangle\left\langle\mathbf{P}_{h}, \mathbf{S}_{h}\left|\bar{\psi}_{j}(\mathbf{0})\right| \mathbf{0}\right\rangle
$$

describing the way the struck quark evolves into a hadronic final state of which the hadron $h$ is detected. This process is called hadronisation or fragmentation of the quark. Decomposition of the correlation function $\Xi$ in the basis of Dirac matrices (2.4.3) including final quark transverse momentum $\vec{k}_{T}$, yields eight fragmentation functions in leading twist,

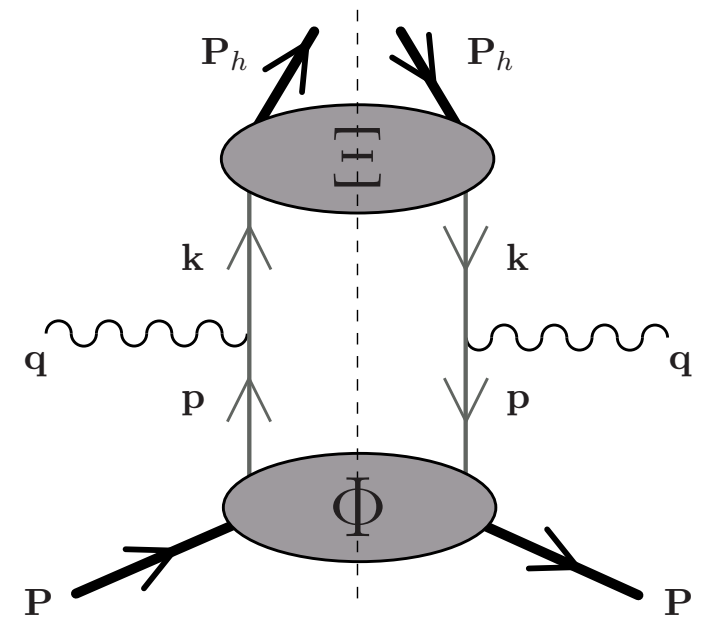

Figure 2.5.1: Extended handbag diagram for semi-inclusive DIS. 
dependent on the hadron energy fraction $z$ and on $z^{2} k_{T}^{2}=z^{2}\left|\vec{k}_{T}\right|^{2}$. After summation over the spin of the produced hadron $\mathbf{S}_{h}$, only two FFs remain, the unpolarised FF $D_{1}^{q \rightarrow h}\left(z, z^{2} k_{T}^{2}\right)$ and the so-called Collins function $H_{1}^{\perp q \rightarrow h}\left(z, z^{2} k_{T}^{2}\right)$ [Col93]. The index $q \rightarrow h$, which indicates the fragmentation of a quark with flavour $q$ into a hadron of type $h$, is often omitted for clarity. Like twist-two DFs, also twist-two FFs have a probabilistic interpretation. The unpolarised FF $D_{1}\left(z, z^{2} k_{T}^{2}\right)$ is the probability density that a struck quark of flavour $q$ with transverse momentum $\vec{k}_{T}$ fragments into a certain hadron of type $h$ with energy fraction $z$. The Collins FF $H_{1}^{\perp}\left(z, z^{2} k_{T}^{2}\right)$ is the difference of the probability densities for quarks with transverse spin state $\uparrow$ and $\downarrow$ to fragment into a hadron $h$ (cf. Figure 2.5.2).

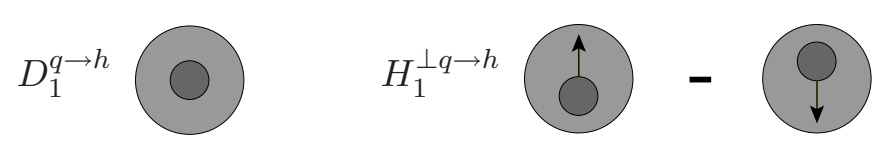

Figure 2.5.2: Leading twist transverse-momentum dependent quark fragmentation functions independent of the produced hadron spin. Both FFs depend on $z$ and $z^{2} k_{T}^{2}$. The struck quark (produced hadron) is indicated as a dark (light) grey circle.

$D_{1}\left(z, z^{2} k_{T}^{2}\right)$ does not change sign under chirality and time-reversal operations while the Collins function is chiral-odd and T-odd. In contrast to the T-odd DFs described in Section 2.4.3, T-odd FFs are not constrained by time-reversal invariance because of the unknown hadronic final state $X$ accompanying the hadron $h$. It has been shown that final-state interactions, which happen solely in the upper soft part of the extended handbag diagram described by the correlation function $\Xi$, are sufficient for the existence of T-odd FFs [Bac01, Bac02a]. Therefore, a soft gluon exchange between the initial quark and the correlator $\Xi$ is not necessary.

As for the DFs also for the unpolarised FF-and equivalently for the Collins functionthe introduction of moments is convenient:

$$
\begin{aligned}
D_{1}^{(1 / 2)}(z) & \equiv z^{2} \int \mathrm{d}^{2} \vec{k}_{T} D_{1}^{(1 / 2) q}\left(z, z^{2} k_{T}^{2}\right) \equiv z^{2} \int \mathrm{d}^{2} \vec{k}_{T} \frac{\left|\vec{k}_{T}\right|}{2 M_{h}} D_{1}^{q}\left(z, z^{2} k_{T}^{2}\right), \\
D_{1}^{(n)}(z) & \equiv z^{2} \int \mathrm{d}^{2} \vec{k}_{T} D_{1}^{(n) q}\left(z, z^{2} k_{T}^{2}\right) \equiv z^{2} \int \mathrm{d}^{2} \vec{k}_{T}\left(\frac{k_{T}^{2}}{2 M_{h}^{2}}\right)^{n} D_{1}^{q}\left(z, z^{2} k_{T}^{2}\right),
\end{aligned}
$$

where $n$ is an integer. The following inequality arises from the positivity constraint [Bac02b]:

$$
\left|H_{1}^{\perp(1)}\left(z, z^{2} k_{T}^{2}\right)\right| \leq D_{1}^{(1 / 2)}\left(z, z^{2} k_{T}^{2}\right) .
$$

Different phenomenological models have been developed to describe the unpolarised fragmentation process. A very successful model for the description of experimental data is the LUND string fragmentation model [And83, And97]. In this model, the colour field connecting the initial quarks is assumed to possess a constant field energy density. This results in a potential which increases linearly with the distance between the quarks. After one of the quarks is struck and moves away, the energy stored in the colour 
string rises. As soon as it exceeds the rest mass of a quark-antiquark pair the string may break up and create such a pair. The partners of this pair are then connected to the initial quarks by two new strings. They continue to break independently until a stringconnected quark-antiquark pair is close to the mass shell of a colour singlet hadron.

The number of independent FFs for quarks of flavour $q$ fragmenting in hadrons of type $h$, decreases significantly when charge conjugation and isospin symmetry is applied, which is valid for a strong interaction process like fragmentation. In case of three different quark flavours which hadronise into pions, only three independent unpolarised FFs remain:

$$
\begin{aligned}
D_{1, \mathrm{fav}}(z) & =D_{1}^{u \rightarrow \pi^{+}}(z)=D_{1}^{\bar{u} \rightarrow \pi^{-}}(z)=D_{1}^{\bar{d} \rightarrow \pi^{+}}(z)=D_{1}^{d \rightarrow \pi^{-}}(z), \\
D_{1, \mathrm{dis}}(z) & =D_{1}^{u \rightarrow \pi^{-}}(z)=D_{1}^{\bar{u} \rightarrow \pi^{+}}(z)=D_{1}^{\bar{d} \rightarrow \pi^{-}}(z)=D_{1}^{d \rightarrow \pi^{+}}(z), \\
D_{1, \mathrm{~s}}(z) & =D_{1}^{s \rightarrow \pi^{+}}(z)=D_{1}^{\bar{s} \rightarrow \pi^{-}}(z)=D_{1}^{\bar{s} \rightarrow \pi^{+}}(z)=D_{1}^{s \rightarrow \pi^{-}}(z) .
\end{aligned}
$$

They are called favoured, disfavoured, and strange unpolarised FFs. The FF of the neutral pion is obtained by:

$$
\begin{aligned}
D_{1}^{q \rightarrow \pi^{0}}(z) & =\frac{1}{2}\left[D_{1}^{q \rightarrow \pi^{+}}(z)+D_{1}^{q \rightarrow \pi^{-}}(z)\right] & & \\
& =\frac{1}{2}\left[D_{1, \text { fav }}(z)+D_{1, \text { dis }}(z)\right] & & \text { for } \quad q=u, d, \bar{u}, \bar{d}, \\
& =D_{1, \mathrm{~s}}(z) & & \text { for } \quad q=s, \bar{s} .
\end{aligned}
$$

Applying the same symmetry constraints to the Collins function yields three independent functions: the favoured $H_{1, \text { fav }}^{\perp}$, the disfavoured $H_{1, \text { dis }}^{\perp}$, and the strange $H_{1, \mathrm{~s}}^{\perp}$ Collins function.

\subsubsection{Cross Sections and Azimuthal Asymmetry Moments}

The differential cross section,

$$
\frac{\mathrm{d}^{6} \sigma}{\mathrm{d} x \mathrm{~d} y \mathrm{~d} z \mathrm{~d} \phi_{S} \mathrm{~d}^{2} \vec{P}_{h \perp}}=\frac{\alpha^{2}}{4 z s^{2} x^{2} y} L_{\mu \nu} W^{\mu \nu},
$$

can be calculated using the hadronic tensor (2.5.2). Here, $\vec{P}_{h \perp}$ is the transverse momentum of the produced hadron and $P_{h \perp}^{2}=\left|\vec{P}_{h \perp}\right|^{2}$ must be small compared to $Q^{2}$ to avoid the introduction of further hard scales [Bac02b]. The azimuthal angle $\phi_{S}$ is defined as the angle around the virtual photon between the target spin $\vec{S}_{T}$ and the plane spanned by the incoming and outgoing lepton, as depicted in Figure 2.5.3. It is convenient to split the cross section into unpolarised and polarised terms:

$$
\mathrm{d} \sigma^{6}=\mathrm{d} \sigma_{\mathrm{UU}}^{6}+\mathrm{d} \sigma_{\mathrm{LU}}^{6}+\mathrm{d} \sigma_{\mathrm{UL}}^{6}+\mathrm{d} \sigma_{\mathrm{LL}}^{6}+\mathrm{d} \sigma_{\mathrm{UT}}^{6}+\mathrm{d} \sigma_{\mathrm{LT}}^{6} .
$$

Two subscripts are used to indicate beam and target polarisation where U means unpolarised, L longitudinally polarised, and T transversely polarised. Here and in the following, the differential kinematic variables are omitted for clarity. The unpolarised cross section in leading twist contains two products of DF and FF [Bac02b]:

$$
\begin{aligned}
& \mathrm{d}^{6} \sigma_{\mathrm{UU}}=\frac{2 \alpha^{2}}{s x y^{2}}\left\{A(y) \sum_{q, \bar{q}} e_{q}^{2} \mathcal{I}\left[q\left(x, p_{T}^{2}\right) D_{1}^{q}\left(z, z^{2} k_{T}^{2}\right)\right]-\right. \\
& \left.B(y) \cos 2 \phi \sum_{q, \bar{q}} e_{q}^{2} \mathcal{I}\left[\frac{2\left(\vec{p}_{T} \cdot \hat{P}_{h \perp}\right)\left(\vec{k}_{T} \cdot \hat{P}_{h \perp}\right)-\vec{p}_{T} \cdot \vec{k}_{T}}{M M_{h}} h_{1}^{\perp q}\left(x, p_{T}^{2}\right) H_{1}^{\perp q}\left(z, z^{2} k_{T}^{2}\right)\right]\right\},
\end{aligned}
$$




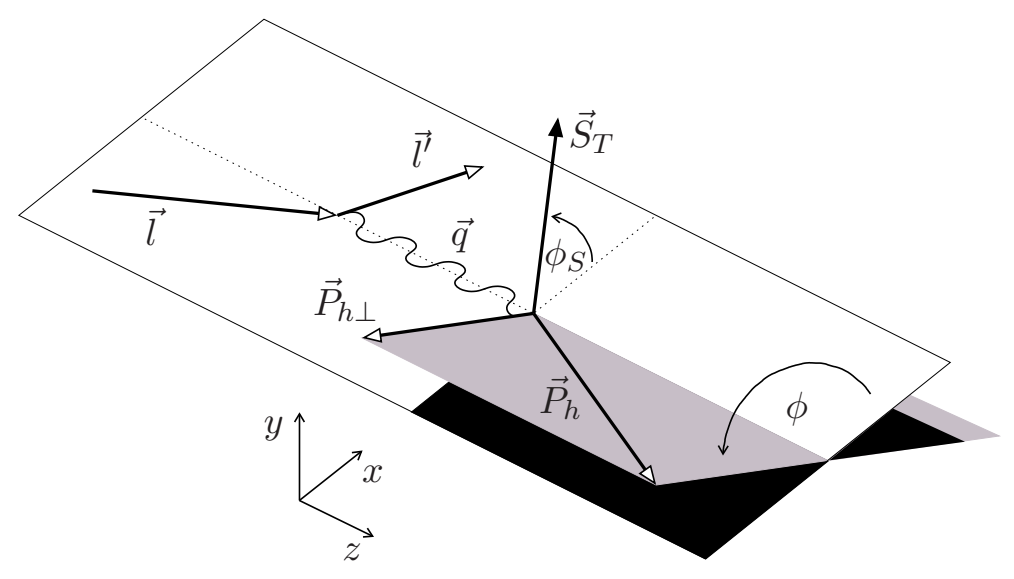

Figure 2.5.3: Definition of the azimuthal angles $\phi$ and $\phi_{S}$ between the scattering plane (white), the production plane (grey), and the transverse nucleon spin component $\vec{S}_{T}$.

where $M_{h}$ is the mass of the produced hadron and $\hat{P}_{h \perp}$ is the unit vector of the hadron transverse momentum. The two products of DF and FF appear in the integral,

$$
\mathcal{I}[\mathcal{W} \cdot d \cdot \mathcal{F}] \equiv \int \mathrm{d}^{2} \vec{p}_{T} \mathrm{~d}^{2} \vec{k}_{T} \delta\left(\vec{p}_{T}-\frac{\vec{P}_{h \perp}}{z}-\vec{k}_{T}\right) \mathcal{W} \cdot d \cdot \mathcal{F},
$$

defined for any combination of DF $d$ and FF $\mathcal{F}$ multiplied by the weight $\mathcal{W}$ dependent on $\hat{P}_{h \perp}, \vec{p}_{T}, \vec{k}_{T}, x$, and $z$. The kinematic factors $A(y), B(y)$ and the $C(y), D(y)$ used later are defined as:

$$
\begin{array}{ll}
A(y)=1-y+\frac{y^{2}}{2}, & B(y)=1-y, \\
C(y)=y\left(1-\frac{y}{2}\right), & D(y)=2(2-y) \sqrt{1-y} .
\end{array}
$$

Using Table 2.5.1, different azimuthal modulations of the unpolarised and polarised cross-section terms can be constructed by:

$$
\mathrm{d}^{6} \sigma_{\text {beam target }}=\frac{2 \alpha^{2}}{s x y^{2}} \cdot \text { factor } \cdot \text { modulation } \cdot \sum_{q, \bar{q}} e_{q}^{2} \mathcal{I}[\mathcal{W} \cdot d \cdot \mathcal{F}] \text {. }
$$

In the table, $\lambda$ is the beam helicity and $\vec{S}_{L}$ and $\vec{S}_{T}$ are the longitudinal and transverse target spin components with respect to the virtual photon. The relevant azimuthal angles are the already introduced angle $\phi_{S}$ and the angle $\phi$ between the scattering and the hadron production plane, as defined in Figure 2.5.3. Table 2.5.1 includes only twist-two and twist-three cross-section terms. The table is not complete since the twist-three terms of $d \sigma_{\mathrm{LL}}^{6}$ and $d \sigma_{\mathrm{LT}}^{6}$ are missing and the cross-section terms of $d \sigma_{\mathrm{LU}}^{6}$ are not listed. The twistthree terms contain several DFs $d$ in conjunction with FFs $\mathcal{F}$ where either $d$ or $\mathcal{F}$ is a twistthree function combined with a twist-two function. The functions $\tilde{D}^{\perp}\left(z, z^{2} k_{T}^{2}\right), \tilde{G}^{\perp}\left(z, z^{2} k_{T}^{2}\right)$, and $\tilde{H}\left(z, z^{2} k_{T}^{2}\right)$ are the interaction dependent parts of the twist-three FFs $D^{\perp}\left(z, z^{2} k_{T}^{2}\right)$, $G^{\perp}\left(z, z^{2} k_{T}^{2}\right)$, and $H\left(z, z^{2} k_{T}^{2}\right)$, respectively. The twist-three DFs $f^{\perp}\left(x, p_{T}^{2}\right), f_{T}^{\perp}\left(x, p_{T}^{2}\right), f_{L}^{\perp}\left(x, p_{T}^{2}\right)$, $h_{T}\left(x, p_{T}^{2}\right)$, and $h_{T}^{\perp}\left(x, p_{T}^{2}\right)$ vanish when integrated over the transverse quark momentum. 


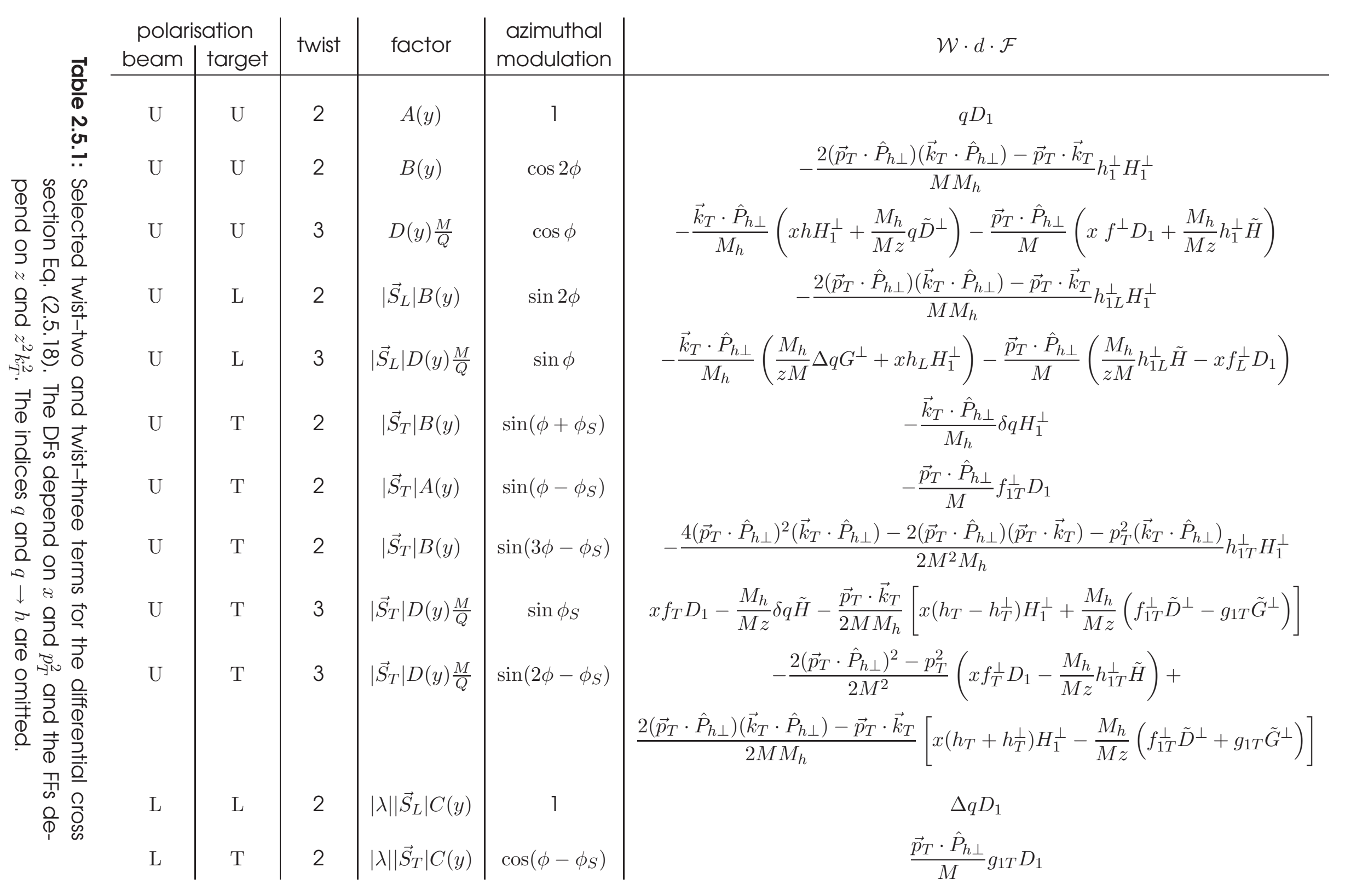


Quark mass terms which are proportional to $m_{q} / M$ or $m_{q} / M_{h}$ are neglected. The subleading-twist component of the unpolarised cross section which exhibits a $\cos \phi$ modulation is also known as Cahn effect [Cah78]. All twist-two and twist-three cross-section terms can be found for instance in [Mul96, Boe98, Bac04c, Bac06].

The product of the transversity DF and the Collins FF appears as a $\sin \left(\phi+\phi_{S}\right)$ modulation in the polarised cross section for an unpolarised beam and a transversely polarised target:

$$
\mathrm{d}^{6} \sigma_{\mathrm{UT}}=-\frac{2 \alpha^{2}}{s x y^{2}}\left|\vec{S}_{T}\right| B(y) \sin \left(\phi+\phi_{S}\right) \sum_{q, \bar{q}} e_{q}^{2} \mathcal{I}\left[\frac{\vec{k}_{T} \cdot \hat{P}_{h \perp}}{M_{h}} \delta q\left(x, p_{T}^{2}\right) H_{1}^{\perp q}\left(z, z^{2} k_{T}^{2}\right)\right] .
$$

The same polarised cross section exhibits also a $\sin \left(\phi-\phi_{S}\right)$ modulation proportional to the product of the Sivers function and the unpolarised FF:

$$
\mathrm{d}^{6} \sigma_{\mathrm{UT}}=-\frac{2 \alpha^{2}}{s x y^{2}}\left|\vec{S}_{T}\right| A(y) \sin \left(\phi-\phi_{S}\right) \sum_{q, \bar{q}} e_{q}^{2} \mathcal{I}\left[\frac{\vec{p}_{T} \cdot \hat{P}_{h \perp}}{M} f_{1 T}^{\perp q}\left(x, p_{T}^{2}\right) D_{1}^{q}\left(z, z^{2} k_{T}^{2}\right)\right] .
$$

Experimentally the measurement of cross-section asymmetries is preferred over the measurement of absolute cross sections or differences thereof as many uncertainties cancel for asymmetry measurements. In order to extract the individual products $d \cdot \mathcal{F}$ with different azimuthal modulations in the cross section, it is common to measure azimuthal moments:

$$
\begin{aligned}
\left\langle\sin \left(n \phi+m \phi_{S}\right)\right\rangle_{\text {beam target }}(x, y, z) & \equiv \frac{\int \mathrm{d} \phi_{S} \mathrm{~d}^{2} \vec{P}_{h \perp} \sin \left(n \phi+m \phi_{S}\right) \mathrm{d}^{6} \sigma_{\text {beam target }}}{\int \mathrm{d} \phi_{S} \mathrm{~d}^{2} \vec{P}_{h \perp} \mathrm{d}^{6} \sigma_{\mathrm{UU}}}, \\
\left\langle\cos \left(n \phi+m \phi_{S}\right)\right\rangle_{\text {beam target }}(x, y, z) & \equiv \frac{\int \mathrm{d} \phi_{S} \mathrm{~d}^{2} \vec{P}_{h \perp} \cos \left(n \phi+m \phi_{S}\right) \mathrm{d}^{6} \sigma_{\text {beam target }}}{\int \mathrm{d} \phi_{S} \mathrm{~d}^{2} \vec{P}_{h \perp} \mathrm{d}^{6} \sigma_{\mathrm{UU}}}
\end{aligned}
$$

where the integers $n$ and $m$ can be positive or negative. For the separation of the individual polarised cross-section terms, cross-section differences of opposite spin states are formed in which the unpolarised cross-section component cancels:

$$
\begin{aligned}
\mathrm{d}^{6} \sigma_{\mathrm{UL}} & \equiv \frac{1}{2}\left(\mathrm{~d}^{6} \sigma_{\left.\mathrm{U} \leftarrow-\mathrm{d}^{6} \sigma_{\mathrm{U} \rightarrow}\right),}\right. & \mathrm{d}^{6} \sigma_{\mathrm{LL}} & \equiv \frac{1}{2}\left(\mathrm{~d}^{6} \sigma_{\rightarrow \leftarrow}-\mathrm{d}^{6} \sigma_{\rightarrow \rightarrow}\right), \\
\mathrm{d}^{6} \sigma_{\mathrm{UT}} & \equiv \frac{1}{2}\left(\mathrm{~d}^{6} \sigma_{\mathrm{U} \uparrow}-\mathrm{d}^{6} \sigma_{\mathrm{U} \downarrow}\right) . & \mathrm{d}^{6} \sigma_{\mathrm{LT}} & \equiv \frac{1}{2}\left(\mathrm{~d}^{6} \sigma_{\rightarrow \uparrow}-\mathrm{d}^{6} \sigma_{\rightarrow \downarrow}\right),
\end{aligned}
$$

The arrows show the direction of the spin orientation, which can be parallel $\rightarrow$ and antiparallel $\leftarrow$ to the direction of the virtual photon and parallel $\uparrow$ and antiparallel $\downarrow$ to the direction specified by the angle $\phi_{S}$.

The so-called Collins moment,

$$
\left\langle\sin \left(\phi+\phi_{S}\right)\right\rangle_{\mathrm{UT}}=-\left|\vec{S}_{T}\right| \frac{\frac{1}{x y^{2}} B(y) \sum_{q, \bar{q}} e_{q}^{2} \int \mathrm{d}^{2} \vec{P}_{h \perp} \mathcal{I}\left[\frac{\vec{k}_{T} \cdot \hat{P}_{h \perp}}{M_{h}} \delta q\left(x, p_{T}^{2}\right) H_{1}^{\perp q}\left(z, z^{2} k_{T}^{2}\right)\right]}{2 \frac{1}{x y^{2}} A(y) \sum_{q, \bar{q}} e_{q}^{2} q(x) D_{1}^{q}(z)},
$$

gives access to transversity and the Collins function. The factor 2 in the denominator remains of the integration over the angle $\phi_{S}$. Unfortunately, the product $\delta q \cdot H_{1}^{\perp}$ is still embedded in a convolution integral over the transverse momenta of the initial and final quarks and of the produced hadron. The integral cannot be factorised like in the case of 
the product of unpolarised DF and FF because of the weight $\mathcal{W}=\frac{\vec{k}_{T} \cdot \hat{P}_{h \perp}}{M_{h}}$. One ansatz to solve the integral is to assume Gaussian transverse momentum distributions in the DF and FF:

$$
\delta q\left(x, p_{T}^{2}\right) \approx \frac{\delta q(x)}{\pi\left\langle p_{T}^{2}(x)\right\rangle} \mathrm{e}^{-\frac{p_{T}^{2}}{\left\langle p_{T}^{2}(x)\right\rangle}}, \quad H_{1}^{\perp}\left(z, K_{T}^{2}\right) \approx \frac{H_{1}^{\perp}(z)}{\pi\left\langle K_{T}^{2}(z)\right\rangle} \mathrm{e}^{-\frac{K_{T}^{2}}{\left\langle K_{T}^{2}(z)\right\rangle}},
$$

where $\vec{K}_{T}=-z \vec{k}_{T}$ is the transverse momentum of the hadron with respect to the quark, and the mean values are defined as:

$$
\left\langle p_{T}^{2}(x)\right\rangle \equiv \frac{\int \mathrm{d}^{2} \vec{p}_{T} p_{T}^{2} q\left(x, p_{T}^{2}\right)}{q(x)}, \quad\left\langle K_{T}^{2}(z)\right\rangle \equiv \frac{\int \mathrm{d}^{2} \vec{K}_{T} K_{T}^{2} D_{1}\left(z, K_{T}^{2}\right)}{D_{1}(z)} .
$$

With this Gaussian ansatz, transversity and Collins function factorise in the Collins moment [Bac02b]:

$$
\left\langle\sin \left(\phi+\phi_{S}\right)\right\rangle_{\mathrm{UT}}=\frac{\left|\vec{S}_{T}\right|}{\sqrt{1+z^{2}\left\langle p_{T}^{2}\right\rangle /\left\langle K_{T}^{2}\right\rangle}} \frac{\frac{1}{x y^{2}} B(y) \sum_{q, \bar{q}} e_{q}^{2} \delta q(x) H_{1}^{\perp(1 / 2) q}(z)}{\frac{1}{x y^{2}} A(y) \sum_{q, \bar{q}} e_{q}^{2} q(x) D_{1}^{q}(z)} .
$$

A different ansatz which avoids any assumption on the transverse momentum distributions, includes $P_{h \perp}$ in the convolution integral for cross-section terms with a weight $\mathcal{W}$ proportional to $\hat{P}_{h \perp}$. In case of the $\sin \left(\phi+\phi_{S}\right)$ modulation, this yields the $P_{h \perp}$-weighted Collins moment:

$$
\begin{aligned}
\left\langle\frac{P_{h \perp}}{z M_{h}} \sin \left(\phi+\phi_{S}\right)\right\rangle_{\mathrm{UT}} & \equiv \frac{\int \mathrm{d} \phi_{S} \mathrm{~d}^{2} \vec{P}_{h \perp} \frac{P_{h \perp}}{z M_{h}} \sin \left(\phi+\phi_{S}\right) \mathrm{d}^{6} \sigma_{\mathrm{UT}}}{\int \mathrm{d} \phi_{S} \mathrm{~d}^{2} \vec{P}_{h \perp} \mathrm{d}^{6} \sigma_{\mathrm{UU}}} \\
& =\left|\vec{S}_{T}\right| \frac{\frac{1}{x y^{2}} B(y) \sum_{q, \bar{q}} e_{q}^{2} \delta q(x) H_{1}^{\perp(1) q}(z)}{\frac{1}{x y^{2}} A(y) \sum_{q, \bar{q}} e_{q}^{2} q(x) D_{1}^{q}(z)} .
\end{aligned}
$$

The combination of Sivers function and unpolarised FF can be extracted from the unweighted and $P_{h \perp}$-weighted Sivers moments:

$$
\begin{aligned}
\left\langle\sin \left(\phi-\phi_{S}\right)\right\rangle_{\mathrm{UT}} & =-\frac{\left|\vec{S}_{T}\right|}{\sqrt{1+\left\langle K_{T}^{2}\right\rangle /\left(z^{2}\left\langle p_{T}^{2}\right\rangle\right)}} \frac{\frac{1}{x y^{2}} A(y) \sum_{q, \bar{q}} e_{q}^{2} f_{1 T}^{\perp(1 / 2) q}(x) D_{1}^{q}(z)}{\frac{1}{x y^{2}} A(y) \sum_{q, \bar{q}} e_{q}^{2} q(x) D_{1}^{q}(z)} \\
\left\langle\frac{P_{h \perp}}{z M} \sin \left(\phi-\phi_{S}\right)\right\rangle_{\mathrm{UT}} & =-\left|\vec{S}_{T}\right| \frac{\frac{1}{x y^{2}} A(y) \sum_{q, \bar{q}} e_{q}^{2}}{\frac{1}{x y^{2}} A(y) \sum_{q, \bar{q}} e_{q}^{2} q(1) q}(x) D_{1}^{q}(z)
\end{aligned}
$$

Note that the factor $\frac{1}{x y^{2}} A(y)$ may not cancel because numerator and denominator are integrated separately over certain $x$ and $y$ ranges in a measurement. Since the Collins and Sivers functions appear in the above introduced azimuthal asymmetry moments, the possible existence of these asymmetry moments are often referred to as Collins and Sivers effect, respectively.

The factorisation of the cross section into the contributions of long-distance and shortdistance interaction which allows the application of perturbative calculations, was proven for transverse-momentum independent functions in leading twist already in the 1980s [Col88]. Only recently proofs were published for the factorisation of twist-two transverse momentum dependent functions at low transverse momenta in semi-inclusive DIS 
and Drell-Yan [JiO4, Ji05]. Whether factorisation holds also for subleading twist is still unclear. The appearance of the functions under the convolution integral and the inclusion of gauge links is challenging. In addition, the $Q^{2}$ evolution for transverse-momentum dependent functions in leading order of the $\alpha_{s}$ expansion became the subject of studies only in the last years [ldiO4].

\subsubsection{The Purity Formalism}

For the extraction of the Sivers DF from the measured azimuthal asymmetry moments, it is convenient to introduce purities [Nic98] which are constructed from unpolarised quantities only:

$$
\mathcal{P}_{q}^{h}(x, z) \equiv \frac{e_{q}^{2} q(x) D_{1}^{q \rightarrow h}(z)}{\sum_{q^{\prime}, \bar{q}^{\prime}} e_{q^{\prime}}^{2} q^{\prime}(x) D_{1}^{q^{\prime} \rightarrow h}(z)} .
$$

Using this definition of the purities, the $P_{h \perp}$-weighted Sivers moment can be rewritten as:

$$
\left\langle\frac{P_{h \perp}}{z} \sin \left(\phi-\phi_{S}\right)\right\rangle_{\mathrm{UT}}=-\left|\vec{S}_{T}\right| \frac{\frac{1}{x y^{2}} A(y)}{\frac{1}{x y^{2}} A(y)} \sum_{q, \bar{q}} \mathcal{P}_{q}^{h}(x, z) \frac{f_{1 T}^{\perp(1) q}(x)}{q(x)} .
$$

Results from a large number of unpolarised DIS experiments with high precision are available for one component of the purities: the unpolarised DF. The second component, the unpolarised FF, can be parametrised based on the LUND string fragmentation model. The parametrisation is implemented in a Monte Carlo simulation of the HERMES experiment which takes into account its acceptance. The parameters included in the simulation are tuned so that hadron multiplicities extracted from the simulation describe results from Hermes data [Hil05]. This Monte Carlo simulation allows the determination of purities for the different quark flavours and hadron types. The azimuthal asymmetry moments of different hadron types can be combined to extract the (1)-moment of the Sivers function for different quark flavours.

The purity formalism was already used for the extraction of the helicity DF $\Delta q(x)$ which appears in the cross-section term for longitudinally polarised beam and target. This crosssection term does not depend on the azimuthal angles and integration over $\vec{P}_{h \perp}$ and $\phi_{S}$ yields the double-spin asymmetry:

$$
\begin{aligned}
A_{\mathrm{LL}}^{h}(x, y, z) \equiv \frac{\int \mathrm{d} \phi_{S} \mathrm{~d}^{2} \vec{P}_{h \perp} \mathrm{d}^{6} \sigma_{\mathrm{LL}}}{\int \mathrm{d} \phi_{S} \mathrm{~d}^{2} \vec{P}_{h \perp} \mathrm{d}^{6} \sigma_{\mathrm{UU}}} & =|\lambda|\left|\vec{S}_{L}\right| \frac{\frac{1}{x y^{2}} C(y) \sum_{q, \bar{q}} e_{q}^{2} \Delta q(x) D_{1}^{q \rightarrow h}(z)}{\frac{1}{x y^{2}} A(y) \sum_{q, \bar{q}} e_{q}^{2} q(x) D_{1}^{q \rightarrow h}(z)} \\
& =|\lambda|\left|\vec{S}_{L}\right| \frac{\frac{1}{x y^{2}} C(y)}{\frac{1}{x y^{2}} A(y)} \sum_{q, \bar{q}} \mathcal{P}_{q}^{h}(x, z) \cdot \frac{\Delta q(x)}{q(x)} .
\end{aligned}
$$

The expression is valid for polarisations with respect to the virtual photon. In experiments where beam and target are polarised with respect to the lepton beam, a correction including the second polarised structure function $g_{2}(x)$ has to be taken into account [Rob90]. The purities determined with the Monte Carlo simulation of the HERMES experiment, allowed the extraction of $\Delta q(x)$ from the HERMES data on longitudinally polarised targets. The double-spin asymmetries for different types of produced hadrons were combined and yield the results for $x \Delta q(x)$ [Air05a] shown in Figure 2.5.4 together with two parametrisations. 


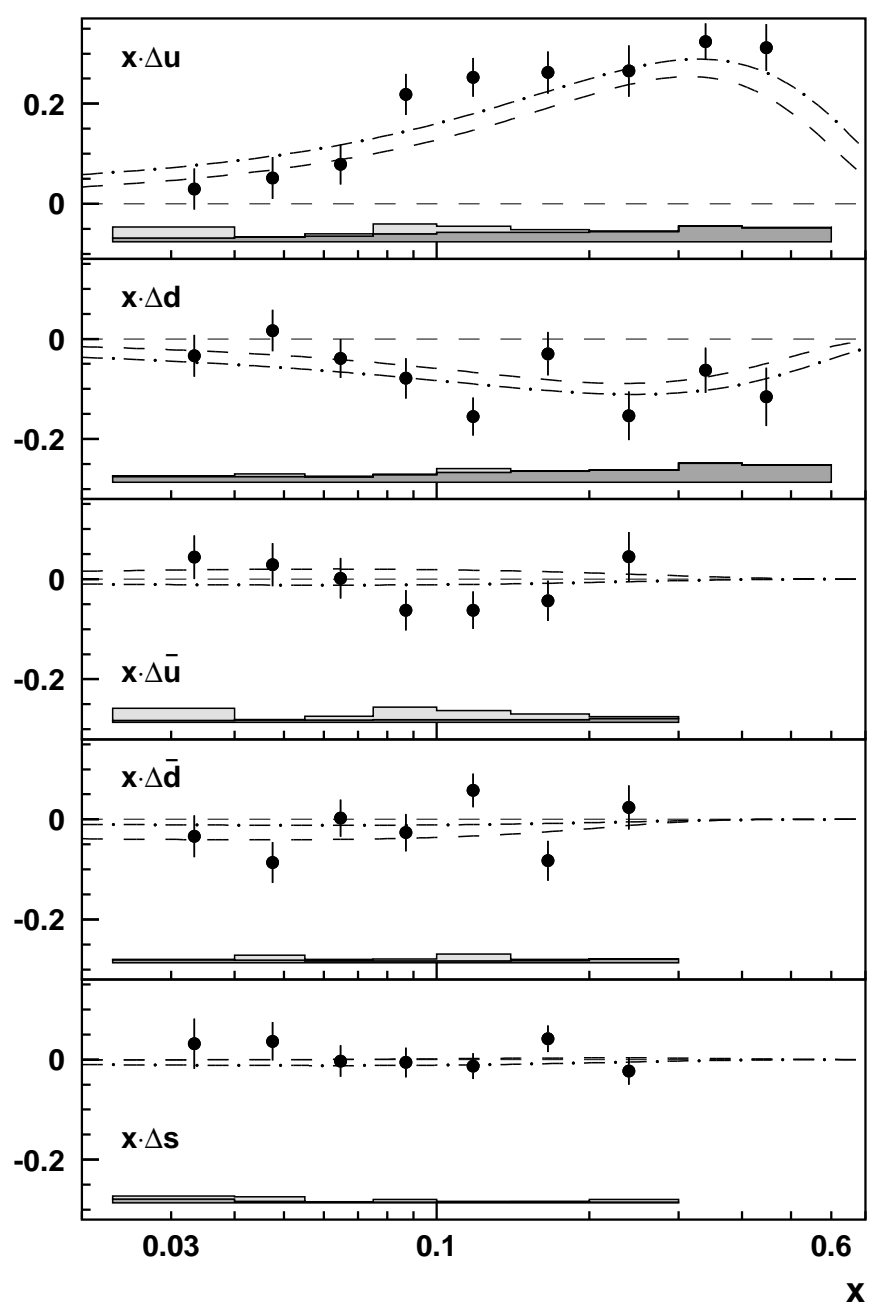

Figure 2.5.4: The quark helicity distributions $x \Delta q\left(x, Q_{0}^{2}\right)$ evaluated at a common value of $Q_{0}^{2}=2.5 \mathrm{GeV}^{2}$ as a function of $x$. The dashed line is the GRSV2000 parametrisation [Glü01] and the dashed-dotted line is the Blümlein-Böttcher parametrisation [BlüO2] (for details see [AirO5a]).

A new type of purity including the Collins function has to be introduced for the extraction of the transversity DF. The determination of transversity from the measured Collins moments will thus be possible after detailed information on the Collins function becomes available.

\subsection{Universality}

In order to measure transversity, in total two hadrons in the initial and final state are necessary where at least one of them must be transversely polarised. The possible processes for a measurement are the semi-inclusive DIS on a transversely polarised target discussed here (see Figure 2.6.1(a)), the scattering of two transversely polarised hadrons, in particular, the transversely polarised Drell-Yan (DY) process (see Figure 2.6. 1(b)), and hadron pro- 


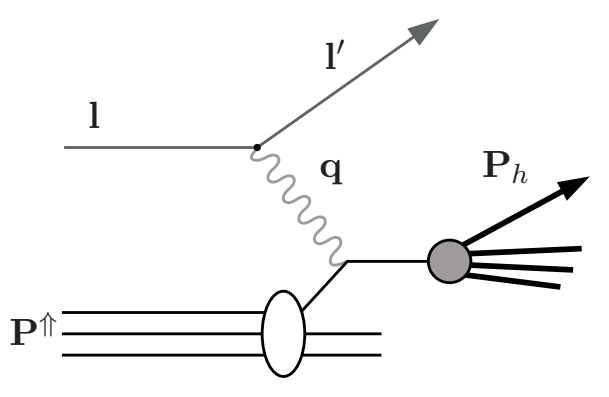

(a)

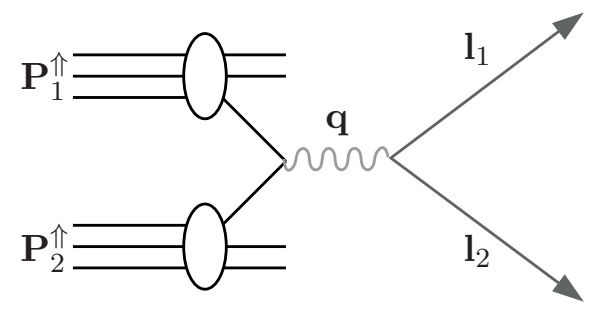

(b)

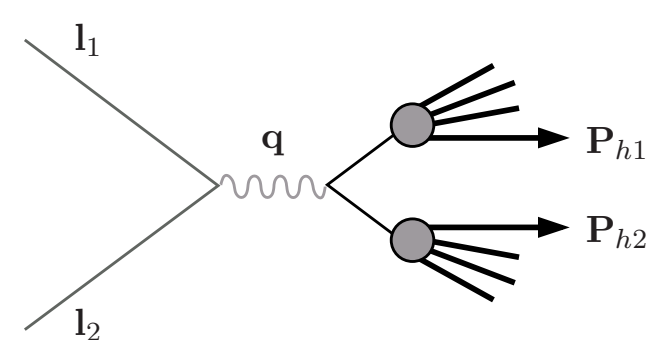

(c)

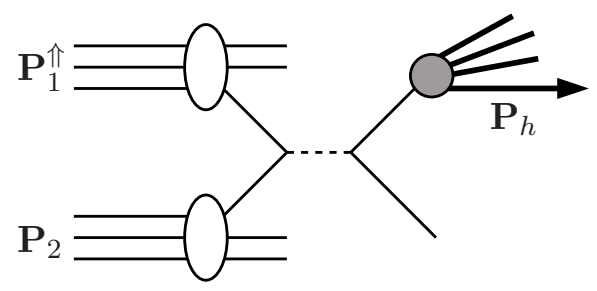

(d)

Figure 2.6.1: The semi-inclusive DIS process (a), the DY process (b), and hadron production in electron-positron annihilation (c), hadron-hadron scattering (d). The white ellipses (gray circles) represent DFs (FFs).

duction with one transversely polarised initial hadron (Figure 2.6.1(d)). The cross sections contain the convolution of one DF and one FF, two DFs, or two DFs and one FF, respectively. For a measurement of the T-odd Sivers function in Drell-Yan processes only one initial hadron needs to be polarised. The Collins function can be accessed in processes involving fragmentation functions like electron-positron annihilation with the production of a quark-antiquark pair which fragments into hadrons (Figure 2.6.1(c)). Here, no DFs are involved but two FFs are convoluted in the cross section. The question arises whether or not the involved DFs and FFs are universal for all the different scattering processes. Thus, can the measurements of different experiments be compared to each other and, furthermore, can they be combined? Such a combined analysis of different processes is often needed for the separation of the different DFs and FFs as at least two of them are usually involved.

As a consequence of factorisation, universality for twist-two transverse-momentum integrated functions was proven in the 1980s [Col88]. The proof of universality for transversemomentum dependent functions was only recently established for T-even functions [Col04] . For twist-two T-odd transverse-momentum dependent DFs, a sign-change between the semi-inclusive DIS and the DY processes,

$$
\begin{aligned}
& \left.f_{1 T}^{\perp q}\left(x, Q^{2}\right)\right|_{\mathrm{DIS}}=-\left.f_{1 T}^{\perp q}\left(x, Q^{2}\right)\right|_{\mathrm{DY}}, \\
& \left.h_{1}^{\perp q}\left(x, Q^{2}\right)\right|_{\mathrm{DIS}}=-\left.h_{1}^{\perp q}\left(x, Q^{2}\right)\right|_{\mathrm{DY}},
\end{aligned}
$$

is caused by the Wilson line [ColO2]. This gauge link which is necessary to allow T-odd functions (cf. Section 2.4.3), is past-pointing in the DY process contrary to the futurepointing Wilson line in the semi-inclusive DIS process. 
Whereas time-reversal can be used to relate T-odd DFs between the semi-inclusive DIS and the DY processes, it is not possible to apply such an argument for T-odd FFs [Boe03, Col04]. A proof of universal FFs was formalised at one-loop [Met02] and generalised to a calculation that shows universality of DFs and FFs in semi-inclusive DIS, electronpositron annihilation and DY processes [Col04]. However, the calculations are model based on Feynman diagrams and might not be an explicit proof of universality [Bom04, Pij04]. Gauge links could spoil universality, and their possible contribution to FFs is not yet clarified. New results in this active field are expected both from the theoretical side as well as from various current and future experimental efforts. 

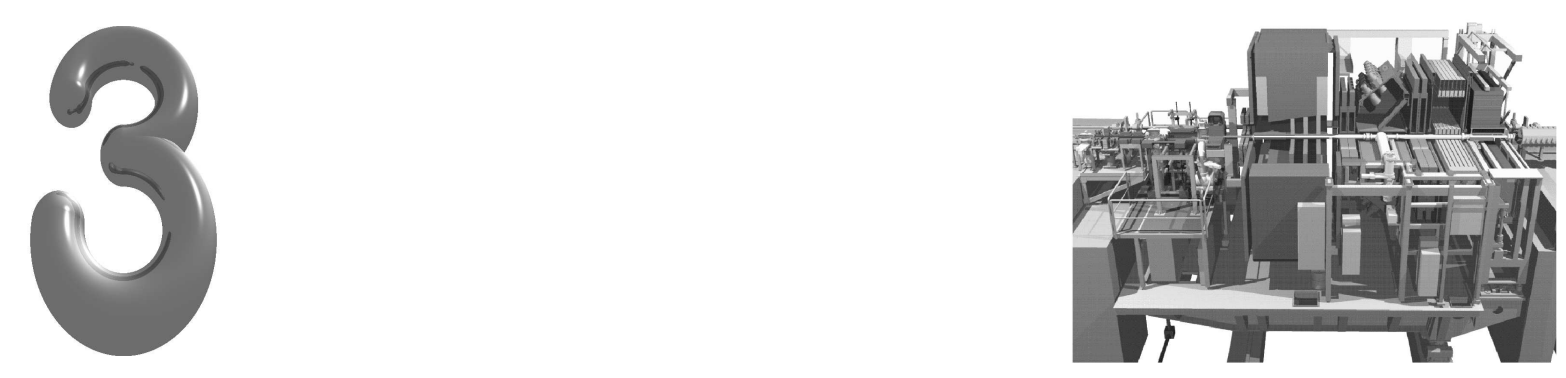

\section{The HERMES Experiment}

A deep-inelastic scattering experiment like HERMES needs three ingredients in order to measure azimuthal asymmetry moments. These are a high-energy polarised or unpolarised lepton beam, a polarised or unpolarised nucleon target, and a spectrometer with reasonable particle identification and tracking resolution. In the following the various components used in the HERMES experiment are described.

\subsection{The Hera Positron Beam}

The HERMES experiment is operated in the East Hall of the storage ring facility HERA, which is part of the DESY accelerator complex located in Hamburg. In the two HERA rings sharing a tunnel with a circumference of $6.3 \mathrm{~km}$ a lepton beam and a proton beam can

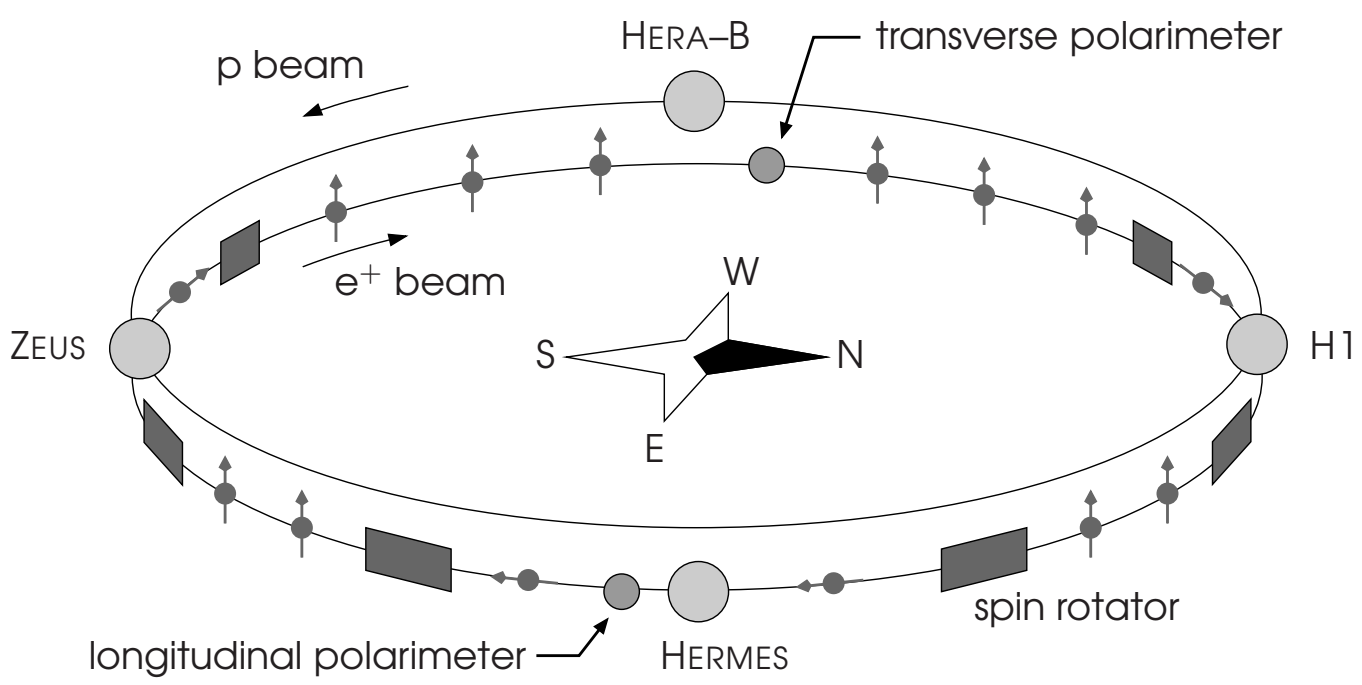

Figure 3.1.1: Schematic view of the two HERA storage rings with the locations of the experiments in the configuration since fall 2001. The spin orientation of the positron beam is indicated by arrows. 
be accelerated to energies of $27.5 \mathrm{GeV}$ and $920 \mathrm{GeV}$, respectively, and stored. HERA is capable of storing electrons or positrons. Positrons were used from 1992 until October 1997 and from June 1999 until the second switch-over to electrons in November 2004. In the following, the term 'beam positrons' will be used when referring to beam leptons in general. The configuration of the HERA facility is sketched in Figure 3.1.1. Both beams are brought into collision in the North and the South experimental halls where the two collider experiments $\mathrm{H} 1$ and ZEUS are located. The HERA-B experiment in the West hall used only the proton beam until the year 2003, whereas in the East hall the HERMES experiment uses the positron beam. Both of these experiments use internal targets in one of the HERA beams.

Due to a small asymmetry in the probability of emission of synchrotron radiation, the initially unpolarised positrons become polarised. This mechanism is called the SokolovTernov effect [Sok64]. The typical time constant of the polarisation rise for the layout and operating beam momentum of HERA is about 30 min. The spin orientation of the positron beam is aligned transversely to the beam direction (see Figure 3.1.1) because of the vertical magnetic field of the HERA dipole bending magnets. The polarisation of the beam is continuously measured by two polarimeters close to the HERA East and West halls. In the East section of HERA the beam spin orientation is turned into the longitudinal direction and back to the transverse direction by a pair of spin rotators located up- and downstream of the HERMES experiment. The longitudinal beam polarisation is measured with the so-called Longitudinal Polarimeter [Bec02]. On the opposite side of the ring the polarisation of the transversely polarised beam is measured by a second device, the socalled Transverse Polarimeter [Bar93]. Until the year 2001 the spin orientation of the beam was rotated only in the HERA East section and longitudinal polarisations up to $70 \%$ were achieved. In 2002, two additional pairs of spin rotators were installed to provide also the experiments $\mathrm{H} 1$ and ZEUS with longitudinally oriented positron spins. After a commissioning phase, maximum polarisation values of $50 \%$ were achieved in March 2003. During regular operation in the years 2003 and 2004 the routinely obtained polarisation values stayed lower at $30-40 \%$. The decrease of the beam currents caused by the scattering of the beam particles requires a refill of the HERA lepton beam every 8-12 h. The longer life time of the HERA proton beam allows usually more than one lepton fill during a proton fill.

\subsection{The Target}

A fixed target in a storage ring needs a special setup to preserve a reasonable life time of the stored beam. In order to fulfil the requirements of HERA, an internal gas target [AirO4] was chosen for the HeRMEs experiment, although achieved area densities are much smaller than for solid targets. However, a polarised gas target provides a high figure of merit as dilutions from unpolarised material typical in liquid or solid state targets are not present in the pure gas target. In addition, background from scattering events at the target material container is not present. Figure 3.2.1 shows the scheme of the HERMES target with its main components: the atomic beam source (ABS), the storage cell, the target gas analyser (TGA), and the Breit-Rabi Polarimeter (BRP). Furthermore, the target magnet (not drawn in Figure 3.2.1) provides a holding field to define the polarisation axis and to prevent spin relaxation by effectively decoupling the magnetic moments 


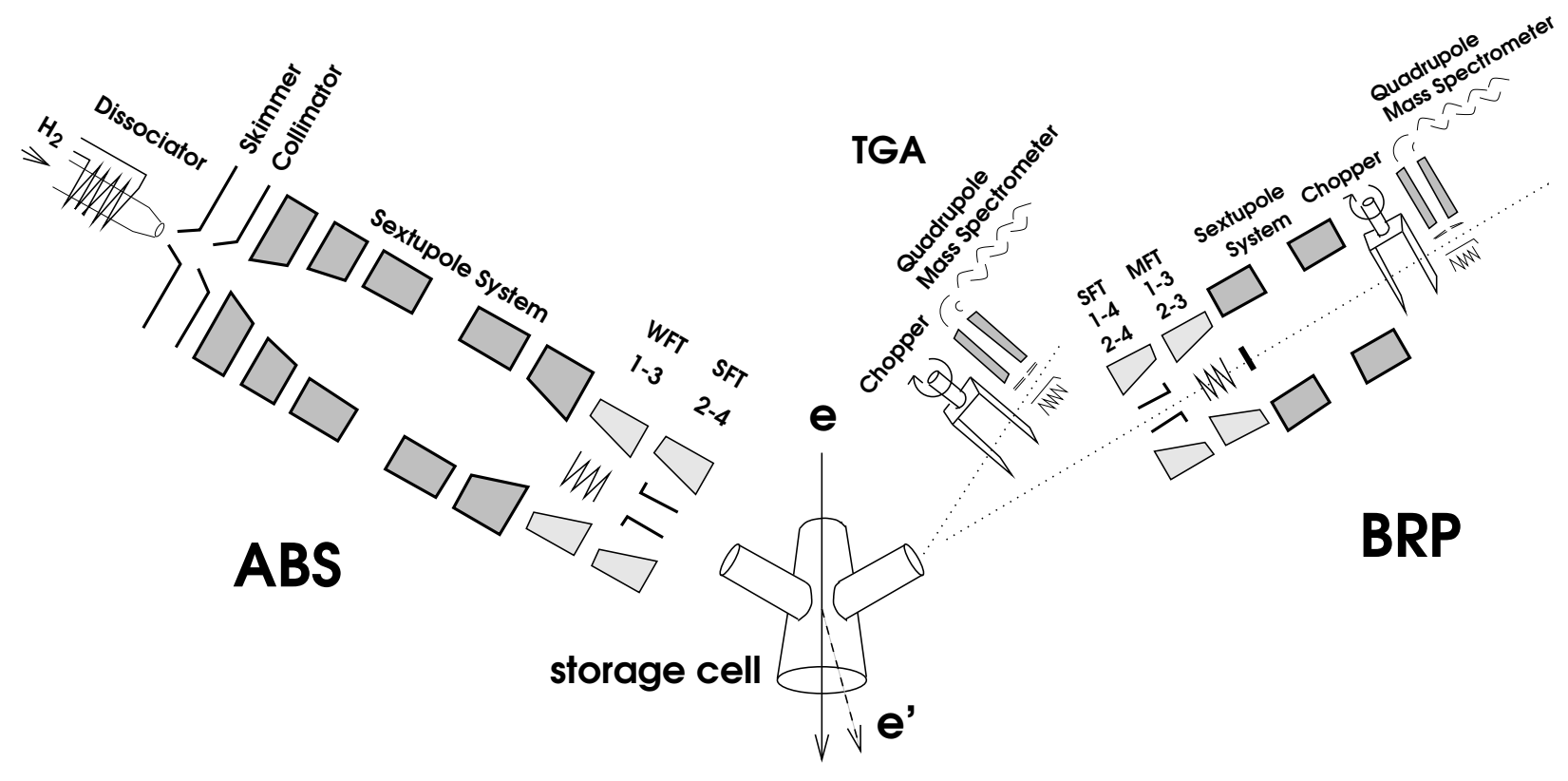

Figure 3.2.1: A schematic view of the HERMES polarised target.

of electrons and nucleons. Two different holding field configurations, longitudinal and transverse with respect to the beam direction, were in use for the HERMES target.

In 1995 the HERMES experiment started data taking with a longitudinally polarised ${ }^{3} \mathrm{He}$ target followed by longitudinally polarised hydrogen in 1996 and 1997 and longitudinally polarised deuterium from 1998 till 2000. During the shutdown in 2001 the longitudinal target magnet was replaced by a transverse target magnet and since 2002 a transversely polarised hydrogen target is in operation. As the analysis described in Chapters 4 and 7 is performed only on hydrogen data, the following description of the polarised target will focus on hydrogen.

\subsubsection{The Atomic Beam Source}

Hydrogen atoms possess four hyperfine energy levels which split up in an external magnetic field, as shown in Figure 3.2.2. In this diagram the field values are given in units of the corresponding critical field $B_{\mathrm{C}}$ and the energy values in units of the hyperfine splitting $E_{\mathrm{HFS}}$. The hyperfine splitting corresponds to the energy difference between the states with total spin $F=0$ and $F=1$ if no external magnet field is present. The critical field is defined as the external field which causes an energy difference between states $|1\rangle$ and $|3\rangle$ equal to the hyperfine splitting $E_{\mathrm{HFS}}$. The interaction between the shell electron and nucleon spins dominates in external fields below $B_{\mathrm{C}}$ whereas the spin-field interaction dominates in stronger external fields $B>B_{\mathrm{C}}$. The four states are combinations of the spin states up and down of the nucleon, $m_{I}= \pm \frac{1}{2}$, and the shell electron, $m_{S}= \pm \frac{1}{2}$.

The different components of the ABS [NasO3] which are necessary to provide the storage cell with Hydrogen atoms in certain hyperfine states, are drawn on the left-hand side of Figure 3.2.1. In a first step molecular hydrogen is dissociated by a radio frequency discharge with a dissociation degree up to $80 \%$. The hydrogen atoms flow through a cooled nozzle with a temperature of $100 \mathrm{~K}$ where a thin layer of frozen water on the nozzle surface 


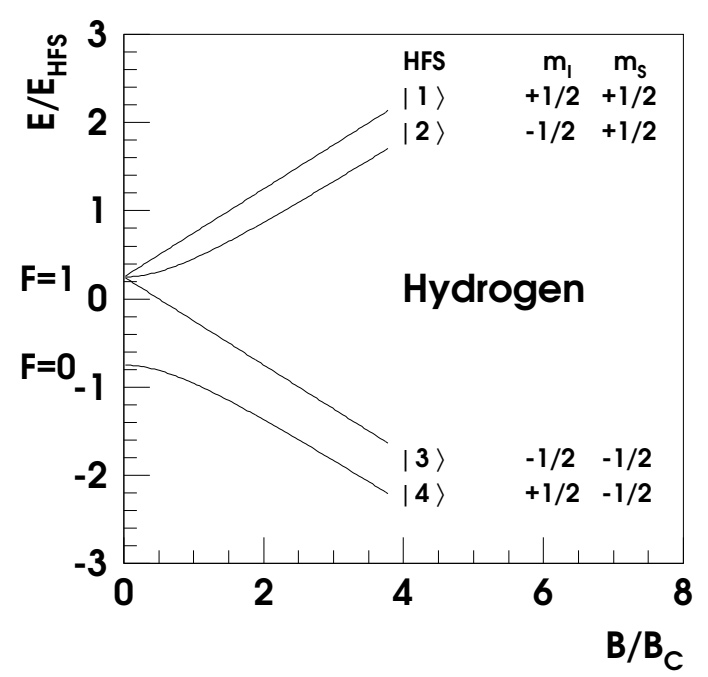

Figure 3.2.2: Hyperfine energy levels of hydrogen atoms as function of an external magnetic field.

helps to prevent recombination.

After passing the nozzle and a beam forming system, hydrogen atoms in all four hyperfine states enter a sextupole magnet system which deflects the states $|3\rangle$ and $|4\rangle$ and focuses atoms in states $|1\rangle$ and $|2\rangle$ onto the central axis of the magnet system. Following the magnet system, the atoms traverse two radio frequency transitions, which can be operated independently. A so-called weak field transition (WFT) interchanges the occupation numbers of hyperfine states $|1\rangle$ and $|3\rangle$ whereas the strong field transition (SFT) acts on states $|2\rangle$ and $|4\rangle$. By operating either the SFT or the WFT, atoms in the hyperfine state combinations $|1\rangle+|4\rangle$ and $|2\rangle+|3\rangle$ are provided at the exit of the ABS such that the electron polarisation vanishes and the nucleon spin projection is either $+\frac{1}{2}$ or $-\frac{1}{2}$ in the high field limit. During the operation of the longitudinally polarised target the nucleon spin state was changed every $60 \mathrm{~s}$. For the transversely polarised target at first a spin flip interval of 60 s was used which was later increased to 90 s in order to reduce the dead time during the roughly $1 \mathrm{~s}$ long configuration changes. The atoms with polarised nucleons and unpolarised shell electrons are injected in the storage cell with fluxes of up to 6.5. $10^{16}$ atoms/s.

\subsubsection{The Storage Cell}

The storage cell [Bau03b] is a $400 \mathrm{~mm}$ long open-ended tube made of $75 \mu \mathrm{m}$ thin pure aluminium. The tube has an elliptical cross section with major and minor axes of $21.0 \mathrm{~mm}$ and $8.9 \mathrm{~mm}^{\mathrm{i}}$ and is embedded in the target chamber inside the ultra high vacuum of the HERA positron machine. The polarised atoms are injected from the ABS through the injection tube which is connected to the storage cell at its centre. They diffuse along the cell axis towards both ends and are pumped away by a very powerful pumping system. A special coating on the aluminium walls minimises depolarisation and recombination

'Until December 1999 a larger tube with dimensions $29.8 \times 9.8 \mathrm{~mm}^{2}$ was installed. 
caused by surface collisions. The cell axis coincides with the positron beam orbit and the target density has a triangular shape with the maximum at the position of the injection tube. The cooling of the storage cell is set to the optimal value for hydrogen of $100 \mathrm{~K}$ where recombination and depolarisation effects are low. In addition to the injection tube a smaller sampling tube exists which extracts about $5 \%$ of the gas for analysis in the TGA and BRP. This sampling tube is installed opposite to the injection tube at an angle of $120^{\circ}$. The distance between injection and sampling tube allows for the thermalization of the gas with the storage cell wall. A vented extension at the downstream end of the tube ensures that all scattered particles in the HERMES acceptance traverse the same amount of material in the cell walls. In front of the storage cell and behind its extension so-called wake-field suppressors provide a gradual electrical transition between the storage cell and the beam pipe. Without the wake-field suppressors the bunched positron beam in HERA would cause strong radio frequency fields to be emitted at the discontinuity of the beam pipe impedance. These wake fields would not only heat up the target cell but also destabilise the beam orbit.

\subsubsection{The Target Gas Analyser}

The main component of the TGA [Bau03a] is a $90^{\circ}$ off-axis quadrupole mass spectrometer (see right-hand side of Figure 3.2.1). It is used for the measurement of the atomic and molecular content of the gas sampled at the centre of the storage cell. In front of the quadrupole mass spectrometer a chopper periodically blocks the sample beam to allow subtraction of the residual gas signal. In order to avoid interference with the BRP measurement the TGA is tilted by $7^{\circ}$ with respect to the sampling tube. The measured normalised nucleon flow rates for atoms $\phi_{a}$ and molecules $\phi_{m}$ yield the degree of dissociation of the sample beam,

$$
\alpha_{\mathrm{T} G \mathrm{~A}} \equiv \frac{\phi_{a}}{\phi_{a}+\phi_{m}}
$$

which is measured roughly once per minute. Together with calibration measurements which are performed during the breaks between fills of the HERA positron ring, two quantities can be calculated [AirO4]. These are the degree of dissociation, also called atomic fraction, in the absence of recombination within the cell, $\alpha_{0}$, and the fraction of atoms surviving recombination in the cell, $\alpha_{r}$. Both values are necessary for the determination of the density-averaged nuclear polarisation $P_{T}$ in the cell.

\subsubsection{The Breit-Rabi Polarimeter}

A second measurement using the gas extracted by the sampling tube is performed by the BRP [Bau02]. As can be seen on the right-hand side of Figure 3.2.1, the BRP consists of a pair of radio frequency transitions - a strong (SFT) and a medium field transition (MFT) - which can be tuned for different hyperfine state transitions. A sextupole magnet system focuses atoms with $m_{S}=+\frac{1}{2}$ towards the detector unit and defocuses atoms with $m_{S}=-\frac{1}{2}$. To prevent atoms which enter on the symmetry axis of the sextupole magnet system (where the field gradient is zero) from entering the detector unit, a beam blocker is installed in front of the first magnet of the sextupole system. As in the TGA, a quadrupole mass spectrometer together with a chopper for background subtraction is used for the 
detection. From the measured relative populations of the hyperfine states of hydrogen atoms, the atomic polarisation $P_{a}$ can be deduced.

The value of the polarisation $P_{a}$ measured with the BRP is the polarisation at the centre of the storage cell. It must be related to the polarisation averaged along the cell, $P_{\mathrm{BRP}}$, by sampling corrections $c_{P}$ :

$$
P_{\mathrm{BRP}}=c_{P} \cdot P_{a} .
$$

The sampling corrections are obtained with the help of Monte Carlo simulations of the ballistic flow of target gas atoms in the storage cell [AirO4]. Using the BRP and TGA measurements the averaged target polarisation $P_{T}$ as seen by the electron beam can be calculated:

$$
P_{T}=\alpha_{0}\left[\alpha_{r}+\left(1-\alpha_{r}\right) \beta\right] P_{\mathrm{BRP}} .
$$

Here, $\beta$ is the ratio of the nuclear polarisation of molecules produced by recombination and the nuclear polarisation of the atoms. A direct measurement of the remnant polarisation contained in the molecules is not possible at HERMES as the BRP is capable only of atomic polarisation measurements. In dedicated measurements at a higher storage cell temperature of $260 \mathrm{~K}$ and by boundary considerations, the range of $\beta$ could be restricted to a range of $\beta=[0.45,0.83]$. The uncertainty on $\beta$ is part of the systematic uncertainty of the target polarisation value.

The HERMES target group provides average target polarisation values for different time periods which can be found in Table 3.2.1. The accumulated data of the HERMES experiment are combined in so-called data productions which cover data taking periods of roughly one year between shut downs of the HERA accelerator for maintenance purposes. Also the target polarisation values are usually averaged over the period of one data production. In the year 2004 the performance of the polarised target became unstable after a period of three months in the beginning of the year. The remaining five months had to be split into smaller periods with average target polarisations between $0.648 \pm 0.090$ and $0.775 \pm 0.044$. During the commissioning of the target in 1996 the TGA was not available for most of the time and the measurement of the target polarisation suffered from a large systematic uncertainty. Therefore the value given in Table 3.2.1 was obtained from a renormalisation of the inclusive DIS cross section to the results from the 97 production [Bec03].

\begin{tabular}{|c|c|c|c|}
\hline production & time period & holding field & polarisation \\
\hline 96 & Aug 1996 - Dec 1996 & $\|$ & $0.759 \pm 0.042$ \\
\hline 97 & Feb 1997 - Oct 1997 & $\|$ & $0.851 \pm 0.031$ \\
\hline 02 & Apr 2002 - Mar 2003 & $\perp$ & $0.783 \pm 0.041$ \\
\hline 03 & Sep 2003 - Dec 2003 & $\perp$ & $0.795 \pm 0.033$ \\
\hline 04 & Jan 2004 - Mar 2004 & $\perp$ & $0.777 \pm 0.039$ \\
04 & Apr 2004 - Aug 2004 & $\perp$ & $0.648 \pm 0.090 \ldots 0.775 \pm 0.044$ \\
\hline
\end{tabular}

Table 3.2.1: Target polarisation values for the different hydrogen productions. The statistical uncertainty is negligible compared to the listed systematic uncertainty. 


\subsubsection{The Target Magnet}

The target magnet surrounding the storage cell provides a holding field defining the polarisation axis. It also suppresses spin relaxation due to the splitting of the hyperfine energy levels. While a holding field parallel to the positron beam has no effect on the beam and a marginal effect on the scattered particle trajectories, for a transverse holding field different effects have to be taken into account. The deflection of the beam requires compensation by correction coils and limits the strength of the magnetic field due to the amount of synchrotron radiation generated by the beam. Not only the beam but also the scattered particles are deflected. Hence, the reconstructed vertex position and scattering angles must be corrected for the deflection.

In addition to the influence on the particle trajectories, depolarisation effects occur due to the bunched structure of the HERA positron beam. The time period of $96.1 \mathrm{~ns}$ between two adjacent positron bunches corresponds to a bunch frequency of $10.41 \mathrm{MHz}$. The induced magnetic high frequency field around the positron beam contains a large number of harmonics because of the short bunch length of $30 \mathrm{ps}$. The energy splitting and hence the resonance frequency between the hyperfine states of the target atoms depends on the strength of the magnetic holding field $B$ (see Figure 3.2.2). If a harmonic of the beam induced field matches such a transition frequency, the target polarisation decreases. In order to avoid depolarisation, the holding field must be set to a value between such resonances. Two kinds of transitions, $\pi$ and $\sigma$, exist for beam induced fields perpendicular and parallel to the holding field, respectively. For nuclear $\pi\left(\Delta m_{I}= \pm 1\right.$, $\Delta m_{S}=0$ ) transitions which are possible for both longitudinal and transverse holding fields, the spacing $\Delta B$ between two resonances is of the order of $50 \mathrm{mT}$ for a field strength around $B=300 \mathrm{mT} . \sigma\left(\Delta m_{I}= \pm 1, \Delta m_{S}=\mp 1\right)$ transitions occur only in case of a transverse holding field and have a very small spacing ( $\Delta B=0.37 \mathrm{mT}$ at $B=300 \mathrm{mT}$ ). Hence, the transverse magnetic holding field needs a good homogeneity over the storage cell to minimise the bunch field induced depolarisation.

The longitudinal target magnet was operated at a field strength $B^{\|}=335 \mathrm{mT}$ with maximum deviations around $10 \mathrm{mT}$ within the storage cell. For the transverse target a homogeneity of $\Delta B^{\perp} \leq 0.15 \mathrm{mT}$ was required at a field value of $297 \mathrm{mT}$. With the magnet configuration in 2002 maximum deviations of $\Delta B_{z}^{\perp}=0.05 \mathrm{mT}, \Delta B_{y}^{\perp}=0.15 \mathrm{mT}$, and $\Delta B_{x}^{\perp}=$ $0.60 \mathrm{mT}$ were achieved. This setup was improved by an additional correction coil installed in 2003 which reduced the deviations to $\Delta B_{y}^{\perp}=0.05 \mathrm{mT}$ and $\Delta B_{x}^{\perp}=0.30 \mathrm{mT}$ [Wan04]. A dedicated measurement showed that the depolarisation because of the $\sigma$ resonances could be reduced by roughly $\frac{1}{3}$ of the total effect [TaiO6] .

\subsubsection{The Unpolarised Gas Feed System}

Alternatively to the injection of polarised atoms from the ABS, the storage cell can be filled with unpolarised gas using the unpolarised gas feed system (UGFS). Adjustable densities and the possibility to inject the gas also into the target chamber (as opposed to the storage cell) furthermore allow various calibration measurements necessary for the determination of the target polarisation and the different contributions to its systematic uncertainty. 


\subsection{The Spectrometer}

The HERMES experiment uses a forward spectrometer for the detection of the scattered positron and a large fraction of the hadronic final state. The spectrometer is capable of the detection in a broad kinematic region with a good angular and momentum resolution [Ack98, Dür95]. It consists of two symmetric halves above and below the horizontal plane in which the HERA beam pipes are located parallel to each other. In Figure 3.3.1 a spectrometer cross section in the $y-z$ plane of the HERMES coordinate system is drawn. The $z$-axis of the right-handed HERMES coordinate system is defined along the lepton beam direction and its $x$-axis is pointing away from the centre of the HERA rings.

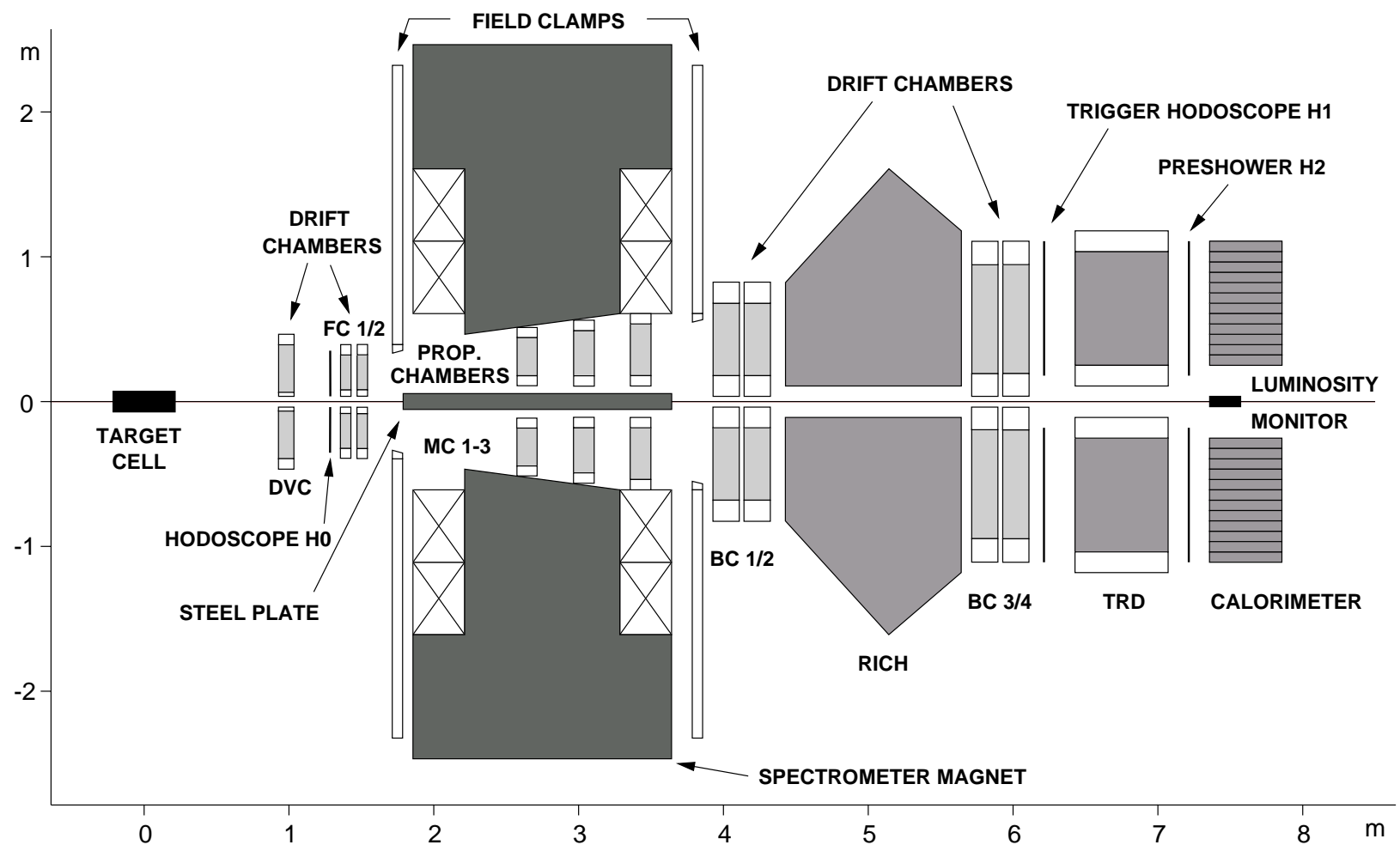

Figure 3.3.1: Side view of the HERMES spectrometer in the configuration since summer 1998.

Located about $2 \mathrm{~m}$ downstream of the target cell the spectrometer magnet provides a vertical field with a deflecting power of $\int B d l=1.3 \mathrm{~T} \cdot \mathrm{m}$. The influence of the field on the HERA beams is minimised by a $11 \mathrm{~cm}$ thick steel plate surrounding the beam pipes. The remaining effects are compensated by a correction coil. Field clamps in front of and behind the magnet reduce fringe fields in the adjacent detectors. The horizontal acceptance of the spectrometer is \pm 170 mrad. The vertical acceptance $\pm(40 \ldots$. 140) mrad has a gap due to the beam pipes embedded in the steel plate.

\subsubsection{The Tracking System}

In front of the spectrometer magnet two drift chambers (FC 1/2) [Bra01] are in operation for the reconstruction of the event vertex and the scattering angles with respect to the 
lepton beam. Each detector consists of $2 \times 3$ planes, two planes with wires arranged in the vertical direction, and four wire planes tilted by $\pm 30^{\circ}$ with respect to the vertical direction. In order to enhance the front-track reconstruction an additional drift chamber, the drift vertex chamber (DVC) [Ack98], with 6 wire planes in the same configuration was installed in 1997. Unfortunately, due to a less than optimal alignment the reconstruction resolution improved only slightly, and therefore consistently for all data productions only the FCs are used for the front track reconstruction. For the track reconstruction behind the magnet two pairs of drift chambers (BC 1/2 and BC 3/4) [Ber98] are located in front of and behind the Čerenkov ( $\mathrm{RICH}$ ) detector. Also these drift chambers consist of 6 planes each which are identically to the FCs arranged, thereby simplifying the reconstruction code significantly [Wan96]. For low-momentum particles which do not reach the rear of the spectrometer three multi-wire proportional chambers (MC1-3) [And01] are installed in the gap inside the magnet. Each chamber contains three planes and they are used for momentum determination of the low-energy particles which are not included in the analysis presented in the following chapters.

The front and back tracks are reconstructed from the drift chamber hits by a pattern recognition algorithm based on a fast tree search. They are combined if both tracks intersect in the centre of the magnet within a given tolerance. The precision of the front track is increased by adding an additional virtual point at the intersection of the forward extrapolation of the back track with the $x y$-plane at the middle of the magnet (force bridge technique). Using look-up tables the momentum of a charged particle is determined from the front and back track parameters. With the threshold Čerenkov counter installed a momentum resolution of $\Delta P / P<2.2 \%$ and an angular resolution of $\Delta \theta<$ $1.4 \mathrm{mrad}$ for particles with $P>2 \mathrm{GeV}$ could be obtained. In 1998 the resolutions became worse with the installation of a ring-imaging Čerenkov detector in place of the threshold Čerenkov counter because of increased multiple scattering. The resolutions increased to values of $\Delta P / P<2.6 \%$ and $\Delta \theta<1.8 \mathrm{mrad}$.

In case of the transverse target holding field the vertex and scattering angle reconstruction has to be corrected for the deflection. To do this the transverse magnet field has to be mapped which was only possible in the shutdown of 2003 after the implementation of additional correction coils. The measured field map can be used for the productions 03 and 04 whereas for the 02 production a theoretical field map was calculated with the help of the magnetostatic program MAFIA [Wei97]. A survey of the magnet field along the $z$-axis and at certain positions along the $x$ direction served as input for this calculation. Two different methods for the target magnet correction (TMC) are available, both using the track position information from the DVC and the FCs [Aug06].

In method 1 the correction on the particle track is applied based on reference tracks from a database. In a detailed (and time-consuming) tracking calculation a grid of trajectories covering the HERMES acceptance is computed in small steps of momentum, $z$-vertex and vertical and horizontal angles. From this set the trajectory closest to a measured particle track is selected, based on the tracking information from the DVC and FCs. The remaining deviations from the reference track in the data base are used in a linear interpolation to yield the corrected $z$-vertex and vertical scattering angle of the measured track. The true horizontal angle can then be computed from the position on FC2 by a simple relationship. 
Method 2 is based on a ray tracing procedure. Using the reasonable assumption that a trajectory which is in the beginning close to a reference trajectory will also be close to the reference trajectory at the end, a Taylor expansion for the final position in terms of the initial position can be performed. The coefficients provide a quick way to relate the initial position of a track to its final position with the help of a transfer function. For the determination of these transfer coefficients several reference particles had to tracked through the magnet field using the MIT-RAYTRACE program [Kow87]. In order to correct the $z$-vertex position and the scattering angles the right transfer function, which depends on the particle momentum and the $z$-position from which the particle is assumed to originate, has to be found iteratively until convergence is achieved.

Using a Monte Carlo simulation of the HERMES detector (cf. Chapter 5) resolutions of the relevant azimuthal angles $\phi$ and $\phi_{S}$ (cf. Chapter 2), the angle between the virtual photon and the outgoing hadron $\theta_{\gamma^{*} h}$, and the transverse momentum of the produced hadrons were compared with and without transverse target magnet field. Especially for the azimuthal angle $\phi_{S}$ the resolution degrades with the magnet field as can be seen in Figure 3.3.2 (for the case of positive pions). Also shown in this figure is the resolution after correction method 2 is applied which almost fully recovers the resolution without the transverse target magnet. Similar results are obtained using correction method 1 and for negative and neutral pions and charged kaons.

The measured field map of the magnet configuration after 2003 is not yet imple-
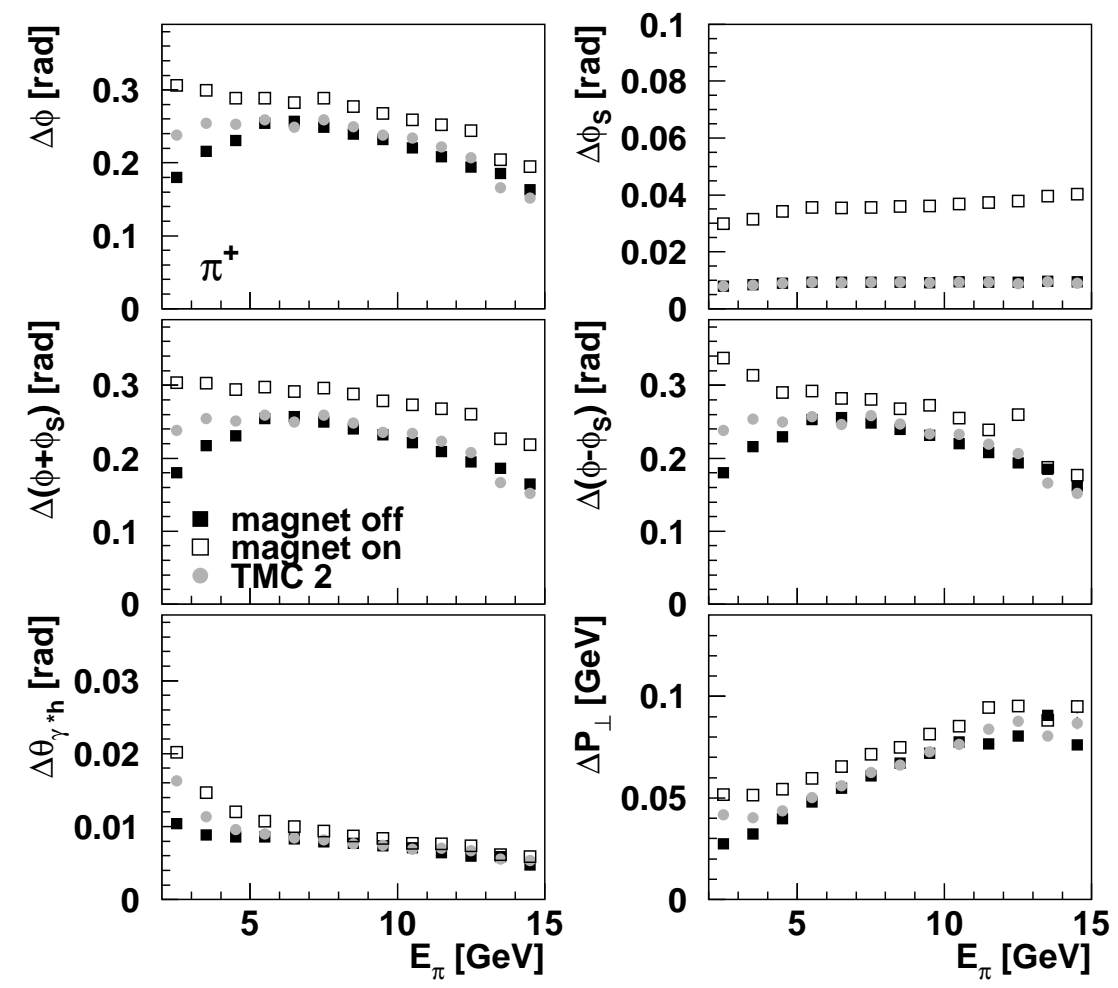

Figure 3.3.2: The resolution of the azimuthal angles and the transverse momentum for positive pions as a function of the pion energy. Compared are the values for the transverse target magnet switched off and on, once with and without correction method 2. 
mented in method 2. Therefore method 1 is used for the 03 and 04 production. This method was not yet working properly when the 2002 data was produced and method 2 was applied for the correction in the 02 production.

\subsubsection{Particle Identification Detectors}

The HERMES experiment utilises four detectors for the particle identification (PID): a threshold Čerenkov counter which was replaced by a ring-imaging Čerenkov (RICH) detector in 1998, a transition radiation detector (TRD), a preshower detector, and an electromagnetic calorimeter. Typical PID detector responses are plotted in Figure 3.3.3.
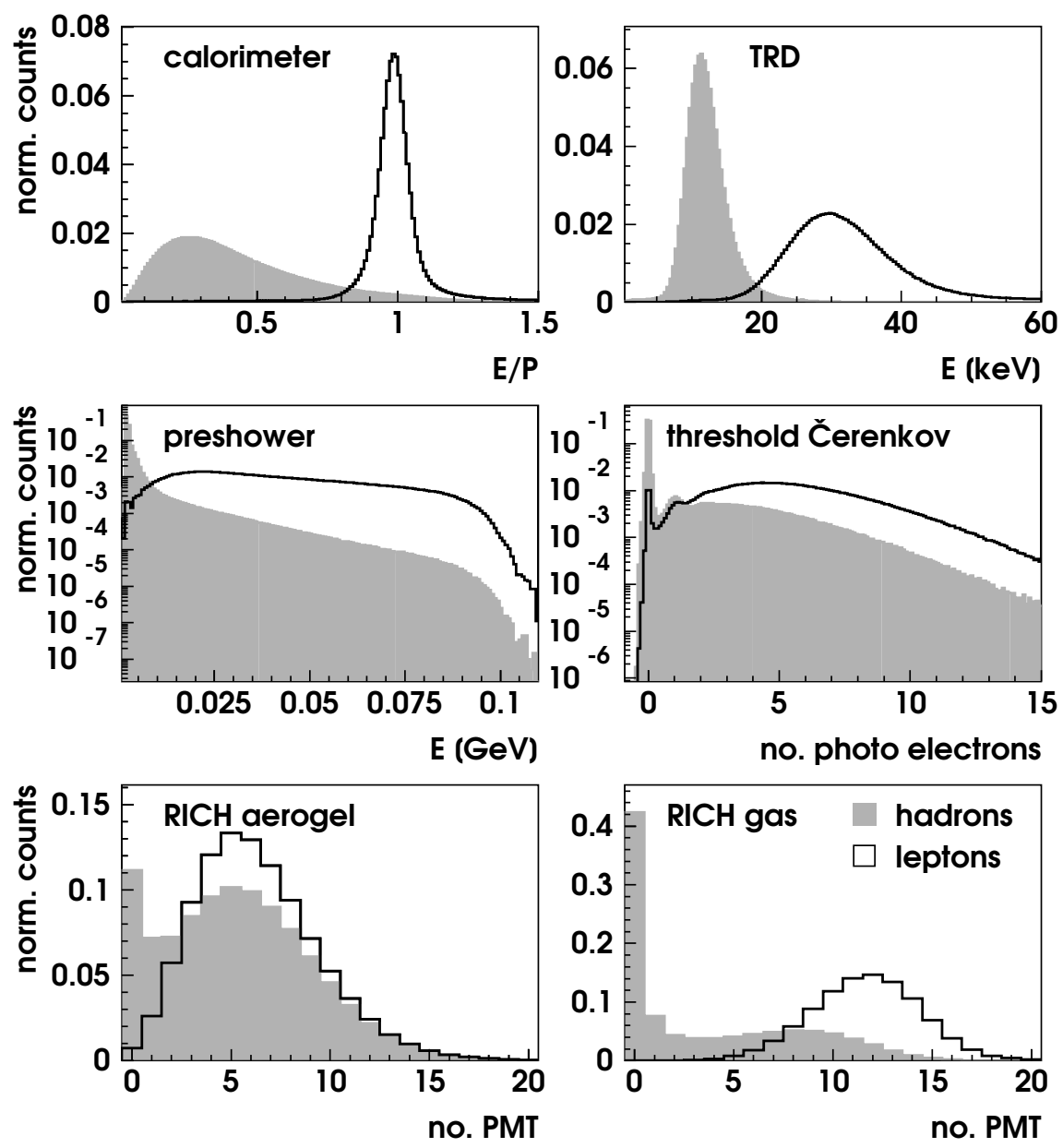

Figure 3.3.3: The responses of the different PID detectors for hadrons and leptons. In order to plot the responses for a given detector, the particles were identified with cuts on the responses of the remaining PID detectors. Except for the threshold Čerenkov counter histogram which was extracted from the 96 and 97 production, the histograms were computed from the 04 production.

The first PID detector on the particle's way through the detector is a Čerenkov detector located between the two pairs of BCs. If a particle passes through a material with a velocity larger than the phase velocity of light in the material it emits Čerenkov radiation 


\begin{tabular}{|c|c|c|c|c|}
\hline & $n$ & $P_{\text {thresh }}^{\pi}(\mathrm{GeV})$ & $P_{\text {thresh }}^{K}(\mathrm{GeV})$ & $P_{\text {thresh }}^{p}(\mathrm{GeV})$ \\
\hline Aerogel & 1.0304 & 0.6 & 2.0 & 3.8 \\
$\mathrm{C}_{4} \mathrm{~F}_{10}$ & 1.00137 & 2.7 & 9.4 & 17.9 \\
\hline
\end{tabular}

Table 3.3.1: Refractive indices and Čerenkov threshold momenta for the two radiators of the $\mathrm{RICH}$ detector.

on a cone with a characteristic opening angle $\theta_{c}$ given by:

$$
\cos \theta_{c}=\frac{1}{\beta n},
$$

where $n$ is the refractive index of the material and $\beta=\frac{v}{c}$ is the ratio of the velocity $v$ of the particle and the speed of light in vacuum $c$. Particles with

$$
\beta<\beta_{\text {thresh }}=\frac{1}{n} \quad \text { or } \quad P<P_{\text {thresh }}=\frac{m}{\sqrt{n^{2}-1}}
$$

do not emit Čerenkov light. Until 1998 a threshold Čerenkov counter was installed which was filled with a radiator gas. The gas consisted of $70 \%$ vol. $\mathrm{N}_{2}$ and $30 \%$ vol. $\mathrm{C}_{4} \mathrm{~F}_{10}$ and had a refractive index of $n=1.000629$. Eq. (3.3.2) yields the threshold momenta 0.014, 3.8, 13.9, and $26.4 \mathrm{GeV}$ for leptons, pions, kaons, and protons, respectively. Hence, all leptons traversing the threshold Čerenkov counter radiate as can be seen in Figure 3.3.3. The peak for the lepton sample at zero photo-electrons is due to detector inefficiencies. Only hadrons with momenta larger than $3.8 \mathrm{GeV}$ radiate, resulting in a larger peak at zero for hadrons. With the threshold Čerenkov counter pion identification in a hadron sample is possible in the momentum range between 3.8 to $13.9 \mathrm{GeV}$ where only pions emit Čerenkov light.

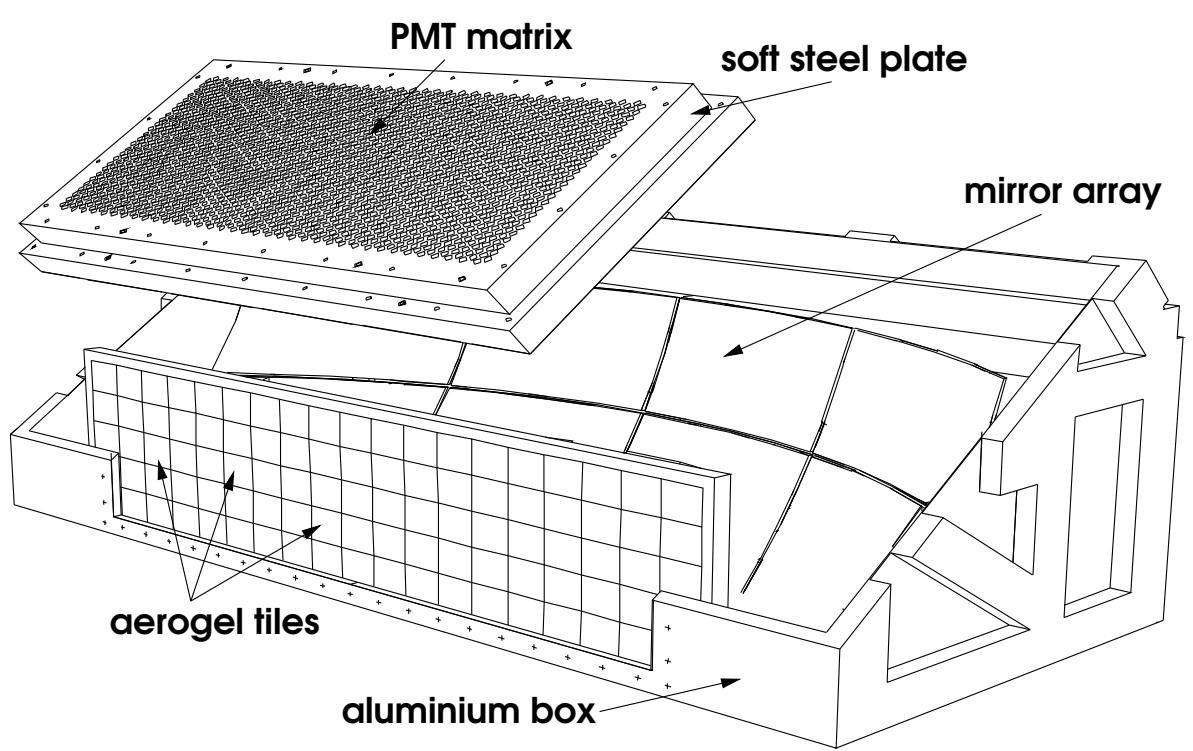

Figure 3.3.4: The upper half of the $\mathrm{RICH}$ detector schematically drawn. 
In order to improve the hadron identification the threshold Čerenkov counter was replaced by a dual-radiator RICH detector [Ako02] in 1998. Particles with the same momentum but with different masses emit Čerenkov light in cones with different opening angles $\theta_{c}$. The RICH detector measures the pattern of the Čerenkov photons which permits the reconstruction of the Čerenkov angle $\theta_{c}$ and thus enables the identification of pions, kaons, and protons in the hadron sample. Like the threshold Čerenkov counter also the $\mathrm{RICH}$ detector is used for the separation of leptons and hadrons. Figure 3.3.4 shows the configuration of the upper half of the $\mathrm{RICH}$ detector with its two radiator materials. After entering the detector volume through a thin entrance window foil (not shown in the sketch) particles pass a first radiator made of $5.5 \mathrm{~cm}$ thick aerogel tiles. This is a colloidal suspension of quartz in air. The second radiator is the heavy $\mathrm{C}_{4} \mathrm{~F}_{10}$ gas which fills the interior of the detector. The refractive indices of the radiators and threshold momenta can be found in Table 3.3.1. The emitted Čerenkov photons are focused by a spherical mirror array on a photo-multiplier tube (PMT) matrix, consisting of 1934 PMTs in both top and bottom part of the RICH detector.

As the second PID detector located between the two hodoscopes $\mathrm{H} 1$ and $\mathrm{H} 2$, also the TRD serves to distinguish between lepton and hadrons. When a highly relativistic charged particle crosses a dielectric boundary it emits electromagnetic radiation. This can be explained by the required continuity of the Coulomb fields at the boundary. Due to the different dielectric constants, the induced Coulomb fields differ between the two media at the boundary and do not fulfil the continuity requirement. This gives rise to an additional field observed as the transition radiation. The total energy of the emitted radiation for a transition between a medium and vacuum,

$$
E=\frac{2}{3} \alpha \omega_{p} \gamma
$$

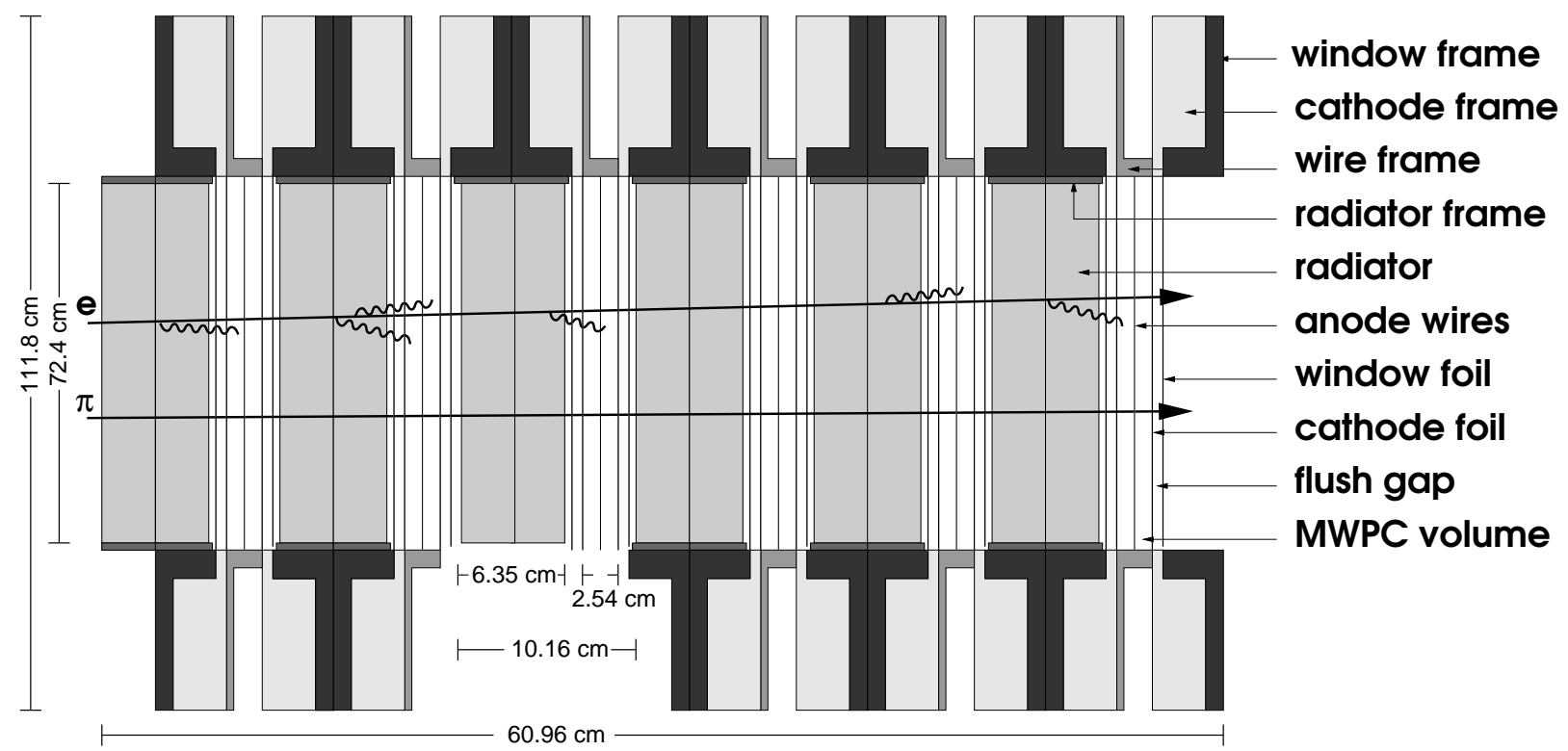

Figure 3.3.5: The upper half of the TRD with a positron and a pion track. The opening angles of the transition radiation produced by the lepton are exaggerated. 
is proportional to the Lorentz factor $\gamma=1 / \sqrt{1-\beta^{2}}$ and the plasma frequency of the medium $\omega_{p}$ [Art75]. At high particle energies the radiation consists mainly of $\mathrm{X}$-rays. It is emitted in a narrow cone with an opening angle $\theta \sim 1 / \gamma$. Since the probability for the emission of transition radiation is small for a single boundary many material boundaries are necessary for the realisation of a TRD.

For the HERMES TRD an irregular mesh of very thin polyethylene/polypropylene fibres was chosen as radiator, arranged in a plane to form a $6.35 \mathrm{~cm}$ thick module. Behind the module a multi-wire proportional chamber, filled with a mixture of $90 \%$ Xenon and $10 \%$ $\mathrm{CH}_{4}$ gas, detects the deposited energy. The top and bottom part of the TRD consists of six consecutive modules each with an active area of $72.4 \times 325 \mathrm{~cm}^{2}$ (see Figure 3.3.5).

At HERMES energies only leptons emit a large enough amount of transition radiation to be detected. The emitted energy depends linearly on $\gamma$ which for a lepton with the same energy as a pion is about 270 times larger. Due to ionisation losses $\mathrm{d} E / \mathrm{d} x$ in the chamber gas all particles deposit some energy in the wire chamber. In general, leptons leave approximately 2.5 times the amount of energy of pions, allowing for a separation by the energy deposition in the TRD modules. In Figure 3.3.3 a typical response to leptons and hadrons is shown. Plotted is the truncated mean which is the averaged signal calculated from five modules, discarding the module with the largest response. This procedure reduces significantly the Landau tail of the hadronic response that originates from $\delta$ electrons while leaving the mean values of the distributions almost unaffected.

The preshower detector is a scintillating hodoscope with a $11 \mathrm{~mm}$ thick curtain of lead in front. The hodoscope $(\mathrm{H} 2)$ is build from 42 vertical panels of scintillator with a thickness of $1 \mathrm{~cm}$. The panels have an area of $9.3 \times 91 \mathrm{~cm}^{2}$ and overlap by $2-3 \mathrm{~mm}$ for maximum efficiency. The individual panels are read out by PMTs away from the beam pipe as sketched in Figure 3.3.6. For traversing leptons, the passive lead radiator initiates electromagnetic showers which deposit more energy than minimum ionising particles. This results in energy losses of $20-40 \mathrm{MeV}$ for leptons whereas hadrons deposit only around $2 \mathrm{MeV}$, as shown in Figure 3.3.3.

Behind the preshower an electromagnetic calorimeter [Ava98] is installed in which leptons lose almost all of their energy in an electromagnetic shower. The depth of $50 \mathrm{~cm}$ or 18 radiation lengths ensures that the shower is almost completely contained in the calorimeter. Each half of the detector is a wall of 420 lead-glass blocks with a cross section of $9 \times 9 \mathrm{~cm}^{2}$ (cf. Figure 3.3.6). These radiation-resistant F101 blocks are coupled to PMTs at the back by means of silicone glue. A $3 \times 3$ array of blocks was measured in a test beam resulting in an energy resolution of $\sigma(E) / E[\%]=5.1 / \sqrt{E[\mathrm{GeV}]}+1.5$ and a linear energy response for electrons to within $1 \%$ in the range from 1 to $30 \mathrm{GeV}$ [Ava96] . The energy resolution of the full scale calorimeter in operation turned out to be slightly worse because of the presence of the preshower detector and imperfections in the gain matching of the lead-glass block/PMT units.

Contrary to leptons, hadrons lose only a small fraction of their energy, due to nuclear interactions and ionisation losses $\mathrm{d} E / \mathrm{d} x$. Since electrons lose almost all of their energy, the total deposited energy $E$ measured by the calorimeter is close to the momentum $P$ of the lepton measured with the spectrometer. This allows a good discrimination of leptons from hadrons in the distribution of $E / P$ as can be seen in Figure 3.3.3.

The calorimeter is also capable of the detection of energetic photons. The photons 


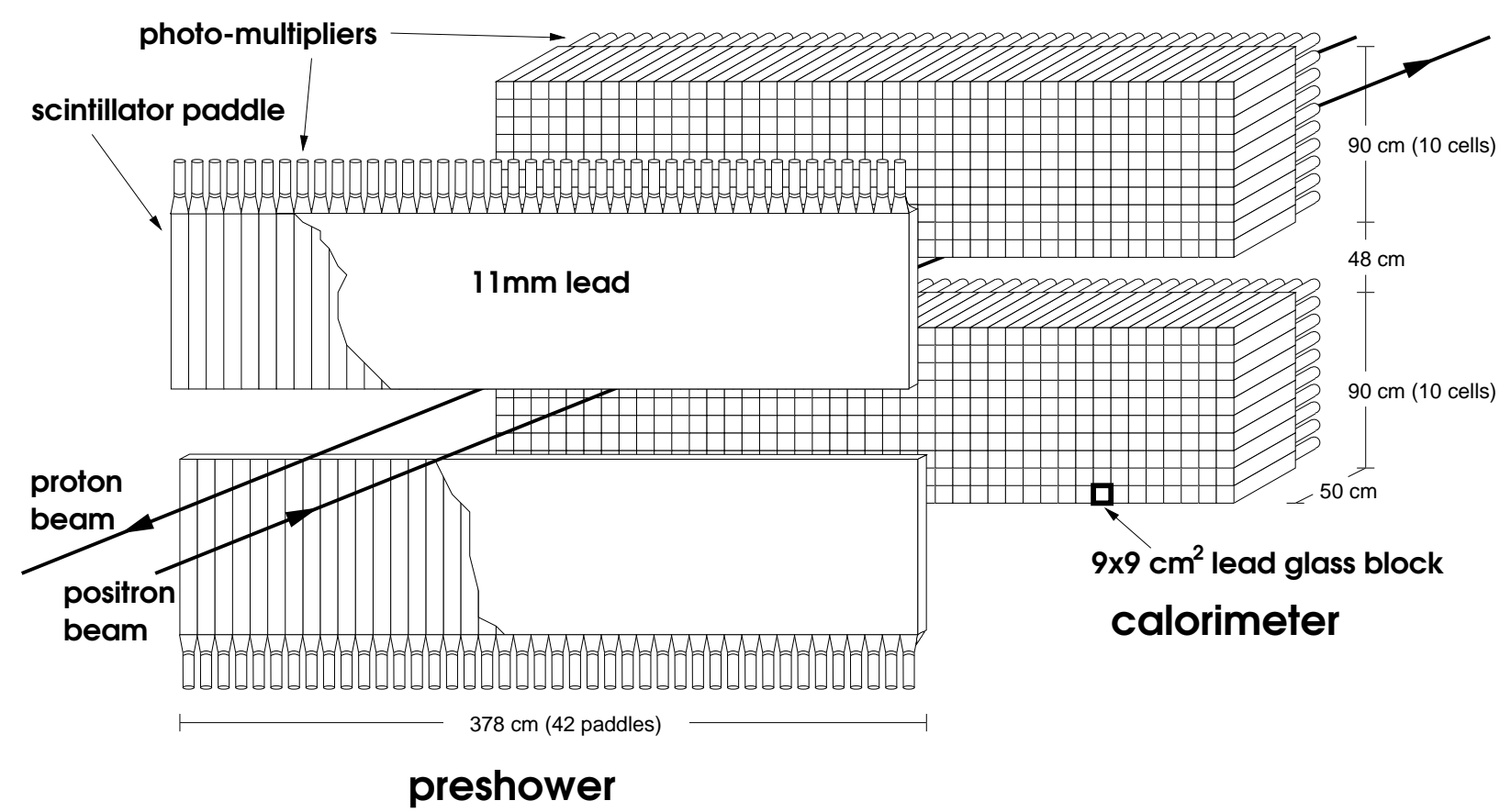

Figure 3.3.6: The preshower detector and the calorimeter setup of the HERMES experiment.

leave a signal in a cluster of blocks which does not coincide with a track reconstructed in the HERMES acceptance. By weighting the centroid of each block of a cluster with the logarithm of the corresponding energy, a position resolution of about $0.5 \mathrm{~cm}$ for photons is achieved [Ely01].

\subsubsection{The Luminosity Monitor}

The measurement of absolute and relative luminosities is necessary for the calculation of cross sections and cross section asymmetries. In the HERMES experiment the luminosity is measured by means of a reference scattering process which is the scattering of beam leptons off shell electrons in the target gas. In case of a positron beam these are the annihilation and Bhabha scattering processes. For electrons as beam particles Møller scattering takes place. The luminosity can be obtained from the ratio of the scattering process rate and its effective cross section. The effective cross section is the theoretical cross section which is known to high orders in quantum electrodynamics, multiplied by the efficiency of the luminosity monitor and integrated over its acceptance.

For high energetic beam positrons which scatter off or annihilate with fixed target shell electrons, the scattering angles of both leptons or the two photons, respectively, are small. Therefore the luminosity monitor [Ben01] consists of two small calorimeters located $7.2 \mathrm{~m}$ downstream of the target, mounted as close as possible on both sides of the beam pipe. The calorimeters are built from $12 \mathrm{NaBi}\left(\mathrm{WO}_{4}\right)_{2}$ crystals arranged in a $3 \times 4$ matrix. Each crystal is $20 \mathrm{~cm}$ long and has a cross section of $2.2 \times 2.2 \mathrm{~cm}^{2}$. At the back side a PMT is coupled to each crystal for the read out of the signal. The two scattered particles are detected in coincidence in the two calorimeters. A required energy deposition above 
4.5 GeV in both calorimeters minimises background events.

The large systematic uncertainty of $6 \%$ in the measurement of absolute luminosities due to the dependence of the luminosity monitor acceptance on the beam position and slope [ElsO1] cancels in the calculation of relative luminosities. Their uncertainties are negligible.

\subsubsection{The Trigger System}

The trigger system is used for the very fast discrimination of interesting candidate events from background events and initiates the readout of all spectrometer components. While the detectors are read out, no new events can be recorded, resulting in a dead time $\delta_{\text {dead }}$ of the spectrometer. The dead time is defined as the ratio of trigger requests which had to be rejected and the total number of readout requests. During standard running it is typically well below $10 \%$.

In the trigger system, two additional hodoscopes $\mathrm{HO}$ and $\mathrm{H} 1$ are used together with the hodoscope $\mathrm{H} 2$ of the preshower detector described above. The hodoscope $\mathrm{H} 1 \mathrm{is}$ constructed in the same way as $\mathrm{H} 2$ except for the lead sheet in front, while $\mathrm{HO}$ is not segmented horizontally as it is only used in the trigger. Each half of the hodoscope $\mathrm{HO}$ is build from a single sheet of $3.2 \mathrm{~mm}$ thick plastic scintillator and read out by two PMTs. The hodoscopes $\mathrm{HO}$ and $\mathrm{H} 1$ are located in front of $\mathrm{FCl}$ and the TRD, respectively (see Figure 3.3.1). The aim of the forward hodoscope $\mathrm{HO}$ is to suppress backward-going particles from the HERA proton beam.

Various first-level triggers are used in the HERMES experiment for DIS events, photoproduction events and for calibration and monitoring of the detectors. The trigger decision is made within about 400 ns of an event. The DIS trigger selects lepton events as candidates for a DIS event. A lepton traversing the spectrometer is identified by requiring hits in the three hodoscopes $\mathrm{HO}, \mathrm{H1}$, and $\mathrm{H} 2$ together with an energy deposition in two adjacent calorimeter columns above a threshold energy $E_{\text {thresh, }}$ all in coincidence with the HERA beam bunch signal. The threshold energy of the calorimeter is set to $1.4 \mathrm{GeV}$ for data taking with the polarised target and $3.5 \mathrm{GeV}$ for the unpolarised target'. $E_{\text {thresh }}=$ $3.5 \mathrm{GeV}$ corresponds to a cut on $y<0.87$. The lower threshold relaxes the cut to a higher $y$ value of 0.95 , but it increases significantly background from the collimator just upstream of the target. Only around $13 \%$ of the DIS trigger events have at least one identified lepton (cf. Figure 4.2.2) and approximately $4-6 \%$ of the recorded events are identified as DIS events in the offline analysis.

\subsection{Data Acquisition and Processing}

The readout of the detector is carried out by specific readout electronics hosted in FastBus crates which are located in an electronic trailer close to the spectrometer. For the timing and analogue information LeCroy 1877 Multihit FastBus TDCs (time-to-digital converter) and LeCroy $1881 \mathrm{M}$ FastBus ADCs (analogue-to-digital converter) are in use. The magnet chambers and the $\mathrm{RICH}$ are read out by the LeCroy PCOS4 system. The data

\footnotetext{
'In the beginning of the 96 production the higher threshold of $3.5 \mathrm{GeV}$ was also used for polarised data taking.
} 
from the FastBus crates are bundled by event builder modules and sent over fast optical links to a Linux cluster (a DEC Alpha cluster before 2002), where they are stored on staging disks and on data tapes. So-called slow control data, like information from the luminosity monitor, the polarimeters, the target, detector temperatures, voltage settings, etc., is recorded in addition. The slow control data are read out once every $\sim 10 \mathrm{~s}$, independent of triggers from the spectrometer. All raw data is buffered in EPIO format on hard disks in the Linux cluster and backed up regularly on data tapes. It is transferred to a taping robot at the DESY main site after the end of each HERA positron fill using a FDDI (fast distributed data interface) link.

From the electronic detector signals, the hit positions, energy depositions, etc. are determined with the HERMES decoder (HDC) using mapping, geometry and calibration of the individual detectors. All required information is stored in an ADAMO [CER93b] database, which is an entity-relationship database allowing structured and portable data storage. In a next step the HERMES reconstruction (HRC) program finds tracks in the spectrometer. Using a timing signal that is written to the event data and slow control data streams, both data streams can be synchronised. All synchronised data which is useful for physics analyses, is stored in data summary tables - the so-called $\mu$ DST files.

Different time scales are used in the HERMES data. The shortest time interval is the event containing all reconstructed tracks which are observed when a trigger is generated. All events recorded within approximately 10 s are grouped into a burst. This is the time scale on which the slow control information is synchronised to the event data. In order to split up the raw data into small enough pieces for storage, bursts are combined into a run with a size of about $450 \mathrm{MB}$. Dependent on the luminosity, a run lasts around $10 \mathrm{~min}$. The longest time scale, the fill, is determined by the $8-12 \mathrm{~h}$ storage time of the HERA positron beam. 

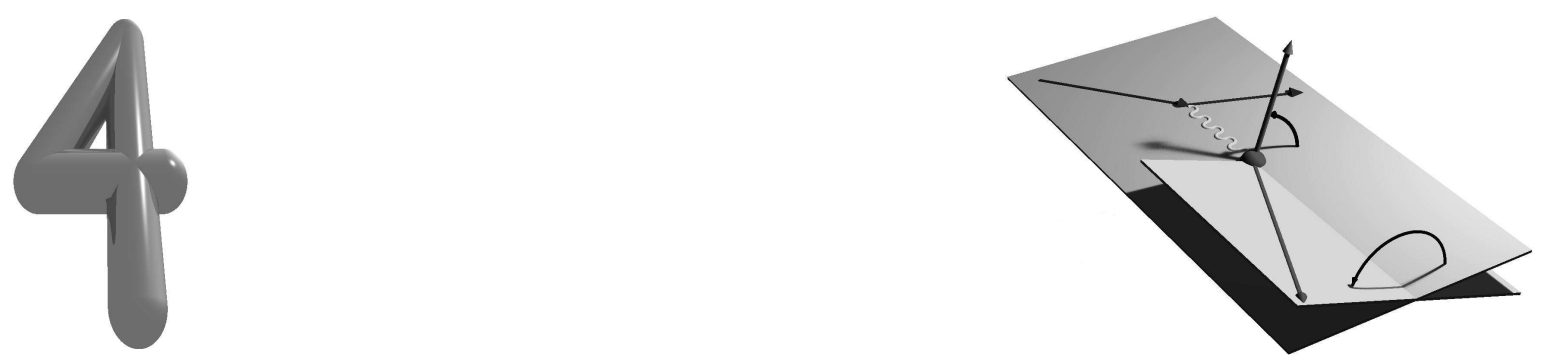

\section{Azimuthal Asymmetry Moments}

In the years 2002 to 2004, the HERMES experiment collected data with a transversely polarised hydrogen target. The extraction of azimuthal single-spin asymmetry moments from the accumulated semi-inclusive deep-inelastic scattering events is presented in this chapter. In the analysis, several checks are applied in order to ensure a high quality of the data before semi-inclusive events are identified from the reconstructed tracks by certain geometry and kinematic requirements. The obtained count rates of semi-inclusive events are used to form a single-spin asymmetry depending on two azimuthal angles. With a two-dimensional fit the sine modulations of the Collins and Sivers effect are extracted. Systematic studies are performed to estimate influences on the extraction from different sources.

\subsection{Data Quality}

The produced $\mu$ DST-files of the different data taking periods are labelled by the last two digits of the corresponding year of data taking, a letter to indicate the production, and a cypher. In the first production of $\mu$ DST-files for a new data taking period (a-production), detector calibrations are used which are based on the data of the preceding period. The a-production allows detailed detector calibrations which serve as input for a reproduction of the $\mu$ DST-files (b-production). In the subsequent c-production, additional corrections which rely on proper calibrations are taken into account. The cypher which completes the production name, is increased for each reproduction without a new track reconstruction. Such a reproduction usually is carried out when improved slow control information such as beam or target polarisation values becomes available. The analysis presented here is based on the data productions $02 \mathrm{c0}, 03 \mathrm{c0}$, and $04 \mathrm{bO}$.

Although some measurements are performed on longer time scales, the information of the different components of the HERMES experiment and of the relevant HERA parameters is available on burst level (cf. Section 3.4). To ensure a high data quality in each recorded burst, several measured quantities are checked for consistency. Data quality checks on

- the rate of the luminosity monitor,

- the beam current and the beam polarisation, 
- the status of the target and of the data acquisition system,

- the information from the shift logbook

and for

- malfunctioning PID detectors and

- high voltage trips in the wire chambers

are performed. For each data production, a burst list with the information of the data quality checks is provided by the HERMES data quality group. Details about the data quality checks can be found in [Wen03] and on the HERMES $\mu$ DST data quality web page [HDQb].

The analysis of target-spin asymmetries does not require a polarised lepton beam. The outcome of the data quality checks on the beam polarisation and the status of its measurement can therefore be ignored in this analysis. Furthermore, one single out of all PMTs connected to a calorimeter block is allowed to be dead in the year 2002, in which the calorimeter suffered from ageing which caused several failures of scintillator-PMT units. An elaborate study showed that one dead unit does not influence semi-inclusive azimuthal asymmetries of charged hadrons [Has03]. For the identification of neutral pions, which is based on the calorimeter signals solely, all PMTs are required to work properly.

\subsection{Event Selection}

In all DIS trigger events of bursts passing the above mentioned quality cuts, a particle type is assigned to each track that fulfils certain requirements on its geometry as described in more detail later. After identification of the scattered positron and the produced hadrons, several cuts are applied on the kinematics for the selection of semi-inclusive DIS events. The cuts are described in the order as they are applied in the analysis chain.

\begin{tabular}{l|c|c} 
& charged particles & photons \\
\hline vertex position & $-18 \mathrm{~cm} \leq z_{\text {vertex }} \leq 18 \mathrm{~cm}$ & \\
calorimeter position & $\left|x_{\text {calo }}\right| \leq 175 \mathrm{~cm}$ & $\left|x_{\text {calo }}\right| \leq 125 \mathrm{~cm}$ \\
front field clamp position & $30 \mathrm{~cm} \leq y_{\text {calo }} \leq 108 \mathrm{~cm}$ & $33 \mathrm{~cm} \leq y_{\text {calo }} \leq 105 \mathrm{~cm}$ \\
septum plate position & $\left|x_{\mathrm{ffc}}\right|<31 \mathrm{~cm}$ & \\
rear field clamp position & $\left|y_{\mathrm{sp}}\right|>7 \mathrm{~cm}$ & \\
rear clamp position & $\left|y_{\mathrm{rfc}}\right|<54 \mathrm{~cm}$ & \\
\hline
\end{tabular}

Table 4.2.1: The geometry cuts which are applied to the detected charged particles and photons. 


\subsubsection{Geometry Cuts}

Before the identification of the particles, several geometry cuts on the particle tracks are applied to ensure that the track reconstruction was not influenced by the edges of the HERMES spectrometer and to suppress background. The longitudinal vertex coordinate $z_{\text {vertex }}$ at which the scattering occurred, is restricted to the dimension of the target cell. In addition, the vertical and horizontal positions of the track are checked at the locations of several detector components which limit the HERMES acceptance. All geometry cuts are listed in Table 4.2.1 for charged particles and photons. The latter are required to hit a smaller area of the calorimeter front because they are not deflected by the spectrometer magnet and to avoid transverse shower leakage as photon identification is exclusively based on the calorimeter signal.

\subsubsection{Particle Identification}

\section{Lepton-Hadron Discrimination}

Combining the responses of the four PID detectors described in Section 3.3.2, a separation between leptons - positrons and electrons - and hadrons is possible. The combination of several PID detectors reduces the contamination significantly compared to a particle identification with only one detector. For each PID detector D, the conditional probability $\mathcal{L}_{\mathrm{D}}^{i}(\xi)$ that a given particle of type $i$ ( $i=$ lepton, hadron) generates a detector response $\xi$, can be extracted using very restrictive cuts on the remaining three PID detector responses. The conditional probability is related by Bayes' theorem to the probability,

$$
\mathcal{P}_{\mathrm{D}}^{i}(\xi)=\frac{\Phi^{i} \mathcal{L}_{\mathrm{D}}^{i}(\xi)}{\sum_{j} \Phi^{j} \mathcal{L}_{\mathrm{D}}^{j}(\xi)},
$$

that a given signal $\xi$ was caused by a particle of type $i . \Phi^{i}$ is the incident flux of particles of type $i$ and the sum is performed over all particle types $j$ to be distinguished.

The extracted conditional probabilities of each PID detector serve as input for the socalled PID value, which is defined as the decadic logarithm of the ratio of the conditional probabilities for leptons $(l)$ and hadrons $(h)$,

$$
\mathrm{PID}_{\mathrm{D}} \equiv \log _{10} \frac{\mathcal{L}_{\mathrm{D}}^{l}(\xi)}{\mathcal{L}_{\mathrm{D}}^{h}(\xi)} .
$$

It is common to combine the three PID values of the preshower detector, the calorimeter, and the Čerenkov detector (threshold Čerenkov counter before and RICH detector since the year 1998) into one quantity:

$$
\mathrm{PID}_{3} \equiv \log _{10} \frac{\mathcal{L}_{\mathrm{Pre}}^{l} \mathcal{L}_{\mathrm{Cal}}^{l} \mathcal{L}_{\mathrm{Cer}}^{l}}{\mathcal{L}_{\mathrm{Pre}}^{h} \mathcal{L}_{\mathrm{Cal}}^{h} \mathcal{L}_{\mathrm{Cer}}^{h}} .
$$

In the special case of the RICH detector, the conditional probabilities for gas and aerogel radiators are multiplied. Also the signals of the six TRD modules are combined into one PID value:

$$
\mathrm{PID}_{5} \equiv \log _{10} \frac{\prod_{m=1}^{6} \mathcal{L}_{\mathrm{TRD} m}^{l}}{\prod_{m=1}^{6} \mathcal{L}_{\mathrm{TRD} m}^{h}}
$$




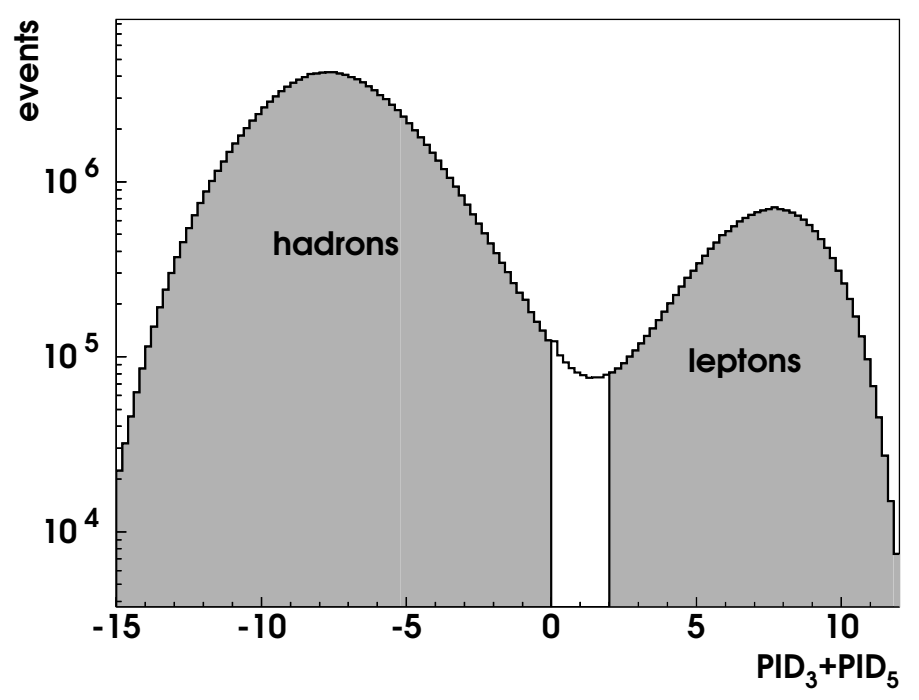

Figure 4.2.1: Sum of $\mathrm{PID}_{3}$ and $\mathrm{PID}_{5}$ for the three analysed data productions. The applied cuts for the separation of hadrons and leptons are plotted as vertical lines.

$\mathrm{PID}_{3}$ and $\mathrm{PID}_{5}$ values are assigned to each detected particle dependent on the responses in the different PID detectors. Once the $\mathcal{L}_{\mathrm{D}}^{i}$ have been determined for each particle type $i$ and detector $\mathrm{D}$, the restrictive cuts for the extraction of the conditional probabilities can be lifted and instead a cut is applied on the quantity

$$
\mathrm{PID}_{3}+\mathrm{PID}_{5}-\log _{10} \frac{\Phi^{h}}{\Phi^{l}}=\log _{10} \frac{\mathcal{P}^{l}}{\mathcal{P}^{h}} .
$$

This quantity can be interpreted as the logarithm of the probability ratio that a given particle is a lepton or a hadron. The hadron and lepton fluxes, $\Phi^{h}$ and $\Phi^{l}$, can be calculated iteratively, which has not yet been done for all of the transverse target data productions. However, the impact of the flux factor $\log _{10}\left(\Phi^{h} / \Phi^{l}\right)$ is small for semi-inclusive DIS event kinematics and an analysis using only the sum of $\mathrm{PID}_{3}$ and $\mathrm{PID}_{5}$ is a good approximation [Wen03]. Figure 4.2.1 shows the $\mathrm{PID}_{3}+\mathrm{PID}_{5}$ distribution of tracks in DIS trigger events with a local minimum around 1.5. As indicated by the shaded areas, leptons and hadrons are

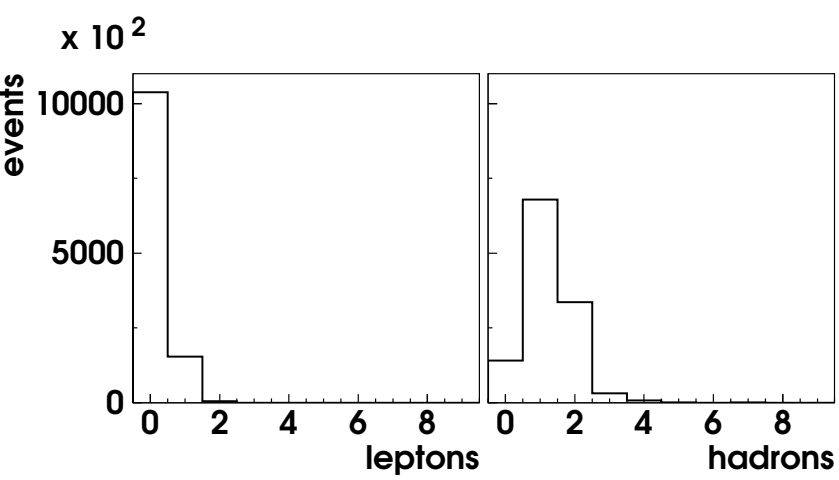

Figure 4.2.2: Number of leptons and hadrons in DIS trigger events of a subset of the 02 production after applying data quality cuts. 
identified with the following cutsi:

$$
\begin{array}{ll}
\mathrm{PID}_{3}+\mathrm{PID}_{5}>2.0 & \text { for leptons } \\
\mathrm{PID}_{3}+\mathrm{PID}_{5}<0.0 & \text { for hadrons. }
\end{array}
$$

Most of the DIS trigger events contain only hadrons and no leptons, as can be seen in Figure 4.2.2.

\section{Charged Hadron Identification}

When passing through the two $\mathrm{RICH}$ radiators, charged particles emit a certain pattern of Čerenkov photons (cf. Section 3.3.2). This allows the separation of the individual charged hadron types. The photon pattern which is focused on the PMT matrix by the spherical mirror array, is associated with a track in the spectrometer using an inverse ray tracing algorithm [Jun00]. Combining all measured Čerenkov angles in a range around the theoretically expected angle, averaged Čerenkov angles are computed for the two radiators, aerogel ( $a$ ) and gas $(g)$, and for each of the three hadron hypotheses, $h=\pi, K, p$. From these, two likelihoods $\mathcal{L}_{h}^{a, g}$ can be calculated from the averaged Čerenkov angles and combined to the total likelihood $\mathcal{L}_{h}^{\text {tot }}=\mathcal{L}_{h}^{a} \cdot \mathcal{L}_{h}^{g}$. The hadron type hypothesis with the largest combined likelihood is assigned to the track.

The success of the identification is assured by requiring a positive value of the quality parameter $\mathrm{Qp}$ which is defined as the logarithm of the likelihood ratio of the most and the second most likely hadron types, $h_{1}$ and $h_{2}$ :

$$
\mathrm{Qp}=\log _{10} \frac{\mathcal{L}_{h_{1}}^{\text {tot }}}{\mathcal{L}_{h_{2}}^{\text {tot }}}>0 .
$$

If the identification algorithm could not find a most probable hadron type the quality parameter is set to zero.

Using a Monte Carlo simulation of the RICH detector, contamination and efficiency of the hadron identification are estimated. The analysis of the Monte Carlo data allows the determination of the conditional probability $P\left(h_{\text {id }} \mid h_{\text {true }}\right)$ that a given hadron of true type $h_{\text {true }}$ is identified as particle of type $h_{\mathrm{id}}$. For $h_{\text {true }}=h_{\mathrm{id}}$, the conditional probability is equivalent to the efficiency of the identification. However, for the interpretation of $P\left(h_{\text {id }} \mid h_{\text {true }}\right)$ as contamination in case of $h_{\text {true }} \neq h_{\text {id }}$, the relative hadron fluxes must be taken into account in analogy to Eq. (4.2.1). These conditional probabilities are combined into a matrix,

$$
\mathcal{P}=\left(\begin{array}{lll}
P\left(\pi_{\text {id }} \mid \pi_{\text {true }}\right) & P\left(\pi_{\text {id }} \mid K_{\text {true }}\right) & P\left(\pi_{\text {id }} \mid p_{\text {true }}\right) \\
P\left(K_{\text {id }} \mid \pi_{\text {true }}\right) & P\left(K_{\text {id }} \mid K_{\text {true }}\right) & P\left(K_{\text {id }} \mid p_{\text {true }}\right) \\
P\left(p_{\text {id }} \mid \pi_{\text {true }}\right) & P\left(p_{\text {id }} \mid K_{\text {true }}\right) & P\left(p_{\text {id }} \mid p_{\text {true }}\right)
\end{array}\right),
$$

dependent on the track momentum and the topology of the photon patterns on the PMT matrix. The more tracks are detected in one detector half (top or bottom), the higher is the probability for patterns to overlap. Therefore, the $\mathcal{P}$-matrix elements are extracted for one, two, and three or more tracks in the given detector half and in $1 \mathrm{GeV}$ momentum bins. The results are plotted in Figure 4.2.3, arranged in the same way as Eq. (4.2.9).

'For both particle types, $\left|\mathrm{PID}_{3}+\mathrm{PID}_{5}\right|<80.0$ is requested in order to reject events with unreliable PID values. 


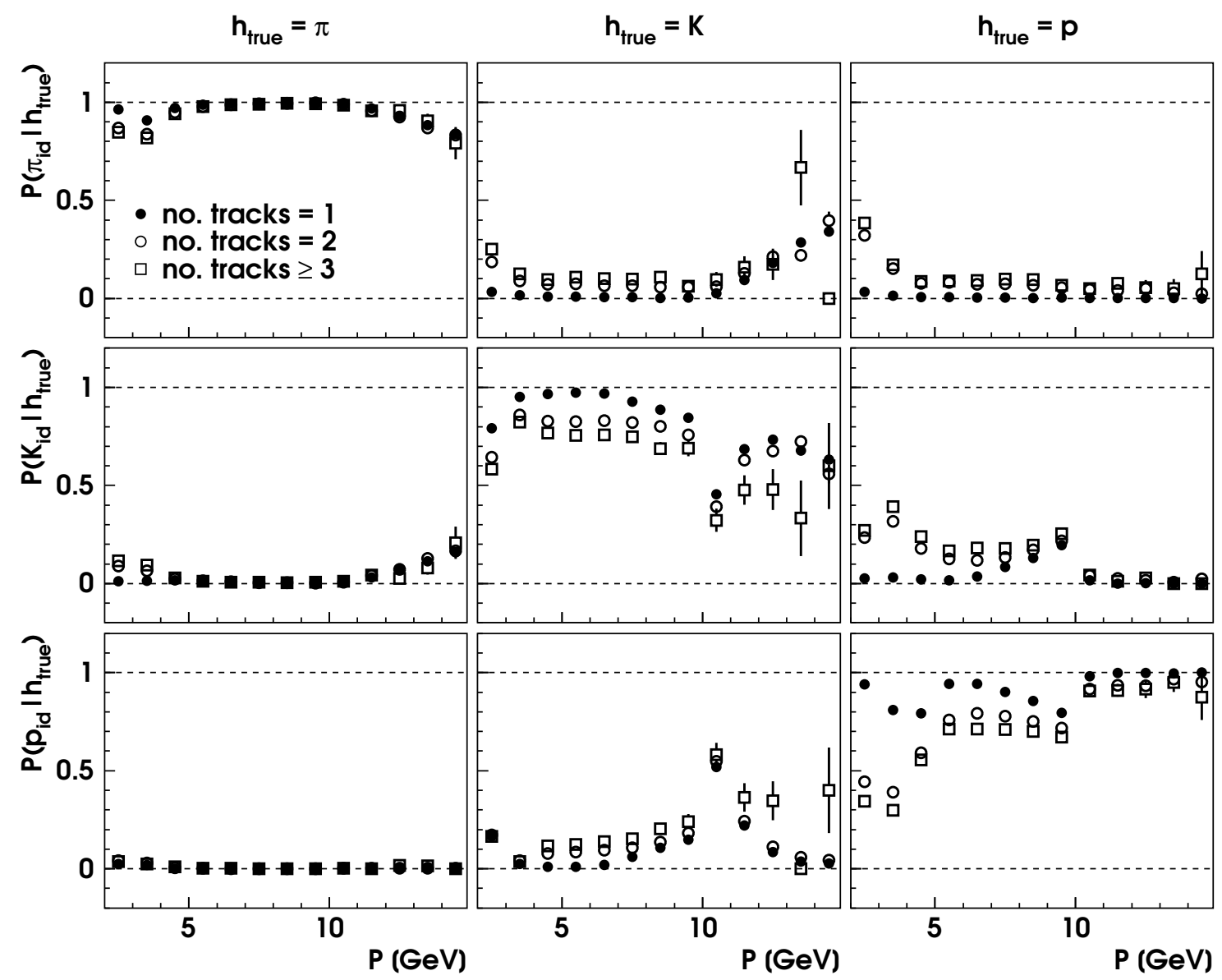

Figure 4.2.3: The $\mathcal{P}$-matrix, dependent on the momentum of the particle, for the HERMES RICH detector obtained from a Monte Carlo simulation [Hom04]. Plotted are the conditional probabilities that a given hadron of true type $h_{\text {true }}$ is identified as a pion, kaon, or proton, respectively. The different plot symbols represent the dependence on the number of charged particle tracks per detector half.

The $\mathrm{RICH}$ pion identification has a large efficiency and the probability to misidentify a kaon or proton as a pion is small over almost the entire momentum range. For kaons the momentum threshold of the gas radiator is visible as a discontinuity at around $10 \mathrm{GeV}$.

The $\mathcal{P}$-matrix relates the flux of identified hadrons, $\vec{I}=\left(N_{\pi}^{\mathrm{id}}, N_{K}^{\mathrm{id}}, N_{p}^{\mathrm{id}}\right)$, to the true flux of hadrons, $\vec{N}=\left(N_{\pi}^{\text {true }}, N_{K}^{\text {true }}, N_{p}^{\text {true }}\right)$, by $\vec{I}=\mathcal{P} \cdot \vec{N}$. In order to obtain the true flux from the measured flux of identified hadrons, the $\mathcal{P}$-matrix must be inverted:

$$
\vec{N}=\mathcal{P}^{-1} \cdot \vec{I}
$$

In the analysis of semi-inclusive events, a weight dependent on the identified hadron type is assigned to each particle track according to the inverse $\mathcal{P}$-matrix. A track identified as $h_{\text {id }}$ is weighted by $\left(\mathcal{P}^{-1}\right)_{\pi_{\text {true }}, h_{\text {id }}}$ in the true pion count rate and by $\left(\mathcal{P}^{-1}\right)_{K_{\text {true }}, h_{\text {id }}}$ in the true kaon count rate. The sum of these weights over all tracks $l$ with identified hadron type $\left(h_{\mathrm{id}}\right)_{l}$ yields the number of true hadrons:

$$
N_{h}^{\text {true }}=\sum_{l}\left(\mathcal{P}^{-1}\right)_{h_{\text {true }},\left(h_{\mathrm{id}}\right)_{l}} .
$$


It is common to present a count number of weighted events in terms of an equivalent count number $N_{\text {eq }}$ which is defined as the number of unweighted events with the same relative error as the sum of weighted events $N=\sum_{i} w_{i}$ :

$$
\frac{\sigma\left(N_{\mathrm{eq}}\right)}{N_{\mathrm{eq}}}=\frac{\sqrt{N_{\mathrm{eq}}}}{N_{\mathrm{eq}}} \equiv \frac{\sqrt{\sum_{i} w_{i}^{2}}}{\sum_{i} w_{i}}=\frac{\sigma(N)}{N},
$$

with the elements of the inverse $\mathcal{P}$-matrix as weights $w_{i}$ in case of the $\mathrm{RICH}$ hadron identification.

\section{Neutral Pion Identification}

The short life time of the neutral pion allows its identification by the decay into a pair of photons with a branching ratio of almost 99\% [Eid04]. In the HERMES spectrometer, the energies and positions of photons are measured by the calorimeter, as described in Section 3.3.2. The reconstructed photon position on the calorimeter front is connected to the vertex $z$-coordinate of the scattered positron by a straight line for the calculation of the photon momentum. Two photons with energies larger than $1 \mathrm{GeV}$ are combined and the invariant mass $M_{\gamma \gamma}$ is computed. For events with more than two photons and less than eleven, all possible combinations are considered. Events with more than ten photons yield more than $\sum_{n=1}^{9} n=45$ combinations and are therefore considered as background events. They are rejected in order to limit the combinatorial background.

Figure 4.2.4 shows the invariant mass distribution of all photon combinations after applying the geometry and kinematic cuts described below. A fit is superimposed on the histogram using a Gaussian for the decay peak and a third order polynomial to describe the background. The fit results in a $\pi^{0}$ mass $M_{\pi^{0}}=(134.29 \pm 0.06) \mathrm{MeV}$ which is close to the value of (134.98 \pm 0.06 ) MeV obtained by the PARTICLE DATA Group [Eid04]. The $5 \sigma$ deviation is caused by the energy calibration of the calorimeter and the position reconstruction

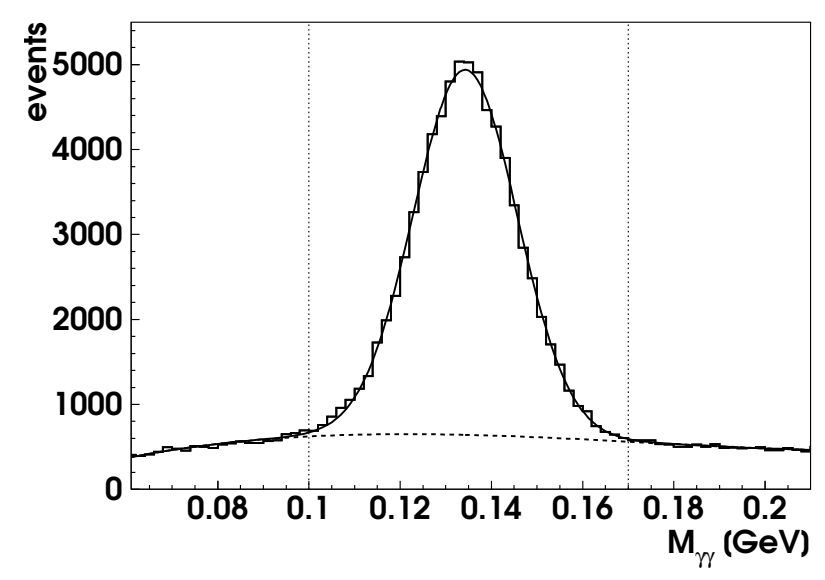

Figure 4.2.4: Invariant mass distribution of the photon pairs with geometry and kinematic cuts applied. The solid line is a fit to the spectrum using a Gaussian and a third order polynomial. The dashed line is the third order polynomial only which parametrises the combinatorial background. 
of the photons which is solely based on the calorimeter signals. The width of the peak $\sigma=$ (1 1.37 \pm 0.06$) \mathrm{MeV}$ reflects the resolution of the calorimeter. In the range $[0.10,0.17] \mathrm{GeV}$ which corresponds approximately to $M_{\pi^{0}} \pm 3 \sigma$, the fraction of signal events $N_{\text {sig }} / N_{\text {tot }}$ can be deduced from the fit. While this fraction is independent of $x$ it is strongly correlated to the energy fraction $z$ (cf. Section 4.5). The fraction of signal events,

$$
\frac{N_{\text {sig }}}{N_{\text {tot }}}=0.736 \pm 0.003
$$

is obtained in the semi-inclusive range $0.2<z<0.7$ and $0.023<x<0.4$.

In the analysed data productions, the energy calibration of the calorimeter yielded a constant correction factor for the measured photon energies. An energy calibration dependent on the signal in the preshower, which will improve the resolution of the calorimeter, is under study [Ave04b] but is not yet implemented in this analysis. The new calibration is also planned to improve the reconstruction of the $\pi^{0}$ mass.

\subsubsection{Kinematic Cuts}

The scattered DIS positron is selected by several kinematic cuts listed in Table 4.2.2. A four-momentum transfer of more than $1 \mathrm{GeV}^{2}$, i.e., larger than the squared proton mass, is required for scattering processes in the deep-inelastic region. Events from the resonance region ( $W \lesssim 2 \mathrm{GeV}$ ) are excluded by a cut on the invariant mass of the final hadronic state. For inclusive DIS events which can be used for the luminosity normalisation described below, this cut is lower than for semi-inclusive DIS events. For the latter, the higher cut improves the separation of the observed hadrons carrying the information of the struck quark from the observed hadrons which come from the target remnant [Bec00]. The upper limit on the fractional energy transfer $y$ eliminates DIS events from a region with a large contribution to the cross section by higher order QED effects, e.g., bremsstrahlung. This limit discards leptons with momenta lower than 4.1 GeV. Due to the restrictions on $W^{2}$ and $Q^{2}$, the lowest possible value of the fractional energy transfer in semi-inclusive events is around 0.18. For inclusive events, in contrast, the lowest possible value of 0.07 is excluded by a lower cut of $y>0.1$. The chosen range in the Bjorken scaling variable is determined by the HERMES acceptance and the cuts on $Q^{2}$ and $W^{2}$.

\begin{tabular}{l|c|c} 
& inclusive DIS & semi-inclusive DIS \\
\hline four momentum transfer & $Q^{2}>1 \mathrm{GeV}^{2}$ & $Q^{2}>1 \mathrm{GeV}^{2}$ \\
squared mass of the final state & $W^{2}>4 \mathrm{GeV}^{2}$ & $W^{2}>10 \mathrm{GeV}^{2}$ \\
fractional energy transfer & $0.1<y<0.85$ & $y<0.85$ \\
Bjorken scaling variable & $0.023<x<0.4$ & $0.023<x<0.4$ \\
virtual photon - hadron angle & & $\theta_{\gamma^{*} h}>0.02 \mathrm{rad}$ \\
hadron momentum & & $2 \mathrm{GeV}<P_{h}<15 \mathrm{GeV}$ \\
energy fraction (extended range) & & $0.2<z<0.7(0.7<z<1.2)$ \\
\hline
\end{tabular}

Table 4.2.2: The kinematic cuts for the selection of inclusive and semi-inclusive DIS events. 

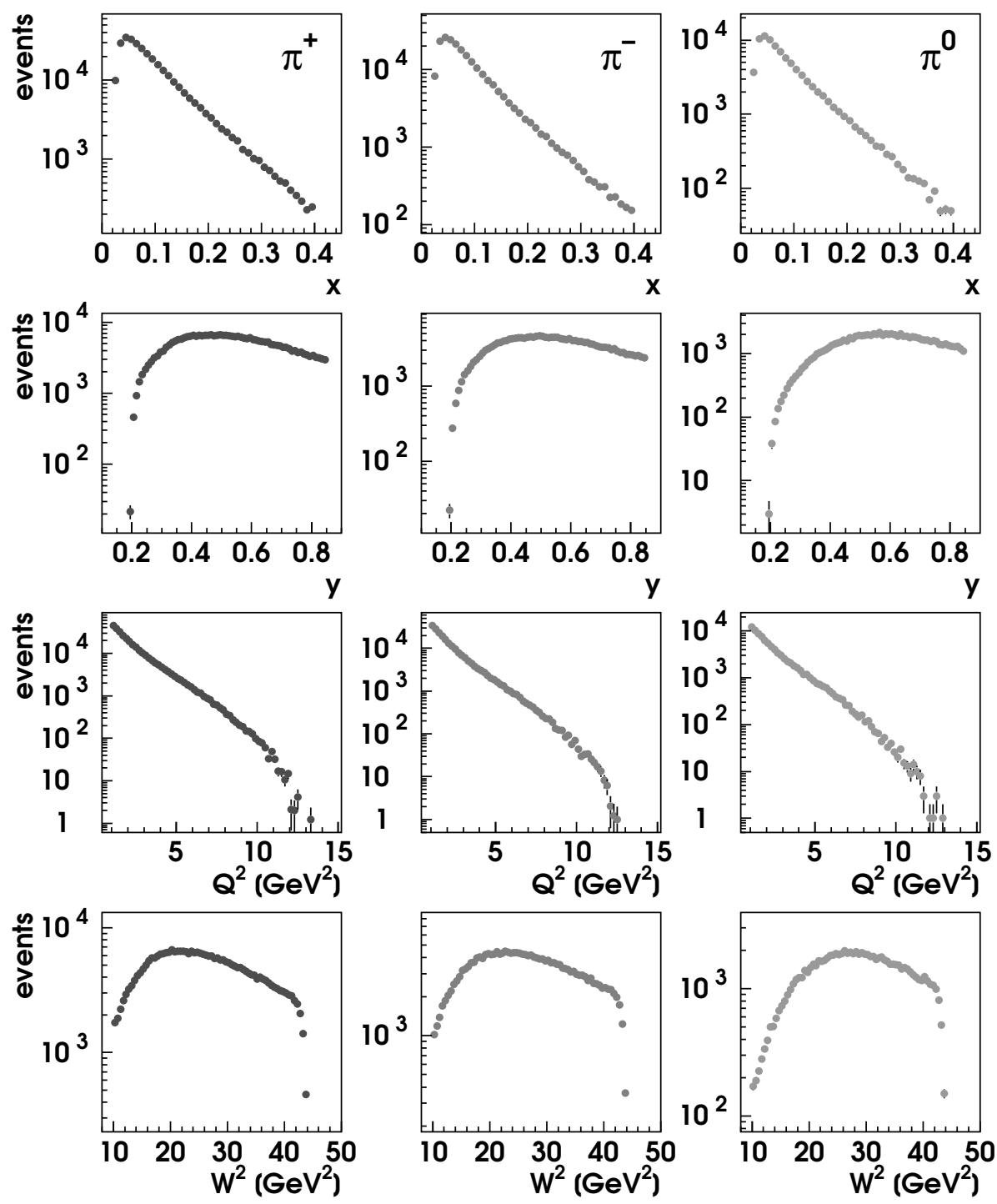

Figure 4.2.5: Distributions of the inclusive variables $x, y, Q^{2}$, and $W^{2}$ for the $\pi^{+}$, $\pi^{-}$, and $\pi^{0}$ event samples after all cuts are applied.

If more than one positron track remains in the event after the geometry and kinematic cuts the one with the highest momentum is identified with the DIS positron.

Further cuts are applied on those hadrons that are detected in coincidence with the identified DIS positron. In order to limit the uncertainty in the determination of the azimuthal angles $\phi$ and $\phi_{S}$ (for the definition see Figure 2.5.3), events with an angle $\theta_{\gamma^{*} h}$ between the virtual photon $\left(\gamma^{*}\right)$ and the hadron $(h)$ smaller than 0.02 rad are discarded. This value reflects the resolution of the reconstruction of $\theta_{\gamma^{*} h}$ (cf. Figure 3.3.2). The constraint on the angle between photon and hadron limits the transverse momentum $P_{h \perp}=\left|\vec{P}_{h \perp}\right|$ of the hadron, e.g., for $2 \mathrm{GeV}$ hadrons, transverse momenta below $0.05 \mathrm{GeV}$ are excluded. In addition, the absolute momenta of the hadrons are restricted to a range between $2 \mathrm{GeV}$ and $15 \mathrm{GeV}$ for a reliable hadron identification with the $\mathrm{RICH}$ detector. The upper limit of the energy fraction $z<0.7$ rejects scattering events in a region which is dominated by exclusively produced vector mesons. For the investigation of the $z$ dependence, this 

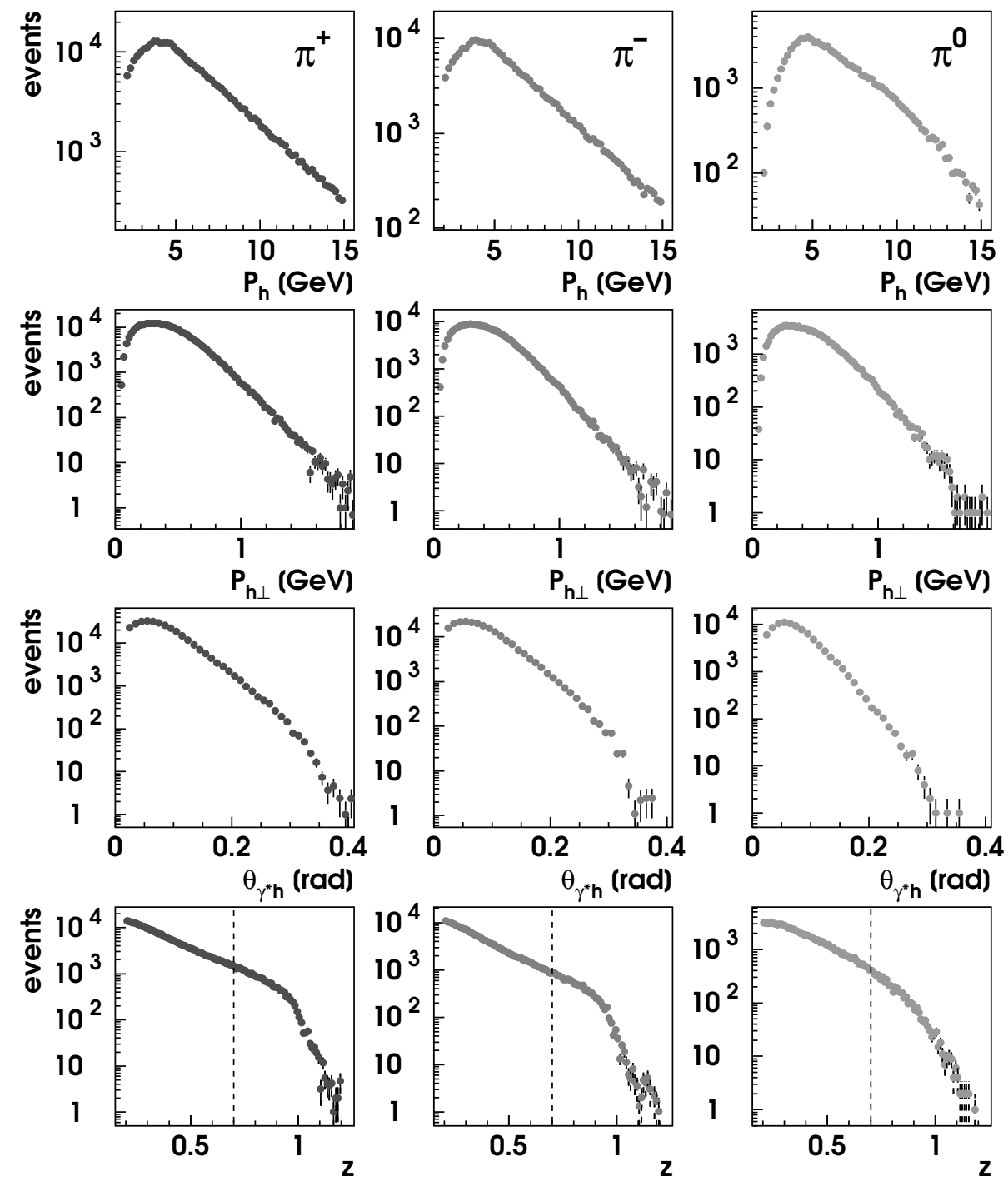

Figure 4.2.6: Distributions of the semi-inclusive variables $P_{h}, P_{h \perp}, \theta_{\gamma^{*} h}$, and $z$ for the $\pi^{+}, \pi^{-}$, and $\pi^{0}$ event samples after all cuts are applied. The extended $z$ range is indicated by a dashed line. This range is not included in the distributions of $P_{h}, P_{h \perp}$, and $\theta_{\gamma^{*} h}$.

cut is lifted and the range in energy fraction is extended to 1.2. The lower limit of 0.2 in combination with the $W^{2}$ cut enhances the fraction of observed hadrons which carry the information of the struck quark. All hadrons passing the kinematic cuts are included in the analysis.

A correction for charge symmetric background processes, e.g., electron-positron pair production, is applied to the particle count rates. The positron from such a process might be misidentified as the scattered beam positron from the DIS process. The background rate is estimated by considering electron tracks which pass the kinematic cuts for inclusive or semi-inclusive events. These electrons are assumed to originate from charge symmetric background events. The rate of electrons passing the DIS cuts can be used as estimate since the rates of electrons and positrons from charge symmetric background events are equivalent. For the correction, the number of DIS events with electrons instead 


\begin{tabular}{|l|c|c|c|c|c|}
\hline & & incl. DIS & $\pi^{0}$ & $\pi_{\text {sig }}^{0}$ & $\pi_{\text {bkg }}^{0}$ \\
\hline count number & $N$ & 3352199 & 81011 & 59624 & 21387 \\
\hline
\end{tabular}

\begin{tabular}{|l|c|c|c|c|c|}
\hline & & $\pi^{+}$ & $\pi^{-}$ & $K^{+}$ & $K^{-}$ \\
\hline number of most likely type & $N_{h}^{\text {id }}$ & 268219 & 190069 & 58132 & 23074 \\
true count number & $N_{h}^{\text {true }}$ & 281497 & 200650 & 48802 & 16450 \\
equivalent count number & $N_{\text {eq }}$ & 257049 & 184085 & 23654 & 6742 \\
\hline
\end{tabular}

Table 4.2.3: Count numbers of DIS positrons and semi-inclusive DIS hadrons for the three productions $02 \mathrm{c0}, 03 \mathrm{c0}$, and $04 \mathrm{b0}$ combined.

of positrons is subtracted from the particle count rates. The resulting particle count rates for inclusive and semi-inclusive DIS events are listed in Table 4.2.3. The signal and background count numbers of $\pi^{0}$ events are determined using the fraction of signal events (4.2.13) extracted from the fit to the invariant mass spectrum. For the charged hadrons, the number of hadrons identified by the $\mathrm{RICH}$ as most likely hadron type together with the true hadron and the equivalent count numbers as defined in (4.2.11) and (4.2.12), are given.

The distributions of inclusive and semi-inclusive variables relevant for this analysis, are plotted for charged and neutral pions in Figures 4.2.5 and 4.2.6. As explained in Section 2.5.2, the squared transverse momentum of the hadron must be small compared to $Q^{2}$. This is approximately fulfilled as can be seen in Figure 4.2.7 which shows the distribution of $P_{h \perp}^{2} / Q^{2}$ for the charged and neutral pions. Only a negligible event fraction of around $0.1 \%$ has a squared transverse momentum which is larger than $Q^{2}$.
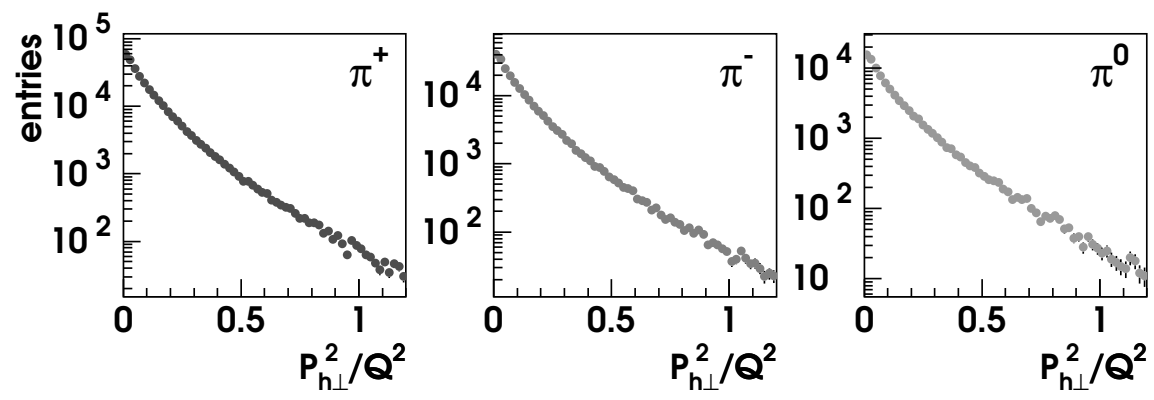

Figure 4.2.7: Distribution of $P_{h \perp}^{2} / Q^{2}$ for the three different pion types.

\subsection{The Extraction of Asymmetry Moments}

The azimuthal target-spin asymmetry is derived from the cross-section asymmetry between two opposite spin states of the transversely polarised target:

$$
A_{\mathrm{UT}}\left(\phi, \phi_{S}\right)=\frac{1}{\langle P\rangle} \frac{N^{\uparrow}\left(\phi, \phi_{S}\right) L^{\downarrow}-N^{\downarrow}\left(\phi, \phi_{S}\right) L^{\uparrow}}{N^{\uparrow}\left(\phi, \phi_{S}\right) L^{\downarrow}+N^{\downarrow}\left(\phi, \phi_{S}\right) L^{\uparrow}},
$$

where $N^{\uparrow / \downarrow}$ are the count numbers of true hadrons (4.2.11) for the two target spin states parallel $(\uparrow)$ and antiparallel $(\downarrow)$ to the target magnet field, binned in the angles $\phi$ and $\phi_{S}$ 


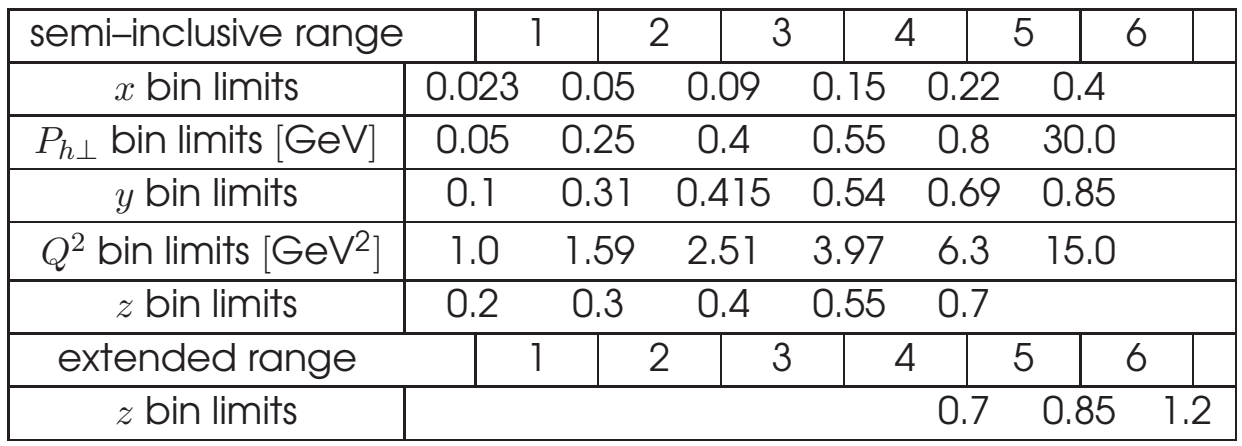

Table 4.3.1: Bin limits of the kinematic quantities used in the analysis.

(see Figure 2.5.3). The count numbers are normalised to the integrated luminosities $L^{\uparrow / \downarrow}$, which can either be calculated from the integrated rates of the luminosity monitor or from the count rates of inclusive DIS events. The different polarisation values are weighted by the integrated luminosity to obtain the averaged target polarisation $\langle P\rangle=0.75 \pm 0.05$. The calculation of the azimuthal angle $\phi_{S}$ is with respect to the nucleon spin direction in target spin state $\uparrow$. This results in a cancellation of the unpolarised (polarised) cross-section terms in the numerator (denominator).

The asymmetry is computed in bins of one kinematic quantity while integrated over the other kinematic quantities. In Table 4.3. 1 the bin limits of the different kinematic quantities used for the analysis, are listed. The binning in the energy fraction $z$ is extended with two bins between 0.7 and 1.2 in order to investigate the behaviour of the asymmetry moments beyond the semi-inclusive range. The HERMEs spectrometer acceptance causes a strong coupling of $x$ and $Q^{2}$ which results in an increasing mean value $\left\langle Q^{2}\right\rangle$ in consecutive $x$ bins and vice versa. For the pion asymmetries each kinematic bin is split

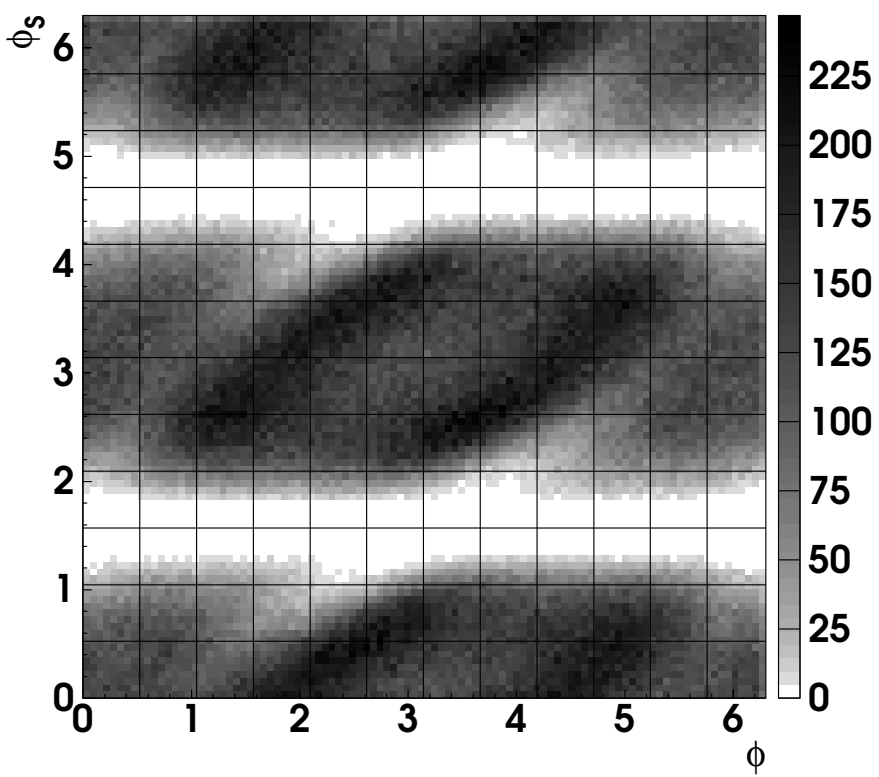

Figure 4.3.1: Distribution of the azimuthal angles $\phi$ and $\phi_{S}$ of charged pions in the kinematic range $0.023<x<0.4$ and $0.2<z<1.2$. 
up into $12 \times 12$ equidistant bins in the azimuthal angles $\phi$ and $\phi_{S}$ as superimposed on the two-dimensional distribution of the azimuthal angles in Figure 4.3.1. In the distribution of $\phi_{S}$ the separation between top and bottom half of the HERMES spectrometer is clearly visible. The number of azimuthal bins is adjusted to obtain sensible statistical precision in each bin using a Monte Carlo simulation (see Section 5.1.2). For the kaons with their lower statistics an $8 \times 8$ binning is chosen.

The 144 (64) azimuthal asymmetries are fitted by a two-dimensional function including all bins with at least one event in each target spin state. The parameters of the fit function:

$$
\begin{aligned}
A_{\mathrm{UT}}\left(\phi, \phi_{S}\right)= & A_{\mathrm{UT}}^{\sin \left(\phi+\phi_{S}\right)} \cdot \sin \left(\phi+\phi_{S}\right)+A_{\mathrm{UT}}^{\sin \left(\phi-\phi_{S}\right)} \cdot \sin \left(\phi-\phi_{S}\right)+ \\
& A_{\mathrm{UT}}^{\sin \left(2 \phi-\phi_{S}\right)} \cdot \sin \left(2 \phi-\phi_{S}\right)+A_{\mathrm{UT}}^{\sin \phi_{S}} \cdot \sin \phi_{S}+C,
\end{aligned}
$$

are the amplitudes $A_{\mathrm{UT}}^{\sin \left(n \phi \pm \phi_{S}\right)}$ of different sine modulations $(n=0,1,2)$ and a constant $C$ which should vanish for a correct luminosity normalisation of the asymmetries. This is in fact found in all the fits performed in this analysis. Neglecting the coupling between subleading-twist terms of the cross section and remaining acceptance effects, the extracted amplitudes are related to the asymmetry moments $\left\langle\sin \left(n \phi \pm m \phi_{S}\right)\right\rangle_{\mathrm{UT}}$ defined in Section 2.5 .2 by:

$$
A_{\mathrm{UT}}^{\sin \left(n \phi \pm m \phi_{S}\right)}=2\left\langle\sin \left(n \phi \pm m \phi_{S}\right)\right\rangle_{\mathrm{UT}}=2 \frac{\int \mathrm{d} \phi_{S} \mathrm{~d}^{2} \vec{P}_{h \perp} \sin \left(n \phi \pm m \phi_{S}\right)\left(\mathrm{d}^{6} \sigma^{\uparrow}-\mathrm{d}^{6} \sigma^{\downarrow}\right)}{\int \mathrm{d} \phi_{S} \mathrm{~d}^{2} \vec{P}_{h \perp}\left(\mathrm{d}^{6} \sigma^{\uparrow}+\mathrm{d}^{6} \sigma^{\downarrow}\right)}
$$

In addition to the Collins and Sivers moments, $\left\langle\sin \left(\phi+\phi_{S}\right)\right\rangle_{\mathrm{UT}}$ and $\left\langle\sin \left(\phi-\phi_{S}\right)\right\rangle_{\mathrm{UT}}$, two other sine modulations are included in the fit. They are subleading-twist terms of the

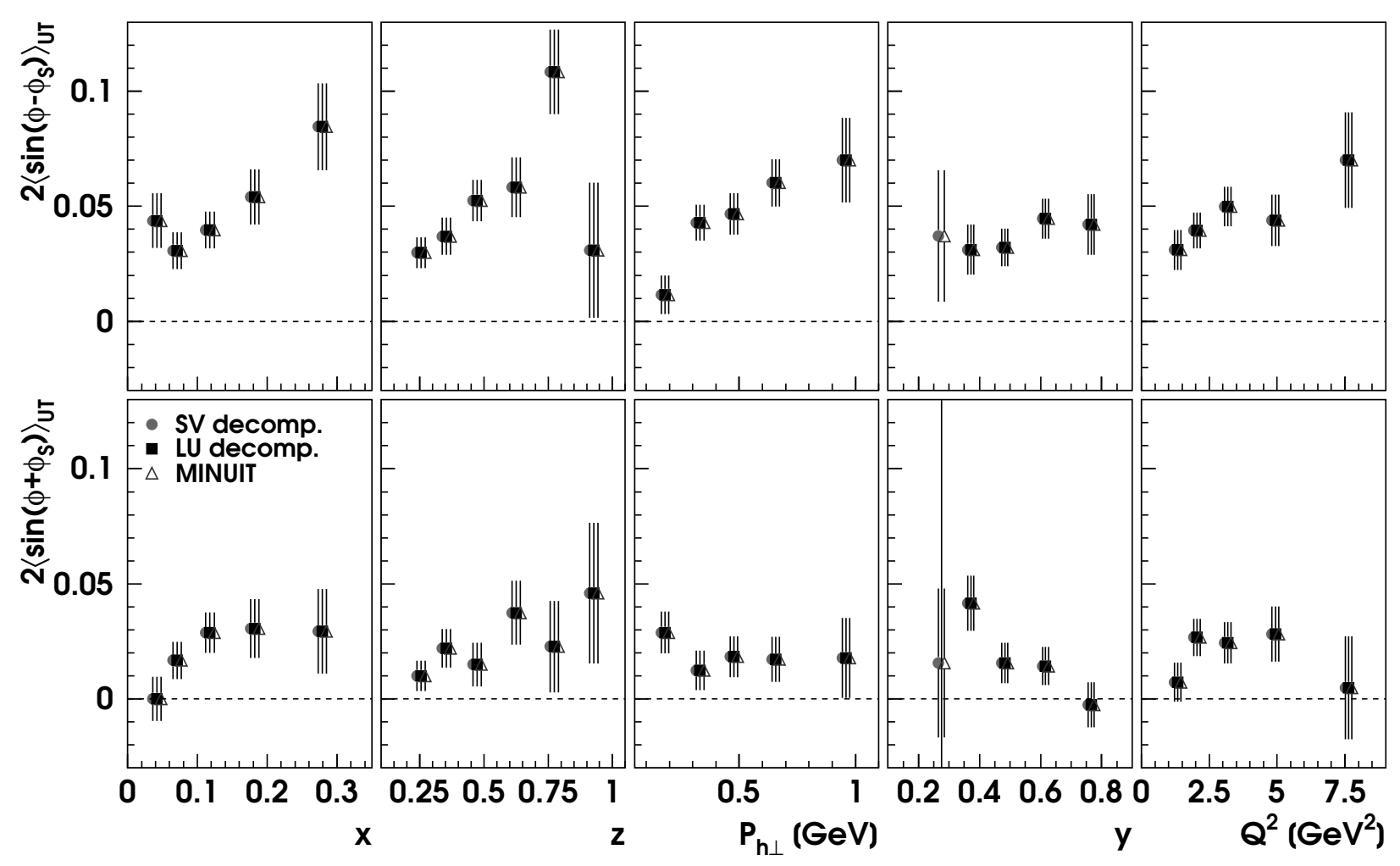

Figure 4.3.2: Comparison of the results of three different fit methods with the function (4.3.2) considering as example the positive pions. 


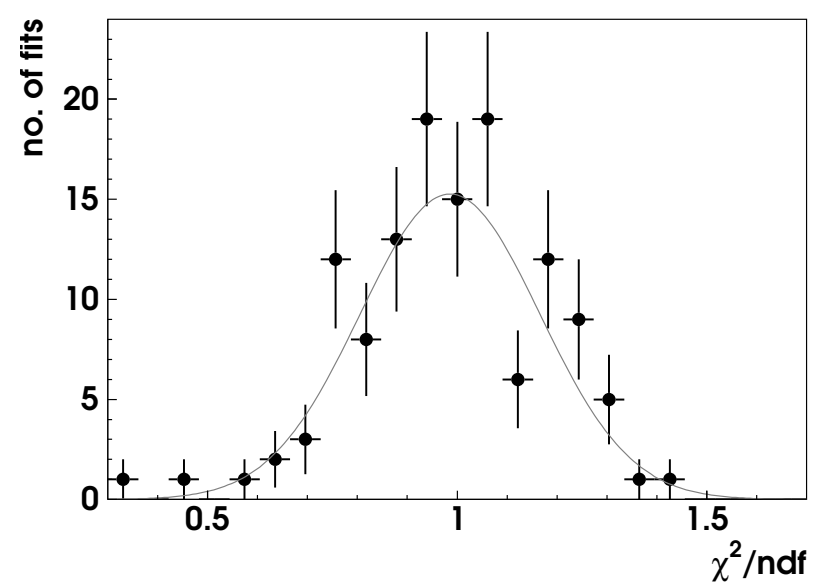

Figure 4.3.3: Distribution of the reduced $\chi^{2}$ of the various two-dimensional fits for the different hadron types and kinematic bins. The grey line shows a Gaussian fit to the distribution.

cross section (cf. Chapter 2) which cannot be omitted because they are significantly non-zero and influence the fit results of the Collins and Sivers moments, as shown later on.

Three different fit routines based on the least-squares method are used for the extraction of the fit parameters. The fit function (4.3.2) is a linear function in the fit parameters. Hence, the minimum of the figure-of-merit function $\chi^{2}$ can be calculated analytically involving decomposition and back substitution of a matrix. Two different decomposition methods, the single value (SV) decomposition and the LU decomposition which is based on a lower and an upper triangular matrix [Pre94], are implemented in the fit routines. As a third method, the program MiNuIT [Jam75] is used which determines the minimum of the figure-of-merit function by scanning the parameter space. Figure 4.3.2 shows the comparison of the extracted asymmetries of positive pions using LU and SV decomposition and the program MINUIT. If the matrices involved in the fit are numerically close to being singular the LU decomposition can fail to give satisfactory results, as can be seen in the first $y$ bin. In the following, the SV decomposition is used if not indicated otherwise.

The goodness of the fits can be estimated by the reduced $\chi^{2}$ which is the minimum of $\chi^{2}$ divided by the number of degrees of freedom (ndf). For a good fit it should be close to 1. The distribution of the reduced $\chi^{2}$ of the various fits used to extract the amplitudes for the different hadron types in each kinematic bin, is plotted in Figure 4.3.3. In addition, a Gaussian fit is shown which yields a mean value close to 1. The correlation between the extracted Collins and Sivers amplitudes varies over the different kinematic bins. On average it is around -0.5 .

In experiments the target is polarised along an axis that is fixed with respect to the lepton beam, while the direction of the virtual photon generally changes in each scattering event. In the case of a transversely polarised target this causes a small longitudinal target spin component with respect to the virtual photon besides the dominant transverse spin component. The influence of the longitudinal target spin component on the measured transverse asymmetry moments is investigated in Chapter 7.

As explained in Section 2.5.2, asymmetry moments that include the transverse mo- 
menta of the produced hadrons in the convolution integral, can be interpreted in terms of distribution and fragmentation functions without assumptions on the distributions of the quark transverse momenta. Besides the so-called unweighted asymmetry moments (4.3.3), the $P_{h \perp}$-weighted asymmetry moments,

$$
\left\langle\frac{P_{h} \perp}{z M_{h}} \sin \left(\phi+\phi_{S}\right)\right\rangle_{\mathrm{UT}} \text { and }\left\langle\frac{P_{h} \perp}{z M} \sin \left(\phi-\phi_{S}\right)\right\rangle_{\mathrm{UT}},
$$

are therefore of interest. For the extraction of the latter, an asymmetry is formed in which the events in the numerator are weighted by $P_{h \perp} / z$ :

$$
A_{\mathrm{UT}}^{P_{h \perp} / z}\left(\phi, \phi_{S}\right)=\frac{1}{\langle P\rangle} \frac{L^{\downarrow} \sum_{i=0}^{N^{\uparrow}\left(\phi, \phi_{S}\right)}\left(P_{h \perp} / z\right)_{i}-L^{\uparrow} \sum_{i=0}^{N^{\downarrow}\left(\phi, \phi_{S}\right)}\left(P_{h \perp} / z\right)_{i}}{N^{\uparrow}\left(\phi, \phi_{S}\right) L^{\downarrow}+N^{\downarrow}\left(\phi, \phi_{S}\right) L^{\uparrow}} .
$$

The calculation of the statistical uncertainty of this asymmetry requires the assumption that the sum over the events $\sum_{i=0}^{N^{\uparrow \downarrow}}$ and the total amount of events $N^{\uparrow \downarrow}$ are uncorrelated. The $P_{h \perp}$-weighted asymmetry amplitudes or moments, respectively, are extracted by a two-dimensional fit using the fit function:

$$
\begin{aligned}
A_{\mathrm{UT}}^{P_{h \perp} / z}\left(\phi, \phi_{S}\right)= & 2 M_{h}\left\langle\frac{P_{h \perp}}{z M_{h}} \sin \left(\phi+\phi_{S}\right)\right\rangle_{\mathrm{UT}} \cdot \sin \left(\phi+\phi_{S}\right)+ \\
& 2 M\left\langle\frac{P_{h \perp}}{z M} \sin \left(\phi-\phi_{S}\right)\right\rangle_{\mathrm{UT}} \cdot \sin \left(\phi-\phi_{S}\right)+ \\
& 2\left\langle\frac{P_{h \perp}}{z} \sin \left(2 \phi-\phi_{S}\right)\right\rangle_{\mathrm{UT}} \cdot \sin \left(2 \phi-\phi_{S}\right)+ \\
& 2\left\langle\frac{P_{h \perp}}{z} \sin \phi_{S}\right\rangle_{\mathrm{UT}} \cdot \sin \phi_{S}+C
\end{aligned}
$$

where the hadron and proton masses assure that the Collins and Sivers $P_{h \perp}$-weighted moments are dimensionless.

\subsection{Systematic Studies}

In this section, systematic studies performed with the HERMES data of the three productions $02 \mathrm{c0}, 03 \mathrm{c0}$, and $04 \mathrm{b0}$ are presented. While only example plots of unweighted asymmetry amplitudes for certain hadron types are shown here, the studies have been performed on unweighted and $P_{h \perp}$-weighted asymmetry amplitudes and all hadron types and the conclusions presented in this chapter are valid throughout.

\subsubsection{Event Number Distributions in the Azimuthal Bins}

The uncertainty of the asymmetries in the azimuthal bins is calculated under the assumption of the validity of Poissonian statistics which implies an uncertainty $\sqrt{N}$ on a number of counts $N$. To ensure a reliable $\chi^{2}$ minimisation, the number of entries in each azimuthal bin is required to exceed 20 . The additional requirement in the analysis results in a decrease of the number of azimuthal bins taken into account, especially in kinematic bins with low count rates. Therefore, the largest deviations of the asymmetry amplitudes appear in the kinematic bins with the largest statistical errors, as can be seen for the amplitudes of 


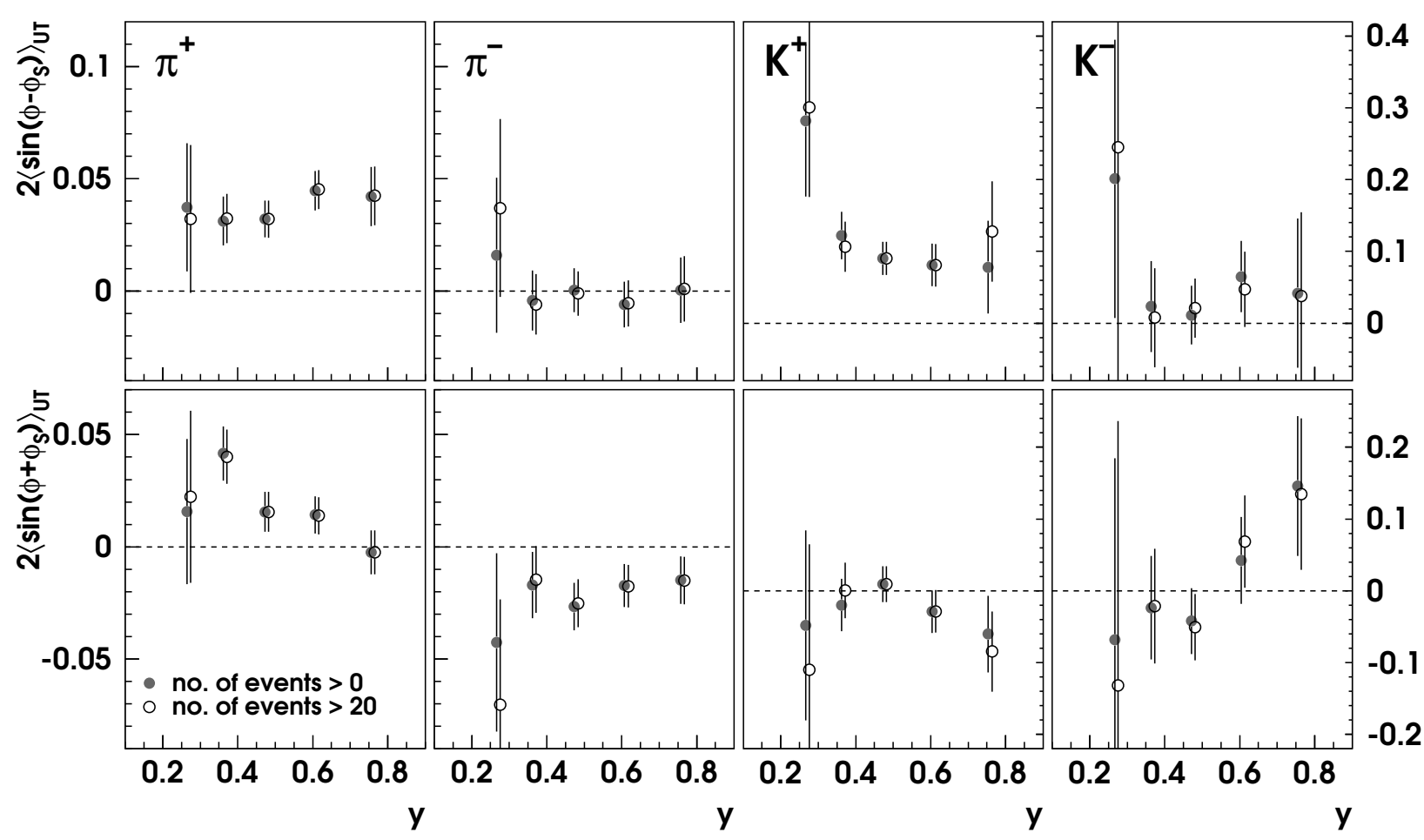

Figure 4.4.1: Comparison of the asymmetry amplitudes of charged hadrons dependent on $y$. In general, the event numbers of the two target spin states in each azimuthal bin are required to be larger than zero. For the results plotted with the open symbols, also the sum of the event numbers in both spin states must be larger than 20.

the charged hadrons in bins of $y$, as plotted in Figure 4.4.1. While this approach results in a slight underestimate of the statistical uncertainty, the requirement $N>20$ has not been applied in the following as no systematic bias on the amplitudes is observed and the analysis is based on a larger event sample without the cut. In general, an extraction of the asymmetry amplitudes with an unbinned maximum likelihood fit is not affected by low count numbers. However, such a fit is not yet implemented.

\subsubsection{Compatibility of Data Taking Periods}

The data analysed in this chapter were recorded during a period that extends over three years. Within this long period changes of the HERMES spectrometer, e.g., in the alignment or the efficiencies of the detectors, may occur which can influence the extracted asymmetry amplitudes. As a check results from two shorter distinct periods are compared.

The 02 production does not only contain data of the year 2002 but also data that were taken during the first month of the year 2003 before a long shutdown of the HERA accelerator. The 03 production covers only the data taking period of the last four month of the year 2003 and hence has a lower statistics than the 02 and 04 productions. The break between the short data taking period at the end of 2003 and the long period in 2004 lasted only a couple of days and no major maintenance work was carried out on the spectrometer components during this period. This legitimates the combination of 

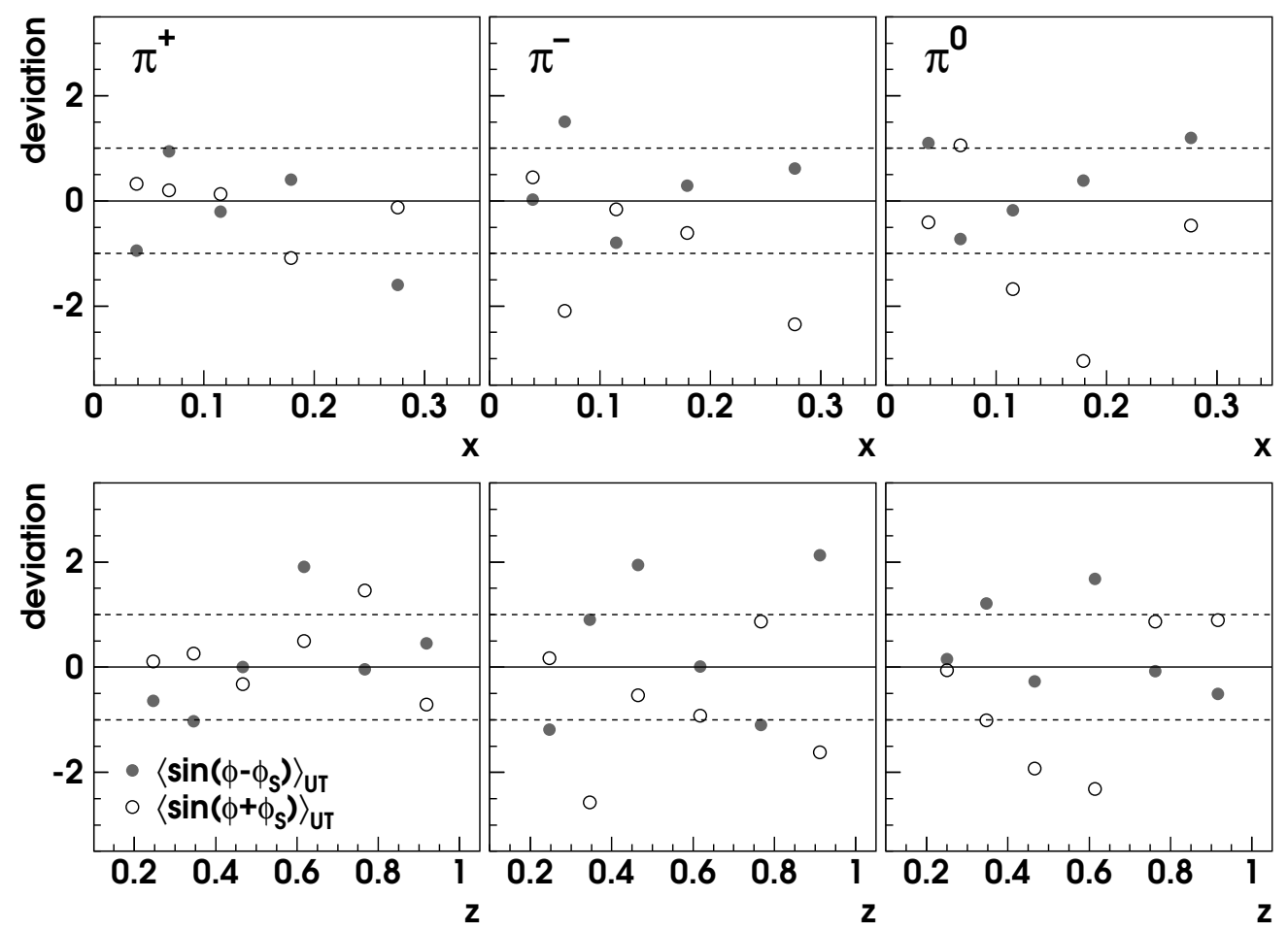

Figure 4.4.2: Deviations as defined in Eq. (4.4.1) of the asymmetry amplitudes between the independent data productions $02 \mathrm{cO}$ and $03 \mathrm{cO} \mathrm{com}$ bined with $04 \mathrm{bO}$. The neutral pions are not corrected for combinatorial background.

the $03 \mathrm{c0}$ and the $04 \mathrm{b0}$ productions for a comparison to the $02 \mathrm{c0}$ production. The results of the $02 \mathrm{co}$ production were presented in the first publication of single-spin asymmetry amplitudes measured on a transversely polarised target with the HERMES experiment [Air05b]. To allow the identification of systematic discrepancies, deviations between the two data taking periods are computed as:

$$
\text { deviation }=\frac{A_{\mathrm{UT}}^{\sin \left(\phi \pm \phi_{S}\right)}(02 \mathrm{c} 0)-A_{\mathrm{UT}}^{\sin \left(\phi \pm \phi_{S}\right)}(03 \mathrm{c} 0,04 \mathrm{~b} 0)}{\sqrt{\sigma^{2}(02 \mathrm{c} 0)+\sigma^{2}(03 \mathrm{c} 0,04 \mathrm{~b} 0)}} .
$$

For the kinematic variables $x$ and $z$ the deviations of the pion Collins and Sivers moments are plotted in Figure 4.4.2. They fluctuate around zero and do not show any systematic trend. The reduced $\chi^{2}$ (which is the sum of the squared deviations) for the amplitudes of the three pion types in all $z$ bins, i.e., three times six amplitudes, is 1.20 (1.43) in case of the Sivers (Collins) amplitudes. The 15 amplitudes in the different $x$ bins result in a reduced $\chi^{2}$ of 0.75 for the Sivers amplitudes and 1.69 for the Collins amplitudes. Consequently, these two different data taking periods are consistent with each other.

\subsubsection{Luminosity Normalisation}

The integrated luminosities $L^{\uparrow / \downarrow}$ appear in the definition of the azimuthal asymmetry (4.3. 1) for the normalisation of the count numbers in the opposite target spin states. They can be determined by means of the luminosity monitor measurement which is based on a 


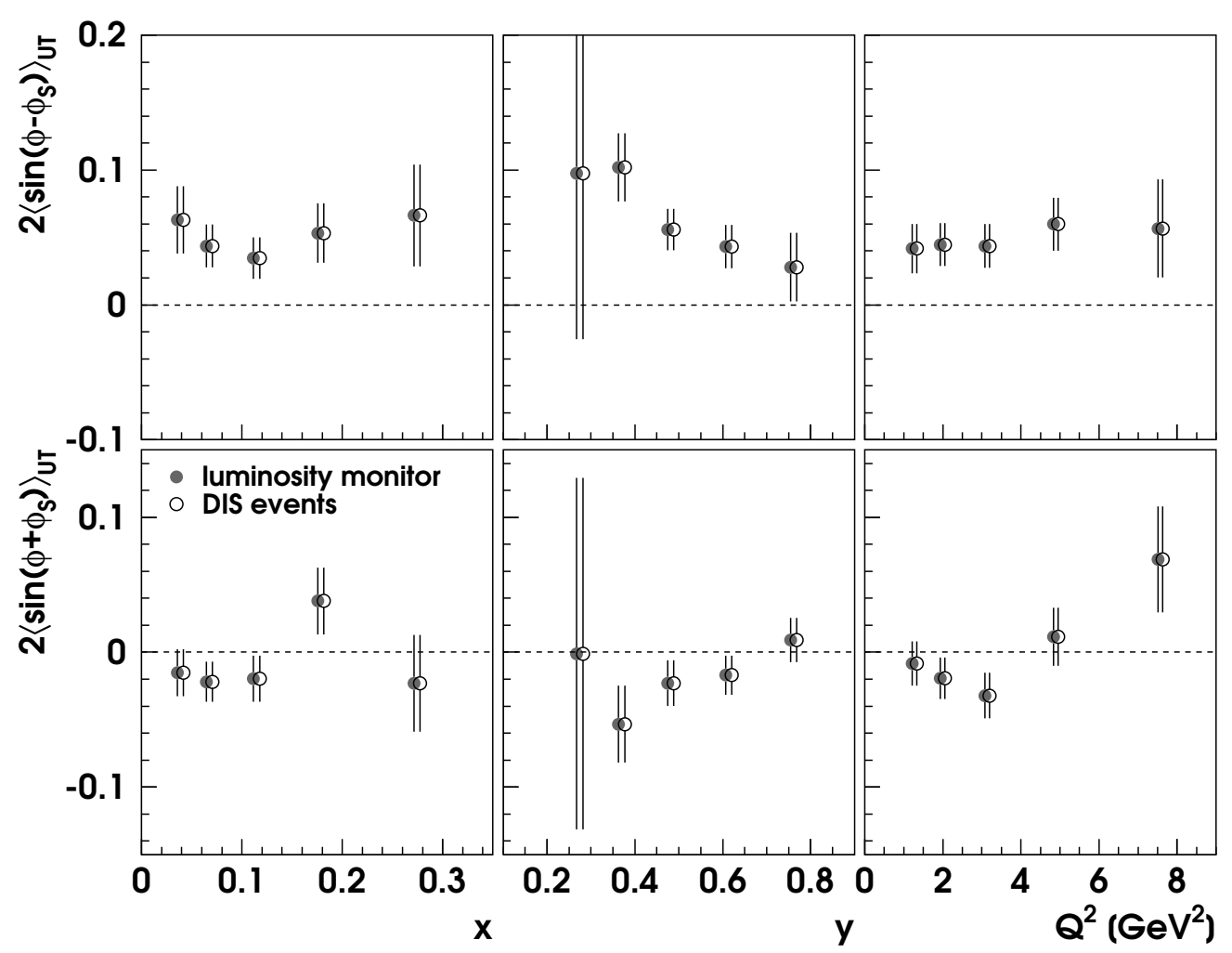

Figure 4.4.3: Comparison of the asymmetry amplitudes computed for two different luminosity measurements (see text). Shown are the amplitudes extracted for neutral pions without combinatorial background correction.

reference scattering process, as described in Section 3.3.3. Before integration, the rate of the reference scattering measured by the luminosity monitor must be corrected for the trigger efficiency of the data acquisition system $1-\delta_{\text {dead }}$ (see Section 3.3.4). The integration is performed by adding up the measured luminosity monitor rates in the different bursts multiplied by the correspondent burst length.

Instead of the scattering of beam leptons off shell electrons, also the inclusive DIS process can be used as reference scattering for the measurement of the luminosity'. Since this measurement is performed with the HERMES spectrometer its trigger efficiency is already accounted for. Both the number of inclusive DIS events as well as the integrated rate of the luminosity monitor are only proportional to the absolute luminosities. However, in the calculation of the azimuthal asymmetry, these proportionality factors drop out. In case of the normalisation with the inclusive DIS rate, the dependence on the kinematic variable $x$ is taken into account by using the binning defined in Table 4.3.1. The comparison of the extracted asymmetry amplitudes for luminosity normalisation with the luminosity monitor rate multiplied by the burst length and the inclusive DIS count number shows no difference, as can be seen, e.g., for neutral pions in Figure 4.4.3. In the following the semi-inclusive count rates are normalised with the integrated rates of the luminosity monitor.

'The influence of the inclusive polarised cross section (2.1.11) which does not vanish in case of unbalanced beam helicity states, is neglected since the averaged beam polarisation is approximately zero. 


\subsubsection{Additional Sine Moments and Unpolarised Cosine Moments}

To suppress the influence of additional sine modulations in the cross section on the extracted Collins and Sivers moments, these modulations can be included in the fit function. In addition to the Collins and Sivers moments, a $\left\langle\sin \left(3 \phi-\phi_{S}\right)\right\rangle_{\mathrm{UT}}$ moment exists at leading twist and two subleading-twist moments exhibit $\sin \phi_{S}$ and $\sin \left(2 \phi-\phi_{S}\right)$ modulations in the cross section, respectively (cf. Table 2.5.1). Although no net longitudinal target spin component with respect to the virtual photon exists, components of the cross section for longitudinally polarised nucleons are considered. These are the twist-two $\langle\sin 2 \phi\rangle_{\mathrm{UL}}$ and the twist-three $\langle\sin \phi\rangle_{\mathrm{UL}}$ moments.

The asymmetries in the azimuthal bins are fitted by a three parameter fit function including only the Collins and Sivers amplitudes and a constant as fit parameters. Compared to the results from this fit are amplitudes extracted with a five and six parameter fit function. On the left-hand side of Figure 4.4.4, the sine modulations $\sin \left(3 \phi-\phi_{S}\right)$, $\sin \phi$, and $\sin 2 \phi$ are accounted for by three additional fit parameters. All three amplitudes are consistent with zero and they do not alter the fit results for the azimuthal modulations of the Collins and Sivers effect. The two subleading-twist moments $\left\langle\sin \left(2 \phi-\phi_{S}\right)\right\rangle_{\mathrm{UT}}$ and $\left\langle\sin \phi_{S}\right\rangle_{\mathrm{UT}}$ are included in the five parameter fit. Here, a change in the results for the Collins and Sivers moments is visible, especially for the negative pions where the $\sin \phi_{S}$ amplitude is of the order of -0.05 . Therefore, the standard fit comprises five fit parameters, as already introduced in the previous section.

Not only the polarised cross sections contain azimuthal modulations but also the unpolarised cross section. The $\langle\cos 2 \phi\rangle_{\mathrm{UU}}$ moment in leading twist and the $\langle\cos \phi\rangle_{\mathrm{UU}}$ moment in subleading twist appear in the denominator of the azimuthal asymmetry (4.3.1). A
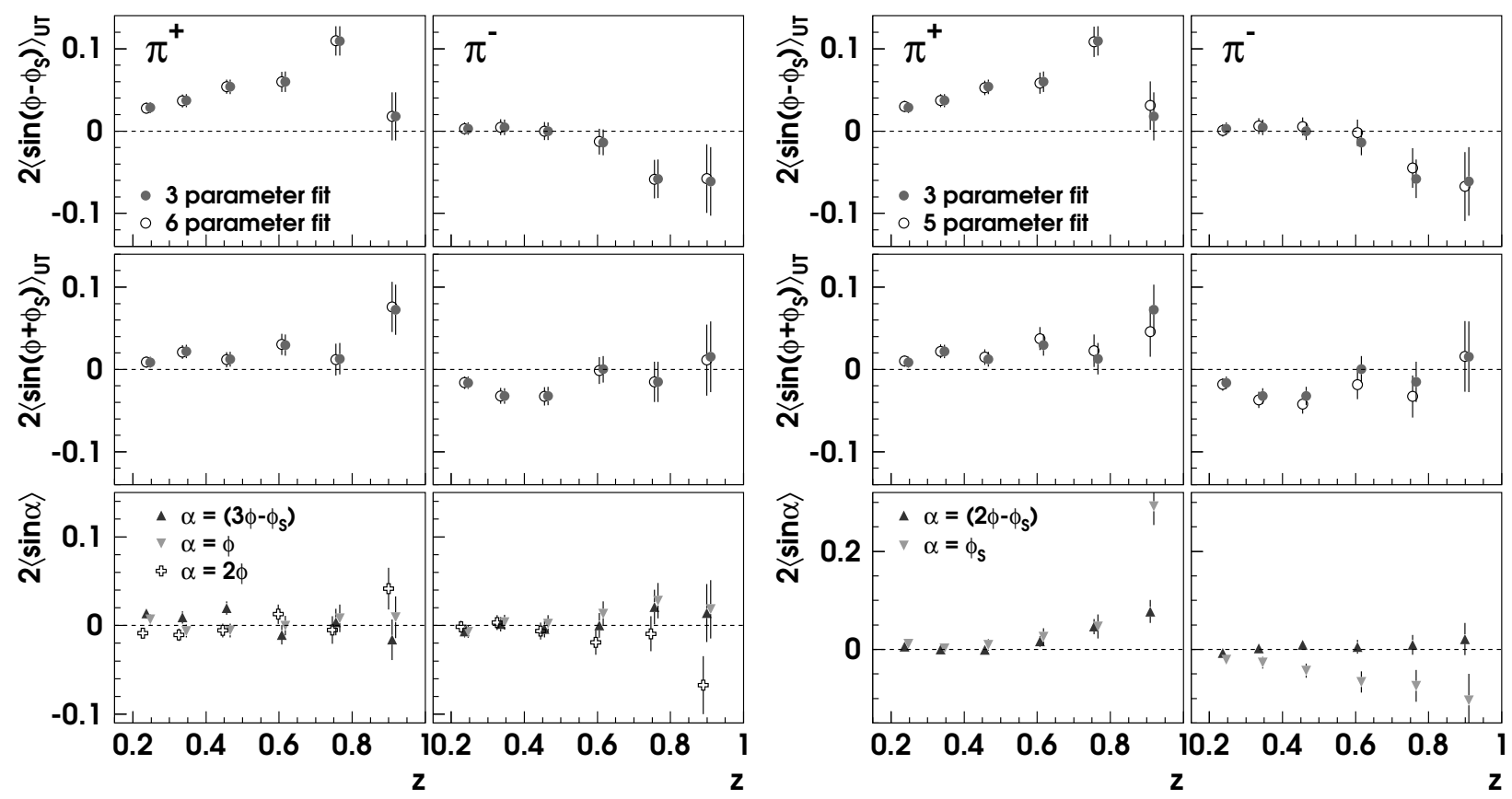

Figure 4.4.4: Additional sine amplitudes extracted by five (right) and six (left) parameter fits. The results for the Collins and Sivers amplitudes are compared to an extraction with a three parameter fit. 
Taylor expansion of the denominator yields for the azimuthal asymmetry:

$$
\begin{aligned}
A_{\mathrm{UT}} \approx & \left(2\left\langle\sin \left(\phi \pm \phi_{S}\right)\right\rangle_{\mathrm{UT}} \sin \left(\phi \pm \phi_{S}\right)+2\left\langle\sin \phi_{S}\right\rangle_{\mathrm{UT}} \sin \phi_{S}+\ldots\right) \times \\
& \left(1-2\langle\cos \phi\rangle_{\mathrm{UU}} \cdot \cos \phi-2\langle\cos 2 \phi\rangle_{\mathrm{UU}} \cdot \cos 2 \phi+\ldots\right) .
\end{aligned}
$$

Since $\sin \phi_{S} \cdot \cos \phi=\frac{1}{2}\left[\sin \left(\phi+\phi_{S}\right)+\sin \left(\phi-\phi_{S}\right)\right]$, the coupling of the unpolarised cosine moment $\langle\cos \phi\rangle_{\mathrm{UU}}$ and the twist-three cross-section term proportional to $\sin \phi_{S}$ contributes to the extracted amplitudes $A_{\mathrm{UT}}^{\sin \left(\phi \pm \phi_{S}\right)}$. Thus, the determination of the unpolarised cosine moments is necessary for the estimate of their contribution to the systematic uncertainty.

A nonlinear fit to the azimuthal asymmetries including the unpolarised cosine moments in the denominator as fit parameters:

$$
A_{\mathrm{UT}}\left(\phi, \phi_{S}\right)=\frac{A_{\mathrm{UT}}^{\sin \left(\phi+\phi_{S}\right)} \cdot \sin \left(\phi+\phi_{S}\right)+A_{\mathrm{UT}}^{\sin \left(\phi-\phi_{S}\right)} \cdot \sin \left(\phi-\phi_{S}\right)+\ldots}{1+A_{\mathrm{UU}}^{\cos \phi} \cdot \cos \phi+A_{\mathrm{UU}}^{\cos 2 \phi} \cdot \cos 2 \phi},
$$

cannot be performed using the LU or SV decomposition but only by the program MINUIT. Unfortunately, the nonlinear fit produces unstable results dependent on the start values of the parameters. Fixed values of the two parameters $A_{\mathrm{UU}}^{\cos \phi}$ and $A_{\mathrm{UU}}^{\cos 2 \phi}$ are necessary in order to allow a reliable linear fit. The HERMEs data summed over both target spin states together with an unpolarised Monte Carlo simulation of the HERMES spectrometer can be used to estimate the cosine moments. The measured cross section of the spin averaged data sample,

$$
\begin{aligned}
& \sigma_{\text {meas }}(\phi, x, y, z)= \\
& \quad \sigma_{0}(x, y, z)\left(1+2\langle\cos \phi\rangle_{\mathrm{UU}} \cdot \cos \phi+2\langle\cos 2 \phi\rangle_{\mathrm{UU}} \cdot \cos 2 \phi\right) \epsilon_{\mathrm{acc}}(\phi, x, y, z) \epsilon_{\mathrm{rad}}(\phi, x, y, z),
\end{aligned}
$$

is influenced by the acceptance and radiative effects taken into account by the acceptance function $\epsilon_{\mathrm{acc}}$ and the radiative correction function $\epsilon_{\mathrm{rad}}$. Here, the integrated unpolarised cross section is $\sigma_{0}(x, y, z)=\int \mathrm{d} \phi_{S} \mathrm{~d}^{2} \vec{P}_{h \perp} \mathrm{d}^{6} \sigma_{\mathrm{UU}}$ with $\mathrm{d}^{6} \sigma_{\mathrm{UU}}$ taken from Eq. (2.5.15). The azimuthal dependence of the acceptance function may be expanded in a Fourier series $\epsilon_{\text {acc }}=a_{0}+\sum_{n=1}^{\infty} a_{n} \cos (n \phi)+\sum_{k=1}^{\infty} b_{k} \sin (k \phi)$. The radiative effects change the total measured cross section because elastic events are smeared into and out of the inelastic region. In addition, they also exhibit cosine modulations because in the peaking approximation the real photon is radiated collinear to the lepton momentum and the virtualphoton momentum is changed within the scattering plane. This effect is symmetric in the azimuthal angle $\phi$ about the incident lepton beam and hence the radiative correction function can be expressed as: $\epsilon_{\mathrm{rad}}=r_{0}+\sum_{m=1}^{\infty} r_{m} \cos (m \phi)$. From the measured unpolarised cross sections or hadron yields, respectively, cosine amplitudes can be extracted by a combination of the obtained parameters of a fit with the function $p_{0}+\sum_{j=1}^{4} p_{j} \cos (j \phi)$ :

$$
2\langle\cos \phi\rangle_{\text {meas }}=\frac{p_{1}}{p_{0}}, \quad 2\langle\cos 2 \phi\rangle_{\text {meas }}=\frac{p_{2}}{p_{0}} .
$$

They contain the following components of the measured cross section (4.4.4):

$$
2\langle\cos \phi\rangle_{\text {meas }}=2\langle\cos \phi\rangle_{\mathrm{UU}}+\frac{a_{1}}{a_{0}}+\frac{r_{1}}{r_{0}}, \quad 2\langle\cos 2 \phi\rangle_{\text {meas }}=2\langle\cos 2 \phi\rangle_{\mathrm{UU}}+\frac{a_{2}}{a_{0}}+\frac{r_{2}}{r_{0}} .
$$




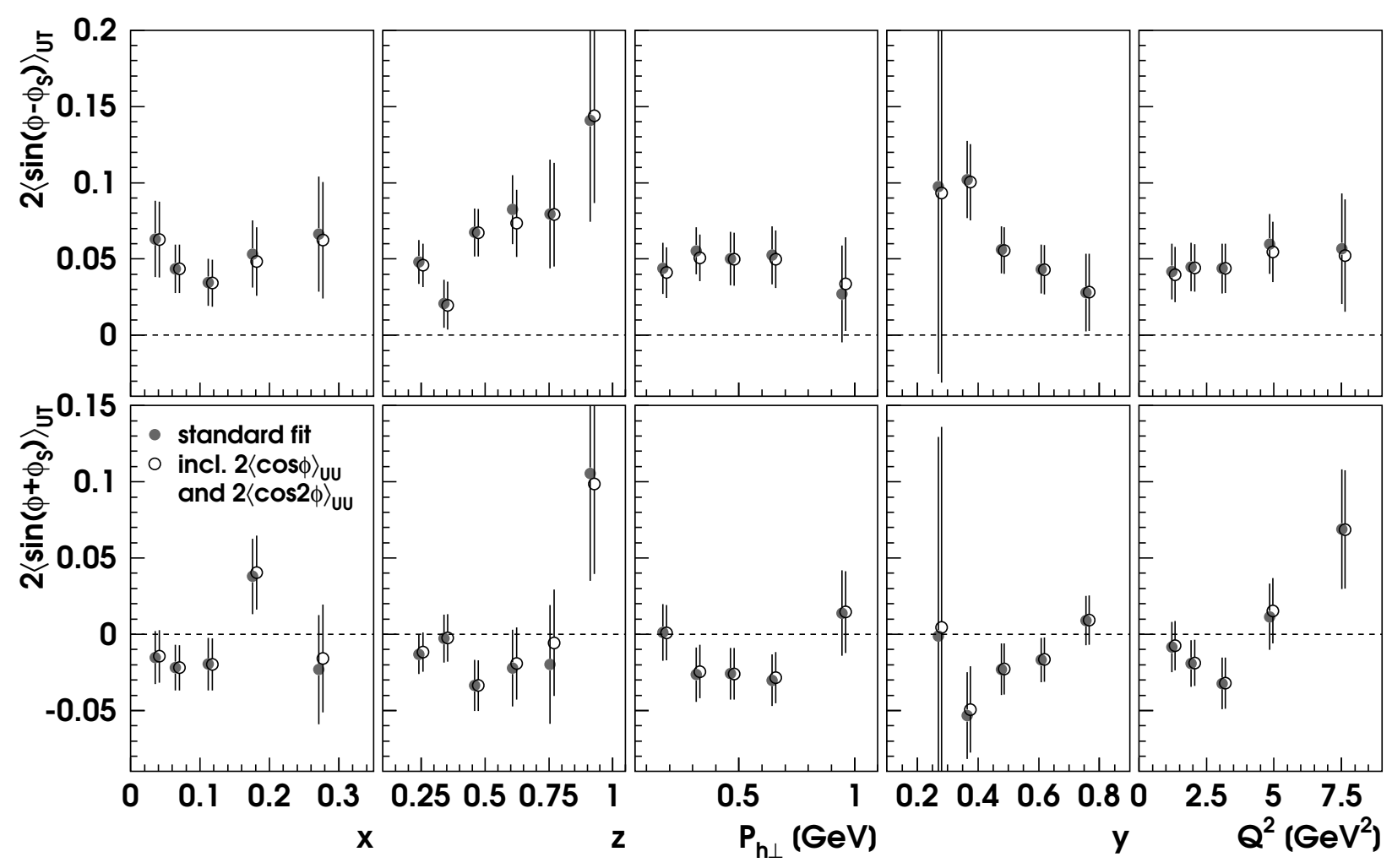

Figure 4.4.5: Comparison of the extracted asymmetry amplitudes for $\pi^{0}$ using the standard fit function (4.3.2) and an extended fit function with the unpolarised cosine moments included in the denominator.

The choice of the number of orders of cosine modulations included in the fit does influence the goodness of the fit but not the results of the two extracted cosine amplitudes.

The unpolarised cosine moments, $\langle\cos \phi\rangle_{\mathrm{UU}}$ and $\langle\cos 2 \phi\rangle_{\mathrm{UU}}$, are not implemented in a PYTHIA Monte Carlo simulation including exclusively produced vector mesons and the full simulation of the HERMES spectrometer (see Section 5.1). Therefore, extracted cosine amplitudes from reconstructed PYTHIA Monte Carlo events contain only acceptance and radiative effects:

$$
2\langle\cos \phi\rangle_{\mathrm{MC}}=\frac{a_{1}}{a_{0}}+\frac{r_{1}}{r_{0}}, \quad 2\langle\cos 2 \phi\rangle_{\mathrm{MC}}=\frac{a_{2}}{a_{0}}+\frac{r_{2}}{r_{0}} .
$$

If acceptance and radiative effects are well described by the Monte Carlo simulation the extracted moments can be subtracted from the HERMES data results in order to estimate the unpolarised cosine moments. The difference of cosine moments extracted from HeRMES data and the PYTHIA Monte Carlo simulation is shown in Figures E.2 and E.3 for the three pion types. The obtained unpolarised cosine moments are used to fix the additional fit parameters of the fit function (4.4.3): $A_{\mathrm{UU}}^{\cos \phi}=2\langle\cos \phi\rangle_{\text {meas }}-2\langle\cos \phi\rangle_{\mathrm{MC}}$ and $A_{\mathrm{UU}}^{\cos 2 \phi}=2\langle\cos 2 \phi\rangle_{\text {meas }}-2\langle\cos 2 \phi\rangle_{\mathrm{MC}}$. The changes to the extracted Sivers and Collins moments give an estimate of the systematic uncertainty due to the unpolarised cosine moments. This estimate is included in the systematic uncertainties of the extracted amplitudes. The results of both fits are compared in Figure 4.4.5, as an example for neutral pions uncorrected for combinatorial background. 


\subsubsection{Beam Polarisation}

In contrast to the year 2002 when the beam polarisation was very small, the HERA lepton beam was longitudinally polarised in the years 2003 and 2004 with an average polarisation of around $30.8 \%$ for helicity $\lambda=+1$, i.e., spin orientation parallel to the beam momentum, and $-39.8 \%$ for helicity $\lambda=-1$. The existence of a polarised beam gives rise to additional cross-section terms, e.g., in leading twist a $\cos \left(\phi-\phi_{S}\right)$ modulation (see Table 2.5.1). The asymmetry amplitudes $A_{\mathrm{LT}}^{\cos \left(\phi-\phi_{S}\right)}$ of the two helicities have opposite signs leaving a non-zero overall amplitude if the polarisations of the two beam spin states differ.

In order to study the influence of the beam polarisation on the extraction of Collins and Sivers moments, the productions $03 \mathrm{c0}$ and $04 \mathrm{b0}$ are split into two independent data sets with positive and negative helicity. As for the comparison of the two data taking periods, the deviations between the extracted asymmetry amplitudes for positive and negative beam helicity,

$$
\text { deviation }=\frac{A_{\mathrm{UT}}^{\sin \left(\phi \pm \phi_{S}\right)}(\lambda=+1)-A_{\mathrm{UT}}^{\sin \left(\phi \pm \phi_{S}\right)}(\lambda=-1)}{\sqrt{\sigma^{2}(\lambda=+1)+\sigma^{2}(\lambda=-1)}},
$$

are calculated for the different kinematic bins. No systematic deviations are visible, as shown for the $x$ and $z$ bins in Figure 4.4.6. The evaluation of the reduced $\chi^{2}$ for all pion types and $z(x)$ bins yields 0.69 (1.20) for the Sivers amplitudes and $1.16(0.90)$ for the Collins
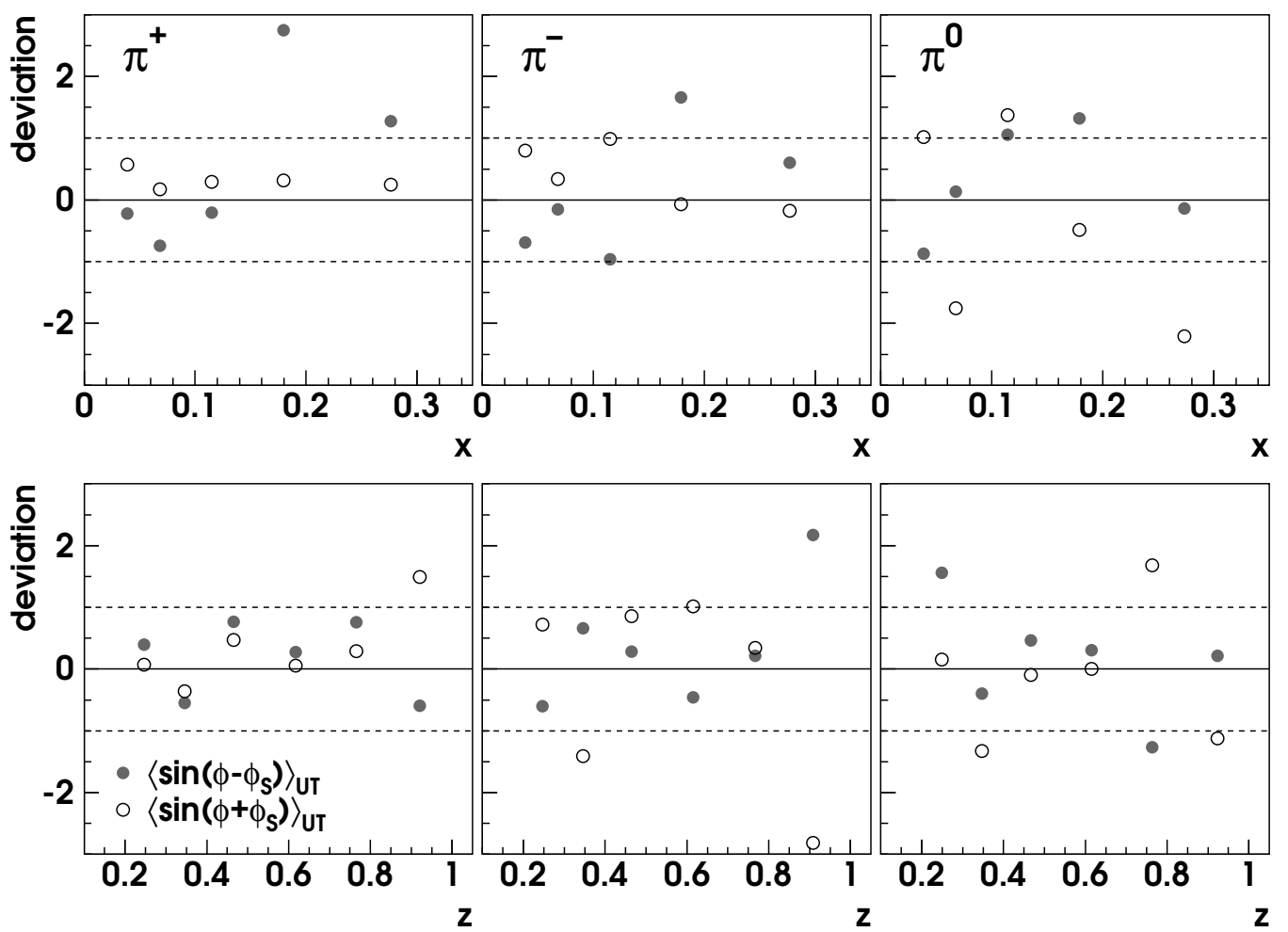

Figure 4.4.6: Deviations (4.4.8) of the asymmetry amplitudes for the independent data sets with positive and negative beam helicity. Plotted is the dependence on the kinematic variables $x$ and $z$ for all pion types. The neutral pions are not corrected for combinatorial background. 
amplitudes, respectively. Therefore, no effort is made to balance the helicity of the two data samples. Furthermore, the beam helicity in the total data sample is already almost balanced since the combination of all three productions yields a luminosity averaged beam polarisation of only $0.2 \%$.

\subsubsection{Hadron Classification with the RICH}

In order to clarify the importance of the application of the inverse $\mathcal{P}$-matrix especially for the kaon identification by the $\mathrm{RICH}$ detector, asymmetry amplitudes are compared which are obtained from true count numbers (4.2.11) and count numbers of identified hadrons, i.e., of the most likely hadron type. Pions are identified by the RICH detector with a large efficiency leading to a small difference in the true count number of pions and the count number of identified pions. This difference is larger for kaons due to an efficiency drop to values below $80 \%$ in some momentum bins. In contrast to the pion asymmetry amplitudes, the kaon amplitudes therefore vary when the inverse $\mathcal{P}$-matrix elements are assigned as event weights instead of weighting the most likely hadron type in each event with weight 1 and the other hadron types with weight 0. In Figure 4.4.7 the results of both methods are compared in the different $x$ bins. The amplitudes for pions on the left-hand side are almost identical whereas the lower true kaon count numbers result in fluctuations and larger statistical uncertainties in the kaon amplitudes on the right side. For the negative kaons, the lower true kaon count numbers do not allow the amplitude

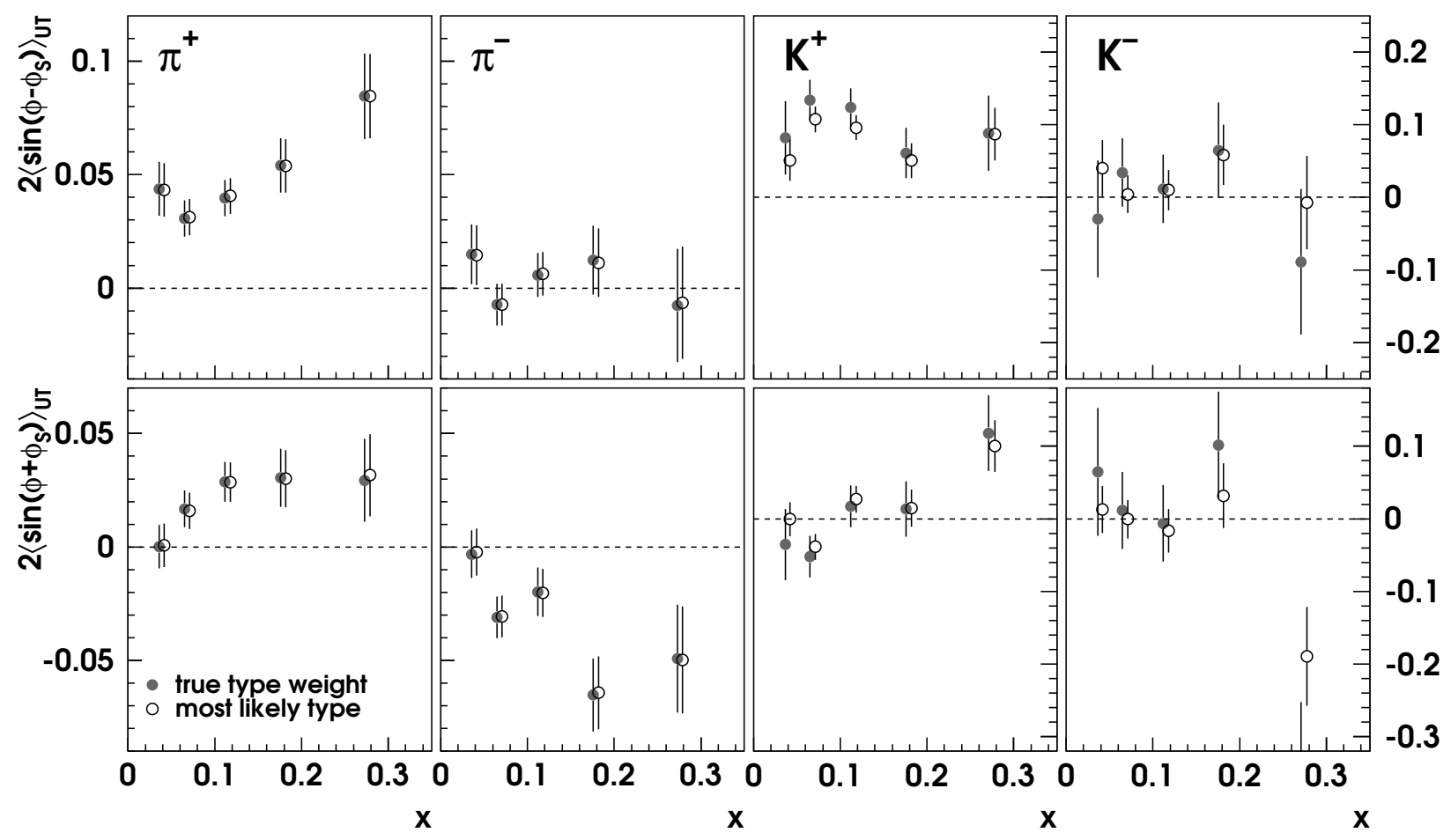

Figure 4.4.7: Comparison of the asymmetry amplitudes of charged hadrons dependent on $x$. The events are either weighted by the inverse $\mathcal{P}$-matrix elements in order to obtain the true hadron count numbers or the most likely hadron type is assigned to the particle. 


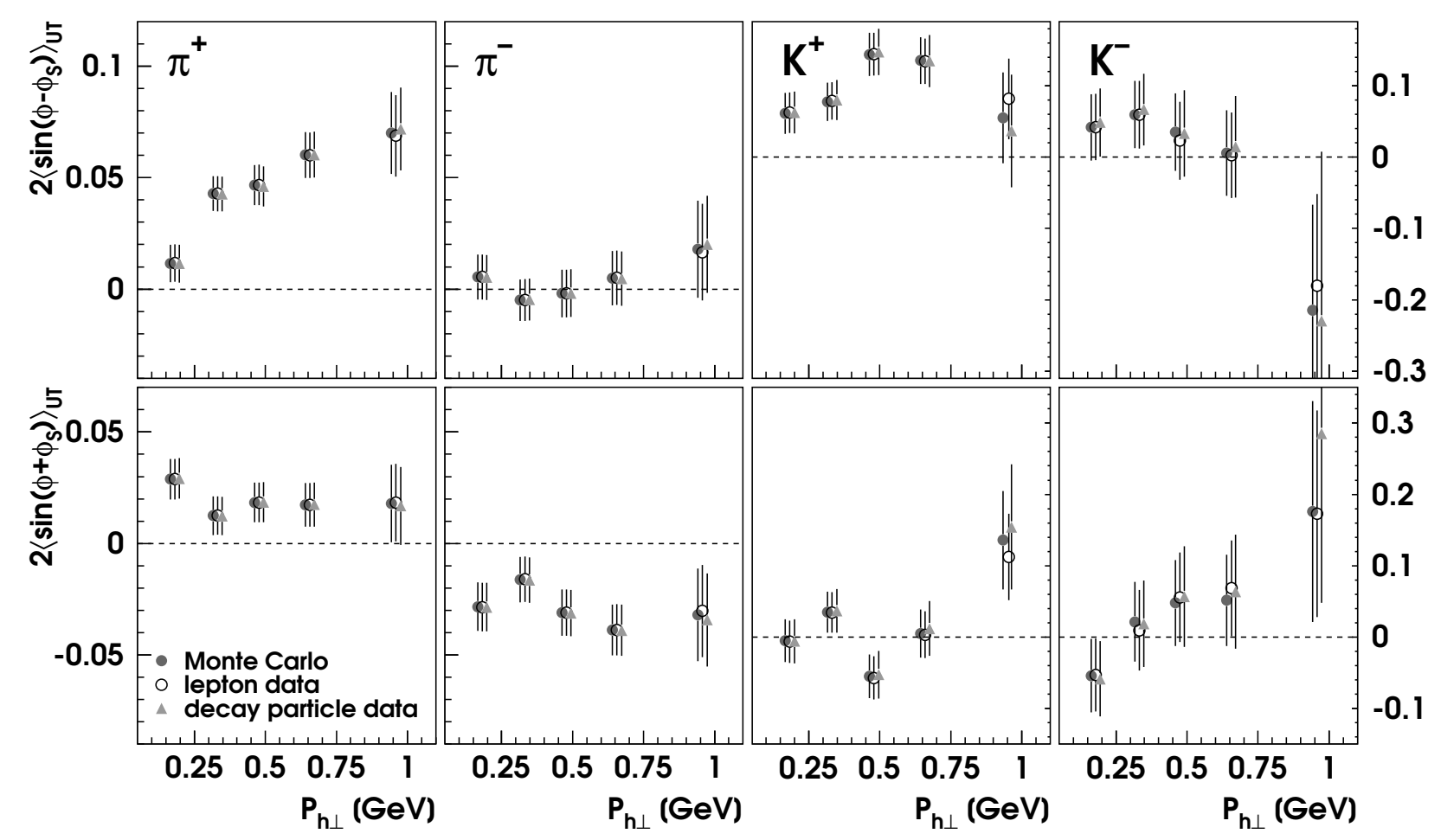

Figure 4.4.8: Comparison of the asymmetry amplitudes of charged hadrons dependent on $P_{h \perp}$. The events are weighted by the elements of three different inverse $\mathcal{P}$-matrices extracted from a Monte Carlo simulation and from HeRMEs lepton and decay particle data.

extraction in the last two $z$ bins.

For the estimate of the systematic uncertainty in the true hadron numbers, two additional $\mathcal{P}$-matrices [Hom04] are used for the amplitude extraction. One of the matrices is purely derived from the analysis of HERMES data. Only decaying particles like for instance $\rho^{0}$ and $\phi$ mesons, are considered in the analysis. By constraining the invariant mass of the decay particles to the mass of the parent particle, they can be identified, e.g., as charged pions for the $\rho^{0} \rightarrow \pi^{+} \pi^{-}$decay. The disadvantage of this procedure is the restricted topology of the decay events. For the second matrix, the simulation of the RICH detector implemented in the Monte Carlo simulation of the HERMES spectrometer (HMC, cf. Section 5.1) is tuned to responses from leptons in the HERMES data which can be identified by the other PID detectors. In Figure 4.4.8 the extracted asymmetry amplitudes for all three $\mathcal{P}$-matrices are compared to each other. Deviations are found especially in the kinematic bins with low statistics. The maximum difference between results using the Monte Carlo $\mathcal{P}$-matrix and the $\mathcal{P}$-matrices obtained from lepton or decay particle data, is assigned as systematic uncertainty in each kinematic bin.

\subsubsection{Target Magnet Field Correction}

The measurement of the azimuthal target-spin asymmetry (4.3.1) requires a transversely polarised target. The transverse holding field which provides the polarisation axis for the nuclei (see Section 3.2), causes a bending of the trajectories of the scattered positron 


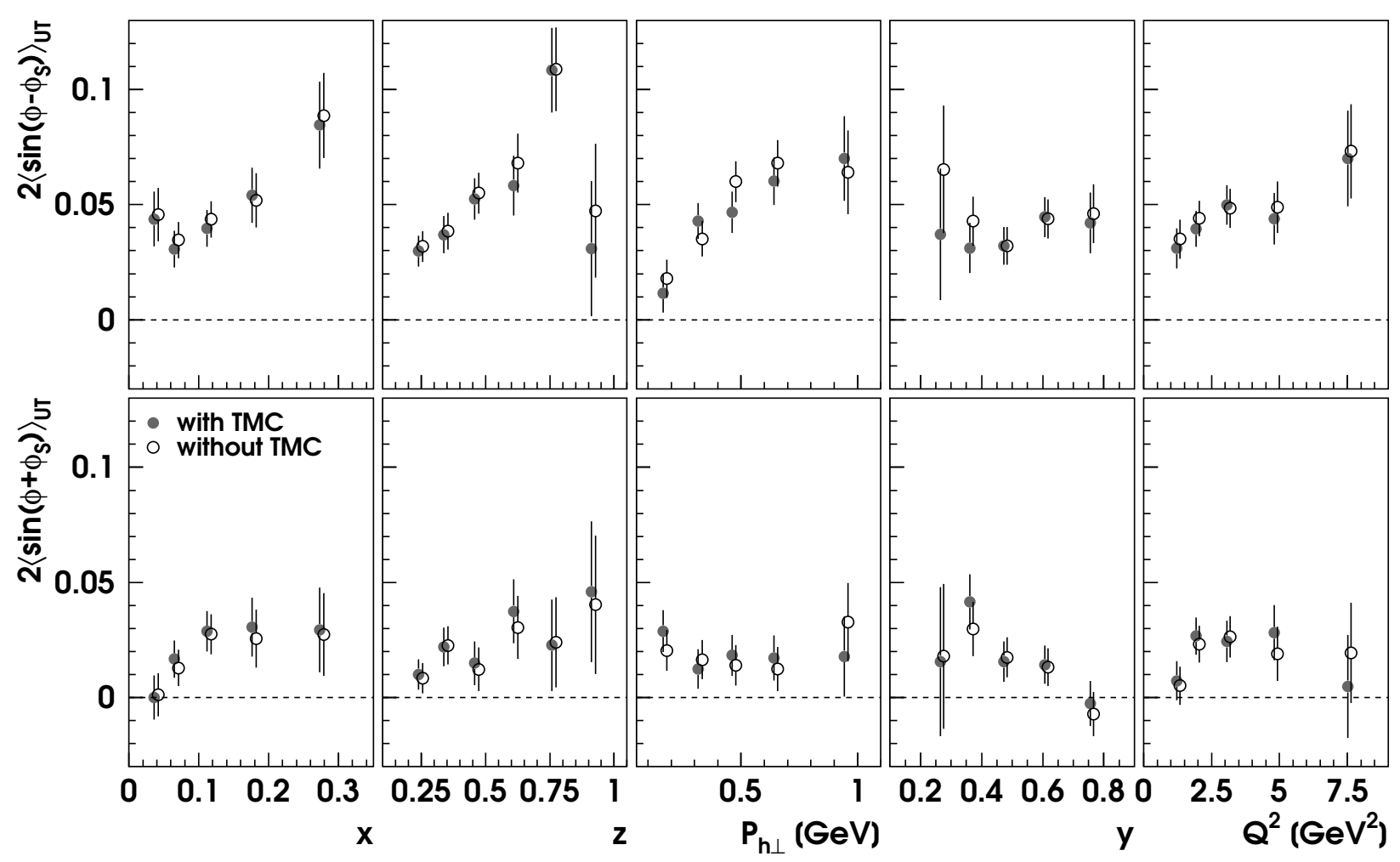

Figure 4.4.9: Comparison of the asymmetry amplitudes for $\pi^{+}$with and without tracking corrections for the transverse target field.

and the produced hadrons due to the Lorentz force. The reconstructed partial particle tracks in front of the spectrometer magnet do not yield the correct vertex positions and scattering angles when they are extrapolated into the target cell by a straight line. As described in Section 3.3.1, two different TMC methods are used for the correction of the obtained positions and angles which regain the reconstruction resolution without transverse magnet field almost completely. Even though the correction of the vertex positions and scattering angles is possible and always applied, the influence of uncorrected particle tracks on the extracted asymmetry amplitudes is studied. The amplitudes with and without tracking corrections are compared, e.g., for positive pions in Figure 4.4.9. Although difference up to $1 / \sqrt{2}$ of the statistical uncertainties are visible, the conclusion of the extracted amplitudes remains unchanged. Thus, the correction of the influence of the magnetic field is necessary but has not a strong impact on the extracted asymmetry amplitudes. No additional systematic uncertainty on the amplitudes has been applied.

\subsubsection{Misalignment}

A measurement of azimuthal quantities can also be biased by a misaligned spectrometer leading to a misreconstruction of the particle tracks, dependent on the traversed spectrometer half. In an elaborate analysis of the HERMES data, the tilt and offset of each spectrometer half was estimated [Brü03]. The analysis shows a change in the obtained values after the long shutdown in 2001 in which the detector was moved out of the HERA beam line orbit and major maintenance work was carried out. All values for top and bottom spectrometer halves are listed in Table 4.4.1. The slopes and offsets listed need 


\begin{tabular}{|l|c|c|c|c|}
\hline before 2001 & $x$-slope [mrad] & $y$-slope $[\mathrm{mrad}]$ & $x$-offset $[\mathrm{cm}]$ & $y$-offset $[\mathrm{cm}]$ \\
\hline top & 0.44 & -1.20 & -0.09 & -0.08 \\
bottom & 0.24 & 0.02 & -0.11 & 0.11 \\
\hline \hline after 2001 & $x$-slope [mrad] & $y$-slope [mrad] & $x$-offset $[\mathrm{cm}]$ & $y$-offset $[\mathrm{cm}]$ \\
\hline top & -0.18 & -0.62 & 0.30 & -0.08 \\
bottom & -0.42 & 0.49 & 0.29 & 0.11 \\
\hline
\end{tabular}

Table 4.4.1: Estimated misalignment of the spectrometer halves before and after 2001 [BrüO3].

not come from a real tilt and offset in the spectrometer halves. Part of the misalignment could be artificially introduced by the force bridge technique of the HERMES reconstruction code described in Section 3.3.1.

In order to study the effect of an angular misalignment on the extraction of azimuthal asymmetries, the reconstructed scattering angles of the particles are corrected for the angular misalignment of the spectrometer half by subtracting the correspondent $x$ - and $y$-slopes. This results in the migration of some events between azimuthal bins. The influence on the extracted amplitudes is more pronounced in kinematic bins with lower count numbers but no systematic deviations are visible, e.g., for the negative pion asymmetry amplitudes shown in Figure 4.4.10.

A misaligned spectrometer results in a $\cos \phi$ modulation in the hadron count rates, i.e.,

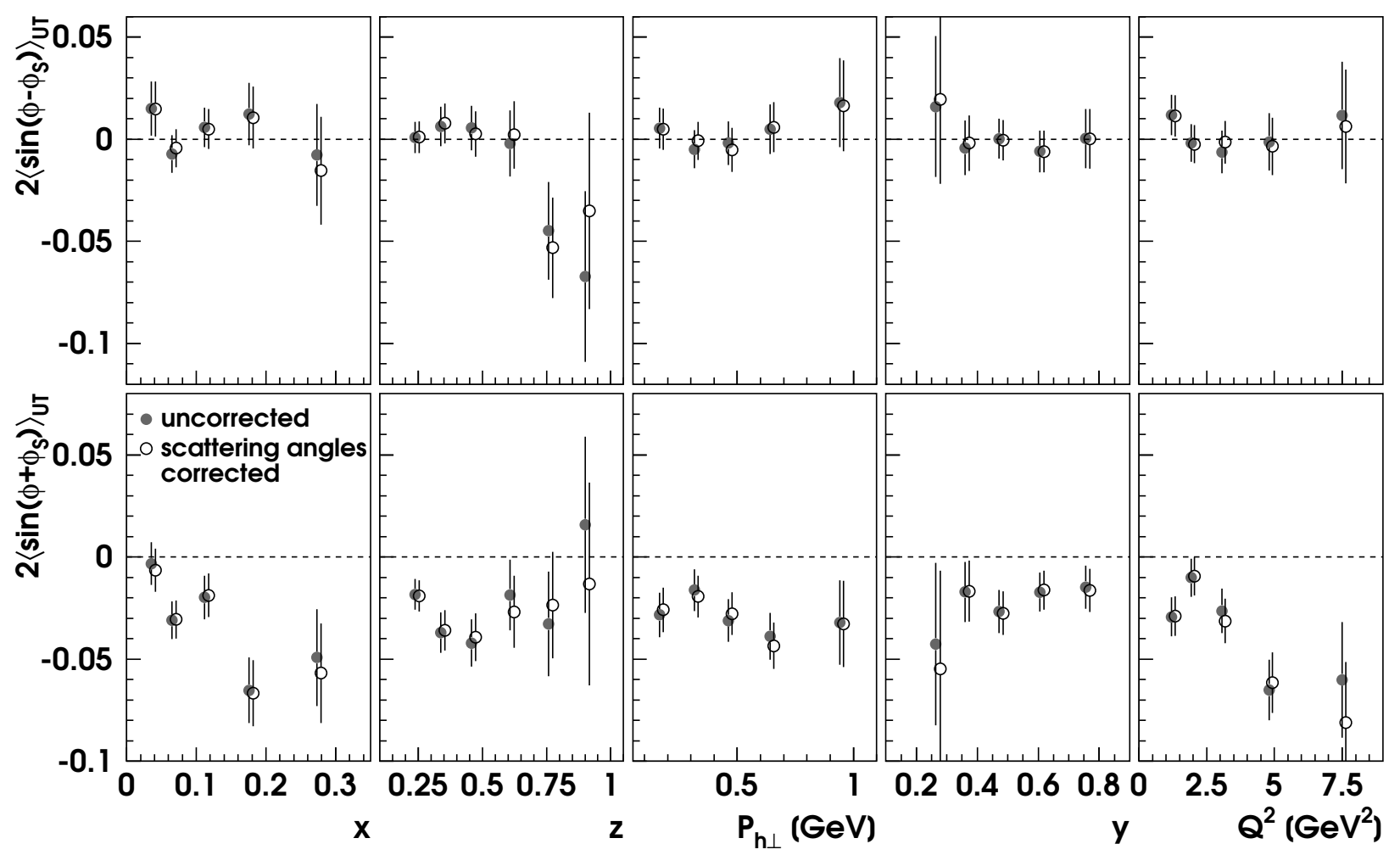

Figure 4.4.10: Comparison of the $\pi^{-}$asymmetry amplitudes with uncorrected and corrected scattering angles for the possible detector misalignment. 
in the denominator of the azimuthal asymmetry. Like the unpolarised $\cos \phi$ moment, it can couple to subleading-twist modulations of the polarised cross section and contribute to the Collins and Sivers amplitudes. The misalignment of the detector is estimated to produce a negative $\cos \phi$ moment with an absolute value smaller than 0.03 [Mai05]. The implementation of a $\cos \phi$ modulation with an amplitude of -0.06 in the fit results in a negligible change of the extracted asymmetry amplitudes. Furthermore, the amplitude of the cosine modulation caused by the misalignment appears as additional term in Eq. (4.4.6) but not in the extracted cosine moment from the Monte Carlo simulation based on a perfectly aligned spectrometer. Hence, the effect is already accounted for in the systematic uncertainty estimated in Section 4.4.4 and the corrections for the angular misalignment described above are not applied in the following.

A new alignment procedure based on a recent survey of the spectrometer, is under study and will decrease the uncertainty of the detector alignment to values of the order of $200 \mu \mathrm{m}$ and $100 \mu \mathrm{rad}$ [Kis05]. The new alignment procedure will be implemented in the reconstruction code and will therefore necessitate a reproduction of the data taken. This advanced alignment correction is not included in the presented analysis.

\subsubsection{Fake Asymmetry Amplitudes}

In order to study other effects of the spectrometer acceptance and efficiency on azimuthal asymmetries, fake asymmetry amplitudes are investigated. These amplitudes are extracted from unpolarised data by assigning the target spin state randomly. The unpo-

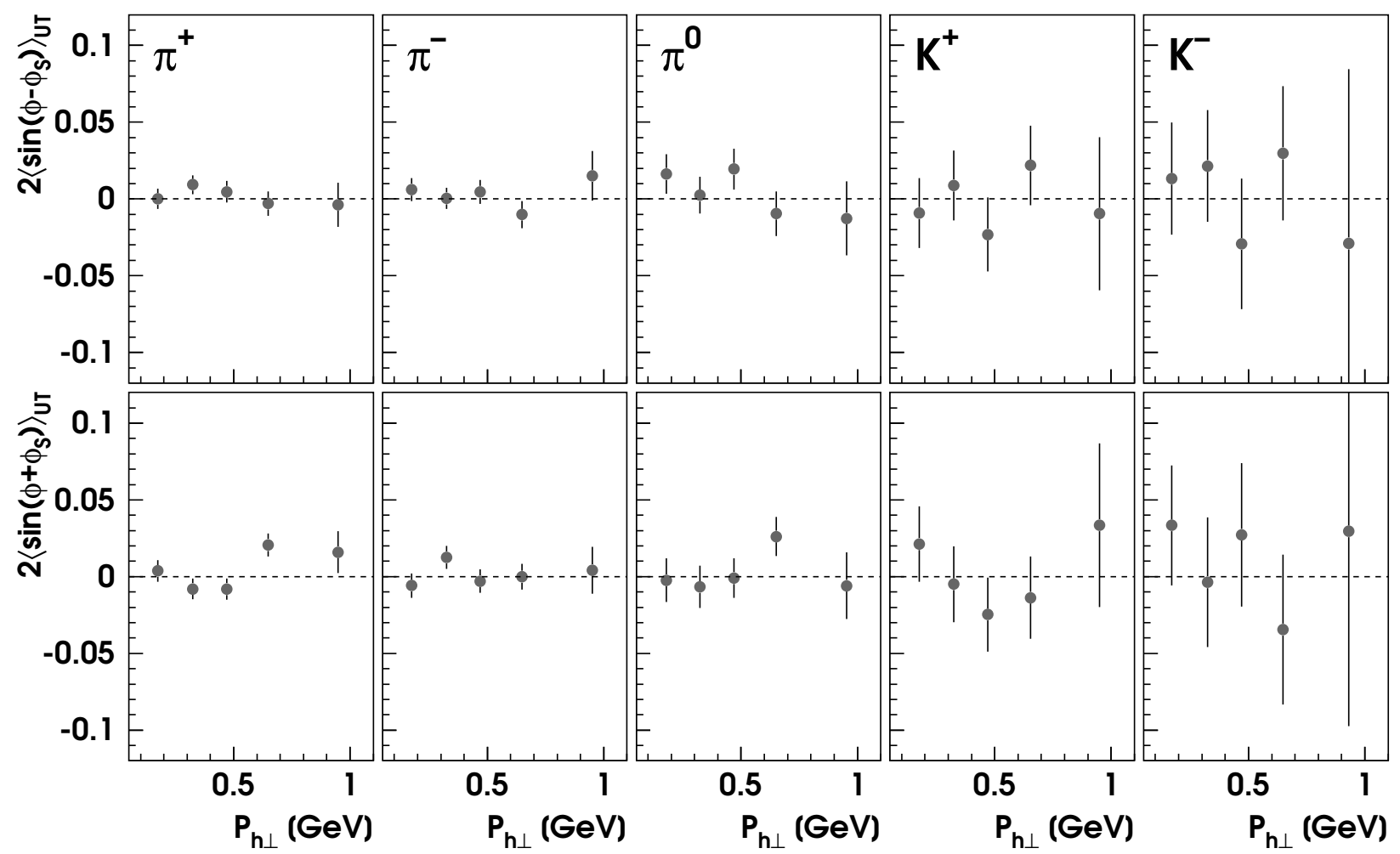

Figure 4.4.11: Asymmetry amplitudes extracted from unpolarised deuterium data by assigning the target spin state randomly. 
larised deuterium data recorded in the year 2004 are analysed. The data have statistics comparable to the three productions 02c0, 03c0, and 04b0 combined. As expected for unpolarised data, only statistical fluctuations of the amplitudes around zero are found, shown for the $P_{h \perp}$ bins as example in Figure 4.4.11. Although some data points deviate by more than one $\sigma$ from zero, the overall reduced $\chi^{2}$ is 0.56 in case of the Sivers amplitudes and 0.96 for the Collins amplitudes. The fluctuations seem to be more pronounced in the amplitudes of the pions with their smaller statistical uncertainties. But also here, a reasonable reduced $\chi^{2}$ of 0.71 (1.33) is obtained when all Sivers (Collins) amplitudes of the pions are combined. The extracted asymmetry of the data with the transversely polarised target is hence not biased by a fake asymmetry introduced by the HERMES spectrometer.

\subsection{The Correction for Neutral Pion Combinatorial Background}

The invariant mass spectrum of photon pairs in Figure 4.2.4 shows a $\pi^{0}$ peak on top of a rather flat combinatorial background. With the knowledge of the signal fraction in the measurement region between $0.1 \mathrm{GeV}$ and $0.17 \mathrm{GeV}$ and the asymmetry amplitude of the background $A_{\mathrm{bkg}}$, the measured amplitudes $A_{\text {meas }}$ for neutral pions can be corrected for combinatorial background:

$$
A_{\text {cor }}=\frac{N_{\text {tot }}}{N_{\text {sig }}} A_{\text {meas }}-\frac{N_{\text {bkg }}}{N_{\text {sig }}} A_{\text {bkg }} .
$$
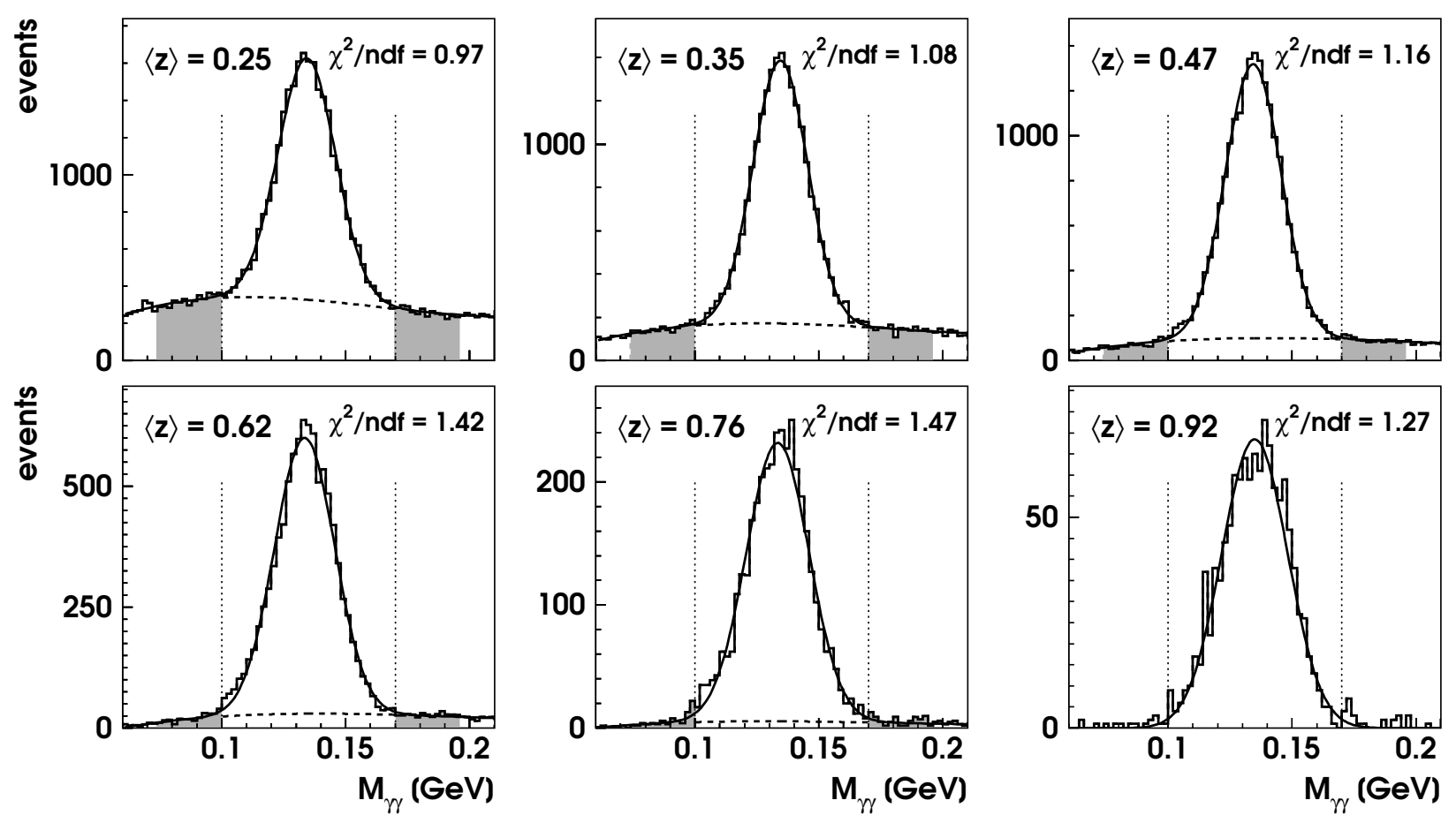

Figure 4.5.1: Invariant mass distributions of photon pairs for the six different $z$ bins. The sidebands used for the determination of the background amplitudes are shaded. 


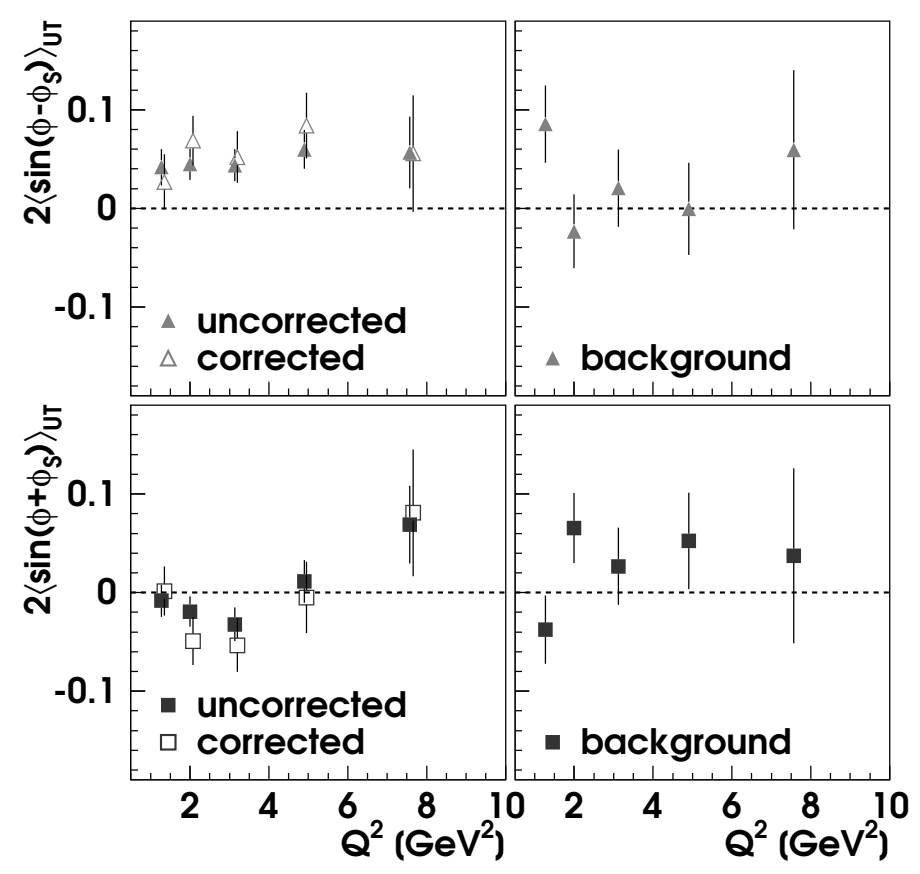

Figure 4.5.2: Measured and corrected $\pi^{0}$ asymmetry amplitudes dependent on $Q^{2}$ and the background amplitudes used for correction.

In all kinematic bins, the background asymmetry amplitudes can be extracted from two-photon events in the $0.025 \mathrm{GeV}(\approx 2 \sigma)$ sidebands-indicated as grey areas in Figure 4.5. 1-of the invariant mass peak outside the signal region. Due to the lower statistics in the sidebands, an $8 \times 8$ azimuthal binning as for kaons is applied and only a three parameter fit is performed. For the determination of the ratio $N_{\text {tot }} / N_{\text {sig }}$, invariant mass spectra are fitted for each kinematic bin. In order to take into account the correlation of the individual fit parameters for the signal and background parts of the spectrum, the fit is performed including $N_{\text {tot }} / N_{\text {sig }}$ as one of the fit parameters. The fits to the invariant mass spectra are shown in Figure 4.5.1 for the six $z$ bins. In the last bin, a fit omitting the third order polynomial for the background description, gives a reasonably good reduced $\chi^{2}$. Thus, no background correction is necessary in this particular bin. The obtained ratios $N_{\text {tot }} / N_{\text {sig }}$ and Gaussian means and widths are listed for all kinematic bins in Table F. 1. The variation of the Gaussian widths is caused by the energy dependence of the calorimeter resolution. In Figure 4.5.2, the corrected and uncorrected neutral pion asymmetry amplitudes are plotted dependent on $Q^{2}$. In addition, the amplitudes of the combinatorial background in the sidebands are shown. 

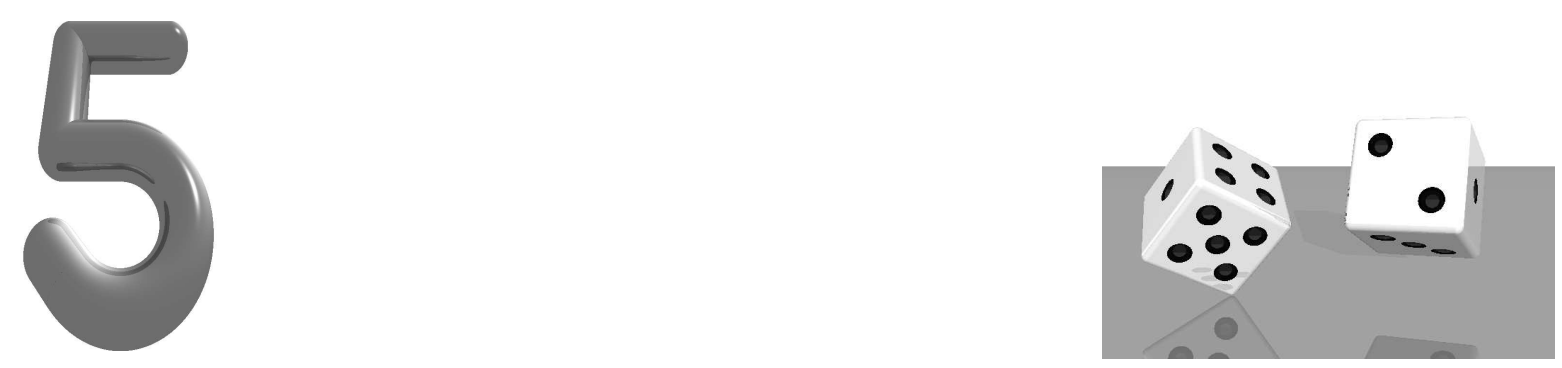

\section{Monte Carlo Studies}

The main advantage of forming cross section asymmetries over the direct measurement of polarised cross sections is that most of the acceptance effects cancel. In order to study the remaining influence of the acceptance on the extraction of azimuthal asymmetry amplitudes, a Monte Carlo generator can be used. Additionally, effects of other sources in the HERMES experiment such as the target magnet field or the smearing in the measured kinematic variables due to the detector resolutions can be investigated. Owing to the lack of a Monte Carlo generator for a transversely polarised target, the first Monte Carlo studies were performed with two different generators for DIS events on an unpolarised target [ElsO3]. The azimuthal asymmetries are implemented by assigning randomly a transverse target spin state and weighting the events by the asymmetry dependent on this target state. The unpolarised Monte Carlo generators are used for the estimate of the systematic uncertainty due to the extraction method and the different sources in the experiment. Later a Monte Carlo generator gmc_trans was developed which can simulate azimuthal distributions due to transverse-momentum dependent quark distribution and fragmentation functions [MakO3b]. It shows a good reproduction of the implemented amplitudes in case of unweighted asymmetries and reveals large acceptance effects in the extracted $P_{h \perp}$-weighted asymmetries.

\subsection{The Unpolarised Monte Carlo Generators}

The two different unpolarised Monte Carlo generators which are used for the studies are PYTHIA [SjöO3] and DISNG. As indicated in the flow chart in Figure 5.1.1, PYTHIA generates first the kinematics of the scattered lepton according to the cross section. The generated cross sections of all processes that are implemented in PYTHIA, were tuned to HERMES data [Lie04]. If radiative effects, i.e., bremsstrahlung in the initial or final state of the scattering process, are to be taken into account in the Monte Carlo production, the generated kinematics is passed to the program RADGEN [Aku98]. This program decides whether or not a photon is radiated off and calculates the true kinematics at the lepton vertex, leaving the generated kinematics unchanged. After that the scattering process is chosen by PYTHIA and in case of semi-inclusive DIS the quark, the diquark, and the string are generated. Based on the LUND string model (cf. Section 2.5.1) the following program JETSET 


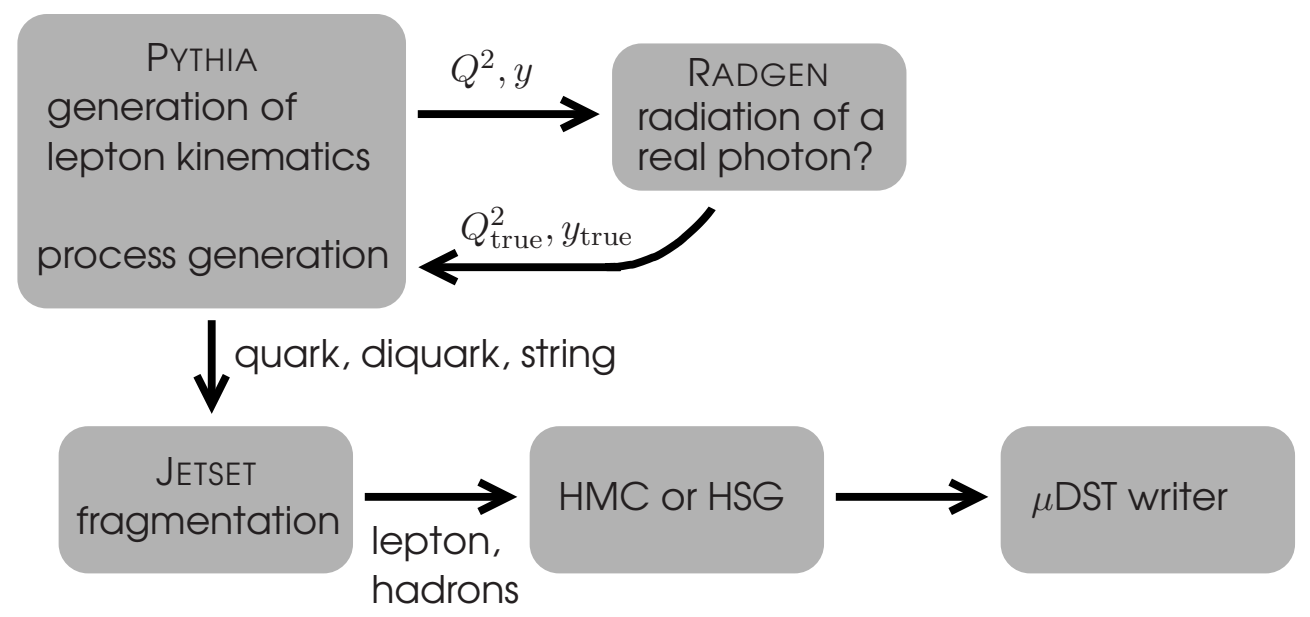

Figure 5.1.1: The PYTHIA Monte Carlo production chain.

[Sjö94] creates the hadronic final state. The fragmentation process can be influenced by means of JETSET parameters which were tuned to describe hadron multiplicities measured at HERMES [Hil05]. The hadronic final state can be passed either to a GEANT Monte Carlo simulation [CER93a] of the HERMES spectrometer (HMC) or the HERMES Smearing Generator (HSG) [MenO1, Hil05]. In HSG, a parametrisation of all smearing and resolution effects is used instead of the time consuming full GEANT simulation. The final step is performed by the $\mu$ DST writer which writes all relevant information into ADAMO tables with a similar layout as the tables of the real HERMES data.

The second Monte Carlo generator DISNG generates the lepton kinematics uniformly distributed in a $\nu, Q^{2}$ box and not according to the cross section like PYTHIA. Therefore, each event has to be assigned an event weight dependent on the cross section whereas the events generated with PYTHIA have weights unity. Also in the DISNG Monte Carlo chain, RADGEN applies the radiative corrections to the lepton kinematics before the decision on the scattering process by the program LEPTO [Ing97] (see Figure 5.1.2). The following steps are performed by JETSET, HMC or HSG, and the $\mu$ DST writer as in the PYTHIA Monte Carlo simulation chain.

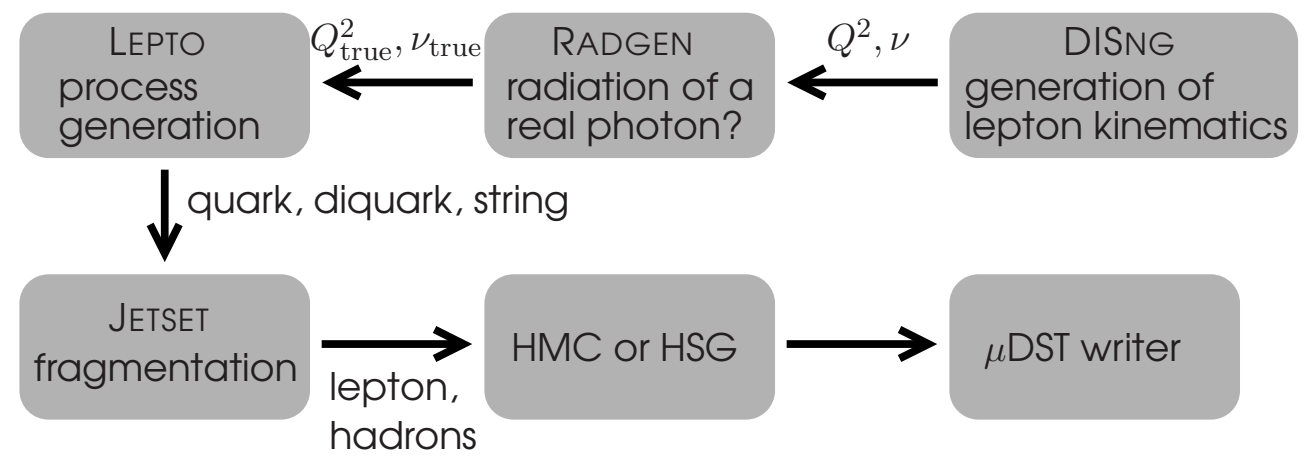

Figure 5.1.2: The DISNG Monte Carlo production chain. 
The PYTHIA and DISNG Monte Carlo generators differ in the generated processes. PYTHIA does not include elastic processes which are irrelevant for studies of semi-inclusive DIS, and in the DISNG generator, exclusive meson production is not implemented. Although the generation of Monte Carlo events with PYTHIA needs significantly more time than with DISNG for comparable statistics, productions with fully tracked particle trajectories using HMC are available for both generators in addition to the faster simulated HSG Monte Carlo productions.

The semi-inclusive pion production cross sections of the $02 \mathrm{c} 0$ production are determined for a cross section comparison between HERMES data and the fully tracked Monte Carlo productions. The cross section of the HERMES data is calculated in each kinematic bin using:

$$
\sigma_{\pi}=\frac{N_{\pi}}{\mathcal{R} \cdot C_{\mathrm{LUMI}}^{02}}
$$

where $N_{\pi}$ is the number of events in the bin, $\mathcal{R}$ is the integrated rate of the luminosity monitor corrected for the dead time of the spectrometer (see Appendix $\mathrm{B}$ ), and $C_{\mathrm{LUMI}}^{02}$ is the luminosity constant for the data taking period 2002 which is different for unpolarised and polarised data due to the influence of the transverse target magnet field and the different target densities ( $c$ f. Appendix B). The luminosity constant relates the count rate of the luminosity monitor to the luminosity. The pion count numbers are not corrected for

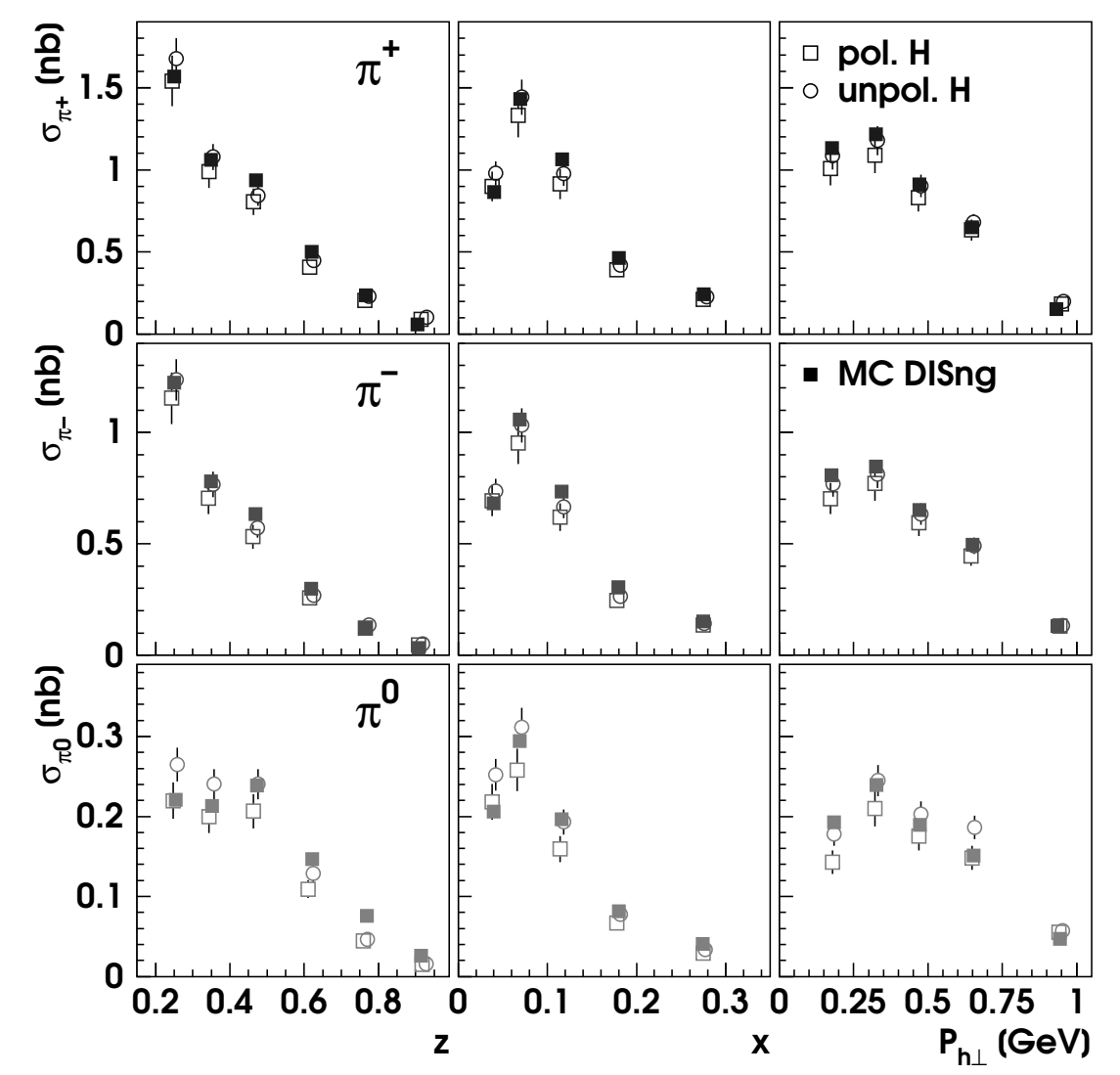

Figure 5.1.3: Pion cross section comparison between HERMES data and the DISNG Monte Carlo. The systematic uncertainty of the luminosity constant is included in the error bar. 


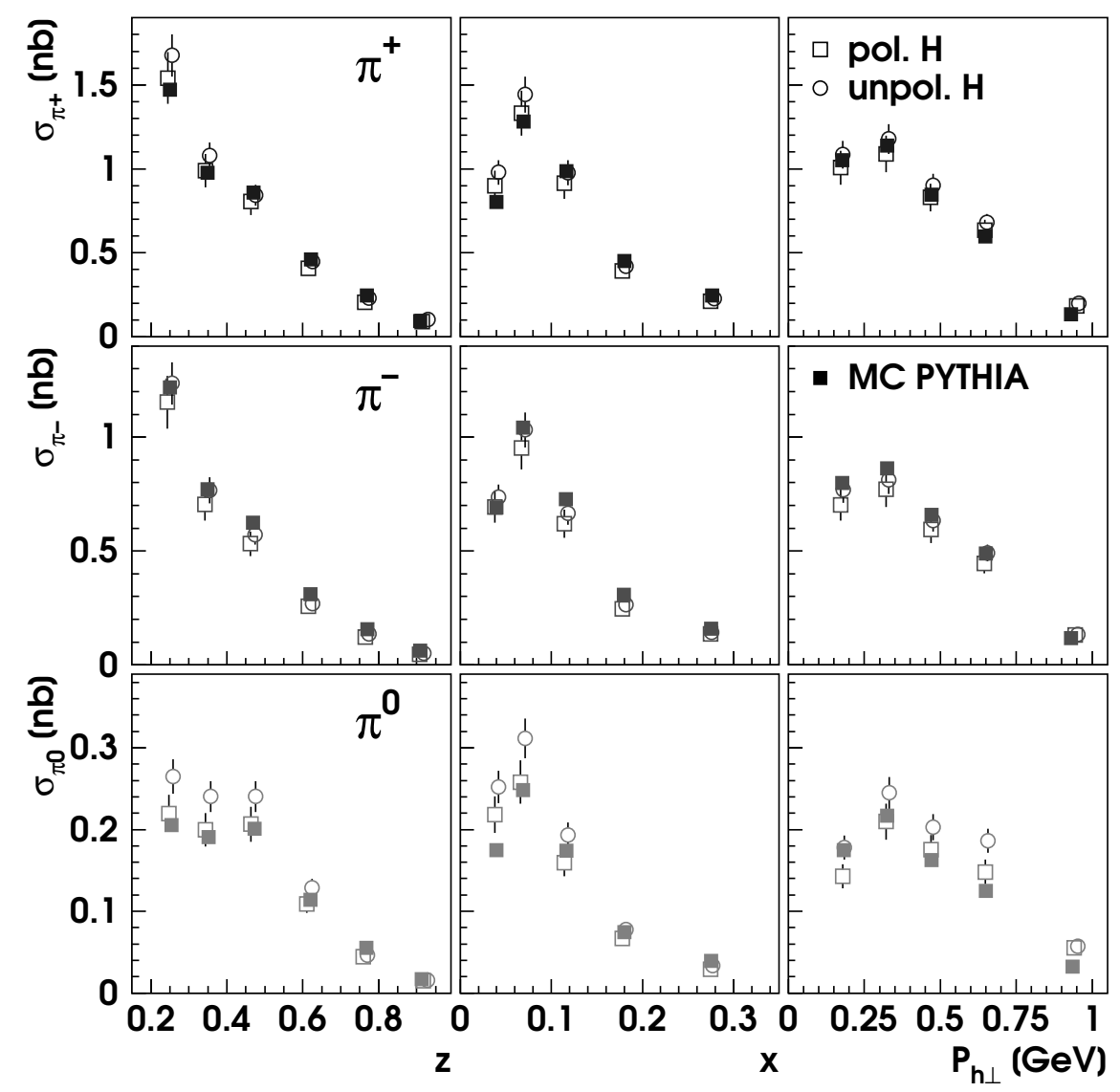

Figure 5.1.4: Pion cross section comparison between HERMES data and the PYTHIA Monte Carlo. The systematic uncertainty of the luminosity constant is included in the error bar.

detector inefficiencies.

Figures 5.1.3 and 5.1.4 show the cross section comparisons for the two unpolarised Monte Carlo generators. The latest JETSET tune 2004c is used in both Monte Carlo productions [HilO5]. The systematic uncertainty of the luminosity constants is contained in the error bars. In the HERMES data, the cross sections of the $\pi^{0}$ events are obtained from the integrated Gaussian of the fit to the invariant mass spectra, as described in Section 4.5 .

The charged pion cross sections are described very well with both generators. The slightly worse description of neutral pions compared to charged pions by DISNG can be explained by an imperfect simulation of the HERMES calorimeter. This affects only the $\pi^{0}$ because charged particles are identified in a Monte Carlo production not by the detector responses but by the generated particle types. The PYTHIA Monte Carlo events were later produced using an $\mathrm{HMC}$ version with an improved calorimeter simulation. The agreement between HERMES data and both Monte Carlo productions is suitable for the study of cross section ratios used in the asymmetry calculation. The differences in the cross sections of polarised and unpolarised HERMES data is caused by the uncertainty in the determination of the two luminosity constants (cf. Appendix B) which is not yet based on a Monte Carlo simulation of the luminosity monitor. 

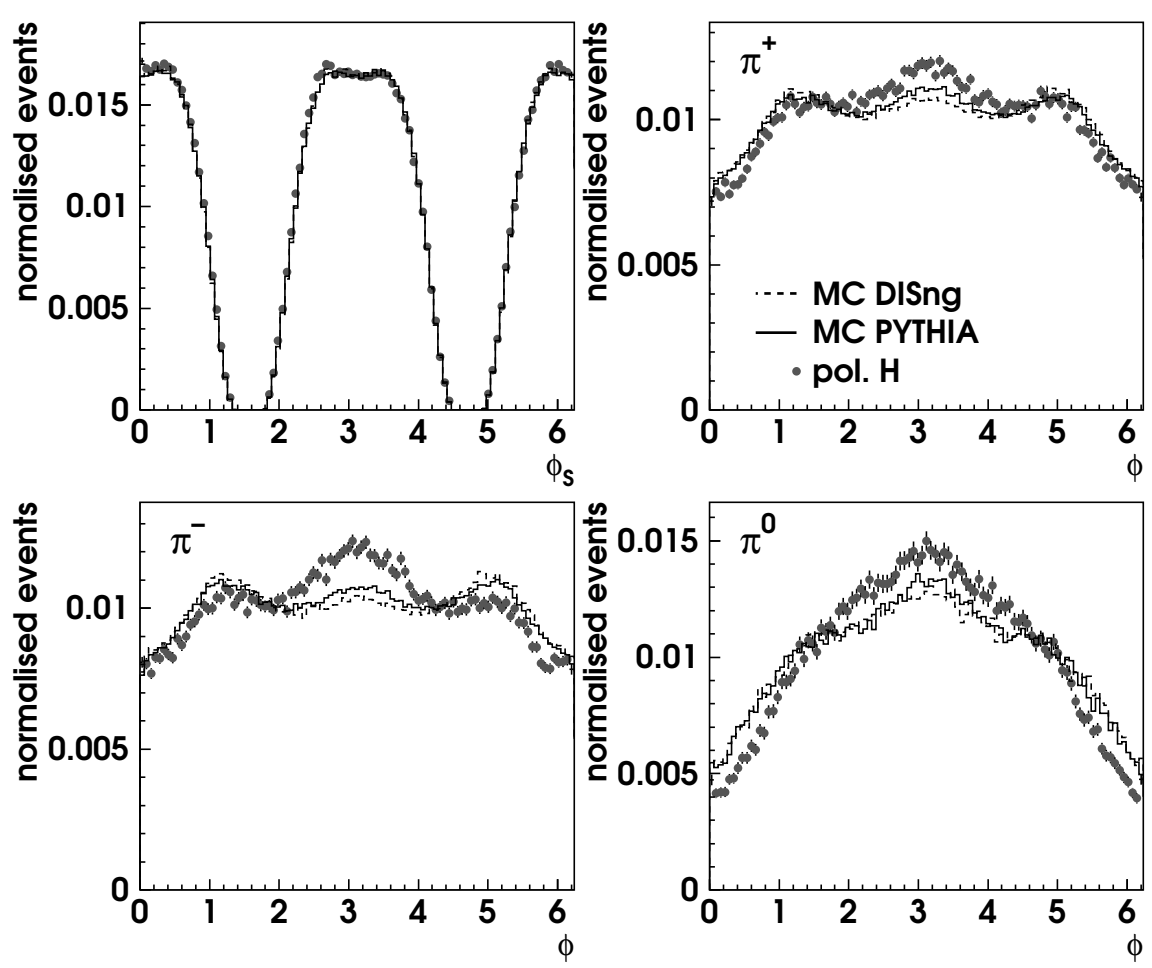

Figure 5.1.5: Comparison of the azimuthal angular distributions between HERMES data of all three productions (02c0, 03c0, 04b0) and the two unpolarised Monte Carlo generators. All histograms are normalised to unity.

The study of azimuthal asymmetries with Monte Carlo events requires also a good description of the distributions of the azimuthal angles $\phi$ and $\phi_{S}$. In Figure 5. 1.5 the shapes of the angular distributions are compared, for all three pions in case of the angle $\phi$. The angular distribution of $\phi_{S}$ is simulated very well by both generators. The Monte Carlo $\phi$ distributions of all three pion types are broader than in the HERMES data but of roughly similar shape. Since the unpolarised cosine moments are not implemented in the Monte Carlo generators, a perfect agreement of the $\phi$ distributions is not expected (see Section 4.4.4).

\subsubsection{Asymmetry Amplitude Scan}

The before mentioned unpolarised Monte Carlo generators do not contain azimuthal modulations of the cross section. Instead, the azimuthal asymmetries are implemented after the event generation and tracking by assigning at random a transverse target spin state $\uparrow$ or $\downarrow$ and weighting the events with $1 \pm\left[A_{\mathrm{UT}}^{\sin \left(\phi+\phi_{S}\right)} \sin \left(\phi+\phi_{S}\right)+A_{\mathrm{UT}}^{\sin \left(\phi-\phi_{S}\right)} \sin \left(\phi-\phi_{S}\right)\right]$, dependent on the assigned target spin state. This is possible since the polarised cross sections for the two target spin states can be written as $\sigma_{\mathrm{UT}}^{\uparrow \downarrow}\left(\phi, \phi_{S}\right)=\sigma_{\mathrm{UU}} \cdot\left(1 \pm A_{\mathrm{UT}}\left(\phi, \phi_{S}\right)\right)$. The influence of different biases on the extracted asymmetry amplitudes is modelled by a relative correction $f$ of the measured amplitude $A_{\text {meas }}$ :

$$
A_{\text {cor }}=(1+f) \cdot A_{\text {meas }} \text {. }
$$


For the simultaneous extraction of two amplitudes, both have to be accounted for in the correction:

$$
\begin{aligned}
& A_{\mathrm{cor}}^{\sin \left(\phi+\phi_{S}\right)}=A_{\mathrm{meas}}^{\sin \left(\phi+\phi_{S}\right)}+F_{c c} \cdot A_{\mathrm{meas}}^{\sin \left(\phi+\phi_{S}\right)}+F_{c s} \cdot A_{\text {meas }}^{\sin \left(\phi-\phi_{S}\right)}, \\
& A_{\mathrm{cor}}^{\sin \left(\phi-\phi_{S}\right)}=A_{\text {meas }}^{\sin \left(\phi-\phi_{S}\right)}+F_{s c} \cdot A_{\text {meas }}^{\sin \left(\phi+\phi_{S}\right)}+F_{s s} \cdot A_{\text {meas }}^{\sin \left(\phi-\phi_{S}\right)},
\end{aligned}
$$

yielding a relative correction matrix $\boldsymbol{F}$ :

$$
\vec{A}_{\text {cor }}=(1+\boldsymbol{F}) \cdot \vec{A}_{\text {meas }} .
$$

Here, the indices $c$ and $s$ denote the Collins and Sivers amplitudes, respectively.

For the determination of the matrix $\boldsymbol{F}$, a constant asymmetry amplitude $A_{\mathrm{imp}}^{\sin \left(\phi \pm \phi_{S}\right)}$ is implemented in the unpolarised Monte Carlo production independent of any kinematic variable and any hadron type. This constant amplitude is scanned between the values -1 and 1 with a step size of 0.1 and the Monte Carlo production is analysed for each setting. When the extracted amplitudes $A_{\text {ext }}^{\sin \left(\phi \pm \phi_{S}\right)}$ are plotted versus the implemented amplitudes a linear dependence is expected: $A_{\text {ext }}^{\sin \left(\phi \pm \phi_{S}\right)}=S \cdot A_{\mathrm{imp}}^{\sin \left(\phi \pm \phi_{S}\right)}$ (see Figure 5. 1.6). Without any influence of the extraction method, the acceptance, the transverse target magnet field or other possible sources the slope $S$ of a fitted straight line is supposed to be 1. Taking into account mixing effects, i.e., the influence of an implemented Sivers amplitude on the extracted Collins amplitude and vice versa, the extracted amplitudes are related to the implemented ones by:

$$
\begin{aligned}
& A_{\mathrm{ext}}^{\sin \left(\phi+\phi_{S}\right)}=S_{c c} \cdot A_{\mathrm{imp}}^{\sin \left(\phi+\phi_{S}\right)}+S_{c s} \cdot A_{\mathrm{imp}}^{\sin \left(\phi-\phi_{S}\right)}, \\
& A_{\mathrm{ext}}^{\sin \left(\phi-\phi_{S}\right)}=S_{s c} \cdot A_{\mathrm{imp}}^{\sin \left(\phi+\phi_{S}\right)}+S_{s s} \cdot A_{\mathrm{imp}}^{\sin \left(\phi-\phi_{S}\right)} .
\end{aligned}
$$

All four slopes $S_{c c}, S_{c s}, S_{s c}$, and $S_{s s}$ can be combined in a matrix $\boldsymbol{S}$ :

$$
\vec{A}_{\mathrm{ext}}=\boldsymbol{S} \cdot \vec{A}_{\mathrm{imp}}
$$

which allow the determination of the relative correction matrix $\boldsymbol{F}$ by:

$$
\boldsymbol{F}=\boldsymbol{S}^{-1}+\mathbb{1 1}
$$

because the corrected amplitudes should recover the implemented amplitudes and the extracted amplitudes correspond the measured ones. Here, $\mathbb{1 1}$ is the two-dimensional unity matrix.

\subsubsection{Binning Effect}

The extracted amplitudes of the generated events covering the whole range $(4 \pi)$ of the solid angle, are neither affected by the track reconstruction nor the spectrometer acceptance. They can be used to estimate a possible bias of the extraction method on the extracted amplitudes. The generated events are analysed in different azimuthal binnings for each of the 21 implemented amplitudes of the scan between -1 and 1. The binnings are the $8 \times 8 \phi \times \phi_{S}$ binning which was applied for the published results [Air05b], and the binnings with increased numbers of $12 \times 12,16 \times 16$, and $20 \times 20$ bins in $\phi$ and $\phi_{S}$. In Figure 5. 1.6, the extracted Collins and Sivers amplitudes from a PYTHIA Monte Carlo production are plotted versus the implemented amplitudes. These are Sivers type amplitudes in the 


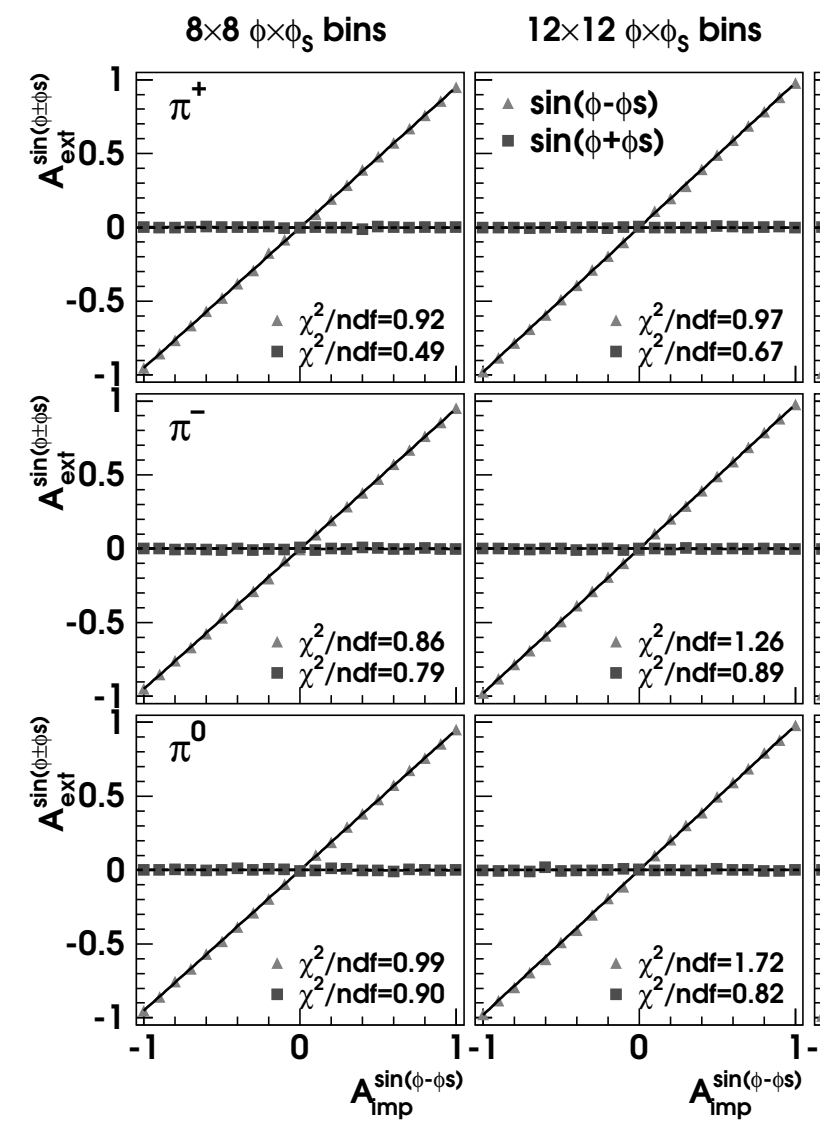

$16 \times 16 \phi \times \phi_{s}$ bins

$20 \times 20 \phi \times \phi_{S}$ bins
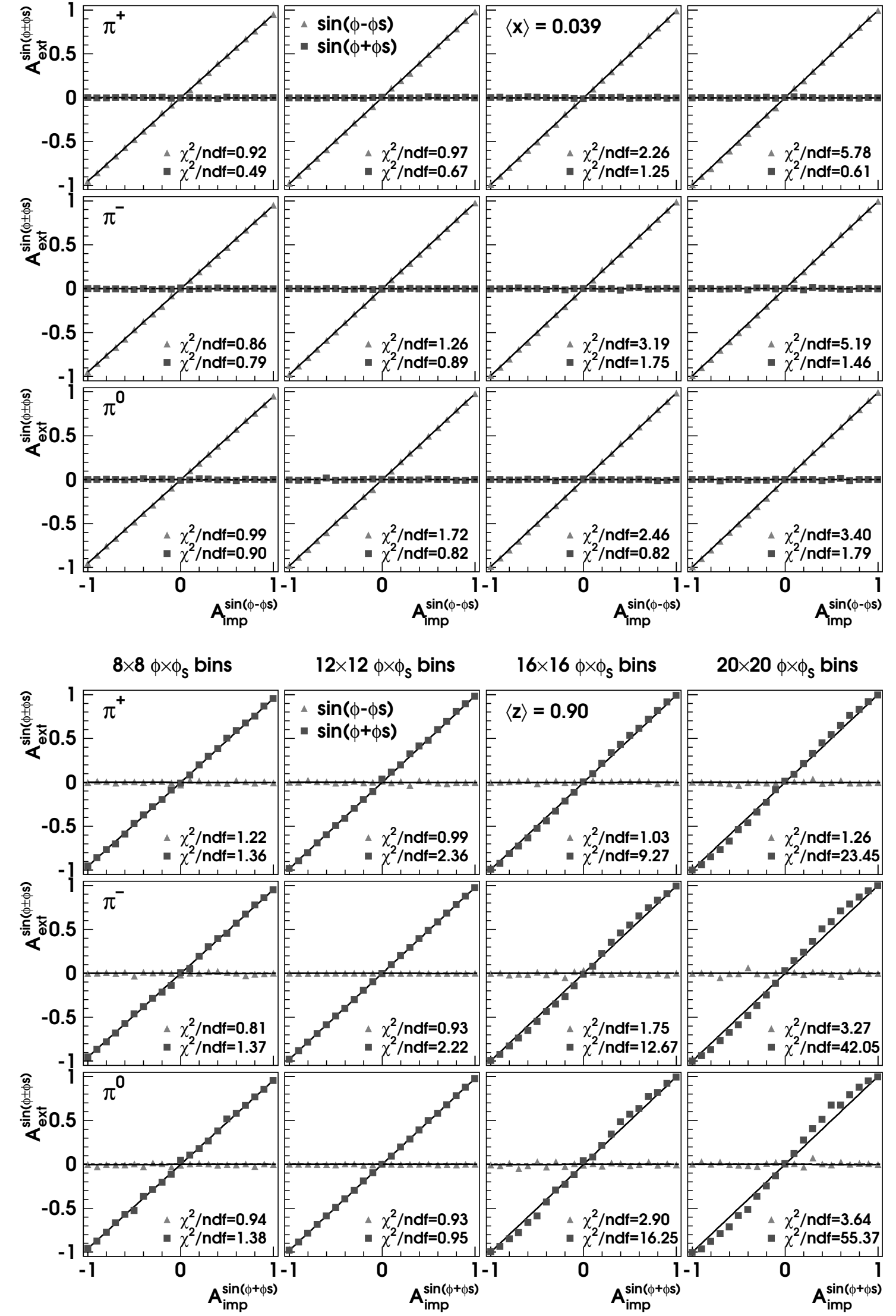

$16 \times 16 \phi \times \phi_{s}$ bins

$20 \times 20 \phi \times \phi_{s}$ bins

Figure 5.1.6: Sivers (top) and Collins (bottom) amplitude scans in the first $x$ bin (top) and the last $z$ bin (bottom) for different azimuthal binnings. 
top part of the figure and Collins type amplitudes in the lower part. The amplitudes are shown for all three pion types and for the four different binnings. In addition, a fit with a straight line, which was constrained through the origin, is indicated. The statistics of
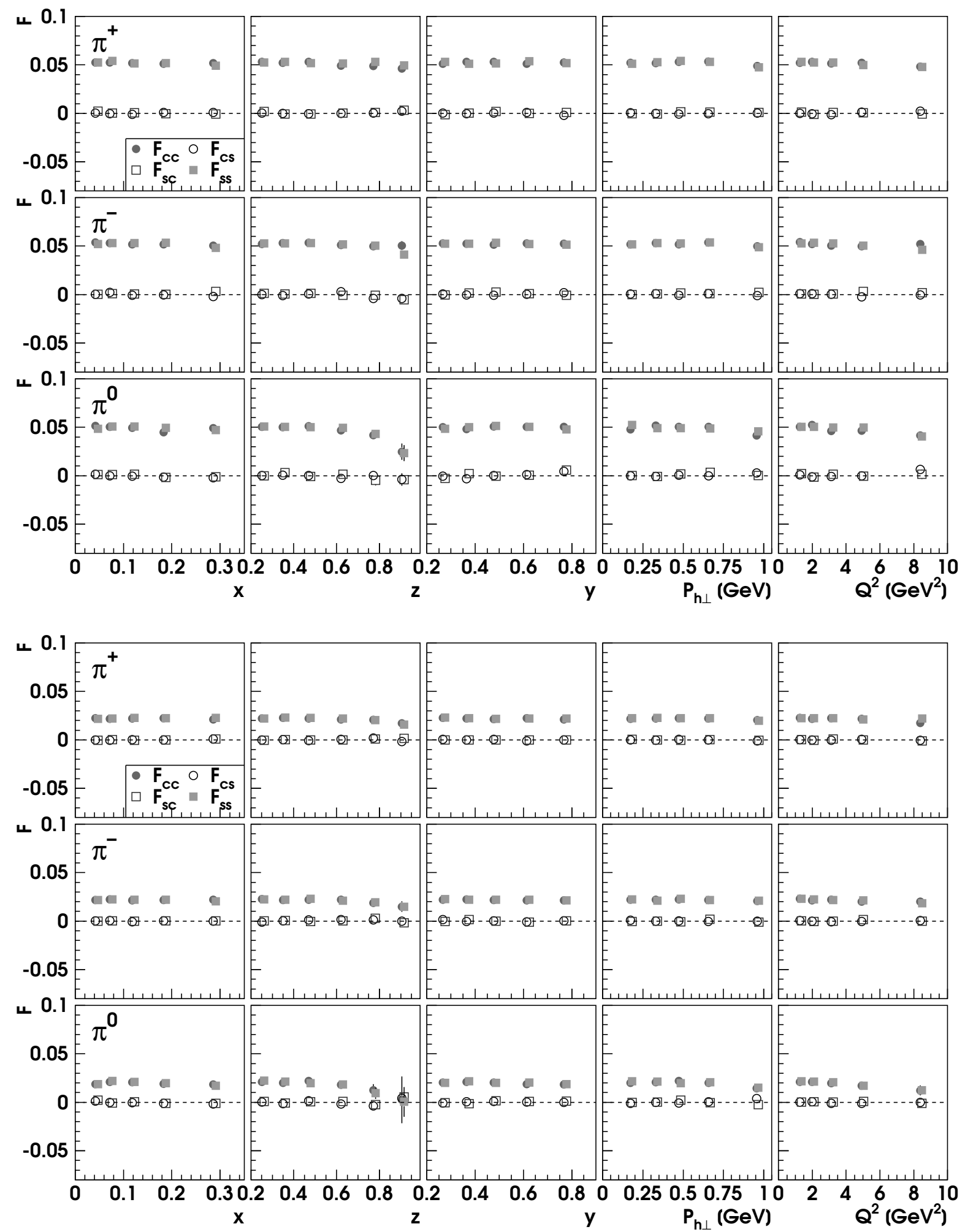

Figure 5.1.7: Elements of the relative correction matrix $\boldsymbol{F}$ for two different azimuthal binnings with $8 \times 8$ (top) and $12 \times 12$ bins (bottom). 
the generated events is adjusted to the statistics of the reconstructed events in the three HERMES data productions listed in Table 4.2.3.

For the scan of the Sivers amplitude, the first $x$ bin with a high statistics is chosen as example whereas the Collins amplitude scan is plotted for the last $z$ bin with the lowest statistics. The reduced $\chi^{2}$ of the straight line fits becomes worse with a higher number of azimuthal bins and with lower statistics. The reason is a nonlinear behaviour of the extracted amplitudes clearly visible for the $20 \times 20$ binning in the last $z$ bin. This nonlinear effect is caused by a non-Gaussian event distribution in the azimuthal bins and could be avoided for instance with a cut on the total number of events, as performed with the HERMES data for a study in Section 4.4.1. However, this strategy results in event losses which prevent the amplitude extraction in some kinematic bins.

With the obtained slopes of the fits, the relative correction factor $\boldsymbol{F}$ can be determined. The four elements are plotted in Figure 5.1.7 dependent on the different kinematic quantities for 8 and 12 azimuthal bins. No mixing effect is visible, i.e., a given Sivers amplitude does not influence the extraction of the Collins amplitude and vice versa. The extracted amplitudes are around $5 \%$ smaller than the implemented amplitudes in case of a $8 \times 8$ binning and only around $2 \%$ smaller for the larger bin number of 12 . For kinematic bins with lower statistics, the diagonal elements $F_{c c}$ and $F_{s s}$ of the relative correction matrix are lower due to the onset of a nonlinear dependence between extracted and implemented amplitudes. The obtained values for $F_{c c}$ and $F_{s s}$ correspond to the relative difference $\Delta$ between the fit function at the centre values of the bin, $\phi_{c}$ and $\phi_{S c}$, and the mean value of the fit function in the bin:

$$
\Delta=\frac{\sin \left(\phi_{c} \pm \phi_{S c}\right)-\left\langle\sin \left(\phi \pm \phi_{S}\right)\right\rangle_{\text {bin }}}{\left\langle\sin \left(\phi \pm \phi_{S}\right)\right\rangle_{\text {bin }}}
$$

where the mean value is defined as:

$$
\left\langle\sin \left(\phi \pm \phi_{S}\right)\right\rangle_{\text {bin }}=\frac{\int_{\text {bin }} \mathrm{d} \phi \mathrm{d} \phi_{S} \sin \left(\phi \pm \phi_{S}\right)}{\int_{\text {bin }} \mathrm{d} \phi \mathrm{d} \phi_{S}} .
$$

In Figure 5. 1.8, the function $\Delta$ describing this binning effect is plotted versus the number of azimuthal bins. In addition, the results of a fit with a constant to the diagonal elements of

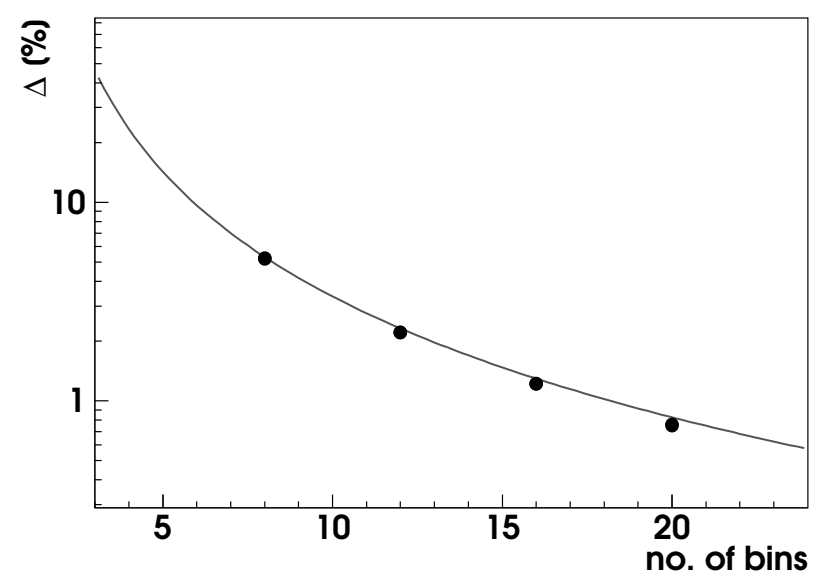

Figure 5.1.8: The effect of the azimuthal binning $\Delta$ (solid line) and the results of a fit with a constant to $F_{c c}(x)$ and $F_{s s}(x)$ for positive pions $(\bullet)$ dependent on the number of azimuthal bins. 
$\boldsymbol{F}$ are shown. For these results, the $x$ dependence of positive pions was fitted. Eq. (5. 1.8) yields binning effects of $5.3 \%, 2.3 \%, 1.3 \%$, and $0.8 \%$ for $8,12,16$, and 20 azimuthal bins, respectively. In principle, a larger number of azimuthal bins decreases the effect of the finite bin size but results in event distributions in the bins which are not Gaussian. In the analysis of the semi-inclusive DIS data, a $12 \times 12 \phi \times \phi_{S}$ binning was chosen, which corresponds to a good compromise to avoid biases on the extracted Collins and Sivers amplitudes (too many bins) and systematic offsets introduced by too few bins.

\subsubsection{Spectrometer Acceptance Effects}

As a next step, the reconstructed events of the Monte Carlo productions are analysed using $12 \times 12$ azimuthal bins. For reconstructed events, the extracted amplitudes contain effects from the acceptance and the smearing in the angles due to the reconstruction resolution. Monte Carlo productions which do not include a target magnet field, are analysed at first. Again the elements of the relative correction matrix $\boldsymbol{F}$ are computed, now with the number of reconstructed events equivalent to the combined statistics of the three HERMES data productions.

For both unpolarised generators, DISNG and PYTHIA, fully tracked productions are available without a transverse target magnet field using the latest JETSET tune. The implementation of asymmetry amplitudes of \pm 1 results very often in unreliable slope parameters which spoil the linear fit. This might be caused by resolution effects distorting the sine behaviour of the asymmetry. Furthermore, the existence of such large asymmetries is very unlikely due to the positivity limits of the involved quark distribution and fragmentation functions. For the reconstructed events, only amplitudes between -0.9 and 0.9 are therefore used in the linear fits. Figures 5.1.9 and 5.1.10 show the elements of $\boldsymbol{F}$ obtained from the DISNG and the PYTHIA productions, respectively. The diagonal elements that contain the $2.3 \%$ effect due to the finite azimuthal bin size (see previous Section 5.1.2) increase to values up to $5 \%$. The off-diagonal elements describing the mixing effect between the two amplitudes, become non-zero in general but they remain small (less than $2 \%$ in most kinematic bins).

Both Monte Carlo productions give similar results for the relative correction matrix $\boldsymbol{F}$. The small deviations can be explained by the differences in the generated processesdifferent processes have different event kinematics-and in the calorimeter simulation. Also the slightly different azimuthal angular distributions as shown in Figure 5.1.5 might cause minute variations in the elements of the correction matrix for the DISNG and the PYTHIA productions. Furthermore, a comparison of results from a fully tracked Monte Carlo production with results from a production with HSG shows a good agreement between both spectrometer simulation methods.

\subsubsection{Target Magnet Field Correction}

Another influence on the extracted amplitudes can arise from the transverse target magnet field, although the resolution in the reconstruction of scattering angles and vertex is almost completely recovered by the target magnet field correction as shown in Figure 3.3.2 on page 42. The transverse magnetic field together with the applied corrections of method 1 and 2 (cf. Section 3.3.1) is implemented in an existing DISNG production. For 


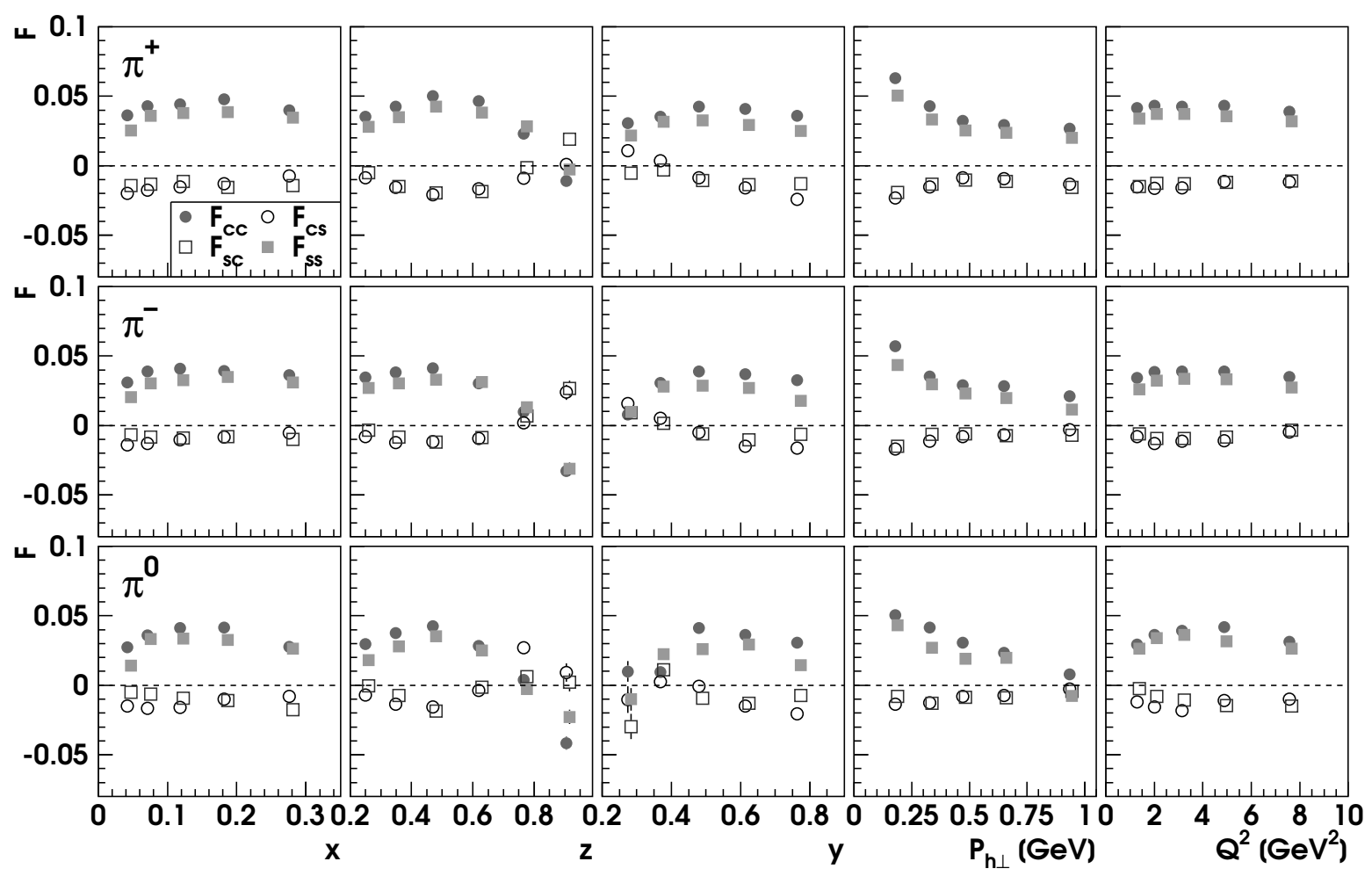

Figure 5.1.9: Elements of the relative correction matrix $\boldsymbol{F}$ for a DISNG production without a transverse target magnet field. The elements are computed for fully tracked Monte Carlo events and $12 \times 12$ azimuthal bins.

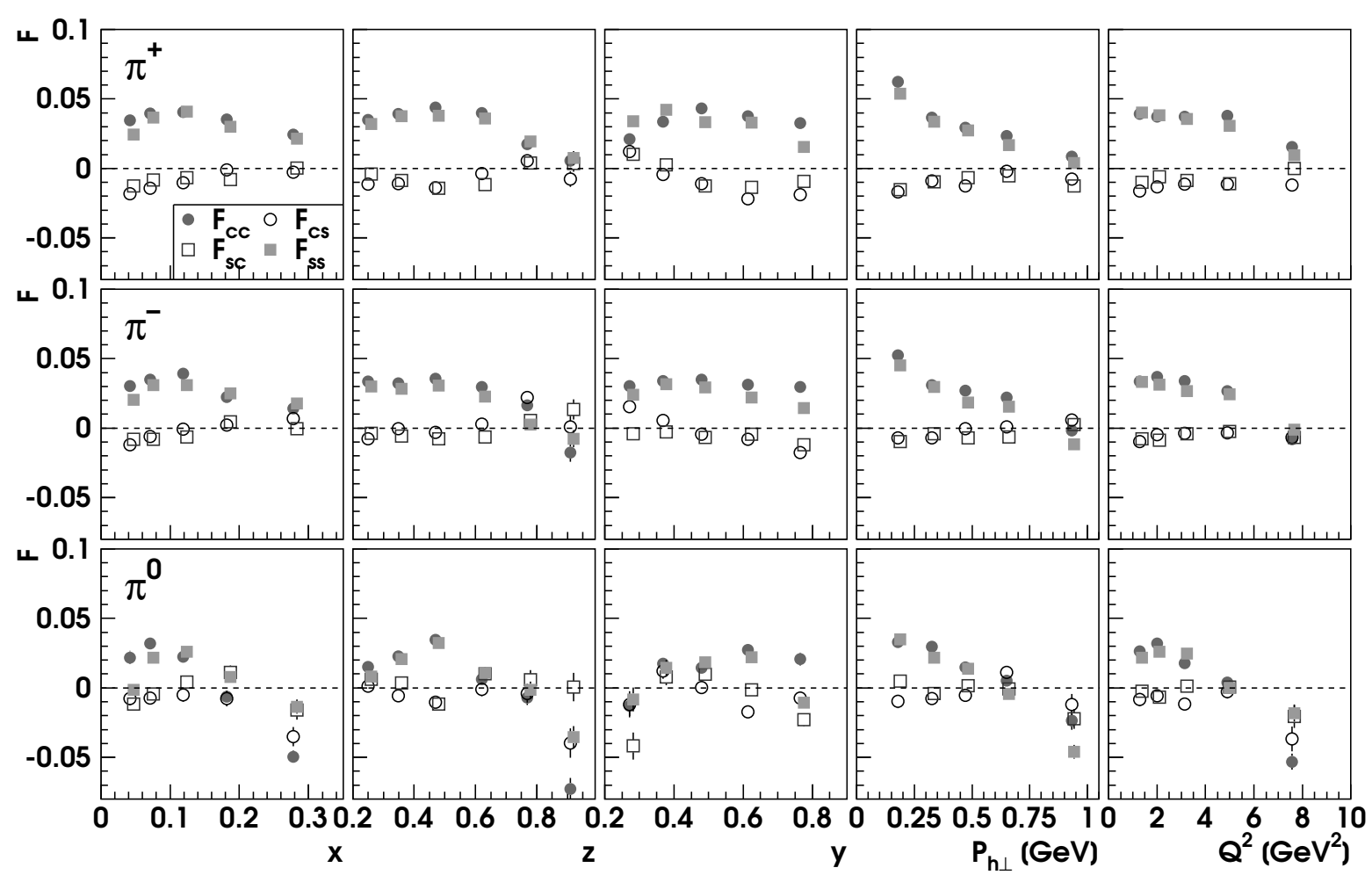

Figure 5.1.10: Elements of the relative correction matrix $\boldsymbol{F}$ for a PYTHIA production without a transverse target magnet field. The elements are computed for fully tracked Monte Carlo events and $12 \times 12$ azimuthal bins. 
this production, an older JETSET tune was used which gives slightly different semi-inclusive cross sections compared to the latest tune. However, the effect on the cross section asymmetries is negligible so that studies performed with this Monte Carlo production remain valid for the present purpose. Figure 5.1.11 shows the extracted elements of the relative correction matrix $\boldsymbol{F}$ for this production. TMC method 2 was used for the correction of the scattering angles and the vertex. As expected no significant change is visible compared to the elements without transverse target field in Figure 5. 1.9. The same conclusion is obtained when applying TMC method 1.

\subsubsection{Radiative Effects}

In the studies described above, radiative effects are not taken into account because the program RADGEN provides only true kinematic quantities such as $x_{\text {true }}, Q_{\text {true }}^{2}$ and not the true azimuthal angles $\phi_{\text {true }}$ and $\phi_{S \text {,true }}$. Both azimuthal angles are influenced by the radiation of a real photon because the virtual-photon momentum defines the $z$-axis of the coordinate system in which the angles are calculated. With the information of the true kinematic quantities only, it is not possible to compute the true azimuthal angles because RADGEN does not define the moment when the photon is radiated. The real photon can be radiated in the initial state (ISR) or in the final state (FSR) of the scattering process. Thus, the momentum of the lepton either before or after the scattering has to be corrected by the momentum of the photon. In contrast to the DISNG generator, PYTHIA stores the four-momentum $\mathrm{q}_{\text {real }}$ of the radiated real photon. With this information the momentum of the virtual photon can be corrected without the knowledge about ISR or FSR: $\mathbf{q}=\mathbf{l}-\mathbf{l}^{\prime}-\mathbf{q}_{\text {real }}$. In the peaking approximation used the real photon is radiated collinear to the lepton momentum and the virtual-photon momentum changes only within the scattering plane. Although the correction of the incoming and outgoing lepton momenta differs for ISR and FSR, it is irrelevant for the definition of the scattering plane. Hence, the PYTHIA generator can be used for the estimate of the influence of the radiative effects.

Figure 5.1.12 shows the obtained correction matrix elements for the PYTHIA Monte Carlo production. Here, the radiated real photons are taken into account as described above. The absolute values of both the diagonal and the off-diagonal elements of $\boldsymbol{F}$ increase compared to the results without the inclusion of radiative effects (cf. Figure 5.1.10). In addition, a difference is visible between the influence on the amplitude extraction of the two sine modulations: The absolute values of the elements $F_{c c}$ and $F_{c s}$ which are relevant for the extracted Collins amplitude, are larger than the absolute values of $F_{s s}$ and $F_{s c}$ needed for the Sivers amplitude correction.

\subsubsection{Detector Misalignment}

In the full simulation of the HERMES spectrometer in HMC a database is used in which the detector geometry is stored. In the studies above, the Monte Carlo events are produced with a database that contains perfectly aligned detectors. In addition, a second database is available with a misaligned detector geometry based on the values for the years before 2001, listed in Table 4.4.1. This misaligned detector geometry was implemented in a DISNG production and the relative correction matrix extracted. The matrix elements are plotted in Figure 5.1.13 and have to be compared to the results in Figure 


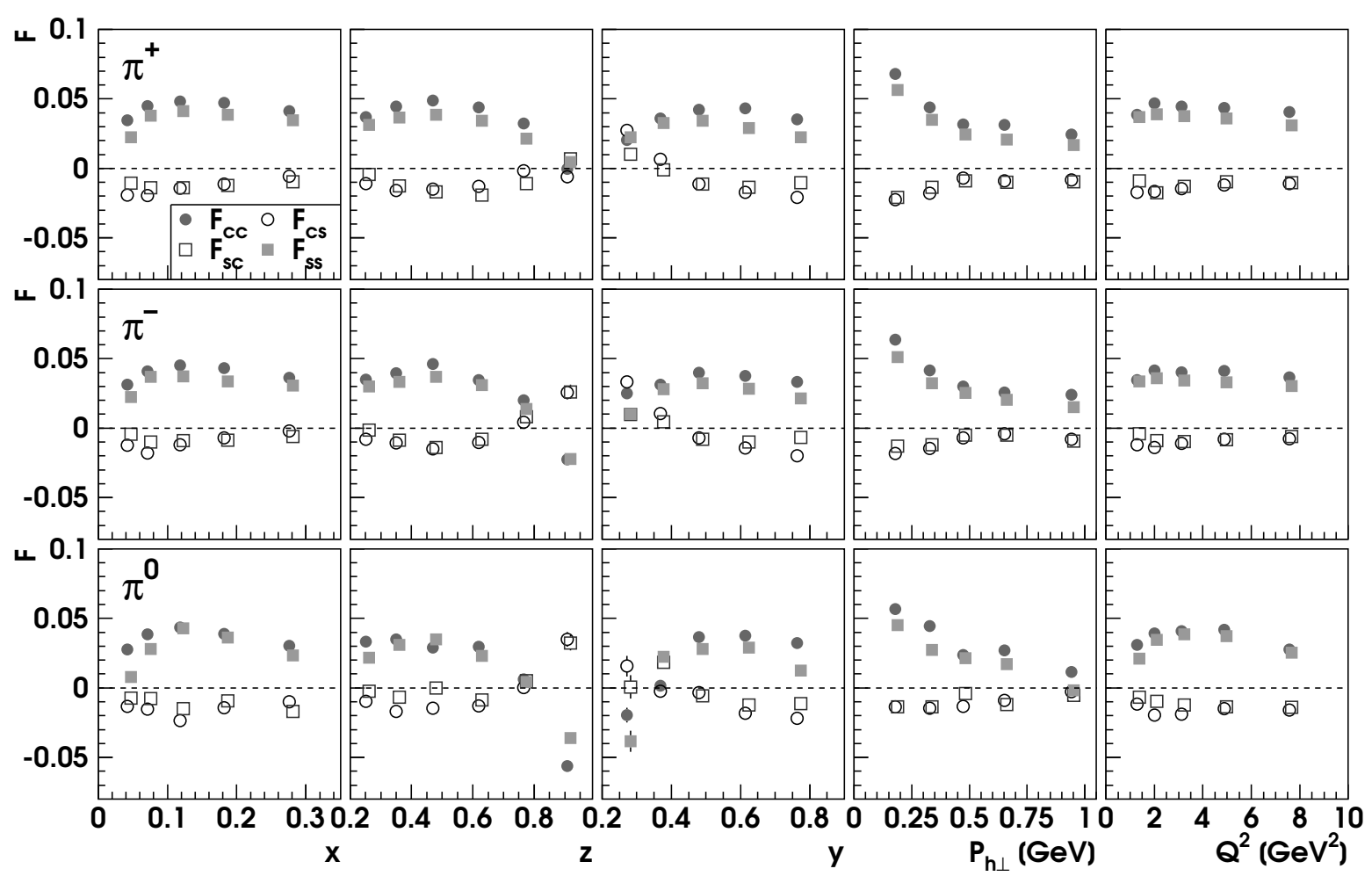

Figure 5.1.11: Elements of the relative correction matrix $\boldsymbol{F}$ for a DISNG production with a transverse target magnet field.

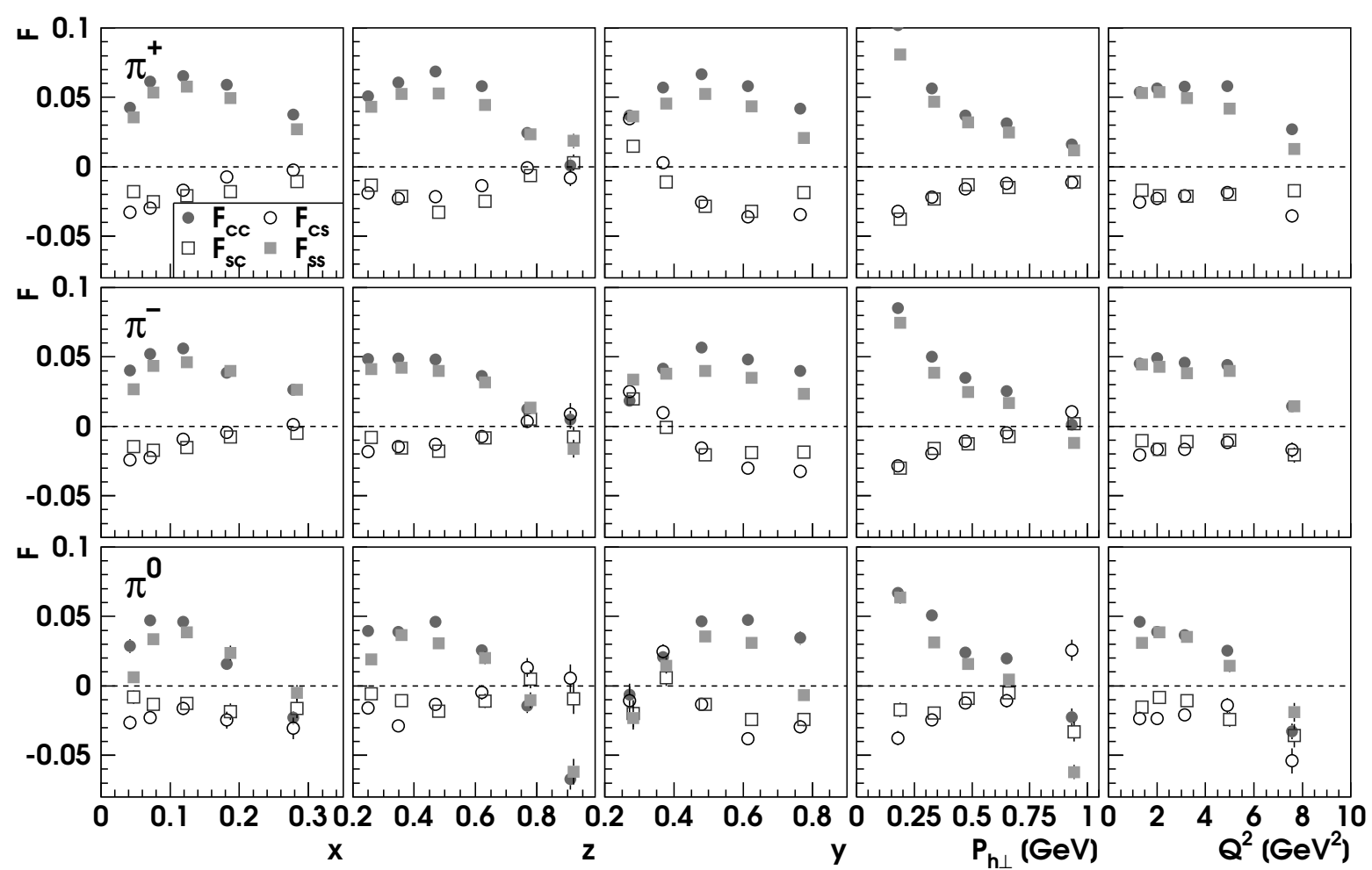

Figure 5.1.12: Elements of the relative correction matrix $\boldsymbol{F}$ for a PYTHIA production when radiative effects are taken into account. 
5.1.11 because in both productions the transverse target magnet field is present. The misaligned geometry influences the extracted Collins asymmetry amplitudes stronger than the Sivers amplitudes yet the changes compared to the results for the perfectly aligned detectors are small. Furthermore, the effect of a misaligned HERMES spectrometer is already accounted for in the systematic uncertainty as described in Section 4.4.8.

Note that the detector misalignment has a similar effect as the radiative effects discussed in the previous section. While a detector misalignment is constant for all events in the data set, the radiation of a real photon leads to a kink in the lepton trajectory which can also be considered as a detector misalignment, albeit for the lepton track only and varying from event to event. The Collins amplitudes are more susceptible to these changes of the lepton kinematics as they are based on the sum of the two azimuthal angles $\phi$ and $\phi_{S}$ (see Figure 2.5.3 on page 25) while for the Sivers amplitudes the difference between these two angles enters and a change in the orientation of the lepton scattering plane cancels.

\subsection{7 $P_{h \perp}$-Weighted Asymmetry Amplitudes}

Also $P_{h \perp}$-weighted asymmetry amplitudes can be investigated with the unpolarised Monte Carlo productions. In this case, the events are weighted by:

$$
1 \pm\left[A_{\mathrm{UT}}^{\sin \left(\phi+\phi_{S}\right)}(z M) / P_{h \perp} \sin \left(\phi+\phi_{S}\right)+A_{\mathrm{UT}}^{\sin \left(\phi-\phi_{S}\right)}(z M) / P_{h \perp} \sin \left(\phi-\phi_{S}\right)\right],
$$

dependent on the assigned target spin state. The kinematic variables $z$ and $P_{h \perp}$ of the generated event are used for the event weight and the amplitudes $A_{\mathrm{UT}}^{\sin \left(\phi \pm \phi_{S}\right)}$ are again scanned between -0.9 and 0.9 . The extracted elements of the relative correction matrix $\boldsymbol{F}$ are shown in Figure 5.1.14 for the DISNG production with implemented transverse target field. Note the enlarged scale compared to the previous plots. The influence of the track reconstruction and the spectrometer acceptance is much stronger for the $P_{h \perp}-$ weighted amplitudes compared to the unweighted amplitudes. The diagonal elements have maximum values up to $10 \%$ and show a different dependence on the kinematic variables. In addition, the mixing effects between the two extracted amplitudes, i.e., the off-diagonal elements, are larger for $P_{h \perp}$-weighted than for the unweighted amplitudes: they also reach $10 \%$ in some kinematic bins. This sizable effect is further investigated using a Monte Carlo generator for the simulation of azimuthal distributions due to transversemomentum dependent DFs and FFs in Section 5.2.

\subsubsection{Estimated Systematic Uncertainty}

The absolute size of the relative correction $F_{i c} \cdot A_{\text {meas }}^{\sin \left(\phi+\phi_{S}\right)}+F_{i s} \cdot A_{\text {meas }}^{\sin \left(\phi-\phi_{S}\right)}$ for $i=c, s$ obtained from the Monte Carlo studies with the two unpolarised generators is used to estimate a relative systematic uncertainty for the unweighted asymmetry amplitudes. This uncertainty includes the influence of the extraction method and effects from the spectrometer such as geometric acceptance and detector resolutions. The contributions from the two sine modulations to the absolute size of the correction have opposite signs, i.e., $F_{c c}$ and $F_{s s}$ are positive while $F_{c s}$ and $F_{s c}$ are negative. Thus, for extracted Collins and Sivers amplitudes with like sign the resulting correction is smaller than ech of its two contributions. 


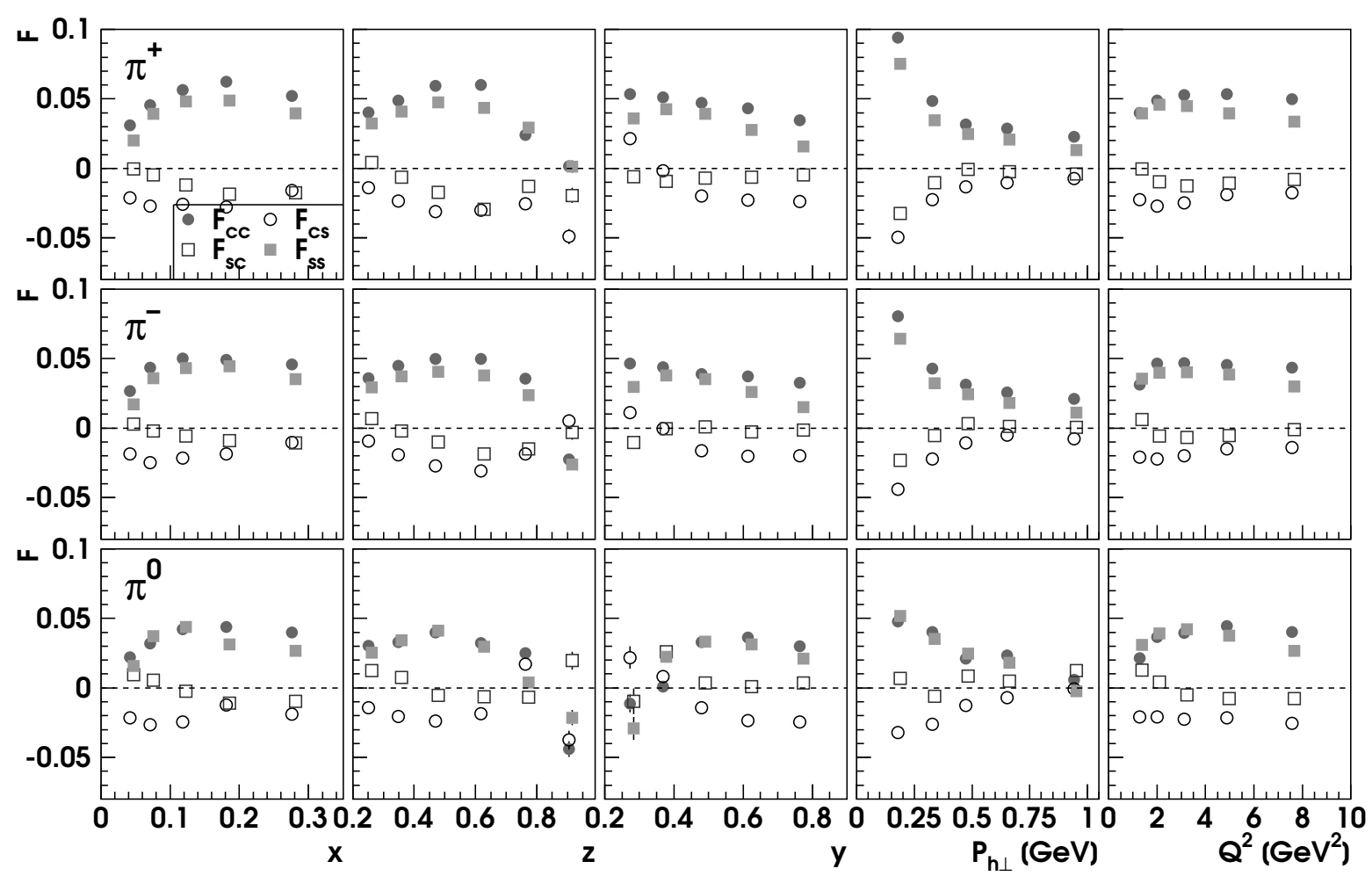

Figure 5.1.13: Elements of the relative correction matrix $\boldsymbol{F}$ for a DISNG production with a misaligned spectrometer.

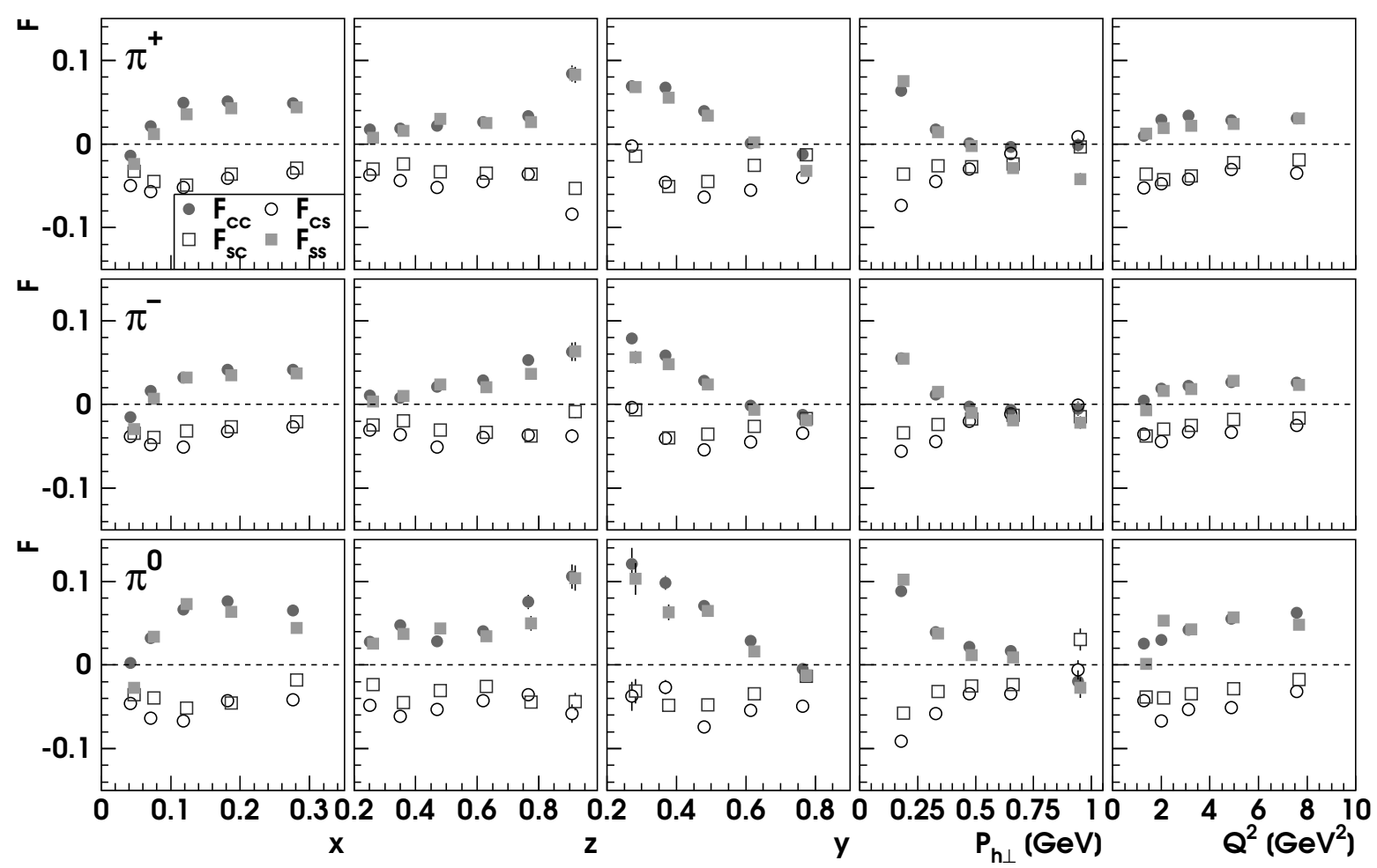

Figure 5.1.14: Elements of the relative correction matrix $\boldsymbol{F}$ for the $P_{h \perp}$-weighted asymmetry amplitudes extracted from a DISNG production. 
Therefore, for charged pions an uncertainty of $5 \%$ was estimated. In case of the neutral pions the extracted Collins and Sivers amplitudes have opposite sign in most of the kinematic bins (see Figure 4.4.3) and the two contributions of the correction matrix add up. Therefore, the systematic uncertainty of the $\pi^{0}$ amplitudes is estimated as $7 \%$. No studies were performed for the charged kaons but results similar to the charged pions are expected regarding the acceptance and spectrometer effects. Since the $8 \times 8$ azimuthal binning causes a higher binning effect of around $5 \%$, a relative systematic uncertainty of $7 \%$ is applied to the kaon asymmetry amplitudes.

The studies performed with the two unpolarised Monte Carlo generators reveal only part of the influences from the extraction method, radiative effects, and the HERMES spectrometer, because the implemented amplitudes do not depend on any kinematic quantity. While it would be also possible to implement asymmetry amplitudes with kinematic dependencies the correlation with the transverse quark momenta can only be accounted for in a Monte Carlo generator including transverse-momentum dependent DFs and FFs which will be described in the following section.

\subsection{The Monte Carlo Generator gmc_trans}

The unpolarised Monte Carlo generators do not include the transverse quark momentum dependency of the distribution and fragmentation functions. Therefore, a new Monte Carlo generator gmc_trans was developed [MakO3b] for the simulation of azimuthal distributions due to intrinsic transverse quark momenta. In this generator, a Gaussian ansatz is used for the transverse momentum distributions with a factorised form of the $x(z)$ and $p_{T}^{2}\left(k_{T}^{2}\right)$ dependencies in the quark distribution (fragmentation) functions:

$$
\begin{gathered}
q\left(x, p_{T}^{2}\right)=q(x) \cdot \frac{1}{\pi\left\langle p_{T}^{2}\right\rangle} e^{-\frac{p_{T}^{2}}{\left\langle p_{T}^{2}\right\rangle}}, \\
D_{1}\left(z, z^{2} k_{T}^{2}\right)=D_{1}(z) \cdot \frac{1}{\pi z^{2}\left\langle k_{T}^{2}\right\rangle} e^{-\frac{k_{T}^{2}}{\left\langle k_{T}^{2}\right\rangle}} .
\end{gathered}
$$

Using this same ansatz for the Sivers and Collins function as for the unpolarised functions:

$$
\begin{gathered}
f_{1 T}^{\perp q}\left(x, p_{T}^{2}\right)=f_{1 T}^{\perp q}(x) \cdot \frac{1}{\pi\left\langle p_{T}^{2}\right\rangle} e^{-\frac{p_{T}^{2}}{\left\langle p_{T}^{2}\right\rangle}}, \\
H_{1}^{\perp}\left(z, z^{2} k_{T}^{2}\right)=H_{1}^{\perp}(z) \cdot \frac{1}{\pi z^{2}\left\langle k_{T}^{2}\right\rangle} e^{-\frac{k_{T}^{2}}{\left\langle k_{T}^{2}\right\rangle}},
\end{gathered}
$$

and inserting all four functions (5.2.1) - (5.2.4) into the positivity limits (2.4.22) and (2.5.6), the problem is encountered that the left-hand sides of the inequalities rise linearly with $\left|\vec{p}_{T}\right|$ and $\left|\vec{k}_{T}\right|$ :

$$
\begin{aligned}
& \frac{\left|\vec{p}_{T}\right|}{M} f_{1 T}^{\perp q}(x)<q(x), \\
& \frac{\left|\vec{k}_{T}\right|}{M_{h}} H_{1}^{\perp}(z)<D_{1}(z) .
\end{aligned}
$$

Note that this problem does not appear for the transversity positivity limit (2.4.12) which also holds for the $p_{T}^{2}$-dependent DFs as it can be easily satisfied by $|\delta q(x)| \leq q(x)$. 


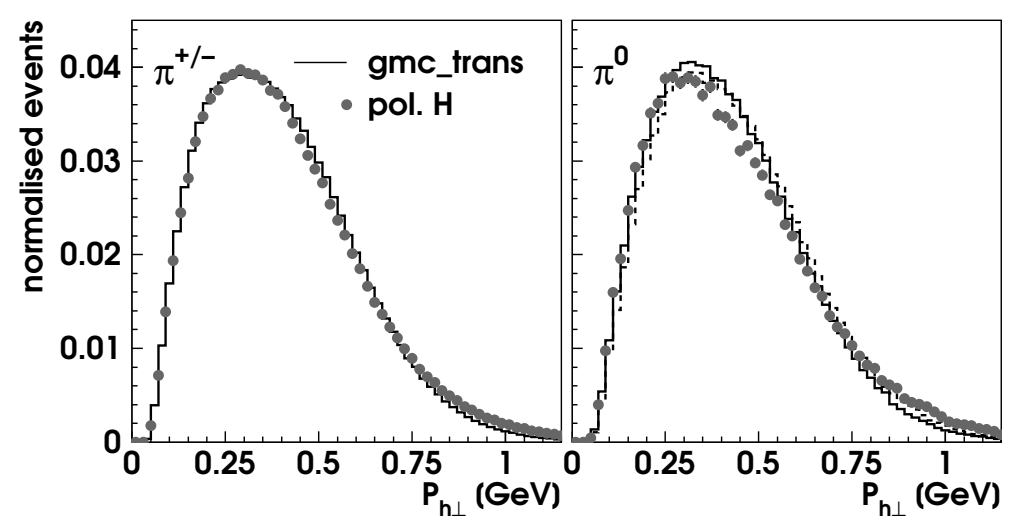

Figure 5.2.1: Comparison of the transverse momentum distributions between the HERMES data on transversely polarised hydrogen and the polarised Monte Carlo generator gmc_trans in the HERMES acceptance. For the $\pi^{0}$ distribution the dashed line shows the result from an earlier Monte Carlo production neglecting smearing of the photons (see text). Both histograms are normalised to unit area.

A solution for the Sivers and Collins functions is to modify Eq. (5.2.3) and (5.2.4) and to use smaller widths for the transverse momentum distributions:

$$
\begin{gathered}
f_{1 T}^{\perp q}\left(x, p_{T}^{2}\right)=f_{1 T}^{\perp q}(x) \cdot \frac{1}{\pi\left(1-C_{s}\right)\left\langle p_{T}^{2}\right\rangle} e^{-\frac{p_{T}^{2}}{\left(1-C_{s}\right)\left\langle p_{T}^{2}\right\rangle}}, \\
H_{1}^{\perp}\left(z, z^{2} k_{T}^{2}\right)=H_{1}^{\perp}(z) \cdot \frac{1}{\pi z^{2}\left(1-C_{c}\right)\left\langle k_{T}^{2}\right\rangle} e^{-\frac{k_{T}^{2}}{\left(1-C_{c}\right)\left\langle k_{T}^{2}\right\rangle}},
\end{gathered}
$$

introducing the two parameters $C_{s}$ and $C_{c}\left(0<C_{s}, C_{c}<1\right)$. With this ansatz one avoids unphysical cut-offs in the $\vec{p}_{T}$ and $\vec{k}_{T}$ distributions, which would further complicate the analytical expression of cross sections.

Even though the Sivers function (5.2.7) fulfils the positivity limit (2.4.22), the sum of the cross section components including the Sivers and transversity functions can still exceed the unpolarised cross section. A more stringent requirement on the Sivers function takes into account the contribution from other DFs, e.g., $\delta q^{i}[$ Bac04b]ii:

$$
\frac{p_{T}^{2}}{M^{2}}\left(f_{1 T}^{\perp q}\left(x, p_{T}^{2}\right)\right)^{2} \leq q\left(x, p_{T}^{2}\right)\left[q\left(x, p_{T}^{2}\right)-2\left|\delta q\left(x, p_{T}^{2}\right)\right|\right] .
$$

The main reason for the Gaussian ansatz is the possibility to calculate the unweighted and $P_{h \perp}$-weighted asymmetry moments analytically for the kinematics of each generated event. These values are stored in a table for the comparison of extracted and implemented moments. This allows the systematic study of biases on the extracted asymmetry moments from different sources, including the dependence on intrinsic transverse quark momenta, in particular with respect to the limited $P_{h \perp}$ acceptance of the spectrometer.

In the gmc_trans generator, certain parameters can be adjusted for a good description of the data. Here, the two parameters $C_{s}$ and $C_{c}$ are set to a value of 0.25 which

'Here, other DFs than $q\left(x, p_{T}^{2}\right), \delta q\left(x, p_{T}^{2}\right)$, and $f_{1 T}^{\perp q}\left(x, p_{T}^{2}\right)$ are set to 0 .

"A missing factor of 2 in the reference is added here. 

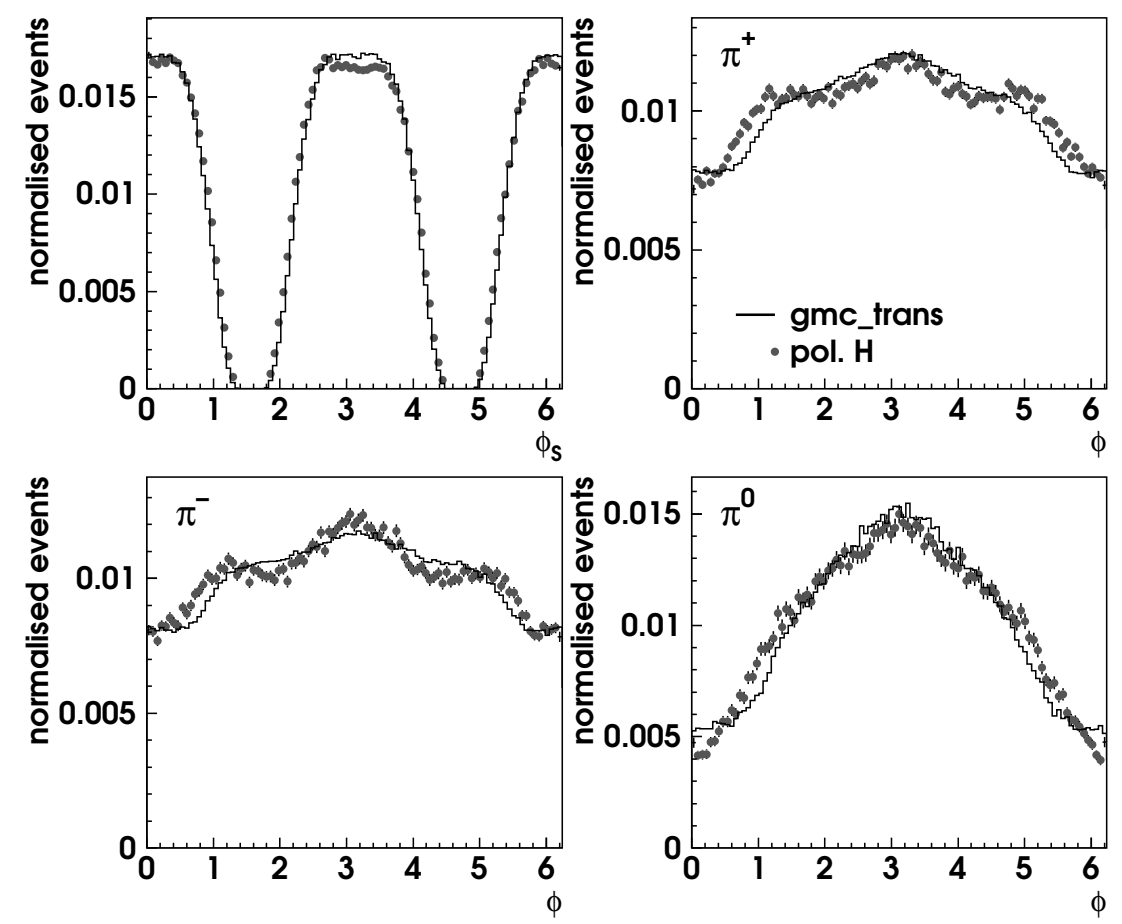

Figure 5.2.2: Comparison of the azimuthal angular distributions between HERMES data and the polarised Monte Carlo generator gmc_trans. All histograms are normalised to unit area.

allows the generation of maximum absolute values for Sivers and Collins $P_{h \perp}$-weighted moments. The mean values $\left\langle p_{T}^{2}\right\rangle$ and $\left\langle K_{T}^{2}\right\rangle=z^{2}\left\langle k_{T}^{2}\right\rangle$, which are assumed to be the same for all quark flavours, are chosen to be independent of $x$ and $z$, respectively. These parameters were varied iteratively for a good description of hadron transverse momentum distributions obtained from data. In Figure 5.2.1 the resulting distributions are plotted for $\left\langle p_{T}^{2}\right\rangle=\left\langle K_{T}^{2}\right\rangle=0.18 \mathrm{GeV}^{2}\left(\left\langle\left|\vec{p}_{T}\right|\right\rangle=\left\langle\left|\vec{K}_{T}\right|\right\rangle=0.38 \mathrm{GeV}\right)$. To speed up the simulation of detector events, the effects of tracking are parametrised with a new version of HSG, which includes the smearing of photons, instead of the time consuming tracking using HMC. The smearing of the photons is caused by the limited energy resolution of the calorimeter which leads to an uncertainty in the determination of the impact position and photon energies. The inclusion of the smearing results in an improved description of the $\pi^{0}$ distributions compared to earlier generators, which neglected the smearing of the photons. In Figure 5.2.1, the distributions of neutral pions reconstructed from the smeared photons (solid line) and from generated photons (dashed line) are compared in addition to HERMES data. The agreement for charged pions is better than for neutral pions and the rise of the $P_{h \perp}$ distribution for $\pi^{0}$ is better described by taking smearing into account also for photons. Also the azimuthal angular distributions are well described by the Monte Carlo, as can be seen in Figure 5.2.2. Here, the distribution of neutral pions reconstructed from photons neglecting any smearing (with the kinematics at generator level) is virtually indistinguishable from the distribution for smeared photons and therefore not shown in the figure. Note that neither the unpolarised cosine moments nor radiative effects are implemented in gmc_trans while the influence from the transverse target magnet is simulated 
in the analysed Monte Carlo production.

\subsubsection{Unweighted Asymmetry Amplitudes}

In the Monte Carlo generator gmc_trans, leading order parametrisations of the unpolarised FFs [Kre00] and the unpolarised and helicity DFs [Glü96], which are based on fits to world data, are implemented as functions of $x$ and $z$. Different models for the $x$ and $z$ dependencies of the transversity, Sivers, and Collins functions are available for the event generation. In most of the models a given moment of the DF or FF is proportional to $q(x)$, $\Delta q(x)$, or $D_{1}(z)$. The model parameters were chosen such that the extracted asymmetry amplitudes are comparable to the amplitudes observed in the data. The transversity functions are proportional to the helicity DF:

$$
\begin{aligned}
& \delta u(x)=0.7 \cdot \Delta u(x), \\
& \delta d(x)=0.7 \cdot \Delta d(x), \\
& \delta q(x)=0.3 \cdot \Delta q(x) \quad \text { for } \quad q=\bar{u}, \bar{d}, s, \bar{s} .
\end{aligned}
$$

In contrast, Sivers functions are modelled proportional to the unpolarised DF:

$$
\begin{array}{ll}
f_{1 T}^{\perp u}(x) & =-0.3 \cdot u(x), \\
f_{1 T}^{\perp d}(x) & =0.9 \cdot d(x), \\
f_{1 T}^{\perp q}(x) & =0.0 \quad \text { for } q=\bar{u}, \bar{d}, s, \bar{s} .
\end{array}
$$

The parametrisation of the unpolarised FFs fulfil isospin and charge conjugation symmetry [Kre00] leaving three independent FFs: the favoured, disfavoured, and strange function (cf. Section 2.5.1). In addition, disfavoured and strange FFs are equal in the parametrisation. The first moments of the Collins function are constructed proportional to the unpolarised FFs. The coefficient for the disfavoured FF is twice as large as for the favoured FF but has the opposite sign:

$$
\begin{aligned}
& H_{1, \text { fav }}^{\perp(1)}(z)=0.65 \cdot D_{1, \text { fav }}(z), \\
& H_{1, \text { dis }}^{\perp(1)}(z)=-1.30 \cdot D_{1, \text { dis }}(z) .
\end{aligned}
$$

Hence, on average favoured and disfavoured Collins function are of similar magnitude but of opposite sign.

In each kinematic bin four different asymmetry amplitudes can be determined. One

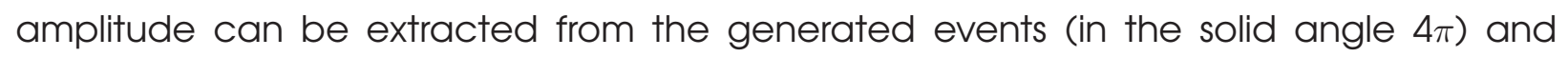
another one from the reconstructed events (in the HERMES acceptance) with the twodimensional fit procedure described in Chapter 4. Furthermore, for the reconstructed and generated events, the implemented asymmetry amplitudes $A_{\mathrm{imp}}^{\sin \left(\phi \pm \phi_{S}\right)}$, which are stored for each event $j$ in a data table, can be averaged over all events in a kinematic bin:

$$
\left\langle A_{\mathrm{imp}}^{\sin \left(\phi \pm \phi_{S}\right)}\right\rangle_{\text {rec }}=\frac{\sum_{j=0}^{N_{\text {rec }}} A_{\mathrm{imp}, j}^{\sin \left(\phi \pm \phi_{S}\right)}}{N_{\text {rec }}},\left\langle A_{\mathrm{imp}}^{\sin \left(\phi \pm \phi_{S}\right)}\right\rangle_{\text {gen }}=\frac{\sum_{j=0}^{N_{\text {gen }}} A_{\mathrm{imp}, j}^{\sin \left(\phi \pm \phi_{S}\right)}}{N_{\text {gen }}} .
$$

The implemented asymmetry amplitudes are integrated over $P_{h \perp}$ and can therefore not be compared to the extracted asymmetries in the individual $P_{h \perp}$ bins. In this case, the 

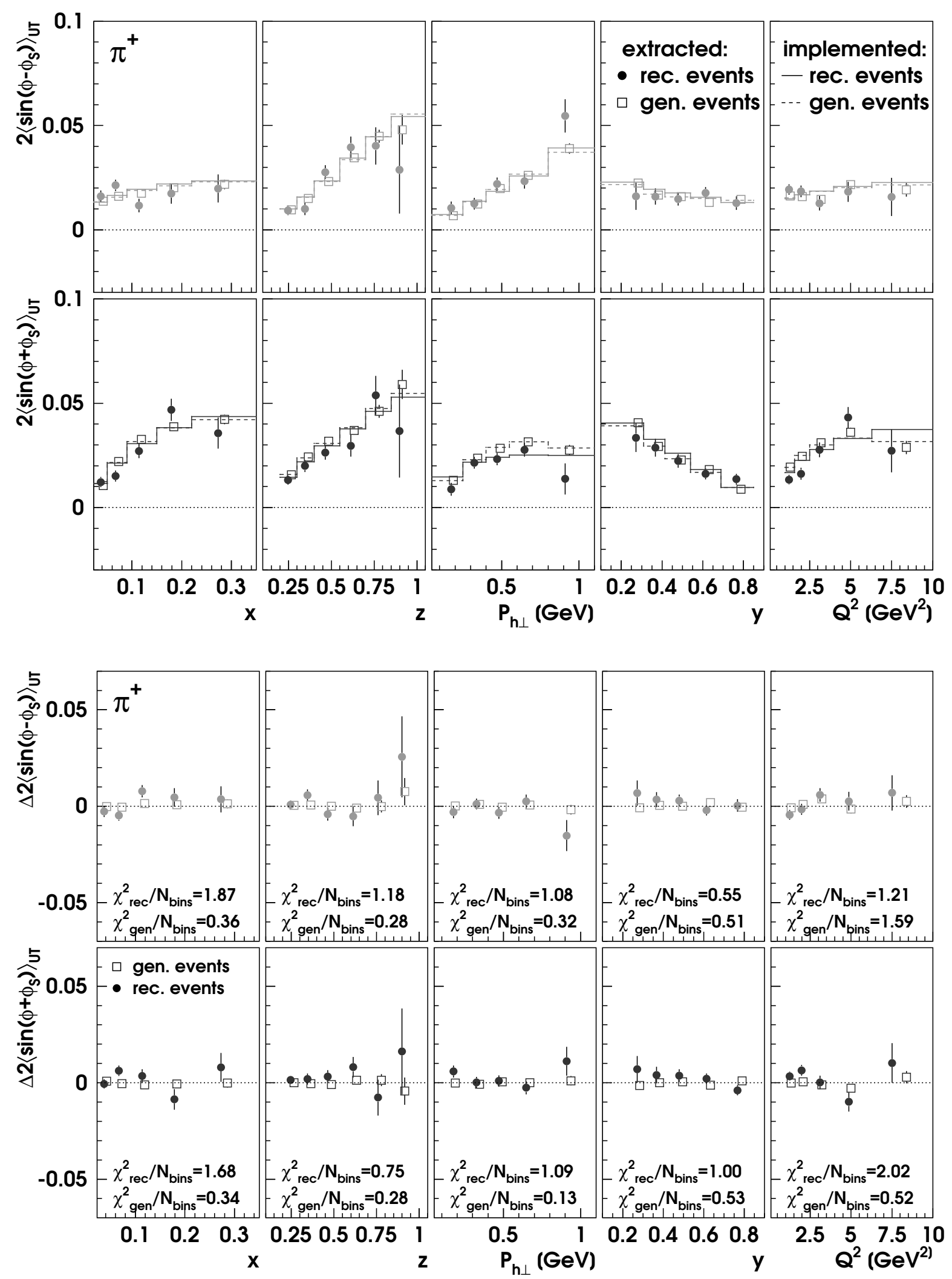

Figure 5.2.3: The implemented and extracted asymmetry amplitudes (upper half) and the differences between implemented and extracted amplitudes (lower half) for generated and reconstructed positive pion events. The generated events cover the whole range of the solid angle. 

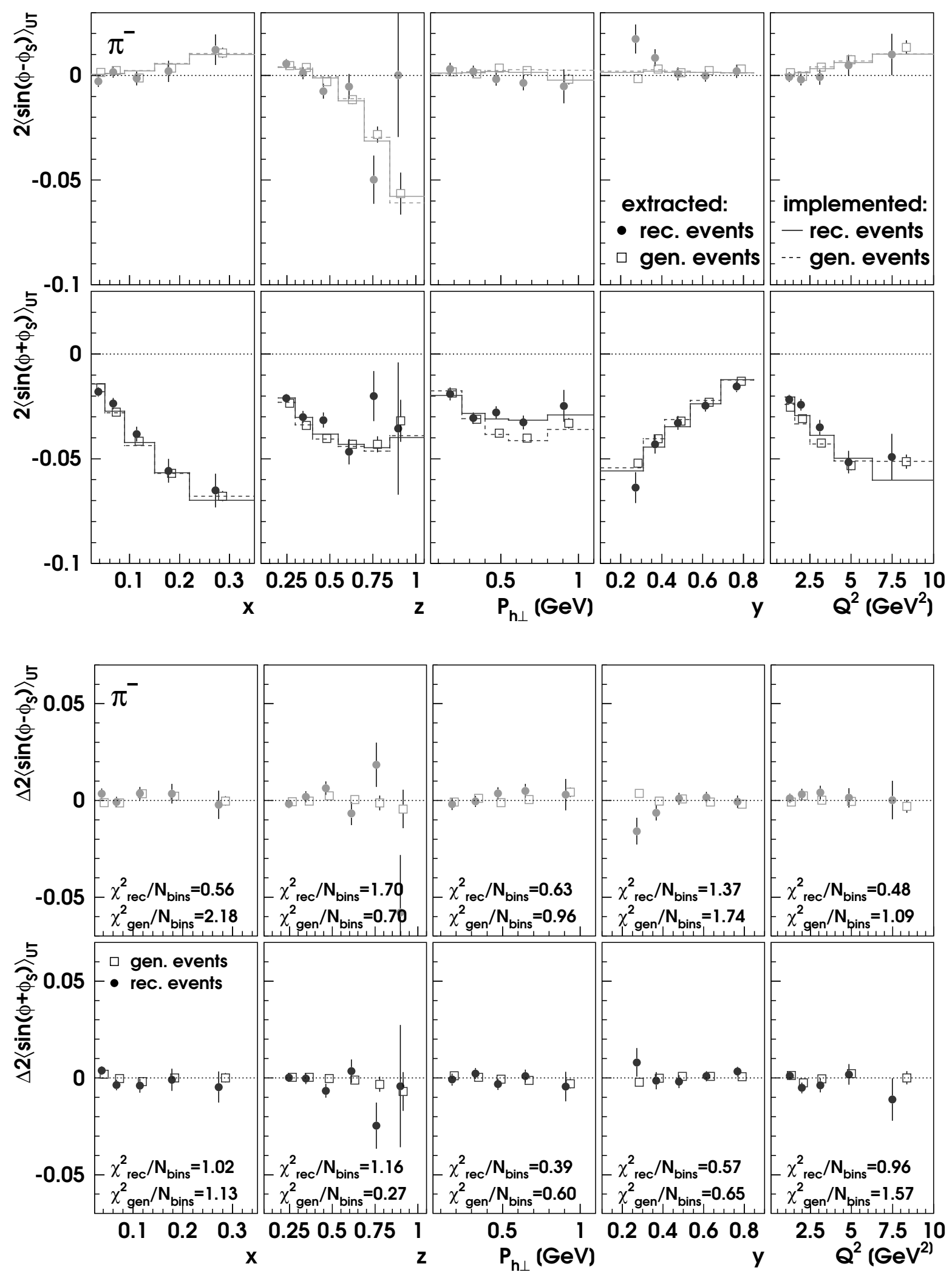

Figure 5.2.4: The implemented and extracted asymmetry amplitudes (upper half) and the differences between implemented and extracted amplitudes (lower half) for generated and reconstructed negative pion events. The generated events cover the whole range of the solid angle. 

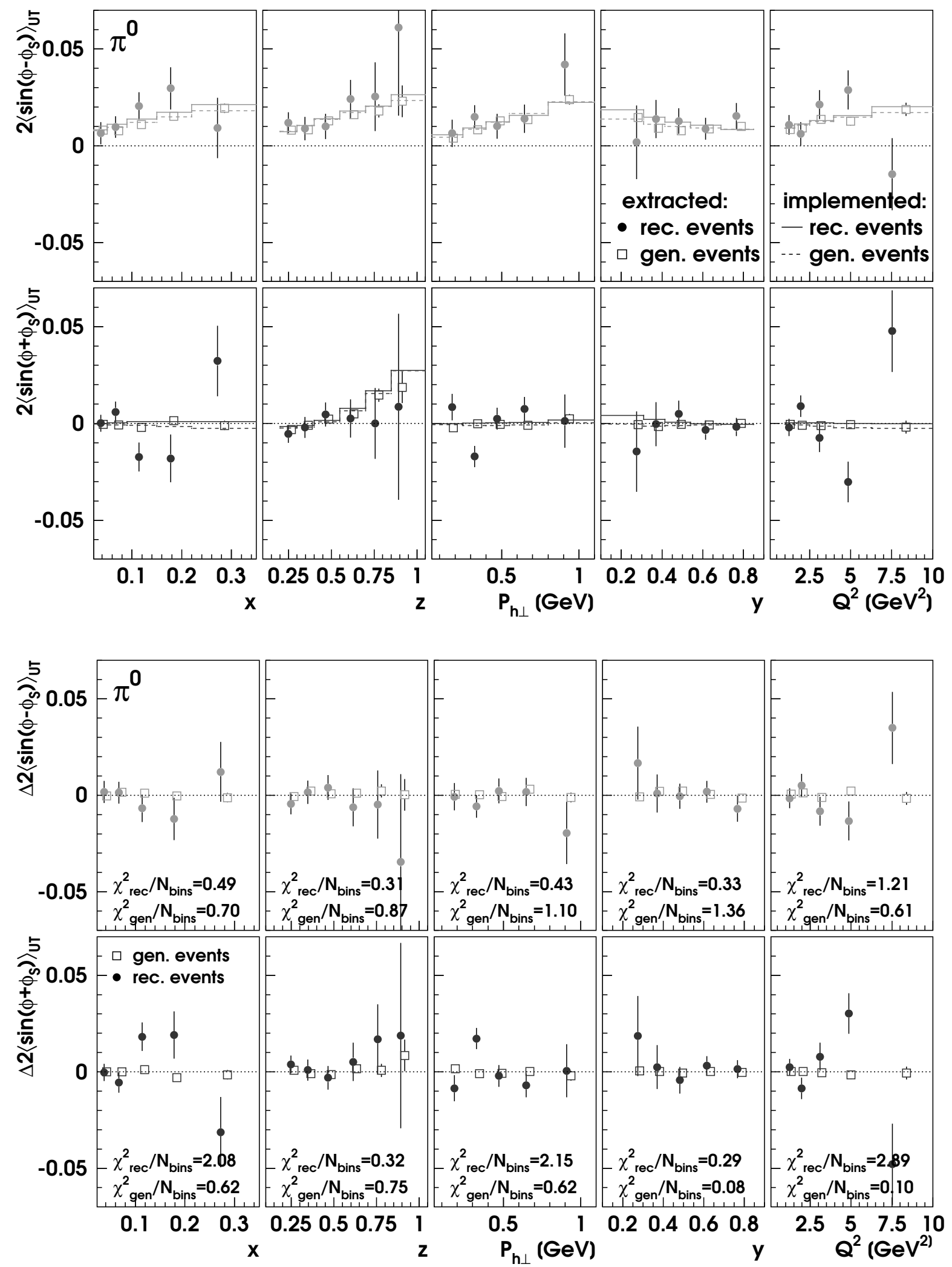

Figure 5.2.5: The implemented and extracted asymmetry amplitudes (upper half) and the differences between implemented and extracted amplitudes (lower half) for generated and reconstructed neutral pion events. The generated events cover the whole range of the solid angle. 
$P_{h \perp}$-dependent cross section ratios, which are also stored in the data table, are averaged in the different $P_{h \perp}$ bins. In the upper halves of Figures 5.2.3 - 5.2.5, all four asymmetry amplitudes are plotted dependent on the different kinematic variables $x, z, P_{h \perp}, y$, and $Q^{2}$ for the three different pion types. The extracted Sivers and Collins amplitudes are shown as open symbols for generated and as closed symbols for reconstructed events. The lines in these plots represent the implemented asymmetries averaged over the generated (dashed line) and the reconstructed events (solid line). The small differences between the implemented amplitudes for generated and reconstructed events are due to the different phase spaces of the two event samples resulting in different kinematic mean values $\langle x\rangle,\langle z\rangle,\langle y\rangle$, and $\left\langle Q^{2}\right\rangle$. The values of the extracted amplitudes of the generated events are corrected for the finite size of the 12 azimuthal bins by multiplication with 1.023 (see Section 5.1.2). In order to identify systematic deviations of the extracted amplitudes from the implemented amplitudes, a large Monte Carlo data set of reconstructed events was produced with around four times the statistics of the HERMES data set on transversely hydrogen recorded in the years 2002 to 2004.

In the lower halves of the figures, the difference between implemented and extracted asymmetry amplitudes:

$$
\Delta 2\left\langle\sin \left(\phi \pm \phi_{S}\right)\right\rangle_{\mathrm{UT}}=2\left\langle\sin \left(\phi \pm \phi_{S}\right)\right\rangle_{\mathrm{imp}}-2\left\langle\sin \left(\phi \pm \phi_{S}\right)\right\rangle_{\mathrm{ext}}
$$

is shown for generated and reconstructed events. Additionally, the $\chi^{2}$ is given which is determined as:

$$
\chi^{2}=\sum_{i=0}^{N_{\mathrm{bins}}}\left(\frac{\Delta 2\left\langle\sin \left(\phi \pm \phi_{S}\right)\right\rangle_{\mathrm{UT}, i}}{\sigma_{\mathrm{ext}, i}^{\sin \left(\phi \pm \phi_{S}\right)}}\right)^{2}
$$

where $\sigma_{\text {ext, } i}^{\sin \left(\phi \pm \phi_{S}\right)}$ is the uncertainty on the extracted amplitudes in each kinematic bin $i$.

For all three pion types, the extracted amplitudes of the generated events reproduce the implemented amplitudes. In order to achieve this, the $\theta_{\gamma^{*} h}$ cut had to be omitted. For a pure Monte Carlo data set on the generator level this cut is obsolete as the azimuthal angles are not influenced by the limited spectrometer resolution in that case. The cut on $\theta_{\gamma^{*} h}$ rejects events with low $P_{h \perp}$ contributing to the integration of the asymmetry over the transverse hadron momentum. Since for the Gaussian ansatz the polarised cross sections are proportional to $P_{h \perp}$ [Bac04b], the contribution from the low $P_{h \perp}$ events to the integrated polarised cross section is lower than to the integrated unpolarised cross section. Hence, the asymmetry increases with an applied cut on $P_{h \perp}$ or $\theta_{\gamma^{*} h}$, respectively. The effect is of the order of a couple of percent and compensates the effects of the finite azimuthal bin size and of the spectrometer (the latter result in an asymmetry amplitude which is around $5 \%$ smaller, as described in Sections 5.1.2 and 5.1.3). This explains the nice agreement of the extracted and implemented asymmetry amplitudes in case of the reconstructed events where the cut on $\theta_{\gamma^{*} h}$ is applied and the results are not corrected for the finite azimuthal bin size. However, the size of the effect of the $\theta_{\gamma^{*} h}$ cut is model dependent and the finite bin size effect decreases with increasing number of azimuthal bins so that the cancellation of the two effects is a mere coincidence. 

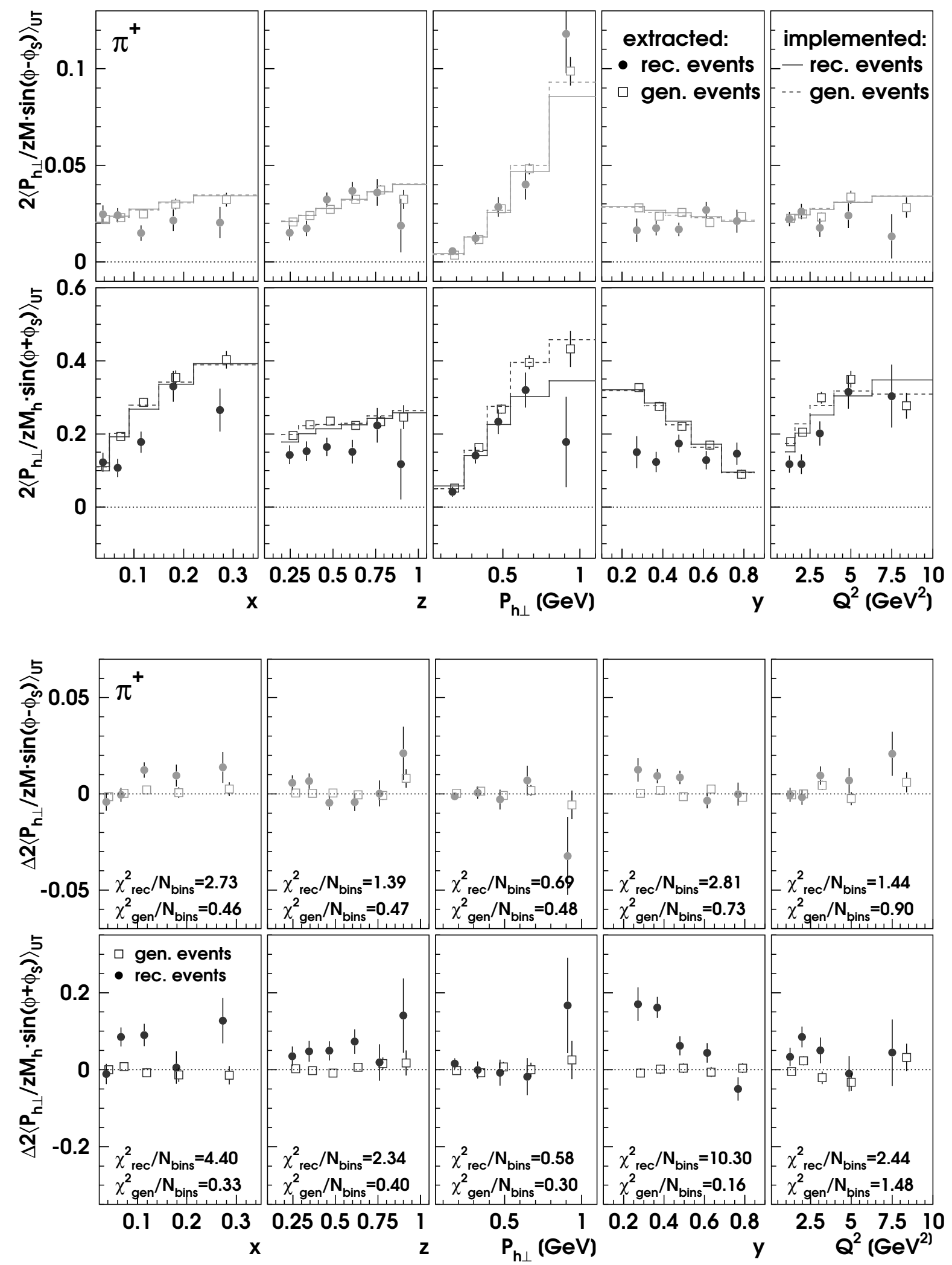

Figure 5.2.6: The implemented and extracted $P_{h \perp}$-weighted asymmetry amplitudes (upper half) and the differences between implemented and extracted amplitudes (lower half) for generated and reconstructed positive pion events. The generated events cover the whole range of the solid angle. 

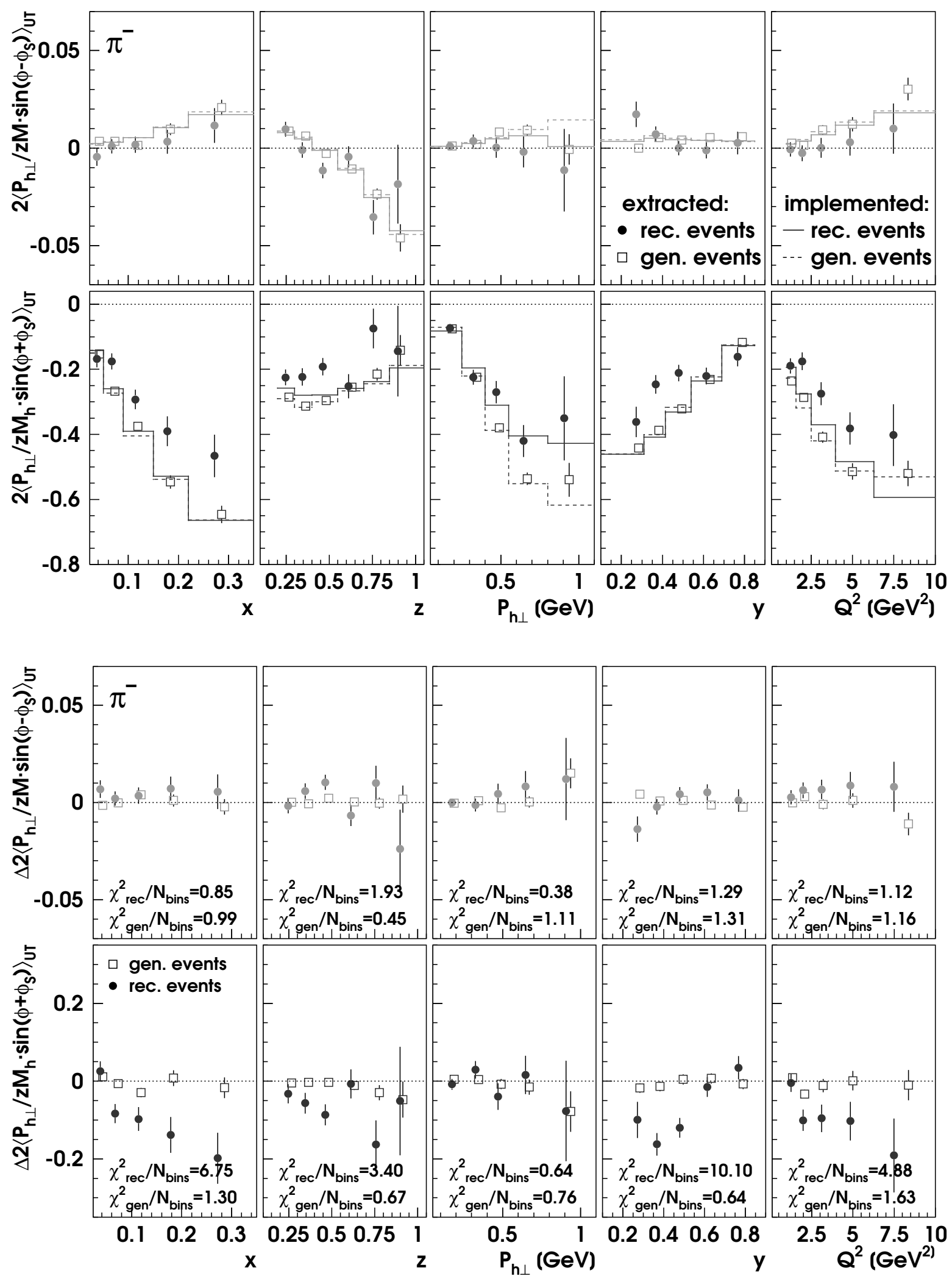

Figure 5.2.7: The implemented and extracted $P_{h \perp}$-weighted asymmetry amplitudes (upper half) and the differences between implemented and extracted amplitudes (lower half) for generated and reconstructed negative pion events. The generated events cover the whole range of the solid angle. 

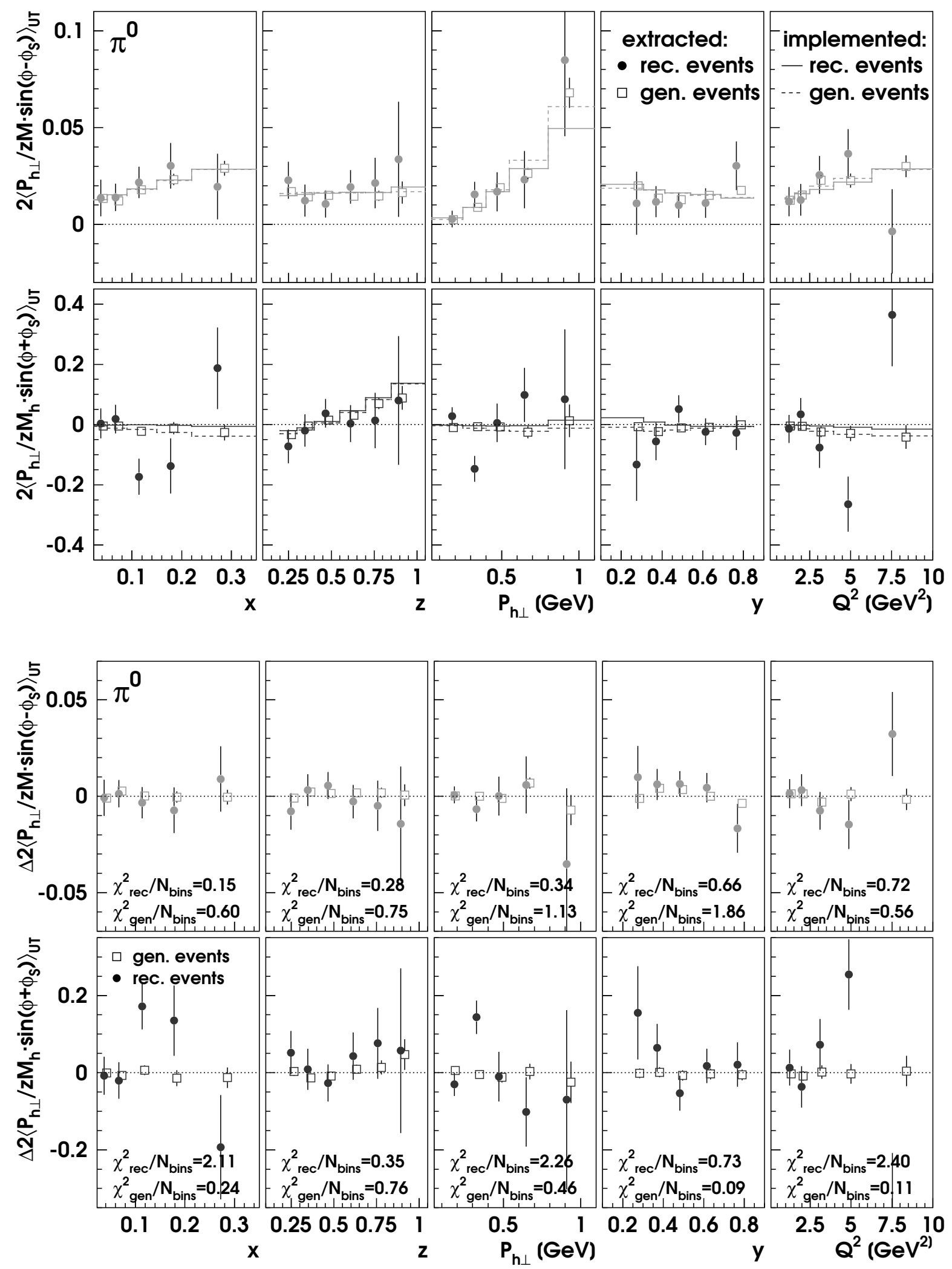

Figure 5.2.8: The implemented and extracted $P_{h \perp}$-weighted asymmetry amplitudes (upper half) and the differences between implemented and extracted amplitudes (lower half) for generated and reconstructed neutral pion events. The generated events cover the whole range of the solid angle. 


\subsection{2 $\quad P_{h \perp}$-Weighted Asymmetry Amplitudes}

For the $P_{h \perp}$-weighted asymmetry amplitudes, the studies with the unpolarised Monte Carlo generators showed large acceptance effects which can be further investigated with the gmc_trans generator. With the same settings for the DFs and FFs, implemented and extracted asymmetry amplitudes of the generated and reconstructed events are obtained. They are shown in Figures 5.2.6 - 5.2.8 for all pion types together with the difference between implemented and extracted amplitudes. For the charged pions, the absolute values of the extracted Collins and Sivers amplitudes for the reconstructed events are systematically lower in most of the kinematic bins. The deviation is larger in case of the Collins amplitudes, as can be inferred from the reduced $\chi^{2}$ given in the figures. As the only kinematic distribution, the $P_{h \perp}$ dependence is well reproduced because the integration over $P_{h \perp}$ is restricted to the rather small range within each bin. However, it is difficult to interpret the results since the separation of DF and FF is not achieved with an incomplete integration over $P_{h \perp}$. The nice reproduction of the amplitudes in the individual $P_{h \perp}$ bins in contrast to the large deviations for the binning in the other kinematic variables suggests that an insufficient integration over $P_{h \perp}$ causes the problem. The asymmetry amplitudes of the neutral pions are well reproduced which is expected only in case of the Collins amplitudes due to the small values for the implemented amplitudes.

For a correction of the $P_{h \perp}$-weighted asymmetry amplitudes, the acceptance function which depends on the five quantities $x, z, P_{h \perp}, \phi$, and $\phi_{S}$, has to be determined. In an attempt to approximate the acceptance function by only three out of the five kinematic quantities no improvements compared to the results shown in Figures 5.2.6 - 5.2.8 could be achieved. Further progress will either require the determination of a five-dimensional acceptance correction function or a new extraction method which is insensitive to acceptance effects. Such a new extraction method using an unbinned maximum likelihood fit is under study [Mil05a, Mil05b]. The interpretation of $P_{h \perp}$-weighted asymmetry amplitudes has to await the resolution of this issue.

\subsection{Concluding Remarks}

With the unpolarised Monte Carlo generators, the relative systematic uncertainty on the unweighted asymmetry amplitudes could be estimated. The values of $5 \%$ for charged pions and $7 \%$ for kaons and neutral pions include the influence of the extraction method and effects from the spectrometer, e.g., detector resolutions and geometric acceptance. Since the implemented asymmetry amplitudes did not contain the correlation with the transverse quark momenta, additional studies were performed with the Monte Carlo generator gmc_trans which simulates azimuthal distributions due to intrinsic quark transverse momenta. In case of the unweighted amplitudes, the implemented amplitudes could be well reproduced and the systematic uncertainty did not have to be increased. The large acceptance effects visible in the extracted $P_{h \perp}$-weighted amplitudes were already indicated by the results of a study with the unpolarised Monte Carlo generators. The interpretation of $P_{h \perp}$-weighted amplitudes will be possible either with a correction of these effects or with a new extraction method which is not influenced by the HERMES acceptance. 

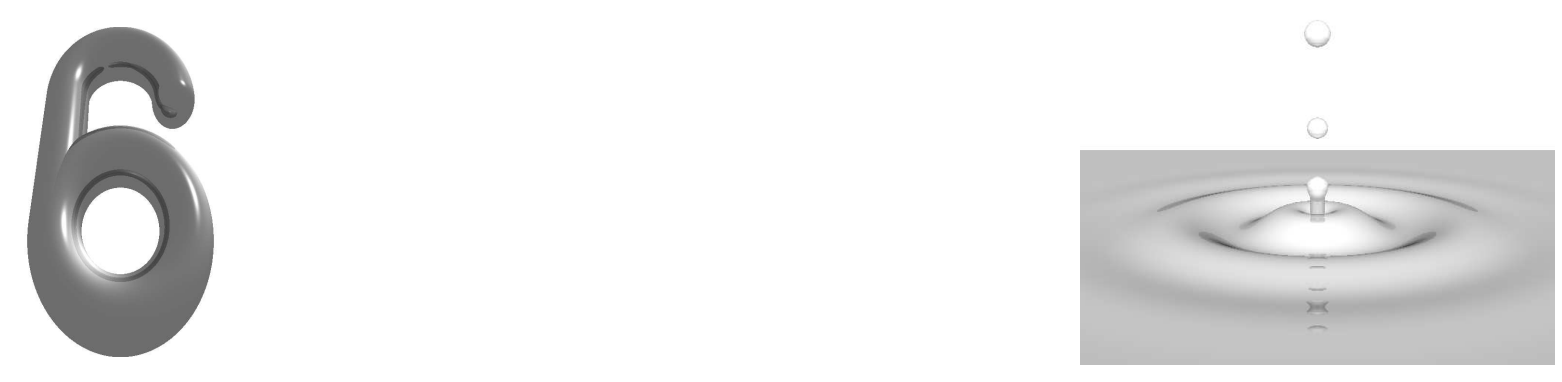

\section{Contribution from Exclusively Produced Vector Mesons}

The pion and kaon samples of semi-inclusive deep-inelastic scattering events detected by the HERMES spectrometer contain decay particles of exclusively produced vector mesons. For the interpretation of the measured asymmetry amplitudes in terms of quark distribution and fragmentation functions, these events might be considered as background contributions. Certain fragmentation models, e.g., the LUND string fragmentation model (see Section 2.5.1), do not include exclusively produced vector mesons. In contrast, in cross section models based on Feynman diagrams, exclusive vector meson production contributes among other diagrams to the semi-inclusive deep-inelastic scattering cross section. However, the applicability of these models becomes questionable as soon as this process starts to dominate the cross section, for instance in certain kinematic regimes.

In this chapter, the fraction of exclusively produced vector mesons is determined with the help of the PYTHIA Monte Carlo generator. This contribution of exclusive vector mesons cannot be excluded entirely from the analysis as the second (and or third) decay particle is not observed in most of these events. The two possible sources of asymmetry amplitudes of the decay hadrons are investigated. In addition, asymmetry amplitudes of exclusively produced $\rho^{0}$ mesons are extracted from the HERMES data, once for the kinematics of the reconstructed vector meson and once for its decay pions. Both asymmetry amplitudes are consistent with zero. Furthermore, an asymmetry in the yield difference of positive and negative pions is constructed in which the exclusive vector meson contributions cancel. The significantly positive Collins and Sivers amplitudes in this asymmetry prove that amplitudes extracted from the semi-inclusive pion samples are not predominantly caused by decay pions of exclusive vector mesons.

\subsection{The Vector Meson Dominance Model}

In the deep-inelastic scattering process, the exchanged virtual photon interacts with a quark of the nucleon. Besides the so-called bare part of the photon, which couples to the electromagnetic charge of the quarks, the virtual photon can also fluctuate into a 


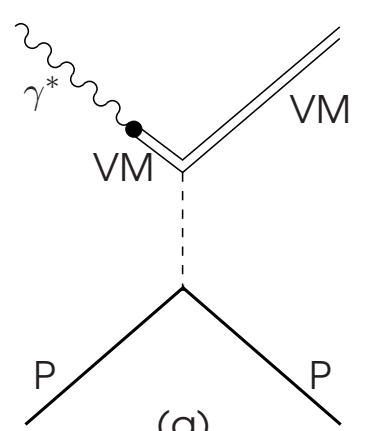

(a)

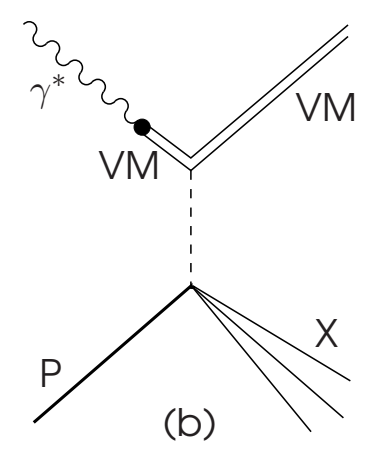

(b)

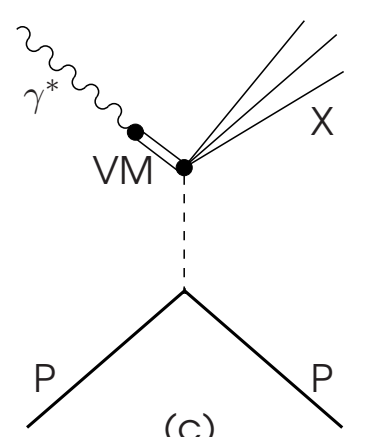

(c)

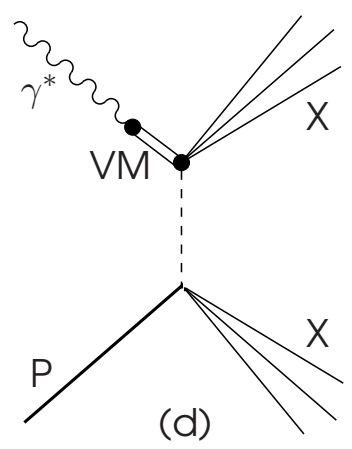

(d)

Figure 6.1.1: The elastic (a), proton single diffractive (b), vector meson single diffractive (c), and double diffractive (d) scattering processes for vector meson production.

quark-antiquark pair. This pair can live long enough to create a cloud of gluons around the quarks, such that the state develops into a hadronic state which then interacts with the nucleon. This interaction of the hadronic part of the photon with the nucleon can be described only phenomenologically in terms of models for hadron-hadron interactions. The vector meson dominance (VMD) model [Sak69], which describes the experimental data over a wide energy range successfully, is the most prominent model. It is implemented in the PYTHIA Monte Carlo generator described in Section 5. 1. In the VMD model, the hadronic part of the photon is interpreted as fluctuations into the vector mesons $\rho^{0}, \omega$, and $\phi$ that carry the same quantum numbers as the photon. The scattering process then factorises into the coupling of the virtual photon to the vector meson and the interaction of the vector meson with the nucleon by exchange of a particle with quantum numbers of the vacuum. It can be classified in elastic, single, and double diffractive scattering processes which are sketched in Figure 6.1.1. In single diffractive processes either the proton or the vector meson gives rise to a resonance or a number of final state particles whose combined quantum numbers match the quantum numbers of the initial particle. Processes in which both the proton and the vector meson break up are called double diffractive. The term diffractive has its origin in the analogy to optical diffraction where light waves give a characteristic intensity pattern behind an opaque obstacle of a size comparable to the wave length. The maximum of the intensity pattern is observed on an axis through the centre of the obstacle. Similar to optical diffraction, in diffractive hadron scattering the final hadrons or fragments move approximately in the same direction as the incident hadron because of only a small transverse momentum transfer.

Besides the hadronic part, the photon exhibits also a so-called anomalous part which is a fluctuation into a quark-antiquark pair with a larger virtuality than the vector meson. Alternatively, this can be viewed as an excited higher resonance of a vector meson which scatters from the nucleon into a vector meson. These states are taken into account by the Generalised VMD (GVMD) model [Sak72]. In the PYTHIA generator the anomalous or GVMD states are assigned to vector mesons of type $\rho^{0}, \omega$, and $\phi$ with a high virtuality. If the mass difference between this highly virtual state and the corresponding ground state vector meson exceeds a threshold, which can be set by parameter in the Monte Carlo, these states fragment into other, lighter mesons. Otherwise they are forced to decay isotropically into a two-body channel. 


\subsection{Exclusively Produced Vector Mesons as Background Events}

If the decay particles of exclusively produced vector mesons are treated as a background contribution to the semi-inclusive DIS sample, the measured asymmetry amplitudes $A_{\text {meas }}$ can be corrected for this contribution by:

$$
A_{\text {cor }}=\frac{N_{\text {tot }}}{N_{\text {sig }}} A_{\text {meas }}-\frac{N_{\mathrm{VM}}}{N_{\text {sig }}} A_{\mathrm{VM} \rightarrow \mathrm{h}}=\frac{1}{1-f_{\mathrm{VM}}} A_{\text {meas }}-\frac{f_{\mathrm{VM}}}{1-f_{\mathrm{VM}}} A_{\mathrm{VM} \rightarrow \mathrm{h}},
$$

where the vector meson fraction $f_{\mathrm{VM}}$ is defined as the number of hadrons from vector meson decays $N_{\mathrm{VM}}$ divided by the total number of hadrons $N_{\text {tot }}$. The difference between $N_{\text {tot }}$ and $N_{\mathrm{VM}}$ is the number of hadrons $N_{\text {sig }}$ which do not originate from an exclusively produced vector meson. Two sources contribute to the amplitude $A_{\mathrm{VM} \rightarrow \mathrm{h}}$ of the decay particles. On the one hand, a Collins or Sivers type asymmetry amplitude $A_{\mathrm{VM}}$ of the vector meson is partially transferred to the hadron. On the other hand, the decay angular distributions can introduce asymmetries in the detected hadron sample. In general, the asymmetry amplitude of the decay particles can be expressed as:

$$
A_{\mathrm{VM} \rightarrow \mathrm{h}}=T \cdot A_{\mathrm{VM}}+A_{\text {decay }},
$$

where the transfer coefficient $T$ describes how much of the vector meson asymmetry amplitude is transferred to its decay particles. Hence, for a correction of the measured asymmetries, four quantities are needed: the vector meson fraction $f_{\mathrm{VM}}$, the transfer coefficient $T$, the asymmetry amplitude $A_{\mathrm{VM}}$ of the exclusively produced vector mesons, and the asymmetry amplitude $A_{\text {decay }}$ acquired in the decay process.

\subsubsection{The Vector Meson Fraction}

The fraction of elastic and diffractive vector meson events in the semi-inclusive DIS event sample of a PYTHIA Monte Carlo production is calculated in each kinematic bin. In the PYTHIA generator, the cross-section ratio between exclusive $\omega$ and $\rho^{0}$ production is set to approximately 0.1 [Lie04], while from HERMES data the exclusive $\omega$ cross section could be estimated to be $15 \%$ of the exclusive $\rho^{0}$ cross section [Tyt01]. Therefore, all exclusive $\omega$ events generated by PYTHIA are scaled by $0.15 / 0.10$ to account for the cross-section ratio measured at HERMES. The obtained vector meson fractions of the different meson types and their dependence on the kinematic variables $x, P_{h \perp}$, and $z$ can be found in Fig. 6.2.1. The full symbols show the vector meson fraction for all mesons taken into account $\left(\rho^{0}, \omega\right.$, and $\left.\phi\right)$. In addition, the open symbols give the main contribution in each distribution. These are $\rho^{0}$ meson decays for the charged pions, $\omega$ meson decays for the neutral pion, and $\phi$ meson decays for the charged kaons. The vector meson fractions for all kinematic bins used in the analysis can be found in Tables F.8 - F. 12. For all hadron types, an increase of the vector meson fraction with decreasing $x$ is observed. This is equivalent to decreasing $Q^{2}$ due to the strong correlation of $x$ and $Q^{2}$ in the HERMES acceptance. The fractions increase also with increasing $z$ except for (positive) kaons. Especially for the charged pions, the exclusive vector meson events dominate in the highest $z$ region. On average, every second detected $\pi^{ \pm}$with $z$ close to 1 is a $\rho^{0}$ decay particle. The strong rise of the vector meson fraction for high $z$ is caused by the boost from the vector meson rest frame into the laboratory system which results in a low and high energetic 

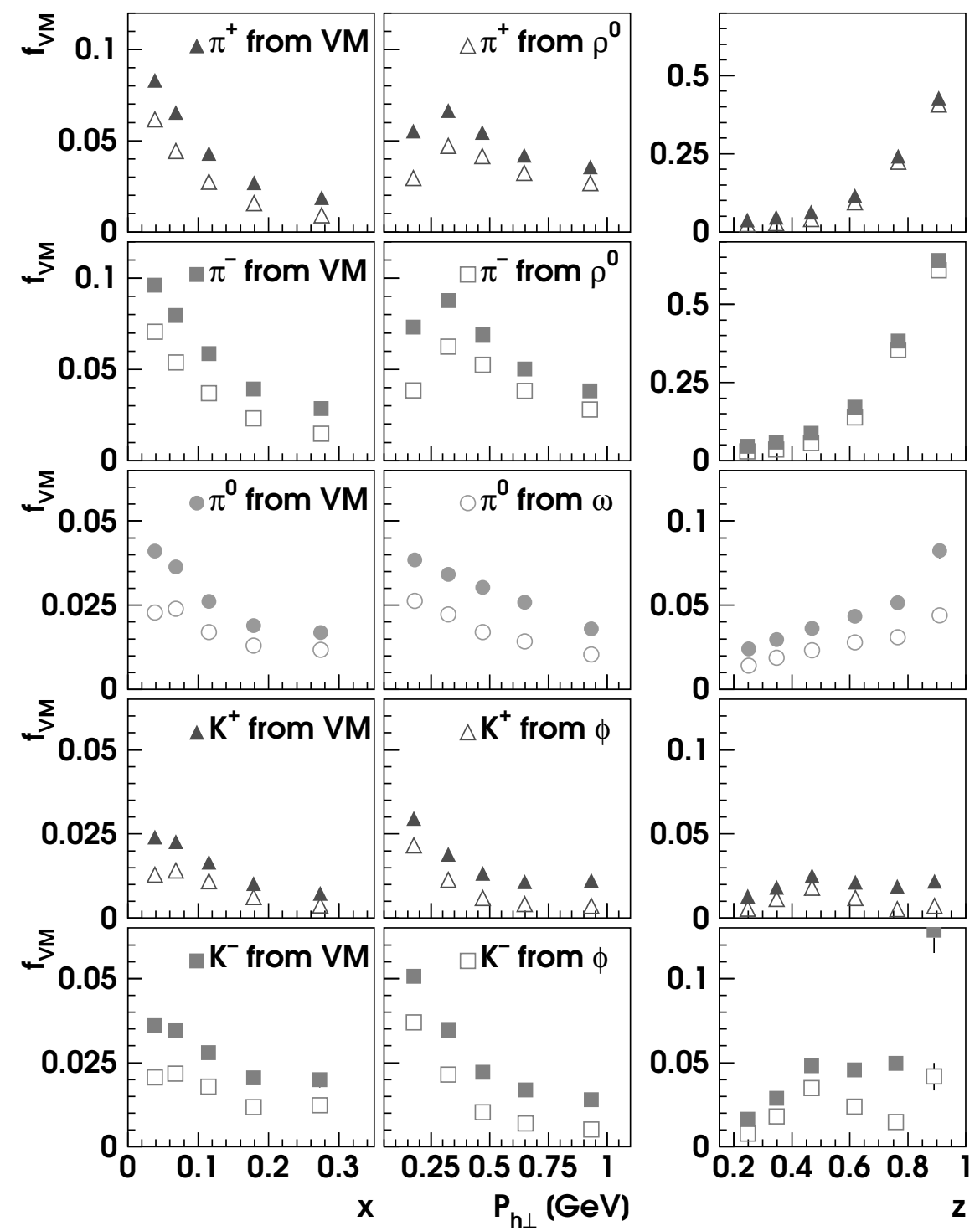

Figure 6.2.1: Vector meson fractions for pion and kaon sample in semi-inclusive DIS as functions of $x, P_{h \perp}$, and $z$. Note the different vertical scales in the panels.

pion. However, the high $z$ range is not included in the analysis of semi-inclusive hadrons with $0.2<z<0.7$. In the other kinematic bins and for the other hadron types, the fractions stay below $10 \%$. The lowest amount of vector meson decays is seen in the $K^{+}$sample.

One possibility to decrease the large vector meson fractions in the charged pion samples is to raise the cut on $Q^{2}$ because the vector meson production cross section is proportional to $Q^{-6}$ for large $Q^{2}$ [Tyt01]. In fact, a cut of $Q^{2}>2 \mathrm{GeV}^{2}$ reduces the vector meson fractions by a factor of somewhat less than 2. Yet this approach is not adopted as at the same time more than $50 \%$ of the overall data are rejected, mainly affecting the low $x$ region.

$20 \%$ of the $\rho^{0}$ states contributing to the charged pion sample and $35 \%$ of the $\phi$ states contributing to the charged kaon sample are anomalous states. An even larger fraction of $\sim 60 \%$ of the $\omega$ mesons contributing to the neutral pion sample stem from the anoma- 
lous states, according to the PYTHIA Monte Carlo generator. Note, however, that the PYTHIA generator has been tuned to HERMES data only for $\rho^{0}$ and $\phi$ mesons [Lie04]. So far, the $\omega$ meson has not been taken into account in the tuning process except for in the additional scale factor of $0.15 / 0.10$ mentioned above.

\subsubsection{The Transfer Coefficients}

Only part of a possible azimuthal asymmetry of the vector meson will be transferred to the decay particles because in general their momentum vectors deviate from the direction of the vector meson. In case of the simultaneous extraction of two asymmetry amplitudes, besides the transfer of the individual amplitudes also a cross transfer can appear: A vector meson Sivers amplitude can cause a Collins amplitude in the decay particle sample and vice versa. The relation between the vector meson amplitudes $A_{\mathrm{VM}}$ and the decay hadron amplitudes $A_{h}$ can be expressed by a transfer matrix $\boldsymbol{T}$ :

$$
\left(\begin{array}{c}
A_{h, s} \\
A_{h, c}
\end{array}\right)=\left(\begin{array}{ll}
T_{s s} & T_{c s} \\
T_{s c} & T_{c c}
\end{array}\right)\left(\begin{array}{c}
A_{\mathrm{VM}, s} \\
A_{\mathrm{VM}, c}
\end{array}\right),
$$

where the characters $c$ and $s$ in the subscript indicate the Collins and Sivers type amplitudes. Like the relative correction matrix (see Section 5.1.1), the transfer matrix can be determined by means of an asymmetry amplitude scan. For exclusively produced vector mesons, asymmetry amplitudes are implemented using the azimuthal angle of the vector meson $\phi_{h=\mathrm{VM}}$. From the azimuthal angles of the decay particles, the amplitudes are extracted and fitted with a straight line dependent on the implemented amplitudes. The obtained slopes correspond to the transfer $\left(T_{s s}, T_{c c}\right)$ and cross transfer coefficients $\left(T_{c s}, T_{s c}\right)$. The PYTHIA Monte Carlo generator for unpolarised nucleons is used for the determination of $\boldsymbol{T}$ for exclusively produced $\rho^{0}$ mesons which decay into a $\pi^{+} \pi^{-}$pair at a branching ratio of almost 100\% [Eid04]. A good description of the experimentally observed decay angular distributions for exclusive $\rho^{0}$ mesons is achieved with this unpolarised generator [Lie04]. Due to the small cross section for $\omega$ production, the available PYTHIA Monte Carlo productions do not have enough statistics to determine transfer coefficients for the $\omega$ decay. A second unpolarised Monte Carlo generator DIPSI [Arn97b], including only elastic but no single or double diffractive events, allows the estimate of transfer coefficients for neutral pions. Also this generator provides a fair description of the $\omega$ decay angular distributions on unpolarised targets [Tyt01]. The determination of the transfer coefficients for exclusive $\phi$ mesons is impeded by the small amount of reconstructed decay kaons in the PYTHIA Monte Carlo production. Also for the $\phi$ decay into a $K^{+} K^{-}$pair, the DIPSI generator could be used. However, with the small vector meson fractions for kaons and the present level of precision in the HERMES data for kaon production, no attempt was made to determine transfer coefficients for exclusive $\phi$ mesons.

\section{Transfer Coefficients of Exclusively Produced $\rho^{0}$ Vector Mesons}

For the determination of the transfer matrix of exclusive $\rho^{0}$ mesons, a PYTHIA Monte Carlo production with HSG is used which reproduces the HERMES data on charged pions at the same level of precision as a fully tracked production (see Chapter 5). Again a $12 \times 12$ 

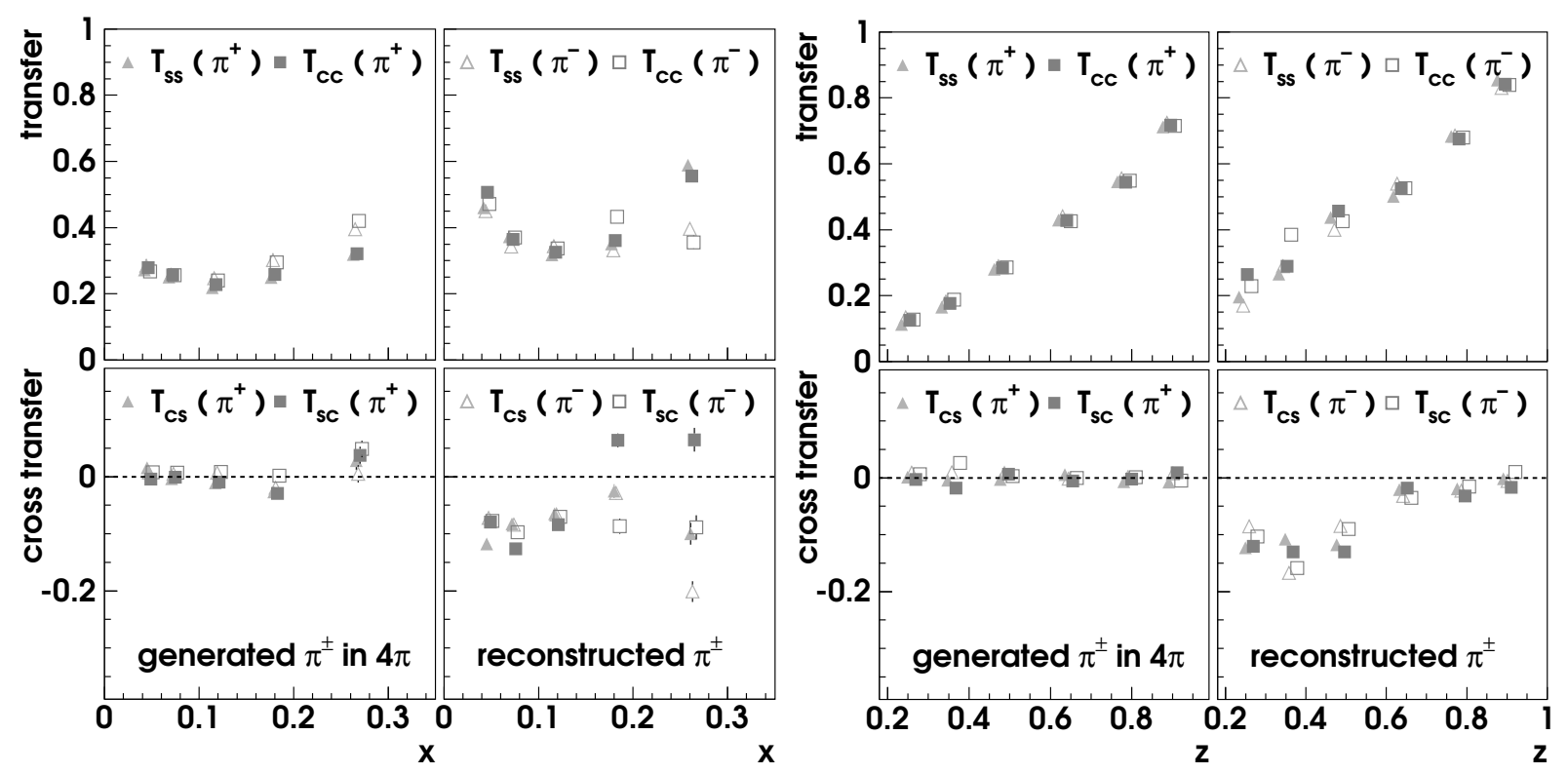

Figure 6.2.2: Transfer matrix elements for charged pions from $\rho^{0}$ decays in $4 \pi$ and in the HERMES acceptance, dependent on $x$ and $z$.

azimuthal binning for the extraction of the pion asymmetry amplitudes is applied. The amplitudes are calculated once for the generated pions in the complete $4 \pi$ solid angle and once for the reconstructed pions in the HERMES acceptance. The transfer coefficients $T_{c c}$ and $T_{s s}$ and the cross transfer coefficients $T_{s c}$ and $T_{c s}$ are shown in Figure 6.2.2, on the left-hand side for the different $x$ bins and on the right-hand side dependent on $z$. No cross transfer is present for the generated pion sample in $4 \pi$. Yet for the recon-
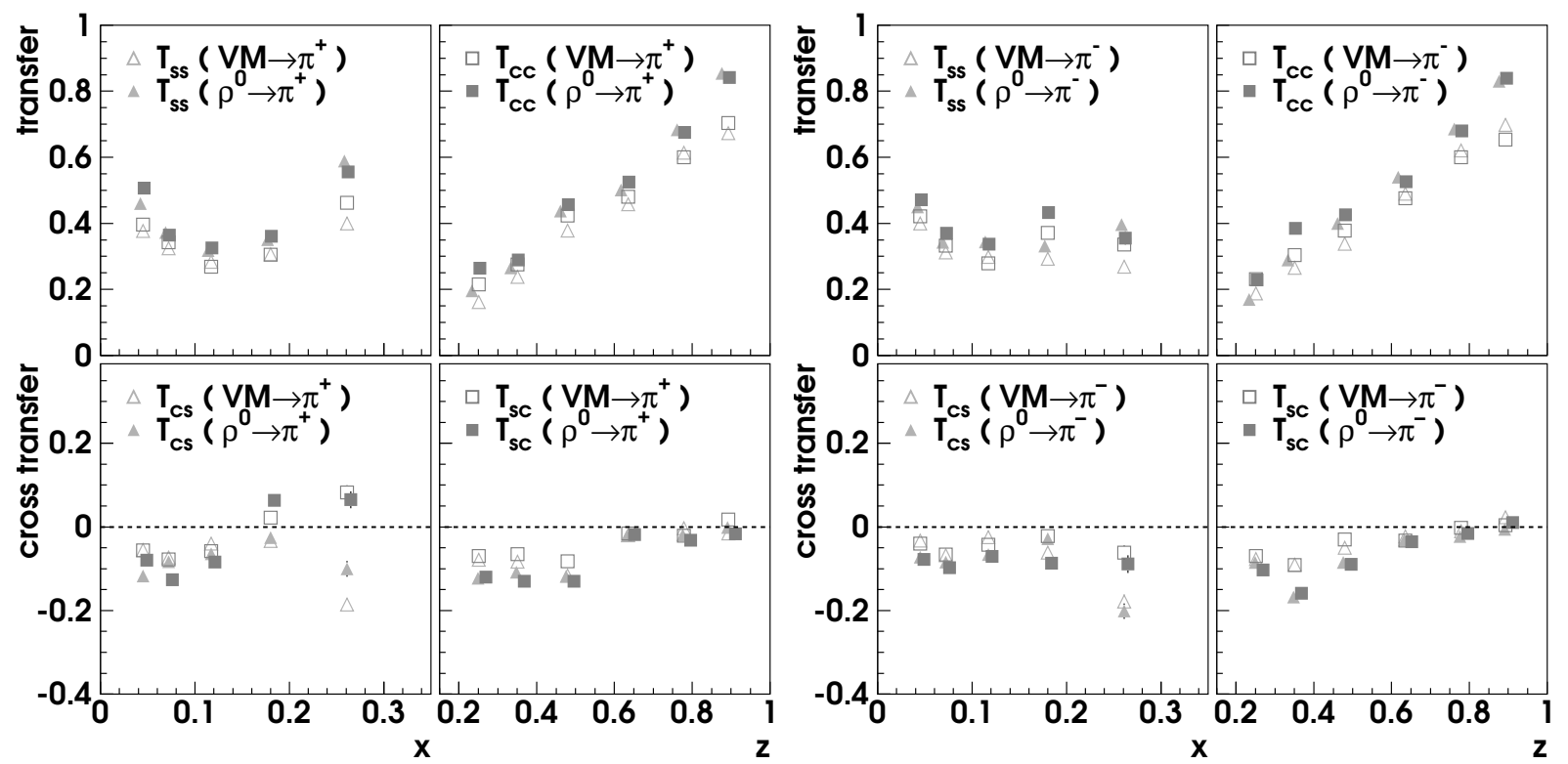

Figure 6.2.3: Comparison of the transfer coefficients for reconstructed charged pions ( $\pi^{+}$on the left, $\pi^{-}$on the right side) from $\rho^{0}$ decays and from all exclusively produced vector mesons dependent on $x$ and $z$. 


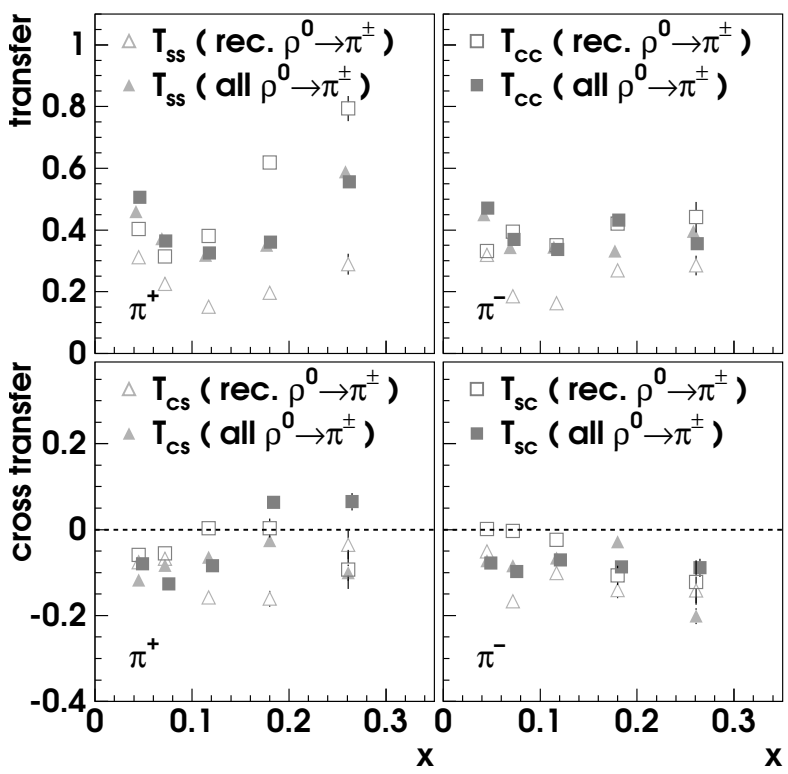

Figure 6.2.4: Elements of $\boldsymbol{T}$ for charged pions as decay particles of all $\rho^{0}$ events and of reconstructed $\rho^{0}$ events dependent on $x$.

structed events, the finite acceptance introduces a non-zero cross transfer. Furthermore, the transfer coefficients are lower for pions generated in $4 \pi$ than for the reconstructed event sample. Pions with high $z$ inherit a large energy fraction and hence are almost collinear with the parent vector meson which causes the rise of the transfer coefficient with increasing $z$. For the reconstructed decay pions, the transfer and cross transfer coefficients in all kinematic bins used in the analysis are listed in Tables F.2 and F.3.

The vector meson contribution originates mainly from $\rho^{0}$ decays but also from other vector meson and anomalous state decays. Model asymmetry amplitudes can be implemented in the Monte Carlo for vector meson events. Transfer coefficients can then be determined for pions originating from all states and not only from $\rho^{0}$ mesons. In Figure 6.2.3, the obtained coefficients are compared for decay pions of $\rho^{0}$ mesons and for pions originating from elastic and diffractive vector meson production. The transfer values for all vector meson events are slightly lower than the transfer values of exclusive $\rho^{0}$ mesons. In order to estimate the vector meson contribution to the measured asymmetry amplitudes of the pion sample the assumption was made that the asymmetry amplitudes for the full vector meson ensemble can be approximated by those of the exclusive $\rho^{0}$ sample.

A measurement of asymmetry amplitudes of $\rho^{0}$ decay pions is only possible for events in which the $\rho^{0}$ is identified, i.e., both pions are in the HERMES acceptance. However, in most of the $\rho^{0}$ events one of the pions escapes the spectrometer undetected. In the subsample of events in which the $\rho^{0}$ is detected, the transferred asymmetry amplitudes can differ from the amplitudes for all decay pions. In fact, the determination of the transfer values for the pion subsamples in which both of the $\rho^{0}$ decay pions are identified by the HERMES spectrometer, yields lower transfer values for the Sivers amplitudes for positive and negative pions than for events where only one pion is detected (see Figure 6.2.4). For negative pions both from the fully reconstructed $\rho^{0}$ sample and all decay pions, the same Collins moments are extracted. In contrast, for the positive pions, the amplitudes extracted from the fully reconstructed sample are significantly larger in the high $x$ bins. 


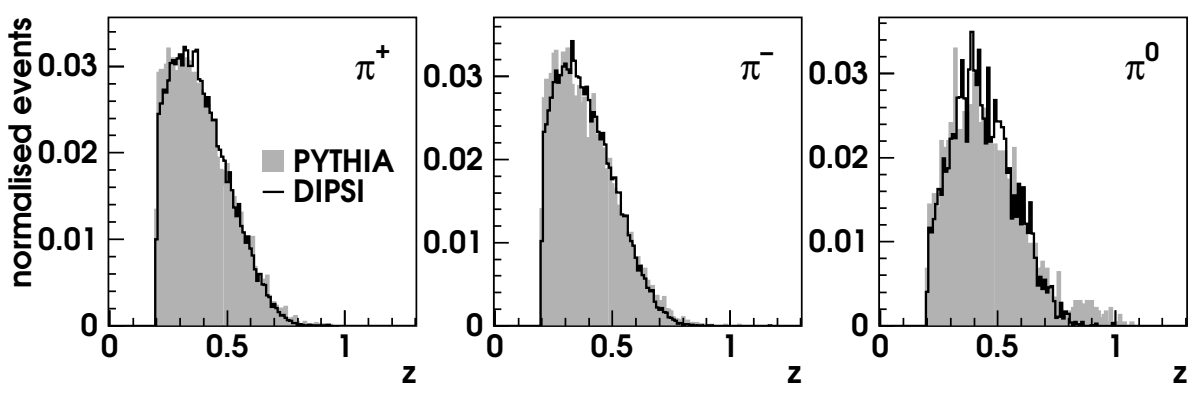

Figure 6.2.5: Comparison of $z$ distributions of the Monte Carlo generators PYTHIA and DIPSI for charged and neutral pions produced in $\omega$ decays. The histograms are normalised to unity.

\section{Transfer Coefficients of Exclusively Produced $\omega$ Vector Mesons}

The dominating decay channel of the $\omega$ meson is the decay into three pions $\pi^{+} \pi^{-} \pi^{0}$ with a branching ratio of around $89 \%$ [Eid04]. A smaller fraction of roughly $9 \%$ decays into a neutral pion and a photon. The statistics of generated $\omega$ mesons with the PYTHIA Monte Carlo generator is not large enough for the determination of the transfer coefficients. Instead, a vector meson Monte Carlo generator DIPSI is used, in which the generation of different vector mesons can be selected individually. Unfortunately, the DIPSI generator is only capable of the three-body decay of the $\omega$ while the two-body $\pi^{0} \gamma$ decay is not implemented. In Figure 6.2.5, the $z$ distributions of reconstructed pions from exclusively produced $\omega$ mesons are compared for the two Monte Carlo generators. All histograms are normalised to unity for the comparison of the distribution shapes. Since the energy of the vector meson has to be distributed among three particles in the dominating decay
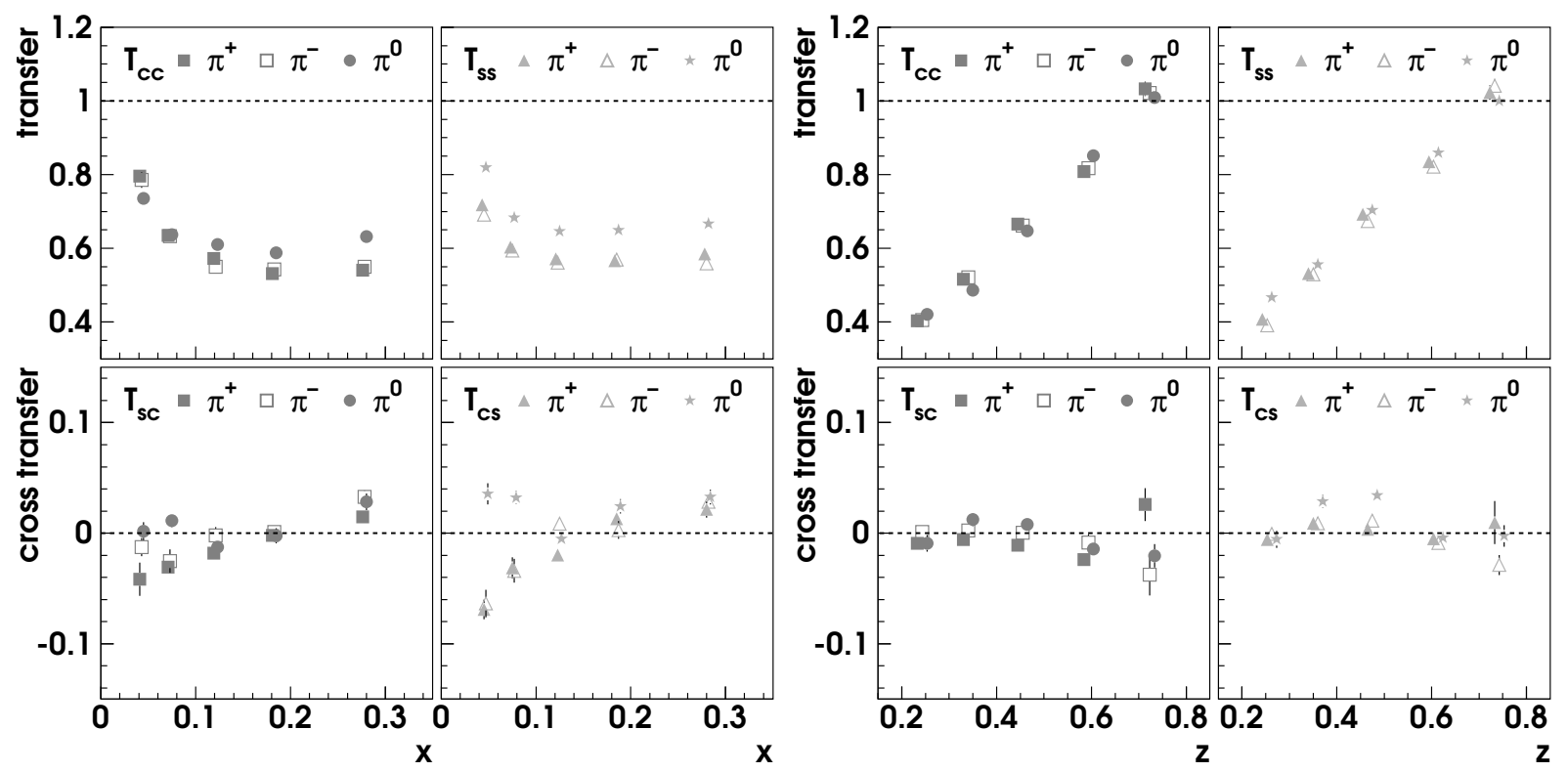

Figure 6.2.6: Transfer values for charged and neutral pions coming from $\omega$ decays in the HERMES acceptance dependent on $x$ and $z$. 
channel, only a small number of pions is found at $z$ values above 0.7. Due to the implementation of the two-body decay in PYTHIA, for neutral pions the $z$ distributions differ at large $z$.

The lack of events at high $z$ prevents the transfer coefficient determination in the last $z$ bin. The obtained coefficients for the lower $z$ bins and the $x$ bins are shown in Figure 6.2.6 comparing all three pion types. In the HERMES acceptance, the $\pi^{0}$ tends to have a larger energy fraction than the charged pions (see Figure 6.2.5) resulting in higher transferred asymmetry amplitudes in most of the $x$ bins. For all three pion types, the transfer coefficients are larger than for the $\rho^{0}$ two-body decay. In the HERMES data, the vector meson contribution for neutral pions in the last $z$ bin is mainly due to $\omega$ decays into $\pi^{0} \gamma$. Like for the $\rho^{0}$ two-body decay, in this kinematic bin a transfer value of 1 can be assumed. The obtained transfer values for neutral pions produced in exclusive $\omega$ decays can be found in Table F.4 for all kinematic bins.

\subsubsection{Asymmetry Amplitudes Acquired during the Decay Process}

Apart from a Collins or Sivers type asymmetry amplitude for the vector meson, which is transferred to the decay particles, also the decay angular distribution can exhibit azimuthal modulations which can contribute to the extracted Collins and Sivers moments. Vector mesons carry a total angular momentum of $J=1$ in their rest frame. Since angular momentum is conserved, also in the decay particle system the sum of spin $S$ and angular momentum $L$ is $J=S+L=1$. For $\rho^{0}, \omega$, and $\phi$ mesons with their dominant decays into two or respectively three scalar mesons, the total angular momentum is completely transferred into the angular momentum $L$ of the decay system reflected in the angular decay distribution of the scalar mesons.

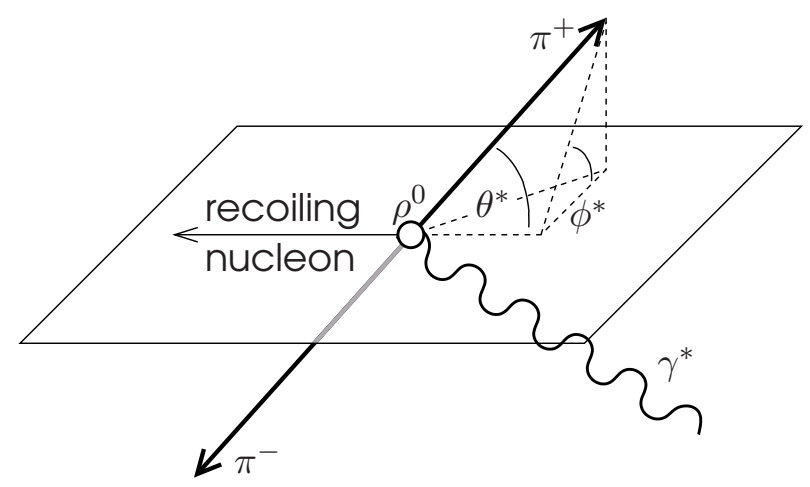

Figure 6.2.7: Definition of the azimuthal and polar decay angles, $\phi^{*}$ and $\theta^{*}$, in the centre-of-mass system of the $\rho^{0}$ vector meson.

It is common to describe the angular decay distribution in the vector meson rest frame using the so-called $s$-channel helicity system, in which the $z$-axis - the quantisation axis is pointing in the opposite direction of the outgoing nucleon. For a two-body decay, the decay angles, $\phi^{*}$ and $\theta^{*}$, are defined as azimuthal and polar angles of one of the decay mesons, which is the positive pion in case of $\rho^{0}$ decay, as shown in Figure 6.2.7. In case of a three-body decay, the decay angles are defined by the normal vector of the decay 
plane.

In the $s$-channel helicity system, the decay angular distribution $W\left(\cos \theta^{*}, \phi^{*}\right)$ can be written as [Sch70, Fra74]:

$$
\begin{aligned}
\frac{\mathrm{d} N}{\mathrm{~d} \cos \theta^{*} \mathrm{~d} \phi^{*}} \equiv W( & \left.\cos \theta^{*}, \phi^{*}\right) \\
=\frac{3}{4 \pi}\left\{\frac{1}{2}\left(\tilde{\rho}_{\mathrm{VM}}^{++}+\tilde{\rho}_{\mathrm{VM}}^{--}\right) \sin ^{2} \theta^{*}+\tilde{\rho}_{\mathrm{VM}}^{00} \cos ^{2} \theta^{*}-\right. & \\
& \sin ^{2} \theta^{*}\left(\cos 2 \phi^{*} \cdot \operatorname{Re} \tilde{\rho}_{\mathrm{VM}}^{+-}-\sin 2 \phi^{*} \cdot \operatorname{Im} \tilde{\rho}_{\mathrm{VM}}^{+-}\right)- \\
& \left.\frac{1}{\sqrt{2}} \sin 2 \theta^{*}\left[\cos \phi^{*} \cdot \operatorname{Re}\left(\tilde{\rho}_{\mathrm{VM}}^{+0}+\tilde{\rho}_{\mathrm{VM}}^{0-}\right)-\sin \phi^{*} \cdot \operatorname{Im}\left(\tilde{\rho}_{\mathrm{VM}}^{+0}+\tilde{\rho}_{\mathrm{VM}}^{0-}\right)\right]\right\} .
\end{aligned}
$$

The decay angular distribution depends on the elements $\rho_{\mathrm{VM}}^{\alpha \beta}$ of the $3 \times 3$ spin density matrix $\rho_{\mathrm{VM}}$ which describes the spin state of the vector meson. The indices $\alpha$ and $\beta$ run over the three helicity states,+ 0 , - of the vector meson. In the expression (6.2.4), the elements of the matrix are normalised by the trace of the spin density matrix: $\tilde{\rho}_{\mathrm{VM}}^{\alpha \beta}=$ $\rho_{\mathrm{VM}}^{\alpha \beta} / \operatorname{Tr} \rho_{\mathrm{VM}}$.

The spin density matrix can be written in terms of $s$-channel helicity amplitudes $f_{j \alpha}^{j^{\prime} \alpha^{\prime}}$ describing the transfer of the virtual-photon helicity $\alpha$ to the vector meson helicity $\alpha^{\prime}$ for an initial nucleon helicity $j= \pm$ and a recoil nucleon helicity $j^{\prime}= \pm$ [Fra74]:

$$
\rho_{\mathrm{VM}}^{\alpha^{\prime} \beta^{\prime}}=\sum_{\alpha, \beta ; j^{\prime}, j, k} e^{i \phi_{\mathrm{VM}}(\alpha-\beta)} \cdot f_{j \alpha}^{j^{\prime} \alpha^{\prime}} \rho_{N}^{j k} \rho_{\gamma^{*}}^{\alpha \beta} f_{k \beta}^{j^{\prime} \beta^{\prime} *} .
$$

Here, $\phi_{\mathrm{VM}}$ is the azimuthal angle $\phi$ of the vector meson in the target nucleon centre-ofmass system, as defined in Figure 2.5.3. The $3 \times 3$ spin density matrix of the virtual photon $\rho_{\gamma^{*}}$ depends on the ratio of the longitudinal to transverse photon flux $\epsilon=(1-y) /(1-y+$ $y^{2} / 2$ ), on $Q^{2}$, and on the lepton beam helicity $\lambda$. The $2 \times 2$ spin density matrix of the target nucleon $\rho_{N}$ contains three polarisation parameters: the degree of transverse polarisation in the production plane $\zeta_{1}$, the degree of polarisation normal to the production plane $\zeta_{2}$, and the degree of longitudinal polarisation $\zeta_{3}$ :

$$
\rho_{N}=\frac{1}{2}\left(\begin{array}{cc}
1+\zeta_{3} & \zeta_{1}-i \zeta_{2} \\
\zeta_{1}+i \zeta_{2} & 1-\zeta_{3}
\end{array}\right) .
$$

The transverse polarisation parameters $\zeta_{1,2}$ are given by the azimuthal angles of the vector meson $\phi_{\mathrm{VM}}$ and of the target spin vector $\phi_{S}$ :

$$
\zeta_{1}=\cos \left(\phi_{\mathrm{VM}}-\phi_{S}\right), \quad \zeta_{2}=\sin \left(\phi_{\mathrm{VM}}-\phi_{S}\right)
$$

assuming a target nucleon polarisation of 1.

In general, a decay angular distribution can be decomposed into unpolarised and polarised terms:

$$
\begin{aligned}
W\left(\cos \theta^{*}, \phi^{*}\right)= & W_{\mathrm{UU}}\left(\cos \theta^{*}, \phi^{*}\right)+W_{\mathrm{LU}}\left(\cos \theta^{*}, \phi^{*} ; \lambda\right)+W_{\mathrm{UL}}\left(\cos \theta^{*}, \phi^{*} ; \zeta_{3}\right)+ \\
& W_{\mathrm{LL}}\left(\cos \theta^{*}, \phi^{*} ; \lambda, \zeta_{3}\right)+W_{\mathrm{UT}}\left(\cos \theta^{*}, \phi^{*} ; \zeta_{1}, \zeta_{2}\right)+ \\
& W_{\mathrm{LT}}\left(\cos \theta^{*}, \phi^{*} ; \lambda, \zeta_{1}, \zeta_{2}\right)
\end{aligned}
$$


where the subscripts indicate the beam and target polarisation states like in the previous chapters. The two contributions $W_{\mathrm{UL}}$ and $W_{\mathrm{LL}}$ vanish in the case of the $\rho^{0}$ decay. Thus, no new information is gained with a longitudinally polarised target compared to an unpolarised nucleon target. Furthermore, $W_{\mathrm{LT}}$ becomes independent of $\zeta_{1}$. For the azimuthal single-spin asymmetry calculation, only the term $W_{\mathrm{UT}}$ is relevant in case of a transversely polarised target. It contains 16 terms with different modulations in the decay angles $\theta^{*}$ and $\phi^{*}$ and in the azimuthal angles $\phi_{\rho^{0}}$ and $\phi_{S}$ which are listed in Table C. 1.

In order to identify contributions to the sine modulations of the Collins and Sivers effects, the azimuthal decay angle $\phi^{*}$ has to be expressed in terms of the azimuthal and polar angles of the $\rho^{0}$ and the decay $\pi^{+}$in the nucleon rest frame (see Appendix C. 1):

$$
\begin{aligned}
& \sin \phi^{*} \approx p_{4}\left(\theta_{\pi^{+}}, \theta_{\rho^{0}}\right) \sin \left(\phi_{\rho^{0}}-\phi_{\pi^{+}}\right), \\
& \cos \phi^{*} \approx p_{5}\left(\theta_{\pi^{+}}, \theta_{\rho^{0}}\right) \cos \left(\phi_{\rho^{0}}-\phi_{\pi^{+}}\right)-p_{6}\left(\theta_{\pi^{+}}, \theta_{\rho^{0}}\right) .
\end{aligned}
$$

These expressions are valid under the assumption of small polar angles ${ }^{i} \theta_{\rho^{0}}, \theta_{\pi^{+}} \ll \frac{\pi}{2}$. The factors $p_{4-6}$ and $p_{1-3}$ used below contain the dependence on the polar angles:

$$
\begin{array}{ll}
p_{1}=\sin ^{2} \theta^{*}, & p_{4}=\frac{\sin \theta_{\pi^{+}}}{\sqrt{1-\cos ^{2} \theta_{\rho^{0}} \cos ^{2} \theta_{\pi^{+}}}}, \\
p_{2}=\cos ^{2} \theta^{*}, & p_{5}=\frac{\cos \theta_{\rho^{0}} \sin \theta_{\pi^{+}}}{\sqrt{1-\cos ^{2} \theta_{\rho^{0}} \cos ^{2} \theta_{\pi^{+}}}}, \\
p_{3}=\frac{\sin 2 \theta^{*}}{\sqrt{2}}, & p_{6}=\frac{\sin \theta_{\rho^{0}} \cos \theta_{\pi^{+}}}{\sqrt{1-\cos ^{2} \theta_{\rho^{0} \cos ^{2} \theta_{\pi^{+}}}} .}
\end{array}
$$

Since the decay pion is detected instead of the vector meson itself, the decay angular distribution can be integrated over the angle $\phi_{\rho^{0}}$. In the integrated decay angular distribution, the sine modulations of the Collins and Sivers effects appear among other modulations with the following amplitudes:

$$
\begin{aligned}
& A_{W}^{\sin \left(\phi_{\pi^{+}}+\phi_{S}\right)}=\frac{1}{2} \pi p_{3}\left(A_{15}-A_{7}\right)\left(p_{4}-p_{5}\right)+\pi p_{1} p_{6}\left(A_{3}-\epsilon A_{8}\right)\left(p_{4}-p_{5}\right), \\
& A_{W}^{\sin \left(\phi_{\pi^{+}}-\phi_{S}\right)}=\pi p_{3}\left(A_{5} p_{4}+A_{13} p_{5}\right)-2 \pi p_{1} p_{6}\left(A_{1} p_{4}+A_{9} p_{5}\right),
\end{aligned}
$$

where the amplitudes $A_{1-16}$ are defined in Table C. 1.

Since only a small transverse momentum is transferred in elastic and diffractive processes, the momentum of the produced vector meson is almost collinear with the virtualphoton direction, i.e., $\theta_{\rho^{0}} \approx 0$. For a vanishing polar angle of the $\rho^{0}$, the parameters $p_{4}$ and $p_{5}$ both approach unity and $p_{6}$ becomes small. In the limit of collinearity, the Collins amplitude (6.2.11) vanishes and only the $\sin 2 \theta^{*}$ modulation of the Sivers amplitude (6.2.12) remains. In order to check the influence of this Sivers modulation, it has been implemented in the PYTHIA Monte Carlo production with amplitudes between -0.9 and 0.9. The Collins and Sivers amplitudes are extracted for the $\rho^{0}$ decay pions and from the ratio between implemented and reconstructed amplitudes the transfer values $T_{\mathrm{Collins}}^{\sin 2 \theta^{*}}$ and $T_{\mathrm{Sivers}}^{\sin 2 \theta^{*}}$, respectively, are computed which are shown in Figure 6.2.8 on the left side. The transfer

'The polar angle $\theta_{\rho^{0}}$ is equivalent to the later used $\theta_{\gamma^{*} \rho^{0}}$. For sake of consistency to the other angles, the notation without the $\gamma^{*}$ is used in this section. 
of the implemented Sivers asymmetry amplitudes to the extracted Collins amplitudes is very small and consistent with zero in most bins and the transfer to the Sivers amplitudes remains below $20 \%$ in all $x$ bins. However, in the smallest and largest $z$ bins, the fraction of the amplitude transferred to the pion Sivers amplitude, becomes large, which is compensated in the lower $z$ bins by a vector meson fraction below $5 \%$. The larger transfer values for positive pions compared to negative pions might be caused by the approximation of small polar angles (see Appendix C.1) together with the arbitrary choice of the positive pion for the definition of the decay angles, i.e., using the kinematics of the positive pion for the implementation of the asymmetry amplitudes.

The reconstructed events of the PYTHIA Monte Carlo production do not reach the limit of collinearity. Instead, the mean values of the parameters are $p_{6} \approx 0.4$ and $p_{4} \approx p_{5} \approx 0.9$. However, since the two parameters $p_{4}$ and $p_{5}$ are comparable, the Collins modulation still vanishes. In the Sivers modulation, an extra term remains which is proportional to $\sin ^{2} \theta^{*}$ and suppressed by $p_{6}$.

The integration over the azimuthal angle $\phi_{\rho^{0}}$ is incomplete because of the restricted acceptance of the HERMES spectrometer. An asymmetry amplitude scan of a $\cos \phi_{\rho^{0}}$. $\sin \left(\phi_{\pi^{+}}-\phi_{S}\right)$ modulation shows indeed non-zero amplitude transfer values, which are mostly below $10 \%$, as shown on the right-hand side of Figure 6.2.8. Before the integration over $\phi_{\rho^{0}}$, the following modulations appear in the Collins and Sivers amplitudes of the decay angular distribution for $p_{5} \approx p_{4}$ :

$$
\begin{aligned}
A_{W}^{\sin \left(\phi_{\pi^{+}}+\phi_{S}\right)}= & \sin \phi_{\rho^{0}} \frac{1}{4} p_{1}\left(1-p_{6}\right) A_{2}+\cos \phi_{\rho^{0}}\left(3 \sin ^{2} \phi_{\rho^{0}}-\cos ^{2} \phi_{\rho^{0}}\right) 4 p_{4}^{2} p_{1} A_{10}+ \\
& \cos \phi_{\rho^{0}} \frac{1}{2}\left[p_{2}\left(A_{4}-A_{12}\right)+p_{3} p_{6}\left(A_{14}+A_{16}-A_{6}\right)-\frac{1}{2} p_{1}\left(1-p_{6}^{2}\right) A_{10}\right]+ \\
& \left(\sin ^{2} \phi_{\rho^{0}}-\cos ^{2} \phi_{\rho^{0}}\right)\left[\frac{1}{2} p_{3} p_{4}\left(A_{5}-A_{13}\right)+p_{1} p_{4} p_{6}\left(A_{1}-A_{9}\right)\right] \\
A_{W}^{\sin \left(\phi_{\pi^{+}}-\phi_{S}\right)}= & \frac{1}{2} p_{3} p_{4}\left(A_{5}+A_{13}\right)-p_{1} p_{4} p_{6}\left(A_{1}+A_{9}\right)+\sin \phi_{\rho^{0}} 4 p_{1}\left(p_{6}^{2}-1\right) A_{2}+ \\
& \cos \phi_{\rho^{0}} \frac{1}{2}\left[p_{2}\left(A_{4}+A_{12}\right)-p_{3} p_{6}\left(A_{14}+A_{16}+A_{6}\right)+\right. \\
& \left.8 p_{1}\left(1+p_{4}^{2}+p_{6}^{2}\right) A_{10}\right]
\end{aligned}
$$

In order to estimate the effect on the extracted Sivers and Collins amplitudes of semiinclusive DIS events, information about the $\rho^{0}$ spin density matrix for a transversely polarised target is necessary. So far, only measurements of $\rho^{0}$ spin density matrix elements for unpolarised targets exist [Tyt01, Pro99]. The decay angular distribution of an unpolarised target is implemented in the Monte Carlo and shows no influence on the Collins and Sivers amplitudes of the $\rho^{0}$ decay pions. Also the decay angular distribution of the $\omega$ meson is described well with the unpolarised Monte Carlo generators and causes no Collins and Sivers amplitudes in the decay pions.

In the Collins and Sivers amplitudes, $(6.2 .13)$ and (6.2.14), the modulations in $\phi_{\rho^{0}}$ always contain dependencies on $\theta^{*}$ as well. The product of the two dependencies should result in small transfer values of the asymmetry amplitudes of the order of a few percent. Therefore, no large effect is expected for the extracted Collins amplitudes of $\rho^{0}$ decay pions. For the Sivers amplitudes, the decay angular distribution might affect only the extracted 

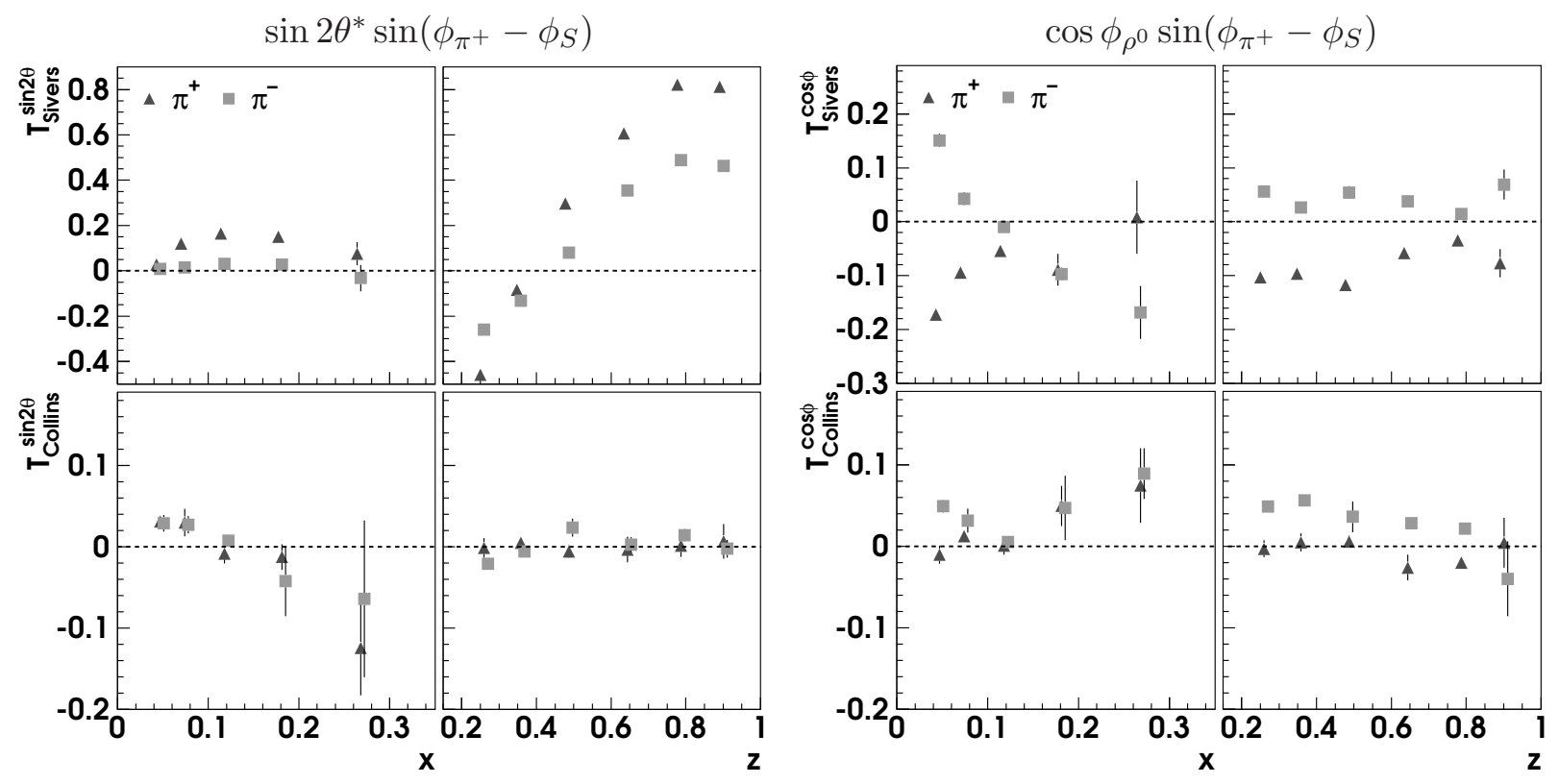

Figure 6.2.8: Transfer from implemented asymmetries with $\sin 2 \theta^{*} \sin \left(\phi_{\pi^{+}}-\phi_{S}\right)$ (left panels) and $\cos \phi_{\rho^{0}} \sin \left(\phi_{\pi^{+}}-\phi_{S}\right)$ (right panels) modulations to Sivers and Collins amplitudes of decay pions.

amplitudes in the highest $z$ bins outside the semi-inclusive range $(z>0.7)$. Furthermore, all 16 amplitudes $A_{1-16}$ vanish under the assumption of $s$-channel helicity conservation and natural parity exchange (see Appendix C.2).

\subsubsection{Asymmetry Amplitudes of Exclusively Produced $\rho^{0}$}

In the HERMES experiment, the clean identification of exclusively produced vector mesons is limited to the detection of elastically produced vector mesons (see Figure 6.1.1(a)). Since the target nucleon recoils outside the HERMES acceptance, single diffractive processes in which the proton is transferred into an excited state (see Figure 6.1.1(b)) also contribute to the measurement. For the selection of $\rho^{0}$ candidate events, the same kinematic cuts are imposed on the scattered lepton as for the semi-inclusive analysis, listed in Table 4.2.2. Several quantities are computed for each candidate event with exactly two pions of opposite charge, which are identified by the RICH detector. Their invariant mass $M_{\pi \pi}$ is restricted to the range $0.6 \mathrm{GeV}$ to $1.0 \mathrm{GeV}$ around the $\rho^{0}$ mass of $775.8 \pm 0.5 \mathrm{MeV}$ [Eid04]. A second quantity,

$$
\Delta E=\frac{M_{X}^{2}-M_{p}^{2}}{2 M_{p}}
$$

vanishes in exclusive events where the rest mass of the recoiling baryonic system $M_{X}$ is equal to the target proton mass $M_{p}$. Accounting for the finite detector resolutions, an upper cut of $0.6 \mathrm{GeV}$ is applied on $\Delta E$ to enhance the exclusive event sample. In elastic and diffractive processes only a small transverse momentum is transferred to the hadronic vertex. For the selection of these processes, the transverse momentum transfer,

$$
-t^{\prime}=-\left(t-t_{0}\right)
$$



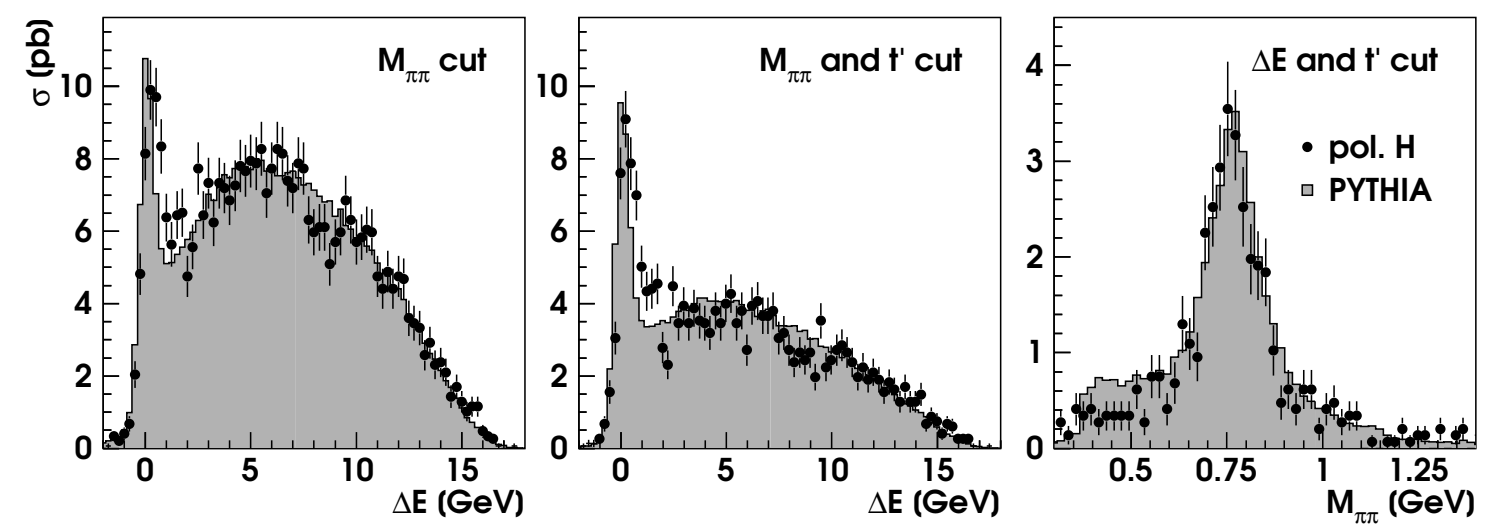

Figure 6.2.9: The $\Delta E$ and invariant mass total cross-section distributions for $\rho^{0}$ candidate events from the 02 data production compared to a PYTHIA Monte Carlo simulation.

is therefore restricted to values below $0.4 \mathrm{GeV}^{2}$. Here, $t$ is the squared four-momentum transfer to the hadronic vertex and $\left|t_{0}\right|$ is the minimum kinematically allowed value of $|t|$. Since in the hadron rest frame, the subtraction of $t_{0}$ removes the longitudinal component of the momentum transfer, $t^{\prime}$ is a measure of the transverse momentum transfer.

In Figure 6.2.9, the $\Delta E$ distribution is shown for the 02 data production which was extracted once with the cut on the invariant mass only and once with the additional cut on $-t^{\prime}$. In addition, the invariant mass distribution is plotted with imposed $\Delta E$ and $-t^{\prime}$ cuts. The distributions are compared to a PYTHIA Monte Carlo production whose yields are decreased by $10 \%$. However, the lower cross sections found in the HERMES data are consistent with the PYTHIA cross sections within the systematic uncertainty of the luminosity constant (see Appendix B). Although the PYTHIA generator reproduces the exclusive $\rho^{0}$ cross section on a $10 \%$ level [Lie04], the Monte Carlo using HSG does not completely reproduce the broadening of the peak of exclusively produced $\rho^{0}$ mesons around $\Delta E=0$, which is caused by increased multiple scattering after the replacement of the threshold Čerenkov counter by the RICH detector. Clearly visible in the HERMES data and the Monte Carlo production is the reduction of background events using the cut on the transverse momentum transfer while the exclusive peak stays almost unaffected. At lower invariant mass, the PYTHIA MC distribution might exceed the data because of the event generation according to a non-relativistic Breit-Wigner shape whereas the HERMES data is better described by a relativistic $p$-wave Breit-Wigner including a skewing parameter [Tyt01].

A cut is imposed on the angle $\theta_{\gamma^{*} h}=\theta_{\gamma^{*} \rho^{0}}$ like for the asymmetry amplitude extraction in case of semi-inclusive pion and kaon production in order to reject events with misreconstructed azimuthal angles (see Table 4.2.2 on page 58). However, the resolution in the angle $\theta_{\gamma^{*} \rho^{0}}$ might differ for $\rho^{0}$ vector mesons compared to charged hadrons. In the combined three HERMES data productions, 1292 exclusive $\rho^{0}$ candidate events are found and filled into $8 \times 8$ azimuthal bins. Because most of the vector mesons are produced at small angles, the number of events increases by roughly $67 \%$ without the cut on $\theta_{\gamma^{*} \rho^{0}}$. The azimuthal asymmetry is formed according to Eq. (4.3.1) and fitted by the function:

$$
A_{\mathrm{UT}}\left(\phi, \phi_{S}\right)=A_{\mathrm{UT}}^{\sin \left(\phi+\phi_{S}\right)} \cdot \sin \left(\phi+\phi_{S}\right)+A_{\mathrm{UT}}^{\sin \left(\phi-\phi_{S}\right)} \cdot \sin \left(\phi-\phi_{S}\right)+C .
$$

The $\sin \left(\phi \pm \phi_{S}\right)$ amplitudes of the azimuthal asymmetry of exclusively produced $\rho^{0}$ mesons 

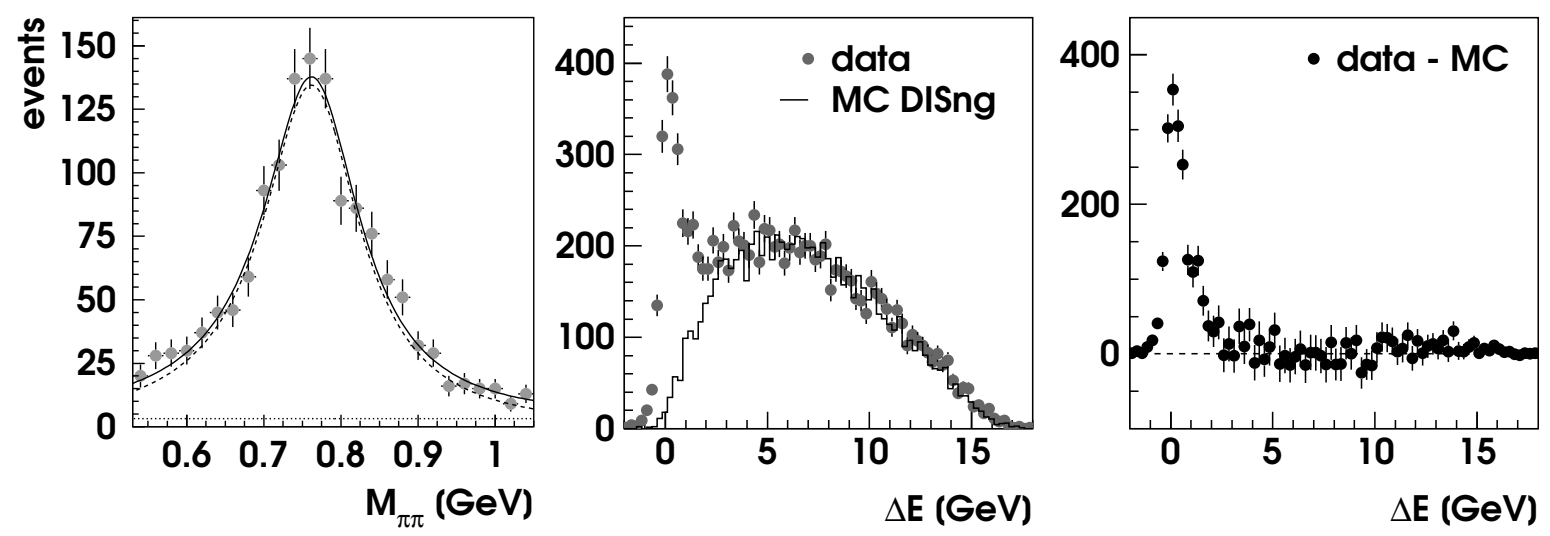

Figure 6.2.10: Invariant mass distribution of HERMES data of the combined productions 02, 03, and 04 fitted with a skewed Breit-Wigner function (left plot) and $\Delta E$ distribution of the three productions compared to a normalised DISNG Monte Carlo production (middle plot) and the difference of the two distributions (right plot).

are not related to the Sivers and Collins function. Nevertheless, the terms Sivers and Collins moments refer to the extracted moments $\left\langle\sin \left(\phi \pm \phi_{S}\right)\right\rangle_{\mathrm{UT}}$ when used in the following. As in the fit to the semi-inclusive pion asymmetries (cf. Eq. (4.3.2) on page 63), the constant $C$ vanishes for a correct luminosity normalisation of the azimuthal asymmetry $A_{\mathrm{UT}}\left(\phi, \phi_{S}\right)$.

Two sources of background contributions exist in the exclusive $\rho^{0}$ candidate sample. These are non-resonant pion-pair events, which are exclusively produced $\pi^{+} \pi^{-}$pairs with an invariant mass in the considered mass range around the $\rho^{0}$ mass, and non-exclusive $\rho^{0}$ events, which are accompanied by particles leaving the HERMEs spectrometer undetected. These background fractions can be determined by means of the invariant mass and the $\Delta E$ distributions, respectively. In the left plot in Figure 6.2.10, the invariant mass spectrum which is obtained after the $\Delta E$ and $-t^{\prime}$ cuts are applied, is plotted together with a fit (solid line). The fit function is composed of a skewed relativistic Breit-Wigner function (dashed line) based on a phenomenological model by Ross and Stodolsky [Ros66]

\begin{tabular}{|c|c|c|c|c|c|c|c|}
\hline \multicolumn{8}{|c|}{$\theta_{\gamma^{*} \rho^{0}}, \theta_{\gamma^{*} \pi}>0.02$} \\
\hline & $\langle x\rangle$ & $\langle z\rangle$ & $\left\langle-t^{\prime}\right\rangle\left[\mathrm{GeV}^{2}\right]$ & $f_{\mathrm{nr}}$ & $f_{\text {ne }}$ & $2\left\langle\sin \left(\phi+\phi_{S}\right)\right\rangle_{\mathrm{UT}}$ & $2\left\langle\sin \left(\phi-\phi_{S}\right)\right\rangle_{\mathrm{UT}}$ \\
\hline$\rho^{0}$ & 0.086 & 0.98 & 0.19 & $0.05 \pm 0.09$ & $0.11 \pm 0.05$ & $-0.03 \pm 0.06$ & $0.03 \pm 0.06$ \\
\hline$\pi^{+}$ & 0.087 & 0.44 & 0.13 & $0.05 \pm 0.09$ & $0.11 \pm 0.05$ & $-0.01 \pm 0.07$ & $-0.06 \pm 0.07$ \\
\hline$\pi^{-}$ & 0.089 & 0.44 & 0.13 & $0.05 \pm 0.09$ & $0.11 \pm 0.05$ & $0.03 \pm 0.08$ & $0.07 \pm 0.07$ \\
\hline \multicolumn{8}{|c|}{ no cut on $\theta_{\gamma^{*} \rho^{0}}$} \\
\hline & $\langle x\rangle$ & $\langle z\rangle$ & $\left\langle-t^{\prime}\right\rangle\left[\mathrm{GeV}^{2}\right]$ & $f_{\mathrm{nr}}$ & $f_{\text {ne }}$ & $2\left\langle\sin \left(\phi+\phi_{S}\right)\right\rangle_{\mathrm{UT}}$ & $2\left\langle\sin \left(\phi-\phi_{S}\right)\right\rangle_{\mathrm{UT}}$ \\
\hline$\rho^{0}$ & 0.085 & 0.98 & 0.13 & $0.07 \pm 0.05$ & $0.09 \pm 0.04$ & $0.00 \pm 0.04$ & $0.03 \pm 0.05$ \\
\hline
\end{tabular}

Table 6.2.1: Background fractions and corrected asymmetry amplitudes integrated over all kinematic variables for exclusively produced $\rho^{0}$ and the decay pions. 
and a constant (dotted line) for the non-resonant background estimate. The integrals of the fit function and of the background contribution only in the range $0.6 \mathrm{GeV}$ to $1.0 \mathrm{GeV}$ yield a non-resonant background fraction of $f_{\mathrm{nr}}=0.05 \pm 0.09$ taking into account the correlation of the different fit parameters. The middle plot of the figure shows the $\Delta E$ distribution with the cuts on the invariant mass and the transverse momentum transfer given above. Superimposed in the plot is the same distribution from a DISNG Monte Carlo simulation that does not include exclusively produced vector mesons (see Section 5. 1). The Monte Carlo distribution is normalised to the data in the region $4.0<\Delta E<8.0 \mathrm{GeV}$. Only the exclusive peak around $\Delta E=0$ remains in the $\Delta E$ spectrum after subtraction of the Monte Carlo distribution, which is shown in the right-hand panel of the figure. A background fraction of $f_{\text {ne }}=0.11 \pm 0.05$ is obtained from the ratio of the integrated yields in the Monte Carlo and the data in the region up to $\Delta E=0.6 \mathrm{GeV}$.

Besides the background fractions $f_{\mathrm{nr}}$ and $f_{\text {ne }}$ also the background asymmetries of the two sources $A_{\mathrm{nr}}$ and $A_{\mathrm{ne}}$ are necessary for the correction of the measured asymmetry amplitudes $A_{\text {meas }}$ :

$$
A_{\mathrm{cor}}=\frac{1}{1-f_{\mathrm{ne}}-f_{\mathrm{nr}}} A_{\mathrm{meas}}-\frac{f_{\mathrm{nr}}}{1-f_{\mathrm{ne}}-f_{\mathrm{nr}}} A_{\mathrm{nr}}-\frac{f_{\mathrm{ne}}}{1-f_{\mathrm{ne}}-f_{\mathrm{nr}}} A_{\mathrm{ne}} .
$$

The background asymmetry of non-resonant pion pairs can be determined from the events in the side bands of the invariant mass peak. The two ranges $[0.2,0.6] \mathrm{GeV}$ and $[1.0,1.4] \mathrm{GeV}$ are chosen for the extraction of the asymmetry amplitudes. The results of both side bands agree within their uncertainties and are combined to yield the background amplitude $A_{\mathrm{nr}}$. As can be seen in the right plot of Figure 6.2.10, no exclusive events contribute to the $\Delta E$ spectrum for $\Delta E>4 \mathrm{GeV}$. The non-exclusive events in this region can be used for the determination of the background asymmetry amplitude $A_{\text {ne }}$. Both background asymmetry amplitudes $A_{\text {nr }}$ and $A_{\text {ne }}$ are of the order of a few percent and statistically consistent with zero. The corrected asymmetry amplitudes for exclusive $\rho^{0}$ events are listed together with some kinematic mean values and the two background fractions in Table 6.2.1, once with imposed cut on the angle $\theta_{\gamma^{*} \rho^{0}}$ and once for the larger statistics data sample without this cut. All amplitudes are consistent with zero and have a statistical uncertainty of the order of a few percent. Among the kinematic average values, only $\left\langle-t^{\prime}\right\rangle$ seems to be influenced by the cut on $\theta_{\gamma^{*} \rho^{0}}$, which is expected since $t^{\prime}$ is a measure of the transverse momentum transfer. The extracted $\rho^{0}$ Sivers moments of the order of a couple of percents are consistent with a prediction based on generalised parton distributions [Goe01]. The Collins moment is suppressed by $1 / Q^{2}$ and is therefore expected to be small [Die05a].

Asymmetry amplitudes can be extracted also for the decay pions of the identified exclusive $\rho^{0}$ events. As in the analysis for semi-inclusive pion and kaon production, the additional kinematic cuts on $z$ and $P_{h}$ listed in Table 4.2.2 are applied. $1510 \pi^{+}$and $1514 \pi^{-}$remain after the additionally imposed cuts. These are more decay pions than exclusive $\rho^{0}$ events when applying the cut on $\theta_{\gamma^{*} \rho^{0}}$ since the cut on $\theta_{\gamma^{*} \pi}$-now applied to the pion kinematics - is less restrictive as the pions gain additional transverse momenta in the decay. Also for the decay pions the amplitudes $2\left\langle\sin \left(\phi \pm \phi_{S}\right)\right\rangle_{\mathrm{UT}}$, which can be found in Table 6.2.1, are consistent with zero. The pion Sivers amplitudes are larger than the Sivers amplitude of exclusive $\rho^{0}$ events. The asymmetry amplitudes of the two different pions have opposite signs which cannot be caused by the transferred $\rho^{0}$ amplitude but only 


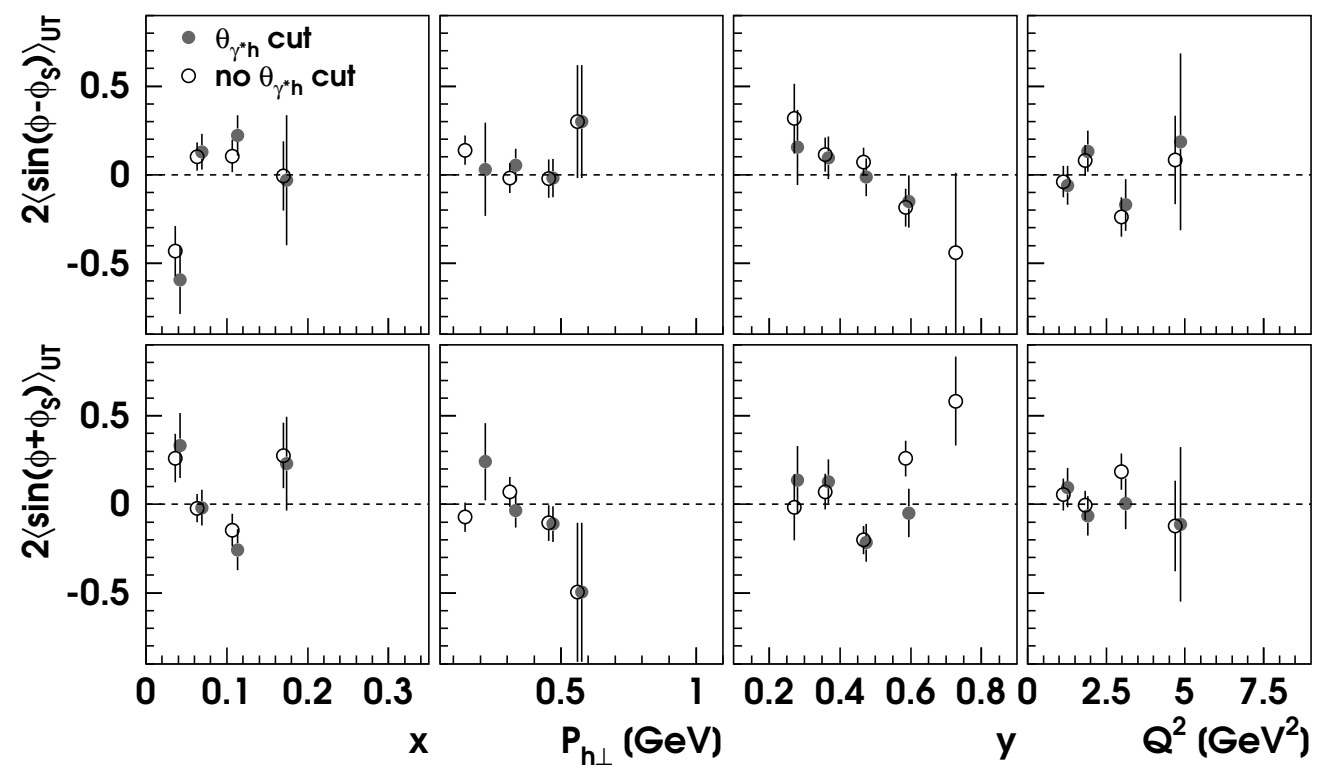

Figure 6.2.11: Asymmetry amplitudes of exclusive $\rho^{0}$ events, once with and once without a cut on the angle between the directions of the virtual photon and the exclusive $\rho^{0}$. No corrections for the nonresonant and non-exclusive backgrounds are performed.

by an additional asymmetry amplitude acquired in the decay process. Nevertheless, the differences between the three amplitudes are statistically not significant.

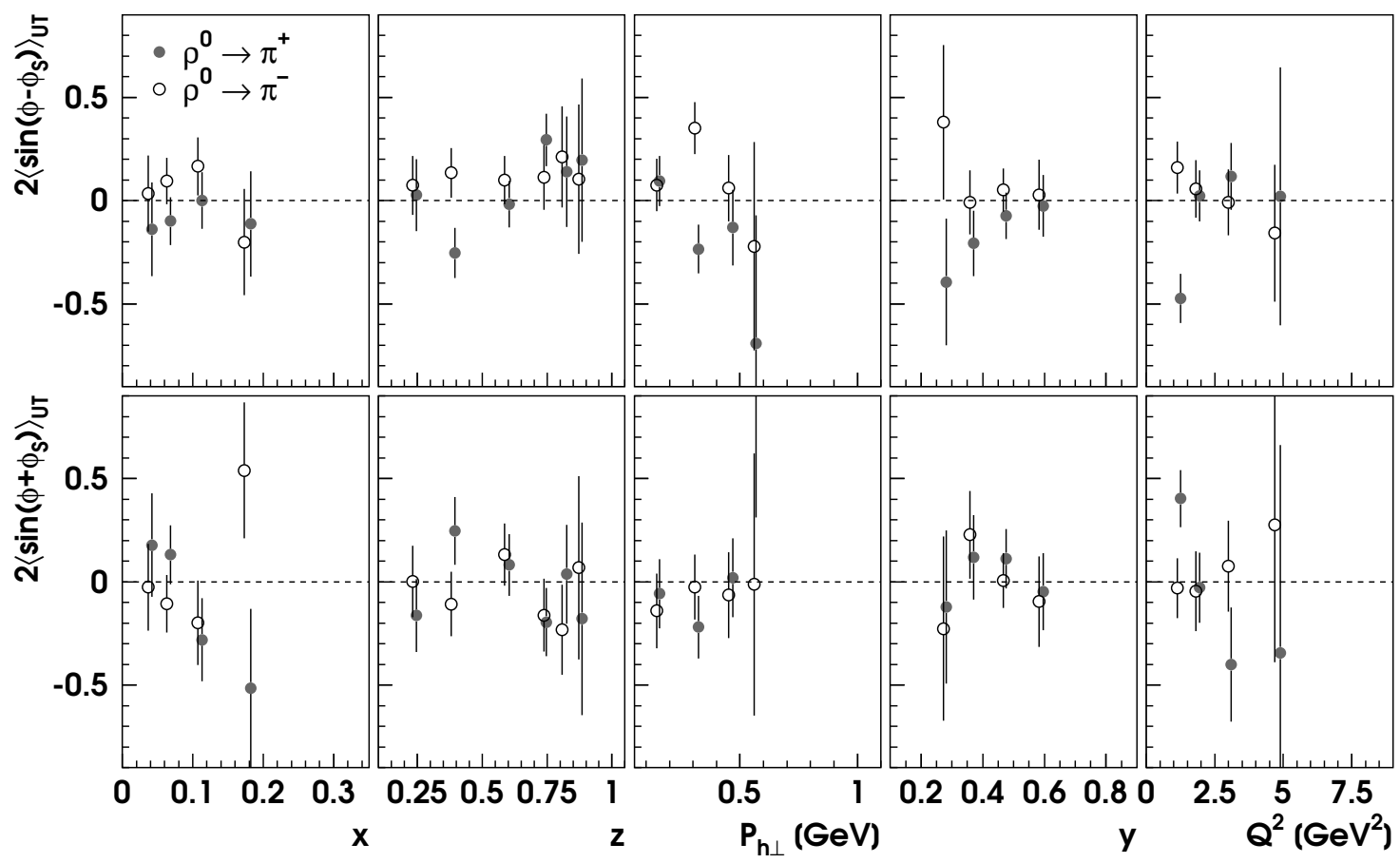

Figure 6.2.12: Asymmetry amplitudes of pions produced in the decay of exclusive $\rho^{0}$ (neither corrected for non-resonant nor for non-exclusive background). 
In a next step, an attempt is made to bin the asymmetry amplitudes in the different kinematic variables, using the bin limits listed in Table 4.3. 1. Due to the lower event counts in the individual bins, no background correction is possible. Furthermore, the amplitude extraction fails in the highest $x, P_{h \perp}, y$, and $Q^{2}$ bins. Yet, omitting the cut on the angle $\theta_{\gamma^{*}} \rho^{0}$, an asymmetry amplitude extraction becomes possible also in the highest $y$ bin. In exclusive processes, all the energy of the virtual photon is transferred to the produced vector mesons, resulting in values of $z$ close to 1 . Hence, a binning in $z$ is not reasonable for exclusive $\rho^{0}$ events. The extracted $\rho^{0}$ amplitudes are plotted in Figure 6.2.11 as functions of $x, P_{h \perp}, y$, and $Q^{2}$. Again results with and without a cut on the angle $\theta_{\gamma^{*} h}$ are compared. No clear trend is visible in any of the kinematic dependencies. The amplitudes are rather fluctuating around zero in the different kinematic bins.

Also the extracted amplitudes of the decay pions in Figure 6.2.12 do not show a trend in any of the kinematic dependencies. The deviation from zero of both Collins and Sivers amplitudes in some bins might be caused by the anti-correlation of the two amplitudes, i.e., if a statistical fluctuation gives rise to a negative amplitude $A_{\mathrm{UT}}^{\sin \left(\phi-\phi_{S}\right)}$ the amplitude $A_{\mathrm{UT}}^{\sin \left(\phi+\phi_{S}\right)}$ becomes positive and vice versa.

\subsubsection{Interpretive Uncertainties}

A measurement of the asymmetry amplitudes of the $\omega$ vector meson is not feasible with the present level of statistics while the measured asymmetry amplitudes of the $\rho^{0}$ vector meson have large statistical uncertainties. Hence, estimates of these vector meson asymmetry amplitudes are employed to determined the possible effect on the semi-inclusive pion asymmetry amplitudes. The estimate of Collins $(c)$ and Sivers $(s)$ amplitudes can be derived from the positivity limit, i.e., that the polarised cross section cannot exceed the
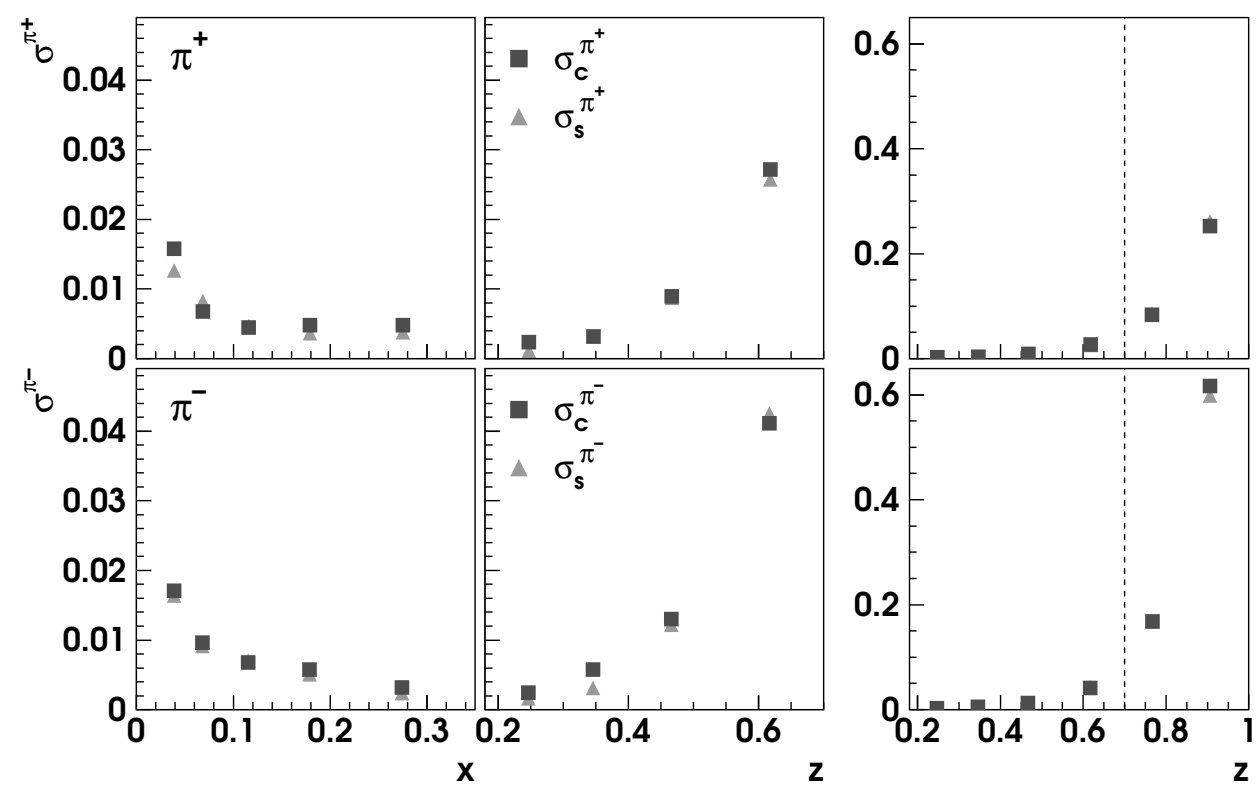

Figure 6.2.13: Interpretive uncertainties for charged pions dependent on the kinematic variables $x$ and $z$. To show the highest two $z$ bins, the scale in the right column is enlarged. 


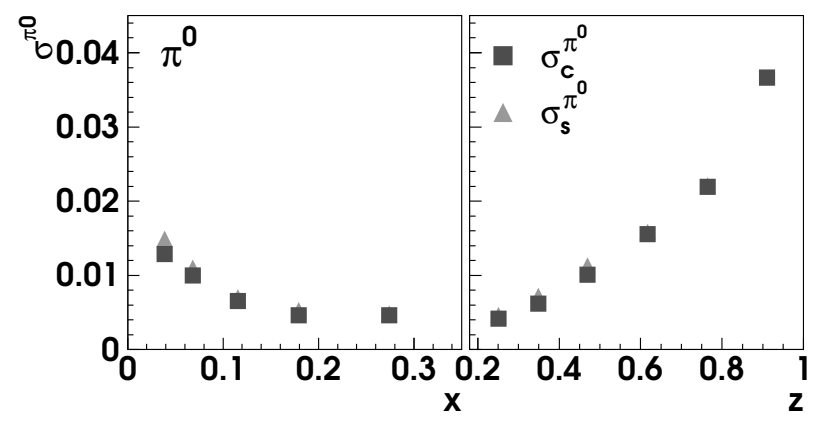

Figure 6.2.14: Interpretive uncertainties of neutral pions dependent on the kinematic variables $x$ and $z$.

unpolarised cross section:

$$
\left|A_{\mathrm{VM}, c} \pm A_{\mathrm{VM}, s}\right| \leq 1 .
$$

Here, other sine and cosine modulations of the cross section are omitted. This absolute limit results in a standard deviation $\sigma=1 / \sqrt{6}$ of each amplitude (see Appendix D).

With the obtained vector meson fractions, the transfer matrix, and the standard deviation of the vector meson asymmetry amplitudes, an interpretive uncertainty on the semi-inclusive asymmetries due to exclusively produced vector mesons can be determined by

$$
\sigma_{i}^{\pi}=\frac{f_{\mathrm{VM}}}{1-f_{\mathrm{VM}}}\left(T_{i c} A_{\mathrm{VM}, c}+T_{i s} A_{\mathrm{VM}, s}\right)=\frac{f_{\mathrm{VM}}}{1-f_{\mathrm{VM}}} \frac{1}{\sqrt{6}}\left(T_{i c}+T_{i s}\right),
$$

with $i=c, s$. Furthermore the assumption is made that no asymmetry amplitudes are acquired by the pions in the decay process, i.e., that $s$-channel helicity conservation and natural parity exchange is valid in case of charged pions. Note that this estimate yields an uncertainty on the interpretation of the experimental results in terms of Collins and Sivers functions. The question whether or not to exclude exclusive vector meson production events from the analysis is still under debate [Die05b].

In Figure 6.2.13, the interpretive uncertainties of the Collins and Sivers amplitudes for $\pi^{+}$and $\pi^{-}$are shown in bins of $x$ and $z$. The central panels show the results for the four lowest $z$ bins while the right panels show the full $z$ range on a larger vertical scale. The absolute amplitude uncertainties of the two highest $z$ bins are larger than $5 \%$ and exceed the measured asymmetries. Due to the lower vector meson fractions, the interpretive uncertainties for neutral pions, which are plotted in Figure 6.2.14, are below $4 \%$ in all kinematic bins. In the following section the possibility to reduce the interpretive uncertainties by the suppression of the vector mesons is investigated.

\subsection{Vector Meson Suppression}

Instead of the correction for the vector meson contribution, the identification of decay pions from exclusive vector mesons would allow the exclusion of these events already before the asymmetry amplitude extraction. Without the complete detection of all final state particles, the decay pion identification is not possible in principle. However, elastically produced vector mesons can be suppressed by cuts on the event kinematics. A 
smaller vector meson fraction in the data sample decreases the interpretive uncertainty. On the other hand, a poor efficiency of the suppression can cause a large increase of the statistical uncertainty. With a successful vector meson suppression the larger statistical uncertainty must be overcompensated by the smaller interpretive uncertainty.

\subsubsection{Suppression of Exclusively Produced $\rho^{0}$ Mesons}

In a strategy for the suppression of the exclusive $\rho^{0}$ vector mesons, the events have to be treated differently dependent on the number of reconstructed pions. Events with more than two pions cannot contain an exclusive $\rho^{0}$ meson. In case of just two oppositely charged pions in the detector, the invariant mass $M_{\pi \pi}$ and $\Delta E$ can be computed and events with $M_{\pi \pi}$ close to the $\rho^{0}$ mass and $\Delta E$ in the exclusive peak around zero can be rejected. Events with exactly one pion can be included for the amplitude extraction if the second decay pion from a potential exclusive $\rho^{0}$ production would be inside the HERMES acceptance. With the knowledge of the energy $E_{\pi 1}$ and the momentum $\vec{P}_{\pi 1}$ of the reconstructed pion, the energy $E_{\pi 2}$ and the momentum $\vec{P}_{\pi 2}$ of the potential second decay pion can be calculated. For the determination of the four unknowns $\left(E_{\pi 2}, \vec{P}_{\pi 2}\right)$, four constraints are necessary:

1: The rest mass of the second pion is the pion mass $M_{\pi 2}=M_{\pi}$.

2: The invariant mass of the two pions is the $\rho^{0}$ meson mass $M_{\pi \pi}=M_{\rho^{0}}$.

3: The missing mass is the proton mass $M_{X}=M_{p}$, corresponding to $\Delta E=0$.

4: Estimate the squared four-momentum transfer $t$ to the hadronic vertex by its kinematically allowed maximum, $t_{0}$, which can be computed from the three other constraints.

In reality, the constraints $\mathbf{2}$ and $\mathbf{3}$ are inequalities because the invariant mass is distributed around the $\rho^{0}$ mass according to the decay width, and the detector resolution smears the missing mass around the proton mass. Yet, taking into account the $\rho^{0}$ decay width and the finite detector resolution would go beyond the practical applicability of this method, so that both of these values are therefore treated as zero.

The four constraints result in the absolute value of the momentum $\left|\vec{P}_{\pi 2}\right|$ and the cosines of two angles $\alpha$ and $\beta$, where $\alpha(\beta)$ is the angle between the sought-after pion and the
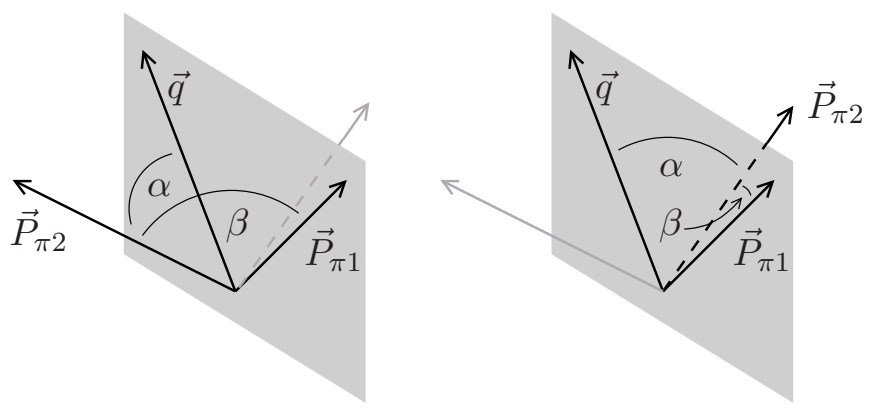

Figure 6.3.1: The two possible solutions (which are mirror symmetric) for a vector $\vec{P}_{\pi 2}$ with a given absolute value and the two angles $\alpha$ and $\beta$. 

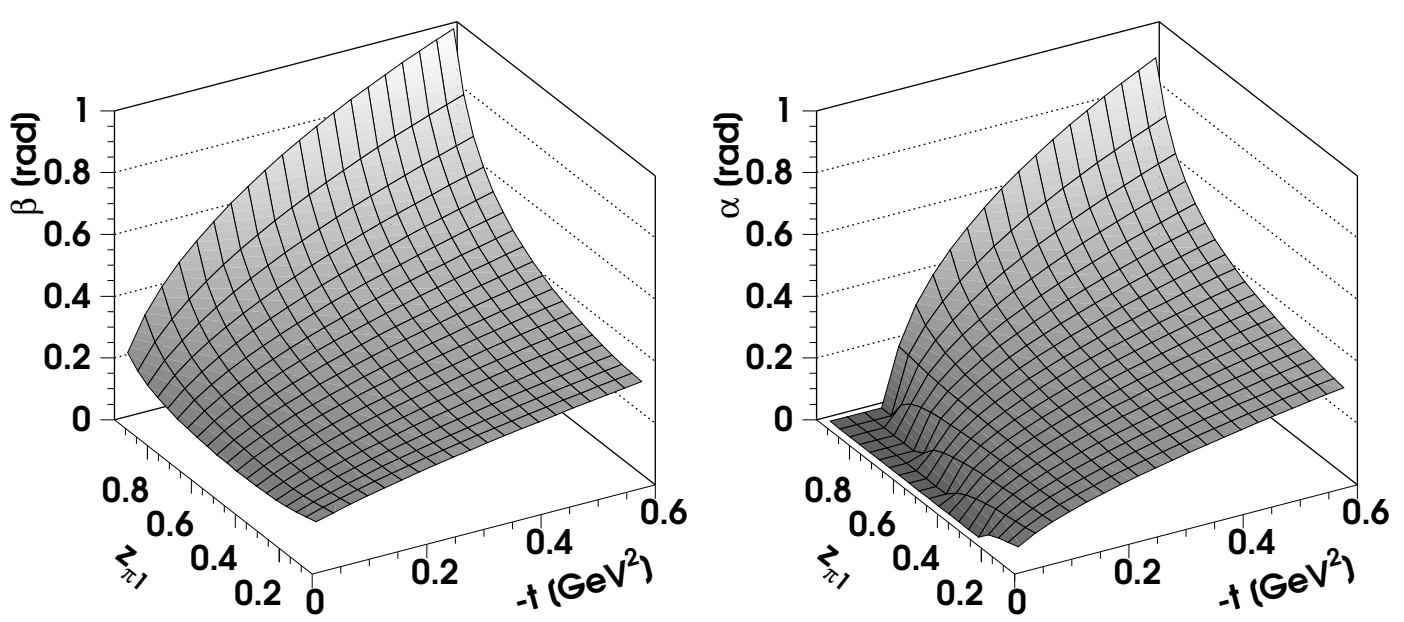

Figure 6.3.2: The dependence of the angles $\alpha$ and $\beta$ on $-t$ and $z_{\pi 1}$ for $Q^{2}=$ $2.4 \mathrm{GeV}^{2}, \nu=15 \mathrm{GeV}$, and $\theta_{\gamma^{*} h}=0.02 \mathrm{rad} . \alpha$ is set to 0 , if it is not calculable due to an argument of the arc cosine function outside its definition range $[-1,1]$. In that case, a single detected pion cannot result from an exclusive $\rho^{0}$ decay event.

virtual photon $\vec{q}$ (the detected pion $\vec{P}_{\pi 1}$ ). This yields two solutions for the orientation of the momentum vector of the second pion as sketched in Figure 6.3.1. Only if both solutions fall inside the detector acceptance the detected single pion event cannot originate from an exclusive $\rho^{0}$ meson decay and is included in the analysis.

Furthermore, constraint $\mathbf{4}$ is only an approximation because the squared momentum transfer $t$ is the sum of $t_{0}$ and $t^{\prime}$ and deviates from the kinematically allowed maximum $t_{0}$. However, the transverse momentum transfer $t^{\prime}$ is small in elastic and diffractive vector meson events. In most of the events, the value of $t^{\prime}$ is between -0.4 and $0 \mathrm{GeV}^{2}$ (see Section 6.2.4). Hence, the search for the second pion has to cover the range $t_{0}-0.4 \mathrm{GeV}$ $<t<t_{0}$. Figure 6.3.2 shows the behaviour of the angles $\alpha$ and $\beta$ dependent on $-t$ and the energy fraction of the detected hadron $z_{\pi 1}$ for the mean values of the data in $Q^{2}$ and $\nu$. The value of $\theta_{\gamma^{*} h}$ is set to the lower limit of $0.02 \mathrm{rad}$ in the analysis. Because of the small absolute values of $t, t_{0}$, and $t^{\prime}$, the plotted range of $-t$ between 0.0 and $0.6 \mathrm{GeV}^{2} \mathrm{covers}$ the complete range of $t_{0}-0.4 \mathrm{GeV}^{2}<t<t_{0}$.

The angles $\alpha(t)$ and $\beta(t)$ increase with increasing $-t$ (decreasing $t$ ), which entails that in events with $t_{0}+t^{\prime}<t<t_{0}$ the second potential pion is closer to the direction of the detected pion than in events with $t=t_{0}+t^{\prime}$ and therefore more likely in the detector acceptance. Hence, for single pion events, a check on the direction of a possible second pion for $t=t_{0}+t^{\prime}=t_{0}-0.4 \mathrm{GeV}^{2}$ does already include the check for $t^{\prime}>-0.4 \mathrm{GeV}^{2}$.

Both functions $\alpha(t)$ and $\beta(t)$ also depend on the kinematic variables $Q^{2}, \nu$, and $z_{\pi 1}$. The angle $\alpha(t)$ contains in addition the cosine of the angle between the virtual photon and the detected pion $\theta_{\gamma^{*} h}$. In order to check if the functions $\alpha(t)$ and $\beta(t)$ decrease monotonically with increasing $t$ within the ranges covered by the kinematic variables, the derivatives of $\alpha(t)$ and $\beta(t)$ with respect to $t$ are computed for energy transfers 0 $<\nu<27.5 \mathrm{GeV}$, four-momentum transfers $1<Q^{2}<13 \mathrm{GeV}^{2}$ (cf. Figure 4.2.5), pion energy fractions $0<z_{\pi 1}<1$, and photon-pion angles $0.02<\theta_{\gamma^{*} h}<0.4 \mathrm{rad}$ (cf. Figure 4.2.6). In the entire kinematic range and for $0>t>-0.6 \mathrm{GeV}^{2} \frac{\mathrm{d} \alpha}{\mathrm{d} t}<0$ and $\frac{\mathrm{d} \beta}{\mathrm{d} t}<0$ so that the angles 

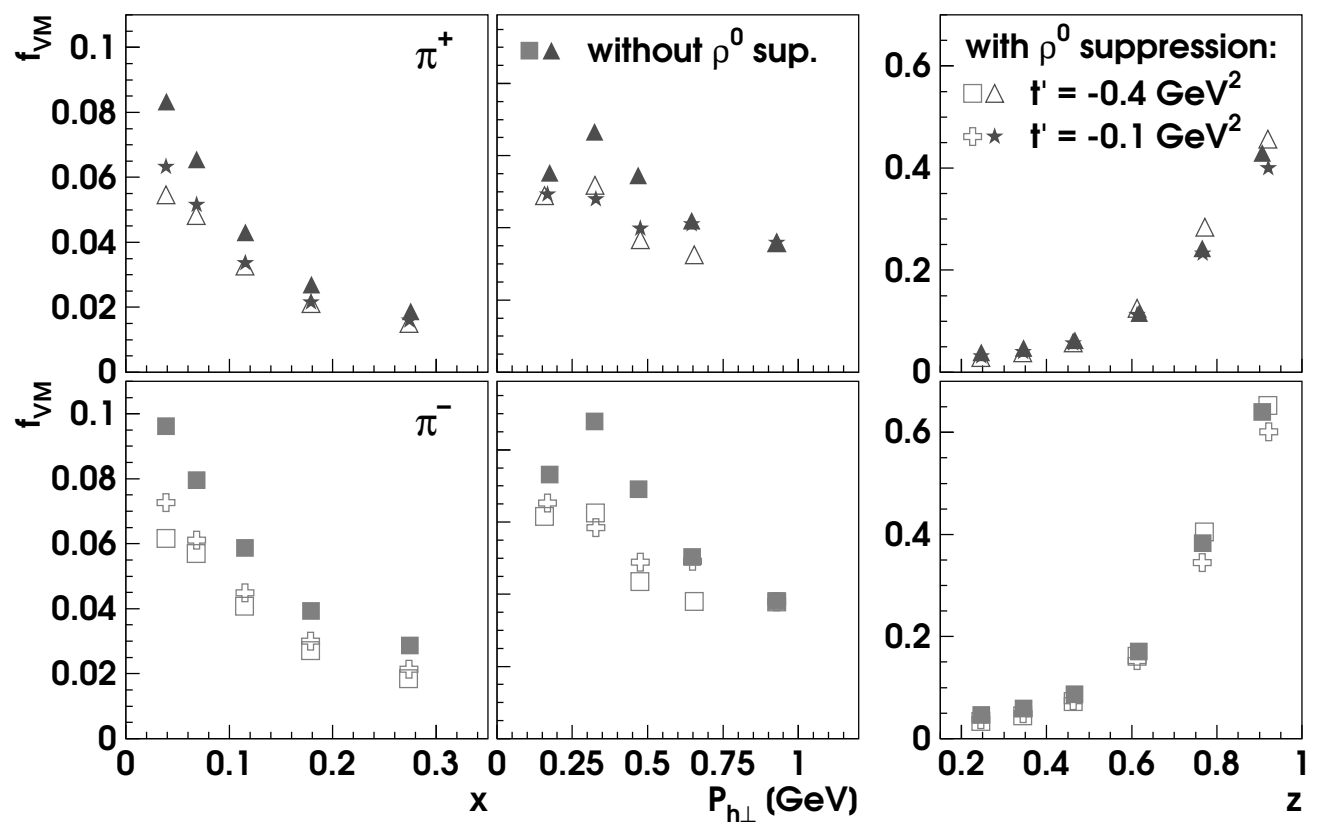

Figure 6.3.3: Dilution of the charged pions with and without the suppression of $\rho^{0}$ vector mesons.

are monotonically decreasing in that range. Therefore, it is sufficient to check $t=t_{0}-$ $0.4 \mathrm{GeV}^{2}$ instead of $t_{0}-0.4 \mathrm{GeV}^{2}<t<t_{0}$ also for values of $Q^{2}, x$, and $\theta_{\gamma^{*} h}$ that differ from the values used to plot the functions $\alpha\left(z_{\pi 1}, t\right)$ and $\beta\left(z_{\pi 1}, t\right)$ in Figure 6.3.2.

The efficiency of the suppression of exclusive $\rho^{0}$ vector mesons is investigated with the help of a fully tracked PYTHIA Monte Carlo production. In case of one-pion events, $t^{\prime}$ is varied in six steps of $0.1 \mathrm{GeV}^{2}$ between $-0.5 \mathrm{GeV}^{2}$ and zero. For the check of twopion events, the invariant mass window is set to $[0.6,1.0] \mathrm{GeV}$ and the upper $\Delta E$ limit to $0.6 \mathrm{GeV}$. Too wide cuts cause a large amount of discarded non-exclusive events and in turn an increase of the vector meson fraction. Figure 6.3.3 shows the obtained vector meson fractions for charged pions with and without applying the $\rho^{0}$ suppression for values of $t^{\prime}$ of $-0.4 \mathrm{GeV}^{2}$ and $-0.1 \mathrm{GeV}^{2}$. For all six values of $t^{\prime}$, the largest reduction in $f_{\mathrm{VM}}$ is found at small $x$. Here, a lower $t^{\prime}$ value improves the suppression efficiency. In the two highest $z$ bins where the vector meson fraction is more than $40 \%$, a reduction is obtained only for $t^{\prime} \geq-0.3 \mathrm{GeV}^{2}$. The smallest values of $f_{\mathrm{VM}}$ in these two bins are obtained for $t^{\prime}=-0.1 \mathrm{GeV}^{2}$.

Since the event samples are dominated by one-pion events the check of the twopion events has less influence on the resulting dilutions. Small variations of the $\Delta E$ limit cause only minor differences in the dilutions and the total amount of rejected events. The remaining exclusive vector meson events, which still dilute the charged pion samples, contain $\rho^{0}$ mesons which were not rejected by the method described above because of the neglected $\rho^{0}$ decay width and detector smearing, and the fixed values for $t^{\prime}$ and $\Delta E$.

The improvement of reduced vector meson fractions is counteracted by an enlargement of the statistical uncertainties due to the event rejection by the suppression method. In Table 6.3.1, the fractions of discarded events in the Monte Carlo and in the data productions are listed for one and two-pion events as well as for the full event sample. The 


\begin{tabular}{|l|c|c|c|c|}
\hline & \multicolumn{2}{|c|}{$t^{\prime}=-0.4 \mathrm{GeV}^{2}$} & \multicolumn{2}{c|}{$t^{\prime}=-0.1 \mathrm{GeV}^{2}$} \\
& Monte Carlo & $\begin{array}{c}\text { 02 - 04 data } \\
\text { productions }\end{array}$ & Monte Carlo & $\begin{array}{c}\text { 02 - 04 data } \\
\text { productions }\end{array}$ \\
\hline \hline 1 pion events & $69.11 \pm 0.05 \%$ & $65.96 \pm 0.09 \%$ & $42.31 \pm 0.05 \%$ & $39.04 \pm 0.09 \%$ \\
\hline 2 pion events & $3.13 \pm 0.03 \%$ & $2.89 \pm 0.06 \%$ & $3.13 \pm 0.03 \%$ & $2.89 \pm 0.06 \%$ \\
\hline all events & $43.45 \pm 0.08 \%$ & $42.82 \pm 0.13 \%$ & $26.52 \pm 0.09 \%$ & $27.69 \pm 0.16 \%$ \\
\hline
\end{tabular}

Table 6.3.1: Fractions of discarded semi-inclusive DIS events for the different event classes in the range $0.2<z<1.2$, performing $\rho^{0}$ suppression. The fractions are given for two different values of $t^{\prime}$.

amount of rejected pion events is comparable in the Monte Carlo and real data productions for all event classes. The remaining small deviations could be further decreased by an improved PYTHIA tune for the exclusive $\rho^{0}$ production. The inflation of the interpretive uncertainty without suppression can be expressed in terms of the vector meson fractions only,

$$
\frac{\sigma_{\text {intpr }}}{\sigma_{\text {intpr }}^{\text {sup }}}=\frac{f_{\mathrm{VM}}\left(1-f_{\mathrm{VM}}^{\text {sup }}\right)}{f_{\mathrm{VM}}^{\text {sup }}\left(1-f_{\mathrm{VM}}\right)},
$$

because the transfer values and vector meson asymmetry values cancel. The increase of the statistical uncertainty $\sigma_{\text {stat }}^{\text {sup }} / \sigma_{\text {stat }}$ when suppressing $\rho$ mesons can be determined for Collins and Sivers amplitudes. All three uncertainty ratios are plotted in Figure 6.3.4 for the different $x$ and $z$ bins. For $t^{\prime}=-0.4 \mathrm{GeV}^{2}$, the interpretive uncertainty ratio becomes

$t^{\prime}=-0.4 \mathrm{GeV}^{2}$

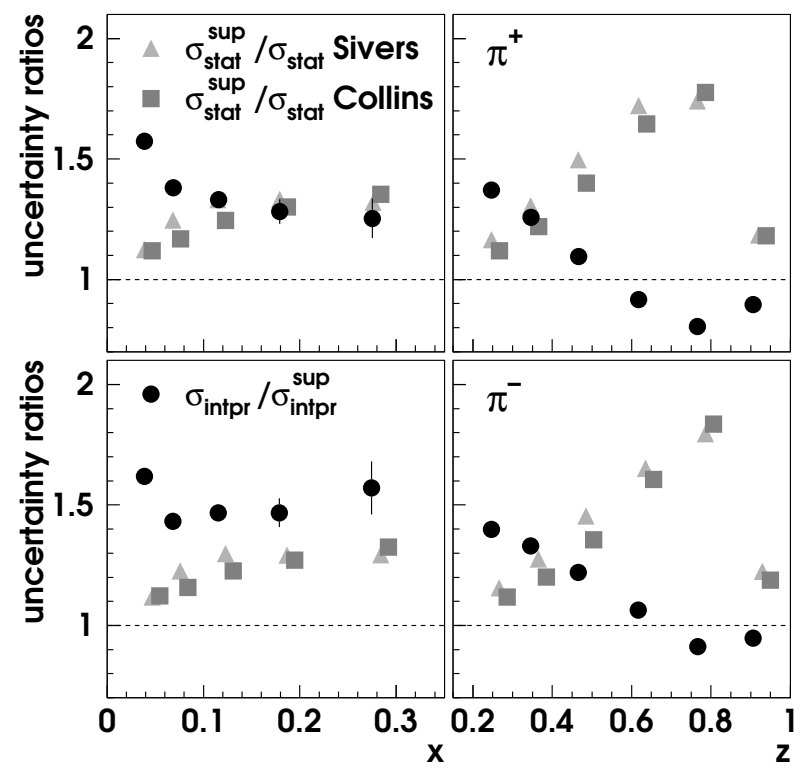

$t^{\prime}=-0.1 \mathrm{GeV}^{2}$

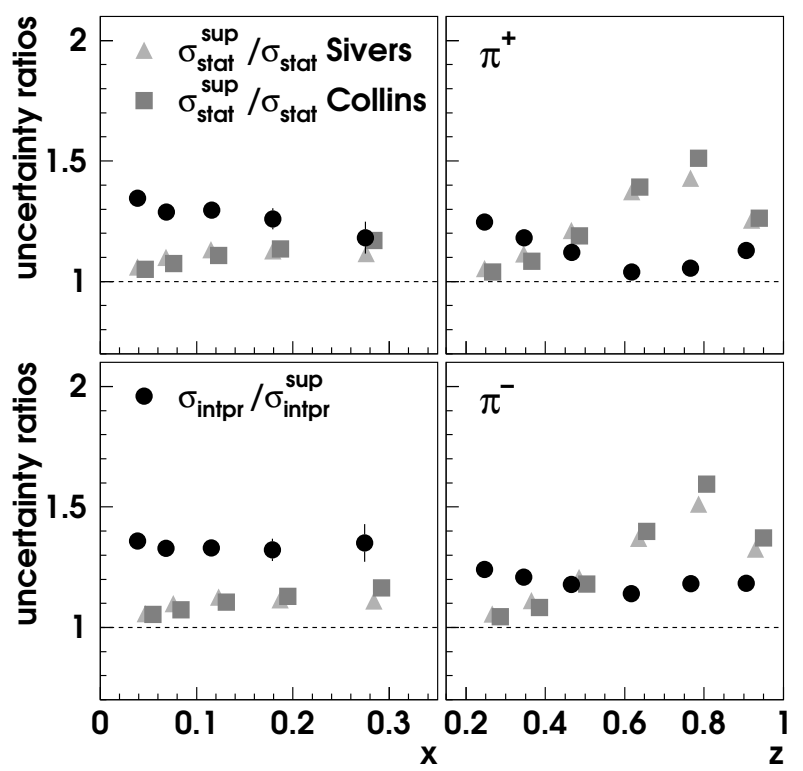

Figure 6.3.4: Relative decrease of the interpretive uncertainties and relative increase of the statistical uncertainties with and without $\rho^{0}$ suppression for $t^{\prime}=-0.4 \mathrm{GeV}^{2}$ (left panels) and $t^{\prime}=-0.1 \mathrm{GeV}^{2}$ (right panels). 

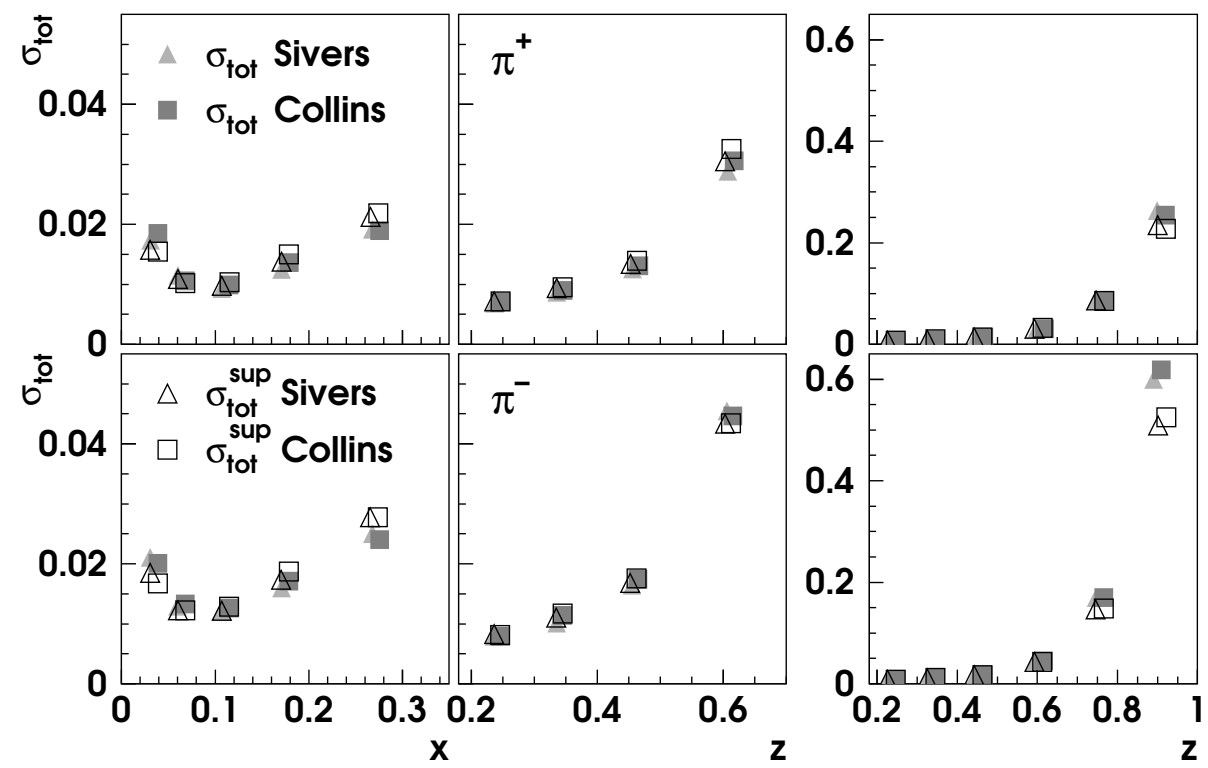

Figure 6.3.5: Total uncertainty with and without $\rho^{0}$ suppression for the Sivers and Collins asymmetries. The transverse momentum transfer $t^{\prime}$ is set to $-0.1 \mathrm{GeV}^{2}$.

even smaller than 1 in some $z$ bins, i.e., the interpretive uncertainty is smaller without the attempt to suppress the $\rho^{0}$ vector mesons. In all $x$ bins, the relative increase of the interpretive uncertainty without the suppression is at least compatible to the relative increase of the statistical uncertainty due to the suppression.

However, the change of the total uncertainty,

$$
\sigma_{\text {tot }}=\sqrt{\sigma_{\text {stat }}^{2}+\sigma_{\text {intpr }}^{2}}
$$

is not only determined by the uncertainty ratios but also by the absolute values of the uncertainties. In particular, for a large statistical uncertainty the total uncertainty can increase despite of the relative change in the interpretive uncertainty being bigger than the relative change of the statistical uncertainty. Figure 6.3.5 shows that only a small improvement of the total uncertainty can be achieved for the present statistics in the first $x$ and in the last $z$ bin. The total uncertainties are computed for the value $-1 \mathrm{GeV}^{2}$ for $t^{\prime}$ which results in the smallest vector meson fractions in the highest $z$ bins. In the latter, however, the total uncertainties still exceed the measured Collins and Sivers asymmetry amplitudes.

Even with the enhanced statistics in the full experimental data set including the data recorded in the year 2005, the suppression of $\rho^{0}$ vector mesons will reduce the total uncertainty only for the lower $x$ and higher $z$ bins. In the two highest $z$ bins where exclusive vector meson production is the dominating scattering process, the suppression method described above is still not able to decrease the vector meson fraction to values that result in interpretive uncertainties smaller than the measured Collins and Sivers amplitudes themselves. 


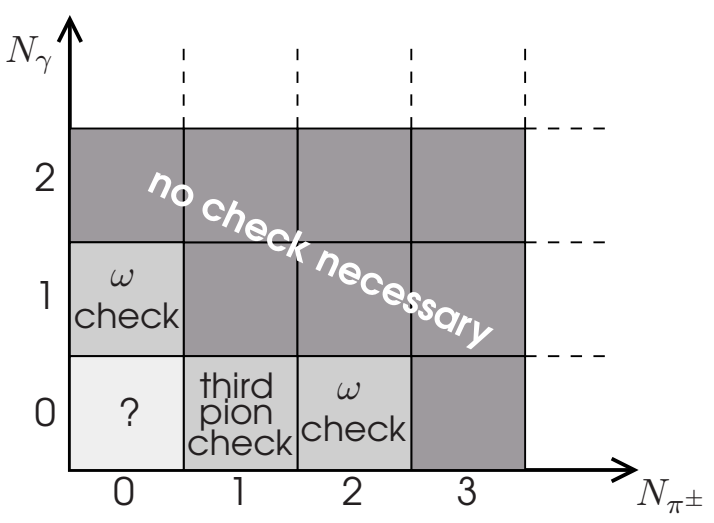

Figure 6.3.6: The classification of $\pi^{0}$ events dependent on the number of additional charged pions $N_{\pi^{ \pm}}$and photons $N_{\gamma}$. For further explanations see text.

\subsubsection{Suppression of Exclusively Produced $\omega$ Mesons}

The two main decay branches of the $\omega$ vector meson, $\omega \rightarrow \pi^{+} \pi^{-} \pi^{0}$ and $\omega \rightarrow \pi^{0} \gamma$, give rise to either two pions and two photons, or three photons, respectively. No attempt is made to suppress the contribution of $\omega$ mesons in the charged pion samples as it is small compared to the contribution of $\rho^{0}$ mesons (see Section 6.2.1). For the neutral pion sample figure 6.3.6 shows the different $\pi^{0}$ event classes which have to be considered for the $\omega$ suppression. Events with more than two charged pions or more than one photon detected together with the $\pi^{0}$ cannot be exclusive $\omega$ events. Also events with exactly one photon and at least one charged pion in addition to the $\pi^{0}$ do not have to be checked for a possible exclusive $\omega$ event. The remaining four event classes are single $\pi^{0}$ events without any additional pion or photon (light-grey field) and events in which the $\pi^{0}$ is accompanied by one photon or one or two charged pions (mid-grey fields). Two of these four classes allow a complete reconstruction of the $\omega$ vector meson, i.e., the calculation of the invariant mass and $\Delta E$ : For events with one $\pi^{0}$ and one additional charged pion, the kinematics of a potential third pion from a $\omega$ decay can be computed with the same method as described in the previous section. Unfortunately, the largest part of the data sample belongs to the single $\pi^{0}$ event class which cannot be checked for a possible exclusive $\omega$ event as not enough information is available to constrain the kinematics of the other decay particles in the three-body decay. Hence, all the single $\pi^{0}$ events can either be discarded or included in the analysis.

The efficiency of both suppression methods is determined in the same way as for the $\rho^{0}$

\begin{tabular}{|l|c|c|}
\hline & Monte Carlo & 02 - 04 data productions \\
\hline \hline with single $\pi^{0}$ event rejection & $21.92 \pm 0.27 \%$ & $46.44 \pm 0.31 \%$ \\
\hline without single $\pi^{0}$ event rejection & $2.79 \pm 0.32 \%$ & $3.89 \pm 0.47 \%$ \\
\hline
\end{tabular}

Table 6.3.2: Fractions of discarded events in the range $0.2<z<1.2$, with and without the rejection of single $\pi^{0}$ events in the $\omega$ suppression. 


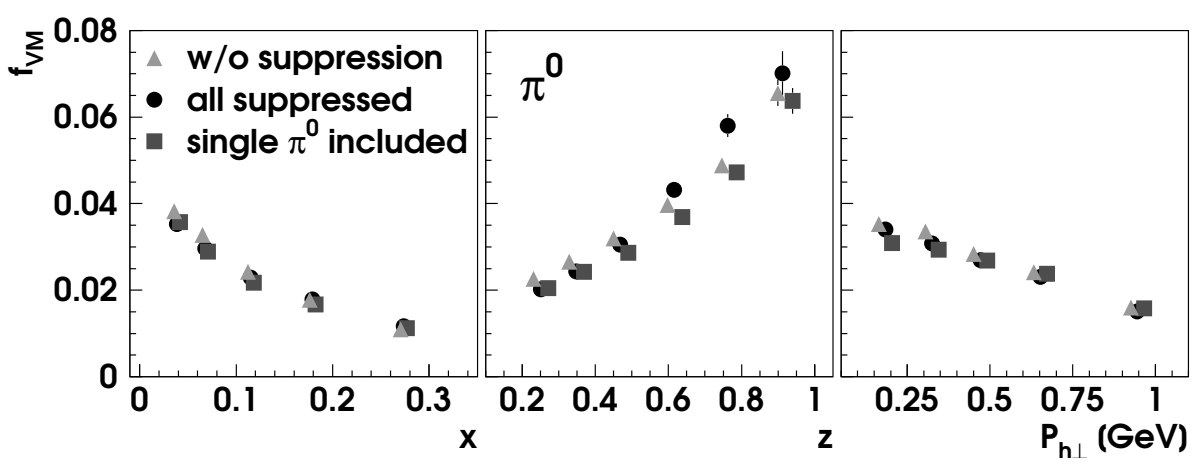

Figure 6.3.7: Dilution of the neutral pion sample with and without the suppression of $\omega$ vector mesons.

mesons with a fully tracked PYTHIA Monte Carlo production. A slightly wider cut on $-t^{\prime}=$ $0.5 \mathrm{GeV}^{2}$ and on $\Delta E=1.0 \mathrm{GeV}$ and a more narrow invariant mass window between 0.72 and $0.88 \mathrm{GeV}$ is used, taken from a previous analysis of exclusively produced $\omega$ mesons [Tyt01]. The width of the invariant mass window is determined by the longer life time of the $\omega$ meson compared to the $\rho^{0}$ meson. The reason for the wider $-t^{\prime}$ and $\Delta E$ cuts is the worse energy resolution for photons as compared to charged particles. In Table 6.3.2, the fractions of discarded events in the Monte Carlo and in the combined experimental data set are listed for the two suppression methods, i.e., with and without the rejection of single $\pi^{0}$ events.

The disagreement of the values is enormous in particular for the case of single $\pi^{0}$ rejection. One possible explanation might be combinatorial background events in the experimental data but not in the Monte Carlo simulation. However, the combinatorial background ratios of the HERMES data analysed with and without $\omega$ suppression are compatible and hence cannot explain the large discrepancy. Alternatively the large contribution of anomalous states to the vector meson fraction in the neutral pion sample seems questionable as the PYTHIA generator has not been funed to match HERMES data for exclusive $\omega$ production (cf. Sections 6.1 and 6.2.1).

The vector meson fractions with and without $\omega$ suppression are compared in Figure
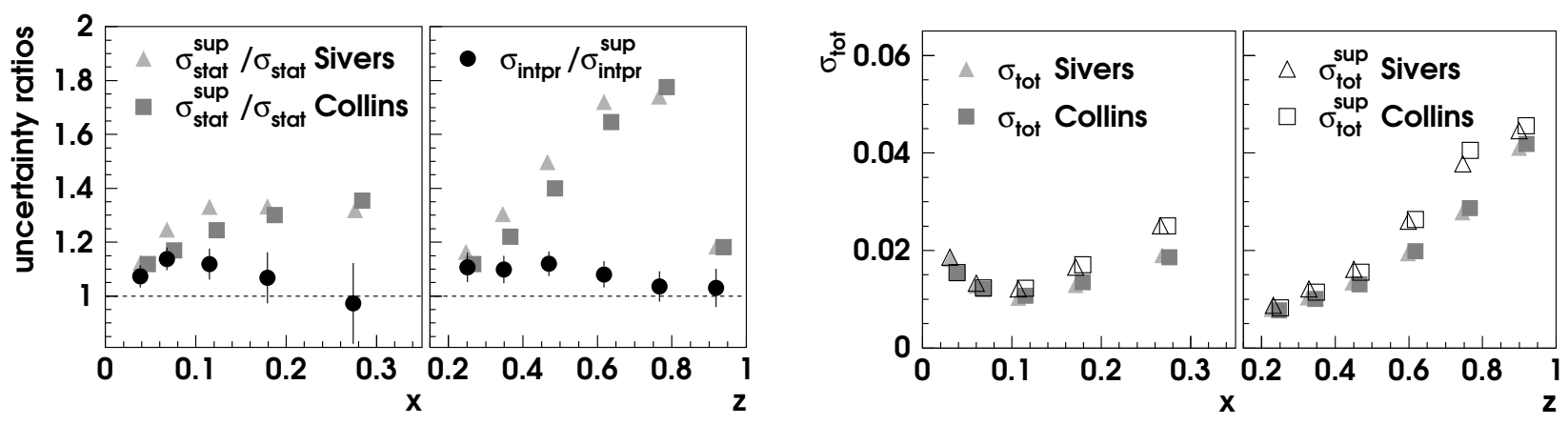

Figure 6.3.8: Relative decrease of the interpretive uncertainties and relative increase of the statistical uncertainties with and without $\omega$ suppression (left panels) and the total uncertainty with and without $\omega$ suppression (right panels). Single $\pi^{0}$ events are not rejected. 
6.3.7. With the inclusion of single $\pi^{0}$ events, the fractions decrease slightly in the highest $z$ bins, whereas they even increase somewhat if all single $\pi^{0}$ events are rejected. This can be understood if more semi-inclusive events than exclusive vector meson events are discarded. Although the vector meson fractions become slightly smaller using one of the suppression methods, the resulting relative and total uncertainties with this method are not reduced, as can be seen in Figure 6.3.8. Even after a successful optimisation of the PYTHIA generator for exclusive $\omega$ production in the HERMES kinematic region, the $\omega$ suppression will probably not be efficient enough for the justification of its application. Neither of the suppression methods described here has consequently been applied in the analysis.

\subsection{Amplitudes of the Pion-Yield-Difference Asymmetry}

Another approach to exclude pions from exclusively produced $\rho^{0}$ vector mesons is to form an asymmetry with the difference in the count rates of positive and negative pions. Disregarding the restriction of a finite detector acceptance, the number of $\rho^{0}$ decay pions is equal in the two count rates and cancels. In the HERMES acceptance, the remaining vector meson fraction in the count rate difference can be determined from the vector meson count numbers in the individual pion samples of a PYTHIA Monte Carlo production by:

$$
f_{\mathrm{VM}}=\frac{N_{\mathrm{VM} \rightarrow \pi^{+}}-N_{\mathrm{VM} \rightarrow \pi^{-}}}{N_{\pi^{+}}-N_{\pi^{-}}} .
$$

Figure 6.4.1 shows that also in the HERMES acceptance the vector meson contribution to this yield difference is consistent with zero. Consequently, asymmetries derived from this pion-yield difference are unaffected by potential vector meson biases. The moments of the pion-yield-difference asymmetry are defined as

$$
\left\langle\sin \left(\phi \pm \phi_{S}\right)\right\rangle_{\mathrm{UT}}^{\pi \pi}=\frac{\int \mathrm{d} \phi_{S} \mathrm{~d}^{2} \vec{P}_{h \perp} \sin \left(\phi \pm \phi_{S}\right)\left[\mathrm{d}^{6} \sigma_{\mathrm{UT}}^{\pi^{+}}-\mathrm{d}^{6} \sigma_{\mathrm{UT}}^{\pi^{-}}\right]}{\int \mathrm{d} \phi_{S} \mathrm{~d}^{2} \vec{P}_{h \perp}\left[\mathrm{d}^{6} \sigma_{\mathrm{UU}}^{\pi^{+}}-\mathrm{d}^{6} \sigma_{\mathrm{UU}}^{\pi^{-}}\right]},
$$

where the definitions of the differential cross sections $\mathrm{d}^{6} \sigma_{\mathrm{UT}}$ and $\mathrm{d}^{6} \sigma_{\mathrm{UU}}$ can be found in Section 2.5.2. In order to obtain these asymmetry moments, the following azimuthal

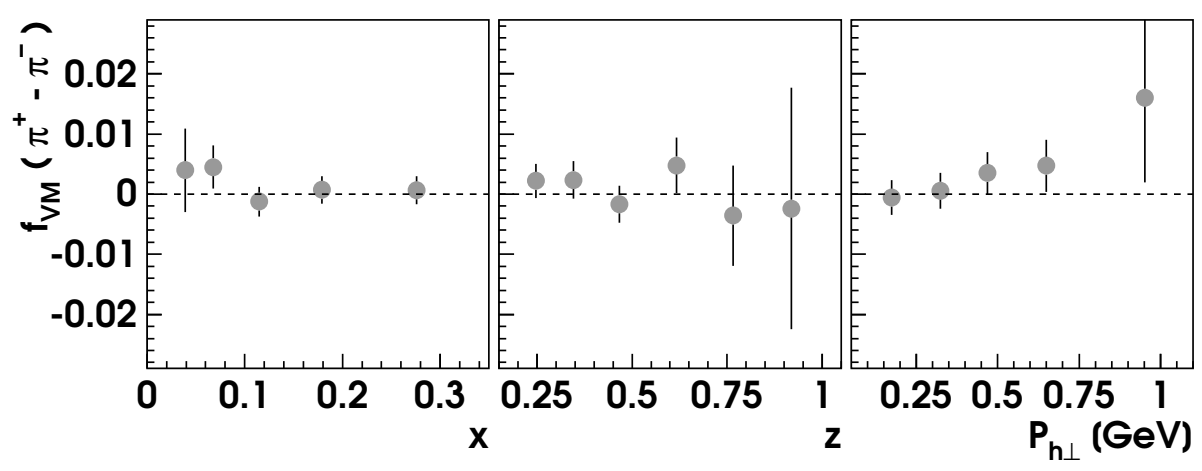

Figure 6.4.1: Vector meson fractions for the amplitudes of the pion-yielddifference asymmetry. 


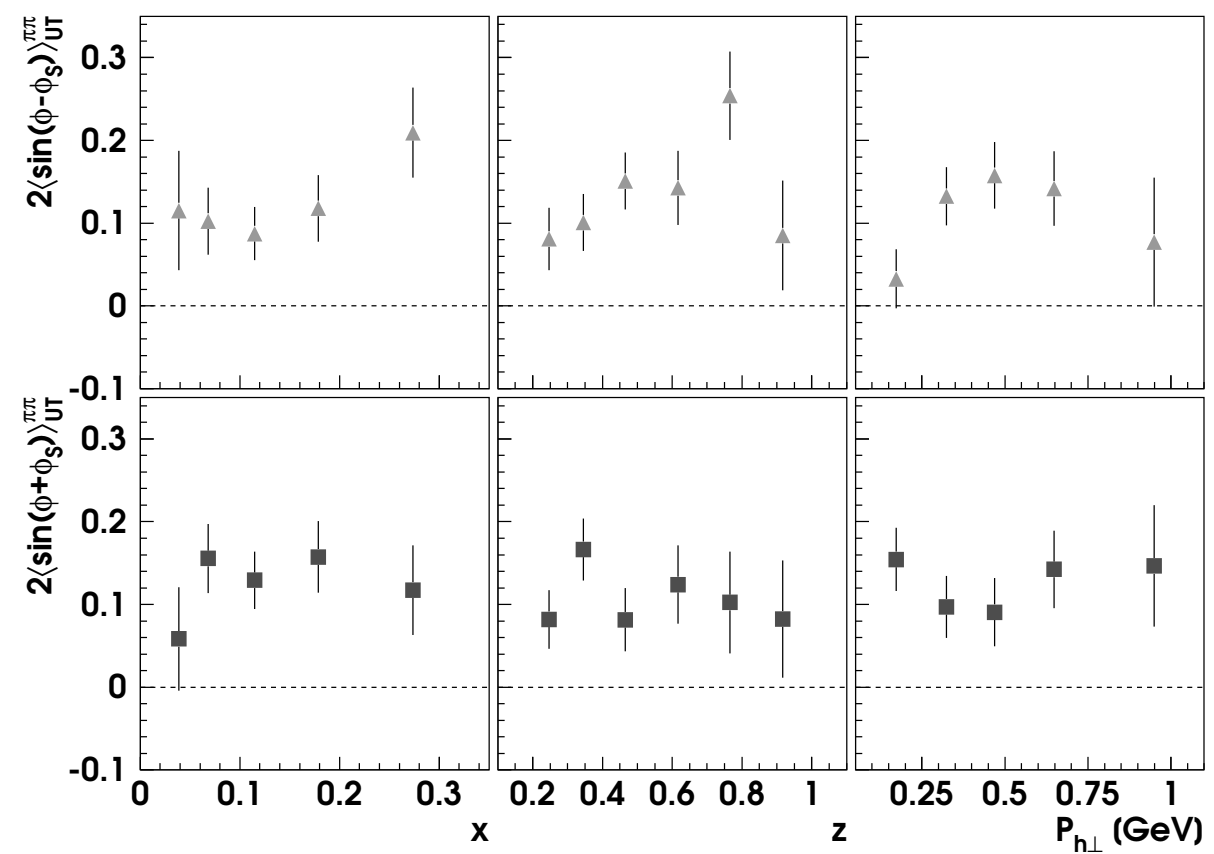

Figure 6.4.2: Sivers and Collins amplitudes of the pion-yield-difference asymmetry dependent on $x, z$, and $P_{h \perp}$.

asymmetry is formed with the count numbers of positive and negative pions:

$$
\begin{aligned}
A_{\mathrm{UT}}^{\pi \pi}\left(\phi, \phi_{S}\right) & =\frac{1}{\langle P\rangle} \frac{\left[L^{\downarrow} N_{\pi^{+}}^{\uparrow}\left(\phi, \phi_{S}\right)-L^{\uparrow} N_{\pi^{+}}^{\downarrow}\left(\phi, \phi_{S}\right)\right]-\left[L^{\downarrow} N_{\pi^{-}}^{\uparrow}\left(\phi, \phi_{S}\right)-L^{\uparrow} N_{\pi^{-}}^{\downarrow}\left(\phi, \phi_{S}\right)\right]}{\left[L N_{\pi^{+}}^{\uparrow}\left(\phi, \phi_{S}\right)+L^{\uparrow} N_{\pi^{+}}^{\downarrow}\left(\phi, \phi_{S}\right)\right]-\left[L \downarrow N_{\pi^{-}}^{\uparrow}\left(\phi, \phi_{S}\right)+L^{\uparrow} N_{\pi^{-}}^{\downarrow}\left(\phi, \phi_{S}\right)\right]} \\
& =\frac{1}{\langle P\rangle} \frac{L^{\downarrow} \Delta N^{\uparrow}\left(\phi, \phi_{S}\right)-L^{\uparrow} \Delta N^{\downarrow}\left(\phi, \phi_{S}\right)}{L^{\downarrow} \Delta N^{\uparrow}\left(\phi, \phi_{S}\right)+L^{\uparrow} \Delta N^{\downarrow}\left(\phi, \phi_{S}\right)},
\end{aligned}
$$

where $\Delta N=N_{\pi^{+}}-N_{\pi^{-}}$. As for the individual pion asymmetries, the amplitudes are extracted by a two-dimensional fit with the function (4.3.2). Figure 6.4.2 shows the obtained pion-yield-difference amplitudes. They are significantly positive which proves that the extracted asymmetry moments for the individual pions are not predominantly caused by decay pions of exclusive $\rho^{0}$ mesons. A second evidence in case of the Sivers effect is that the Sivers $K^{+}$amplitudes are significantly positive while the $K^{+}$sample has a negligible contribution from exclusive vector mesons (see Figures 4.4.8 and 6.2.1).

\subsection{Correction for the Vector Meson Contribution}

Based on the above results for the small decay pion asymmetries (see Section 6.2.4) and on the significantly positive amplitudes for the pion-yield-difference as well as for the $K^{+}$ asymmetries, one can safely assume that exclusively produced vector mesons do not significantly bias the extracted Collins and Sivers amplitudes. The decay hadrons from vector meson production processes rather dilute the semi-inclusive hadron samples. This dilution can be corrected for by scaling both the extracted asymmetry moments and their statistical uncertainties by $1 /\left(1-f_{\mathrm{VM}}\right)$.

These corrected asymmetry amplitudes for charged and neutral pions are compared to the uncorrected amplitudes in Figure 6.5. 1. Only in the last two $z$ bins, where the vector 

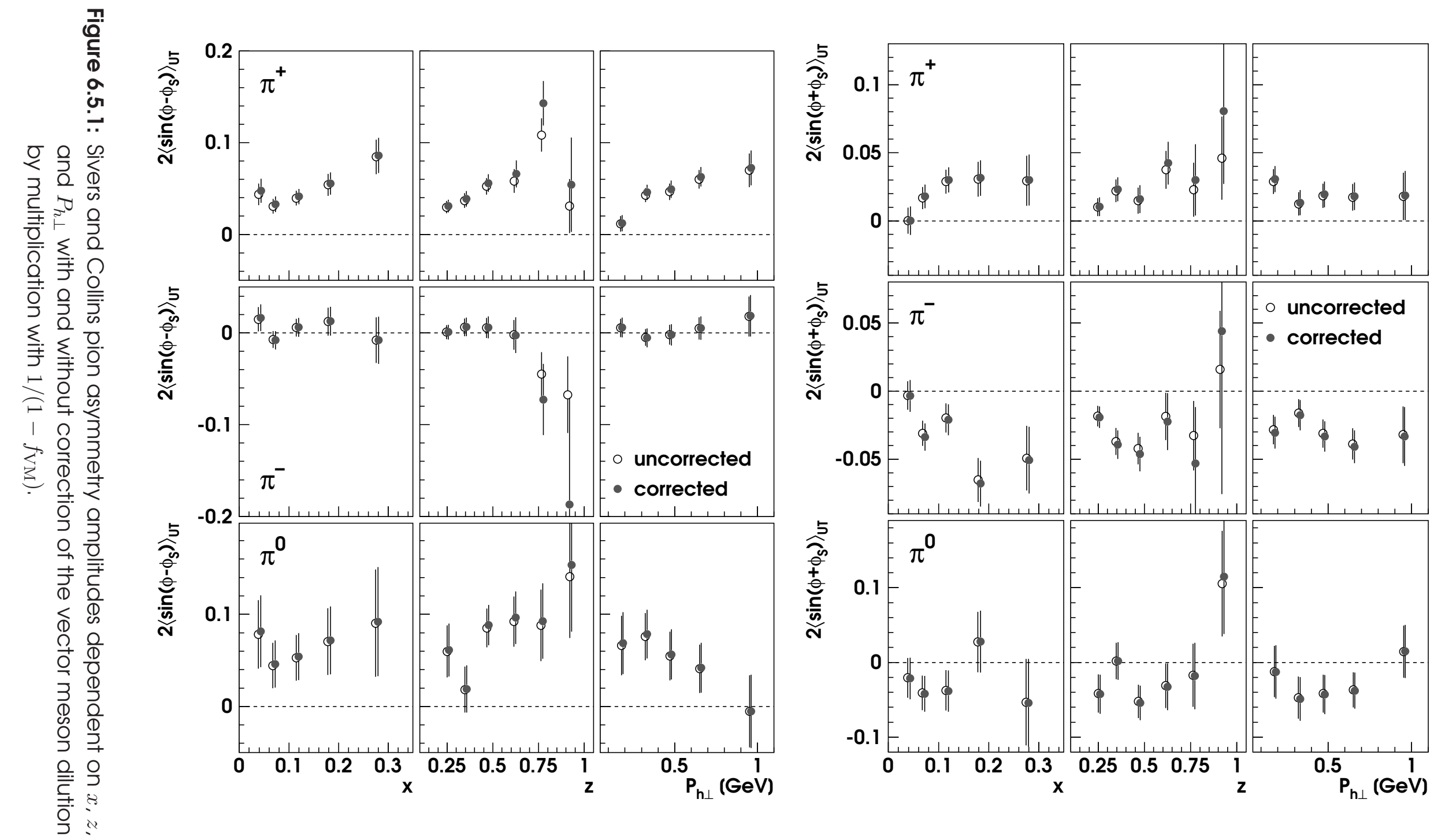
meson fractions of the charged pion samples exceed $10 \%$, a change of the amplitudes is visible. In all the other kinematic bins, the small vector meson fraction does not influence the measured amplitudes significantly. Also for the charged kaons, the measured amplitudes are not affected by the vector meson correction because of the even smaller vector meson fractions. The systematic uncertainty of the corrected amplitudes which is not shown in the plot, has to be increased by a $15 \%$ relative systematic uncertainty on the vector meson fractions which is based on the description of the measured cross sections of exclusive vector meson production by the PYTHIA Monte Carlo generator [Lie04]. 

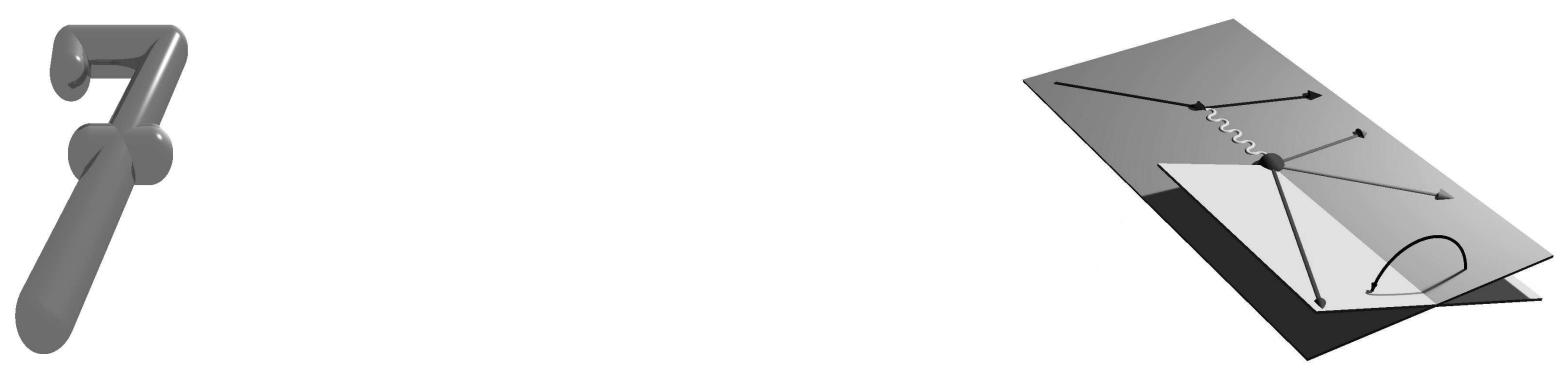

\section{Separation of the Photon-Axis Asymmetry Moments}

The asymmetry moments (2.5.21) are defined for particles polarised with respect to the virtual-photon direction. Experimentally, however, the particle spin is oriented with respect to the lepton beam while the virtual-photon direction changes in every scattering event. Longitudinal and transverse spin asymmetry moments defined with respect to the virtual-photon direction, in the following referred to as photon-axis asymmetry moments $\left\langle\sin \left(\phi \pm \phi_{S}\right)\right\rangle_{\mathrm{UT}}^{\mathrm{q}}$, contribute to the measured so-called lepton-axis asymmetry moments $\left\langle\sin \left(\phi \pm \phi_{S}\right)\right\rangle_{\mathrm{UT}}^{1}$ introduced in Chapter 4. They also appear in the measurement on a longitudinally polarised target with respect to the lepton beam. This chapter presents the separation of the individual cross-section contributions in a frame where the target polarisation is defined with respect to the direction of the virtual photon.

\subsection{Spin Components in the Two Reference Systems}

As shown in Figure 2.5.3, a nucleon transversely polarised with respect to the incoming lepton beam has a dominant transverse spin component [Die05a]:

$$
S_{T}=\frac{\cos \theta_{\gamma^{*}}}{\sqrt{1-\sin ^{2} \theta_{\gamma^{*}} \sin ^{2} \phi_{S}}},
$$

where $\theta_{\gamma^{*}}$ is the polar angle between the momenta of incoming lepton and the virtual photon. The small longitudinal spin component,

$$
S_{L}=\frac{\sin \theta_{\gamma^{*}} \cos \phi_{S}}{\sqrt{1-\sin ^{2} \theta_{\gamma^{*}} \sin ^{2} \phi_{S}}},
$$

is not indicated in Figure 2.5.3. For sufficiently high beam momenta, the angle between the incoming lepton and the virtual photon is small and $\sin ^{2} \theta_{\gamma^{*}}$ can be neglected. The components can then be approximated by $S_{T} \approx \cos \theta_{\gamma^{*}}$ and $S_{L} \approx \sin \theta_{\gamma^{*}} \cos \phi_{S}$ where the longitudinal component can be as large as $15 \%$ at HERMES kinematics (see Tables F.5 F.7). When integrated over $\phi_{S}$ the longitudinal spin component vanishes and the resulting 


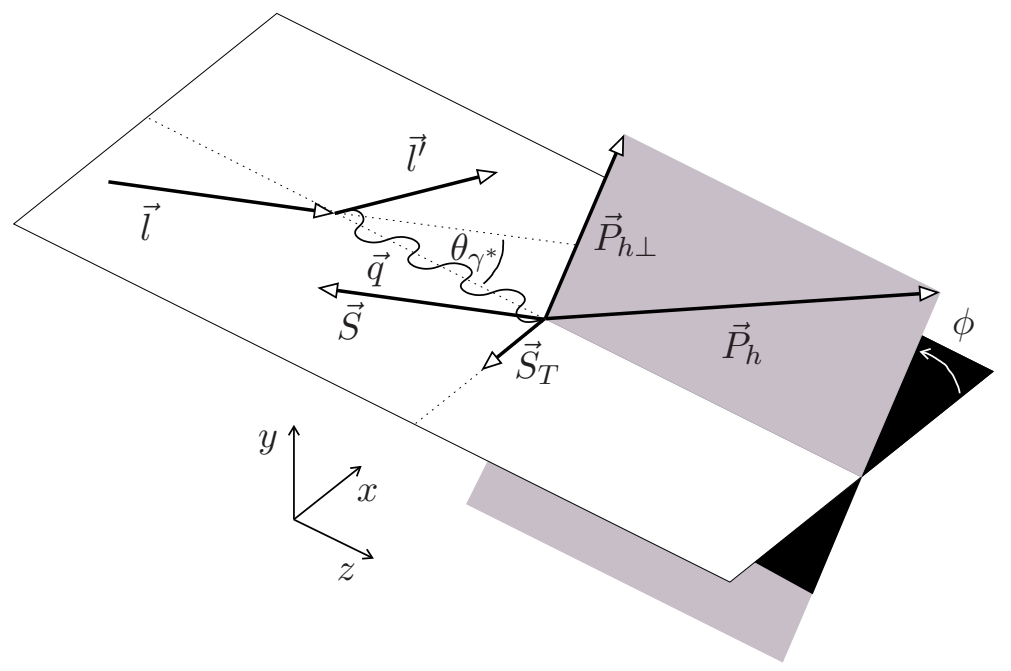

Figure 7.1.1: Configuration of angles and spin components in case of a longitudinally polarised target.

longitudinal polarisation for all scattering events is zero. However, the polarised cross section $\mathrm{d}^{6} \sigma_{\mathrm{UL}}$ contains a $\sin \phi$ modulation (see Table 2.5.1) which couples to the $\cos \phi_{S}$ modulation of the longitudinal spin component since $\sin \phi \cos \phi_{S}=\frac{1}{2}\left[\sin \left(\phi+\phi_{S}\right)+\sin (\phi-\right.$ $\left.\phi_{S}\right)$ ]. Hence, the longitudinal twist-three cross-section term contributes to the measured Collins and Sivers moments:

$$
\left\langle\sin \left(\phi \pm \phi_{S}\right)\right\rangle_{\mathrm{UT}}^{1}=\cos \theta_{\gamma^{*}}\left\langle\sin \left(\phi \pm \phi_{S}\right)\right\rangle_{\mathrm{UT}}^{\mathrm{q}}+\frac{1}{2} \sin \theta_{\gamma^{*}}\langle\sin \phi\rangle_{\mathrm{UL}}^{\mathrm{q}} .
$$

For a target which is polarised longitudinally with respect to the lepton beam, also a small transverse spin component exists, as indicated in Figure 7.1.1. In this case, however, the azimuthal angle $\phi_{S}$ of the transverse component is constant for all scattering events and the sine modulations of the Collins and Sivers effect occur as $\sin \phi$ contributions:

$$
\langle\sin \phi\rangle_{\mathrm{UL}}^{\mathrm{L}}=\cos \theta_{\gamma^{*}}\langle\sin \phi\rangle_{\mathrm{UL}}^{\mathrm{q}}-\sin \theta_{\gamma^{*}}\left[\left\langle\sin \left(\phi+\phi_{S}\right)\right\rangle_{\mathrm{UT}}^{\mathrm{q}}+\left\langle\sin \left(\phi-\phi_{S}\right)\right\rangle_{\mathrm{UT}}^{\mathrm{q}}\right],
$$

where the minus sign results from the fact that $\phi_{S}=\pi$. The three measured lepton-axis azimuthal moments can be related to the three corresponding photon-axis moments by a matrix, dependent on the angle $\theta_{\gamma^{*}}$ :

$$
\left(\begin{array}{c}
\langle\sin \phi\rangle_{\mathrm{UL}}^{1} \\
\left\langle\sin \left(\phi-\phi_{S}\right)\right\rangle_{\mathrm{UT}}^{1} \\
\left\langle\sin \left(\phi+\phi_{S}\right)\right\rangle_{\mathrm{UT}}^{1}
\end{array}\right)=\left(\begin{array}{ccc}
\cos \theta_{\gamma^{*}} & -\sin \theta_{\gamma^{*}} & -\sin \theta_{\gamma^{*}} \\
\frac{1}{2} \sin \theta_{\gamma^{*}} & \cos \theta_{\gamma^{*}} & 0 \\
\frac{1}{2} \sin \theta_{\gamma^{*}} & 0 & \cos \theta_{\gamma^{*}}
\end{array}\right)\left(\begin{array}{c}
\langle\sin \phi\rangle_{\mathrm{UL}}^{\mathrm{q}} \\
\left\langle\sin \left(\phi-\phi_{S}\right)\right\rangle_{\mathrm{UT}}^{\mathrm{q}} \\
\left\langle\sin \left(\phi+\phi_{S}\right)\right\rangle_{\mathrm{UT}}^{\mathrm{q}}
\end{array}\right),
$$

valid up to corrections of order $\sin ^{2} \theta_{\gamma^{*}}[$ Die05a].

\subsection{The Reviewed Analysis of the Longitudinal Data}

During the years 1996 and 1997, the HERMES experiment took data with a longitudinally polarised hydrogen target. Azimuthal asymmetry moments extracted from these data 
can be used for the separation of the photon-axis moments together with the measured asymmetry moments presented in Chapter 4. Different kinematic bins and cuts were used for the publications [AirO0, AirOl] of the longitudinal spin asymmetry moments requiring a reanalysis of the data with the same kinematic cuts and the same binning as in the analysis of the transverse spin asymmetry moments, listed in Tables 4.2.2 and 4.3. 1. For the data of the years 1996 and 1997, the threshold Čerenkov detector was used to identify charged pions (see Section 3.3.2). For clean identification, the pions are required to have a momentum well in between the two threshold momenta yielding a cut on the pion momentum of $4 \mathrm{GeV}<P_{\pi}<13.8 \mathrm{GeV}$. In addition, the signal in the threshold Čerenkov counter must be larger than 0.5 photo electrons.

Similar to the extraction of the Sivers and Collins amplitudes, the $\sin \phi$ amplitude of the luminosity normalised azimuthal asymmetry,

$$
A_{\mathrm{UL}}(\phi)=\frac{1}{\langle P\rangle} \frac{N^{\rightarrow}(\phi) L^{\leftarrow}-N^{\leftarrow}(\phi) L^{\rightarrow}}{N^{\rightarrow}(\phi) L^{\leftarrow}+N^{\leftarrow}(\phi) L^{\rightarrow}},
$$

is extracted by a fit. Here, $\langle P\rangle$ is the averaged longitudinal target polarisation which can be found in Table 3.2.1, and $\rightarrow(\leftarrow)$ indicates the target polarisation state antiparallel (parallel) to the beam momentum. $L \leftrightarrows$ are the luminosities in each target spin state and $N^{\leftrightarrows}(\phi)$ are the hadron count numbers in each target spin state and $\phi$ bin. The events are filled in 8 equidistant $\phi$ bins, i.e., a one-dimensional azimuthal binning is applied. The fit function,

$$
A_{\mathrm{UL}}(\phi)=A_{\mathrm{UL}}^{\sin \phi} \cdot \sin \phi+A_{\mathrm{UL}}^{\sin 2 \phi} \cdot \sin 2 \phi+C,
$$

includes_besides the $\sin \phi$ amplitude and a constant-also the leading twist $\sin 2 \phi$ cross section term (cf. Table 2.5.1) as a fit parameter. In the former analysis, a different approach was used for the extraction of the $\sin \phi$ moment by weighting the events with $\sin \phi$. This method is affected by the mixing of the two cross-section sine modulations which the extracted moments have been corrected for.

For the published results on neutral pions [AirO1], the combinatorial background is accounted for in the systematic uncertainty whereas a correction is applied in the present reanalysis like it is done for the $\pi^{0}$ asymmetry moments of the transversely polarised target.

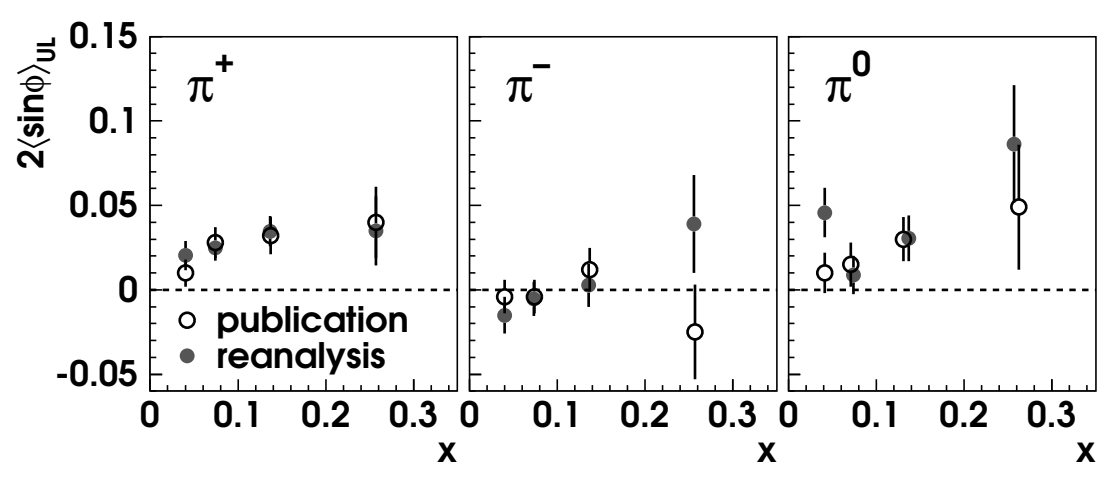

Figure 7.2.1: Comparison of the published asymmetry amplitudes of the longitudinally polarised hydrogen data [AirO0, AirO1] and the results of the reanalysis of the two data productions. Shown are the amplitudes as a function of $x$. 
The cut on the number of photons per event is lowered to $N_{\gamma} \leq 5$ in order to obtain combinatorial background fractions which are similar to the fractions obtained in the years 2002 to 2004.

In Figure 7.2.1 the published asymmetry amplitudes are compared to the amplitudes obtained in this reanalysis. For the comparison the same $x$ binning and the same kinematic cuts as in the publication are applied and the neutral pions are not corrected for combinatorial background. The general behaviour of the extracted amplitudes using Eq. (7.2.2) and the published results is consistent. The differences are mainly due to the reproductions of the 1996 and 1997 data samples which include, among others, a new calibration of the detectors and an improved reconstruction algorithm for the photon position on the calorimeter front. In addition, the published results are corrected both for acceptance effects and mixing between the $\sin \phi$ and $\sin 2 \phi$ modulations. The latter correction is not necessary for the fit that extracts both modulations at the same time. The acceptance effects on the asymmetry amplitudes obtained in the reanalysis are estimated to be $5 \%$ which is the observed effect for a two-dimensional azimuthal binning. It can be used as an upper limit for the effects in case of the one-dimensional azimuthal binning. The uncertainty of the target polarisation measurement yields a $4.2 \%$ scale uncertainty.

\subsection{The Subleading-Twist Cross Section Component}

Inverting to Eq. (7.1.5), the photon-axis twist-three asymmetry moment $\langle\sin \phi\rangle_{\mathrm{UL}}^{\mathrm{q}}$ can be written as function of the measured lepton-axis asymmetry moments:

$$
\langle\sin \phi\rangle_{\mathrm{UL}}^{\mathrm{q}}=\cos \theta_{\gamma^{*}}\langle\sin \phi\rangle_{\mathrm{UL}}^{1}+\sin \theta_{\gamma^{*}}\left[\left\langle\sin \left(\phi+\phi_{S}\right)\right\rangle_{\mathrm{UT}}^{1}+\left\langle\sin \left(\phi-\phi_{S}\right)\right\rangle_{\mathrm{UT}}^{1}\right],
$$

where the deviation from unity of $\cos \theta_{\gamma^{*}}$ is of the order of a few tenths of a percent for the HERMES kinematics and can therefore be neglected. From the inclusive quantities $x$, $Q^{2}$, and $y, \sin \theta_{\gamma^{*}}$ can be evaluated as:

$$
\sin \theta_{\gamma^{*}}=\frac{2 x M}{Q} \sqrt{\frac{1-y-y^{2} x^{2} M^{2} / Q^{2}}{1+4 x^{2} M^{2} / Q^{2}}} .
$$

The transformation into the photon-axis reference system is only an approximation because the cross sections are integrated over the kinematic variables and an average value of $\sin \theta_{\gamma^{*}}$ is used instead of the actual value for each event.

Also the transversely polarised hydrogen data has to be reanalysed using the hadron momentum cuts of the pion identification with the threshold Čerenkov counter instead of the $\mathrm{RICH}$. Otherwise, the kinematic mean values in the individual bins differ slightly. A tighter restriction of the momentum range causes lower hadron count numbers and larger statistical uncertainties. However, the extraction of $\langle\sin \phi\rangle_{\mathrm{UL}}^{\mathrm{q}}$ is clearly dominated by the statistical uncertainty of the longitudinally polarised hydrogen data set due to the kinematic suppression of the moments $\left\langle\sin \left(\phi \pm \phi_{S}\right)\right\rangle_{\mathrm{UT}}^{1}$ and thus their statistical uncertainties by $\sin \theta_{\gamma^{*}}$.

The obtained photon-axis asymmetry amplitudes $2\langle\sin \phi\rangle_{\mathrm{UL}}^{\mathrm{q}}$ are shown in Figure 7.3.1 together with the measured lepton-axis amplitudes for the two perpendicular target polarisations. Solving Eq. (7.3.1) for $\langle\sin \phi\rangle_{\mathrm{UL}}^{1}$ yields the two components in the measurement 


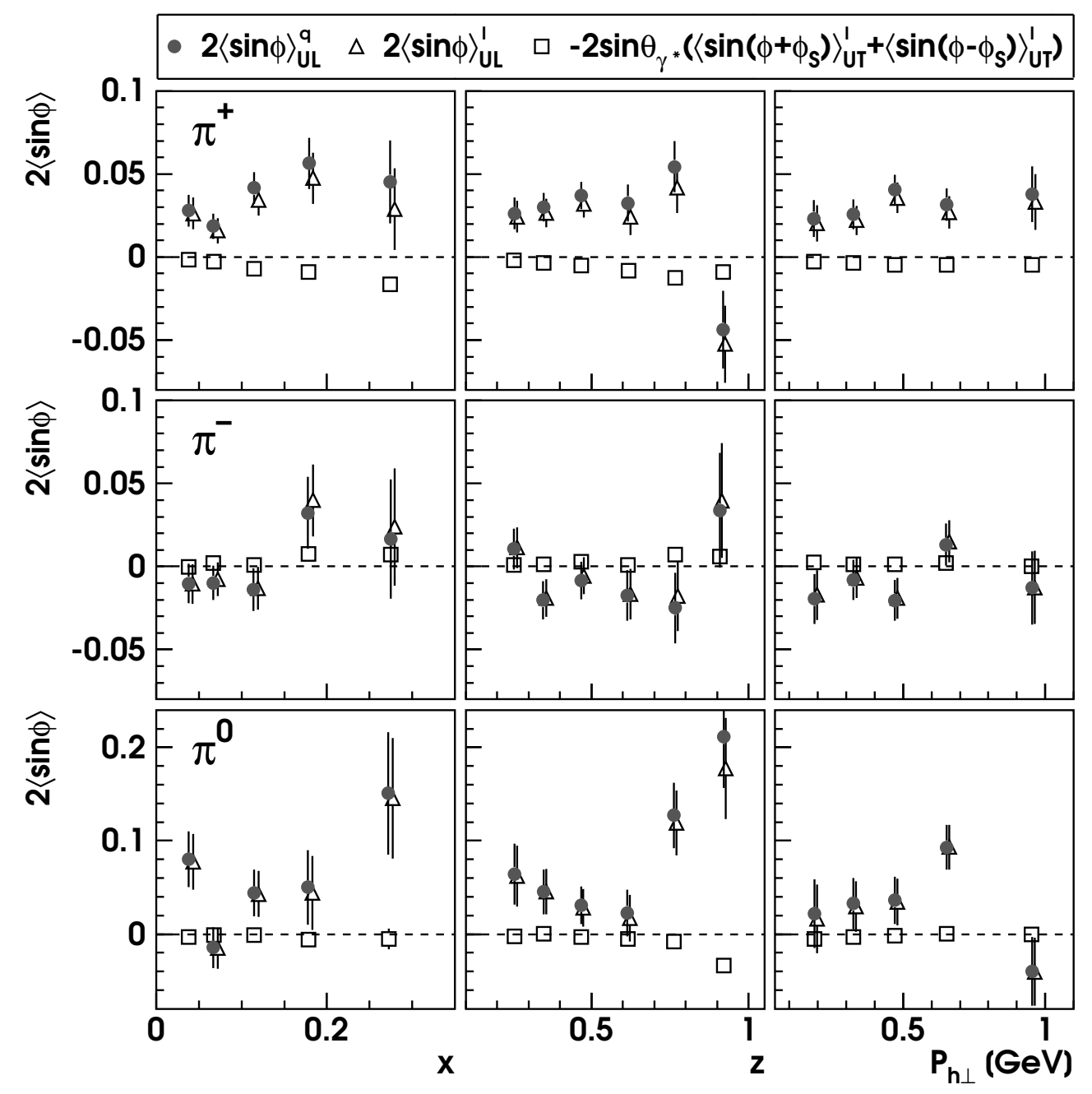

Figure 7.3.1: The measured lepton-axis asymmetry amplitudes on a longitudinally polarised target (open triangles) and their two contributions: the photon-axis asymmetry amplitudes in case of a longitudinally polarised target (closed circles) and the kinematically suppressed lepton-axis amplitudes for a transversely polarised target (open squares).

on a longitudinally polarised target. The transverse spin component is the sum of the measured Collins and Sivers amplitudes multiplied by $-\sin \theta_{\gamma^{*}}$ which is also plotted in Figure 7.3.1. The measurement on a longitudinally polarised target is dominated by the subleading-twist term in the longitudinal cross section. Therefore, the twist-three amplitude $2\langle\sin \phi\rangle_{\mathrm{UL}}^{\mathrm{q}}$ shows the same behaviour as the measured asymmetry amplitude on the longitudinally polarised target, i.e., the term is positive for positive and neutral pions and compatible with zero in case of the negative pions. The systematic uncertainty of the extracted twist-three term is negligible compared to the statistical uncertainty. The results of the photon-axis and lepton-axis $\langle\sin \phi\rangle_{\mathrm{UL}}$ moments dependent on the various kinematic quantities together with statistical and systematic uncertainties can be found in the Tables F.5 - F.7. Note that the $x$ dependence is influenced by the $Q^{2}$ dependence due to the strong correlation of $x$ and $Q^{2}$ in the HERMES acceptance. 
The mean asymmetry amplitudes $2\langle\sin \phi\rangle_{\mathrm{UL}}^{\mathrm{q}}$ in the range $0.023<x<0.04$ and $0.2<z<0.7$ are $0.031 \pm 0.005 \pm 0.002$ for positive pions, $-0.007 \pm 0.006 \pm 0.001$ for negative pions, and $0.035 \pm 0.013 \pm 0.003$ for neutral pions. They are obtained by averaging over the $x$ bins. The first uncertainty value given arises from the statistics and the second one is the systematical uncertainty.

Several contributions appear in the subleading-twist cross section term (cf. Table 2.5. 1), yielding a photon-axis asymmetry moment:

$$
\begin{aligned}
\langle\sin \phi\rangle_{\mathrm{UL}}^{\mathrm{q}}=-\left|\vec{S}_{L}\right| \frac{(2-y) \sqrt{1-y}}{1-y+\frac{y^{2}}{2}} \frac{M}{Q}\left\{\frac{\int \mathrm{d}^{2} \vec{P}_{h \perp} \mathcal{I}\left[\frac{\hat{P}_{h \perp} \cdot \vec{k}_{T}}{M_{h}}\left(\frac{M_{h}}{z M} \Delta q G^{\perp}+x h_{L} H_{1}^{\perp}\right)\right]}{q D_{1}}+\right. \\
\left.\frac{\int \mathrm{d}^{2} \vec{P}_{h \perp} \mathcal{I}\left[\frac{\hat{P}_{h \perp \cdot} \vec{p}_{T}}{M}\left(\frac{M_{h}}{z M} h_{1 L}^{\perp} \tilde{H}-x f_{L}^{\perp} D_{1}\right)\right]}{q D_{1}}\right\} .
\end{aligned}
$$

Here, the summation over quark flavours and the $x, z, p_{T}^{2}$, and $k_{T}^{2}$ dependencies of the DFs and FFs are implied. The integral $\mathcal{I}[\ldots]$ over $\vec{k}_{T}$ and $\vec{p}_{T}$ is defined in Eq. (2.5.16). The extracted asymmetry amplitudes $2\langle\sin \phi\rangle_{\mathrm{UL}}^{\mathrm{q}}$ allow no statement about the size of the individual contributions. Yet, the significantly positive amplitudes for $\pi^{+}$and $\pi^{0}$ show that subleading-twist terms can be large at HERMEs kinematics and that an interpretation of azimuthal asymmetry moments solely in terms of leading-twist functions is not appropriate in all cases. Some of the DFs and FFs in Eq. (7.3.3) appear also in other azimuthal asymmetry moments. The DF $h_{1 L}^{\perp}$ is present in the $\sin 2 \phi$ moment of the longitudinally polarised target (cf. Table 2.5.1), which is extracted by the fit (7.2.2) together with the $\sin \phi$ modulation. For HERMEs kinematics this moment is found to be compatible with zero. Nevertheless, a recent measurement in a different kinematic region by the CLAS experiment yielded non-vanishing $\langle\sin 2 \phi\rangle_{\mathrm{UL}}^{\mathrm{l}}$ moments [Ava04a]. The FF $G^{\perp}$ in conjunction with the unpolarised DF is present in the cross section for a longitudinally polarised beam and an unpolarised target [Bac04c]. Non-zero values of this beam spin asymmetry moment were reported by CLAS [Ava04b] and HERMES [Ave04a], but also here additional twistthree DFs or FFs contribute. Like the Sivers function, the T-odd DF $f_{L}^{\perp}$ was thought to be forbidden and therefore neglected for a long time. At the moment, no firm information about any of the subleading-twist functions in $\langle\sin \phi\rangle_{\mathrm{UL}}^{\mathrm{q}}$ exists.

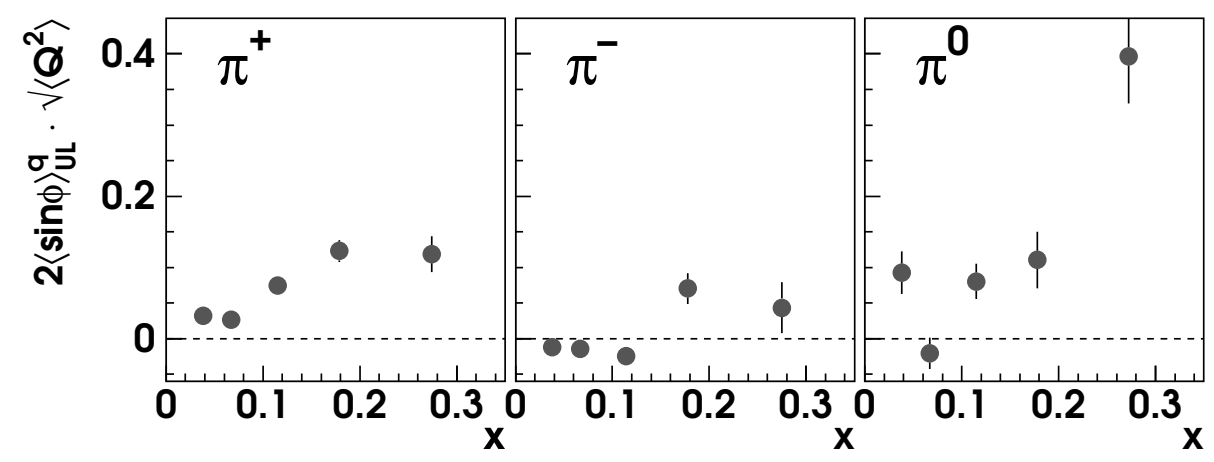

Figure 7.3.2: The $x$ dependence of the photon-axis asymmetry amplitudes approximately corrected for the $1 / Q$ dependence by multiplication with $\sqrt{\left\langle Q^{2}\right\rangle}$. 
In an attempt to gain more information from the measured asymmetries by separating their $x$ and $Q^{2}$ dependencies a simple study was performed. For this, the asymmetry moments were multiplied with the average $Q \approx \sqrt{\left\langle Q^{2}\right\rangle}$ in each $x$ bin. In Figure 7.3.2 the resulting $x$ dependence of the asymmetries can be seen, showing a clear hint for a rise towards larger $x$. However, whether this is due to the explicit $x$ dependence of some of the terms in Eq. (7.3.3) or due to the $x$ dependence of the DFs cannot be concluded. Speculating, the term involving $f_{L}^{\perp}$ could produce the measured $x$ dependence if both the unpolarised DF $q(x)$ (denominator of the asymmetry) and $f_{L}^{\perp}$ (numerator) depend similarly on $x$. As both occur in $\langle\sin \phi\rangle_{\mathrm{UL}}^{\mathrm{q}}$ in combination with the same FF, the latter would-in first approximation-cancel and only the relative size of $f_{L}^{\perp}$ would matter besides the contribution from the convolution integral.

\subsubsection{Exclusively Produced Vector Meson Contribution}

Also in case of the $\langle\sin \phi\rangle_{\mathrm{UL}}^{\mathrm{q}}$ moments, the contribution from the decay of exclusively produced vector mesons to the analysed pion sample causes a possible uncertainty in the interpretation in terms of DFs and FFs. Since no $\sin \phi$ modulation for the vector meson pro-

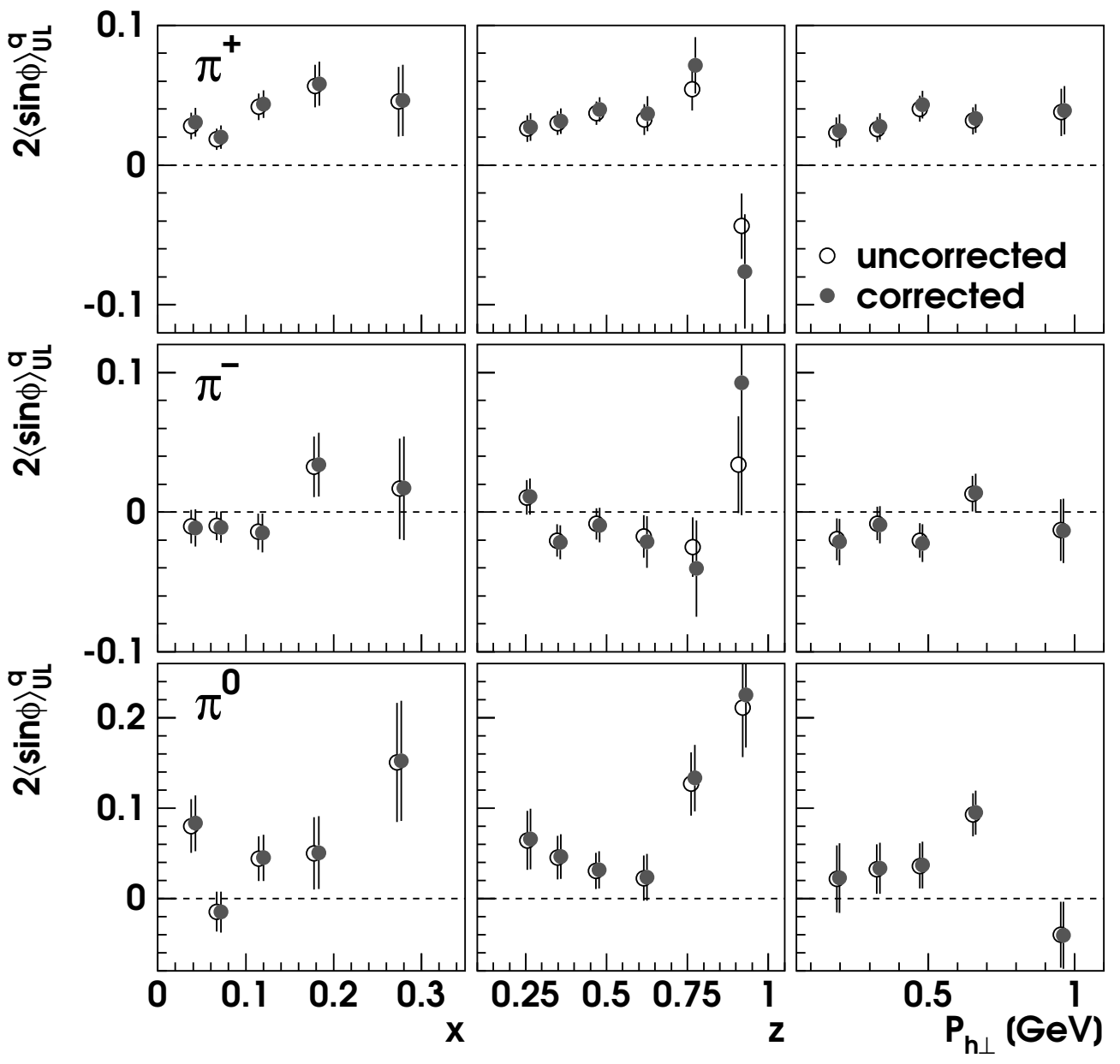

Figure 7.3.3: The photon-axis amplitudes $2\langle\sin \phi\rangle_{\mathrm{UL}}^{\mathrm{q}}$ uncorrected and corrected for the vector meson contribution. 
duction or its decay distribution is expected in case of a longitudinally polarised nucleon with respect to the virtual photon [Fra74], their contribution can be treated as a dilution. For an estimate of such an effect, the correction for the vector meson contribution is applied by dividing the asymmetry amplitudes by $1-f_{\mathrm{VM}}$. The vector meson fractions $f_{\mathrm{VM}}$ are determined for the tighter hadron momentum cut and can be found in Tables F.5 F.7. They differ only slightly from the values obtained for the full RICH momentum range shown in Figure 6.2.1. The comparison between corrected and uncorrected amplitudes is plotted in Figure 7.3.3. Differences between corrected and uncorrected amplitudes are only visible for kinematic bins with a significantly large vector meson fraction like in the highest $z$ bins. Hence, the vector meson dilution does not significantly influence an interpretation of $\langle\sin \phi\rangle_{\mathrm{UL}}^{\mathrm{q}}$ in terms of Eq. (7.3.3) in the semi-inclusive range $0.2<z<0.7$.

\subsection{Contribution to the Lepton-Axis Collins and Sivers Amplitudes}

The small longitudinal target spin component that contributes to the measured leptonaxis Collins and Sivers amplitudes presented in Chapter 4 , is proportional to the extracted

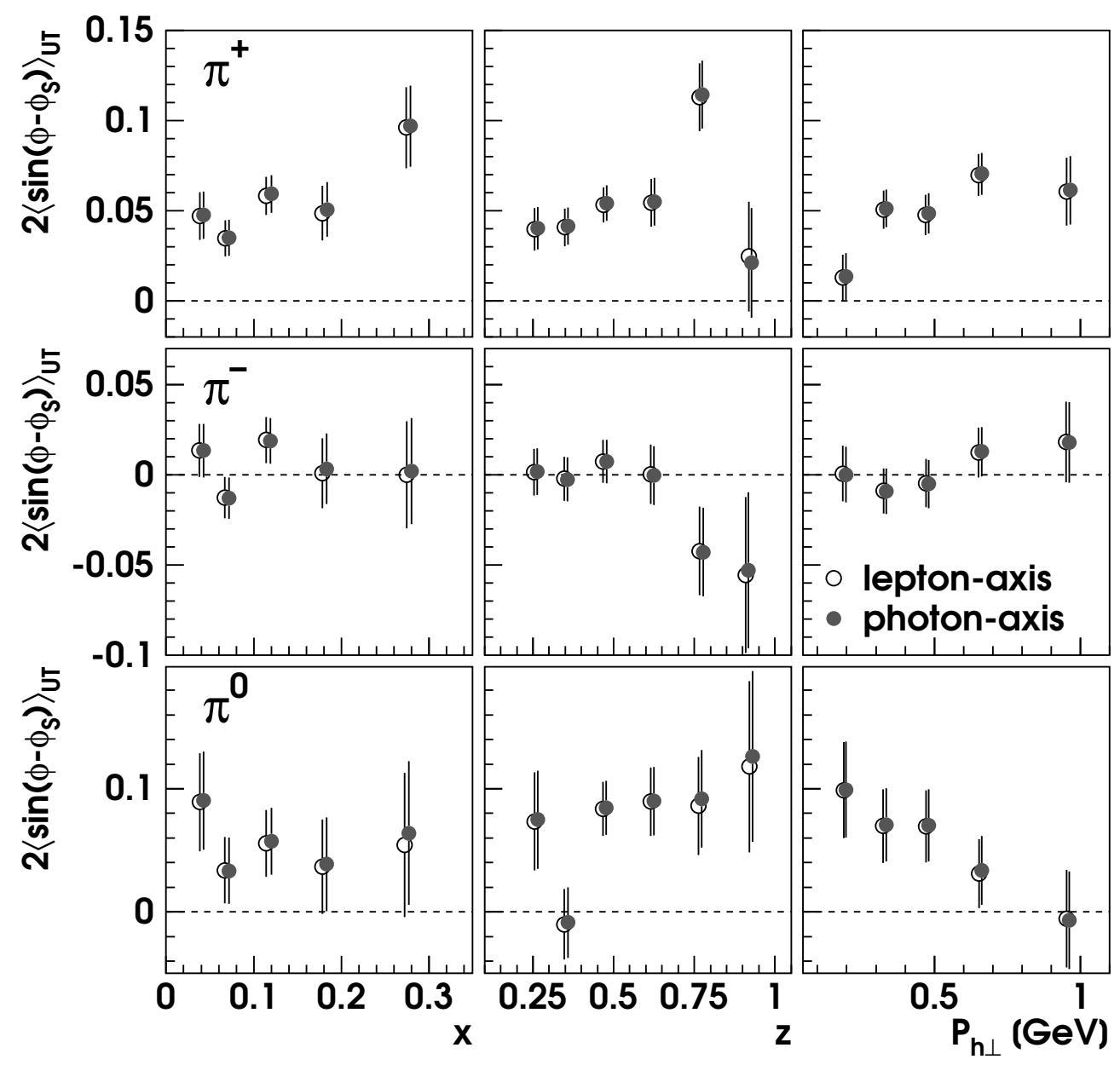

Figure 7.4.1: Comparison of photon-axis and lepton-axis Sivers amplitudes for all three pion types dependent on $x, z$, and $P_{h \perp}$. 
subleading-twist terms (see Eq. (7.1.5)):

$$
\left\langle\sin \left(\phi \pm \phi_{S}\right)\right\rangle_{\mathrm{UT}}^{\mathrm{l}}=\cos \theta_{\gamma^{*}}\left\langle\sin \left(\phi \pm \phi_{S}\right)\right\rangle_{\mathrm{UT}}^{\mathrm{q}}+\frac{1}{2} \sin \theta_{\gamma^{*}}\langle\sin \phi\rangle_{\mathrm{UL}}^{\mathrm{q}} .
$$

Again, the kinematic factor $\cos \theta_{\gamma^{*}} \approx 1$. Using the $\langle\sin \phi\rangle_{\mathrm{UL}}^{\mathrm{q}}$ moments from above, the photon-axis Collins and Sivers amplitudes can be determined for the momentum range of the threshold Čerenkov counter. They can be compared to the lepton-axis amplitudes in the same momentum range as shown in Figure 7.4.1. For the comparison neither the twist-three component $\langle\sin \phi\rangle_{\mathrm{UL}}^{\mathrm{q}}$ nor the measured amplitudes for the transversely polarised target are corrected for the vector meson contribution. The differences between the results in the two reference systems are negligible. Therefore, in order to recover the full statistics of the RICH momentum range, the assumption,

$$
\left\langle\sin \left(\phi \pm \phi_{S}\right)\right\rangle_{\mathrm{UT}}^{1} \approx\left\langle\sin \left(\phi \pm \phi_{S}\right)\right\rangle_{\mathrm{UT}}^{\mathrm{q}} \equiv\left\langle\sin \left(\phi \pm \phi_{S}\right)\right\rangle_{\mathrm{UT}},
$$

is used in the following and the superscript is omitted.

A measurement of kaon asymmetry amplitudes with a longitudinally polarised hydrogen target does not exist due to the poor kaon identification with the threshold Čerenkov detector. The separation of photon-axis and lepton-axis asymmetry moments can thus not be performed for kaons. However, supported by the results for pions and by a measurement with a longitudinally polarised deuterium target that gave similar amplitudes for positive pions and kaons [AirO3], also the photon-axis and lepton-axis kaon asymmetry amplitudes are assumed to be equal. 

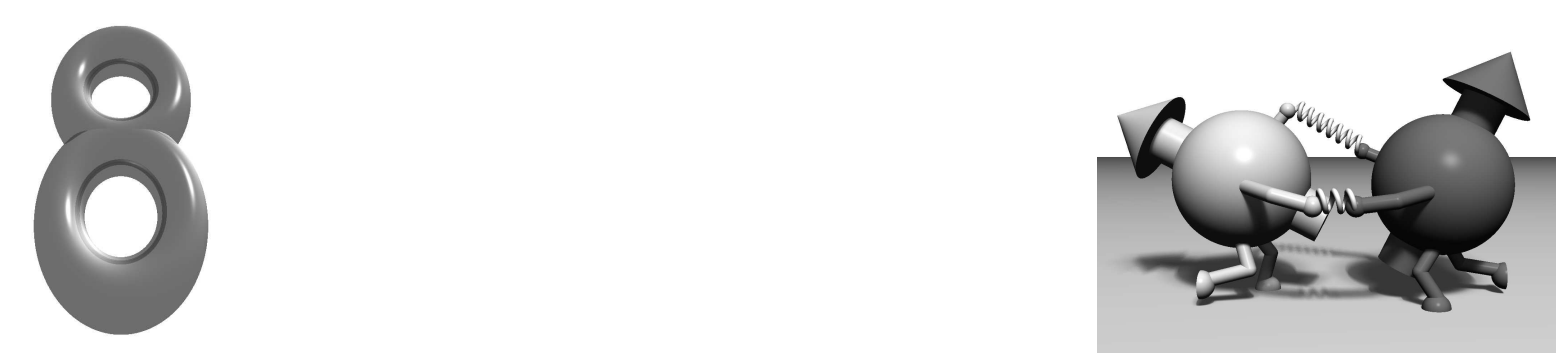

\section{Interpretation of the Asymmetry Moments}

When the measured asymmetry moments are interpreted in terms of distribution and fragmentation functions, a relation between the moments of the three pion types can be derived which is investigated for Monte Carlo and HERMES results. The measured Collins and Sivers amplitudes are discussed and compared to model predictions and to fits of the results for charged pions. The ratio of the disfavoured to the favoured Collins fragmentation functions can be constrained by the extracted Collins amplitudes to negative values with a most probable value of about -1. A first extraction of the Sivers function is attempted which yields a negative $u$ quark and a positive $d$ quark function. In order to extract the transversity distribution functions from the results, the Collins fragmentation function needs to be known. This quantity could be measured by the BeLLE experiment, for instance. The concept of such a measurement is outlined and discussed. Also other possibilities to access transversity and the Sivers function in current and future experiments are discussed as an outlook.

\subsection{The Makins Relation}

For a closer look at the measured Collins asymmetry moments, the following minimal assumptions are made:

- The transverse polarisations of the strange quarks, $\delta s / s$ and $\delta \bar{s} / \bar{s}$, are negligible in the kinematic range of the HERMES experiment like the longitudinal polarisations, $\Delta s / s$ and $\Delta \bar{s} / \bar{s}[$ AirO5a].

- Charge conjugation and isospin symmetry are assumed to hold so that only the two independent favoured and disfavoured FFs have to be considered (cf. Section 2.5.1).

With these assumptions, the $P_{h \perp}$-weighted Collins moments (2.5.27) of the three pion types can be written as:

$$
\left\langle\frac{P_{h \perp}}{z M_{h}} \sin \left(\phi+\phi_{S}\right)\right\rangle_{\mathrm{UT}}^{\pi^{+}}=\mathcal{K} \frac{(4 \delta u+\delta \bar{d}) H_{1, \mathrm{fav}}^{\perp(1)}+(\delta d+4 \delta \bar{u}) H_{1, \mathrm{dis}}^{\perp(1)}}{(4 u+\bar{d}) D_{1, \mathrm{fav}}+(d+4 \bar{u}) D_{1, \mathrm{dis}}}
$$




$$
\begin{aligned}
& \left\langle\frac{P_{h \perp}}{z M_{h}} \sin \left(\phi+\phi_{S}\right)\right\rangle_{\mathrm{UT}}^{\pi^{-}}=\mathcal{K} \frac{(4 \delta u+\delta \bar{d}) H_{1, \mathrm{dis}}^{\perp(1)}+(\delta d+4 \delta \bar{u}) H_{1, \mathrm{fav}}^{\perp(1)}}{(4 u+\bar{d}) D_{1, \mathrm{dis}}+(d+4 \bar{u}) D_{1, \mathrm{fav}}}, \\
& \left\langle\frac{P_{h \perp}}{z M_{h}} \sin \left(\phi+\phi_{S}\right)\right\rangle_{\mathrm{UT}}^{\pi^{0}}=\mathcal{K} \frac{(4 \delta u+\delta \bar{d}+\delta d+4 \delta \bar{u})\left(H_{1, \mathrm{fav}}^{\perp(1)}+H_{1, \mathrm{dis}}^{\perp(1)}\right)}{(4 u+\bar{d}+d+4 \bar{u})\left(D_{1, \mathrm{dis}}+D_{1, \mathrm{fav}}\right)},
\end{aligned}
$$

where $\mathcal{K}$ is a kinematic factor common to all of the asymmetry moments and the $x$ and $z$ dependencies are omitted. Introducing the quantities

$$
r \equiv \frac{d+4 \bar{u}}{u+\frac{1}{4} \bar{d}}, \quad \delta r \equiv \frac{\delta d+4 \delta \bar{u}}{\delta u+\frac{1}{4} \delta \bar{d}}, \quad \eta \equiv \frac{D_{1, \mathrm{dis}}}{D_{1, \mathrm{fav}}}, \quad \eta_{H} \equiv \frac{H_{1, \mathrm{dis}}^{\perp(1)}}{H_{1, \mathrm{fav}}^{\perp(1)}},
$$

two relations can be obtained for the moment ratios of negative and positive pions and of neutral and positive pions, respectively:

$$
\begin{array}{r}
\frac{\left\langle\frac{P_{h \perp}}{z M_{h}} \sin \left(\phi+\phi_{S}\right)\right\rangle_{\mathrm{UT}}^{\pi^{-}}}{\left\langle\frac{P_{h} \perp}{z M_{h}} \sin \left(\phi+\phi_{S}\right)\right\rangle_{\mathrm{UT}}^{\pi^{+}}} \cdot \frac{4 \eta+r}{4+r \eta}=\frac{4 \eta_{H}+\delta r}{4+\delta r \eta_{H}}, \\
\frac{\left\langle\frac{P_{h \perp}}{z M_{h}} \sin \left(\phi+\phi_{S}\right)\right\rangle_{\mathrm{UT}}^{\pi^{0}}}{\left\langle\frac{P_{h \perp}}{z M_{h}} \sin \left(\phi+\phi_{S}\right)\right\rangle_{\mathrm{UT}}^{\pi^{+}}}\left(1+\frac{4 \eta+r}{4+r \eta}\right)-1=\frac{4 \eta_{H}+\delta r}{4+\delta r \eta_{H}} .
\end{array}
$$

In these ratios the kinematic factor $\mathcal{K}$ cancels. From Eq. (8. 1.5) and (8.1.6) follows directly that the asymmetry moments are correlated which leads to a constraint on the asymmetry moments, the so-called Makins Relation [MakO3a]:

$$
\begin{aligned}
\mathrm{MR}^{P_{h \perp} \sin \left(\phi+\phi_{S}\right)} \equiv & \left\langle\frac{P_{h \perp}}{z M_{h}} \sin \left(\phi+\phi_{S}\right)\right\rangle_{\mathrm{UT}}^{\pi^{+}}+C\left\langle\frac{P_{h \perp}}{z M_{h}} \sin \left(\phi+\phi_{S}\right)\right\rangle_{\mathrm{UT}}^{\pi^{-}}- \\
& (1+C)\left\langle\frac{P_{h \perp}}{z M_{h}} \sin \left(\phi+\phi_{S}\right)\right\rangle_{\mathrm{UT}}^{\pi^{0}} \\
= & 0,
\end{aligned}
$$

including only the unpolarised quantity $C$ :

$$
C=\frac{4 \eta+r}{4+r \eta} \text {. }
$$

More general, the Makins Relation is valid for any single-spin or double-spin asymmetry in semi-inclusive DIS at twist-two and twist-three and in leading and next-to-leading order in $\alpha_{s}$ when $C$ is replaced by the unpolarised cross-section ratio for semi-inclusive negative and positive pion production: $C=\sigma_{\mathrm{UU}}^{\pi^{+}} / \sigma_{\mathrm{UU}}^{\pi^{-}}$[Die05c]. The Makins Relation is therefore also valid for the $P_{h \perp}$-weighted Sivers moments. Besides the factorisation of the semi-inclusive cross section only the following isospin relation between the FFs $\mathcal{F}$ for quarks and antiquarks is required for the validity of the Makins Relation:

$$
\mathcal{F}^{q \rightarrow \pi^{0}}=\frac{1}{2}\left(\mathcal{F}^{q \rightarrow \pi^{+}}+\mathcal{F}^{q \rightarrow \pi^{-}}\right) .
$$

The validity of the Makins Relation is unaffected by a convolution integral over the transverse quark and pion momenta so that it also holds for the unweighted Collins and Sivers moments:

$$
\mathrm{MR}^{\sin \left(\phi \pm \phi_{S}\right)} \equiv\left\langle\sin \left(\phi \pm \phi_{S}\right)\right\rangle_{\mathrm{UT}}^{\pi^{+}}+C\left\langle\sin \left(\phi \pm \phi_{S}\right)\right\rangle_{\mathrm{UT}}^{\pi^{-}}-(1+C)\left\langle\sin \left(\phi \pm \phi_{S}\right)\right\rangle_{\mathrm{UT}}^{\pi^{0}}=0 .
$$


In a first step, the Makins Relation is checked on the same gmc_trans Monte Carlo production used for the studies discussed in Section 5.2 since all requirements for the validity of the Makins Relation are implemented in the generator. The Makins Relation can be evaluated for the extracted overall amplitudes of the generated and reconstructed events, where the overall amplitudes are obtained by averaging the amplitudes over the individual $x$ bins. The following values of the Makins Relation are found for generated (gen) and reconstructed (rec) events:

$$
\begin{array}{ll}
\mathrm{MR}_{\text {gen }}^{\sin \left(\phi+\phi_{S}\right)}=0.0013 \pm 0.0006, & \mathrm{MR}_{\mathrm{rec}}^{\sin \left(\phi+\phi_{S}\right)}=-0.002 \pm 0.003, \\
\mathrm{MR}_{\text {gen }}^{\sin \left(\phi-\phi_{S}\right)}=0.0004 \pm 0.0006, & \mathrm{MR}_{\text {rec }}^{\sin \left(\phi-\phi_{S}\right)}=-0.002 \pm 0.003 .
\end{array}
$$

The quantity $C$ was evaluated using parametrisations of the unpolarised DFs [Pum02] and FFs [Kre00]. Although the obtained values are very small, this study yields a deviation from zero by $2 \sigma$ for the generated Collins amplitudes. However, for other gmc_trans productions with similar statistics smaller deviations are found, indicating that the $2 \sigma$ deviation of the Collins amplitudes is a statistical fluctuation.

Despite the fact that the $P_{h \perp}$-weighted asymmetry amplitudes are subject to large acceptance effects (cf. Section 5.2.2), not only the asymmetries for the generated events but also for the reconstructed events fulfil the Makins Relation within $1.25 \sigma$ in the worst case:

$$
\begin{array}{ll}
\mathrm{MR}_{\text {gen }}^{P_{h \perp} \sin \left(\phi+\phi_{S}\right)}=0.003 \pm 0.007, & \mathrm{MR}_{\text {rec }}^{P_{h \perp} \sin \left(\phi+\phi_{S}\right)}=0.007 \pm 0.027 \\
\mathrm{MR}_{\text {gen }}^{P_{h \perp} \sin \left(\phi-\phi_{S}\right)}=0.000 \pm 0.001, & \mathrm{MR}_{\text {rec }}^{P_{h \perp} \sin \left(\phi-\phi_{S}\right)}=-0.005 \pm 0.004 .
\end{array}
$$

For the combined three data productions, the overall unweighted and $P_{h \perp}$-weighted amplitudes can be found in Tables 8.1.1 and 8.1.2. An additional scale uncertainty of $6.6 \%$ given by the target polarisation measurement has not been included. Adding the systematic and statistical uncertainties quadratically, the values of the Makins Relation read:

$$
\begin{aligned}
\mathrm{MR}^{\sin \left(\phi+\phi_{S}\right)} & =0.024 \pm 0.013, & \mathrm{MR}^{\sin \left(\phi-\phi_{S}\right)} & =-0.030 \pm 0.014, \\
\mathrm{MR}^{P_{h \perp} \sin \left(\phi+\phi_{S}\right)} & =0.257 \pm 0.125, & \mathrm{MR}^{P_{h \perp} \sin \left(\phi-\phi_{S}\right)} & =-0.035 \pm 0.017,
\end{aligned}
$$

\begin{tabular}{|c|c|c|c|c|c|}
\hline & $\langle z\rangle$ & $\langle x\rangle$ & $\left\langle P_{h \perp}\right\rangle[\mathrm{GeV}]$ & $2\left\langle\sin \left(\phi+\phi_{S}\right)\right\rangle_{\mathrm{UT}}$ & $2\left\langle\sin \left(\phi-\phi_{S}\right)\right\rangle_{\mathrm{UT}}$ \\
\hline$\pi^{+}$ & 0.36 & 0.096 & 0.40 & $0.019 \pm 0.005 \pm 0.001$ & $0.042 \pm 0.005 \pm 0.002$ \\
\hline$\pi^{-}$ & 0.35 & 0.091 & 0.40 & $-0.026 \pm 0.005 \pm 0.002$ & $0.003 \pm 0.005 \pm 0.001$ \\
\hline$\pi^{0}$ & 0.37 & 0.089 & 0.42 & $-0.028 \pm 0.013 \pm 0.003$ & $0.059 \pm 0.014 \pm 0.004$ \\
\hline$K^{+}$ & 0.37 & 0.100 & 0.42 & $-0.001 \pm 0.016 \pm 0.008$ & $0.108 \pm 0.015 \pm 0.009$ \\
\hline$K^{-}$ & 0.33 & 0.096 & 0.39 & $0.002 \pm 0.030 \pm 0.015$ & $0.016 \pm 0.027 \pm 0.009$ \\
\hline
\end{tabular}

Table 8.1.1: Kinematic mean values $\langle z\rangle,\langle x\rangle$, and $\left\langle P_{h \perp}\right\rangle$ and overall unweighted moments $\left\langle\sin \left(\phi \pm \phi_{S}\right)\right\rangle_{\mathrm{UT}}$ of the combined HERMES data productions together with their statistical and systematic uncertainties in the semi-inclusive range $0.2<z<0.7$. 


\begin{tabular}{|c|c|c|c|c|}
\hline & $\langle y\rangle$ & $\left\langle Q^{2}\right\rangle\left[\mathrm{GeV}^{2}\right]$ & $2\left\langle\frac{P_{h \perp}}{z M_{h}} \sin \left(\phi+\phi_{S}\right)\right\rangle_{\mathrm{UT}}$ & $2\left\langle\frac{P_{h \perp}}{z M} \sin \left(\phi-\phi_{S}\right)\right\rangle_{\mathrm{UT}}$ \\
\hline$\pi^{+}$ & 0.53 & 2.43 & $0.138 \pm 0.045 \pm 0.006$ & $0.052 \pm 0.006 \pm 0.001$ \\
\hline$\pi^{-}$ & 0.54 & 2.35 & $-0.252 \pm 0.054 \pm 0.009$ & $0.007 \pm 0.008 \pm 0.001$ \\
\hline$\pi^{0}$ & 0.58 & 2.49 & $-0.321 \pm 0.135 \pm 0.016$ & $0.071 \pm 0.018 \pm 0.003$ \\
\hline$K^{+}$ & 0.51 & 2.50 & $-0.041 \pm 0.044 \pm 0.018$ & $0.138 \pm 0.022 \pm 0.010$ \\
\hline$K^{-}$ & 0.52 & 2.40 & $-0.001 \pm 0.087 \pm 0.038$ & $0.022 \pm 0.040 \pm 0.013$ \\
\hline
\end{tabular}

Table 8. 1.2: Kinematic mean values $\langle y\rangle$ and $\left\langle Q^{2}\right\rangle$ and overall $P_{h \perp}$-weighted moments $\left\langle\frac{P_{h \perp}}{z M_{(h)}} \sin \left(\phi \pm \phi_{S}\right)\right\rangle_{\text {UT }}$ of the combined HERMES data productions together with their statistical and systematic uncertainties in the semi-inclusive range $0.2<z<0.7$. The acceptance effect is not included in the systematic uncertainty.

showing a $2 \sigma$ deviation from zero for both Collins and Sivers amplitudes. For the interpretation of this apparent violation of the Makins Relation note that the extracted Collins and Sivers moments are strongly anti-correlated. Furthermore, the unweighted and $P_{h \perp_{-}}$ weighted moments are correlated so that a statistical fluctuation necessarily influences all four results in (8.1.13) simultaneously. Hence, these observed deviations from zero might all arise from the same statistical fluctuation. As a cross check, the sum of the Makins relations for the two unweighted moments is indeed consistent with zero: $-0.006 \pm 0.014$, where the anti-correlation has been taken into account in the computation of the combined uncertainty. The different mass factors $M$ and $M_{\pi}$ entering the moments prevent the simple summation in case of the $P_{h \perp}$-weighted amplitudes. The results of the Makins Relation are not influenced by the combinatorial background correction of the neutral pions. The absolute values of the relation and their uncertainties are smaller without the correction but they still deviate from zero by $2 \sigma$.

Another possible explanation of the deviation could be the contribution from exclusively produced vector mesons since the Makins Relation does not hold for exclusive production channels. In case that the contribution from vector meson production can be treated as a dilution, a correction of the measured amplitudes for the vector meson fraction (see Figure 6.2.1 on page 112) does not change the results of the Makins Relation. The asymmetry amplitudes of decay pions from exclusive $\rho^{0}$ production are consistent with zero within the semi-inclusive range (cf. Table 6.2.1 on page 123). Allowing non-zero asymmetry amplitudes of the neutral decay pions from exclusive $\omega$ mesons, large amplitudes of the order of unity are required to fulfil the Makins Relation for both Sivers and Collins amplitudes. Although the Sivers amplitudes of exclusive $\omega$ mesons are predicted to be of the order of $10 \%$ and hence larger than the $\rho^{0}$ amplitudes [Goe01], it seems very unlikely that the neutral decay pions acquire an amplitude at their positivity limit. The Collins amplitude is suppressed by $1 / Q^{2}$ and therefore not expected to be larger than the Sivers amplitude. Thus, the vector meson contribution with an asymmetry amplitude in the decay pions is very unlikely to cause the violation of the Makins Relation.

Furthermore, exclusive $\pi^{+}$production, which is the only possible exclusive pion production in electron-proton scattering, violates the Makins Relation. However, exclusive 


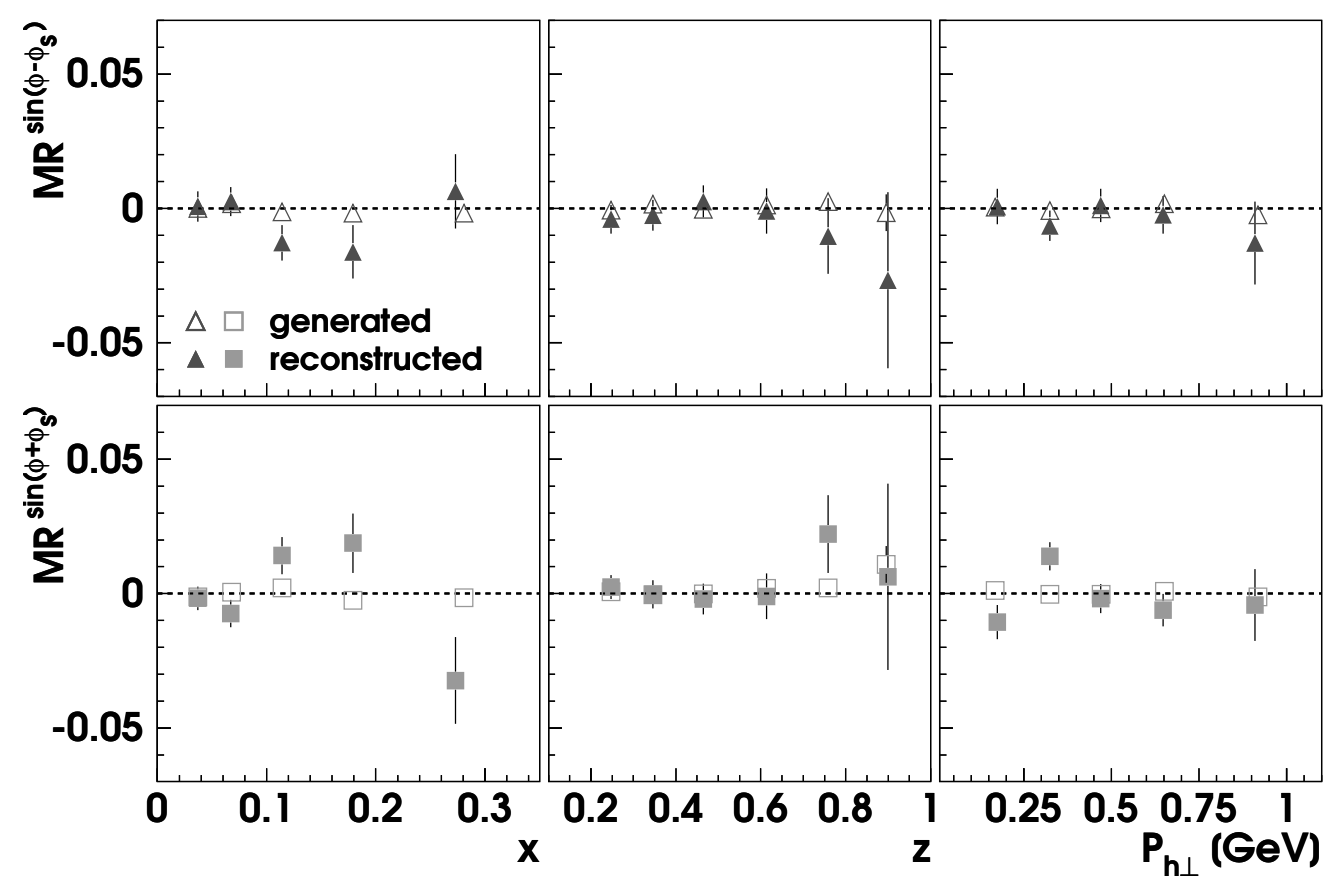

Figure 8.1.1: Makins Relation for Sivers and Collins unweighted amplitudes of the gmc_trans Monte Carlo production dependent on $x, z$, and $P_{h \perp}$.

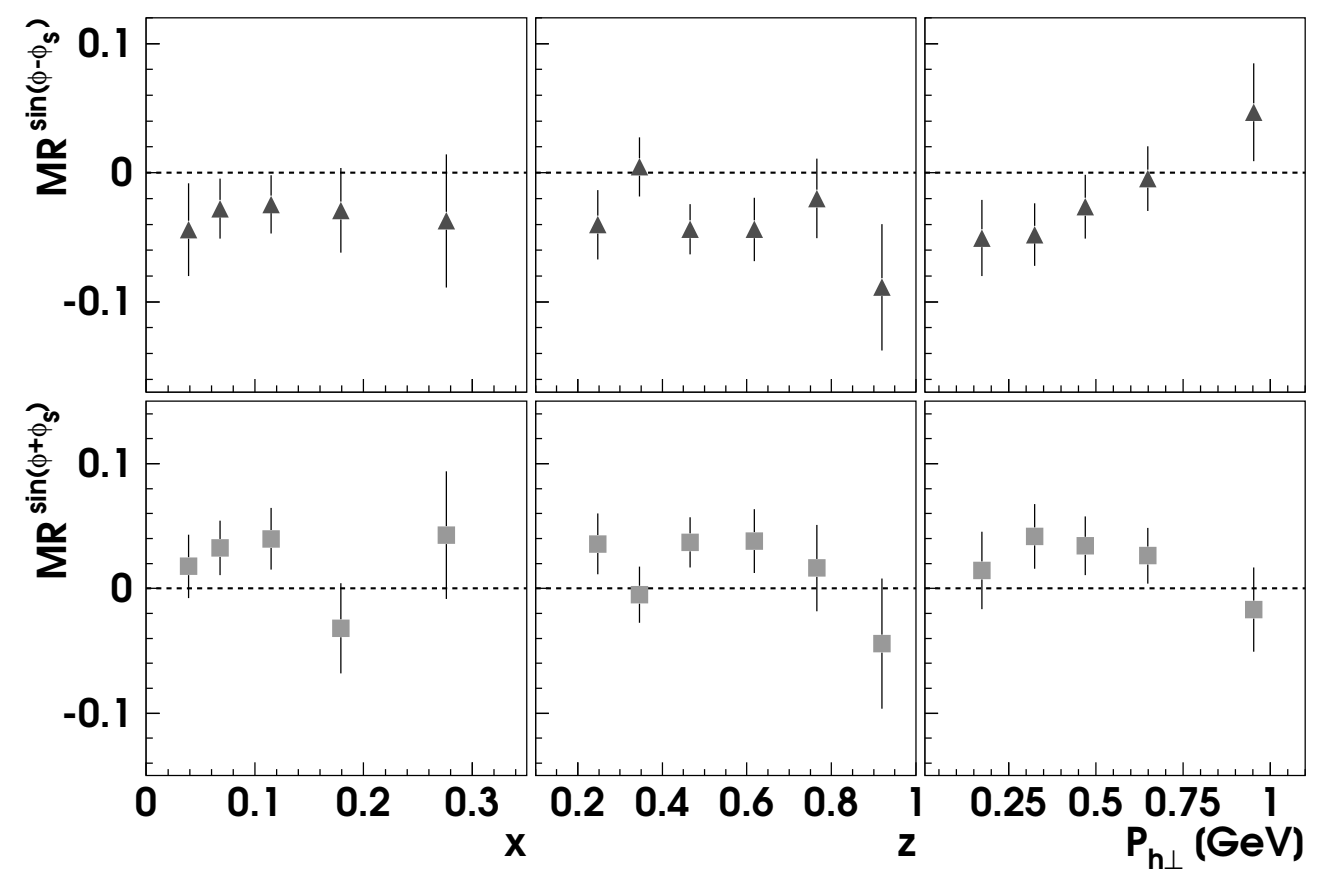

Figure 8.1.2: Makins Relation for Sivers and Collins unweighted amplitudes of the combined three data productions dependent on $x, z$, and $P_{h \perp}$. 
pions have an energy fraction $z=1$ and even taking into account smearing they do not contribute to the semi-inclusive range $0.2<z<0.7$.

The Makins Relation can also be evaluated in the individual kinematic bins, e.g. , in the $x, z$, and $P_{h \perp}$ bins. These results are shown for the unweighted asymmetry amplitudes in Figures 8.1.1 and 8.1.2 for the Monte Carlo and the combined HERMES data productions, respectively. The kinematic dependencies of the Makins Relation for the $P_{h \perp}$-weighted amplitudes are shown in Figures E.4 and E.5. Reflecting the deviation of the overall amplitudes of the HERMES data, the values of the Makins Relation in the different kinematic bins are systematically above or below zero for the Sivers or Collins amplitudes, respectively.

Although the measured unweighted Collins and Sivers amplitudes violate the Makins relation, the interpretation of the amplitudes in terms of DFs and FFs is assumed to be valid since the violation might be caused by a single statistical fluctuation in the azimuthal asymmetry of the neutral pions. Due to the large statistical uncertainty of the amplitudes for the neutral pion compared to the amplitudes for the charged pions, the $\pi^{0}$ amplitudes have only a small impact on the estimate of the ratio of the favoured to disfavoured Collins functions and on the results of the extracted Sivers functions which both will be presented in the following.

\subsection{Virtual-Photon Asymmetry Moments}

In the measured asymmetry amplitudes (2.5.26) and (2.5.28), a dependence on the fractional energy transfer $y$ is contained which has to be eliminated for the extraction of the DFs. Since the amplitudes are integrated over a certain range in $y$ in each kinematic bin, the events had to be weighted with the reciprocal of the corresponding $y$ dependence in the numerator and denominator separately. For practical reasons, a different approach is used where the kinematic factors $A(y) /\left(x y^{2}\right)$ and $B(y) /\left(x y^{2}\right)$ are moved out of the integral and approximated by $A(\langle y\rangle) /\left(\langle x\rangle\langle y\rangle^{2}\right)$ and $B(\langle y\rangle) /\left(\langle x\rangle\langle y\rangle^{2}\right)$. The latter approximation results in a small bias of less than $4 \%$ due to the finite bin size [Mil04], whereas the weighting procedure is complicated by the statistical correlations of the kinematic factors in numerator and denominator.

The kinematic factors cancel in case of the Sivers amplitude when they are approximated by their mean values in each kinematic bin. The Collins amplitude and its uncertainty have to be multiplied by $A(\langle y\rangle) / B(\langle y\rangle)$ in order to remove the $y$ dependence. In analogy to the depolarisation factor $C(y) / A(y)$ in case of the double-spin asymmetry (2.5.32), also $B(y) / A(y)$ is called depolarisation factor and $A(\langle y\rangle) / B(\langle y\rangle)\left\langle\sin \left(\phi+\phi_{S}\right)\right\rangle_{\mathrm{UT}}$ is called virtual-photon asymmetry moment. The factors $C(y) / A(y)$ and $B(y) / A(y)$ give the circular and linear polarisations of the transverse virtual photons, respectively.

The expressions of the kinematic factors $A(y), B(y), C(y)$, and $D(y)$, given in Eq. (2.5.17), are valid only in the Bjorken limit when $R \rightarrow 0$ and $\gamma^{2} \rightarrow 0$. Taking into account mass corrections of the order $\gamma^{2} \sim 1 / Q^{2}$ in the leptonic tensor, the kinematic factors read [Bac06]:

$$
\begin{array}{ll}
A(y)=\frac{y^{2}}{2}+\left(1-y-\frac{y^{2} \gamma^{2}}{4}\right) \frac{1}{1+\gamma^{2}}, & B(y)=\left(1-y-\frac{y^{2} \gamma^{2}}{4}\right) \frac{1}{1+\gamma^{2}} \\
C(y)=\frac{1}{\sqrt{1+\gamma^{2}}} y\left(1-\frac{y}{2}\right), & D(y)=\frac{2(2-y)}{1+\gamma^{2}} \sqrt{1-y-\frac{y^{2} \gamma^{2}}{4}}
\end{array}
$$


Corrections of the same order $\left(1 / Q^{2}\right)$ are not taken into account in the hadronic tensor since the quark-quark-correlation matrix is only known up to twist-three so far, i.e., up to the order $1 / Q$.

Higher order contributions in $\alpha_{s}$, resulting in a non-vanishing $R$, change the factor $A(y)$ of the unpolarised cross-section terms to:

$$
A(y, R)=\frac{y^{2}}{2}+\left(1-y-\frac{y^{2} \gamma^{2}}{4}\right) \frac{1+R\left(x, Q^{2}\right)}{1+\gamma^{2}} .
$$

The equivalent higher order corrections in the kinematic factors $B(y)$ and $A(y)$ are unknown in case of the cross-section terms for a transversely polarised target. Although the ratio $R$ of the photo-absorption cross sections of longitudinal and transverse photons could depend on the produced hadron type in semi-inclusive DIS events, the only parametrisations available are based on fits to world data on inclusive DIS [Abe99]. So far, $R$ has not been measured in semi-inclusive DIS.

The values of $A(\langle y\rangle, R) / A(\langle y\rangle)$ and $A(\langle y\rangle, R) / B(\langle y\rangle)$ in the different kinematic bins for the calculation of the Sivers and Collins virtual-photon asymmetry moments can be found in Tables F.8 - F.12. In the following, no higher order corrections are applied to the Sivers amplitudes, i.e., the cancellation of the kinematic factors is assumed, and the Collins amplitudes are multiplied by the factor $A(\langle y\rangle, R) / B(\langle y\rangle)$ only in the comparison to model predictions for virtual-photon asymmetry moments later on (cf. Figure 8.3.6).

\subsection{The Collins Moments}

The unweighted Collins amplitudes for charged and neutral pions and charged kaons are shown in Figure 8.3.1 dependent on the five kinematic variables. Numerical values of the amplitudes and their statistical and systematic uncertainties can be found in Tables F.8 - F.12. In the figure and the tables, the systematic uncertainty does not include the $6.6 \%$ scale uncertainty from the target polarisation measurement. The large systematic uncertainties in the higher bins of the kaon asymmetries are dominated by the contribution from the kaon identification with the RICH detector (cf. Section 4.4.6).

Because of the $u$ quark dominance in DIS, which is caused by the quark-charge factor $e_{q}^{2}$ in the summation over the quark flavours, one could naively expect the amplitudes for positive pions and kaons to be similar. However, the amplitudes for the $\mathrm{K}^{+}$are systematically lower than the $\pi^{+}$amplitudes in all kinematic bins even though the overall amplitudes have overlapping error bars (cf. Table 8.1.1). Like in the case of the unpolarised FFs, the Collins function may differ for fragmentation of $u$ into $K^{+}$and $u$ into $\pi^{+}$. In contrast to the positive pions and kaons, the $\pi^{-}$and $K^{-}$amplitudes are not expected to be similar, not only because of different Collins functions but also since the $K^{-}$is a sea object, i.e., it contains only sea-quark flavours of the proton.

As predicted for a valence-quark object, the maxima of the transversity DFs and hence the Collins amplitudes are at large $x$ values. The transversity distribution of sea quarks is expected to be small since the contribution of the gluons, from which their arise, vanishes (see Section 2.4.1). Transversity is then dominated by valence quarks which have momentum fractions around $x=0.16$. No $Q^{2}$ dependence is expected for leading-twist cross-section terms. The clear trend which is visible for negative pions and positive kaons 


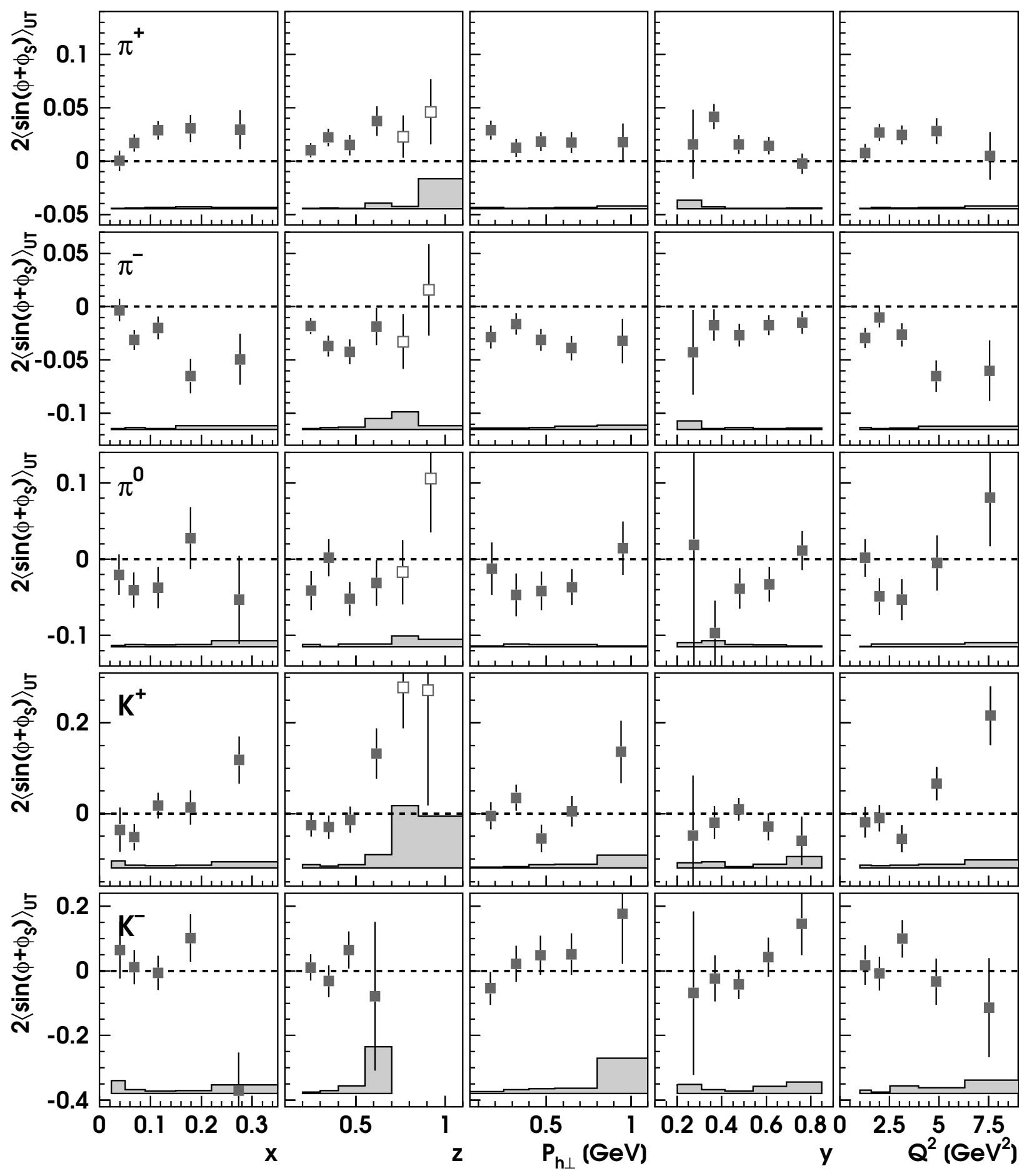

Figure 8.3.1: Unweighted Collins amplitudes for all five hadron types as functions of $x, z, P_{h \perp}, y$, and $Q^{2}$. An additional $6.6 \%$ scale uncertainty from the target polarisation measurement is not included in the shaded systematic uncertainty bands.

is caused by the strong correlation of $Q^{2}$ and $x$ within the HERMES acceptance. The visible rise of the amplitudes with $Q^{2}$ is related to an increase of $\langle x\rangle$ in each $Q^{2}$ bin.

As the right-hand sides of Eq. (8.1.5) and (8.1.6) are equal, they cannot be solved to yield unambiguous solutions for $\delta r$ and $\eta_{H}$ but only a relation between the two quantities. 
This relation can be deduced either from the $\pi^{-}$to $\pi^{+}$moment ratio or from the $\pi^{0}$ to $\pi^{+}$ moment ratio and is unambiguous if the Makins Relation is fulfilled. A large set of relationships between $\delta r$ and $\eta_{H}$ can be constructed by Gaussian sampling of the asymmetry amplitudes of the three pion types, i.e., by three sets of randomly chosen amplitudes distributed according to Gaussians. The measured amplitudes are used as mean values of the Gaussian distributions while their widths are the statistical uncertainties of the amplitudes. The accumulation of these trajectories provides a distribution of likelihood in terms of $\delta r$ and $\eta_{H}$.

Due to the strong influence of the acceptance on the $P_{h \perp}$-weighted amplitudes, the Gaussian sampling is performed with the overall unweighted Collins amplitudes (listed in Table 8.1.1) under the assumption that the kinematic factors cancel in the moment ratios. The (1)-moments of the disfavoured and favoured Collins functions in $\eta_{H}$ are then replaced by the (1/2)-moments (cf. Eq. 2.5.26 and 2.5.27 on page 28). Figure 8.3.2 shows the likelihood distributions obtained from the two unweighted Collins amplitude ratios. The distribution for the $\pi^{-}$to $\pi^{+}$ratio ( $\pi^{0}$ to $\pi^{+}$ratio) is plotted on the left-hand side (the right-hand side). Since the three asymmetry amplitudes violate the Makins Relation by $2 \sigma$, the two likelihood distributions differ. Nevertheless, both distributions favour a negative ratio $\eta_{H}$ of disfavoured and favoured Collins functions.

Since the interpretation of such distributions is only reasonable for a fulfilled Makins Relation, the likelihood distributions are also obtained with the requirement that Eq. (8. 1.10) is satisfied. This is obtained by shifting the three sampled amplitudes by a certain fraction of their statistical uncertainty. To avoid biases in favour of a particular amplitude, the fractions were chosen the same for all three amplitudes and were restricted to absolute values below 1. On the left-hand side of Figure 8.3.3 the obtained likelihood distribution for $\eta_{H}$ as a function of $\delta r$ is shown which is now identical for both amplitude ratios. In the middle and right panels the sampled Gaussian distributions of the Collins amplitudes are plotted for the three pion types as open histograms. The shaded histograms show the dis-

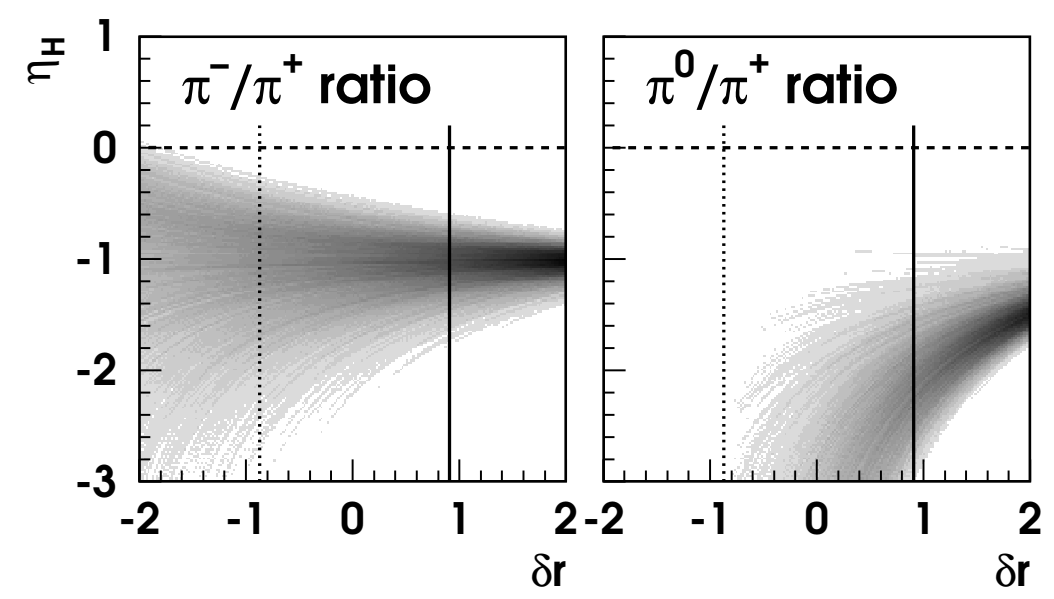

Figure 8.3.2: Likelihood distribution of $\eta_{H}$ as a function of $\delta r$ obtained from the two asymmetry ratios $\pi^{-}$to $\pi^{+}$and $\pi^{0}$ to $\pi^{+}$. The contour scale is linear in likelihood. The values of $\delta r$ for the non-relativistic assumption $\delta q(x)=\Delta q(x)$ and the Soffer limit $\delta q(x)=(\Delta q(x)+q(x)) / 2$ are indicated as dotted and solid lines, respectively. 

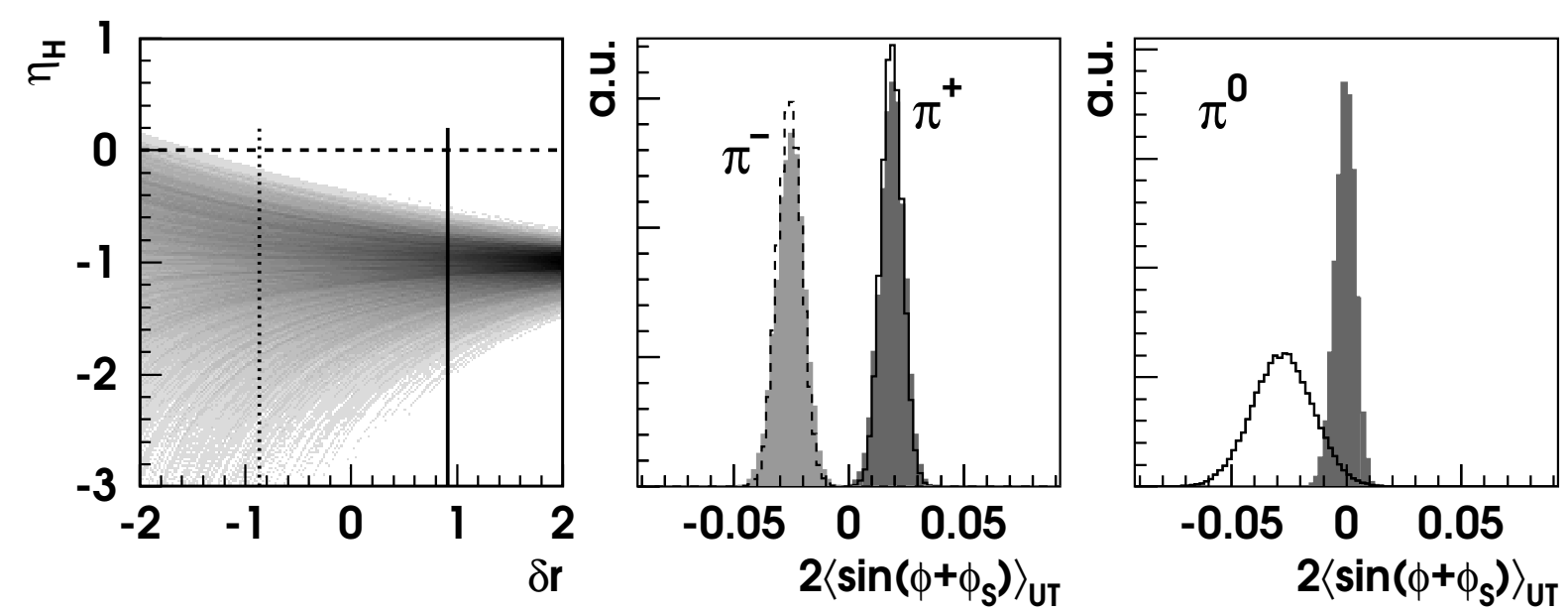

Figure 8.3.3: Left panel: Likelihood distribution on condition that the Makins Relation is satisfied. The contour scale is linear in likelihood. The values of $\delta r$ for the non-relativistic assumption $\delta q(x)=\Delta q(x)$ and the Soffer limit $\delta q(x)=(\Delta q(x)+q(x)) / 2$ are indicated as dotted and solid lines, respectively. Middle and right panels: Distributions of the sampled Collins amplitudes (open histograms) and of the shifted amplitudes which fulfil the Makins Relation (shaded histograms).

tributions of the shifted amplitudes which are bound to satisfy the Makins Relation. Since the statistical uncertainties of the charged pions are much smaller than the uncertainty of the neutral pion, their distributions are hardly affected while the amplitude distribution of the neutral pion is shifted to zero and its width decreases. This decrease is caused by the rejection of part of the sampled amplitudes because a shift within $1 \sigma$ is not enough to fulfil the Makins Relation. The obtained likelihood distribution contains therefore mainly the information of the measured amplitudes for the charged pions.

In the likelihood distributions in Figures 8.3.2 and 8.3.3, two values for $\delta r$ are indicated by a dotted and a solid line. These are the values for the non-relativistic assumption $\delta q(x)$ $=\Delta q(x)$ and the Soffer limit $\delta q(x)=(\Delta q(x)+q(x)) / 2$. For the determination of these values, parametrisations of $\Delta q(x)$ [Blü02] and $q(x)$ [Pum02] were used. Note that the value of $\delta r$ computed from the Soffer limits of the individual quark flavours does not limit the range of $\delta r$ as $\delta r$ is a ratio of different flavours. The likelihood distribution in Figure 8.3.3 does not constrain the quantity $\delta r$, but it favours negative values of $\eta_{H}$. Independent of $\delta r$, the most likely value of the ratio of disfavoured to favoured Collins functions is about -1, i.e., the disfavoured Collins function seems to be of comparable size as the favoured Collins function but has the opposite sign.

In all available model calculations for the Collins function, a simple model for the fragmentation process in lowest order of $\alpha_{s}\left(\alpha_{s}^{0}\right)$ is used, based either on pseudo-scalar or on pseudo-vector pion-quark coupling. The calculation of the Collins function, however, requires the calculation of the imaginary parts of one-loop corrections which are of the order $\alpha_{s}^{1}$. Here, either pion or gluon loop corrections are considered yielding four possible combinations of the two different couplings with the two different one-loop corrections [Bac01, Bac02a, Gam03, Bac03a]. Recently a review of the four model calculations was 


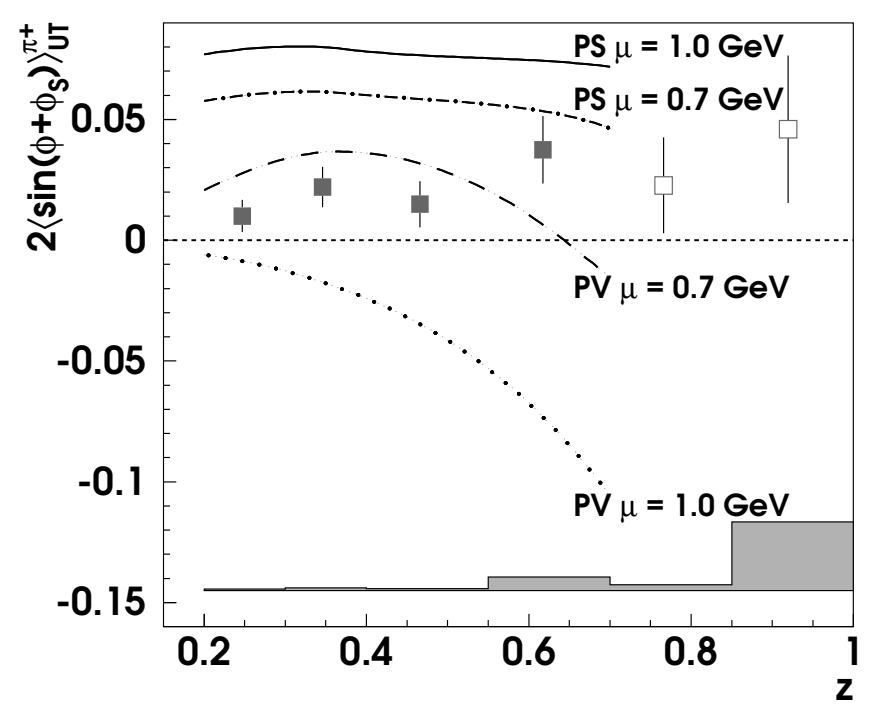

Figure 8.3.4: Unweighted Collins amplitudes of the positive pion as a function of $z$ compared to predictions of different model calculations for the Collins function [Amr05]. For details see text.

presented [Amr05]. The results for the $\pi^{+}$Collins amplitudes are shown in Figure 8.3.4 for pseudo-scalar (PS) and pseudo-vector (PV) pion-quark coupling. In the calculations, pion and gluon loop corrections were summed up. Both kinds of coupling are investigated for two different values of $\mu(0.7 \mathrm{GeV}$ and $1.0 \mathrm{GeV})$, where $\mu^{2}$ is the cut-off on the fragmenting quark virtuality resulting in a cut-off in the $K_{T}^{2}$ integration of the FFs. The non-relativistic assumption is used for the transversity DF. All four curves decrease with increasing $z$ for $z>0.4$ and do not model the increase of the measured amplitudes. The lower $z$ region could be described by a pseudo-vector coupling with a cut-off between $0.7 \mathrm{GeV}$ and $1.0 \mathrm{GeV}$. However, the amplitudes above $z \approx 0.6$ will be negative in contradiction to the measured value which is significantly positive. It should be noted that none of the models considers the disfavoured fragmentation function, which appears to be large and negative from the present analysis. The failure of the models to correctly describe the data might be an indication for a non-vanishing contribution from the disfavoured Collins function.

A physically attractive explanation of the Collins effect based on the string-fragmentation model (cf. Section 2.5.1) was suggested by Artru in 1993 [Art93] (see also [Art97]) and is illustrated in Figure 8.3.5(a) - (c). The first chart (a) shows the absorption of the virtual photon by the struck quark which on average reverses the quark-polarisation component in the lepton scattering plane [Col94]. When the string that connects the struck quark and the nucleon remnant breaks (see Figure 8.3.5(b)), a quark-antiquark pair is produced with vacuum quantum numbers $J^{P}=0^{+}$, i.e., in a ${ }^{3} P_{0}$-state. Since the positive parity of this state requires aligned spins of quark and antiquark, an orbital angular momentum of $L=1$ has to compensate the spins. This non-zero orbital angular momentum creates a transverse momentum of the produced pseudo-scalar meson. Hence, the outgoing meson is deflected with respect to the virtual-photon direction, indicated by an open arrow in Figure 8.3.5(c).

In the special case of a $u$ quark in a proton with $\delta u>0$, most of the $u$ quarks have their 


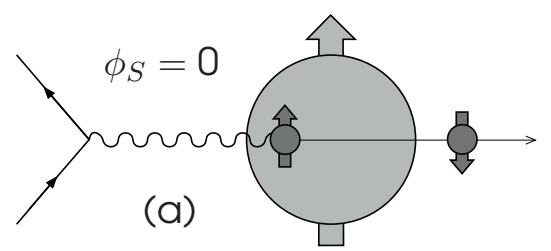

(a)

(b)

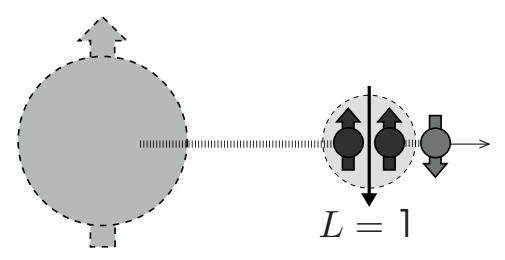

(c)

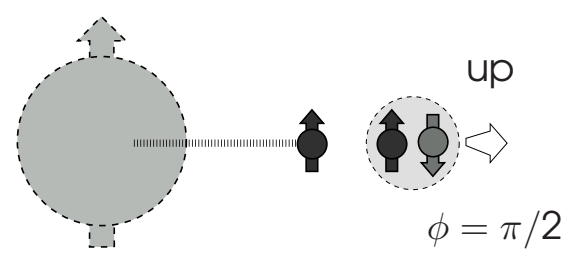

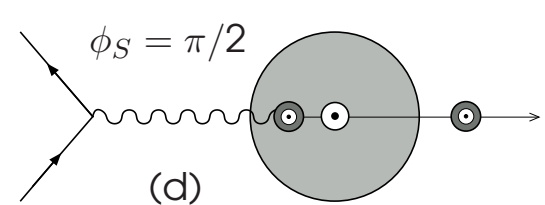

(e)

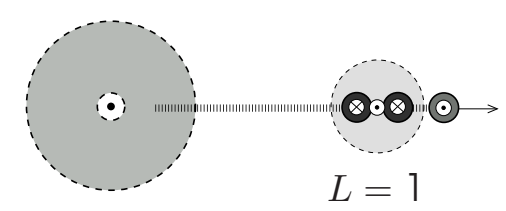

(f)

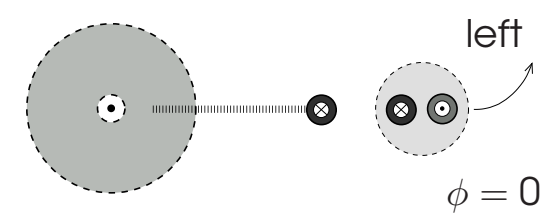

Figure 8.3.5: Collins effect in the string fragmentation model by Artru [Art93] for a transversely polarised nucleon with its spin orientation in (left panels) and perpendicular (right panels) to the lepton scattering plane (see text).

spins aligned with the proton spin. If the azimuthal target spin angle $\phi_{S}=0$, as shown in Figure 8.3.5(a), the spin of the $u$ quark is reversed by the photoabsorption. The creation of a $d-\bar{d}$ pair yields a $\pi^{+}$pseudo-scalar meson which is deflected upwards with respect to the page, i.e., $\phi=\pi / 2$ (see chart (c)). For a target spin perpendicular to the scattering plane $\left(\phi_{S}=\pi / 2\right.$, Figure 8.3.5(d)) the spin of the $u$ quark does not flip and the produced pion is deflected to the left-hand side of the target spin when looking in the direction of the virtual photon, so that $\phi=0$, as illustrated in charts (e) and (f). For both target spin orientations, the azimuthal modulation of the Collins effect $\sin \left(\phi+\phi_{S}\right)=\sin (\pi / 2)$ is therefore positive and consistent with the measured Collins moment for $\pi^{+}$, which is dominated by scattering off $u$ quarks.

The Artru model yields the correct sign for the $\pi^{+}$Collins moment under the condition of a positive transversity DF for $u$ quarks which is predicted by all models for transversity, e.g., by the chiral quark soliton model [EfrO5, WakO1] and the light-cone quarkspectator-diquark model [Ma02]. Both models give a negative transversity DF for the $d$ quark. Lattice QCD calculations [Aok97] yield a positive (negative) first moment of the valence $u(d)$ quark transversity distributions consistent with $\delta u>0$ and $\delta d<0$. A perturbative $Q \mathrm{CD}$ based analysis [Ma02] results also in $\delta u>0$ but the $d$ quark transversity becomes positive for large $x \gtrsim 0.4$.

In Figure 8.3.6, predictions for virtual-photon asymmetry amplitudes from the chiral quark soliton model [Efr05] are compared to the measured Collins amplitudes multiplied by the kinematic factor $A(\langle y\rangle, R) / B(\langle y\rangle)$, as described in Section 8.2. For the good description of the charged pion amplitudes, the ratio of the disfavoured to the favoured 


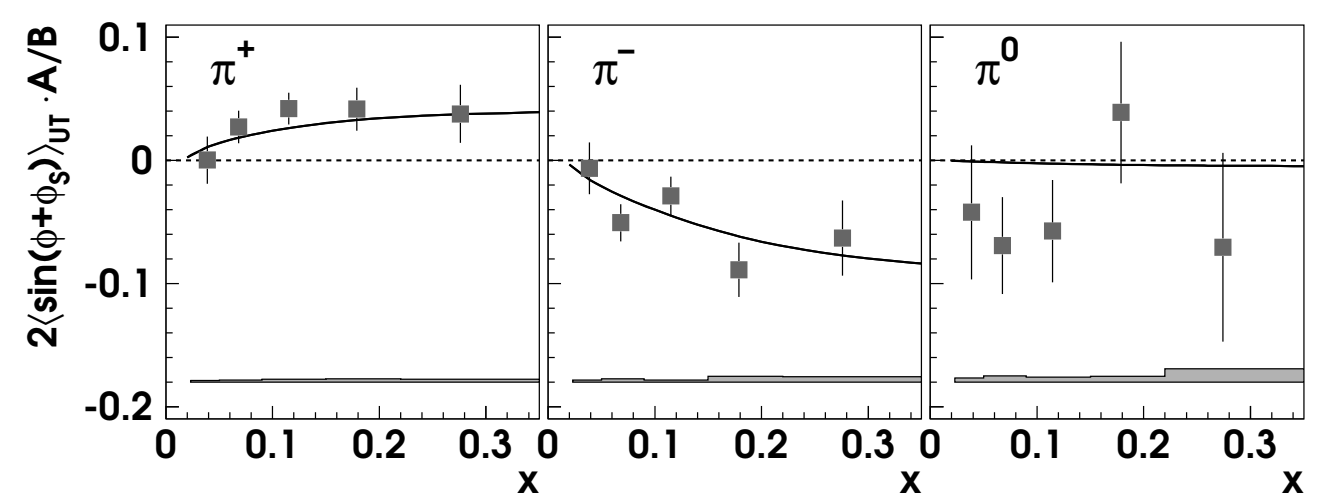

Figure 8.3.6: Unweighted Collins amplitudes of all pion types compared to amplitudes obtained from the chiral quark soliton model for transversity and $\left\langle H_{1, \text { dis }}^{\perp}\right\rangle=-1.2\left\langle H_{1, \text { fav }}^{\perp}\right\rangle<0$ [EfrO5].

Collins functions had to be set to -1.2. For the favoured Collins function a value obtained from an analysis of DELPHI data [Efr99] was taken. Since the prediction satisfies the Makins Relation, the predicted amplitudes for neutral pions have smaller absolute values than the measured amplitudes.

Results of a fit to the unweighted Collins amplitudes of charged pions with parametrisations for the transversity and Collins functions [Vog05] are shown in Figure 8.3.7. The parametrisations are based on the Soffer limit in case of transversity and are proportional to the unpolarised favoured FF for favoured and disfavoured Collins functions. A good
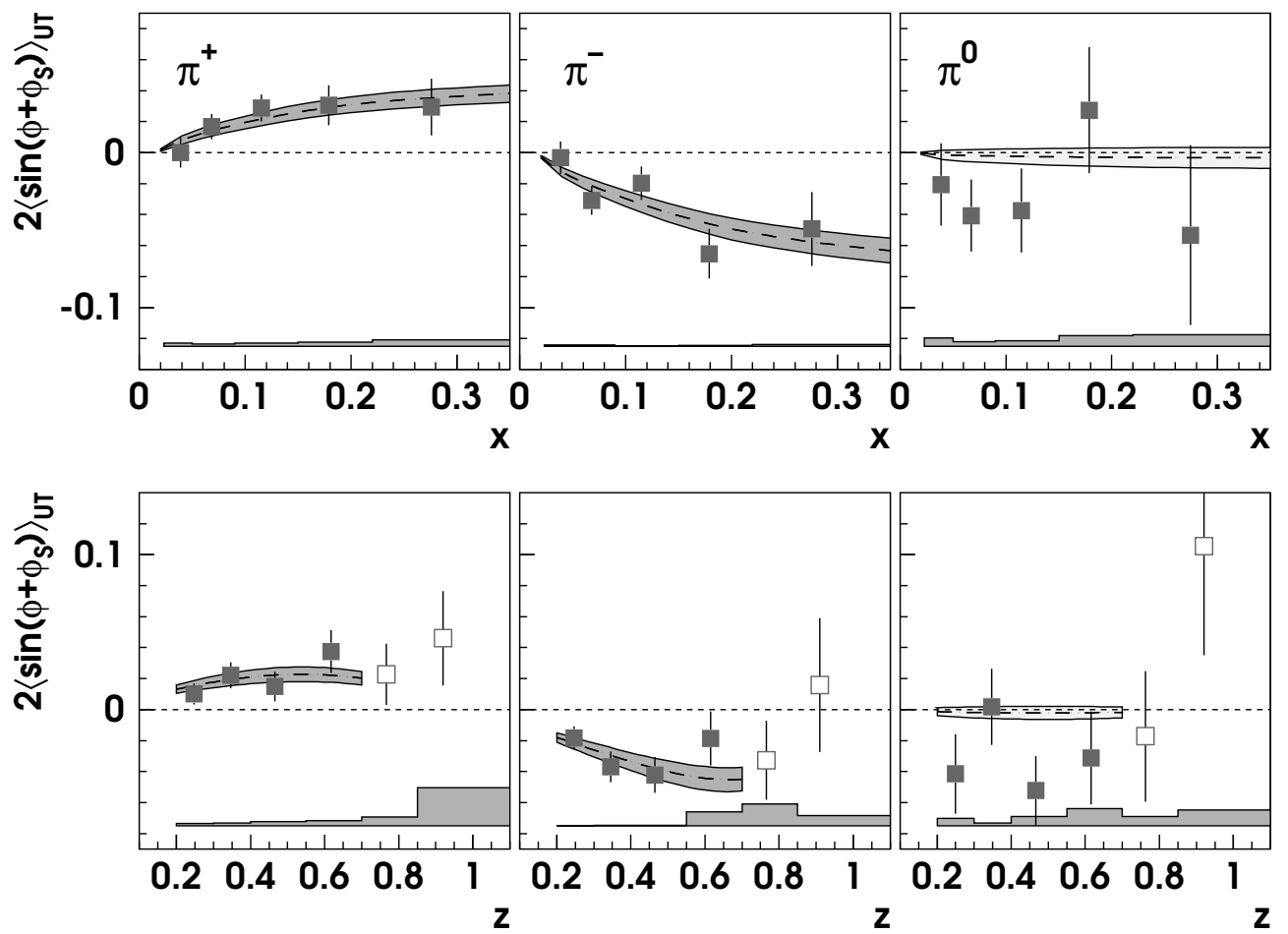

Figure 8.3.7: Unweighted Collins amplitudes of all pion types together with the results of a fit to the charged pion amplitudes [Vog05]. 
description like in Figure 8.3.7 of the charged pion amplitudes is also achieved with a parametrisation of the Collins functions in terms of the unpolarised favoured and disfavoured FFs which have different $z$ dependencies. In both cases, the absolute amplitudes for the neutral pions computed with the obtained fit parameters are smaller than the measured amplitudes. The fit and the prediction of the chiral quark soliton model in Figure 8.3.6 give almost identical $x$ dependencies of the unweighted Collins amplitudes.

In summary, model calculations which do not take into account the disfavoured Collins FF are not able to reproduce the measured unweighted Collins amplitudes for positive pions. Predictions from the chiral quark soliton model describe the measured amplitudes for charged pions well when the disfavoured Collins function is set to a value of similar size but opposite sign compared to the favoured Collins function. This is also the result of a fit to the unweighted Collins amplitudes of the charged pions. These results can be used to predict the unweighted amplitudes of the neutral pions resulting in values of smaller absolute size than the measured amplitudes. The underestimation of the absolute size of the neutral pion amplitudes, which is also present in the predictions from the chiral quark soliton model, is the consequence of the Makins-Relation violation of the measured amplitudes. Both the predictions from the chiral quark soliton model and the parametrisations used in the fit satisfy Eq. (8.1.9) and hence the Makins Relation.

\subsection{The Sivers Moments}

The unweighted Sivers amplitudes as functions of $x, z, P_{h \perp}, y$, and $Q^{2}$ are plotted in Figure 8.4.1 for charged and neutral pions and charged kaons. Also the Sivers amplitudes are listed in Tables F.8 - F.12 together with their statistical and systematic uncertainties. In the latter, the $6.6 \%$ scale uncertainty due to the target polarisation measurement is not included.

Since the Sivers function is combined with the unpolarised FF and because of $u$ quark dominance, the Sivers amplitudes of positive pions and kaons are expected to be similar. However, the kaon amplitudes are twice as large as the pion amplitudes. While it might be important to consider the Sivers function of sea quarks for an explanation of the large difference between the kaon and pion amplitudes the Sivers function had to be much larger for $s$ quarks than for $u$ quarks. In the absence of other known mechanisms, the fact that the kaon Collins amplitudes are smaller than the pion Collins amplitudes (cf. Figure 8.3.1) together with the anti-correlation of the extracted Collins and Sivers functions might indicate that part of the difference is caused by a statistical fluctuation although the real causes are presently not understood. In addition, the unweighted amplitudes contain a kinematic factor dependent on the mean values of $\vec{p}_{T}$ and $\vec{k}_{T}$ (cf. Section 2.5.2) which in principle could be flavour dependent. Like the Collins amplitudes for the negative kaons also the Sivers amplitudes are consistent with zero.

Figure 8.4.2 shows the comparison of the measured unweighted Sivers amplitudes with a model estimate in which the Sivers function is calculated in a spectator model of the nucleon with scalar and axial-vector diquarks [Bac04e]. The gauge link that is necessary to generate the T-odd Sivers function (cf. Section 2.4.3) is approximated by an one-gluon exchange. The obtained Sivers function for $d$ quarks has the opposite sign and a much smaller size compared to the $u$ quark Sivers function. The asymmetry amplitudes esti- 


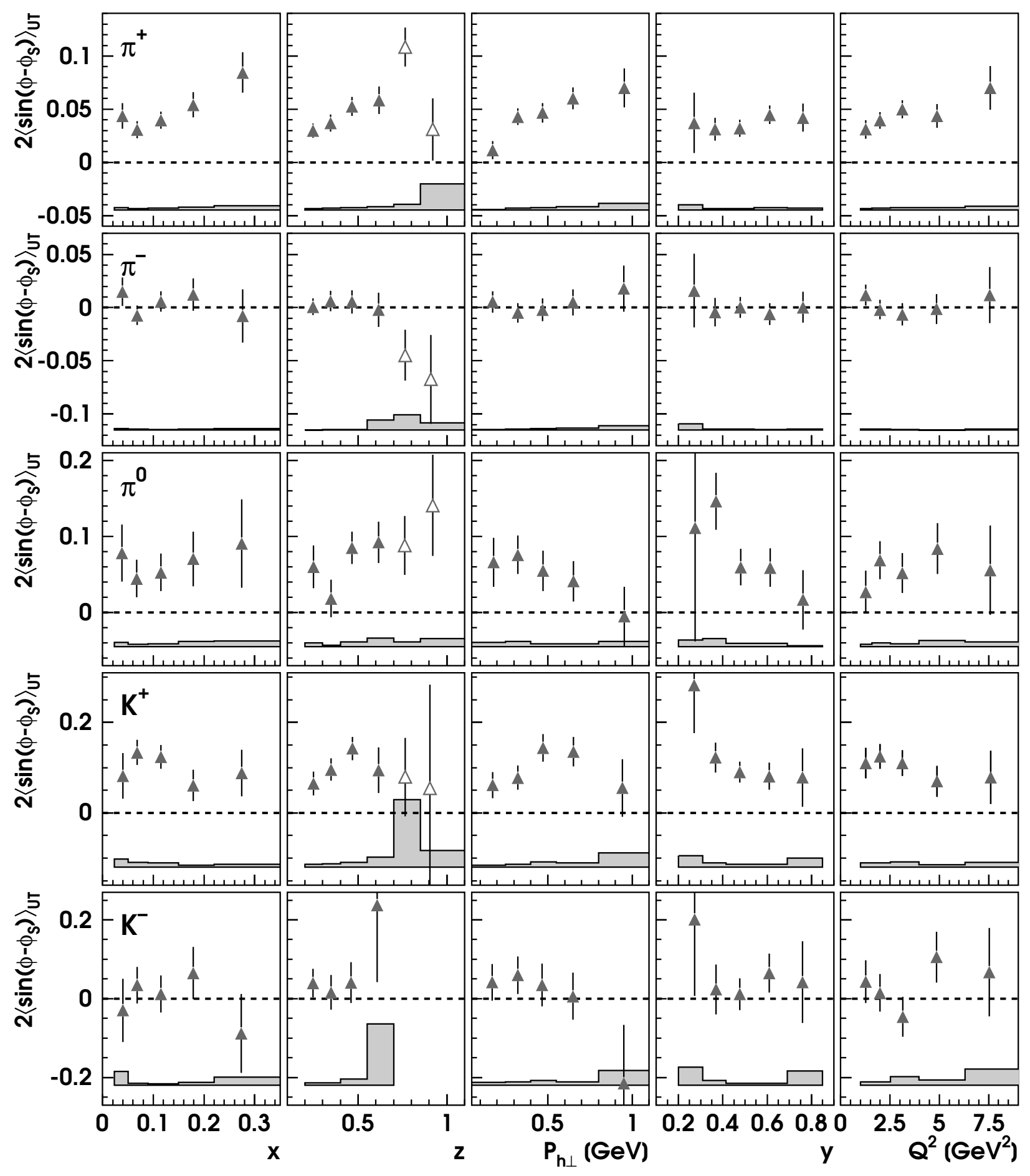

Figure 8.4.1: Unweighted Sivers amplitudes for all five hadron types as functions of $x, z, P_{h \perp}, y$, and $Q^{2}$. An additional $6.6 \%$ scale uncertainty from the target polarisation measurement is not included in the shaded systematic uncertainty bands.

mated from this model describe the order of magnitude of the positive and neutral pion amplitudes. For the negative pions, however, a Sivers amplitude similar to the amplitude of the positive pions is calculated which is in contradiction to the measurement.

Two fits to the amplitudes of the charged pions have been performed so far. A simple 

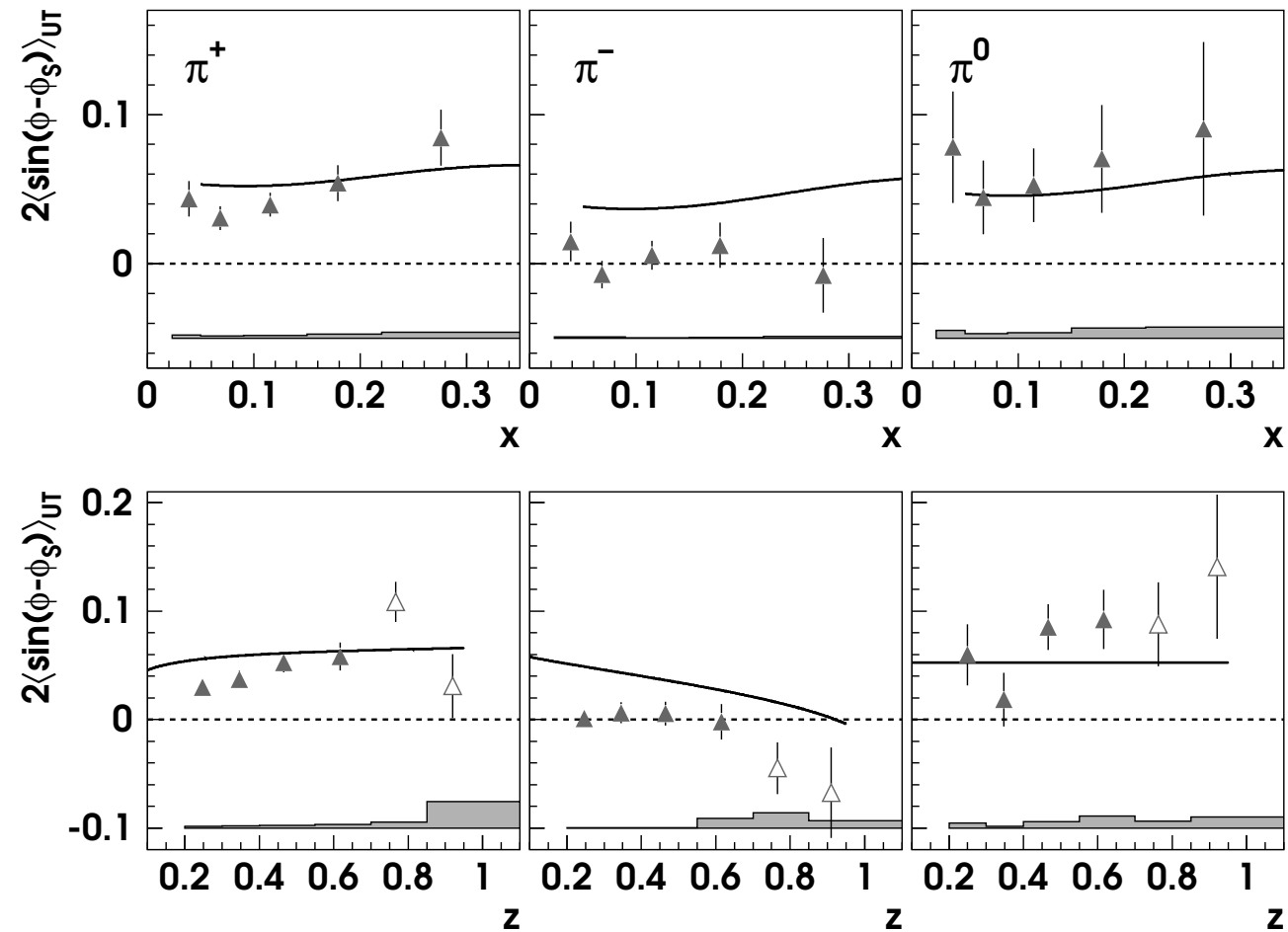

Figure 8.4.2: Unweighted Sivers amplitudes of the three pion types compared to a model estimate in which the Sivers function is calculated in a spectator model of the nucleon [Bac04e].
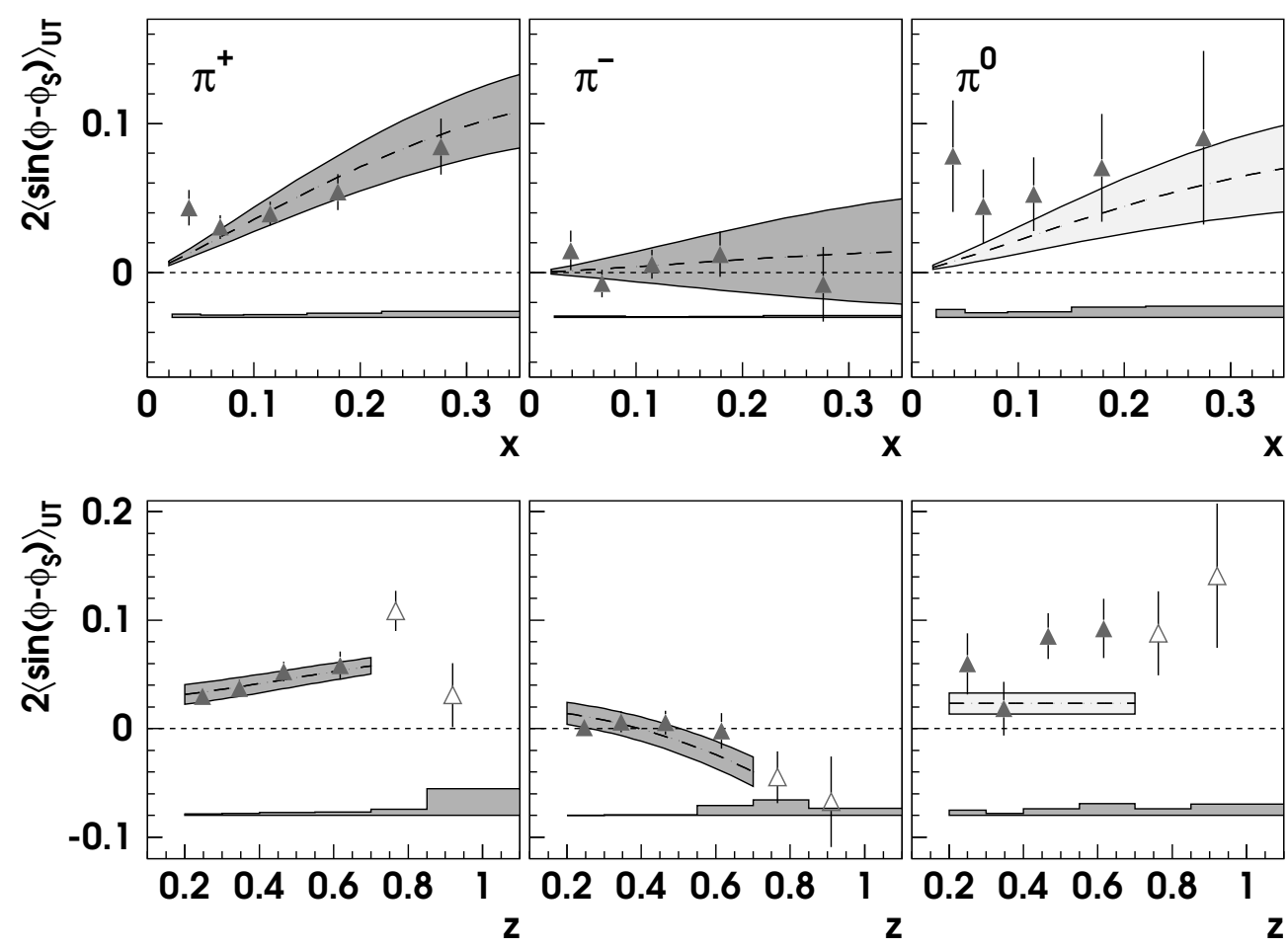

Figure 8.4.3: Sivers amplitudes of pions compared to the results of a fit to the unweighted amplitudes of charged pions [Vog05]. 

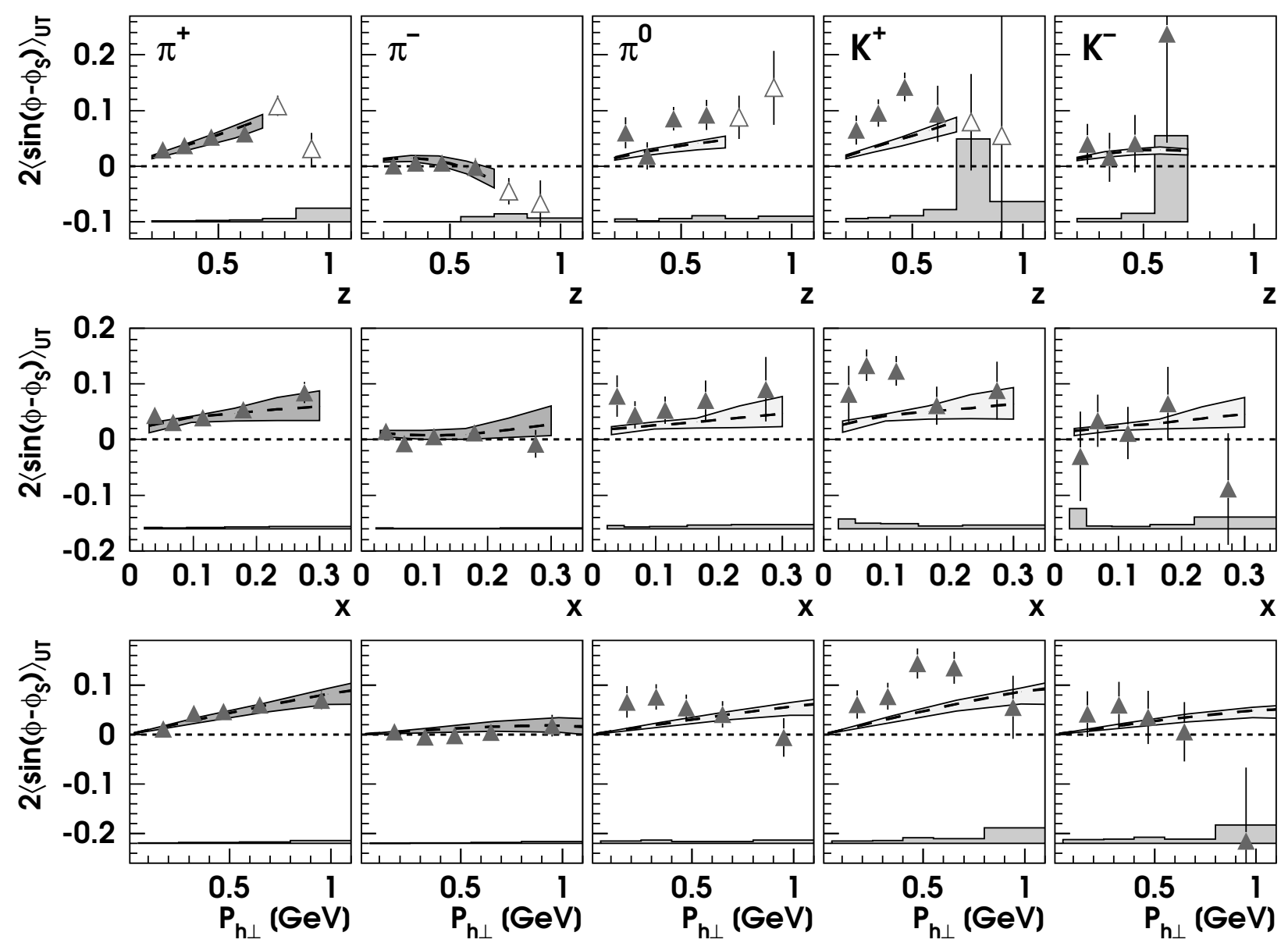

Figure 8.4.4: Sivers amplitudes of pions and kaons compared to a fit to the unweighted amplitudes of charged pions [Ans05, Pro05].

parametrisation of the Sivers function with only one free parameter is used in the fit shown in Figure 8.4.3 [Vog05]. For $u$ and $d$ quarks, the (1/2)-moment of the Sivers function is modelled as $N \cdot x(1-x) \cdot u(x)$. It is assumed to vanish for all other quark and antiquark flavours. A more complex parametrisation with three parameters based on the individual unpolarised DFs is implemented in the fit in Figure 8.4.4. Here, also $\bar{u}$ and $\bar{d}$ antiquarks are considered besides the $u$ and $d$ valence quarks. Both fits describe the amplitudes of the charged pions well and give similar ratios of the $d$ to $u$ quark Sivers functions. The fit in Figure 8.4.3 yields a negative Sivers function for the $u$ quark and $f_{1 T}^{\perp d} \approx-2 \cdot f_{1 T}^{\perp u}$. The second fit shown in Figure 8.4.4 results in the relation $f_{1 T}^{\perp d_{v}} \approx-1.5 \cdot f_{1 T}^{\perp u_{v}}$ for the valence quarks.

From the obtained parametrisations, it is possible to predict the amplitudes of the neutral pions. The amplitudes of the charged kaons can be estimated neglecting $s$ quarks, i.e., $f_{1 T}^{\perp s}=f_{1 T}^{\perp \bar{s}}=0$. Since the measured Sivers pion amplitudes violate the Makins Relation by $2 \sigma$, the measured $\pi^{0}$ amplitudes are above the predicted curves of both parametrisations. Kaon amplitudes are only given for the second fit in Figure 8.4.4. Here, the negative kaons are described well but the measured amplitudes of the positive kaons are larger than the results obtained from the parametrisation which does not include strange quarks. 


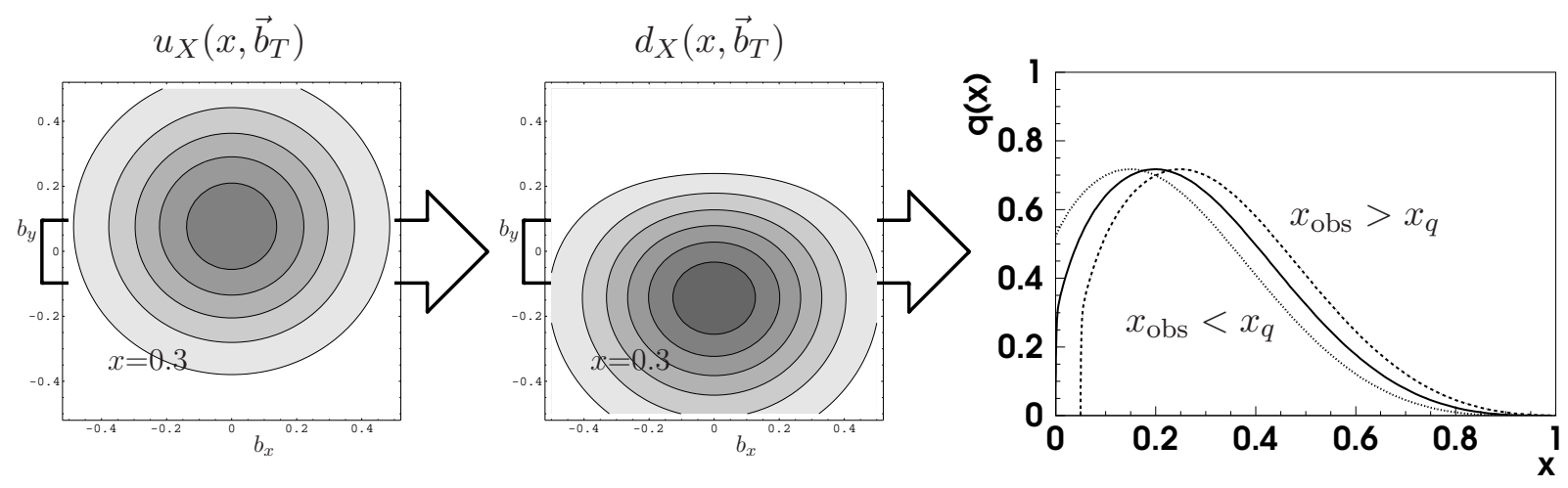

Figure 8.4.5: Impact parameter distribution functions of unpolarised $u$ and $d$ quarks in a transversely polarised nucleon for $x=0.3$ (left and middle panels). Shifted quark DFs for observed quark momenta $x_{\text {obs }}$ smaller and larger than the quark momentum $x_{q}$ (right panel).

A descriptive picture of the relation between the orbital angular momentum of the quarks and the Sivers effect can be obtained when the quark DFs are expressed in impact parameter space [BurO2] so that the unpolarised DF can be written as:

$$
q(x)=\int \mathrm{d}^{2} \vec{b}_{T} q\left(x, \vec{b}_{T}\right)
$$

with the impact parameter $\vec{b}_{T}=\left(b_{x}, b_{y}\right)$. The reference point for the impact parameter is the transverse centre of the longitudinal momentum, i.e., the sum over the transverse positions $\vec{r}_{T, i}$ of all quarks, antiquarks, and gluons in the target weighted by their momentum fractions $x_{i}$ :

$$
\vec{R}_{T}=\sum_{q, \bar{q}, g} x_{i} \vec{r}_{T, i}
$$

The impact parameter dependent DF $q\left(x, \vec{b}_{T}\right)$ of unpolarised quarks is axial symmetric for unpolarised nucleons and nucleons with their spins aligned with the momentum direction ( $z$-axis), i.e., longitudinally polarised nucleons. In case of transversely polarised nucleons, the distribution of unpolarised quarks $q_{X}\left(x, \vec{b}_{T}\right)$ is distorted perpendicular to the spin and the momentum of the nucleon. This distortion vanishes when there is no quark orbital angular momentum parallel to the nucleon spin. Even though the mathematical description of the distortion is model-independent, models for the impact parameter dependencies have to be used in order to visualise these distortions. An example for the distributions of unpolarised $u$ and $d$ quarks in a transversely polarised nucleon is shown in the left two panels of Figure 8.4.5 for a momentum fraction of $x=0.3$. The nucleon spin is in the $x$-direction, i.e., pointing to the right, and the virtual-photon direction is along the negative $z$-axis, i.e., pointing into the page. The signs of the distortions in Figure 8.4.5 are fixed by the signs of the anomalous magnetic moments of the proton and the neutron.

In a semi-classical picture, the superposition of translational and orbital motion of the quarks can be identified as the cause of the distortion of the distribution function. For quarks with an orbital angular momentum parallel to the nucleon spin in the $x$-direction, i.e., pointing to the right in case of the distributions plotted in Figure 8.4.5, the orbital 


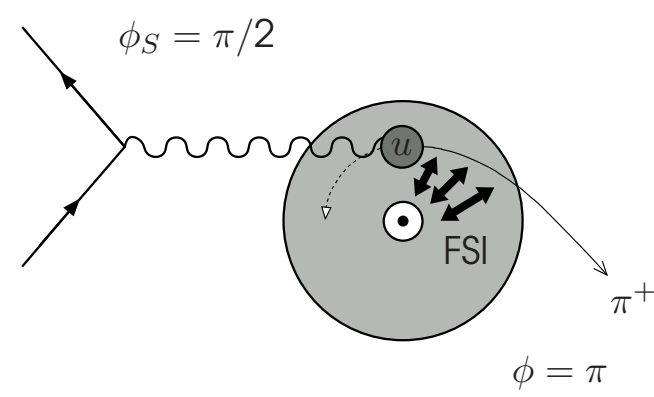

Figure 8.4.6: Illustration of the scattering process off a $u$ quark in the semiclassical picture with the production of a $\pi^{+}$meson.

momentum adds to the quark momentum in the top and subtracts in the bottom. Hence, a quark with a given momentum fraction $x_{q}$ is probed by the virtual photon at a higher momentum fraction $x_{\mathrm{obs}}>x_{q}$ in the top and a smaller fraction $x_{\mathrm{obs}}<x_{q}$ in the bottom. In the top the unpolarised DF is therefore shifted towards higher $x$ values while in the bottom it is shifted to smaller $x$ values as shown in the right panel of Figure 8.4.5. Since the unpolarised DF decreases with increasing values of $x$ in the valence region, the increase of the momentum on one side of the nucleon spin results in a larger number of quarks for a certain observed momentum fraction $x_{\text {obs }}$ at this side. At the opposite side, less quarks are observed at $x_{\mathrm{obs}}$ due to the decrease of the quark momentum, resulting in a distortion of the DF at $x_{\mathrm{obs}}$ towards the top. For quarks with antialigned orbital angular momentum, the DF is distorted towards the bottom. This semi-classical picture thus yields a positive orbital angular momentum for $u$ quarks and a negative orbital angular momentum for $d$ quarks.

In Figure 8.4.6 the scattering process is schematically illustrated for a nucleon spin orientation perpendicular to the scattering plane, i.e., $\phi_{S}=\pi / 2$. For a positive orbital angular momentum of the $u$ quarks, the $u$ quark density is enhanced in the left hemisphere of the nucleon when looking along the virtual-photon direction so that it will be absorbed more likely by a $u$ quark in that region. After the absorption, final-state interactions (FSI) (cf. Section 2.4.3) bend the quark towards the centre. The FSI are attractive since struck quark and the spectators - the remaining quarks from the nucleon-form a colour antisymmetric state. The outgoing positive pion that contains the struck quark is therefore observed on the right-hand side of the nucleon spin, i.e., $\phi=\pi$. Thus, the description of the quark DFs in the impact parameter space yields a positive Sivers moment $\sin \left(\phi-\phi_{S}\right)=\sin \pi>0$ for $u$ quarks fragmenting into $\pi^{+}$. This is consistent with the positive Sivers amplitudes for $\pi^{+}$in the HERMES data which are dominated by the scattering off $u$ quarks. In case of $\pi^{-}$production, both $u$ and $d$ quarks have to be taken into account because of the quark-charge factor $e_{q}^{2}$ and the results cannot be interpreted solely in terms of $d$ quark scattering. Scattering from $d$ quarks alone would yield a negative Sivers moment so that the two quark flavours contribute with opposite sign to the Sivers moment and their contributions might cancel. 


\subsection{Extraction of the Quark Distribution Functions}

\subsubsection{Sivers Distribution Function}

Moving the kinematic factors out of the integrals, using the definition of the purities in Eq. (2.5.30), and assuming Gaussian distributions for the quark transverse momenta, the unweighted Sivers moment (2.5.28) of a hadron type $h$ in a certain $x$ bin $i$ can be written as:

$$
\begin{aligned}
\left\langle\sin \left(\phi-\phi_{S}\right)\right\rangle_{\mathrm{UT}}^{h}\left(x_{i},\left\langle Q^{2}\right\rangle\right) & =-\frac{1}{\sqrt{1+\left\langle K_{T}^{2}\right\rangle /\left(\langle z\rangle^{2}\left\langle p_{T}^{2}\right\rangle\right)}} \frac{1+R\left(x_{i},\left\langle Q^{2}\right\rangle\right)}{1+\gamma^{2}} \sum_{q, \bar{q}} \mathcal{P}_{q}^{h}\left(x_{i}\right) \frac{f_{1 T}^{\perp(1 / 2) q}\left(x_{i}\right)}{q\left(x_{i}\right)} \\
& =-\mathcal{C}_{T} \mathcal{C}_{i} \sum_{q, \bar{q}} \mathcal{P}_{q}^{h}\left(x_{i}\right) \frac{f_{1 T}^{\perp(1 / 2) q}\left(x_{i}\right)}{q\left(x_{i}\right)} .
\end{aligned}
$$

The additional kinematic factor $\mathcal{C}_{i}=\left(1+R\left(x, Q^{2}\right)\right) /\left(1+\gamma^{2}\right)$ arises from the determination of the purities using parametrisations for the unpolarised DFs. Technically, the parametrisations are obtained by fits to the world data on $F_{2}$. In order to relate the functions to the structure function $F_{1}$, this additional factor has to be applied (cf. Eq. (2.1.20)) [Ack99]. The kinematic factor $\mathcal{C}_{T}$ is identical for the different hadron types since the mean values of the quark transverse momenta are assumed to be independent of the quark flavours.

Eq. (8.5.1) can be expressed as a matrix equation for $m$ hadron types and $n$ quark flavours:

$$
\vec{A}\left(x_{i}\right)=-\mathcal{C}_{i} \cdot \boldsymbol{P}\left(x_{i}\right) \vec{Q}\left(x_{i}\right)
$$

with the vectors,

$$
\vec{A}=\left(\begin{array}{c}
\left\langle\sin \left(\phi-\phi_{S}\right)\right\rangle_{\mathrm{UT}}^{h_{1}}\left(x_{i}\right) \\
\vdots \\
\left\langle\sin \left(\phi-\phi_{S}\right)\right\rangle_{\mathrm{UT}}^{h_{m}}\left(x_{i}\right)
\end{array}\right), \quad \vec{Q}=\left(\begin{array}{c}
\mathcal{C}_{T} \frac{f_{1 T}^{\perp(1 / 2) q_{1}}}{q_{1}}\left(x_{i}\right) \\
\vdots \\
\mathcal{C}_{T} \frac{f_{1 T}^{\perp(1 / 2) q_{n}}}{q_{n}}\left(x_{i}\right)
\end{array}\right),
$$

and the purity matrix:

$$
\boldsymbol{P}=\left(\begin{array}{ccc}
\mathcal{P}_{q_{1}}^{h_{1}}\left(x_{i}\right) & \ldots & \mathcal{P}_{q_{n}}^{h_{1}}\left(x_{i}\right) \\
\vdots & \ddots & \vdots \\
\mathcal{P}_{q_{1}}^{h_{m}}\left(x_{i}\right) & \ldots & \mathcal{P}_{q_{n}}^{h_{m}}\left(x_{i}\right)
\end{array}\right)
$$

The Sivers polarisations $f_{1 T}^{\perp(1 / 2) q}(x) / q(x)$ multiplied by the kinematic factor $\mathcal{C}_{T}$ can be obtained for $n \leq m$ by minimising the figure-of-merit function:

$$
\chi^{2}=\left(\vec{A}+\mathcal{C}_{i} \cdot \boldsymbol{P} \vec{Q}\right)^{T} \mathcal{V}_{A}^{-1}\left(\vec{A}+\mathcal{C}_{i} \cdot \boldsymbol{P} \vec{Q}\right)
$$

Here, $\mathcal{V}_{A}$ denotes the covariance matrix of the set of asymmetry moments. For a first attempt to extract the Sivers polarisations, the systematic uncertainties as well as the statistical correlations of the asymmetry moments are neglected. The off-diagonal elements of the covariance matrix then vanish and the diagonal elements are given by

${ }^{\mathrm{i}}\left|\vec{S}_{T}\right| \approx 1$ since the measured azimuthal asymmetry is normalised by the target polarisation and the angle between incoming lepton and virtual photon is small. 

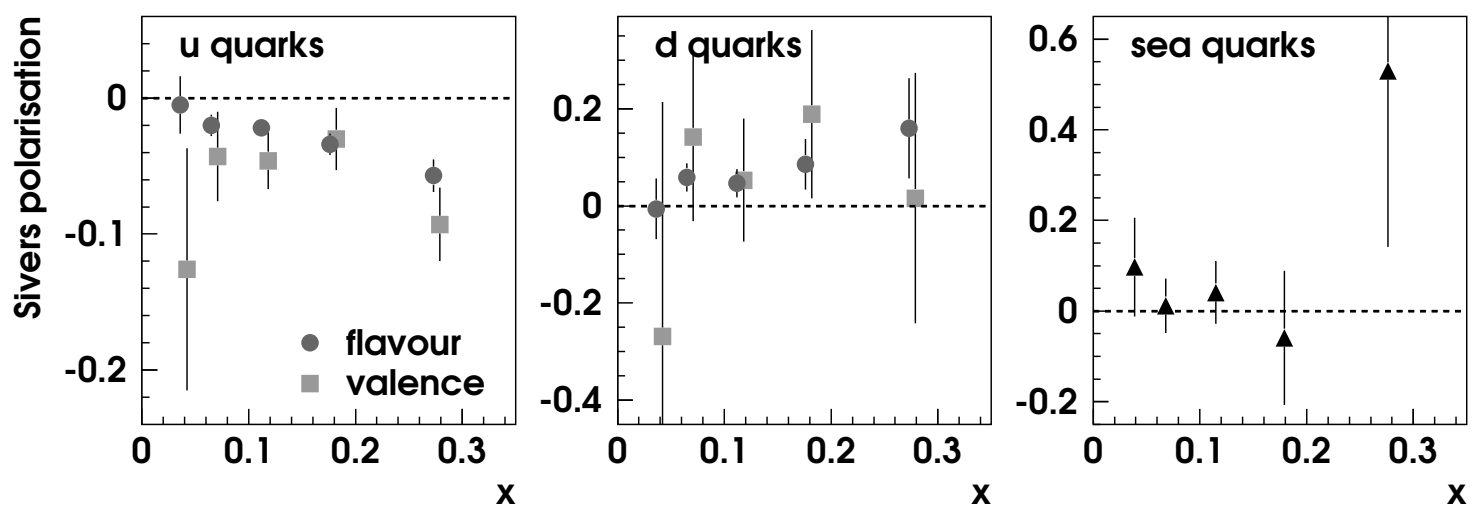

Figure 8.5.1: Extracted Sivers polarisations for flavour and valence decomposition and the assumption of a symmetric sea polarisation.

the squared statistical uncertainty. The figure-of-merit function was implemented in a fit program based on MINUIT.

Six quark and antiquark flavours have to be taken into account for HERMEs kinematics where the $u$ and $d$ quarks can be split up into valence quarks, $u_{v}$ and $d_{v}$, and sea quarks, $u_{s}$ and $d_{s}$. However, asymmetry moments are measured only for five hadron types. Therefore, a symmetric sea polarisation is assumed:

$$
\begin{aligned}
\frac{f_{1 T}^{\perp(1 / 2) q_{s}}(x)}{q_{s}(x)} & \equiv \frac{f_{1 T}^{\perp(1 / 2) u_{s}}(x)}{u_{s}(x)}=\frac{f_{1 T}^{\perp(1 / 2) \bar{u}}(x)}{\bar{u}(x)}=\frac{f_{1 T}^{\perp(1 / 2) d_{s}}(x)}{d_{s}(x)} \\
& =\frac{f_{1 T}^{\perp(1 / 2) \bar{d}}(x)}{\bar{d}(x)}=\frac{f_{1 T}^{\perp(1 / 2) s}(x)}{s(x)}=\frac{f_{1 T}^{\perp(1 / 2) \bar{s}}(x)}{\bar{s}(x)} .
\end{aligned}
$$

Different combinations of the three remaining valence $u$-quark, valence $d$-quark, and sea-quark distributions can be extracted. Two of these are the flavour decomposition:

$$
\mathcal{C}_{T} \frac{f_{1 T}^{\perp(1 / 2) u}+f_{1 T}^{\perp(1 / 2) \bar{u}}}{u+\bar{u}}, \quad \mathcal{C}_{T} \frac{f_{1 T}^{\perp(1 / 2) d}+f_{1 T}^{\perp(1 / 2) \bar{d}}}{d+\bar{d}}, \quad \mathcal{C}_{T} \frac{f_{1 T}^{\perp(1 / 2) s}+f_{1 T}^{\perp(1 / 2) \bar{s}}}{s+\bar{s}},
$$

and the valence decomposition:

$$
\begin{array}{r}
\mathcal{C}_{T} \frac{f_{1 T}^{\perp(1 / 2) u_{v}}}{u_{v}}=\mathcal{C}_{T} \frac{f_{1 T}^{\perp(1 / 2) u}-f_{1 T}^{\perp(1 / 2) \bar{u}}}{u-\bar{u}}, \quad \mathcal{C}_{T} \frac{f_{1 T}^{\perp(1 / 2) d_{v}}}{d_{v}}=\mathcal{C}_{T} \frac{f_{1 T}^{\perp(1 / 2) d}+f_{1 T}^{\perp(1 / 2) \bar{d}}}{d+\bar{d}}, \\
\mathcal{C}_{T} \frac{f_{1 T}^{\perp(1 / 2) q_{s}}}{q_{s}}
\end{array}
$$

For both decompositions the elements of the purity matrix $\boldsymbol{P}$ are combinations of the purities of the individual quark and antiquark flavours, which are shown in Figure E.1. The expressions for the elements of the purity matrices in case of flavour and valence decomposition can be found in [Bec00].

Figure 8.5.1 shows the extracted Sivers polarisations for the flavour and the valence decomposition. In the Sivers asymmetry moments, no higher order $\alpha_{s}$ corrections are applied to the kinematic factor $A(y)$ of the unpolarised cross section, i.e., the $y$ dependence of the Sivers and the unpolarised cross-section terms cancel (cf. Section 8.2). The reduced $\chi^{2}$ of the fit is close to 1 in the lowest and the two highest $x$ bins. The second and 
third $x$ bins with their large $K^{+}$amplitudes have worse values of the reduced $\chi^{2}$ above 5. However, the systematic uncertainties and the statistical correlations are not yet included in the extraction and the assumption of the symmetric sea polarisation might not be appropriate.

A larger sensitivity in the extraction of the sum of quark and antiquark distributions, i.e., in the flavour decomposition, than in the extraction of their differences is visible, as it was already found in case of the helicity distribution function [Dür98]. The obtained flavour symmetric sea polarisation is consistent with zero and hence the $u$ and $d$ quark Sivers polarisations for flavour and valence decompositions are comparable. The Sivers polarisation of the $u$ quark is negative in contrast to the positive $d$ quark polarisation. Since the unpolarised DF for $u$ quarks is about twice as large as for $d$ quarks, the extracted Sivers functions for $u$ and $d$ quarks are of similar size. Furthermore, a negative (positive) Sivers function corresponds to a net motion of the struck quark to the right (left) when looking along the direction of the virtual photon if the proton spin is upwards [Bac04a]. In this case the semi-classical picture described in the previous section suggests a positive (negative) orbital angular momentum for $u$ ( $d$ ) quarks. The Sivers polarisations still contain the kinematic factor $\mathcal{C}_{T}$ which is around 0.7 in the case of $\left\langle k_{T}^{2}\right\rangle \approx\left\langle p_{T}^{2}\right\rangle$. For $\left\langle K_{T}^{2}\right\rangle \approx\left\langle p_{T}^{2}\right\rangle$ and $z \approx 0.36, \mathcal{C}_{T}$ is around 0.3 .

\subsubsection{Transversity}

As shown earlier, the HERMES data alone restrict the ratio of the disfavoured and the favoured Collins functions. However, they do not allow to separate the transversity DFs. To gain information about $\delta q(x)$, the Collins function has to be measured, e.g., in electronpositron annihilation with the production of a quark-antiquark pair that fragments into hadrons (cf. Figure 2.6.1 (c) on page 31). Since there is a non-zero probability that quarks have their spins aligned transversely to the lepton beams, the Collins function causes the correlation of the transverse momenta of the produced hadrons. This leads to an azimuthal modulation in the cross section of inclusive two-hadron production. This modulation can be expressed either by one azimuthal angle $\phi_{0}$, defined with respect to the momentum of one of the produced hadrons, or by two azimuthal angles $\phi_{1}$ and $\phi_{2}$, de-
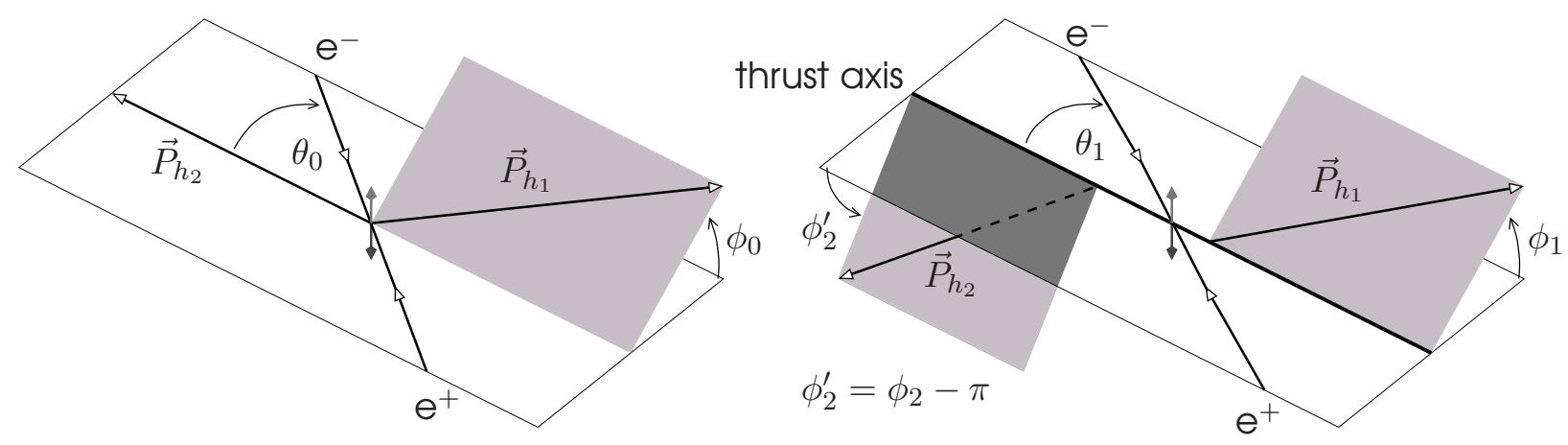

Figure 8.5.2: Definition of the azimuthal angles for two-hadron production in electron-positron annihilation. 
fined with respect to the measured thrust axis which is an approximation of the quarkantiquark axis. These two definitions are depicted in Figure 8.5.2.

In both reference systems, the azimuthal asymmetry contains a cosine modulation: $\cos 2 \phi_{0}$ or $\cos \left(\phi_{1}+\phi_{2}\right)$, respectively. The amplitudes of these modulations are proportional to the product of quark and antiquark Collins functions [Boe97]:

$$
\begin{aligned}
A^{\cos 2 \phi_{0}} & =\frac{\sin ^{2} \theta_{0}}{1+\cos ^{2} \theta_{0}} \frac{1}{M_{1} M_{2}} \frac{\mathcal{F}\left[H_{1}^{\perp q}\left(z_{1}\right) H_{1}^{\perp \bar{q}}\left(z_{2}\right)\right]}{D_{1}^{q}\left(z_{1}\right) D_{1}^{\bar{q}}\left(z_{2}\right)}, \\
A^{\cos \left(\phi_{1}+\phi_{2}\right)} & =\frac{\sin ^{2} \theta_{1}}{1+\cos ^{2} \theta_{1}} \frac{H_{1}^{\perp[1] q}\left(z_{1}\right) H_{1}^{\perp[1] \bar{q}}\left(z_{2}\right)}{D_{1}^{q}\left(z_{1}\right) D_{1}^{\bar{q}}\left(z_{2}\right)},
\end{aligned}
$$

where $\mathcal{F}$ is a convolution integral over transverse quark and hadron momenta, $M_{1}$ and $M_{2}$ are the masses of the two hadrons, and $z_{1}$ and $z_{2}$ are their energy fractions. In contrast to the (1/2)-moment in Eq. 2.5.4 where the integration is over $\vec{k}_{T}$, the [1]-moment of the Collins FF contains the integral over $\left|\vec{k}_{T}\right|$ :

$$
H_{1}^{\perp[1]}(z)=\int \mathrm{d}\left|\vec{k}_{T}\right| \frac{\left|\vec{k}_{T}\right|}{M_{h}} H_{1}^{\perp}\left(z, z^{2} k_{T}^{2}\right) .
$$

The polar angle $\theta_{0}\left(\theta_{1}\right)$ is defined between the hadron momentum (the thrust axis) and the electron momentum (cf. Figure 8.5.2) and determines the transverse polarisation of the quark-antiquark pair: $\sin ^{2} \theta_{0 / 1} /\left(1+\cos ^{2} \theta_{0 / 1}\right)$.

A first measurement of azimuthal asymmetries in the production of charged-pion pairs in electron-positron annihilation was performed with the BELLE detector [Aba02] at an asymmetric electron-positron collider, the KEKB accelerator in Tsukuba City, Japan. Both asymmetry amplitudes were found to be significantly positive for pions with energy fractions between 0.2 and 1.0 [Abe05]. Yet, the extraction of the Collins function from these promising data is only possible with assumptions on the ratio of the disfavoured to favoured Collins function. The restriction on this ratio derived from the HERMES data will provide helpful information for a first attempt of the Collins FF extraction. In addition, future measurements for the production of neutral-pion pairs will allow the determination of this ratio.

\subsection{Other Options to Measure the Transversity and Sivers Functions}

\subsection{Possibilities at HeRmes}

\section{Two-Hadron Production in Semi-Inclusive DIS}

An alternative process to access the transversity function is the semi-inclusive production of two hadrons in DIS. In this process, the transverse spin of the quark inside a transversely polarised nucleon can be correlated to the transverse momentum of the hadron pair, instead of the transverse momentum of a single hadron. This allows to probe transversity without the inclusion of partonic transverse momenta. The study of such two-hadron fragmentation processes was already proposed in 1993 [Col94] and 1998 [Jaf98].

The differential cross section for semi-inclusive two-hadron production in DIS on a transversely polarised target, integrated over the transverse momentum of the hadron 


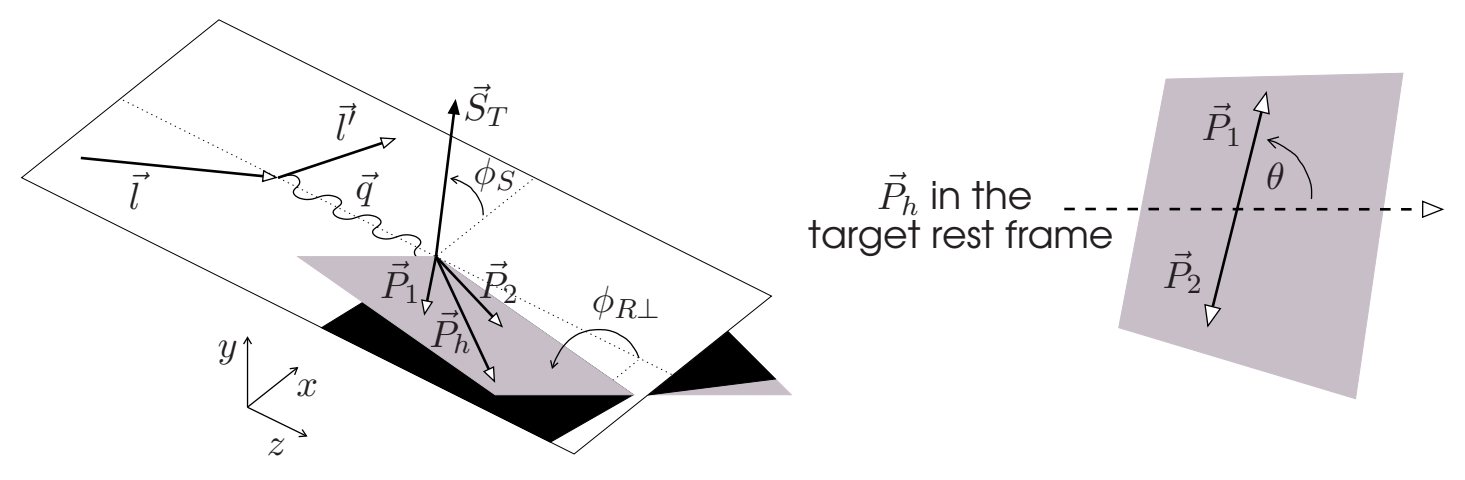

Figure 8.6.1: Definition of the azimuthal angle $\phi_{R \perp}$ (left) and the polar angle $\theta$ (right) for two-hadron production in DIS. The latter is defined in the two-hadron centre-of-mass system.

pair, is [Bac03b, Bac04d]:

$$
\begin{gathered}
\frac{\mathrm{d}^{7} \sigma_{\mathrm{UT}}}{\mathrm{d} x \mathrm{~d} y \mathrm{~d} z \mathrm{~d} \phi_{S} \mathrm{~d} \phi_{R \perp} \mathrm{d} \cos \theta \mathrm{d} M_{\pi \pi}^{2}}= \\
-\frac{\alpha^{2}}{2 \pi s x y^{2}} \sum_{q, \bar{q}} e_{q}^{2} B(y)\left|\vec{S}_{T}\right| \frac{|\vec{R}|}{M_{h h}} \sin \left(\phi_{R \perp}+\phi_{S}\right) \sin \theta \delta q(x)\left[H_{1}^{\varangle s p}\left(z, M_{h h}^{2}\right)+\cos \theta H_{1}^{\varangle p p}\left(z, M_{h h}^{2}\right)\right],
\end{gathered}
$$

where $z$ is the energy fraction of the hadron pair and $M_{h h}$ its invariant mass. For two hadrons with equal mass $M_{h},|\vec{R}|=\frac{1}{2} \sqrt{M_{h h}^{2}-4 M_{h}^{2}}$. The azimuthal angle $\phi_{R \perp}$ and the polar angle $\theta$ are defined in Figure 8.6.1. The transversity DF appears in conjunction with a combination of two-hadron FFs that describe the interference of different production channels of the hadron pair. The chiral-odd and T-odd FFs $H_{1}^{\varangle s p}\left(z, M_{h h}^{2}\right)$ and $H_{1}^{\varangle p p}\left(z, M_{h h}^{2}\right)$ are related to the interference between the $s$-wave and $p$-wave channels and between two $p$-wave channels, respectively. The index $q \rightarrow h h$ is omitted for clarity. As for onehadron production, two terms exist in the $P_{h \perp}$-dependent cross section that include the Sivers function and a two-hadron FF, equivalent to the Collins function for one-hadron production. However, these two terms vanish when the cross section is integrated over the transverse momentum of the hadron pair.

The advantages of such a measurement are the independent access to transversitydifferent FFs than for one-hadron production are involved-and that DF and FF are not embedded in a convolution integral. The disadvantage is a significantly smaller count number of hadron pairs compared to single hadron events; about five times less $\pi^{+} \pi^{-}$ pairs than single $\pi^{+}$events can be identified in the HERMES data on a transversely polarised target. Nevertheless, the high statistics of the 2002-2004 combined data productions allows the determination of the azimuthal asymmetry for semi-inclusive $\pi^{+} \pi^{-}$-pair production. The azimuthal amplitude of interest is extracted by a two-dimensional fit dependent on $\phi_{R \perp}+\phi_{S}$ and $\theta$ and found to be significantly positive [vdN05]:

$$
A_{\mathrm{UT}}^{\sin \left(\phi_{R \perp}+\phi_{S}\right) \sin \theta}=0.040 \pm 0.009 \text { (stat.) } \pm 0.003 \text { (syst.) } .
$$

This azimuthal asymmetry can be evaluated even in four different invariant mass bins between 0.25 and $2.0 \mathrm{GeV}$, yielding four positive asymmetry moments as shown in Figure 


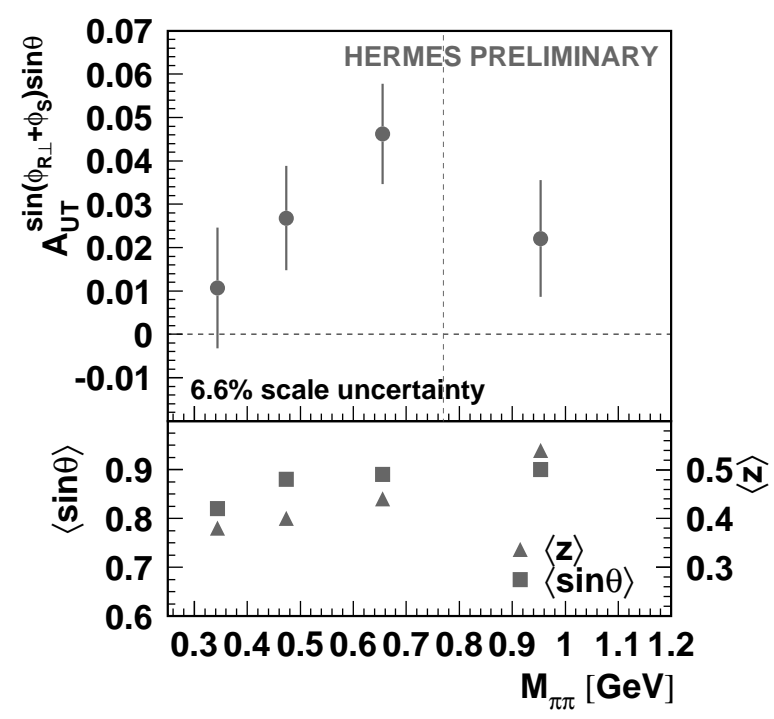

Figure 8.6.2: Asymmetry amplitude $A_{\mathrm{UT}}^{\sin \left(\phi_{R \perp}+\phi_{S}\right) \sin \theta}$ for semi-inclusive $\pi^{+} \pi^{-}$-pair production as a function of the invariant mass $M_{\pi \pi}$ of the pion pair.

8.6.2. This result is clearly inconsistent with a sign change at the mass of the $\rho^{0}$ vector meson, predicted in a two-meson phase shift analysis [Jaf98]. The asymmetry moments rather show a maximum around the $\rho^{0}$ mass, as predicted, e.g., in [Rad05].

Also for the two-hadron production, measurements of the interference FFs by the BELLE collaboration will allow the extraction of the transversity DF. Even though the statistical precision and the number of kinematic bins with sufficient statistics are limited, such a measurement provides an alternative access to transversity, requiring no assumption on the distributions of the quark transverse momenta.

\section{Polarimetry of the Final-State Hadron}

So far, only the production of spin-zero mesons has been considered. However, also the analysis of semi-inclusive production of spin- $\frac{1}{2}$ or spin-1 hadrons can provide information about transversity. The production of transversely polarised hadrons on a transversely polarised target is sensitive to the transversity DF. Like in the case of two-hadron production, the interpretation of this cross-section term in terms of DF and FF is not hampered by the convolution over quark transverse momenta. The challenge of such a measurement is the determination of the polarisation of the produced hadrons. In case of a two-particle decay of the produced hadron, this can be achieved through the analysis of the decay angular distributions.

For spin- $\frac{1}{2}$ hadrons, like the $\Lambda^{0}$ baryon, the transversity function and the conjunct twisttwo FF $H_{1}$ exhibit a $\cos \left(\phi_{S}+\phi_{S h}\right)$ modulation where $\phi_{S h}$ is the azimuthal angle between the transverse $\Lambda^{0}$ polarisation axis and the lepton scattering plane [Boe98]. The azimuthal modulation $\cos \left(\phi_{S}-\phi_{S h}\right)$ is proportional to the Sivers function, yet the DF and FF require the inclusion of transverse quark momenta and are embedded in a convolution integral. Unfortunately, the small acceptance for $\Lambda^{0}$ baryons in the HERMES experiment together with the complex decay angular distribution analysis will not allow the extraction of the azimuthal asymmetries amplitudes from the complete HERMES data sample on a trans- 
versely polarised target, i.e., including the data recorded in the year 2005.

Higher statistics are available for the analysis of semi-inclusive vector mesons, i.e., spin1 hadrons, especially in the case of the $\rho^{0}$ meson decaying into a pion pair. As for the two-hadron production, the amplitude of the $\sin \left(\phi_{R \perp}+\phi_{S}\right)$ modulation is proportional to transversity [Bac02b]. Even though a large amount of semi-inclusive $\rho^{0}$ mesons are present in the data, a huge background of non-resonant pion pairs will probably inhibit such an analysis.

\section{Subleading-Twist Components}

For the semi-inclusive production of a single spin-zero hadron, the transversity DF appears not only together with the Collins function but also in conjunction with the twist-three FF $\tilde{H}\left(z, z^{2} k_{T}^{2}\right)$ in the differential cross section $\mathrm{d} \sigma_{\mathrm{UT}}$ (see Table 2.5.1 on page 26). The latter combination is part of the twist-three cross-section term that exhibits a sin $\phi_{S}$ modulation. The $\sin \phi_{S}$ moment of the cross section,

$$
\begin{aligned}
& \left\langle\sin \phi_{S}\right\rangle_{\mathrm{UT}}(x, y, z)=\left|\vec{S}_{T}\right| \frac{M}{Q} \frac{\frac{1}{x y^{2}} D(y)}{2 \frac{1}{x y^{2}} A(y) \sum_{q, \bar{q}} e_{q}^{2} q(x) D_{1}^{q}(z)} \times \\
& \sum_{q, \bar{q}} e_{q}^{2} \int \mathrm{d}^{2} \vec{P}_{h \perp} \mathcal{I}\left[x f_{T} D_{1}-\frac{M_{h}}{M z} \delta q \tilde{H}-\frac{\vec{p}_{T} \cdot \vec{k}_{T}}{2 M M_{h}}\left\{x\left(h_{T}-h_{T}^{\perp}\right) H_{1}^{\perp}+\frac{M_{h}}{M z}\left(f_{1 T}^{\perp} \tilde{D}^{\perp}-g_{1 T} \tilde{G}^{\perp}\right)\right\}\right],
\end{aligned}
$$

contains besides the product $\delta q \cdot \tilde{H}$ a term proportional to $\vec{p}_{T} \cdot \vec{k}_{T} /\left(2 M M_{h}\right)$ and the product of the unpolarised FF and the twist-three DF $f_{T}$. After performing the convolution integral over the transverse momenta of the initial and final quarks and the produced hadron, $\int \mathrm{d}^{2} \vec{P}_{h \perp} \mathcal{I}[\ldots]$ (cf. Section 2.5.2), only the two terms proportional to the FFs $D_{1}$ and $\tilde{H}$ remain because the third term is odd in both quark transverse momenta. Since the gauge-link (cf. Section 2.4.3) contributes to the quark-quark-correlation function only for $\vec{p}_{T}$-dependent DFs where $\xi_{T} \neq 0$, T-odd DF that are independent of $\vec{p}_{T}$ are expected to be zero. So far, no mechanism has been found which could create non-zero $\vec{p}_{T}-$ independent DF that are T-odd like the twist-three DF $f_{T}$. Hence, Eq. (8.6.3) reduces to:

$$
\left\langle\sin \phi_{S}\right\rangle_{\mathrm{UT}}(x, y, z)=-\left|\vec{S}_{T}\right| \frac{M_{h}}{Q z} \frac{\frac{1}{x y^{2}} D(y) \sum_{q, \bar{q}} e_{q}^{2} \delta q(x) \tilde{H}^{q}(z)}{2 \frac{1}{x y^{2}} A(y) \sum_{q, \bar{q}} e_{q}^{2} q(x) D_{1}^{q}(z)} .
$$

In contrast to the twist-two Collins moment, the convolution integral over the product of transversity and the FF $\tilde{H}$ resolves without any assumption on the quark transverse momenta simplifying the interpretation in terms of DF and FF.

With the two-dimensional fit function (4.3.2) (see page 63), the $\sin \phi_{S}$ moment is extracted from the azimuthal asymmetries together with the Sivers and Collins moments and found to be significantly negative in case of the negative pions, as can be seen in Figure 4.4.4. For the final $\left\langle\sin \phi_{S}\right\rangle_{\mathrm{UT}}$ extraction, the influence of possible biases on the moment extraction has to be studied which will require the implementation of the $\sin \phi_{S}$ moment in the Monte Carlo generator gmc_trans (see Section 5.2).

So far, no experimental information about the twist-three FF $\tilde{H}$ exists that would allow the extraction of transversity from the $\sin \phi_{S}$ moment. The function $\tilde{H}$ has been introduced in 1993 [Jaf93] $^{i}$ and is-like the Collins function and the interference FF-accessible in

\footnotetext{
'Here, $\tilde{H}$ is called $\hat{e}_{\overline{1}}$.
} 
electron-positron annihilation, e.g., in the BELLE experiment. However, since $\tilde{H}$ is suppressed by $Q$ and at BELLE $Q=\sqrt{s}$ is $10.52 \mathrm{GeV}$ in contrast to a mean $Q$ of around $1.5 \mathrm{GeV}$ at HERMES, the expected small values of the asymmetries might require a very large data set to make such a measurement feasible. A measurement at a lower centre-of-mass energy of about $4 \mathrm{GeV}$ could be performed with the BES experiment at the BEPC electronpositron collider in Beijing, China, which will be upgraded to yield higher luminosities.

Another subleading-twist component can in principle be used to measure transversity [Jaf93]. This component exhibits a $\cos \phi_{S}$ modulation in the cross section for a longitudinally polarised lepton beam and a transversely polarised target [Mul96]. Like the $\sin \phi_{S}$ moment (8.6.3), the moment $\left\langle\cos \phi_{S}\right\rangle_{\text {LT }}$ contains terms involving different combinations of subleading-twist DFs or FFs which partially vanish when integrated over transverse momenta. After integration one term remains in addition to the product of transversity and the twist-three FF $\tilde{E}$ :

$$
\left\langle\cos \phi_{S}\right\rangle_{\mathrm{LT}}(x, y, z)=-\left|\vec{S}_{T}\right| \frac{M}{Q} \frac{\frac{2 y \sqrt{1-y}}{x y^{2}} \sum_{q, \bar{q}} e_{q}^{2}\left(\frac{M_{h}}{M z} \delta q(x) \tilde{E}^{q}(z)+x g_{T}^{q}(x) D_{1}^{q}(z)\right)}{2 \frac{1}{x y^{2}} A(y) \sum_{q, \bar{q}} e_{q}^{2} q(x) D_{1}^{q}(z)} .
$$

This term contains the T-even twist-three DF $g_{T}$ which is not constrained to zero by timereversal symmetry arguments like the T-odd function $f_{T}$. In fact, $g_{T}$ is linked to the sum of the structure functions $g_{1}$ and $g_{2}$ (cf. Eq. (2.4.24) on page 21) which both have been shown to be non-zero at least for certain ranges in $x$ (cf. Figure 2.1.4 and [Ant03]). An isolation of this contribution of transversity to $\left\langle\cos \phi_{S}\right\rangle_{\mathrm{LT}}$ will therefore be more difficult than for $\left\langle\sin \phi_{S}\right\rangle_{\mathrm{UT}}$.

\subsubsection{Other Semi-Inclusive DIS Experiments}

\section{The Compass Experiments}

Like the HERMES experiment, the COMPASs experiment [Mal04] at CERN in Geneva, Switzerland, can measure azimuthal asymmetry amplitudes for semi-inclusive hadron production in DIS. Instead of positrons, muons with an energy of $160 \mathrm{GeV}$ are scattered off an alternating transversely and longitudinally polarised solid state target. The COMPASs collaboration probes the structure of the deuteron with a fixed solid state ${ }^{6}$ LiD target.

For positive and negative hadrons, the Sivers and Collins moments were found to be consistent with zero [Ale05]. This is not in contradiction with the significantly non-zero HERMES results, as was shown in a calculation of the asymmetry moments for the deuteron [Vog05, Ans05]. In this calculation the results for the DFs and FFs of the fits to the HERMES data shown in Figures 8.3.7 and 8.4.3 were used.

The high luminosity of the COMPASs experiment will allow a significant improvement in the present level of precision of the Sivers and Collins moments. Furthermore, future results for azimuthal asymmetries in two-hadron production and transversely polarised $\Lambda^{0}$ production are expected [Bra05].

\section{The ClAS Experiments}

The CLAS experiment in Hall B of the Jefferson Lab, Newport News, USA uses the 6 GeV electron beam provided by the CEBAF accelerator. The strength of the CLAS experi- 
ment is its very good geometric coverage. Single-spin asymmetries on a longitudinally polarised solid state proton $\left(\mathrm{NH}_{3}\right)$ target have been presented for neutral and charged pions [Ava05]. Measurements of Collins and Sivers as well as subleading-twist amplitudes with high statistics at larger $Q^{2}$ values will be possible with the proposed upgrade of the accelerator to $12 \mathrm{GeV}$ and the installation of a transversely polarised solid state target.

\subsubsection{Hadron-Hadron Scattering Experiments}

\section{The RHIC Spin Program}

RHIC is the Relativistic Heavy lon Collider at the Brookhaven National Laboratory in Upton, USA. RHIC cannot only be operated with stored heavy ion beams but also with proton beams. They collide with an average centre-of-mass energy up to $500 \mathrm{GeV}$. The successful acceleration of longitudinally or transversely polarised proton beams opened the possibility of spin related measurements at RHIC. Four experiments participate in the RHIC spin program: PHENIX, STAR, BRAHMS, and PP2PPii.

The first data, colliding a transversely polarised with an unpolarised proton beam, were taken in the years 2001 and 2002. Single-spin asymmetries in inclusive neutral-pion and charged-hadron production were measured at STAR and PHENIX [Aid04, Fat04]. Yet, the measured asymmetries do not allow the distinction between Sivers, Collins, and twistthree effects. In the year 2006, additional data with transversely polarised protons will be taken which will increase the precision of the existing results.

Transversity measurements using the Drell-Yan process (see Figure 2.6.1(b)) require transverse polarisation of both proton beams due to chirality conservation. They are sensitive to the product of a quark and an antiquark transversity DF, i.e., the product of a valence-quark and a sea-quark transversity function. For the proton, sea-quark transversity is expected to be small because the gluons which split up into the sea quarkantiquark pairs do not exhibit a transversity distribution (see Section 2.4.1). Hence, only small double-spin asymmetries for the Drell-Yan process are predicted in proton-proton scattering and a very high luminosity is therefore necessary for such a measurement.

More promising are the prospects of measuring the Sivers function in Drell-Yan as here the valence-quark Sivers function can be combined with the unpolarised sea-quark DF which increases for small $x$. Also other possibilities to access the Sivers function have been discussed recently [Boe04].

\section{The Pax Proposal}

The proposed PAx experiment plans to use an asymmetric proton-antiproton collider including the approved High Energy antiproton Storage Ring (HESR) at the GSI in Darmstadt, Germany, to access the transversity DF. In polarised proton-antiproton scattering, larger Drell-Yan asymmetries are predicted compared to proton-proton scattering since two valence-quark distributions enter the cross section. As in the RHIC spin program, also the Sivers function can be accessed in the PAx experiment. Provided the PAx proposal is accepted, the start of the data taking with polarised protons and antiprotons is forseen for the year 2015 [Bar05].

\footnotetext{
iiThe PP2PP experiment investigates elastic proton-proton scattering.
} 

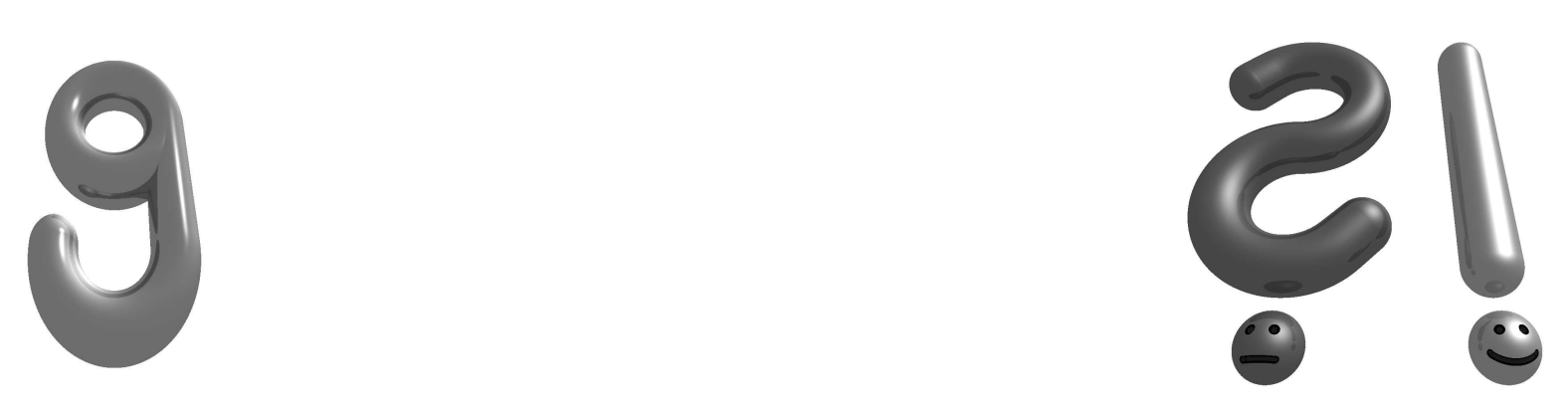

\section{Summary and Conclusion}

In the year 2001 a transversely polarised hydrogen target was installed in the HERMES experiment which allowed the individual measurements of azimuthal asymmetry amplitudes in semi-inclusive deep-inelastic scattering that contain the transversity and the Sivers distribution functions. For this thesis, the data taken with the transversely polarised target in the years 2002 through 2004 were analysed. Unweighted and $P_{h \perp}$-weighted Collins and Sivers amplitudes were extracted for charged and neutral pions and charged kaons by a two-dimensional fit of the azimuthal single-spin asymmetry. In studies systematic influences on these results from various experimental sources, like spectrometer acceptance and resolution, particle misidentification, knowledge of the beam and target polarisations, and the extraction method itself, were investigated. To enable these studies, Collins and Sivers amplitudes were implemented in an unpolarised Monte Carlo data sample by weighting events dependent on a randomly assigned target spin state. Furthermore, a new Monte Carlo generator, gmc_trans, was developed which simulates azimuthal distributions due to transverse-momentum dependent quark distribution and fragmentation functions. Unweighted asymmetry amplitudes implemented into this generator could be reproduced well after the simulated event reconstruction in the HERMES detector. For $P_{h \perp}$-weighted amplitudes, the incomplete integration over $P_{h \perp}$ within the HERMES acceptance strongly affects the reconstructed amplitudes.

Besides mesons from semi-inclusive DIS, the analysed data samples contain a contribution of decay mesons from exclusively produced vector mesons. It is currently still under debate whether or not this contribution has to be treated as a background. The fractions of mesons which stem from exclusive vector mesons were determined for the different hadron samples with the PYTHIA Monte Carlo generator which includes the exclusive production of vector mesons. The largest vector-meson decay fractions-up to $10 \%$ in the kinematic domain used in the analysis-are contained in the charged-pion samples. These fractions, which are dominated by exclusive $\rho^{0}$ decays, even increase to values about $50 \%$ for $z>0.7$, which is outside of the kinematic range regarded in this analysis. Neutral pions and charged kaons have contributions of less than $4 \%$ in the range $0.2<z<0.7$. The decay particles from exclusive vector mesons do not only dilute the extracted amplitudes but they can also exhibit Collins and Sivers type amplitudes. These amplitudes can either arise from an amplitude of the exclusively produced vector mesons which is transferred to the decay mesons or they can be acquired in the de- 
cay process. With the help of the PYTHIA and the DIPSI Monte Carlo generators, transfer coefficients for charged pions coming from exclusive $\rho^{0}$ decays and neutral pions from exclusive $\omega$ decays were determined. Moreover, the statistics of the HERMES data allowed the extraction of Collins and Sivers type amplitudes for exclusive $\rho^{0}$ mesons. The amplitudes were extracted for the kinematics of both the $\rho^{0}$ vector meson and its decay pions. In both cases the obtained amplitudes were small (of the order of a few percent) and statistically consistent with zero. For the estimate of the amplitudes acquired in the decay process, the decay angular distribution of $\rho^{0}$ mesons was investigated. It could be shown that the acquired Collins and Sivers amplitudes are also expected to be small in the semi-inclusive range. The same result is expected from the domination of natural parity exchange and the rather small violation of $s$-channel helicity conservation observed in HERMES data.

A method to suppress the exclusive $\rho^{0}$ and $\omega$ events based on the event kinematics was investigated but not implemented as the small reduction in the vector meson fractions was outweighed by a severe reduction of the event sample due to kinematic requirements. Furthermore, significantly positive amplitudes were extracted from the azimuthal asymmetry in the yield difference of positive and negative pions which has no contribution from exclusive vector mesons. This result proves that the Collins and Sivers amplitudes in the semi-inclusive pion samples are not predominantly caused by decay pions of exclusive vector mesons.

In the years 1996 and 1997, the HERMES experiment took data with a longitudinally polarised hydrogen target. As the spins of the nucleons were aligned with respect to the lepton beam momentum, a small target spin component transverse to the direction of the virtual photon resulted. Hence, besides a subleading-twist component of the cross section for longitudinal polarised nucleons the measured azimuthal asymmetries also contain the Collins and Sivers amplitudes. The extracted Collins and Sivers amplitudes from the measurement on the transversely polarised target were used to separate the subleading-twist amplitude from the measurement on a longitudinally polarised target. Significantly positive amplitudes for positive and neutral pions were found which show that, at the kinematics of the HERMES experiment, the interpretation of azimuthal asymmetry amplitudes solely in terms of leading-twist functions is not appropriate in all cases. The transversely polarised target, which was used for the measurement of the Collins and Sivers amplitudes, has in addition to the dominant transverse spin component also a small spin component longitudinal to the direction of the virtual photon. The subleading-twist contributions to the measured Collins and Sivers amplitudes evaluated for this small longitudinal component were found to be negligible, however.

Based on all the studies performed one can conclude that the significantly positive unweighted Sivers amplitudes for the positive pions and kaons and the neutral pions are the first signal of a non-zero T-odd distribution function in semi-inclusive DIS. Furthermore, the product of the T-odd Collins fragmentation function and the transversity distribution function causes significantly positive (negative) unweighted Collins amplitudes for positive (negative) pions.

The interpretation of the azimuthal asymmetry amplitudes in terms of distribution and fragmentation functions allows the derivation of a relation between the three pion amplitudes. Experimentally, this relation is violated by $2 \sigma$ for both Collins and Sivers amplitudes. Yet, these two amplitudes are strongly anti-correlated because of their simultaneous ex- 
traction and the sum of the two amplitudes satisfies the relation which suggests that the observed violation might arise from a statistical fluctuation. By Gaussian sampling of the overall unweighted Collins amplitudes of the three pion types, a likelihood distribution of the ratio of the disfavoured to favoured Collins functions can be determined as a function of the ratio of transversity for different quark flavours. In the most likely scenario, the disfavoured Collins function is of similar size as the favoured Collins function but has the opposite sign. The obtained most likely ratio of the disfavoured to favoured Collins functions will facilitate the extraction of the Collins function from a measurement of azimuthal asymmetries in the production of charged-pion pairs in electron-positron annihilation by the BELLE experiment. An explanation of the Collins effect based on the string-fragmentation model results in a positive sign of the Collins function for positive pions which is consistent with the measurement. A first extraction of the Sivers function from the measured unweighted Sivers amplitudes yielded a negative function for $u$ quarks and a positive function for $d$ quarks. In a semi-classical model this implies a positive orbital angular momentum for $u$ quarks and a negative orbital angular momentum for $d$ quarks.

The HERMES experiment continued data taking in the year 2005 and was able to more than double the statistics of the combined data of the years 2002 through 2004. In the future, an enhanced energy calibration of the calorimeter will improve its resolution which will result in an even more efficient neutral-pion identification and therefore a smaller uncertainty of the neutral-pion amplitudes. In addition, the higher statistics might allow the determination of the fraction of signal events in each azimuthal bin and spin state. A background subtraction can then be performed before forming the azimuthal asymmetry. Furthermore, a new amplitude extraction method with an unbinned maximum likelihood fit was proposed. This fit is less sensitive to acceptance effects and might enable the analysis of the $P_{h \perp}$-weighted amplitudes, which allows the interpretation in terms of distribution and fragmentation functions without further assumption on the transverse momentum distributions inside the nucleon. 


\section{Appendix A}

\section{Conventions}

The following metric tensor is used

$$
g^{\mu \nu}=\left(\begin{array}{cccc}
1 & 0 & 0 & 0 \\
0 & -1 & 0 & 0 \\
0 & 0 & -1 & 0 \\
0 & 0 & 0 & -1
\end{array}\right),
$$

where the Greek indices run over $0,1,2,3$. In contrast, Latin indices are used for 1, 2, 3. The normalisation of the Levi-Civita tensor is defined by:

$$
\epsilon^{0123}=1=-\epsilon_{0123} .
$$

The Einstein summation convention is applied unless noted otherwise.

\section{A. 1 Light-cone Vectors}

A four-vector with Cartesian contravariant components $a^{\mu}=\left(a^{0}, a^{i}\right)$ can be written as a Lightcone vector:

$$
\begin{aligned}
a^{\mu} & =\left[a^{-}, a^{+}, \vec{a}_{T}\right], \\
& =\left[\frac{a^{0}-a^{3}}{\sqrt{2}}, \frac{a^{0}+a^{3}}{\sqrt{2}}, a^{1}, a^{2}\right],
\end{aligned}
$$

where the \pm components are along the lightcone axes $x^{ \pm}$(see Figure A. 1). The scalar product of two light-cone vectors reads:

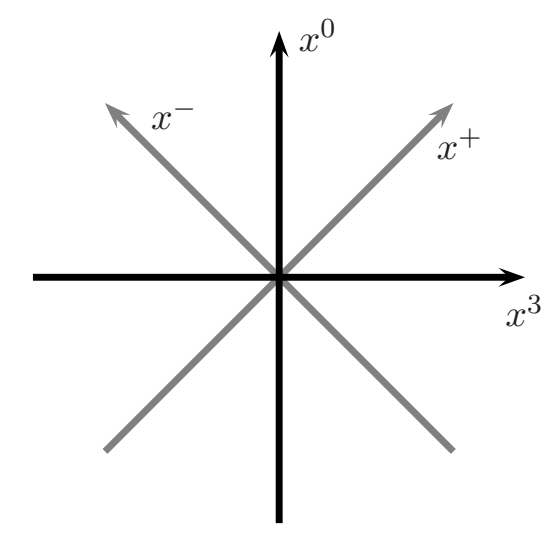

$$
\mathbf{a} \cdot \mathbf{b}=a^{+} b^{-}+a^{-} b^{+}-\vec{a}_{T} \cdot \vec{b}_{T} . \quad \text { (A.4) Figure A.1: The light-cone axes } x^{ \pm} .
$$

In the Bjorken limit ( $Q^{2} \rightarrow \infty, \nu \rightarrow \infty, x$ const.) the four-momentum vectors of the proton and the virtual photon can be parametrised in light-cone coordinates as:

$$
\begin{aligned}
P^{\mu} & =\left[\frac{M^{2}}{2 P^{+}}, P^{+}, \overrightarrow{0}\right] \\
q^{\mu} & =\left[\frac{Q^{2}}{2 x P^{+}},-x P^{+}, \overrightarrow{0}\right] .
\end{aligned}
$$


This parametrisation is valid in any collinear frame, i.e., in any frame of reference in which the virtual-photon direction is antiparallel to the $x^{3}$ axis. For all collinear frames $P^{+}$is the dominant variable in a $1 / Q$ expansion. In the infinite momentum frame the plus component of the nucleon is of the order of $Q$.

\section{A.2 Dirac Matrices}

The Dirac matrices $\gamma^{\mu}$ in the chiral representation are defined by means of the Pauli matrices $\sigma^{i}$ :

$$
\gamma^{0}=\left(\begin{array}{ll}
0 & \mathbb{1} \\
\mathbb{1} & 0
\end{array}\right), \quad \gamma^{i}=\left(\begin{array}{cc}
0 & -\sigma^{i} \\
\sigma^{i} & 0
\end{array}\right),
$$

where $\mathbb{1}$ is the $2 \times 2$ unit matrix. The product,

$$
\gamma_{5}=i \gamma^{0} \gamma^{1} \gamma^{2} \gamma^{3}=\left(\begin{array}{cc}
\mathbb{1} & 0 \\
0 & -\mathbb{1}
\end{array}\right)
$$

anticommutes with all four $\gamma$-matrices: $\gamma^{5} \gamma^{\mu}=-\gamma^{\mu} \gamma^{5}$. As for any other Lorentz vector, the Dirac matrices $\gamma^{\mu}$ can be expressed in the light-cone coordinates with $\gamma^{ \pm}=\left(\gamma^{0} \pm \gamma^{3}\right) / \sqrt{2}$. 


\section{Appendix B}

\section{The Luminosity Constants for the Year 2002}

The luminosity $\mathcal{L}$ is the ratio of the measured scattering rate $\mathcal{R}$ and the effective cross section $\sigma$ of a scattering process. For a fixed target experiment, it can be related to the beam current $I$ and the mean target area density $\bar{\rho}_{T}$

$$
\mathcal{L}=\frac{\mathcal{R}}{\sigma}=\frac{\bar{\rho}_{T} I}{e}
$$

with the elementary charge $e$. In the HERMES experiment the luminosity is measured by the luminosity monitor (cf. Section 3.3.3) using the electron positron scattering as reference scattering. With the knowledge of the effective cross section of this reference process $\sigma_{\text {ref }}$ the luminosity can be calculated from the luminosity monitor coincidence rate $\mathcal{R}_{\text {LUMI }}$ using the following relation:

$$
\mathcal{L}=\frac{\mathcal{R}_{\mathrm{LUMI}}}{\sigma_{\text {ref }}}=C_{\mathrm{LUMI}} \cdot \mathcal{R}_{\mathrm{LUMI}}
$$

introducing the luminosity constant $C_{\mathrm{LUMI}}$. Integrated luminosities are obtained by integrating the luminosity monitor rate over the relevant time period. The effective cross section and hence the luminosity constant depends on the beam settings and the alignment of the luminosity monitor [Els01]. Therefore the 'constant' can vary within the different periods of data taking. Values of $C_{\mathrm{LUMI}}$ for the different periods until the year 2000 are given in [ElsO2] and [HDQa]. Furthermore, the different numbers of nucleons per number of shell electrons for different target gases have to be taken into account when relating the measured coincidence rates at the luminosity monitor to the luminosity.

Due to the installation of the transverse target magnet before the start of the data taking in 2002, the alignment of the luminosity monitor with respect to the target cell has changed. Furthermore, also the transverse target field influences the effective cross section of the reference scattering. For the polarised data of the year 2002 a measurement of the area density by the target group of $\bar{\rho}_{T}=1.1 \cdot 10^{14} \frac{\text { nucleons }}{\mathrm{cm}^{2}}[$ AirO4] could be used to calculate the luminosity constant:

$$
C_{\mathrm{LUMI}}=\frac{I \bar{\rho}_{T}}{\mathcal{R}_{\mathrm{LUMI}} e}
$$

The mean ratio $\frac{\mathcal{R}_{\mathrm{LUMI}}}{I}=0.630$ could be extracted from the data and yields a luminosity constant $C_{\text {LUMI }}=1090 / \mathrm{mb}$. The relative uncertainty of this quantity is around $10 \%$, 
dominated by the measurement of the area density. It is larger than the $6 \%$ systematic uncertainty of the luminosity constants determined with help of a Monte Carlo simulation [ElsO1].

During the unpolarised data taking the luminosity measurement is not influenced by the transverse target field and therefore the luminosity constant differs. A different approach was used to determine the constant $C_{\mathrm{LUMI}}^{2002 \mathrm{unpol}}$ of these periods. For all different data takings in the years before 2002 the ratios of the number of DIS events and the integrated luminosity were compared to the ratio of DIS events and the integrated luminosity monitor rate for the unpolarised data taking periods in the year 2002. From this comparison a luminosity constant $C_{\mathrm{LUMI}}^{2002 \mathrm{unpol}}=(538 \pm 48) / \mathrm{mb}$ was obtained. 


\section{Appendix C}

\section{Angular Decay Distribution for Exclusive $\rho^{0}$ Production}

\section{C.1 The Decay Angles}

The decay angles are defined in the centre-of-mass system of the $\rho^{0}$ vector meson as azimuthal and polar angle of the decay $\pi^{+}$(see Figure 6.2.7). In order to express the decay angles in terms of azimuthal and polar angles in the nucleon rest-mass system, the momentum vectors have to be transformed from the nucleon to the $\rho^{0}$ rest system. In the nucleon rest-mass system with the virtual-photon momentum as $z$-axis, the $\rho^{0}$ vector meson is produced with the angles $\theta_{\rho^{0}}$ and $\phi_{\rho^{0}}$, the momentum $P_{\rho^{0}}$, and the energy $E_{\rho^{0}}$. The positive pion from the $\rho^{0}$ decay is emitted with the angles $\theta_{\pi^{+}}$and $\phi_{\pi^{+}}$, the momentum $P_{\pi^{+}}$, and the energy $E_{\pi^{+}}$. By rotation about the $z$-axis one obtains a system where the vector meson is produced in the $z-x$ plane and the $\pi^{+}$momentum threevector reads:

$$
\vec{P}_{\pi^{+}}=P_{\pi^{+}}\left(\begin{array}{c}
\sin \theta_{\pi^{+}} \cos \left(\phi_{\pi^{+}}-\phi_{\rho^{0}}\right) \\
\sin \theta_{\pi^{+}} \sin \left(\phi_{\pi^{+}}-\phi_{\rho^{0}}\right) \\
\cos \theta_{\pi^{+}}
\end{array}\right)
$$

The vector meson momentum can be chosen colinear with the $z$-direction by an additional rotation about the $y$-axis:

$$
\begin{aligned}
\vec{P}_{\pi^{+}}^{\prime} & =\left(\begin{array}{ccc}
\cos \theta_{\rho^{0}} & 0 & -\sin \theta_{\rho^{0}} \\
0 & 1 & 0 \\
\sin \theta_{\rho^{0}} & 0 & \cos \theta_{\rho^{0}}
\end{array}\right) \vec{P}_{\pi^{+}} \\
& =P_{\pi^{+}}\left(\begin{array}{c}
\cos \theta_{\rho^{0}} \sin \theta_{\pi^{+}} \cos \left(\phi_{\pi^{+}}-\phi_{\rho^{0}}\right)-\sin \theta_{\rho^{0}} \cos \theta_{\pi^{+}} \\
\sin \theta_{\pi^{+}} \sin \left(\phi_{\pi^{+}}-\phi_{\rho^{0}}\right) \\
\sin \theta_{\rho^{0}} \sin \theta_{\pi^{+}} \cos \left(\phi_{\pi^{+}}-\phi_{\rho^{0}}\right)+\cos \theta_{\rho^{0}} \cos \theta_{\pi^{+}}
\end{array}\right) .
\end{aligned}
$$


For the transfer into the $\rho^{0}$ rest frame, this vector must be boosted along the $z$-direction:

$$
\begin{gathered}
\left(\begin{array}{cccc}
\gamma & 0 & 0 & -\gamma \beta \\
0 & 1 & 0 & 0 \\
0 & 0 & 1 & 0 \\
-\gamma \beta & 0 & 0 & \gamma
\end{array}\right)\left(\begin{array}{c}
E_{\pi^{+}} \\
\vec{P}_{\pi^{+}}^{\prime}
\end{array}\right)= \\
P_{\pi^{+}}\left(\begin{array}{c}
\frac{\gamma E_{\pi^{+}}}{P_{\pi^{+}}}-\gamma \beta \sin \theta_{\rho^{0}} \sin \theta_{\pi^{+}} \cos \left(\phi_{\pi^{+}}-\phi_{\rho^{0}}\right)-\gamma \beta \cos \theta_{\rho^{0}} \cos \theta_{\pi^{+}} \\
\cos \theta_{\rho^{0}} \sin \theta_{\pi^{+}} \cos \left(\phi_{\pi^{+}}-\phi_{\rho^{0}}\right)-\sin \theta_{\rho^{0}} \cos \theta_{\pi^{+}} \\
\sin \theta_{\pi^{+}} \sin \left(\phi_{\pi^{+}}-\phi_{\rho^{0}}\right) \\
-\frac{\gamma \beta E_{\pi^{+}}}{P_{\pi^{+}}}+\gamma \sin \theta_{\rho^{0}} \sin \theta_{\pi^{+}} \cos \left(\phi_{\pi^{+}}-\phi_{\rho^{0}}\right)+\gamma \cos \theta_{\rho^{0}} \cos \theta_{\pi^{+}}
\end{array}\right) .
\end{gathered}
$$

Here, $\gamma=\sqrt{1-\beta^{2}}$ with $\beta=\frac{P_{\rho^{0}}}{M_{\rho^{0}}}$ and $M_{\rho^{0}}$ is the rest mass of the $\rho^{0}$ meson. The $x$ - and $y$-components of the pion momentum are unaffected by the Lorentz boost. They yield the cosine and sine of the azimuthal decay angle:

$$
\begin{aligned}
\sin \phi^{*} & \approx \frac{\sin \theta_{\pi^{+}} \sin \left(\phi_{\pi^{+}}-\phi_{\rho^{0}}\right)}{\sqrt{1-\cos ^{2} \theta_{\rho^{0}} \cos ^{2} \theta_{\pi^{+}}}}, \\
\cos \phi^{*} & \approx \frac{\cos \theta_{\rho^{0}} \sin \theta_{\pi^{+}} \cos \left(\phi_{\pi^{+}}-\phi_{\rho^{0}}\right)-\sin \theta_{\rho^{0}} \cos \theta_{\pi^{+}}}{\sqrt{1-\cos ^{2} \theta_{\rho^{0}} \cos ^{2} \theta_{\pi^{+}}}},
\end{aligned}
$$

where the approximation of small polar angles $\theta_{\rho^{0}}, \theta_{\pi^{+}} \ll \frac{\pi}{2}$ is made.

\section{C.2 The Terms of the Angular Decay Distribution}

The different terms of the angular decay distribution of exclusively produced $\rho^{0}$ vector mesons on a transversely polarised nucleon target with an unpolarised lepton beam depend on the two nucleon polarisation parameters $\zeta_{1,2}$ defined in Eq. (6.2.7), on the decay angles $\phi^{*}$ and $\theta^{*}$, on the azimuthal production angle $\phi_{\rho^{0}}$ of the $\rho^{0}$, and on the helicity amplitudes $f_{j \alpha}^{j^{\prime} \alpha^{\prime}}$. Here, the virtual-photon helicity is $\alpha$, the $\rho^{0}$ helicity is $\alpha^{\prime}$, and the initial and final nucleon helicities are $j$ and $j^{\prime}$. The helicity amplitudes can be split up into two terms [Fra74]:

$$
f_{j \alpha}^{j^{\prime} \alpha^{\prime}}=n_{j \alpha}^{j^{\prime} \alpha^{\prime}}+u_{j \alpha}^{j^{\prime} \alpha^{\prime}}
$$

which correspond to the exchange of natural and unnatural parities in the $t$-channel for high energies. In case of the $\rho^{0}$ vector meson, the natural parity exchange helicity amplitude $n_{j \alpha}^{j^{\prime} \alpha^{\prime}}$ can be further split up into the contribution $d_{\alpha}$ conserving $s$-channel helicity and amplitudes $a_{j \alpha}^{j^{\prime} \alpha^{\prime}}$, which violate helicity conservation,

$$
n_{j \alpha}^{j^{\prime} \alpha^{\prime}}=d_{\alpha} \cdot \delta_{\alpha^{\prime} \alpha} \delta_{j^{\prime}, j}+a_{j \alpha}^{j^{\prime} \alpha^{\prime}} .
$$

At HERMES, the violation of $s$-channel helicity conservation is found to be small [Tyt01] . The amplitudes $\left|a_{j \alpha}^{j^{\prime} \alpha^{\prime}}\right|$ are therefore expected to be much smaller than the terms $\left|d_{\alpha}\right|$. In the domain of small momentum transfer, the unnatural parity exchange amplitudes can be identified with the amplitudes of one-pion exchange:

$$
u_{j \alpha}^{j^{\prime} \alpha^{\prime}}=p_{j \alpha}^{j^{\prime} \alpha^{\prime}} .
$$




\begin{tabular}{lll|l}
\multicolumn{2}{c|}{ angular modulation } & \multicolumn{1}{c}{ amplitude $\cdot 4 / 3 \pi B \operatorname{Tr} \rho_{\rho^{0}}$} \\
\hline$\zeta_{1}$ & $\sin ^{2} \theta^{*}$ & $\left(\sin 2 \phi^{*}-\epsilon \sin 2 \phi_{\rho^{0}}\right)$ & $A_{1}=-2 \operatorname{Im}\left(d_{+} p_{--}^{++*}\right)$ \\
$\zeta_{1}$ & $\sin ^{2} \theta^{*}$ & $\sin \phi^{*} \sin \left(\phi_{\rho^{0}}+\phi^{*}\right)$ & $A_{2}=-4 \sqrt{\epsilon(1+\epsilon)} \operatorname{Im}\left(d_{+} p_{-0}^{++*}\right)$ \\
$\zeta_{1}$ & $\sin ^{2} \theta^{*}$ & $\sin 2\left(\phi_{\rho^{0}}+\phi^{*}\right)$ & $A_{3}=2 \epsilon \operatorname{Im}\left(d_{+} p_{-+}^{++*}\right)$ \\
$\zeta_{1}$ & $\cos ^{2} \theta^{*}$ & $\sin \phi_{\rho^{0}}$ & $A_{4}=-4 \sqrt{\epsilon(1+\epsilon)} \operatorname{Im}\left(d_{0} p_{-+}^{+0 *}\right)$ \\
$\zeta_{1}$ & $\sin 2 \theta^{*} / \sqrt{2}$ & $\sin \phi^{*}$ & $A_{5}=2 \operatorname{Im}\left(d_{+} p_{-+}^{+0 *}-2 \epsilon d_{0} p_{-0}^{++*}\right)$ \\
$\zeta_{1}$ & $\sin 2 \theta^{*} / \sqrt{2}$ & $\sin \left(\phi_{\rho^{0}}+\phi^{*}\right)$ & $A_{6}=2 \sqrt{\epsilon(1+\epsilon)} \operatorname{Im}\left(d_{0} p_{-+}^{++*}\right)$ \\
$\zeta_{1}$ & $\sin 2 \theta^{*} / \sqrt{2}$ & $\sin \left(2 \phi_{\rho^{0}}+\phi^{*}\right)$ & $A_{7}=-2 \epsilon \operatorname{Im}\left(d_{+} p_{-+}^{+0 *}\right)$ \\
$\zeta_{2}$ & $\sin ^{2} \theta^{*}$ & $1+\epsilon \sin 2\left(\phi_{\rho^{0}}+\phi^{*}\right)$ & $A_{8}=2 \operatorname{Im}\left(d_{+} a_{-+}^{++*}\right)$ \\
$\zeta_{2}$ & $\sin ^{2} \theta^{*}$ & $\cos 2 \phi^{*}+\epsilon \cos 2 \phi_{\rho^{0}}$ & $A_{9}=-2 \operatorname{Im}\left(d_{+} a_{--}^{++*}\right)$ \\
$\zeta_{2}$ & $\sin ^{2} \theta^{*}$ & $\cos \phi^{*} \cos \left(\phi^{*}-\phi_{\rho^{0}}\right)$ & $A_{10}=-4 \sqrt{\epsilon(1+\epsilon)} \operatorname{Im}\left(d_{+} a_{-0}^{++*}\right)$ \\
$\zeta_{2}$ & $\cos ^{2} \theta^{*}$ & & $A_{11}=4 \operatorname{Im}\left(d_{+} a_{-0}^{+0 *}\right)$ \\
$\zeta_{2}$ & $\cos ^{2} \theta^{*}$ & $\cos \phi_{\rho^{0}}$ & $A_{12}=-4 \sqrt{\epsilon(1+\epsilon)} \operatorname{Im}\left(d_{0} a_{-+}^{+0 *}\right)$ \\
$\zeta_{2}$ & $\sin 2 \theta^{*} / \sqrt{2}$ & $\cos \phi^{*}$ & $A_{13}=2 \operatorname{Im}\left(d_{+} a_{-+}^{+0 *}+2 \epsilon d_{0} a_{-0}^{++*}\right)$ \\
$\zeta_{2}$ & $\sin 2 \theta^{*} / \sqrt{2}$ & $\cos \left(\phi_{\rho^{0}}+\phi^{*}\right)$ & $A_{14}=2 \sqrt{\epsilon(1+\epsilon)} \operatorname{Im}\left(d_{0} a_{--}^{++*}\right)$ \\
$\zeta_{2}$ & $\sin 2 \theta^{*} / \sqrt{2}$ & $\cos \left(2 \phi_{\rho^{0}}+\phi^{*}\right)$ & $A_{15}=2 \epsilon \operatorname{Im}\left(d_{+} a_{-+}^{+0 *}\right)$ \\
$\zeta_{2}$ & $\sin ^{*+*} \theta^{*} / \sqrt{2}$ & $\cos \left(\phi^{*}-\phi_{\rho^{0}}\right)$ & $A_{16}=-2 \sqrt{\epsilon(1+\epsilon)} \operatorname{Im}\left(d_{+} a_{-0}^{+0 *}+d_{0} a_{-+}^{++*}\right)$
\end{tabular}

Table C.1: The decay angular distribution $W_{\mathrm{UT}}\left(\cos \theta^{*}, \phi^{*} ; \zeta_{1}, \zeta_{2}\right)$ of exclusively produced $\rho^{0}$ mesons on a transversely polarised target. Table taken from [Fra74].

The 16 terms of the decay angular distribution for an unpolarised beam and a transversely polarised target $W_{\mathrm{UT}}\left(\cos \theta^{*}, \phi^{*} ; \zeta_{1}, \zeta_{2}\right)$ are listed in Table C. 1 . In the left column the angular modulations and in the right column the amplitudes are given. They depend on the ratio of the longitudinal to transverse photon flux $\epsilon=(1-y) /\left(1-y+y^{2} / 2\right)$ and on the parameter $B=m_{e}^{2} Q^{2}(1-\epsilon) /(4 \pi \alpha)$ with the electron mass $m_{e}$. The amplitudes are normalised by the trace of the $\rho^{0}$ spin density matrix $\rho_{\rho^{0}}$. Natural parity exchange dominates the exclusive $\rho^{0}$ production at HERMES [Tyt01], resulting in small amplitudes $p_{j \alpha}^{j^{\prime} \alpha^{\prime}}$. All 16 amplitudes vanish under the assumption of $s$-channel helicity conservation and natural parity exchange. 


\section{Appendix D}

\section{Asymmetry Estimate Using Positivity Limits}

An unknown asymmetry amplitude can be estimated by its standard deviation from zero, assuming the amplitude is distributed uniformly between -1 and 1. These limits ensure a positive cross section, i.e., that the polarised cross section does not exceed the unpolarised cross section. The squared standard deviation of an amplitude $A$ is defined as:

$$
\sigma^{2}=\int_{-1}^{1} A^{2} f(A) \mathrm{d} A
$$

where $f(A)$ is the distribution function of $A$ which for a uniform distribution is constant, $f(A)=1 / l$, with the integration length $l=2$.

Figure D. 1 shows the uniform distribution of two

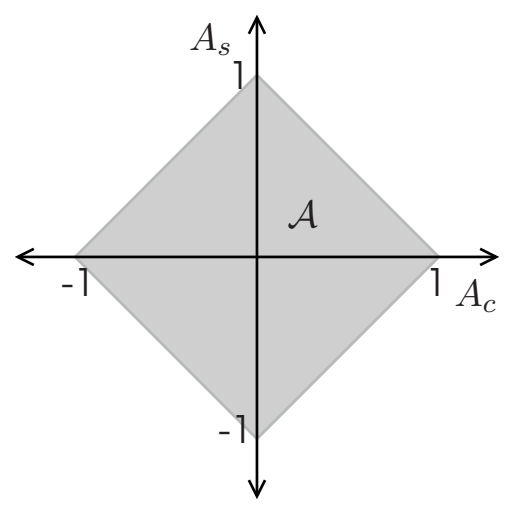

Figure D.1: Asymmetry distribution in the two dimensional case. asymmetry amplitudes in a diamond shape which are subject to the positivity limit:

$$
\left|A_{c} \pm A_{s}\right| \leq 1
$$

For each amplitude, the standard deviation can be calculated performing a two dimensional integration of the squared amplitude, weighted with the distribution function:

$$
\sigma_{i}^{2}=\int_{-\infty}^{\infty} \int_{-\infty}^{\infty} A_{i}^{2} f\left(A_{c}, A_{s}\right) \mathrm{d} A_{c} \mathrm{~d} A_{s}
$$

with $i=c, s$. Here, the two dimensional distribution function $f\left(A_{c}, A_{s}\right)$ is the reciprocal of the diamond shaped area $\mathcal{A}=2$ for values of $A_{c}$ and $A_{s}$ inside the diamond and zero otherwise:

$$
f\left(A_{c}, A_{s}\right)=\left\{\begin{array}{cl}
1 / \mathcal{A} & ,\left|A_{c}+A_{s}\right| \leq 1 \\
0 & , \text { else }
\end{array} .\right.
$$

Splitting the integration over the active area into the upper triangle for positive amplitudes $A_{s}$ and the lower triangle for negative $A_{s}$, the standard deviations for the two am- 
plitudes are obtained by:

$$
\sigma_{i}^{2}=\frac{1}{2} \int_{0}^{1} \int_{-1+A_{c}}^{1-A_{c}} A_{i}^{2} \mathrm{~d} A_{c} \mathrm{~d} A_{s}+\frac{1}{2} \int_{-1}^{0} \int_{-1-A_{c}}^{1+A_{c}} A_{i}^{2} \mathrm{~d} A_{c} \mathrm{~d} A_{s}=\frac{1}{6} .
$$

Both amplitudes have the same standard deviations of $\sigma=1 / \sqrt{6}$ which are smaller than the standard deviation in the one dimensional case of $\sigma=1 / \sqrt{3}$. 


\section{Appendix E}

\section{Additional Figures}

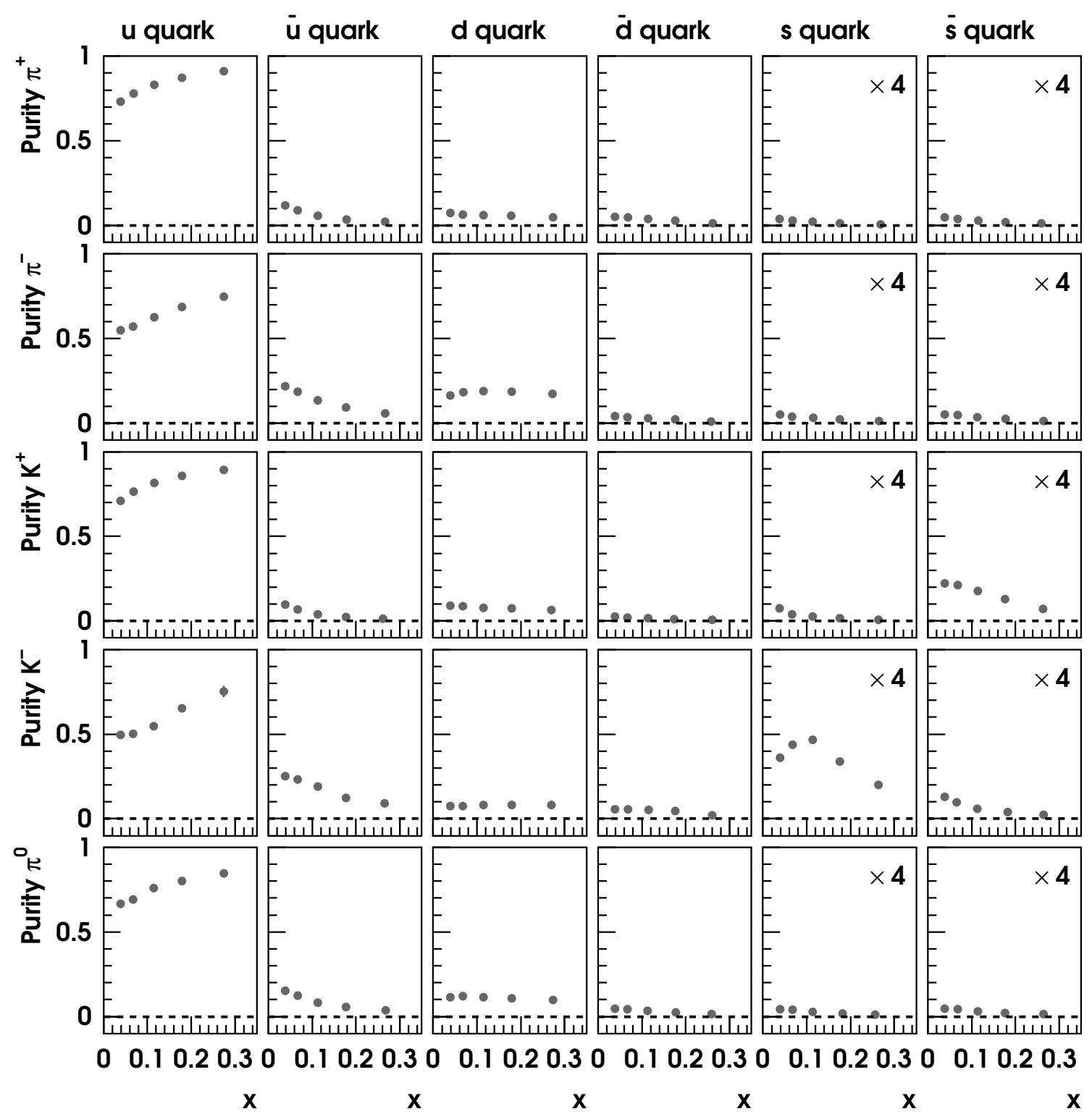

Figure E. 1: Purities for pions and kaons and the three lightest quarks and antiquarks. The purities of the $s$ and $\bar{s}$ quarks are scaled for better visibility. 


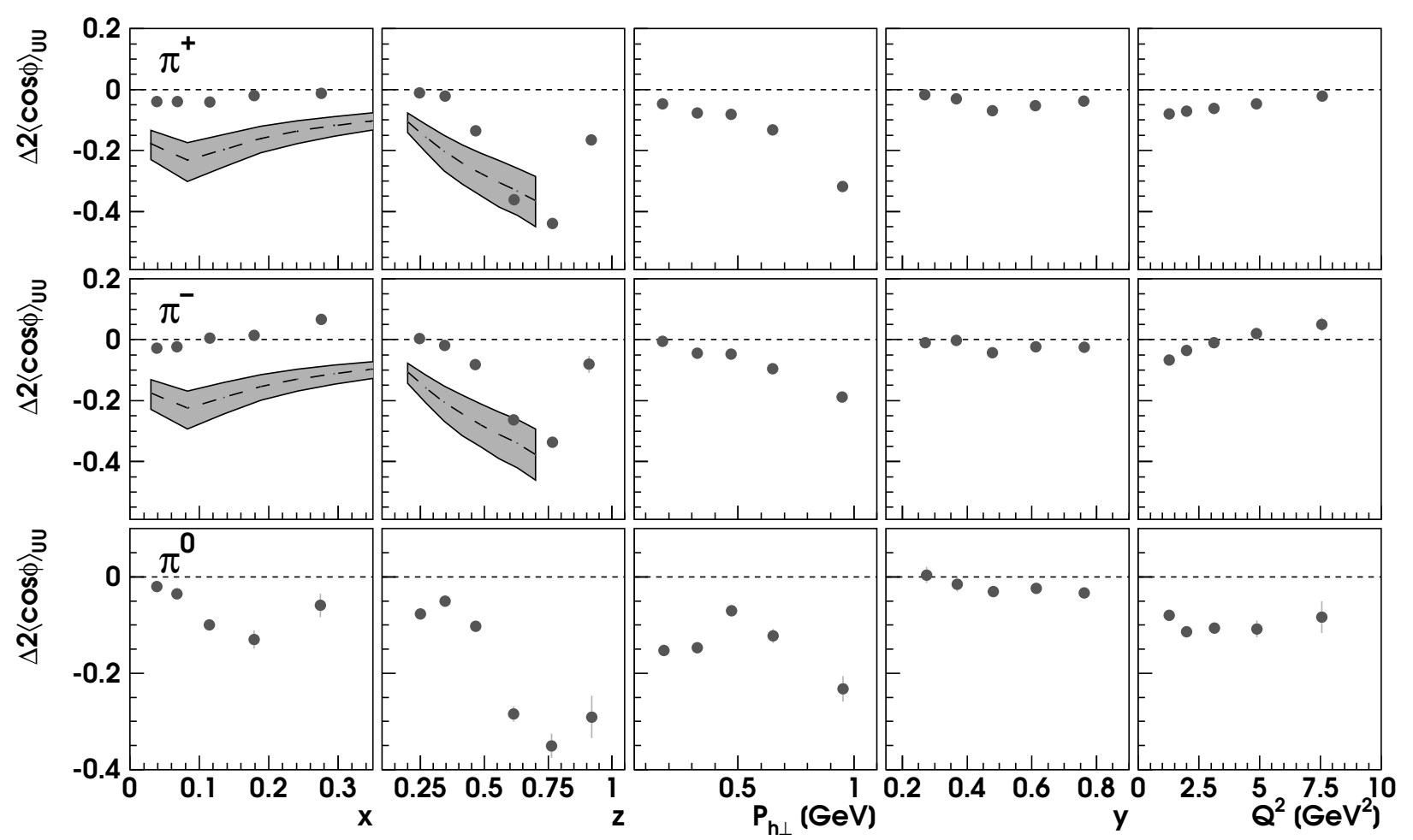

Figure E.2: Difference of the cosine moments extracted from data and a PYTHIA Monte Carlo simulation $2\langle\cos \phi\rangle_{\text {meas }}-2\langle\cos \phi\rangle_{\mathrm{MC}}$ for all three pion types. In addition a calculation within the QCD parton model is shown for the $x$ and $z$ dependence of the charged pions [Ans05, Pro05].

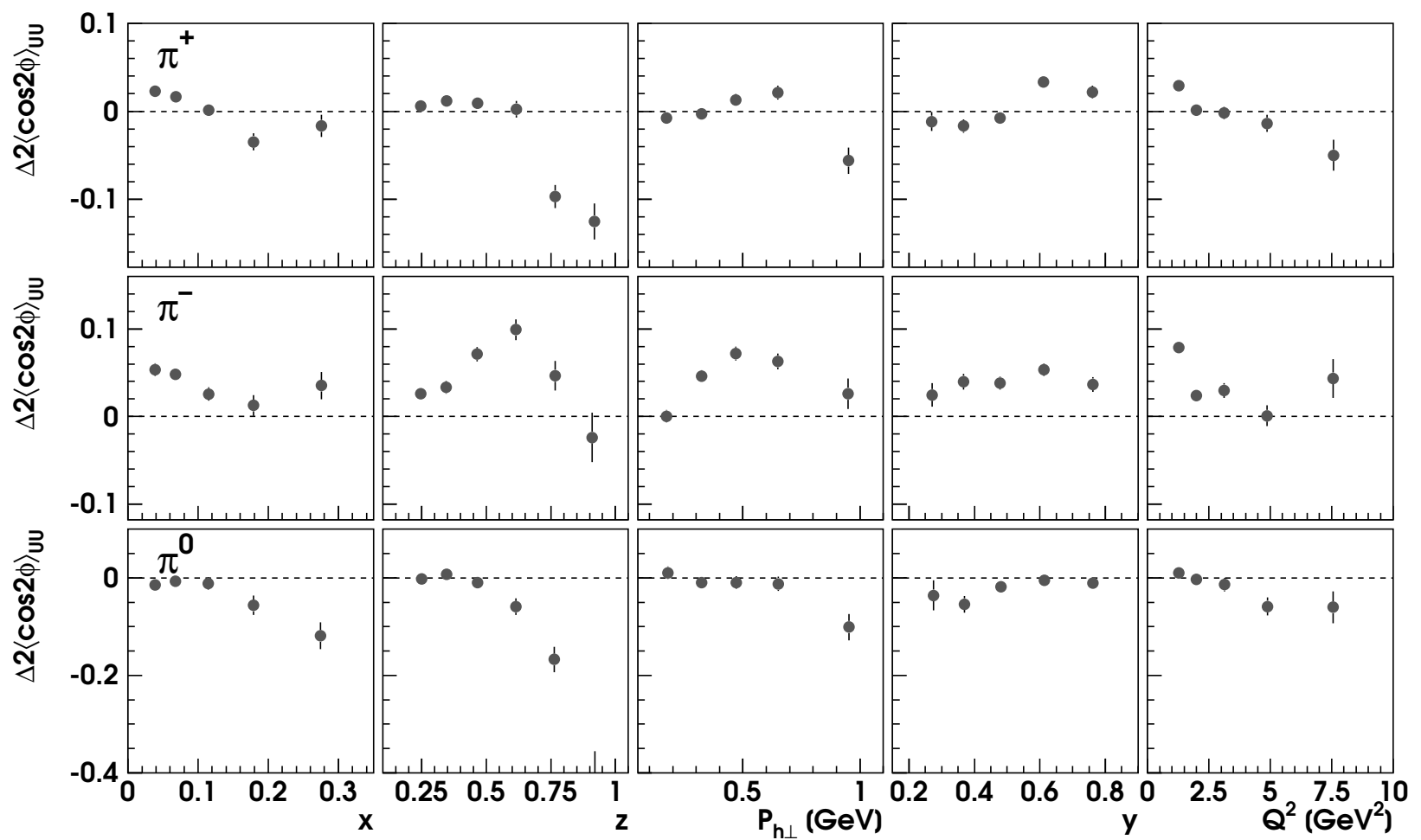

Figure E.3: Difference of the cosine moments extracted from data and a PYTHIA Monte Carlo simulation $2\langle\cos 2 \phi\rangle_{\text {meas }}-2\langle\cos 2 \phi\rangle_{\mathrm{MC}}$ for all three pion types. 


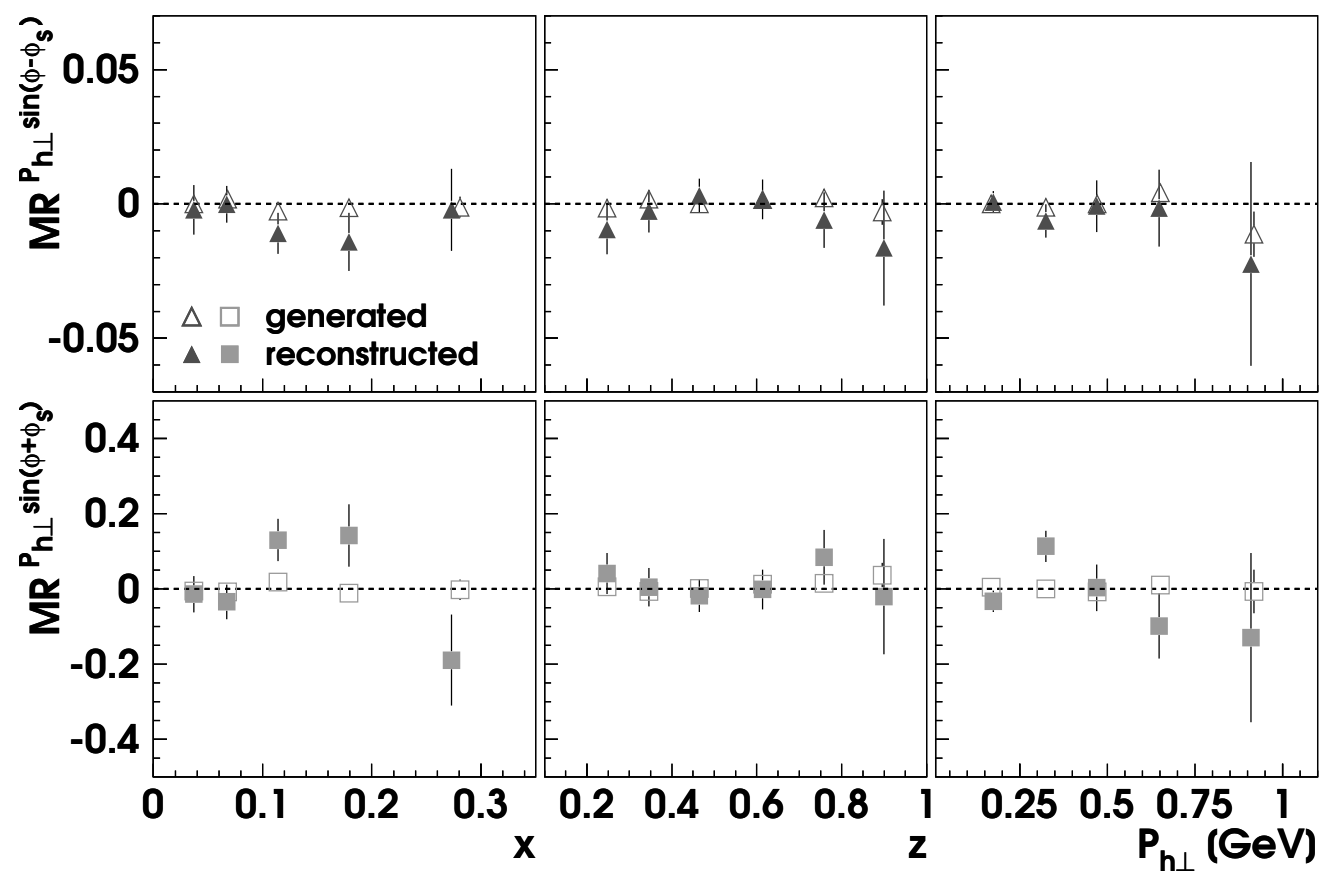

Figure E.4: Makins Relation for Sivers and Collins $P_{h \perp}$-weighted amplitudes of the gmc_trans Monte Carlo production as functions of $x, z$, and $P_{h \perp}$.

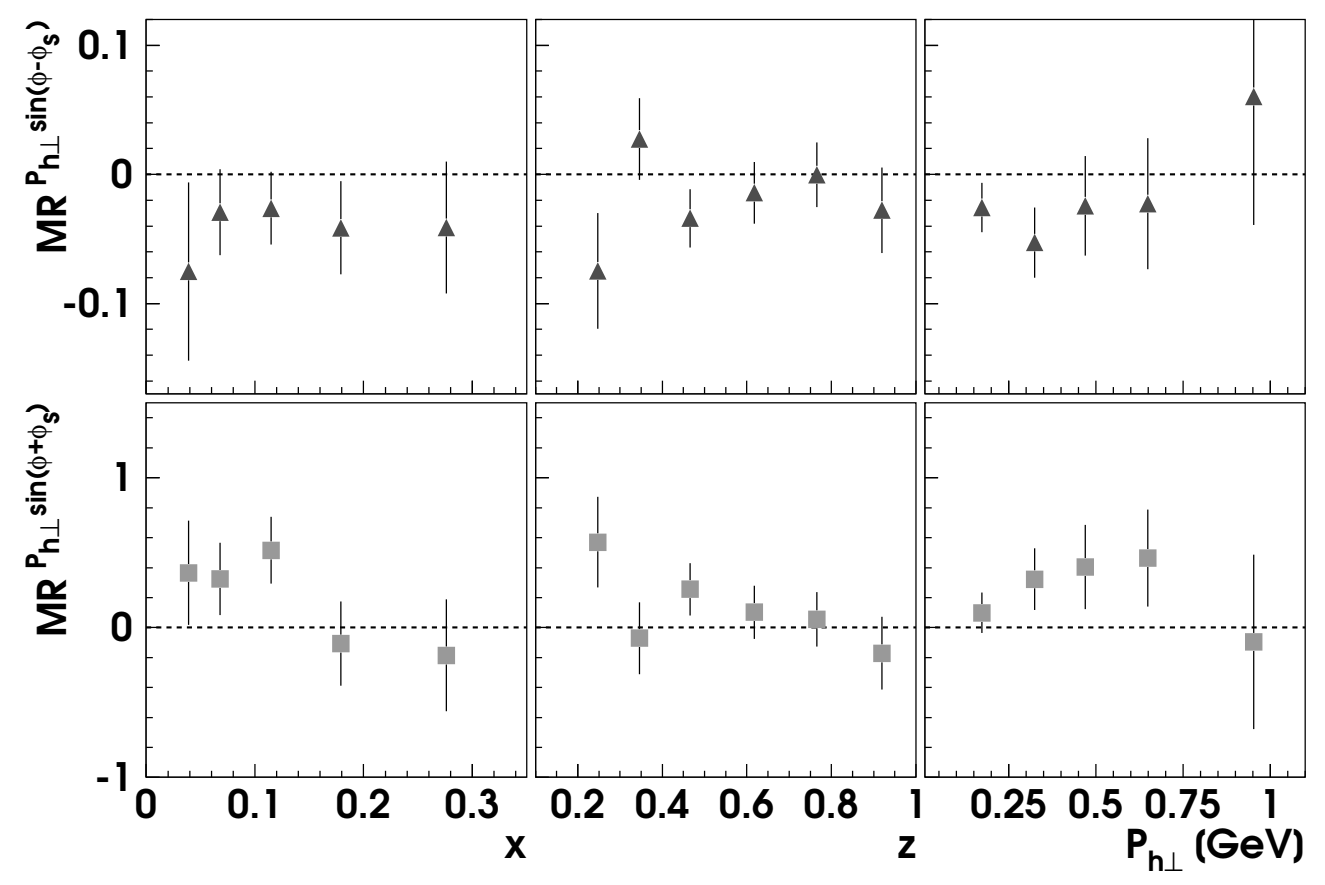

Figure E.5: Makins Relation for Sivers and Collins $P_{h \perp}$-weighted amplitudes of the three HERMES data productions as functions of $x, z$, and $P_{h \perp}$. 


\section{Appendix F}

\section{Additional Tables}

Combinatorial background fit parameters, transfer values and azimuthal asymmetry moments are listed in the following tables. In Tables F.5 - F.7 (F.8 - F.12), the $4.2 \%$ (6.6\%) scale uncertainty from the target polarisation measurement is not included in the systematic uncertainty.

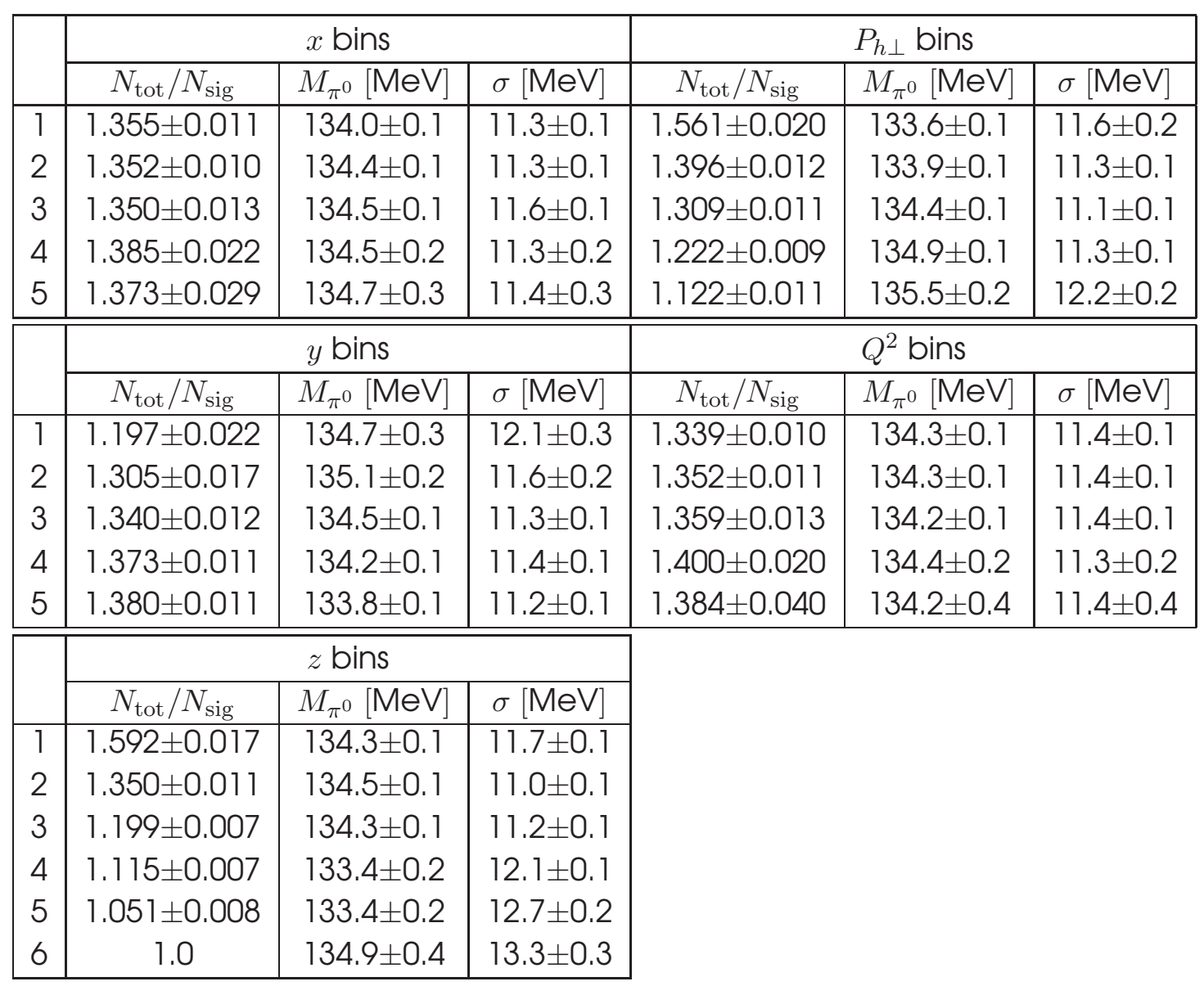

Table F.1: Parameters of a fit with the free parameters $M_{\pi^{0}}$ and $\sigma$ to the invariant mass spectra of photon pairs, including combinatorial background. In the last $z$ bin, only a Gaussian is fitted to the distribution. 


\begin{tabular}{|c|c|c|c|c|}
\hline \multicolumn{5}{|c|}{$z$ bins } \\
\hline & $T_{c c}$ & $T_{c s}$ & $T_{s s}$ & $T_{s c}$ \\
\hline 1 & $0.264 \pm 0.006$ & $-0.120 \pm 0.006$ & $0.195 \pm 0.006$ & $-0.123 \pm 0.006$ \\
\hline 2 & $0.289 \pm 0.007$ & $-0.130 \pm 0.007$ & $0.265 \pm 0.007$ & $-0.108 \pm 0.007$ \\
\hline 3 & $0.457 \pm 0.006$ & $-0.130 \pm 0.006$ & $0.437 \pm 0.006$ & $-0.118 \pm 0.006$ \\
\hline 4 & $0.525 \pm 0.005$ & $-0.018 \pm 0.006$ & $0.501 \pm 0.005$ & $-0.021 \pm 0.005$ \\
\hline 5 & $0.675 \pm 0.004$ & $-0.032 \pm 0.005$ & $0.683 \pm 0.004$ & $-0.019 \pm 0.005$ \\
\hline 6 & $0.841 \pm 0.007$ & $-0.017 \pm 0.007$ & $0.854 \pm 0.006$ & $-0.001 \pm 0.007$ \\
\hline \multicolumn{5}{|c|}{$x$ bins } \\
\hline & $T_{c c}$ & $T_{c s}$ & $T_{s s}$ & $T_{s c}$ \\
\hline 1 & $0.506 \pm 0.005$ & $-0.080 \pm 0.005$ & $0.460 \pm 0.006$ & $-0.117 \pm 0.005$ \\
\hline 2 & $0.364 \pm 0.005$ & $-0.126 \pm 0.005$ & $0.372 \pm 0.005$ & $-0.083 \pm 0.005$ \\
\hline 3 & $0.326 \pm 0.008$ & $-0.084 \pm 0.008$ & $0.318 \pm 0.007$ & $-0.065 \pm 0.007$ \\
\hline 4 & $0.361 \pm 0.013$ & $0.064 \pm 0.012$ & $0.350 \pm 0.011$ & $-0.025 \pm 0.012$ \\
\hline 5 & $0.556 \pm 0.019$ & $0.065 \pm 0.021$ & $0.588 \pm 0.018$ & $-0.100 \pm 0.019$ \\
\hline \multicolumn{5}{|c|}{$P_{h \perp}$ bins } \\
\hline & $T_{c c}$ & $T_{c s}$ & $T_{s s}$ & $T_{s c}$ \\
\hline 1 & $0.285 \pm 0.006$ & $-0.103 \pm 0.006$ & $0.224 \pm 0.006$ & $-0.120 \pm 0.006$ \\
\hline 2 & $0.350 \pm 0.005$ & $-0.101 \pm 0.006$ & $0.293 \pm 0.005$ & $-0.091 \pm 0.005$ \\
\hline 3 & $0.610 \pm 0.006$ & $-0.043 \pm 0.007$ & $0.550 \pm 0.006$ & $-0.066 \pm 0.006$ \\
\hline 4 & $0.626 \pm 0.008$ & $-0.079 \pm 0.009$ & $0.679 \pm 0.008$ & $-0.037 \pm 0.009$ \\
\hline 5 & $0.941 \pm 0.012$ & $0.005 \pm 0.016$ & $0.864 \pm 0.015$ & $-0.103 \pm 0.015$ \\
\hline \multicolumn{5}{|c|}{$y$ bins } \\
\hline & $T_{c c}$ & $T_{c s}$ & $T_{s s}$ & $T_{s c}$ \\
\hline 1 & $0.166 \pm 0.014$ & $-0.029 \pm 0.014$ & $0.284 \pm 0.013$ & $0.071 \pm 0.013$ \\
\hline 2 & $0.223 \pm 0.009$ & $-0.009 \pm 0.009$ & $0.225 \pm 0.008$ & $0.010 \pm 0.008$ \\
\hline 3 & $0.307 \pm 0.007$ & $-0.034 \pm 0.007$ & $0.302 \pm 0.006$ & $-0.033 \pm 0.006$ \\
\hline 4 & $0.439 \pm 0.005$ & $-0.090 \pm 0.006$ & $0.442 \pm 0.006$ & $-0.081 \pm 0.006$ \\
\hline 5 & $0.536 \pm 0.006$ & $-0.099 \pm 0.006$ & $0.566 \pm 0.007$ & $-0.068 \pm 0.008$ \\
\hline \multicolumn{5}{|c|}{$Q^{2}$ bins } \\
\hline & $T_{c c}$ & $T_{c s}$ & $T_{s s}$ & $T_{s c}$ \\
\hline 1 & $0.441 \pm 0.004$ & $-0.070 \pm 0.005$ & $0.351 \pm 0.005$ & $-0.124 \pm 0.004$ \\
\hline 2 & $0.379 \pm 0.006$ & $-0.110 \pm 0.006$ & $0.365 \pm 0.005$ & $-0.064 \pm 0.006$ \\
\hline 3 & $0.430 \pm 0.008$ & $-0.085 \pm 0.008$ & $0.385 \pm 0.008$ & $-0.128 \pm 0.008$ \\
\hline 4 & $0.536 \pm 0.012$ & $-0.034 \pm 0.013$ & $0.487 \pm 0.012$ & $-0.089 \pm 0.013$ \\
\hline 5 & $0.461 \pm 0.023$ & $-0.185 \pm 0.029$ & $0.724 \pm 0.024$ & $-0.601 \pm 0.025$ \\
\hline
\end{tabular}

Table F.2: Elements of the transfer matrix $\boldsymbol{T}$ for positive pions produced in $\rho^{0}$ decays. 


\begin{tabular}{|c|c|c|c|c|}
\hline \multicolumn{5}{|c|}{$z$ bins } \\
\hline & $T_{c c}$ & $T_{c s}$ & $T_{s s}$ & $T_{s c}$ \\
\hline 1 & $0.228 \pm 0.006$ & $-0.103 \pm 0.006$ & $0.169 \pm 0.006$ & $-0.085 \pm 0.006$ \\
\hline 2 & $0.385 \pm 0.007$ & $-0.158 \pm 0.007$ & $0.289 \pm 0.007$ & $-0.167 \pm 0.007$ \\
\hline 3 & $0.426 \pm 0.007$ & $-0.090 \pm 0.006$ & $0.399 \pm 0.006$ & $-0.085 \pm 0.007$ \\
\hline 4 & $0.526 \pm 0.005$ & $-0.035 \pm 0.005$ & $0.539 \pm 0.005$ & $-0.032 \pm 0.005$ \\
\hline 5 & $0.679 \pm 0.004$ & $-0.015 \pm 0.005$ & $0.685 \pm 0.004$ & $-0.022 \pm 0.005$ \\
\hline 6 & $0.839 \pm 0.007$ & $0.010 \pm 0.007$ & $0.830 \pm 0.006$ & $-0.005 \pm 0.007$ \\
\hline \multicolumn{5}{|c|}{$x$ bins } \\
\hline & $T_{c c}$ & $T_{c s}$ & $T_{s s}$ & $T_{s c}$ \\
\hline 1 & $0.471 \pm 0.005$ & $-0.077 \pm 0.005$ & $0.450 \pm 0.006$ & $-0.072 \pm 0.006$ \\
\hline 2 & $0.369 \pm 0.005$ & $-0.097 \pm 0.005$ & $0.343 \pm 0.005$ & $-0.084 \pm 0.005$ \\
\hline 3 & $0.337 \pm 0.007$ & $-0.070 \pm 0.008$ & $0.344 \pm 0.007$ & $-0.066 \pm 0.007$ \\
\hline 4 & $0.432 \pm 0.012$ & $-0.086 \pm 0.013$ & $0.332 \pm 0.012$ & $-0.028 \pm 0.011$ \\
\hline 5 & $0.356 \pm 0.020$ & $-0.089 \pm 0.021$ & $0.396 \pm 0.018$ & $-0.201 \pm 0.018$ \\
\hline \multicolumn{5}{|c|}{$P_{h \perp}$ bins } \\
\hline & $T_{c c}$ & $T_{c s}$ & $T_{s s}$ & $T_{s c}$ \\
\hline 1 & $0.271 \pm 0.006$ & $-0.095 \pm 0.006$ & $0.242 \pm 0.006$ & $-0.094 \pm 0.006$ \\
\hline 2 & $0.353 \pm 0.005$ & $-0.082 \pm 0.006$ & $0.288 \pm 0.005$ & $-0.085 \pm 0.005$ \\
\hline 3 & $0.561 \pm 0.006$ & $-0.065 \pm 0.007$ & $0.570 \pm 0.006$ & $-0.040 \pm 0.007$ \\
\hline 4 & $0.587 \pm 0.008$ & $-0.097 \pm 0.008$ & $0.678 \pm 0.008$ & $-0.019 \pm 0.009$ \\
\hline 5 & $0.900 \pm 0.015$ & $-0.051 \pm 0.017$ & $0.781 \pm 0.015$ & $-0.253 \pm 0.016$ \\
\hline \multicolumn{5}{|c|}{$y$ bins } \\
\hline & $T_{c c}$ & $T_{c s}$ & $T_{s s}$ & $T_{s c}$ \\
\hline 1 & $0.173 \pm 0.014$ & $0.032 \pm 0.014$ & $0.203 \pm 0.013$ & $0.063 \pm 0.013$ \\
\hline 2 & $0.205 \pm 0.009$ & $0.012 \pm 0.009$ & $0.227 \pm 0.008$ & $0.006 \pm 0.008$ \\
\hline 3 & $0.328 \pm 0.007$ & $-0.073 \pm 0.006$ & $0.353 \pm 0.006$ & $-0.034 \pm 0.006$ \\
\hline 4 & $0.446 \pm 0.005$ & $-0.095 \pm 0.005$ & $0.433 \pm 0.006$ & $-0.072 \pm 0.006$ \\
\hline 5 & $0.569 \pm 0.006$ & $-0.107 \pm 0.006$ & $0.567 \pm 0.007$ & $-0.062 \pm 0.007$ \\
\hline \multicolumn{5}{|c|}{$\bar{Q}^{2}$ bins } \\
\hline & $T_{c c}$ & $T_{c s}$ & $T_{s s}$ & $T_{s c}$ \\
\hline 1 & $0.399 \pm 0.004$ & $-0.082 \pm 0.005$ & $0.368 \pm 0.005$ & $-0.083 \pm 0.005$ \\
\hline 2 & $0.379 \pm 0.006$ & $-0.087 \pm 0.006$ & $0.352 \pm 0.005$ & $-0.074 \pm 0.005$ \\
\hline 3 & $0.414 \pm 0.008$ & $-0.123 \pm 0.008$ & $0.439 \pm 0.008$ & $-0.057 \pm 0.008$ \\
\hline 4 & $0.495 \pm 0.013$ & $-0.129 \pm 0.013$ & $0.459 \pm 0.012$ & $-0.145 \pm 0.012$ \\
\hline 5 & $0.648 \pm 0.027$ & $-0.171 \pm 0.026$ & $0.606 \pm 0.023$ & $-0.273 \pm 0.023$ \\
\hline
\end{tabular}

Table F.3: Elements of the transfer matrix $\boldsymbol{T}$ for negative pions produced in $\rho^{0}$ decays. 


\begin{tabular}{|c|c|c|c|c|}
\hline \multicolumn{5}{|c|}{$z$ bins } \\
\hline & $T_{c c}$ & $T_{c s}$ & $T_{s s}$ & $T_{s c}$ \\
\hline 1 & $0.421 \pm 0.007$ & $-0.009 \pm 0.007$ & $0.467 \pm 0.008$ & $-0.005 \pm 0.008$ \\
\hline 2 & $0.487 \pm 0.006$ & $0.012 \pm 0.006$ & $0.556 \pm 0.006$ & $0.029 \pm 0.006$ \\
\hline 3 & $0.647 \pm 0.004$ & $0.008 \pm 0.005$ & $0.703 \pm 0.004$ & $0.034 \pm 0.005$ \\
\hline 4 & $0.852 \pm 0.005$ & $-0.014 \pm 0.005$ & $0.860 \pm 0.005$ & $-0.004 \pm 0.005$ \\
\hline 5 & $1.009 \pm 0.008$ & $-0.021 \pm 0.011$ & $1.000 \pm 0.008$ & $-0.003 \pm 0.010$ \\
\hline \multicolumn{5}{|c|}{$x$ bins } \\
\hline & $T_{c c}$ & $T_{c s}$ & $T_{s s}$ & $T_{s c}$ \\
\hline 1 & $0.735 \pm 0.007$ & $0.002 \pm 0.008$ & $0.819 \pm 0.009$ & $0.036 \pm 0.009$ \\
\hline 2 & $0.637 \pm 0.006$ & $0.011 \pm 0.006$ & $0.683 \pm 0.006$ & $0.032 \pm 0.006$ \\
\hline 3 & $0.610 \pm 0.005$ & $-0.013 \pm 0.006$ & $0.647 \pm 0.005$ & $-0.005 \pm 0.005$ \\
\hline 4 & $0.587 \pm 0.007$ & $-0.002 \pm 0.007$ & $0.649 \pm 0.006$ & $0.024 \pm 0.006$ \\
\hline 5 & $0.631 \pm 0.007$ & $0.028 \pm 0.007$ & $0.666 \pm 0.006$ & $0.033 \pm 0.007$ \\
\hline \multicolumn{5}{|c|}{$P_{h \perp}$ bins } \\
\hline & $T_{c c}$ & $T_{c s}$ & $T_{s s}$ & $T_{s c}$ \\
\hline 1 & $0.271 \pm 0.005$ & $-0.020 \pm 0.005$ & $0.340 \pm 0.005$ & $0.012 \pm 0.005$ \\
\hline 2 & $0.718 \pm 0.004$ & $0.034 \pm 0.004$ & $0.755 \pm 0.004$ & $0.045 \pm 0.004$ \\
\hline 3 & $0.898 \pm 0.005$ & $0.034 \pm 0.005$ & $0.922 \pm 0.004$ & $0.036 \pm 0.005$ \\
\hline 4 & $0.983 \pm 0.007$ & $0.011 \pm 0.007$ & $1.015 \pm 0.005$ & $0.035 \pm 0.008$ \\
\hline 5 & $1.050 \pm 0.015$ & $0.009 \pm 0.018$ & $1.093 \pm 0.015$ & $0.113 \pm 0.019$ \\
\hline \multicolumn{5}{|c|}{$y$ bins } \\
\hline & $T_{c c}$ & $T_{c s}$ & $T_{s s}$ & $T_{s c}$ \\
\hline 1 & $0.684 \pm 0.019$ & $0.062 \pm 0.018$ & $0.787 \pm 0.015$ & $0.112 \pm 0.018$ \\
\hline 2 & $0.591 \pm 0.009$ & $-0.018 \pm 0.009$ & $0.646 \pm 0.007$ & $0.043 \pm 0.008$ \\
\hline 3 & $0.576 \pm 0.005$ & $-0.003 \pm 0.006$ & $0.625 \pm 0.005$ & $0.019 \pm 0.005$ \\
\hline 4 & $0.615 \pm 0.004$ & $0.030 \pm 0.005$ & $0.678 \pm 0.004$ & $0.022 \pm 0.005$ \\
\hline 5 & $0.680 \pm 0.006$ & $0.031 \pm 0.006$ & $0.810 \pm 0.007$ & $0.100 \pm 0.008$ \\
\hline \multicolumn{5}{|c|}{$Q^{2}$ bins } \\
\hline & $T_{c c}$ & $T_{c s}$ & $T_{s s}$ & $T_{s c}$ \\
\hline 1 & $0.719 \pm 0.007$ & $-0.034 \pm 0.008$ & $0.741 \pm 0.008$ & $-0.012 \pm 0.008$ \\
\hline 2 & $0.613 \pm 0.006$ & $0.003 \pm 0.006$ & $0.673 \pm 0.006$ & $0.049 \pm 0.007$ \\
\hline 3 & $0.613 \pm 0.006$ & $0.018 \pm 0.006$ & $0.654 \pm 0.005$ & $0.011 \pm 0.006$ \\
\hline 4 & $0.612 \pm 0.006$ & $-0.005 \pm 0.006$ & $0.658 \pm 0.005$ & $0.014 \pm 0.006$ \\
\hline 5 & $0.642 \pm 0.008$ & $0.059 \pm 0.008$ & $0.692 \pm 0.007$ & $0.037 \pm 0.008$ \\
\hline
\end{tabular}

Table F.4: Elements of the transfer matrix $\boldsymbol{T}$ for neutral pions produced in $\omega$ decays. 


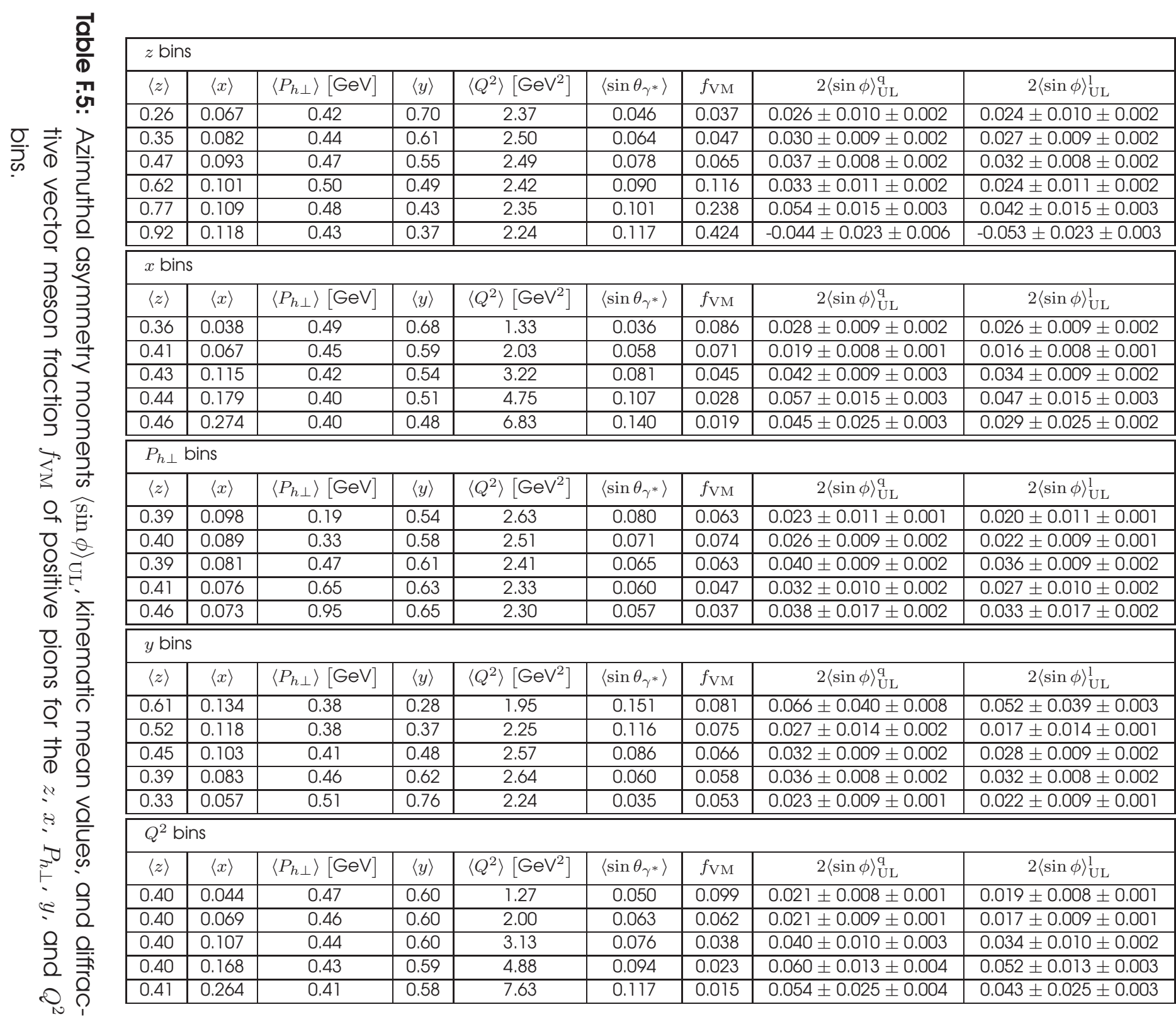




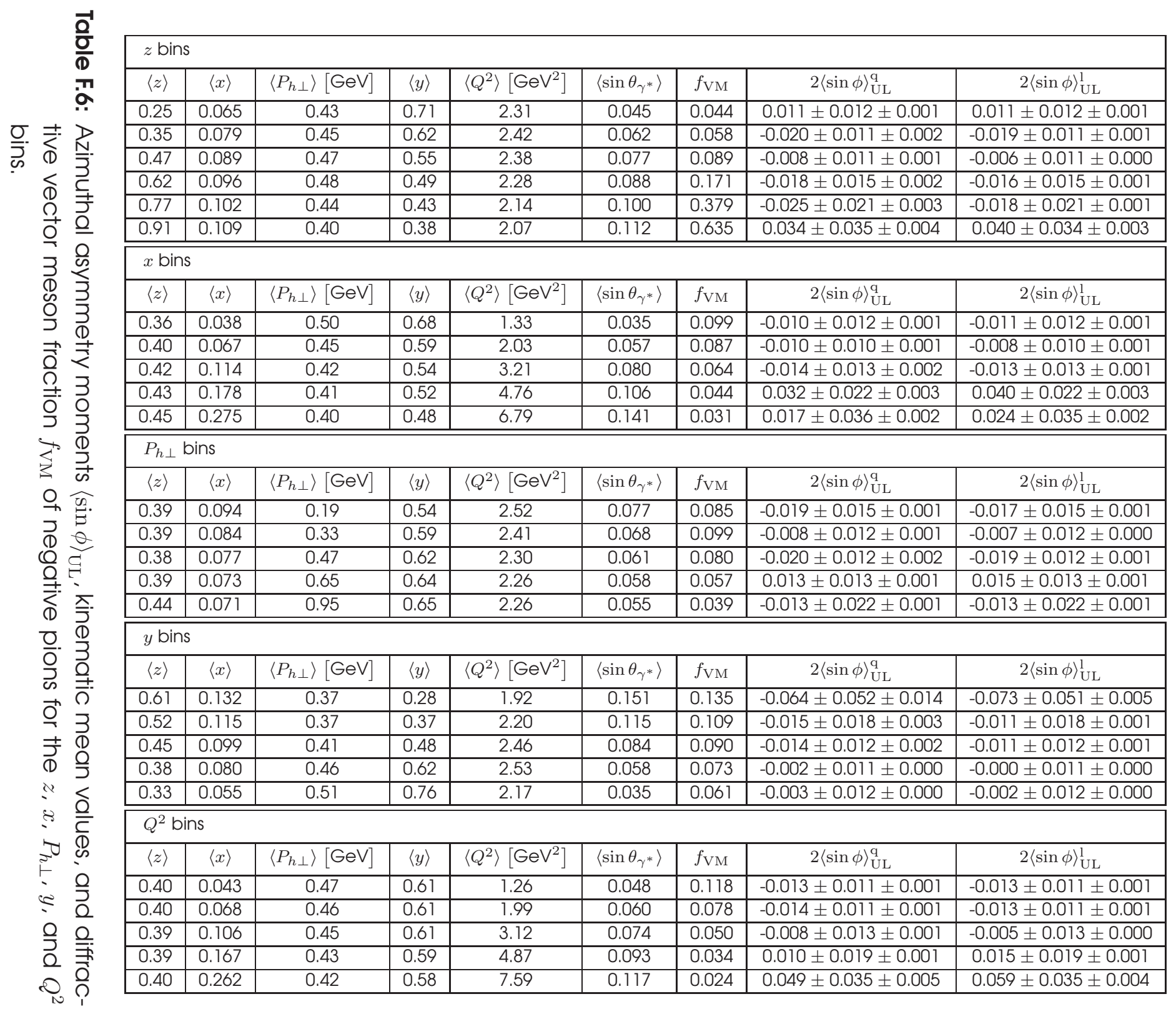




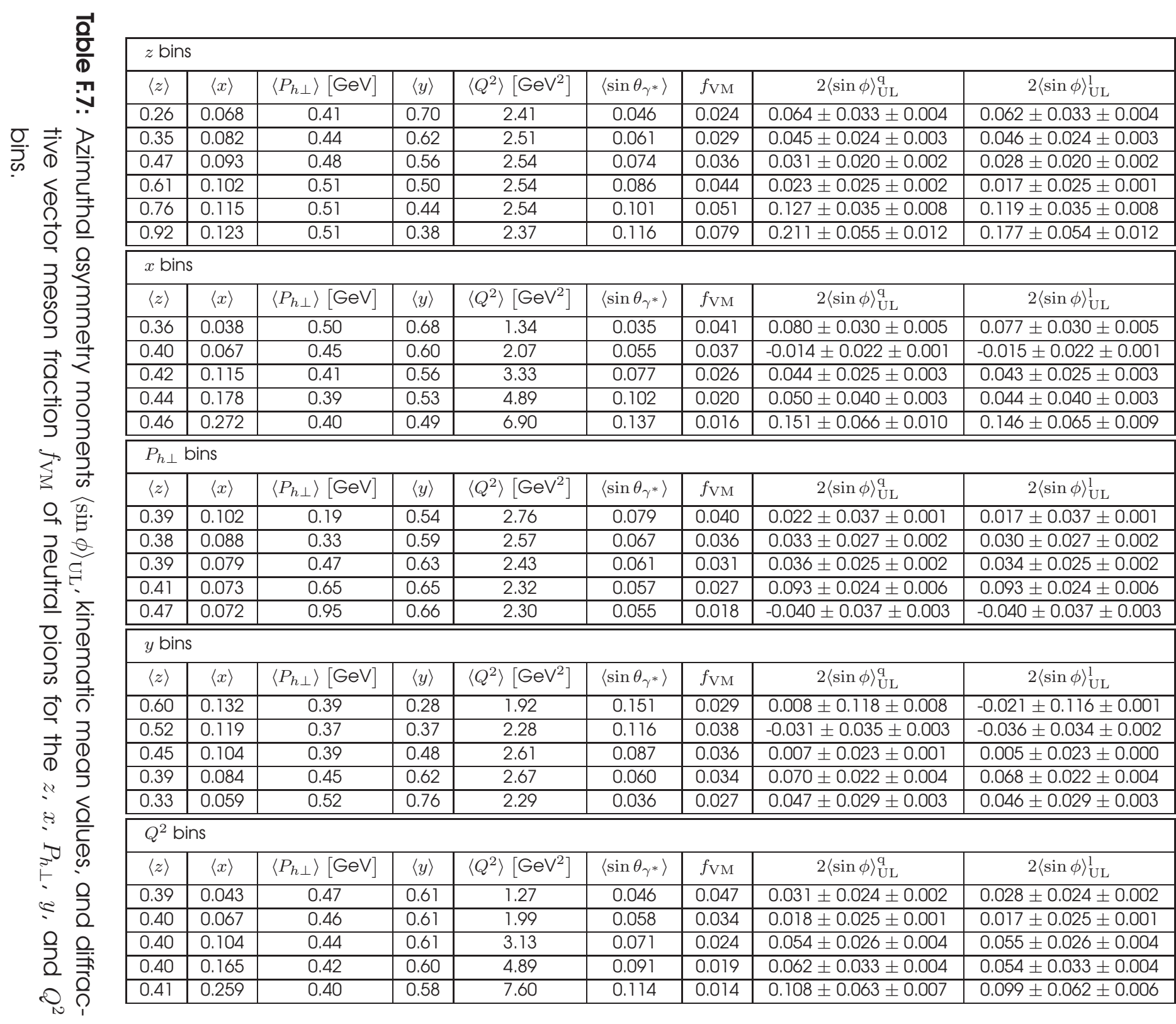




\begin{tabular}{|c|c|c|c|c|c|c|c|c|c|c|}
\hline \multirow{3}{*}{ 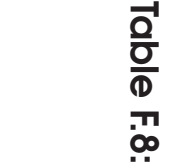 } & \multicolumn{10}{|c|}{$z$ bins } \\
\hline & $\langle z\rangle$ & $\langle x\rangle$ & $\left\langle P_{h \perp}\right\rangle[\mathrm{GeV}]$ & $\langle y\rangle$ & $\left\langle Q^{2}\right\rangle\left[\mathrm{GeV}^{2}\right]$ & $f_{\mathrm{VM}}$ & $\frac{A(\langle y\rangle, R)}{A(\langle y)}$ & $\frac{A(\langle y\rangle, R)}{B(y y)}$ & $2\left\langle\sin \left(\phi-\phi_{S}\right)\right\rangle_{\mathrm{UT}}$ & $2\left\langle\sin \left(\phi+\phi_{S}\right)\right\rangle_{\mathrm{UT}}$ \\
\hline & 0.25 & 0.090 & 0.35 & 0.56 & 2.45 & 0.038 & 1.203 & 1.649 & $0.030 \pm 0.007 \pm 0.002$ & $0.010 \pm 0.007 \pm 0.001$ \\
\hline \multirow{7}{*}{\multicolumn{2}{|c|}{ 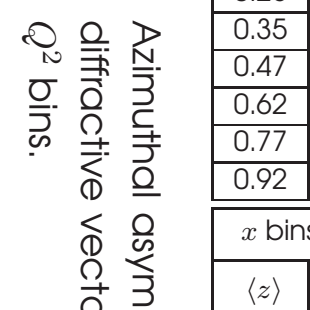 }} & 0.098 & 0.40 & 0.52 & 2.43 & 0.047 & 1.214 & 1.567 & $0.037 \pm 0.008 \pm 0.002$ & $0.022 \pm 0.008 \pm 0.001$ \\
\hline & & 0.100 & 0.45 & 0.51 & 2.41 & 0.063 & 1.219 & 1.538 & $0.052 \pm 0.009 \pm 0.003$ & $0.015 \pm 0.010 \pm 0.001$ \\
\hline & & 0.102 & 0.50 & 0.49 & 2.39 & 0.116 & 1.223 & 1.514 & $0.058 \pm 0.013 \pm 0.003$ & $0.037 \pm 0.014 \pm 0.006$ \\
\hline & & 0.107 & 0.49 & 0.45 & 2.36 & 0.242 & 1.233 & 1.458 & $0.108 \pm 0.018 \pm 0.006$ & $0.023 \pm 0.020 \pm 0.002$ \\
\hline & & 0.119 & 0.45 & 0.39 & 2.34 & 0.429 & 1.242 & 1.398 & $0.031 \pm 0.029 \pm 0.025$ & $0.046 \pm 0.031 \pm 0.028$ \\
\hline & & & & & & & & & & \\
\hline & & $\langle x\rangle$ & $\left\langle P_{h \perp}\right\rangle[\mathrm{GeV}]$ & $\langle y\rangle$ & $\left\langle Q^{2}\right\rangle\left[\mathrm{GeV}^{2}\right]$ & $f_{\mathrm{VM}}$ & $\frac{A(\langle y\rangle, R)}{A(\langle y)}$ & $\frac{A(\langle y\rangle, R)}{B(y))}$ & $2\left\langle\sin \left(\phi-\phi_{S}\right)\right\rangle_{\mathrm{UT}}$ & $2\left\langle\sin \left(\phi+\phi_{S}\right)\right\rangle_{\mathrm{UT}}$ \\
\hline \multirow{7}{*}{ 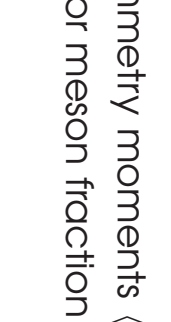 } & 0.34 & 0.039 & 0.47 & 0.66 & 1.31 & 0.083 & 1.220 & 1.997 & $0.044 \pm 0.012 \pm 0.002$ & $0.000 \pm 0.010 \pm 0.001$ \\
\hline & 0.36 & 0.068 & 0.40 & 0.53 & 1.85 & 0.065 & 1.247 & 1.623 & $0.031 \pm 0.008 \pm 0.002$ & $0.017 \pm 0.008 \pm 0.001$ \\
\hline & 0.37 & 0.115 & 0.37 & 0.47 & 2.80 & 0.043 & 1.205 & 1.464 & $0.040 \pm 0.008 \pm 0.002$ & $0.029 \pm 0.009 \pm 0.001$ \\
\hline & 0.37 & 0.179 & 0.36 & 0.45 & 4.15 & 0.027 & 1.147 & 1.362 & $0.054 \pm 0.012 \pm 0.003$ & $0.031 \pm 0.013 \pm 0.002$ \\
\hline & 0.37 & 0.276 & 0.36 & 0.43 & 6.13 & 0.019 & 1.099 & 1.285 & $0.085 \pm 0.019 \pm 0.004$ & $0.029 \pm 0.018 \pm 0.002$ \\
\hline & \multicolumn{10}{|c|}{$P_{h \perp}$ bins } \\
\hline & $\langle z\rangle$ & $\langle x\rangle$ & $\left\langle P_{h \perp}\right\rangle[\mathrm{GeV}]$ & $\langle y\rangle$ & $\left\langle Q^{2}\right\rangle\left[\mathrm{GeV}^{2}\right]$ & $f_{\mathrm{VM}}$ & $\frac{A(\langle y\rangle, R)}{A(\langle y\rangle)}$ & $\frac{A(\langle y\rangle, R)}{B(\langle y\rangle)}$ & $2\left\langle\sin \left(\phi-\phi_{S}\right)\right\rangle_{\mathrm{UT}}$ & $2\left\langle\sin \left(\phi+\phi_{S}\right)\right\rangle_{\mathrm{UT}}$ \\
\hline \multirow{7}{*}{\multicolumn{2}{|c|}{ 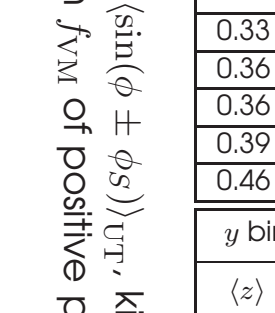 }} & 0.109 & 0.17 & 0.46 & 2.50 & 0.055 & 1.222 & 1.472 & $0.012 \pm 0.008 \pm 0.001$ & $0.029 \pm 0.009 \pm 0.002$ \\
\hline & & 0.098 & & 0.52 & 2.4 & 0.067 & 1.212 & 1.565 & 0.002 & $0.012 \pm 0.009 \pm 0.001$ \\
\hline & & 0.089 & 0.47 & 0.56 & 2.38 & 0.055 & 1.208 & 1.642 & $0.047 \pm 0.009 \pm 0.002$ & $0.018 \pm 0.009 \pm 0.001$ \\
\hline & & \begin{tabular}{|l|}
0.084 \\
\end{tabular} & 0.65 & 0.59 & 2.35 & 0.042 & 1.200 & 1.717 & & $0.017 \pm 0.010 \pm 0.002$ \\
\hline & & 0.077 & 0.95 & 0.63 & 2.34 & 0.036 & 1.187 & 1.845 & $0.070 \pm 0.018 \pm 0.006$ & $0.018 \pm 0.017 \pm 0.003$ \\
\hline & & & & & & & & & & \\
\hline & & $\langle x\rangle$ & $\left\langle P_{h \perp}\right\rangle[\mathrm{GeV}]$ & $\langle y\rangle$ & $\left\langle Q^{2}\right\rangle\left[\mathrm{GeV}^{2}\right]$ & $f_{\mathrm{VM}}$ & $\frac{A(\langle y\rangle, R)}{A(\langle y)}$ & $\frac{A(\langle y\rangle, R)}{B(y y)}$ & $2\left\langle\sin \left(\phi-\phi_{S}\right)\right\rangle_{\mathrm{UT}}$ & $2\left\langle\sin \left(\phi+\phi_{S}\right)\right\rangle_{\mathrm{UT}}$ \\
\hline \multirow{7}{*}{ 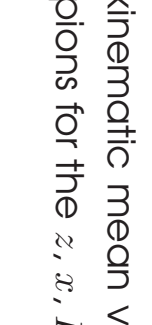 } & 0.43 & 0.141 & 0.33 & 0.27 & 1.97 & 0.061 & 1.271 & 1.337 & $0.037 \pm 0.029 \pm 0.005$ & $0.016 \pm 0.032 \pm 0.008$ \\
\hline & 0.38 & 0.122 & 0.32 & 0.37 & 2.31 & 0.057 & 1.246 & 1.381 & $0.031 \pm 0.011 \pm 0.002$ & $0.042 \pm 0.012 \pm 0.002$ \\
\hline & 0.36 & 0.104 & 0.36 & 0.48 & 2.55 & 0.056 & 1.218 & 1.489 & $0.032 \pm 0.008 \pm 0.002$ & $0.016 \pm 0.009 \pm 0.001$ \\
\hline & 0.35 & 0.084 & 0.44 & 0.61 & 2.64 & 0.054 & 1.183 & 1.758 & $0.045 \pm 0.009 \pm 0.002$ & $0.014 \pm 0.008 \pm 0.001$ \\
\hline & 0.33 & 0.058 & 0.52 & 0.76 & 2.25 & 0.054 & 1.137 & 2.525 & $0.042 \pm 0.013 \pm 0.002$ & $-0.003 \pm 0.010 \pm 0.001$ \\
\hline & $\overline{Q^{2} \mathrm{k}}$ & & & & & & & & & \\
\hline & $\langle z\rangle$ & $\langle x\rangle$ & $\left\langle P_{h \perp}\right\rangle[\mathrm{GeV}]$ & $\langle y\rangle$ & $\left\langle Q^{2}\right\rangle\left[\mathrm{GeV}^{2}\right]$ & $f_{\mathrm{VM}}$ & $\frac{A(\langle y\rangle, R)}{A(\langle y\rangle)}$ & $\frac{A(\langle y\rangle, R)}{B(\langle y\rangle)}$ & $2\left\langle\sin \left(\phi-\phi_{S}\right)\right\rangle_{\mathrm{UT}}$ & $2\left\langle\sin \left(\phi+\phi_{S}\right)\right\rangle_{\mathrm{UT}}$ \\
\hline \multirow{3}{*}{ 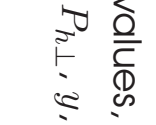 } & 0.36 & 0.052 & 0.41 & 0.53 & 1.27 & 0.087 & 1.274 & 1.658 & $0.031 \pm 0.009 \pm 0.002$ & $0.007 \pm 0.008 \pm 0.001$ \\
\hline & 0.36 & 0.081 & 0.40 & 0.53 & 1.99 & 0.055 & 1.238 & 1.604 & $0.039 \pm 0.008 \pm 0.002$ & $0.027 \pm 0.008 \pm 0.001$ \\
\hline & 0.36 & 0.122 & 0.40 & 0.54 & 3.12 & 0.034 & 1.175 & 1.552 & $0.050 \pm 0.009 \pm 0.003$ & $0.024 \pm 0.009 \pm 0.001$ \\
\hline \multirow{2}{*}{ 을 } & 0.36 & 0.186 & 0.39 & 0.54 & 4.87 & 0.021 & 1.116 & 1.483 & $0.044 \pm 0.011 \pm 0.002$ & $0.028 \pm 0.012 \pm 0.001$ \\
\hline & 0.36 & 0.276 & 0.38 & 0.55 & 7.58 & 0.014 & 1.073 & 1.440 & $0.070 \pm 0.021 \pm 0.004$ & $0.005 \pm 0.022 \pm 0.003$ \\
\hline
\end{tabular}




\begin{tabular}{|c|c|c|c|c|c|c|c|c|c|c|}
\hline \multirow{3}{*}{$\begin{array}{l}\frac{0}{\sigma} \\
\bar{D} \\
\bar{i}\end{array}$} & \multicolumn{10}{|c|}{$z$ bins } \\
\hline & $\langle z\rangle$ & $\langle x\rangle$ & $\left\langle P_{h \perp}\right\rangle[\mathrm{GeV}]$ & $\langle y\rangle$ & $\left\langle Q^{2}\right\rangle\left[\mathrm{GeV}^{2}\right]$ & $f_{\mathrm{VM}}$ & $\frac{A(\langle y\rangle, R)}{A(\langle y\rangle)}$ & $\frac{A(\langle y\rangle, R)}{B(\langle y\rangle)}$ & $2\left\langle\sin \left(\phi-\phi_{S}\right)\right\rangle_{\mathrm{UT}}$ & $2\left\langle\sin \left(\phi+\phi_{S}\right)\right\rangle_{\mathrm{UT}}$ \\
\hline & 0.25 & 0.087 & 0.36 & 0.57 & 2.39 & 0.046 & 1.204 & 1.674 & $0.001 \pm 0.008 \pm 0.000$ & $-0.018 \pm 0.008 \pm 0.001$ \\
\hline \multirow{7}{*}{\multicolumn{2}{|c|}{ 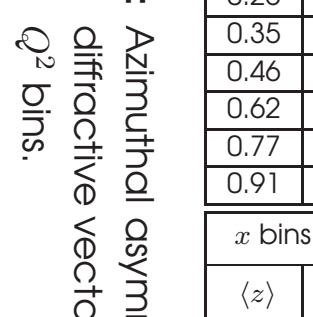 }} & 0.093 & 0.41 & 0.53 & 2.34 & 0.059 & 7.216 & 7.589 & $0.006 \pm 0.010 \pm 0.000$ & $-0.037 \pm 0.010 \pm 0.002$ \\
\hline & & 0.097 & 0.45 & 0.51 & 2.32 & 0.087 & 7.223 & 1.547 & $0.005 \pm 0.011 \pm 0.000$ & $-0.042 \pm 0.012 \pm 0.002$ \\
\hline & & 0.097 & 0.48 & 0.49 & 2.26 & 0.170 & 7.231 & 1.522 & $-0.002 \pm 0.016 \pm 0.009$ & $-0.019 \pm 0.017 \pm 0.010$ \\
\hline & & 0.100 & 0.45 & 0.44 & 2.18 & 0.383 & 7.245 & 1.467 & $-0.045 \pm 0.024 \pm 0.014$ & $-0.033 \pm 0.026 \pm 0.016$ \\
\hline & & 0.108 & 0.41 & 0.39 & 2.12 & 0.640 & 1.256 & 1.419 & $-0.067 \pm 0.042 \pm 0.007$ & $0.016 \pm 0.043 \pm 0.004$ \\
\hline & & \\
\hline & & $\langle x\rangle$ & $\left\langle P_{h \perp}\right\rangle[\mathrm{GeV}]$ & $\langle y\rangle$ & $\left\langle Q^{2}\right\rangle\left[\mathrm{GeV}^{2}\right]$ & $f_{\mathrm{VM}}$ & $\frac{A(\langle y\rangle, R)}{A(\langle y\rangle)}$ & $\frac{A(\langle y\rangle, R)}{B(\langle y)\rangle}$ & $2\left\langle\sin \left(\phi-\phi_{S}\right)\right\rangle_{\mathrm{UT}}$ & $2\left\langle\sin \left(\phi+\phi_{S}\right)\right\rangle_{\mathrm{UT}}$ \\
\hline \multirow{7}{*}{ 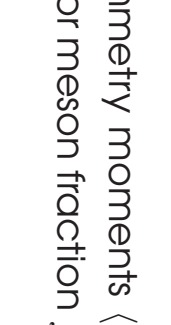 } & 0.33 & 0.039 & 0.47 & 0.66 & 1.30 & 0.096 & 1.217 & 2.015 & $0.015 \pm 0.013 \pm 0.001$ & $-0.003 \pm 0.010 \pm 0.001$ \\
\hline & 0.35 & 0.068 & 0.39 & 0.53 & 7.86 & 0.080 & 1.246 & 7.631 & $-0.007 \pm 0.009 \pm 0.001$ & $-0.031 \pm 0.009 \pm 0.002$ \\
\hline & 0.37 & 0.115 & 0.36 & 0.47 & 2.80 & 0.059 & 1.205 & 1.465 & $0.006 \pm 0.010 \pm 0.000$ & $-0.020 \pm 0.011 \pm 0.001$ \\
\hline & 0.37 & 0.179 & 0.36 & 0.45 & 4.16 & 0.039 & 1.147 & 1.364 & $0.012 \pm 0.015 \pm 0.001$ & $-0.065 \pm 0.016 \pm 0.003$ \\
\hline & 0.36 & 0.276 & 0.37 & 0.43 & 6.10 & 0.029 & 1.099 & 1.284 & $-0.008 \pm 0.025 \pm 0.001$ & $-0.049 \pm 0.024 \pm 0.003$ \\
\hline & \multicolumn{10}{|c|}{$P_{P_{h \perp}}$ bins } \\
\hline & $\langle z\rangle$ & $\langle x\rangle$ & $\left\langle P_{h \perp}\right\rangle[\mathrm{GeV}]$ & $\langle y\rangle$ & $\left\langle Q^{2}\right\rangle\left[\mathrm{GeV}^{2}\right]$ & $f_{\mathrm{VM}}$ & $\frac{A(\langle y\rangle, R)}{A(\langle y\rangle)}$ & $\frac{A(\langle y\rangle, R)}{B(\langle y\rangle)}$ & $2\left\langle\sin \left(\phi-\phi_{S}\right)\right\rangle_{\mathrm{UT}}$ & $2\left\langle\sin \left(\phi+\phi_{S}\right)\right\rangle_{\mathrm{UT}}$ \\
\hline \multirow{7}{*}{\multicolumn{2}{|c|}{ 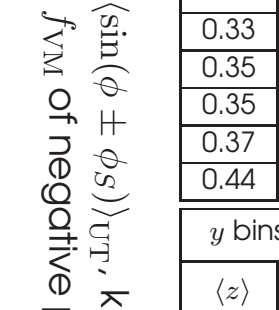 }} & 0.104 & 0.17 & 0.47 & 2.41 & 0.073 & 1.226 & 1.485 & $0.005 \pm 0.010 \pm 0.000$ & $-0.028 \pm 0.011 \pm 0.001$ \\
\hline & & 0.094 & 0.32 & 0.53 & 2.38 & 0.088 & 1.215 & 1.585 & $-0.005 \pm 0.009 \pm 0.001$ & $-0.016 \pm 0.010 \pm 0.001$ \\
\hline & & 0.085 & 0.47 & 0.57 & 2.30 & 0.069 & 1.208 & 1.677 & $-0.002 \pm 0.011 \pm 0.001$ & $-0.031 \pm 0.010 \pm 0.002$ \\
\hline & & 0.081 & 0.65 & 0.60 & 2.30 & 0.050 & 7.199 & 1.749 & $0.005 \pm 0.012 \pm 0.002$ & $-0.039 \pm 0.011 \pm 0.003$ \\
\hline & & 0.075 & 0.95 & 0.64 & 2.29 & 0.038 & 7.187 & 1.868 & $0.018 \pm 0.022 \pm 0.004$ & $-0.032 \pm 0.021 \pm 0.004$ \\
\hline & & & & & & & & & & \\
\hline & & $\langle x\rangle$ & $\left\langle P_{h \perp}\right\rangle[\mathrm{GeV}]$ & $\langle y\rangle$ & $\left\langle Q^{2}\right\rangle\left[\mathrm{GeV}^{2}\right]$ & $f_{\mathrm{VM}}$ & $\frac{A(\langle y\rangle, R)}{A(\langle y\rangle)}$ & $\frac{A(\langle y\rangle, R)}{B(\langle y\rangle)}$ & $2\left\langle\sin \left(\phi-\phi_{S}\right)\right\rangle_{\mathrm{UT}}$ & $2\left\langle\sin \left(\phi+\phi_{S}\right)\right\rangle_{\mathrm{UT}}$ \\
\hline \multirow{7}{*}{ 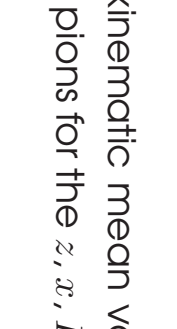 } & 0.42 & 0.138 & 0.32 & 0.27 & 1.94 & 0.089 & 1.273 & 1.340 & $0.016 \pm 0.035 \pm 0.006$ & $-0.043 \pm 0.040 \pm 0.008$ \\
\hline & 0.37 & 0.120 & 0.32 & 0.37 & 2.26 & 0.078 & 7.249 & 7.384 & $-0.004 \pm 0.013 \pm 0.001$ & $-0.017 \pm 0.015 \pm 0.001$ \\
\hline & 0.36 & 0.100 & 0.36 & 0.48 & 2.47 & 0.073 & 7.223 & 1.495 & $0.000 \pm 0.010 \pm 0.001$ & $-0.027 \pm 0.011 \pm 0.002$ \\
\hline & 0.34 & 0.080 & 0.43 & 0.61 & 2.53 & 0.067 & 7.187 & 1.767 & $-0.006 \pm 0.010 \pm 0.000$ & $-0.017 \pm 0.010 \pm 0.001$ \\
\hline & 0.32 & 0.055 & 0.51 & 0.76 & 2.17 & 0.062 & 7.139 & 2.543 & $0.000 \pm 0.015 \pm 0.001$ & $-0.015 \pm 0.011 \pm 0.001$ \\
\hline & \multicolumn{10}{|c|}{$Q^{2}$ bins } \\
\hline & $\langle z\rangle$ & $\langle x\rangle$ & $\left\langle P_{h \perp}\right\rangle[\mathrm{GeV}]$ & $\langle y\rangle$ & $\left\langle Q^{2}\right\rangle\left[\mathrm{GeV}^{2}\right]$ & $f_{\mathrm{VM}}$ & $\frac{A(\langle y\rangle, R)}{A(\langle y\rangle)}$ & $\frac{A(\langle y\rangle, R)}{B(\langle y\rangle)}$ & $2\left\langle\sin \left(\phi-\phi_{S}\right)\right\rangle_{\mathrm{UT}}$ & $2\left\langle\sin \left(\phi+\phi_{S}\right)\right\rangle_{\mathrm{UT}}$ \\
\hline \multirow{5}{*}{ 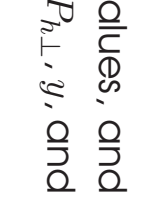 } & 0.36 & 0.051 & 0.41 & 0.54 & 1.27 & 0.104 & 1.270 & 1.677 & $0.012 \pm 0.010 \pm 0.001$ & $-0.029 \pm 0.009 \pm 0.002$ \\
\hline & 0.35 & 0.079 & 0.40 & 0.54 & 1.99 & 0.069 & 7.235 & 1.624 & $-0.002 \pm 0.009 \pm 0.001$ & $-0.010 \pm 0.009 \pm 0.001$ \\
\hline & 0.35 & 0.120 & 0.40 & 0.55 & 3.11 & 0.045 & 7.174 & 7.565 & $-0.006 \pm 0.010 \pm 0.000$ & $-0.026 \pm 0.011 \pm 0.001$ \\
\hline & 0.35 & 0.184 & 0.39 & 0.54 & 4.87 & 0.030 & 7.116 & 1.489 & $-0.001 \pm 0.014 \pm 0.000$ & $-0.065 \pm 0.015 \pm 0.003$ \\
\hline & 0.34 & 0.277 & 0.38 & 0.54 & 7.56 & 0.022 & 1.074 & 1.438 & $0.012 \pm 0.026 \pm 0.001$ & $-0.060 \pm 0.028 \pm 0.003$ \\
\hline
\end{tabular}




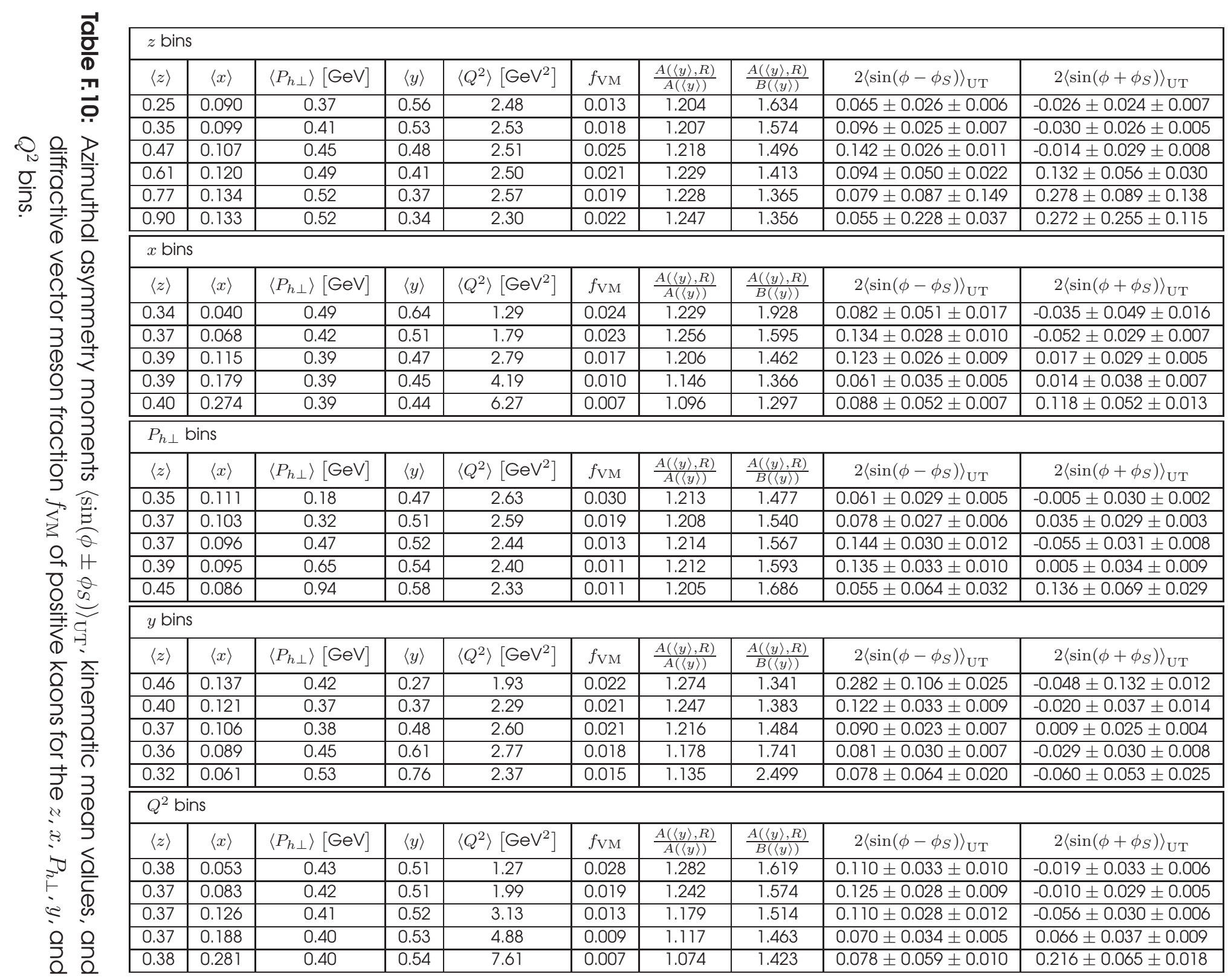




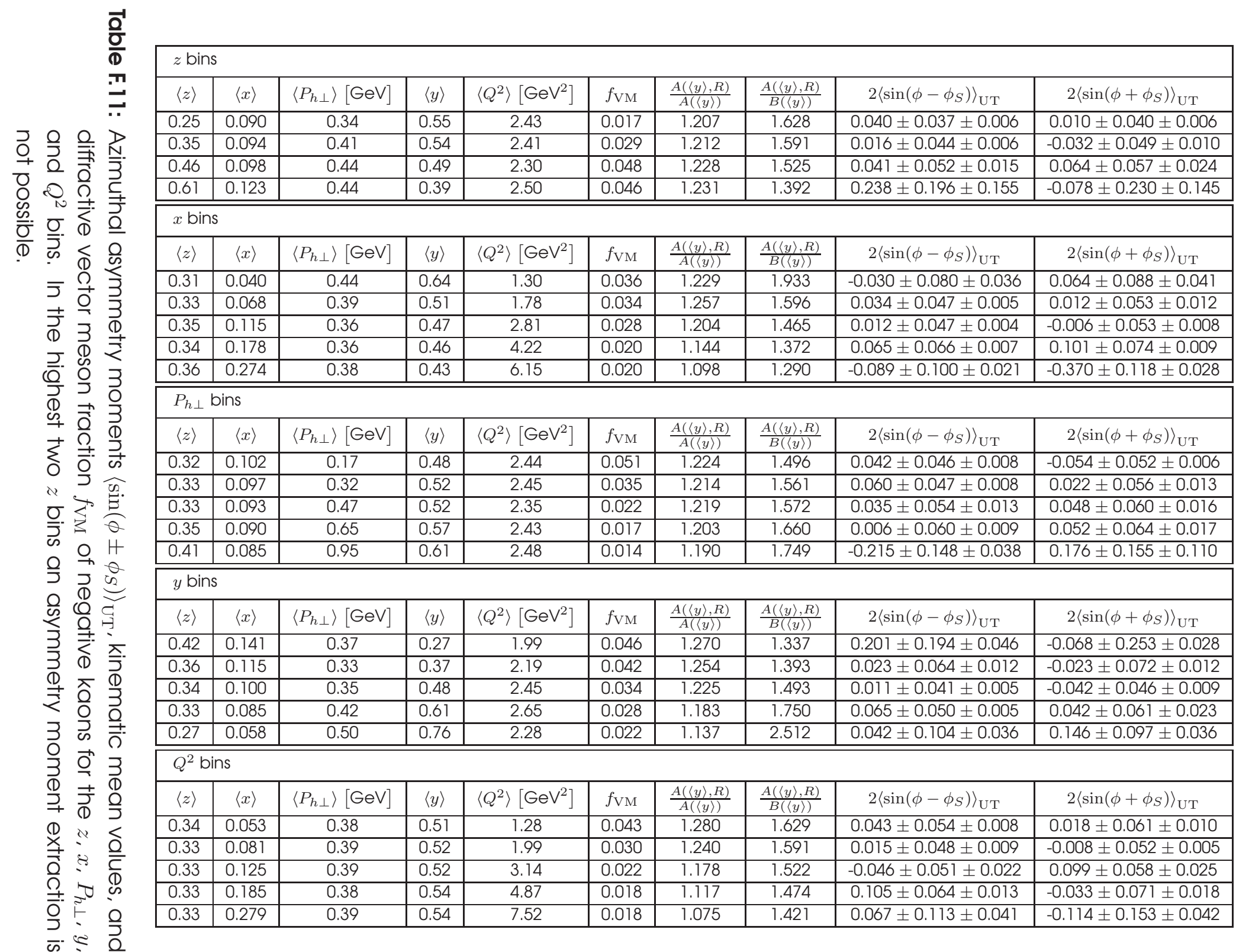




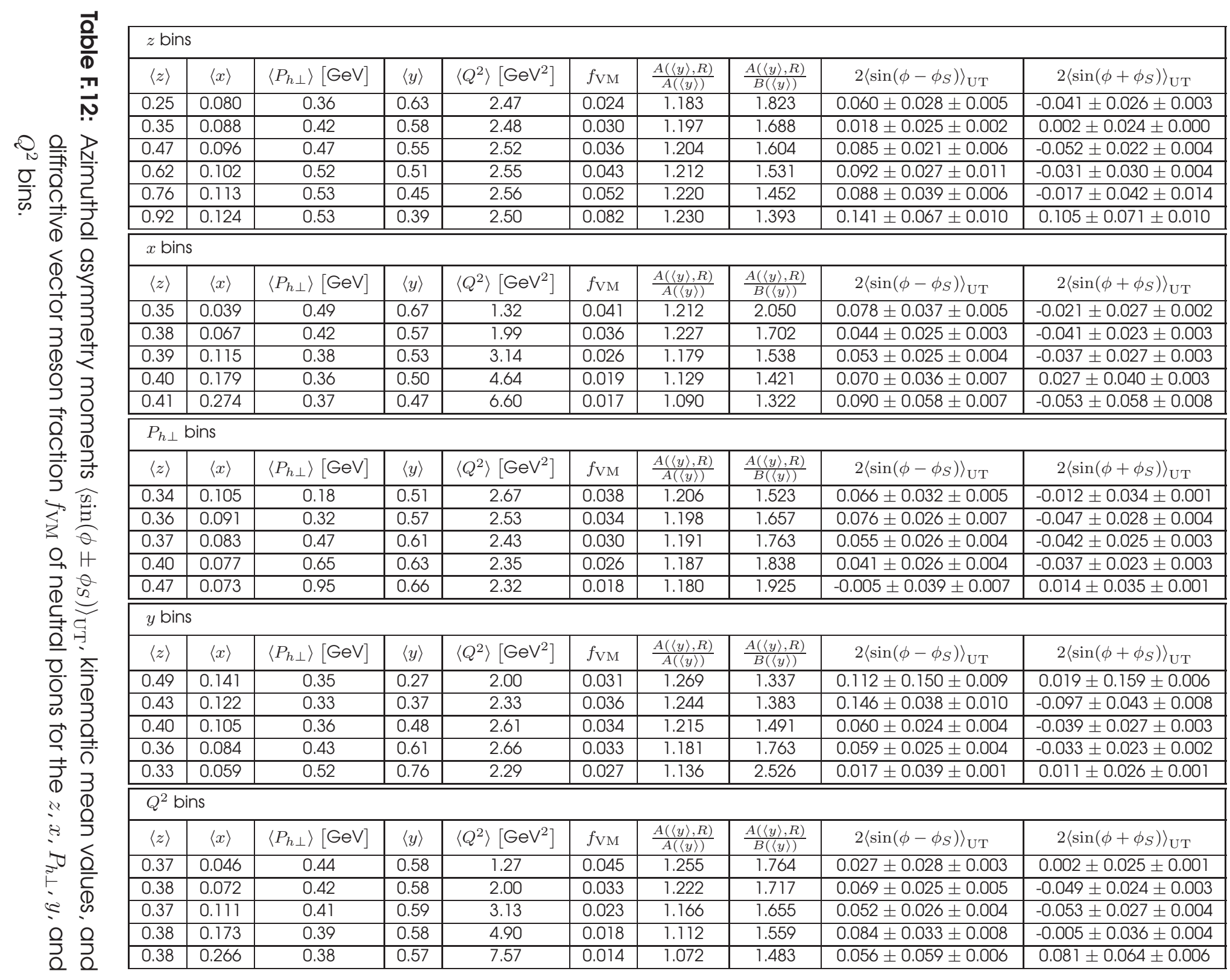




\section{Bibliography}

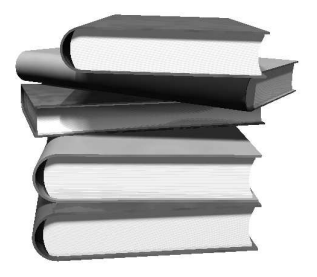

[Aba02] A. Abashian et al., The Belle detector, Nucl. Instrum. Meth. A479, 117-232 (2002).

[Abe98] K. Abe et al. (E143 Collaboration), Measurements of the proton and deuteron spin structure functions $g_{1}$ and $g_{2}$, Phys. Rev. D58, 112003 (1998).

[Abe99] K. Abe et al. (E143 Collaboration), Measurements of $R=\sigma_{L} / \sigma_{T}$ for $0.03<x<$ 0.1 and fit to world data, Phys. Lett. B452, 194-200 (1999).

[Abe05] K. Abe et al. (Belle Collaboration), Measurement of azimuthal asymmetries in inclusive production of hadron pairs in e+e- annihilation at Belle. Presented at 22nd International Symposium on Lepton-Photon Interactions at High Energy, Uppsala, Sweden, 30 Jun - 5 Jul 2005, hep-ex/0507063.

[Ack97] K. Ackerstaff et al. (HERMES Collaboration), Measurement of the neutron spin structure function $g_{1}^{n}$ with a polarized ${ }^{3} \mathrm{He}$ internal target, Phys. Lett. B404, 383-389 (1997).

[Ack98] K. Ackerstaff et al. (HERMES Collaboration), The HERMES spectrometer, Nucl. Instrum. Meth. A417, 230-265 (1998).

[Ack99] K. Ackerstaff et al. (HERMES Collaboration), Flavor decomposition of the polarized quark distributions in the nucleon from inclusive and semi-inclusive deepinelastic scattering, Phys. Lett. B464, 123-134 (1999).

[Ada96] M. R. Adams et al. (E665 Collaboration), Proton and deuteron structure functions in muon scattering at $470 \mathrm{GeV}$, Phys. Rev. D54, 3006-3056 (1996).

[Ade98] B. Adeva et al. (Spin Muon Collaboration), A next-to-leading order QCD analysis of the spin structure function $g_{1}$, Phys. Rev. D58, 112002 (1998).

[Ade99] B. Adeva et al. (Spin Muon Collaboration), Spin asymmetries $A_{1}$ of the proton and the deuteron in the low $x$ and low $Q^{2}$ region from polarized high energy muon scattering, Phys. Rev. D60, 072004 (1999).

[Adl01] C. Adloff et al. (H1 Collaboration), Deep-inelastic inclusive e p scattering at low $x$ and a determination of $\alpha_{s}$, Eur. Phys. J. C21, 33-61 (2001).

[Adl03] C. Adloff et al. (H1 Collaboration), Measurement and QCD analysis of neutral and charged current cross sections at HERA, Eur. Phys. J. C30, 1-32 (2003). 
[Aid04] C. Aidala (PHENIX Collaboration), Single-spin transverse asymmetry in neutral pion and charged hadron production at PHENIX, Prepared for 12th International Workshop on Deep Inelastic Scattering (DIS 2004), Strbske Pleso, Slovakia, 14-18 Apr 2004, hep-ex/0410003.

[AirO0] A. Airapetian et al. (HERMES Collaboration), Observation of a single-spin azimuthal asymmetry in semi- inclusive pion electro-production, Phys. Rev. Lett. 84, 4047-4051 (2000).

[AirO1] A. Airapetian et al. (HERMES Collaboration), Single-spin azimuthal asymmetries in electroproduction of neutral pions in semi-inclusive deep-inelastic scattering, Phys. Rev. D64, 097101 (2001).

[AirO3] A. Airapetian et al. (HERMES Collaboration), Measurement of single-spin azimuthal asymmetries in semi-inclusive electroproduction of pions and kaons on a longitudinally polarised deuterium target, Phys. Lett. B562, 182-192 (2003).

[Air04] A. Airapetian et al. (HERMES Collaboration), The HERMES polarized hydrogen and deuterium gas target in the HERA electron storage ring, Nucl. Instrum. Meth. A540, 68-101 (2004).

[Air05a] A. Airapetian et al. (HERMES Collaboration), Quark helicity distributions in the nucleon for up, down, and strange quarks from semi-inclusive deep-inelastic scattering, Phys. Rev. D71, 012003 (2005).

[Air05b] A. Airapetian et al. (HERMES Collaboration), Single-spin asymmetries in semiinclusive deep-inelastic scattering on a transversely polarized hydrogen target, Phys. Rev. Lett. 94, 012002 (2005).

[Ako02] N. Akopov et al., The HERMES dual-radiator ring imaging Čerenkov detector, Nucl. Instrum. Meth. A479, 511-530 (2002).

[Aku98] I. Akushevich, H. Böttcher and D. Ryckbosch, RADGEN 1.0: Monte Carlo generator for radiative events in DIS on polarized and unpolarized targets, (1998), hep-ph/9906408.

[Ale05] V. Y. Alexakhin et al. (COMPASS Collaboration), First measurement of the transverse spin asymmetries of the deuteron in semi-inclusive deep inelastic scattering, Phys. Rev. Lett. 94, 202002 (2005).

[Alt77] G. Altarelli and G. Parisi, Asymptotic Freedom in Parton Language, Nucl. Phys. B 126, 298-330 (1977).

[Amr05] D. Amrath, A. Bacchetta and A. Metz, Reviewing model calculations of the Collins fragmentation function, Phys. Rev. D71, 114018 (2005).

[And83] B. Andersson, G. Gustafson, G. Ingelman and T. Sjostrand, Parton Fragmentation and String Dynamics, Phys. Rept. 97, 31-145 (1983).

[And97] B. Andersson, The Lund model, Camb. Monogr. Part. Phys. Nucl. Phys. Cosmol. 7, 1-471 (1997). 
[And01] A. Andreev et al., Multiwire proportional chambers in the HERMES experiment, Nucl. Instrum. Meth. A465, 482-497 (2001).

[Ans95] M. Anselmino, A. Efremov and E. Leader, The theory and phenomenology of polarized deep inelastic scattering, Phys. Rept. 261, 1-124 (1995).

[Ans05] M. Anselmino et al., The role of Cahn and Sivers effects in deep inelastic scattering, Phys. Rev. D71, 074006 (2005).

[Ant99] P. L. Anthony et al. (E155 Collaboration), Measurement of the deuteron spin structure function $g_{1}^{d}(x)$ for $1(\mathrm{GeV} / \mathrm{C})^{2}<Q^{2}<40(\mathrm{GeV} / \mathrm{C})^{2}$, Phys. Lett. B463, 339-345 (1999).

[Ant00] P. L. Anthony et al. (E155 Collaboration), Measurements of the $Q^{2}$ dependence of the proton and neutron spin structure functions $g_{1}^{p}$ and $g_{1}^{n}$, Phys. Lett. B493, 19-28 (2000).

[Ant03] P. L. Anthony et al. (E155 Collaboration), Precision measurement of the proton and deuteron spin structure functions $g_{2}$ and asymmetries $A_{2}$, Phys. Lett. B553, 18-24 (2003).

[Aok97] S. Aoki, M. Doui, T. Hatsuda and Y. Kuramashi, Tensor charge of the nucleon in lattice QCD, Phys. Rev. D56, 433-436 (1997).

[Arn97a] M. Arneodo et al. (New Muon Collaboration), Measurement of the proton and deuteron structure functions, $F_{2}^{p}$ and $F_{2}^{d}$, and of the ratio $\sigma_{L} / \sigma_{T}$, Nucl. Phys. B483, 3-43 (1997).

[Arn97b] M. Arneodo, L. Lamberti and M. Ryskin, DIPSl: A Monte Carlo generator for elastic vector meson production in charged lepton proton scattering, Comput. Phys. Commun. 100, 195-214 (1997).

[Art75] X. Artru, G. B. Yodh and G. Mennessier, Practical Theory of the Multilayered Transition Radiation Detector, Phys. Rev. D12, 1289-1306 (1975).

[Art93] X. Artru, Proposals for measuring transversity distributions in deep inelastic electron scattering and a model for E-704 asymmetries, Prepared for the 5th International Workshop on High-energy Spin Physics, Protvino, Russia, Sep 2024, 1993, hep-ph/9310323.

[Art97] X. Artru, J. Czyzewski and H. Yabuki, Single spin asymmetry in inclusive pion production, Collins effect and the string model, Z. Phys. C73, 527-534 (1997).

[Aug06] W. Augustyniak, C. A. Miller, G. Schnell, S. Yen and P. Zupranski, TMC - Vertex Reconstruction in Presence of the HERMES Transverse Target Magnet, HERMES Internal Report, 2006, in preparation.

[Ava96] H. Avakian et al., Performance of F101 radiation resistant lead glass shower counters, Nucl. Instrum. Meth. A378, 155-161 (1996).

[Ava98] H. Avakian et al., Performance of the electromagnetic calorimeter of the HERMES experiment, Nucl. Instrum. Meth. A417, 69-78 (1998). 
[Ava04a] H. Avakian and L. Elouadrhiri (CLAS Collaboration), Single spin asymmetries at CLAS, AIP Conf. Proc. 698, 612-616 (2004).

[Ava04b] H. Avakian et al. (CLAS Collaboration), Measurement of beam-spin asymmetries for deep inelastic pi+ electroproduction, Phys. Rev. D69, 112004 (2004).

[Ava05] H. Avakian, P. Bosted, V. Burkert and L. Elouadrhiri (CLAS Collaboration), New results on SIDIS SSA from Jefferson Lab, Prepared for the 13th International Workshop on Deep-inelastic Scattering (DIS 2005), Madison, Wisconsin, USA, 27 Apr - 1 May 2005, nucl-ex/0509032.

[Ave04a] E. Avetisyan, A. Rostomyan and A. Ivanilov (HERMES Collaboration), Beam-spin azimuthal asymmetries in pion electroproduction at HERMES, Prepared for the 12th International Workshop on Deep-inelastic Scattering (DIS 2004), Strbske Pleso, Slovakia, 14-18 Apr 2004, hep-ex/0408002.

[Ave04b] E. Avetysian, New cluster energy calibration, December 2004, Talk given at the HERMES Collaboration Meeting, DESY, Hamburg, Germany.

[Bac00] A. Bacchetta, M. Boglione, A. Henneman and P. J. Mulders, Bounds on transverse momentum dependent distribution and fragmentation functions, Phys. Rev. Lett. 85, 712-715 (2000).

[Bac01] A. Bacchetta, R. Kundu, A. Metz and P. J. Mulders, The Collins fragmentation function: A simple model calculation, Phys. Lett. B506, 155-160 (2001).

[Bac02a] A. Bacchetta, R. Kundu, A. Metz and P. J. Mulders, Estimate of the Collins fragmentation function in a chiral invariant approach, Phys. Rev. D65, 094021 (2002).

[Bac02b] A. Bacchetta, Probing the transverse spin of quarks in deep inelastic scattering, PhD thesis, Vrije Universiteit Amsterdam, 2002, hep-ph/0212025.

[Bac03a] A. Bacchetta, A. Metz and J.-J. Yang, Collins fragmentation function from gluon rescattering, Phys. Lett. B574, 225-231 (2003).

[Bac03b] A. Bacchetta and M. Radici, Partial-wave analysis of two-hadron fragmentation functions, Phys. Rev. D67, 094002 (2003).

[Bac04a] A. Bacchetta, U. D’Alesio, M. Diehl and C. A. Miller, Single-spin asymmetries: The Trento conventions, Phys. Rev. D70, 117504 (2004).

[Bac04b] A. Bacchetta et al., On the way to a decomposition of the flavor dependent Sivers functions: Transverse asymmetries, the decomposition and the transverse MC generator GMC_TRANS, HERMES Internal Report, 2004, HERMES-04039.

[Bac04c] A. Bacchetta, P. J. Mulders and F. Pijlman, New observables in longitudinal single-spin asymmetries in semi-inclusive DIS, Phys. Lett. B595, 309-317 (2004). 
[Bac04d] A. Bacchetta and M. Radici, Single-spin asymmetries with two-hadron fragmentation functions, Prepared for the 12th International Workshop on Deepinelastic Scattering (DIS 2004), Strbske Pleso, Slovakia, 14-18 Apr 2004, hep$\mathrm{ph} / 0407345$.

[Bac04e] A. Bacchetta, A. Schäfer and J.-J. Yang, Sivers function in a spectator model with axial-vector diquarks, Phys. Lett. B578, 109-118 (2004).

[Bac06] A. Bacchetta, All cross sections for SIDIS, 2006, in preparation.

[Bar93] D. P. Barber et al., The HERA polarimeter and the first observation of electron spin polarization at HERA, Nucl. Instrum. Meth. A329, 79-111 (1993).

[Bar03] V. Barone and P. G. Ratcliffe, Transverse Spin Physics, World Scientific, New Jersey, London, Singapore, Hong Kong, 2003.

[Bar05] V. Barone et al. (PAX Collaboration), Antiproton proton scattering experiments with polarization, May 2005, hep-ex/0505054, Technical Proposal.

[Bau02] C. Baumgarten et al., An atomic beam polarimeter to measure the nuclear polarization in the HERMES gaseous polarized hydrogen and deuterium target, Nucl. Instrum. Meth. A482, 606-618 (2002).

[Bau03a] C. Baumgarten et al., A gas analyzer for the internal polarized target of the HERMES experiment, Nucl. Instrum. Meth. A508, 268-275 (2003).

[Bau03b] C. Baumgarten et al., The storage cell of the polarized H/D internal gas target of the HERMES experiment at HERA, Nucl. Instrum. Meth. A496, 277-285 (2003).

[Bec00] M. Beckmann, Extraction of polarised quark distributions of the nucleon from deep inelastic scattering at the HERMES experiment, PhD thesis, AlbertLudwigs-Universität Freiburg, 2000, DESY-THESIS-2000-029.

[Bec02] M. Beckmann et al., The longitudinal polarimeter at HERA, Nucl. Instrum. Meth. A479, 334-348 (2002).

[Bec03] M. Beckmann, Target polarization values and their uncertainties used by the $\Delta q$ analysis group for the 1996 to 2000 running periods, HERMES Internal Report, 2003, HERMES-03-005.

[Ben89] A. C. Benvenuti et al. (BCDMS Collaboration), A High Statistics Measurement of the Proton Structure Functions $F_{2}\left(x, Q^{2}\right)$ and $R$ from Deep Inelastic Muon Scattering at High $Q^{2}$, Phys. Lett. B223, 485-489 (1989).

[Ben01] T. Benisch et al., The luminosity monitor of the HERMES experiment at DESY, Nucl. Instrum. Meth. A471, 314-324 (2001).

[Ber98] S. Bernreuther et al., The HERMES back drift chambers, Nucl. Instrum. Meth. A4 16, 45-58 (1998).

[Bjo69] J. D. Bjorken, Asymptotic Sum Rules at Infinite Momentum, Phys. Rev. 179, 1547-1553 (1969). 
[Blo69] E. D. Bloom et al., HIGH-ENERGY INELASTIC e p SCATTERING AT 6-degrees AND 10- degrees, Phys. Rev. Lett. 23, 930-934 (1969).

[Blü02] J. Blümlein and H. Böttcher, QCD analysis of polarized deep inelastic scattering data and parton distributions, Nucl. Phys. B636, 225-263 (2002).

[Boe97] D. Boer, R. Jakob and P. J. Mulders, Asymmetries in polarized hadron production in e+ e- annihilation up to order 1/Q. Nucl. Phys. B504, 345-380 (1997).

[Boe98] D. Boer and P. J. Mulders, Time-reversal odd distribution functions in leptoproduction, Phys. Rev. D57, 5780-5786 (1998).

[Boe00] D. Boer and P. J. Mulders, Color gauge invariance in the Drell-Yan process, Nucl. Phys. B569, 505-526 (2000).

[Boe03] D. Boer, P. J. Mulders and F. Pijlman, Universality of T-odd effects in single spin and azimuthal asymmetries, Nucl. Phys. B667, 201-241 (2003).

[Boe04] D. Boer and W. Vogelsang, Asymmetric jet correlations in p p(pol.) scattering, Phys. Rev. D69, 094025 (2004).

[Bom04] C. J. Bomhof, P. J. Mulders and F. Pijlman, Gauge link structure in quark quark correlators in hard processes, Phys. Lett. B596, 277-286 (2004).

[Bra99] A. Bravar (Spin Muon Collaboration), Hadron Azimuthal Distributions and Transverse Spin Asymmetries in DIS of Leptons off Transversely Polarized Targets from SMC, Nucl. Phys. Proc. Suppl. 79, 520-522 (1999).

[Bra01] J. T. Brack et al., The HERMES forward tracking chambers: Construction, operation, and aging effects, Nucl. Instrum. Meth. A469, 47-54 (2001).

[Bra05] F. Bradamante (COMPASS Collaboration), Spin physics at COMPASS, Prog. Part. Nucl. Phys. 55, 270-280 (2005).

[Bre69] M. Breidenbach et al., Observed Behavior of Highly Inelastic Electron - Proton Scattering, Phys. Rev. Lett. 23, 935-939 (1969).

[Bro02] S. J. Brodsky, D. S. Hwang and I. Schmidt, Final-state interactions and singlespin asymmetries in semi-inclusive deep inelastic scattering, Phys. Lett. B530, 99-107 (2002).

[Brü03] A. Brüll and A. Kisselev, Top/bottom misalignment for 1996-2003 data, Mailing list: offline-list@hermes.le.desy.de, June 9, 2003.

[BurO2] M. Burkardt, Impact parameter dependent parton distributions and transverse single spin asymmetries, Phys. Rev. D66, 114005 (2002).

[Bur04] M. Burkardt, Quark correlations and single spin asymmetries, Phys. Rev. D69, 057501 (2004).

[Cah78] R. N. Cahn, Azimuthal Dependence in Leptoproduction: A Simple Parton Model Calculation, Phys. Lett. B78, 269-273 (1978). 
[Cal69] C. G. Callan Jr. and D. J. Gross, High-energy electroproduction and the constitution of the electric current, Phys. Rev. Lett. 22, 156-159 (1969).

[CER93a] CERN Computing and Network Division, GEANT - Detector Description and Simulation Tool, 1993, CERN Program Library Long Writeup W5013.

[CER93b] CERN Programming Techniques Group, ADAMO - Entity-Relationship Programming System, 1993, Users Guide, Version 3.3.

[Che01] S. Chekanov et al. (ZEUS Collaboration), Measurement of the neutral current cross section and $F_{2}$ structure function for deep inelastic e+ $p$ scattering at HERA, Eur. Phys. J. C21, 443-471 (2001).

[Clo79] F. E. Close, The quark parton model, Rep. Prog. Phys. 42, 1285-1335 (1979).

[Col88] J. C. Collins, D. E. Soper and G. Sterman, Factorization of Hard Processes in QCD. Adv. Ser. Direct. High Energy Phys. 5, 1-91 (1988).

[Col93] J. C. Collins, Fragmentation of transversely polarized quarks probed in transverse momentum distributions, Nucl. Phys. B396, 161-182 (1993).

[Col94] J. C. Collins, S. F. Heppelmann and G. A. Ladinsky, Measuring transversity densities in singly polarized hadron-hadron and lepton-hadron collisions, Nucl. Phys.

B420, 565-582 (1994).

[Col02] J. C. Collins, Leading-twist single-transverse-spin asymmetries: Drell-Yan and deep-inelastic scattering, Phys. Lett. B536, 43-48 (2002).

[Col04] J. C. Collins and A. Metz, Universality of soft and collinear factors in hardscattering factorization, Phys. Rev. Lett. 93, 252001 (2004).

[Die05a] M. Diehl and S. Sapeta, On the analysis of lepton scattering on longitudinally or transversely polarized protons, Eur. Phys. J. C41, 515-533 (2005).

[Die05b] M. Diehl, private communication, 2005.

[Die05c] M. Diehl and A. Bacchetta, Question: semi-inclusive isospin symmetry, Mailing list: draft_61@hermes.le.desy.de, January 27, 2005.

[Dok77] Y. L. Dokshitzer, Calculation of the Structure Functions for Deep Inelastic Scattering and e+ e- Annihilation by Perturbation Theory in Quantum Chromodynamics, Sov. Phys. JETP 46, 641-653 (1977).

[Dür95] M. Düren, The HERMES experiment: From the design to the first results, Habilitation thesis, Friedrich-Alexander-Universität Erlangen-Nürnberg, 1995, HERMES95-002.

[Dür98] M. Düren, M. Ruh and B. Tipton, Quark Spin Flavor Decomposition - Cross Checks and First Moments, HERMES Internal Report, 1998, HERMES-98-018.

[Efr99] A. V. Efremov, O. G. Smirnova and L. G. Tkachev, Study of T-odd quark fragmentation function in $Z_{0} \rightarrow$ 2-jet decay, Nucl. Phys. Proc. Suppl. 74, 49-52 (1999). 
[Efr05] A. V. Efremov, K. Goeke and P. Schweitzer, Transversity distribution in spin asymmetries in semi-inclusive DIS and in the Drell-Yan process, Czech. J. Phys. 55, A189-A208 (2005).

[Eid04] S. Eidelman et al. (Particle Data Group), Review of particle physics, Phys. Lett. B592, 1 (2004).

[Ell83] R. K. Ellis, W. Furmanski and R. Petronzio, Unraveling Higher Twists, Nucl. Phys. B212, 29-98 (1983).

[ElsO1] U. Elschenbroich, Bhabha scattering and luminosity measurement at the HERMES experiment, Diploma thesis, Heinrich-Heine-Universität Düsseldorf, 2001, HERMES-01-044.

[Els02] U. Elschenbroich, Analysis of Luminosity Monitor Data for Different Years, HERMES Internal Report, 2002, HERMES-02-013.

[Els03] U. Elschenbroich, G. Schnell and R.-C. Seidl, Single-Spin Azimuthal Asymmetries in SIDIS off a Transversely Polarized Proton Target - MC Studies, HERMES Internal Report, 2003, HERMES-03-007.

[Ely01] J. Ely, Hermes Calorimeter Position Reconstruction Study, HERMES Internal Report, 2001, HERMES-01-056.

[Fat04] R. Fatemi (STAR Collaboration), Constraining the Sivers functions using transverse spin asymmetries at STAR, Prepared for 12th International Workshop on Deep Inelastic Scattering (DIS 2004), Strbske Pleso, Slovakia, 14-18 Apr 2004.

[Fra74] H. Fraas, Vector meson production by Inelastic Scattering of Polarized Electrons off Polarized Nucleons, Annals of Physics 87, 417-456 (1974).

[Gam03] L. P. Gamberg, G. R. Goldstein and K. A. Oganessyan, A mechanism for the T-odd pion fragmentation function, Phys. Rev. D68, 051501 (2003).

[Gel64] M. Gell-Mann, A schematic model of baryons and mesons, Phys. Lett. 8, 214-215 (1964).

[Glü96] M. Glück, E. Reya, M. Stratmann and W. Vogelsang, Next-to-leading order radiative parton model analysis of polarized deep inelastic lepton - nucleon scattering, Phys. Rev. D53, 4775-4786 (1996).

[Glü01] M. Glück, E. Reya, M. Stratmann and W. Vogelsang, Models for the polarized parton distributions of the nucleon, Phys. Rev. D63, 094005 (2001).

[Goe01] K. Goeke, M. V. Polyakov and M. Vanderhaeghen, Hard exclusive reactions and the structure of hadrons, Prog. Part. Nucl. Phys. 47, 401-515 (2001).

[Gri72] V. N. Gribov and L. N. Lipatov, e+ e-pair annihilation and deep inelastic e $p$ scattering in perturbation theory, Sov. J. Nucl. Phys. 15, 675-684 (1972).

[Has03] D. Hasch for the Hermes Calorimeter Group, Effect of a Dead Calo Block in the Analysis of Semi-Inclusive Asymmetries, Mailing list: offlinelist@hermes.le.desy.de, April 23, 2003. 
[HDQa] HERMES data quality group, DIS Statistics of Different Years, http://wwwhermes.desy.de/groups/daqlgrp/OFFLINE_DQ/UDST/general/docu/DISstats.html.

[HDQb] HERMES data quality group, Offline Data Quality - $\mu$ DST Productions, http://www-hermes.desy.de/groups/daglgrp/OFFLINE_DQ/uDST/index.html.

[Hil05] A. Hillenbrand, Measurement and Simulation of the Fragmentation Process at HERMES, PhD thesis, Friedrich-Alexander-Universität Erlangen-Nürnberg, 2005, DESY-THESIS-2005-035.

[Hom04] B. Hommez, H. Jackson, R. Kaiser and Y. Miyachi, Using the RICH detector for Physics Analysis, December 2004, http://www-hermes.desy.de/groups/richgrp/ rich/richanalysis/index.html.

[Idi04] A. Idilbi, X. Ji, J.-P. Ma and F. Yuan, Collins-Soper equation for the energy evolution of transverse-momentum and spin dependent parton distributions, Phys. Rev. D70, 074021 (2004).

[Ing97] G. Ingelman, A. Edin and J. Rathsman, LEPTO 6.5 - A Monte Carlo Generator for Deep Inelastic Lepton-Nucleon Scattering, Comput. Phys. Commun. 101, 108-134 (1997).

[Jaf93] R. L. Jaffe and X.-D. Ji, Novel quark fragmentation functions and the nucleon's transversity distribution, Phys. Rev. Lett. 71, 2547-2550 (1993).

[Jaf96] R. L. Jaffe, Spin, twist and hadron structure in deep inelastic processes, Talk given at Ettore Majorana International School of Nucleon Structure: 1st Course: The Spin Structure of the Nucleon, Erice, Italy, 3-10 Aug 1995, hep-ph/9602236.

[Jaf98] R. L. Jaffe, X.-M. Jin and J. Tang, Interference fragmentation functions and the nucleon's transversity, Phys. Rev. Lett. 80, 1166-1169 (1998).

[Jam75] F. James and M. Roos, 'MINUIT'A System for Function Minimization and Analysis of the Parameter Errors and Correlations, Comput. Phys. Commun. 10, 343-367 (1975).

[Ji04] X. Ji, J.-P. Ma and F. Yuan, QCD factorization for spin-dependent cross sections in DIS and Drell-Yan processes at low transverse momentum, Phys. Lett. B597, 299-308 (2004).

[Ji05] X. Ji, J.-P. Ma and F. Yuan, QCD factorization for semi-inclusive deep-inelastic scattering at low transverse momentum, Phys. Rev. D71, 034005 (2005).

[Jun00] P. Jung, Pion-kaon separation with the HERMES RICH detector, Diploma thesis, Humboldt Universität Berlin, 2000, HERMES-01-025.

[Kis05] A. Kisselev, Alignment at HERMES: progress report, June 2005, Talk given at the HERMES Collaboration Meeting, DESY, Hamburg, Germany.

[Kow87] S. B. Kowalski and H. A. Enge, The ion-optical program raytrace, Nucl. Instrum. Meth. A258, 407 (1987). 
[Kre00] S. Kretzer, Fragmentation functions from flavour-inclusive and flavour-tagged e+ e- annihilations, Phys. Rev. D62, 054001 (2000).

[Lie04] P. Liebing, Can the gluon polarization in the nucleon be extracted from HERMES data on single high-p $p_{T}$ hadrons?, PhD thesis, Universität Hamburg, 2004, DESY-THESIS-2004-036.

[Lip75] L. N. Lipatov, The parton model and perturbation theory, Sov. J. Nucl. Phys. 20, 94-102 (1975).

[Ma02] B.-Q. Ma, I. Schmidt and J.-J. Yang, Azimuthal spin asymmetries of pion electroproduction, Phys. Rev. D65, 034010 (2002).

[Mai05] B. Maiheu, A. Hillenbrand and E. Aschenauer, Monte Carlo study of the influence of possible moments on the HERMES acceptance function for hadrons, HERMES Internal Report, 2005, HERMES-05-004.

[Mak03a] N. Makins, "Back-of-the-Envelope" Interpretation of our First Transversity Data, HERMES Internal Report, 2003, HERMES-03-010.

[Mak03b] N. Makins, GMC_TRANS Manual, HERMES Internal Report, 2003, HERMES-03060.

[Mal04] G. K. Mallot (COMPASS Collaboration), The COMPASS spectrometer at CERN, Nucl. Instrum. Meth. A518, 121-124 (2004).

[Men01] F. M. Menden, Determination of the gluon polarization in the nucleon, PhD thesis, Albert-Ludwigs-Universität Freiburg, 2001, DESY-THESIS-2001-060.

[Met02] A. Metz, Gluon-exchange in spin-dependent fragmentation, Phys. Lett. B549, 139-145 (2002).

[Mil04] C. A. Miller, Effects of $x y^{2} /[A ; B](x, y)$ weighting, Mailing list: transversitylist@hermes.le.desy.de, February 25, 2004.

[Mil05a] C. A. Miller, Extracting Fourier harmonics from sparse distributions, HERMES Internal Report, 2005, HERMES-05-006.

[Mil05b] C. A. Miller, Maximum Likelihood fits of azimuthal Fourier amplitudes, October 2005, Talk given at the HERMES Collaboration Meeting, DESY, Hamburg, Germany.

[Mul96] P. J. Mulders and R. D. Tangerman, The complete tree-level result up to order $1 / Q$ for polarized deep-inelastic leptoproduction, Nucl. Phys. B461, 197-237 (1996).

[NasO3] A. Nass et al., The HERMES polarized atomic beam source, Nucl. Instrum. Meth. A505, 633-644 (2003).

[Nic98] J. M. Niczyporuk and E. E. W. Bruins, Quark flavor tagging in polarized hadronic processes, Phys. Rev. D58, 091501 (1998). 
[Pij04] F. Pijlman, Factorization and universality in azimuthal asymmetries, Presented at 16th International Spin Physics Symposium (SPIN 2004), Trieste, Italy, 10-16 Oct 2004, hep-ph/0411307.

[Pre94] W. H. Press, S. A. Teukolsky, W. T. Vetterling and B. P. Flannery, Numerical Recipes in C, The Art of Scientific Computing, Cambridge University Press, Cambridge, New York, Melbourne, second edition, 1994.

[Pro99] A. Proskuryakov (H1 and ZEUS Collaborations), Light vector meson production at HERA, Prepared for the Ringberg Workshop: New Trends in HERA Physics 1999, Ringberg Castle, Tegernsee, Germany, 30 May - 4 Jun 1999.

[Pro05] A. Prokudin, private communication, October 2005.

[Pum02] J. Pumplin et al., New generation of parton distributions with uncertainties from global QCD analysis, JHEP 07, 012 (2002).

[Rad05] M. Radici, Alternative approaches to transversity: How convenient and feasible are they?, Prepared for the International Workshop on Transverse Polarisation Phenomena in Hard Processes (Transversity 2005), Como, Italy, 7-10 Sep 2005, hep-ph/0510165.

[Ral79] J. P. Ralston and D. E. Soper, Production of Dimuons from High-Energy Polarized Proton Proton Collisions, Nucl. Phys. B 152, 109-124 (1979).

[Reg05] D. Reggiani (HERMES Collaboration), Precise Results on $g_{1}^{p}$ and $g_{1}^{d}$ and First Measurements of the Tensor Structure Function $b_{1}^{d}$ with the HERMES-Experiment, Prepared for the 13th International Workshop on Deep-inelastic Scattering (DIS 2005), Madison, Wisconsin, USA, 27 Apr - 1 May 2005.

[Rob90] R. G. Roberts, The Structure of the proton: Deep inelastic scattering, Cambridge University Press, Cambridge, 1990.

[Ros66] M. Ross and L. Stodolsky, Photon dissociation model for vector meson photoproduction, Phys. Rev. 149, 1172-1181 (1966).

[Sak69] J. J. Sakurai, Vector meson dominance and high-energy electron proton inelastic scattering, Phys. Rev. Lett. 22, 981-984 (1969).

[Sak72] J. J. Sakurai and D. Schildknecht, Generalized vector dominance and inelastic electron - proton scattering, Phys. Lett. B40, 121-126 (1972).

[Sch70] K. Schilling, P. Seyboth and G. E. Wolf, On the Analysis of Vector Meson Production by Polarized Photons, Nucl. Phys. B15, 397-412 (1970).

[Siv90] D. W. Sivers, Single Spin Production Asymmetries from the Hard Scattering of Point-like Constituents, Phys. Rev. D41, 83-90 (1990).

[Sjö94] T. Sjöstrand, High-energy physics event generation with PYTHIA 5.7 and JETSET 7.4, Comput. Phys. Commun. 82, 74-90 (1994). 
[Sjö03] T. Sjöstrand, L. Lonnblad, S. Mrenna and P. Skands, PYTHIA 6.3: Physics and manual, (2003), hep-ph/0308153.

[Sof95] J. Soffer, Positivity constraints for spin dependent parton distributions, Phys. Rev. Lett. 74, 1292-1294 (1995).

[Sok64] A. A. Sokolov and I. M. Ternov, On polarization and spin effects in the theory of synchrotron radiation, Phys. Dokl. 8, 1203-1205 (1964).

[Tai06] P. Tait, Beam-Induced Depolarisation at the HERMES Transversely Polarised Hydrogen Target, PhD thesis, Friedrich-Alexander-Universität Erlangen-Nürnberg, 2006, in preparation.

[Tyt01] M. Tytgat, Diffractive production of $\rho^{0}$ and $\omega$ vector mesons at HERMES, PhD thesis, Universiteit Gent, 2001, DESY-THESIS-2001-018.

[vaN05] P. B. van der Nat (HERMES Collaboration), First measurement of interference fragmentation on a transversely polarized hydrogen target, Prepared for the 13th International Workshop on Deep-inelastic Scattering (DIS 2005), Madison, Wisconsin, USA, 27 Apr - 1 May 2005.

[Vog05] W. Vogelsang and F. Yuan, Single-transverse spin asymmetries: From DIS to hadronic collisions, Phys. Rev. D72, 054028 (2005).

[Wak01] M. Wakamatsu, Chiral-odd distribution functions in the chiral quark soliton model, Phys. Lett. B509, 59-68 (2001).

[Wan96] W. Wander, Reconstruction of high-energy scattering events in the HERMES experiment, PhD thesis, Friedrich-Alexander-Universität Erlangen-Nürnberg, 1996, HERMES-97-031.

[Wan04] S. Wang, Improvements of the HERMES Transverse Polarized Hydrogen Target and Calculations of the Sextupole Magnet, PhD thesis, China Institute of Atomic Energy, 2004, HERMES-04-056.

[Wei97] T. Weiland et al., MAFIA Version 4, AIP Conf. Proc. 391, 65-70 (1997), Prepared for CAP 96: The 1996 Computational Accelerator Physics Conference, Williamsburg, Virginia, USA 24-27 Sep 1996.

[Wen03] J. Wendland, Polarized parton distributions measured at the HERMES experiment, PhD thesis, Simon Fraser University, 2003, DESY-THESIS-2003-032.

[Whi92] L. W. Whitlow, E. M. Riordan, S. Dasu, S. Rock and A. Bodek, Precise measurements of the proton and deuteron structure functions from a global analysis of the SLAC deep inelastic electron scattering cross-sections, Phys. Lett. B282, 475-482 (1992).

[Zwe64] G. Zweig, An SU(3) Model for Strong Interaction Symmetry and its Breaking. II, 1964, CERN-TH-412. 


\section{Samenvatting}

In het onderzoek naar de structuur van nucleonen is de vraag naar de oorsprong van de spin van deze deeltjes nog steeds niet volledig opgelost. De spinbijdrage van de partonen, de nucleonconstituenten, wordt gegeven door de heliciteitsdistributie. Deze beschrijft het verschil in het aantal partonen met een spin parallel en antiparallel met de nucleonspin in een referentiestelsel waarin het nucleon een oneindig grote impuls heeft (infinite momentum frame) en waarbij de richting van zijn spin longitudinaal is t.o.v. de bewegingsrichting. In het naïeve quark-parton model worden de partonen geïdentificeerd met de quarks, waarbij hun spins samengevoegd de totale nucleonspin opleveren. Experimenten in CERN en SLAC leverden echter totale quark heliciteitsdistributies op kleiner dan 30\%. Quantum chromodynamica (QCD) beschrijft de interactie tussen quarks via een uitwisseling van gluonen, de ijkbosonen van de sterke wisselwerking. Deze gluonen bedden de quarks in het nucleon in een zee van virtuele quark-antiquark paren en gluonen. Naast de spin van de quarks kunnen dus ook de spin van de gluonen evenals het baanimpulsmoment van de quarks en gluonen een bijdrage leveren tot de nucleonspin. Tot op de dag van vandaag gaven zowel indirecte als directe metingen slechts vage informatie over de grootte van de gluon heliciteitsdistributie. Daarnaast is een directe meting van de baanimpulsmomenten tot op heden onmogelijk gebleken.

Niet enkel de heliciteitsdistributie maar ook de transversale spin van de constituenten kan informatie over de nucleonstructuur geven. In tegenstelling tot de heliciteitsdistributie is de transversale distributie gedefiniëerd voor een nucleonspin die transversaal is t.o.v. zijn bewegingsrichting. Net zoals het geval is voor een longitudinale nucleonspin stelt de transversale distributie het verschil voor van het aantal partonen met spin parallel of antiparallel met de nucleonspin. De heliciteits- en transversale distributies lijken gelijkaardig te zijn, maar hun interpretatie is slechts geldig in het infinite momentum frame waarin de richting van de impuls van het nucleon de rotatiesymmetrie breekt. Bovendien verbiedt behoud van heliciteit bijdragen van gluonen tot de transversale distributie van het nucleon.

Naast de heliciteit en transversale distributiefunctie bestaan in leading twist nog zes andere quarkdistributiefuncties voor elke quarksmaak. Bij integratie over de intrinsieke quark transversale impulsen blijken echter enkel de ongepolariseerde distributiefunctie, die de quarkdichtheid in het nucleon voorstelt, en de heliciteit en transversale distributies niet te verdwijnen. Deze drie distributies zijn evenwaardig en vormen de fundamentele vrijheidsgraden van de quarks in het nucleon. De transversale distributiefunctie werd eerst vermeld in 1979, maar werd tot op heden nooit gemeten. De reden hiervoor is te vinden in haar chiraal-oneven aard die vereist dat ze gecombineerd wordt met een ander chiraal-oneven object in processen die de heliciteit behouden. Eén mogelijkheid 
om toegang tot transversaliteit te krijgen is het semi-inclusief diep-inelastische verstrooiingsproces waarbij de distributiefuncties gecombineerd worden met fragmentatiefuncties. De combinatie van de transversale distributie met de chiraal-oneven Collins fragmentatiefunctie veroorzaakt een links-rechts asymmetrie in de werkzame doorsnede voor een transversaal gepolariseerd nucleon. De interesse in deze asymmetrie groeide toen een allereerste meting in het SMC-experiment in 1999 een positieve asymmetrie opleverde voor positieve hadronen geproduceerd in diep-inelastische verstrooiing op transversaal gepolariseerde waterstof trefkernen. Semi-inclusieve diep-inelastische verstrooiing laat bovendien toe nog een andere distributiefunctie te meten, nl. de Sivers functie die de correlatie van quark transversale impulsen met de transversale spin van het nucleon beschrijft. De motivatie voor het bestuderen van de Sivers functie wordt gevormd door het feit dat haar bestaan samenhangt met de eis voor het niet nul zijn van het baanimpulsmoment van de quarks. Net zoals er een combinatie optreedt van de transversale en Collins functies, verschijnt de Sivers functie samen met de ongepolariseerde fragmentatiefunctie in de werkzame doorsnede voor een transversaal gepolariseerd nucleon en produceert zij ook een links-rechts asymmetrie.

Het diep-inelastische verstrooiingsexperiment HERMES (HERa MEasurement of Spin) maakt gebruik van de leptonenbundel van HERA (Hadron-Elektron-RingAnlage) te DESY (Deutsches Elektronen-SYnchrotron) in Hamburg te Duitsland. In 2001 werd een transversaal gepolariseerde waterstof target geïnstalleerd die het mogelijk maakte om de individuele Collins en Sivers asymmetrie amplitudes te meten. In dit eindwerk werden de metingen met deze transversaal gepolariseerde target genomen tijdens de periode van 2002 tot 2004 geanalyseerd. Ongewogen en $P_{h \perp}$-gewogen Collins en Sivers amplitudes werden bepaald voor geladen en neutrale pionen en voor geladen kaonen via een twee-dimensionale fit aan de azimutale single-spin asymmetrie. Om verschillende invloeden van b.v. de HERMES spectrometer en target of de HERA bundel te controleren, werden diverse systematische studies uitgevoerd met de HERMES metingen. Zo werd de contributie tot de systematische onzekerheid te wijten aan de hadron identificatie door de RICH detector en aan het ongepolariseerde cosinusmoment in de noemer van de asymmetrie bepaald. Andere bijdragen tot de systematische onzekerheid werden geschat met behulp van data van ongepolariseerde Monte Carlo generatoren. Collins en Sivers asymmetrieën werden geïmplementeerd in de Monte Carlo data door het wegen van de evenementen afhankelijk van een willekeurig toegekende target spintoestand. De schatting van de systematische onzekerheid op de ongewogen amplitudes omvat o.a. effecten van de extractiemethode, de spectrometeracceptantie en de eindige detectorresoluties. De correlatie van de azimutale asymmetrie met de intrinsieke quark transversale impulsen werd gecontroleerd met behulp van een nieuw ontworpen Monte Carlo generator gmc_trans, die azimutale verdelingen te wijten aan de transversale impulsafhankelijkheid van quark distributie- en fragmentatiefuncties genereert. De geïmplementeerde ongewogen amplitudes konden goed gereconstrueerd worden, terwijl $P_{h \perp}$-gewogen amplitudes sterk beïnvloed werden door de onvolledige integratie over $P_{h \perp}$ in de HERMES acceptantie.

De geanalyseerde datasets van semi-inclusieve mesonen bevatten een bijdrage van mesonen afkomstig van het verval van vector mesonen geproduceerd in exclusieve reacties. Vanuit het standpunt van de theorie is het alsnog onduidelijk of deze bijdrage al dan niet als achtergrond beschouwd moet worden. Met behulp van de PYTHIA Monte 
Carlo generator met inbegrip van exclusieve vector mesonproductie werden de fracties van mesonen afkomstig van exclusieve vector mesonen in de verschillende hadron data sets bepaald. De grootste fracties - tot $10 \%$ in het semi-inclusief gebied, dat in de analyse gebruikt werd - werden gevonden in de data sets van de geladen pionen. Deze fracties die gedomineerd worden door verval van exclusieve $\rho^{0}$-mesonen, kunnen zelfs toenemen tot ongeveer $50 \%$ voor $z>0.7$ buiten het semi-inclusief gebied. Voor neutrale pionen en geladen kaonen zijn de bijdragen kleiner dan $4 \%$ in het gebied $0.2<z<$ 0.7. Vervalmesonen van exclusieve vector mesonen verstoren niet alleen de geëxtraheerde amplitudes, ze kunnen eveneens Collins- en Siversachtige amplitudes vertonen. Deze amplitudes kunnen ofwel afkomstig zijn van een vector meson amplitude die getransfereerd werd naar de vervalmesonen of ze kunnen tot stand gekomen zijn tijdens het vervalproces. Met behulp van de PYTHIA en DIPSI Monte Carlo generatoren werden transfercoëfficiënten voor geladen pionen komende van exclusieve $\rho^{0}$-vervallen en voor neutrale pionen van exclusieve $\omega$-vervallen bepaald. De statistische significantie van de HERMES data lieten bovendien een extractie van de Collins- en Siversachtige amplitudes voor exclusieve $\rho^{0}$-mesonen toe, die uiteindelijk consistent met nul bleken. Deze amplitudes werden niet enkel voor de typische kinematica van het $\rho^{0}$ vector meson zelf bepaald, maar ook voor zijn vervalpionen. Net zoals voor de vector mesonen werden deze amplitudes met grootte-orde van enkele procenten consistent met nul bevonden voor zowel positieve als negatieve pionen. Voor de schatting van de amplitudes die verworven worden tijdens het vervalproces werden vervalhoekdistributies van $\rho^{0}$-mesonen onderzocht. Er werd aangetoond dat de verworven Collins and Sivers amplitudes klein mogen verondersteld worden in het semi-inclusieve gebied. Deze conclusie wordt ondersteund door de dominantie van natuurlijke pariteitsuitwisseling en de kleine schending van behoud van $s$-kanaalsheliciteit die gevonden werd in de HERMES data. Een methode om de bijdrage van exclusieve $\rho^{0}$ en $\omega$-mesonen d.m.v. reactiekinematica te onderdrukken werd onderzocht, waarbij aangetoond werd dat dit soort techniek niet werkt, zelfs wanneer de meetgegevens van HERMES van het jaar 2005 in beschouwing genomen worden. Daarnaast werden amplitudes bepaald uit de azimutale asymmetrie van het verschil in opbrengst tussen positieve en negatieve pionen, waarin geen bijdrage van exclusieve vector mesonen optreedt. Deze significant positieve amplitudes tonen aan dat de Collins en Sivers amplitudes van de semi-inclusieve pion data sets zeker niet in hoofdzaak te wijten zijn aan vervalpionen van exclusieve vector mesonen.

In 1996 en 1997 verrichtte het HERMES experiment metingen met een longitudinaal gepolariseerde waterstof target. De spins van de nucleonen waren gealigneerd langs de richting van de impuls van de leptonenbundel, wat resulteerde in een kleine component van de spins transversaal t.o.v. de richting van het virtueel foton. Dit heeft tot gevolg dat de gemeten azimutale asymmetrieën naast een subleading-twist component van de werkzame doorsnede voor longitudinaal gepolariseerde nucleonen ook de Collins en Sivers amplitudes bevatten. De geëxtraheerde Collins en Sivers amplitudes van de metingen op een transversaal gepolariseerde target werden aangewend om de subleading-twist amplitudes te scheiden van de metingen op een longitudinaal gepolariseerde target. Hierbij werden significant positieve amplitudes voor positieve en neutrale pionen gevonden, hetgeen aantoont dat de interpretatie van de azimutale asymmetrieën louter in termen van leading-twist functies niet altijd afdoende is. De transversaal gepolariseerde target die aangewend werd voor de meting van de Collins en Sivers 
amplitudes, heeft naast de dominante transversale spincomponent eveneens een kleine component longitudinaal t.o.v. de richting van het virtueel foton. Er werden echter maar verwaarloosbare bijdragen van de subleading-twist component tot de gemeten Collins en Sivers amplitudes gevonden.

Uit alle ondernomen studies kan besloten worden dat de significant positieve ongewogen Sivers amplitudes voor positieve pionen en kaonen en neutrale pionen de allereerste aanduiding vormen van een niet nul zijnde $T$-odd distributiefunctie van semi-inclusieve diep-inelastische verstrooiing. Bovendien veroorzaakt het product van de $T$-odd Collins fragmentatiefunctie en de transversale distributiefunctie significant positieve (negatieve) ongewogen Collins amplitudes voor positieve (negatieve) pionen. De interpretatie van de azimutale asymmetrie amplitudes in termen van distributie- en fragmentatiefuncties laat toe een verband tussen de drie pionamplitudes af te leiden. Dit verband wordt met $2 \sigma$ geschonden voor zowel de Collins als de Sivers amplitudes. Niettemin zijn beide amplitudes sterk anti-gecorreleerd door hun simultane extractieprocedure. De som van beide amplitudes voldoet aan de relatie hetgeen aantoont dat de schending van de eerdervermelde relatie mogelijkerwijze veroorzaakt werd door een statistische fluctuatie. Via Gaussische sampling van de algemene ongewogen Collins amplitude voor de drie piontypes kan een waarschijnlijkheidsdistributie opgesteld worden voor de verhouding van de disfavoured tot de favoured Collins functie als functie van de verhouding van transversaliteit van verschillende quarksmaken. In het scenario met de hoogste waarschijnlijkheid blijkt de disfavoured Collins functie van dezelfde grootte te zijn maar met een tegengesteld teken als de favoured Collins functie. De verkregen meest waarschijnlijke verhouding van disfavoured tot favoured Collins functie kan mogelijkerwijze nuttig zijn voor het extraheren van de Collins functie uit een meting van azimutale asymmetrieën voor de productie van geladen pionparen in elektron-positron annihilatie in het BELLE experiment. Een verklaring voor het Collinseffect gebaseerd op het stringfragmentatiemodel resulteert in een positief teken van de Collins functie voor positieve pionen, hetgeen consistent is met het meetresultaat. Een allereerste extractie van de Sivers functie uit de gemeten ongewogen Sivers amplitude gaf een negatieve functie voor $u$-quarks en een positieve functie voor $d$-quarks. In een semi-klassiek model impliceert dit een positief baanimpulsmoment voor $u$-quarks en een negatief baanimpulsmoment voor $d$-quarks.

Het HERMES experiment zette zijn metingen verder tijdens het jaar 2005 en was in staat een verdubbeling te behalen van de statistische significantie van de gecombineerde data set genomen van 2002 tot 2004 . Naar de toekomst toe zal een verbeterde energiecalibratie van de calorimeter zijn resolutie verbeteren wat zal leiden tot een grotere fractie van signaal tot achtergrond evenementen in de neutrale pionidentificatie en daardoor tot een kleinere onzekerheid in de amplitudes voor neutrale pionen. Bovendien zal de hogere statistische significantie misschien toelaten om de fractie van signaal evenementen in elke azimutale bin en spintoestand te bepalen. In dat geval kan een achtergrondscorrectie gebeuren vóór de vorming van de azimutale amplitude. Voorts werd een vernieuwde amplitude extractiemethode via een unbinned maximum likelihood fit voorgesteld. Deze fit is minder gevoelig aan acceptantie-effecten en kan misschien de analyse van de $P_{h \perp}$-gewogen amplitudes toelaten. Dit zou de interpretatie in termen van distributie- en fragmentatiefuncties toestaan zonder verdere veronderstellingen over de transversale impulsdistributies binnen het nucleon. 


\section{Acknowledgements}

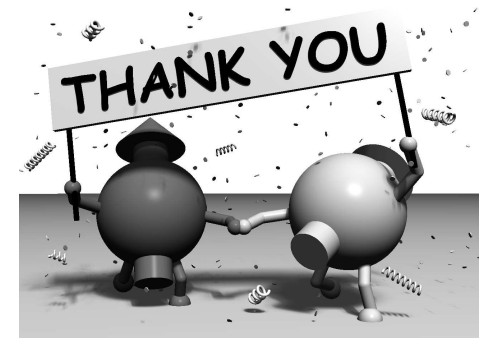

I reached the last pages of my thesis and it is time to look back and thank all the people whose help was indispensable to complete my PhD. First of all I would like to thank my promoter Dirk Ryckbosch who gave me the opportunity to work in the international environment of the DESY research facility where always people are around for fruitful (non-)physical discussions. During the numerous conferences, workshops, and summer schools all around the world which Dirk sent me to, I gained a lot of insight, not only in the interesting subject of spin physics but also in different cultures. Furthermore, Dirk gave me all the freedom in my approach of the analysis I wanted and he had always time for a discussion when I needed one.

Although I spend most of the time in Hamburg, I also made some longer and shorter visits of my home institute in Gent. The Gent groupies Arne Vandenbroucke, Bino Maiheu, Brecht Hommez, Charlotte Van Hulse, Michael Tytgat, Peter Janssens, Tiko Rostomyan, and Yves Van Haarlem showed me the nice aspects of Flanders and helped me a lot with the sometimes laborious administration. Thanks for the possibility to use your guest bed whenever I was in Gent, Bino!

I did not work alone on the still young subject of transverse momentum dependent distribution and fragmentation functions but together with Alessandro Bacchetta, Andy Miller, Francesca Giordano, Gunar Schnell, Hide Tanaka, Luciano Pappalardo, Markus Diefenthaler, Naomi Makins, Paul van der Nat, Ralf Seidl, and Tomo Kobayashi. Without their contributions to the analysis and to the development of the Monte Carlo generator and without the inspiring discussions within the transversity group, I would not have been able to address all the different aspects of the analysis and of the interpretation of the results.

Special thanks also to Achim Hillenbrand, Bino Maiheu, Delia Hasch, Elke Aschenauer, Jürgen Wendland, Marc Beckmann, Markus Diehl, Patricia Liebing, and to all the other physicists on- and off-site which I could bother with my questions concerning theory or the analysis of HERMES data. With my computing problems I could always come to Alexander Kisselev, Eduard Avetisyan, Larry Felawka, and to the other IT specialists. The effort of the secretaries Sabine Krohn, Sörne Möller, and Evelyn Olabisi should not remain unmentioned, too. It was fun to work together with the other DQ mice: Caro Riedl, Dominik Gabbert, Ingrid Gregor, and Vanessa Mexner.

To Shibata-san I would like to express my gratitude for the possibility to work for two months in his group at the Tokyo Institute of Technology. Sincere thanks not only to him but also to Miyashi-san, Tanaka-san, Kobayashi-san, Hasegawa-san, and all the other group members who-together with Gunar and Elba-helped me a lot with the organisation 
and gave me valuable cultural insights.

I am indebted to Achim Hillenbrand (my personal cab driver), Christian Vogel (my ear is not yet bleeding), Didi Zeiler, Erhard Steffens, Fidi Stinzing, Klaus Rith, Martin Raithel, Nils Pickert, Phil Tait, and the others of the Erlangen group for the granting of asylum including interesting coffee-break discussions and fiendish Sudoku fights.

Thanks a lot for the careful proofreading of this thesis, Gunar, Marc, and Markus.

Time for recreation I found during the social activities in Hamburg and on conferences, workshops, summer schools, and skiing tours with Achim, Alessandro, Alex, Ami, Andreas G., Andreas R., Andy, Antje, Arne, Beni, Bino, Caro, Charlotte, Cynthia, Davide, Delia, Dirk, Dominik, Ed, Elba, Elke, Frank, Gunar, Hide, Ingrid, Jim, Jochen, Josh, Jürgen, Larry, Matthias, Marc, Marco, Markus E., Markus D., Martin, Michiel, Mike, Naomi, Pasquale, Patricia, Paul, Phil, Ralf, Riccardo, Sörne, Tomo, Vanessa, Volker, Wouter, Yorck, Yves, and all the others I forgot to mention here. The fun we all have together gives one the motivation to work hard and long during stressful periods.

To all the people I met at DESY and all around the world in the last years: thanks a lot, vielen Dank, hartelijk bedankt, ありがとう, Dange, mille grazie, merci beaucoup, спасибо, ¿đnphuluuınıpjnı, 谢谢, dziękuję.

Meinen Eltern und meiner Schwester möchte ich für ihre Unterstützung und ihr Verständnis während der letzten Jahre danken. Auch wenn meine Aufenthalte in Mönchengladbach meistens kurz und nicht besonders regelmäßig waren, konnte ich mich bei innen immer sehr gut erholen; besonders wenn ich mich beim Abarbeiten diverser Listen mal wieder mit Problemen fern jeglicher quantemechanischer oder relativistischer Effekte befassen konnte.

Der letzte und größte Dank gilt der wichtigsten Person in meinem Leben: Marc. 